\title{
Search for R-parity Violating Supersymmetry in Multilepton Final States with the DØ Detector Dissertation
}

\author{
by \\ Daniela Käfer
}

III. Physikalisches Institut A

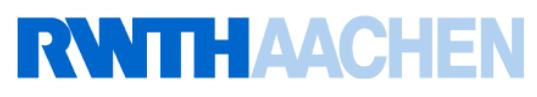

\begin{abstract}
A search for the trilepton signature $\mu \mu \ell$ (with $\ell=e$, or $\mu$ ) is presented. The results are interpreted stand-alone and in combination with two complementary searches for the trilepton signatures eel and eet in the framework of R-parity violating Supersymmetry. Pairwise, R-parity conserving production of the supersymmetric particles is assumed, followed by R-parity violating decays via an $L L \bar{E}$-operator with one dominant coupling $\lambda_{122}$. An $L L \bar{E}$ operator couples two weak isospin doublet and one singlet (s)lepton fields and thus violates lepton number conservation.

The data, corresponding to an integrated luminosity of $\int \mathcal{L} d t=360 \pm 23 \mathrm{pb}^{-1}$, was collected with the D $\varnothing$ detector at the Fermilab Tevatron Collider between April 2002 and August 2004. No event is observed in the data, while $0.41 \pm 0.11$ (stat) \pm 0.07 (sys) events are expected from Standard Model processes. The $95 \%$ confidence level cross section upper limits are in the range of 0.020 to $0.136 \mathrm{pb}$. From these, lower bounds on the masses of the lightest neutralino $\left(\widetilde{\chi}_{1}^{0}\right)$ and chargino $\left(\widetilde{\chi}_{1}^{ \pm}\right)$are extracted and interpreted in two different SUSY models.
\end{abstract}





\section{Search for R-parity Violating Supersymmetry in Multilepton Final States with the DØ Detector}

Von der Fakultät für Mathematik, Informatik und Naturwissenschaften der Rheinisch-Westfälischen Technischen Hochschule Aachen zur Erlangung des akademischen Grades eines

Doktors der Naturwissenschaften genehmigte Dissertation

vorgelegt von

Diplom-Physikerin Daniela Käfer

aus Monschau-Kalterherberg

Berichter: Universitätsprofessor Dr. Thomas Hebbeker Universitätsprofessor Dr. Martin Erdmann

Tag der mündlichen Prüfung: 27. Oktober 2006

Diese Dissertation ist auf den Internetseiten der Hochschulbibliothek online verfügbar. 



\section{Abstract}

Results obtained from a search for the trilepton signature $\mu \mu \ell$ (with $\ell=e$, or $\mu$ ) are combined with two complementary searches for the trilepton signatures eel and eet and interpreted in the framework of R-parity violating Supersymmetry. Pairwise, R-parity conserving production of the supersymmetric particles is assumed, followed by R-parity violating decays via an $L L \bar{E}$-operator with one dominant coupling $\lambda_{122}$. An $L L \bar{E}$-operator couples two weak isospin doublet and one singlet (s)lepton fields and thus violates lepton number conservation.

The data, collected with the $\mathrm{D} \emptyset$ detector at the Fermilab proton-antiproton collider Tevatron, corresponds to an integrated luminosity of $\int \mathcal{L} d t=360 \pm 23 \mathrm{pb}^{-1}$. No event is observed, while $0.41 \pm 0.11$ (stat) \pm 0.07 (sys) events are expected from Standard Model processes. The resulting $95 \%$ confidence level cross section limits on new physics producing a $\mu \mu \ell$ signature in the detector are of the order of 0.020 to $0.136 \mathrm{pb}$. They are interpreted in two different supersymmetry scenarios: the mSUGRA and the MSSM model. The corresponding lower limits on the masses of the lightest neutralino $\left(\widetilde{\chi}_{1}^{0}\right)$ and the lightest chargino $\left(\widetilde{\chi}_{1}^{ \pm}\right)$in case of the mSUGRA model are found to be in the range of:

$$
\begin{array}{llll}
\text { mSUGRA, } \mu>0: & \mathrm{M}\left(\widetilde{\chi}_{1}^{0}\right) \gtrsim 115-128 \mathrm{GeV} & \text { and } & \mathrm{M}\left(\widetilde{\chi}_{1}^{ \pm}\right) \gtrsim 215-241 \mathrm{GeV} \\
\text { mSUGRA }, \mu<0: & \mathrm{M}\left(\widetilde{\chi}_{1}^{0}\right) \gtrsim 101-114 \mathrm{GeV} & \text { and } & \mathrm{M}\left(\widetilde{\chi}_{1}^{ \pm}\right) \gtrsim 194-230 \mathrm{GeV},
\end{array}
$$

depending on the actual values of the model parameters: $m_{0}, m_{1 / 2}, A_{0}, \tan \beta$, and $\mu$. The first and second parameters provide the boundary conditions for the masses of the supersymmetric spin-0 and spin- $1 / 2$ particles, respectively, while $A_{0}$ gives the universal value for the trilinear couplings at the GUT scale. The parameter $\tan \beta$ denotes the ratio of the vacuum expectation values of the two Higgs fields and $\mu$, finally, represents the Higgs mixing parameter.

In the MSSM scenario the lower bound on the mass of the lightest chargino (for fixed neutralino mass) is found to be in the range of:

$$
\begin{aligned}
& \mathrm{M}\left(\widetilde{\chi}_{1}^{ \pm}\right) \gtrsim 205 \mathrm{GeV}, \text { for } \mathrm{M}\left(\widetilde{\chi}_{1}^{0}\right)=30 \mathrm{GeV} \\
& \mathrm{M}\left(\widetilde{\chi}_{1}^{ \pm}\right) \gtrsim 232 \mathrm{GeV}, \text { for } \mathrm{M}\left(\widetilde{\chi}_{1}^{0}\right)=200 \mathrm{GeV} .
\end{aligned}
$$

The parameters of the considered MSSM model are: $M_{1}, M_{2}, M_{3}, A_{0}, \tan \beta, \mu$, and $m_{A}$. The first three parameters define the common masses of the superpartners of the $\mathrm{U}(1)_{Y}, \mathrm{SU}(2)_{L}$, and $\mathrm{SU}(3)_{C}$ bosons at the electroweak scale, respectively. The following three parameters are identical to those defined in the mSUGRA model above, while $m_{A}$ denotes the mass of the pseudoscalar Higgs boson, present in the supersymmetric extension of the Standard Model. In addition all sfermion masses are set to $1000 \mathrm{GeV}$. 



\section{Zusammenfassung}

Die Ergebnisse einer Suche nach der Drei-Leptonen Signatur $\mu \mu \ell$ (mit $\ell=e$, oder $\mu$ ) werden mit zwei weiteren, komplementären Suchen nach den Drei-Leptonen Signaturen eel und ee $\tau$ kombiniert und im Rahmen R-Partitätsverletzender Supersymmetrie interpretiert. Eine paarweise, die R-Parität erhaltende Produktion supersymmetrischer Teilchen wird vorausgesetzt, gefolgt von R-Paritätsverletzenden Zerfällen mittels eines $L L \bar{E}$-Operators mit der dominanten Kopplung $\lambda_{122}$. Ein $L L \bar{E}$-Operator koppelt je zwei (s)fermionische Dublett- und ein Singulettfeld des schwachen Isospins und verletzt so die Leptonzahl-Erhaltung.

Die Daten wurden mit dem DØ-Detektor am Proton-Antiproton-Beschleuniger Tevatron des Fermilab aufgenommen und entsprechen einer integrierten Luminosität von $\int \mathcal{L} d t=360 \pm 23 \mathrm{pb}^{-1}$. In den Daten wird kein Ereignis beobachtet, während $0.41 \pm 0.11$ (stat) $\pm 0.07($ sys $)$ Ereignisse aufgrund von Standardmodell-Prozessen und instrumentellem Untergrund erwartet werden. Die resultierenden Grenzen auf den Wirkungsquerschnitt von Prozessen, die eine $\mu \mu \ell$ Signatur im Detektor hervorrufen sind bei einem Vertrauensniveau von $95 \%$ von der Größenordnung $0.020 \mathrm{pb}$ bis $0.136 \mathrm{pb}$. Diese werden in zwei unterschiedlichen phänomenologischen SupersymmetrieSzenarien, dem mSUGRA und dem MSSM Modell interpretiert. Die entsprechenden unteren Massengrenzen auf die Masse des leichtesten Neutralinos $\left(\widetilde{\chi}_{1}^{0}\right)$ bzw. des leichtesten Charginos $\left(\widetilde{\chi}_{1}^{ \pm}\right)$liegen im Bereich von:

$$
\begin{array}{llll}
\text { mSUGRA, } \mu>0: & \mathrm{M}\left(\widetilde{\chi}_{1}^{0}\right) \gtrsim 115-128 \mathrm{GeV} & \text { und } & \mathrm{M}\left(\widetilde{\chi}_{1}^{ \pm}\right) \gtrsim 215-241 \mathrm{GeV} \\
\text { mSUGRA }, \mu<0: & \mathrm{M}\left(\widetilde{\chi}_{1}^{0}\right) \gtrsim 101-114 \mathrm{GeV} & \text { und } & \mathrm{M}\left(\widetilde{\chi}_{1}^{ \pm}\right) \gtrsim 194-230 \mathrm{GeV},
\end{array}
$$

in Abhängigkeit von den gewählten SUSY Modellparametern: $m_{0}, m_{1 / 2}, A_{0}, \tan \beta$, und $\mu$. Die beiden ersten Parameter geben die Grenzbedingungen für die Massen der supersymmetrischen Spin-0 und Spin-1/2 Teilchen vor, während $A_{0}$ den universellen Wert der trilinearen Kopplungen an der GUT Skala definiert. Der Parameter $\tan \beta$ beschreibt den Quotienten der VakuumErwartungswerte der beiden Higgsboson Felder und der letzte Parameter, $\mu$, ist der HiggsMischungsparameter.

Im MSSM Modell ergibt sich für das leichteste Chargino (bei fester Neutralinomasse) eine untere Massengrenze im Bereich von:

$$
\begin{aligned}
& \mathrm{M}\left(\widetilde{\chi}_{1}^{ \pm}\right) \gtrsim 205 \mathrm{GeV}, \text { für } \mathrm{M}\left(\widetilde{\chi}_{1}^{0}\right)=30 \mathrm{GeV} \\
& \mathrm{M}\left(\widetilde{\chi}_{1}^{ \pm}\right) \gtrsim 232 \mathrm{GeV}, \text { für } \mathrm{M}\left(\widetilde{\chi}_{1}^{0}\right)=200 \mathrm{GeV} .
\end{aligned}
$$

Die Parameter des MSSM Modells sind: $M_{1}, M_{2}, M_{3}, A_{0}, \tan \beta, \mu$, und $m_{A}$, wobei die ersten drei Parameter die entsprechenden universellen Werte für die Massen der Superpartner der $\mathrm{U}(1)_{Y}, \mathrm{SU}(2)_{L}$, und $\mathrm{SU}(3)_{C}$ Eichbosonen definieren. Die folgenden drei Parameter entsprechen denen des mSUGRA Modells, und $m_{A}$ beschreibt die Masse des pseudoskalaren Higgsbosons, das in supersymmetrischen Erweiterungen des Standardmodells auftritt. Zusätzlich werden alle Sfermionmassen auf $1000 \mathrm{GeV}$ festgelegt. 



\section{Contents}

$\begin{array}{ll}\text { Introduction } & 1\end{array}$

1 Theoretical Foundations 5

1.1 Gauge Principles and Local Gauge Invariance . . . . . . . . . . . . . . . . 5

1.2 A Brief Overview of the Standard Model . . . . . . . . . . . . . . 6

1.2.1 The Particle Content of the Standard Model . . . . . . . . . . . . . . 7

1.2.2 Local Symmetry Groups and the SM Lagrangian . . . . . . . . . . . . 9

1.2 .3 The Higgs Mechanism . . . . . . . . . . . . . . . . . . . 12

1.2.4 Open Questions and Limitations . . . . . . . . . . . . . . . 15

1.3 Introduction to Supersymmetry . . . . . . . . . . . . . . . . . . . . 17

1.3.1 Motivating Supersymmetry . . . . . . . . . . . . . . . 17

1.3.2 The Particle Content in Supersymmetric Models . . . . . . . . . . . . 19

1.3.3 The Theoretical Formalism . . . . . . . . . . . . . . . . . . 20

1.3.4 SUSY Breaking and the Higgs Sector . . . . . . . . . . . . . . . 27

1.3.5 Phenomenological SUSY Models . . . . . . . . . . . . . . . . 30

1.3.6 R-parity and Its Consequences . . . . . . . . . . . . . . 35

1.3.7 Gaugino Pair Production and $\not_{p}$-decay of the LSP . . . . . . . . . 37

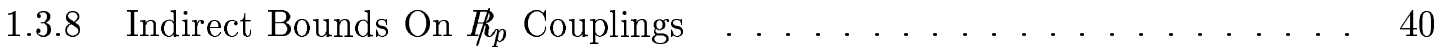

2 The Experimental Setup $\quad 43$

2.1 General Aspects of $\boldsymbol{p} \overline{\boldsymbol{p}}$ Collisions $\ldots \ldots \ldots \ldots \ldots$. . . . . . . . . . . 43

2.1.1 Parton Density Functions $(\mathrm{PDF}) \ldots \ldots \ldots \ldots \ldots$

2.1.2 Hadronisation and Additional Partons . . . . . . . . . . . . . . . . 47

2.1.3 The Underlying Event, Multiple Interactions, and Pile-Up . . . . . . . . 47

2.1.4 Common Variables and Definitions . . . . . . . . . . . . . . . 48 
2.2 The Accelerator Chain at Fermilab . . . . . . . . . . . . . . . . . 51

2.3 The $\mathrm{D} \emptyset$ Detector . . . . . . . . . . . . . . . . 65

2.3.1 The Central Tracking Systems . . . . . . . . . . . . . 67

2.3.2 The Liquid Argon Calorimeter . . . . . . . . . . . . . . . . 72

2.3 .3 The Muon System . . . . . . . . . . . . . . . . . . . 76

2.3 .4 The Luminosity System . . . . . . . . . . . . . . . . . . 84

2.3.5 The Forward Proton Detector: FPD . . . . . . . . . . . 85

2.4 The D $\varnothing$ Trigger and Data Acquisition System . . . . . . . . . . . . . 87

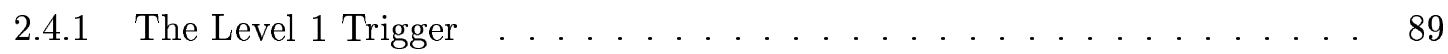

2.4 .2 The Level 2 Trigger . . . . . . . . . . . . . . . . . . . 90

2.4.3 The Level 3 Trigger and Data Acquisition . . . . . . . . . . . . . . 91

2.4.4 Online Monitoring of the Detector and Data Quality . . . . . . . . . . 94

2.5 Data Simulation, Handling, and Storage . . . . . . . . . . . . 98

3 Phenomenology of $\not_{p}$-SUSY and Signal MC Simulation Studies 103

3.1 Gaugino Pair Production at the Tevatron . . . . . . . . . . . . . 103

3.2 Next-to-leading Order Signal Cross Sections . . . . . . . . . . . . . . . 107

3.3 Cascade Decays and Branching Ratios of $\widetilde{\boldsymbol{\chi}}_{\mathbf{2}}^{\mathbf{0}}$ and $\widetilde{\chi}_{\mathbf{1}}^{ \pm} \ldots \ldots \ldots 108$

3.4 Kinematic Properties of the Produced Electrons and Muons . . . . . . . . . 117

4 The Event Selection $\quad 123$

4.1 Data Sample and Luminosity . . . . . . . . . . . . . . . . 123

4.2 Monte Carlo Simulations . . . . . . . . . . . . . . . . . 126

4.2.1 Monte Carlo Simulations of Standard Model Processes . . . . . . . . . . 126

4.2.2 Monte Carlo Simulations of SUSY Signal Processes . . . . . . . . . . 128

4.3 Trigger Efficiency and Object Identification . . . . . . . . . . . . . . 132

4.3.1 The Dimuon Trigger Efficiency and Application to MC . . . . . . . . 132

4.3 .2 Object Identification and Efficiencies . . . . . . . . . . . . 135

4.4 Estimation of the Multijet Background from Data . . . . . . . . . . 156

4.5 The Selection of Dimuon \& Electron-Muon Events . . . . . . . . . . 158

4.5.1 The Dimuon Selection: dimu . . . . . . . . . . . . . . 161

4.5.2 The Electron-Muon Selection: emu . . . . . . . . . . . . . . 163 
4.5.3 The Trilepton Selection . . . . . . . . . . . . . . . 171

4.5.4 The Optimisation of the dimu+emu Selection Cuts . . . . . . . . . 174

4.6 Systematic Uncertainties . . . . . . . . . . . . . . . . . . . . . 176

4.6.1 Uncertainties on the Dimuon Trigger Efficiencies . . . . . . . . . . 176

4.6.2 Uncertainties on Object ID Efficiencies . . . . . . . . . . . . . 176

4.6.3 Uncertainties due to the $\boldsymbol{p}_{\mathbf{T}} / \boldsymbol{E}_{\mathbf{T}}$ Resolution for Muons/Electrons . . . 177

4.6.4 Uncertainties due to the Modeling of the $\boldsymbol{Z}$ boson $\boldsymbol{p}_{\mathbf{T}} \ldots \ldots \ldots 177$

4.6.5 Systematic Uncertainties Specific to the Signal . . . . . . . . . . . 179

4.6 .6 Other Uncertainties . . . . . . . . . . . . . . . . . . 180

4.7 Facts and Figures . . . . . . . . . . . . . . . . . . . . 180

5 The Results and Their Interpretation $\quad 183$

5.1 The Statistical Method . . . . . . . . . . . . . . . . . . . . 183

5.1 .1 The Concept of Hypothesis Tests . . . . . . . . . . . . . . . . 184

$5.1 .2 \quad$ Confidence Levels and Exclusion Limits . . . . . . . . . . . . . . 186

5.1.3 Combining Different Analysis Channels . . . . . . . . . . . . . . 188

5.1.4 Technical Realisation of the $\mathrm{CL}_{S}$ Method . . . . . . . . . . . . . 189

5.2 Interpretation within the mSUGRA Scenario . . . . . . . . . . . . . 191

5.3 Interpretation within the $»$ no-GUT $\ll$ MSSM Scenario . . . . . . . . . . . . 200

$\begin{array}{ll}\text { Conclusion and Outlook } & 203\end{array}$

$\begin{array}{ll}\text { A Simulated mSUGRA Signal Points } & 205\end{array}$

B Simulated MSSM Signal Points (no-GUT Scenario) 209

C Results within the mSUGRA Scenario $\quad 213$

$\begin{array}{ll}\text { D Results within the no-GUT MSSM Scenario } & 217\end{array}$

$\begin{array}{lr}\text { Bibliography } & 225\end{array}$ 



\section{Introduction}

In your hands now, here's my book, between whose pages, if you look, is written verse on verse, in language dry and terse, about some tiny sparticles, obeying mighty principles; for instance supersymmetry, possibly even with »ar-pe-ve«. And while they're not discovered yet gauginos, sleptons, squarks and co. are waiting in this splendid mess, for someone finding them, I guess!

At all times physicists tried to better understand nature, always guided by the idea that it should be possible to explain the inanimate physical world with only a few principles. Thus the ultimate goal of all research in the realm of particle physics is the development of only $\gg$ one « theory of matter, the » Theory of Everything«.

Although it is still a long way to this Theory of Everything, the Standard Model (SM) already represents a milestone in our understanding of nature. It not only describes three of the four fundamental forces, but employs only two quantum field theories to do so. The symmetry group of the SM comprises the $\mathrm{SU}(3)_{C}$ of Quantum Chromodynamics $(\mathrm{QCD})$ and the $\mathrm{SU}(2)_{L} \otimes \mathrm{U}(1)_{Y}$ to describe the strong and electroweak interactions. The latter are due to the weak and the electromagnetic forces, which have already been unified in the Electroweak Theory. As all models, the SM has been tested thoroughly over the last decades and with tremendous success. So far all experimental results agree with the theoretical predictions within a few standard deviations. This is even more astonishing, since it is known that the SM can only be a low energy effective theory. At the latest, at the Planck scale $\left(\approx 10^{19} \mathrm{GeV}\right)$ the fourth fundamental force, gravity, has to be included into the description. At lower energies, e.g. energies that are accessible in today's experiments, gravitational interactions are negligibly small.

Now it is speculated, that at extremely high energies $\left(\approx 10^{16} \mathrm{GeV}\right)$ all interactions could be uniformly described by a single quantum field theory, such that at low energies $\left(\approx 10^{2} \mathrm{GeV}\right)$ the seemingly distinct electromagnetic, weak and strong interactions are only different manifestations of a single overarching force. This consideration together with several open questions in the SM give rise to new »Beyond the $S M$ « theories. One very promising approach is the supersymmetric extension of the SM. Supersymmetry (SUSY) predicts the existence of an additional particle 
for each SM particle, differing by half a unit in spin but otherwise sharing the same quantum numbers. Since no supersymmetric particle has been observed so far, supersymmetry must be broken, leading to different masses of the SM particles and their superpartners. Each particle is characterized by a new internal quantum number, R-parity, defined as $R_{p}=(-1)^{3 B+L+2 S}$ (with $B$ being the baryon, $L$ the lepton and $S$ the spin quantum number), such that SM particles have $R_{p}=+1$ and SUSY particles $R_{p}=-1$. Although most SUSY models assume R-parity conservation in order to conserve the quantum numbers $B$ and $L$, the underlying gauge symmetry also allows $R_{p}$-violating $\left(\not R_{p}\right)$ terms in the Lagrangian density.

So far no evidence for SUSY has been observed, resulting in lower bounds on the masses of these particles. Cosmological observations, e.g. measurements of the relic density, or dark matter searches, lead to further constraints, as do precision measurements of quantities sensitive to higher-order corrections from possible SUSY particles. In 2001 the $»$ hunt « for SUSY particles passed from the Large Electron Positron Collider (LEP) to the Tevatron Proton-AntiprotonCollider, where the second phase of data taking (Run II) started at a center-of-mass energy of $\sqrt{s}=1.96 \mathrm{TeV}$ (compared to $\sqrt{s}=1.8 \mathrm{TeV}$ in Run I). Since the luminosity has also been considerably increased, the Tevatron and its two experiments CDF and $\mathrm{D} \varnothing$ allow to probe a large new SUSY mass range.

In this work, the search for chargino and neutralino pair production in the hypothesis of $\not_{p}$-SUSY is presented. Final states with at least three charged leptons with the flavour combinations $\mu \mu \ell$ $(\ell=e$, or $\mu)$ are selected. This already implies a very low SM background, since there are not many processes which yield up to three charged leptons in the final state. The results are interpreted in two different supersymmetric models: the minimal supergravity (mSUGRA) scenario and a phenomenological Minimal Supersymmetric Standard Model (MSSM). In both scenarios the lightest neutralino $\left(\widetilde{\chi}_{1}^{0}\right)$ is assumed to be the lightest supersymmetric particle (LSP).

Chapter one briefly reviews the theoretical foundations of the Standard Model and gives an introduction to the theory and phenomenology of supersymmetry including R-parity violation. In chapter two, some of the most important terms in $p \bar{p}$ collisions are discussed and an overview of the Tevatron Proton-Antiproton-Collider, as well as of the D $\varnothing$ detector with all its important subsystems is given. Chapter three focuses on the phenomenology of supersymmetric particle production and possible $\not R_{p}$-decays in $p \bar{p}$ collisions, studied with signal Monte Carlo samples for mSUGRA and MSSM scenarios. The event selection and optimisation together with the determination of Monte Carlo corrections for various quality criteria of the charged leptons and the estimation of multijet background from data is discussed in chapter four.

Finally, in chapter five, the results are discussed and interpreted stand alone and in combination with two other, complementary searches for the trilepton signatures ee $(\ell=e$, or $\mu)$ [1] and

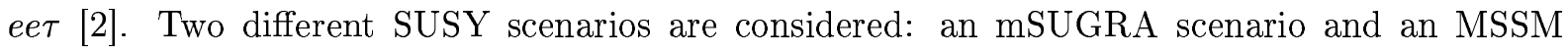
scenario without GUT-relation. A conclusion summarises the results and provides a brief outlook of what could be achieved at the Tevatron with more luminosity and - later on - at the LHC. 
In the following natural units are used, unless noted otherwise. Within this system the reduced Planck constant $\hbar$ and the speed of light $c$ are defined as: $\hbar=c=1$. 



\title{
Chapter 1
}

\section{Theoretical Foundations}

\begin{abstract}
There is a theory which states that if ever anyone discovers exactly what the Universe is for and why it is here, it will instantly disappear and be replaced by something even more bizarre and inexplicable. - There is another theory which states that this has already happened.
\end{abstract}

»The Restaurant at the End of the Universe «, Douglas Adams (1980)

The Standard Model of particle physics (SM) has been tested thoroughly over the last three to four decades, up to energies of approximately $100 \mathrm{GeV}$, and so far an overwhelming number of experimental results is in very good agreement with the theoretical predictions. Although supersymmetric theories and the self-interacting quanta of non-Abelian gauge theories seem to suggest that the traditional distinction between force particles and matter constituents might be wrong and that both kinds should be treated in a unified way, it is imperative that any new, more fundamental theory reproduces the SM as a low energy effective theory.

Before introducing Supersymmetry (SUSY) as one of the possible extensions of the Standard Model, the basic theoretical concepts: gauge principles, local gauge invariance of the symmetry groups in the SM and spontaneous symmetry breaking are briefly reviewed. These fundamentally new ideas have led to the current quantum field theoretical formulation of the Standard Model, and they are also a vital part of many of its extensions.

\subsection{Gauge Principles and Local Gauge Invariance}

In quantum field theories [3] all matter particles (fermions) are described by complex spinors $\psi(x)$ depending on the space-time coordinate $x$. Interactions between fermions are then introduced by requiring the theory to be invariant under local ( $x$-dependent) transformations $U(x)$

$$
\begin{aligned}
\psi(x) & \rightarrow \psi^{\prime}(x)=U(x) \psi(x), \\
\text { with } \quad U(x) & =\exp \left[-i \sum_{j=1}^{n} \alpha_{j}(x) \mathcal{G}_{j}\right] .
\end{aligned}
$$

Only if the equations of motion remain invariant under the gauge transformations $U(x)$, the theory is said to be symmetric under $U(x)$. The set of all unitary $\left(U^{\dagger} U=1\right)$ gauge transformations, describing one symmetry, form a group. In general the transformations are written as in 
Eq. (1.2) using $n$ generators $\mathcal{G}_{j}$, which determine the algebra of this n-dimensional group. The parameters $\alpha_{j}(x)$ are real functions of $x$ and specify the local transformation. If the commutator of any two generators $\mathcal{G}_{j}$ is a linear combination of these (Lie algebra):

$$
\left[\mathcal{G}_{j}, \mathcal{G}_{k}\right]=i \sum_{l=1}^{n} f_{j k l} \mathcal{G}_{l}
$$

the corresponding group is referred to as a Lie group. The $f_{j k l}$ are the totally antisymmetric structure constants, which vanish, if the generators commute; the group is then Abelian.

The equations of motion, e.g. the Dirac equation, contain not only the particle fields $\psi(x)$, but also partial derivatives thereof, $\partial_{\mu}=\partial / \partial x^{\mu}$. An application of the above transformations according to Equation (1.2) generates additional terms due to the transformations $\alpha_{j}(x)$. In order to satisfy the requirement of invariance under local transformations, the partial derivative $\partial_{\mu}$ is extended to the covariant derivative $\mathcal{D}_{\mu}$ (Eq. (1.4)), which contains $n$ additional vector fields $A_{\mu}^{j}(x)$. Later on, the vector fields $A_{\mu}^{j}$ are identified with the vector bosons $W^{ \pm}, \gamma$ and $Z^{0}$ of the Standard Model mediating the interactions between fermions. These new vector fields couple to the fermion fields with specific coupling strengths $g$ (being free parameters), and in this way they introduce interactions between the formerly free particles $\psi(x)$. The vector fields themselves are transformed according to Eq. (1.5).

$$
\begin{aligned}
\partial_{\mu}: & \mathcal{D}_{\mu}=\partial_{\mu}+i \sum_{j=1}^{n} g \mathcal{G}_{j} A_{\mu}^{j}(x) \\
A_{\mu}^{j}(x) \rightarrow & A_{\mu}^{j \prime}(x)=A_{\mu}^{j}(x)-\frac{1}{g} \partial_{\mu} \alpha_{j}(x)-\sum_{k, l}^{n} f_{j k l} \alpha_{k}(x) A_{\mu}^{l}(x),
\end{aligned}
$$

In a Lagrangian density used to describe a physical system, the interactions mediated by gauge fields (gauge bosons) are expressed by field strength tensors $\mathcal{F}_{\mu \nu}^{j}(x)$

$$
\mathcal{F}_{\mu \nu}^{j}(x)=\partial_{\mu} A_{\nu}^{j}-\partial_{\nu} A_{\mu}^{j}-g \sum_{k, l}^{n} f_{j k l} A_{\mu}^{k} A_{\nu}^{l} .
$$

The last term of the field strength tensor describes the self-interactions of the gauge field quanta. It only exists if the group generators do not commute, i.e. if the group has a non-Abelian structure. Gauge theories containing non-Abelian symmetry groups are also called Yang-Mills theories after C.N. Yang and R.L. Mills, who, in 1954 generalised the gauge invariance of electromagnetism (Abelian U(1) symmetry) and constructed a theory based on the non-Abelian SU(2) symmetry group to describe the isospin doublet of protons and neutrons [4].

\subsection{A Brief Overview of the Standard Model}

The Standard Model of particle physics is a renormalisable quantum field theory of the electroweak and strong interactions, based on the symmetry group $\mathrm{SU}(3)_{C} \otimes \mathrm{SU}(2)_{L} \otimes \mathrm{U}(1)_{Y}$.

The electroweak theory (also known as GSW-theory) was developed in the period from 1961 to 1967, primarily by the work of S.L. Glashow, A. Salam and S. Weinberg [5], who were awarded the Nobel Prize in 1979. For the first time, their formalism enabled a consistent, uniform description of electromagnetic and weak interactions, based on the gauge group $\mathrm{SU}(2)_{L} \otimes \mathrm{U}(1)_{Y}$. 
The invention of the Higgs mechanism [6] in 1964, named after P.W. Higgs, solved the problem of mass generation in quantised gauge theories and allowed not only for massive mediators of the short-ranged weak interactions but also for massive fermions. Mass terms generated through the particles' interactions with a scalar background field, the new Higgs field, leave the Lagrangian invariant under $\mathrm{U}(1)_{Y}$ and $\mathrm{SU}(2)_{L}$ transformations, contrary to manually inserted mass terms. In 1964, M. Gell-Mann and, independently, Georg Zweig, postulated the quark model [7], where the name quarks for the constituents of hadrons was coined by Gell-Mann through a reference to the book Finnegans Wake, written by James Joyce. For his contributions concerning the classification of elementary particles and their interactions Gell-Mann was awarded the Nobel Prize in 1969. Nowadays the quark model is part of the theory of strong interactions, Quantum Chromodynamics (QCD), which was developed in the following years by H. Fritzsch, M. Gell-Mann, H. Leutwyler, D.J. Gross, F. Wilczek, S. Weinberg and many others [8]. Quantum Chromodynamics is based on the symmetry group $\mathrm{SU}(3)_{C}$, where the index $C$ stands for $»$ colour «.

D.J. Gross, F. Wilczek and D. Politzer found the phenomenon of asymptotic freedom [9] in 1973 and were awarded the Nobel Prize in physics in 2004. Asymptotic freedom refers to the behaviour of quarks at high energies, or correspondingly, small distances. For quarks bound inside hadrons, the strong force between them is so weak, that they behave almost like free particles. The opposite is true when the quarks move apart, i.e. the force becomes stronger with increasing distance, what came to be known as confinement [10]. This property is widely believed to be realized in nature, because it would take an infinite amount of energy to separate two quarks, which, in turn, would explain why no free quarks have been observed so far. Also recent numerical simulations in lattice QCD confirm, that a string-like flux tube forms between distant static colour charges (quarks) leading to the phenomenon of confinement.

Renormalisability of quantum gauge field theories is fundamental, because it prevents unphysical, infinite quantities in the theoretical description and thus ensures a unique relationship between theoretically predictable quantities and measurable observables. Although renormalisability of Abelian gauge theories (as is QED) was already shown in the late 1940s by S.-I. Tomonaga, J. Schwinger and R.P. Feynman [11], who shared the 1965 Nobel Prize in physics, the proof could not be extended to non-Abelian theories. It took about 25 years more, before M.J.G. Veltman and G. 't Hooft proved in 1971 that also non-Abelian gauge theories were renormalisable [12], a work for which they, too, were awarded the Nobel Prize in physics (1999).

\subsubsection{The Particle Content of the Standard Model}

In general, two different kinds of particles are distinguished: Dirac fermions with spin $1 / 2$ and bosons with spin 1 . While the first kind form the basic building blocks of matter, the latter are the quanta of the gauge fields and mediate the interactions. Additionally, for every fermion, there exists an anti-fermion, differing only by opposite signs of charge-like quantum numbers from its »ordinary « partner. All fermions participate in the Neutral Current electroweak interactions, but only left-chiral fermion states (and right-chiral anti-fermion states) are involved in the Charged Current electroweak interactions. A picture that has been emerging since 1957 when C.S. Wu discovered parity violation in charged current weak interactions [13]. Thus, the left-chiral fermion fields are grouped into weak isospin doublets - in analogy to the proton/neutron doublet in nuclear physics, where proton and neutron are regarded as two states of a single nucleon. The 
fermion fields, $\psi$, can then be written as a linear combination of states with definite chirality

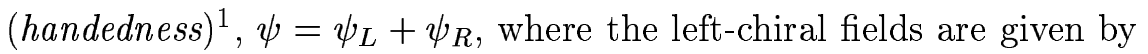

$$
\psi_{\ell_{i}}^{L}=\left(\begin{array}{c}
\nu_{i} \\
\ell_{i}^{-}
\end{array}\right) \quad \psi_{q_{i}}^{L}=\left(\begin{array}{c}
u_{i} \\
d_{i}^{\prime}
\end{array}\right) \quad \text { with } i=1,2,3 \text { fermion generations. }
$$

The eigenstates of the down-type quarks $\left(d^{\prime}\right)$ arise from the mass eigenstates $d_{i}$ by applying the Cabibbo-Kobayashi-Maskawa quark mixing matrix $V_{C K M}: d_{i}^{\prime}=\sum_{j} V_{i j} d_{j}$ [14]. Right-chiral states $\left(\ell_{R}^{-}, u_{R}, d_{R}\right)$ are assumed to transform as singlets under $\mathrm{SU}(2)_{L}$ transformations. Contrary to the »coloured « quarks, leptons do not interact strongly because the gauge bosons of QCD (gluons), only couple to »colour-charge «. As of now, three generations of fermions are known, where each generation consists of two leptons, one charged and one electrically neutral lepton and six quarks, three up-type and three down-type quarks (in red, green and blue). In Table 1.1 all SM-fermions are listed, together with their corresponding values of the conserved quantum numbers: the electric charge $Q / e$, the third component of the weak isospin $T_{3}$ and the weak hypercharge $Y_{w}$, all of which are connected by the Gell-Mann/Nishijima relation $Q_{f}=T_{3}+Y_{w} / 2$, in addition the $\mathrm{SU}(2)_{L}$ and $\mathrm{SU}(3)_{C}$ representations are given.

\begin{tabular}{cccccccc}
\hline \hline Name & \multicolumn{3}{c}{ Fermion fields, $\psi$} & $Q / e$ & $T_{3}$ & $\mathrm{SU}(3)_{C}, \mathrm{SU}(2)_{L}, \mathrm{U}(1)_{Y}$ \\
\hline \hline Leptons & $L:$ & $\left(\begin{array}{c}\nu_{e} \\
e^{-}\end{array}\right)_{L}$ & $\left(\begin{array}{c}\nu_{\mu} \\
\mu^{-}\end{array}\right)_{L}$ & $\left(\begin{array}{c}\nu_{\tau} \\
\tau^{-}\end{array}\right)_{L}$ & -1 & $-1 / 2$ & $(\mathbf{1}, \mathbf{2},-1)$ \\
& $e:$ & $e_{R}$ & $\mu_{R}$ & $\tau_{R}$ & -1 & 0 & $(\mathbf{1}, \mathbf{1},-2)$ \\
\hline \multirow{4}{*}{ Quarks } & $Q:$ & $\left(\begin{array}{c}u \\
d^{\prime}\end{array}\right)_{L}$ & $\left(\begin{array}{c}c \\
s^{\prime}\end{array}\right)_{L}$ & $\left(\begin{array}{c}t \\
b^{\prime}\end{array}\right)_{L}$ & $-1 / 3$ & $-1 / 2$ & $\left(\mathbf{3}, \mathbf{2}, \frac{1}{3}\right)$ \\
& $u:$ & $u_{R}$ & $c_{R}$ & $t_{R}$ & $+2 / 3$ & 0 & $\left(\overline{\mathbf{3}}, \mathbf{1}, \frac{4}{3}\right)$ \\
& $d^{\prime}:$ & $d_{R}^{\prime}$ & $s_{R}^{\prime}$ & $b_{R}^{\prime}$ & $-1 / 3$ & 0 & $\left(\overline{\mathbf{3}}, \mathbf{1},-\frac{2}{3}\right)$ \\
\hline \hline
\end{tabular}

Table 1.1: The fermions of the Standard Model with their quantum numbers: electric charge $Q$, third component of the weak isospin $T_{3}$, weak hypercharge $Y_{w}$, and the $S U(2)_{L}$ and $S U(3)_{C}$ representations.

The heaviest of the quarks, the top-quark, was discovered in 1995 by the CDF (Collider Detector at Fermilab) and $\mathrm{D} \emptyset^{2}$ experiments at the Fermilab $p \bar{p}$ collider Tevatron [15] and the last lepton to be discovered was the tau-neutrino $\left(\nu_{\tau}\right)$ found in 2000 by the DONUT (Direct Observation of $\mathrm{Nu}$ Tau) collaboration which operated experiment E872 at Fermilab [16].

Neutrinos with right chirality are still assumed - at least as far as the theoretical framework of the SM is concerned - not to exist. Nevertheless it is established beyond doubt that neutrinos do possess mass. The deficit in the expected solar $\nu_{e}$-flux was confirmed by several experiments [17]. More recent data indicate the existence of neutrino oscillations, consistent with two different non-zero neutrino mass differences. Transformations of atmospheric and accelerator neutrinos, assuming $\nu_{\mu}-\nu_{\tau}$ oscillations, have been confirmed by Super-Kamiokande [18] and K2K (KEK

\footnotetext{
${ }^{1}$ Massless particles (e.g. photon) have absolute chirality (helicity), independent of the reference frame of the observer, while the handedness of massive particles (e.g. electron, quark) depends on the observer's reference frame, i.e. they have relative chirality. A particle is right-handed (positive chirality), if its spin and momentum vector point in the same direction; left-handed (negative chirality) if the directions of spin and motion are opposite.

${ }^{2}$ The name $\mathrm{D} \varnothing$ originates from the naming scheme of accelerator sections at the Fermilab Tevatron.
} 
to Kamioka - Long Baseline Neutrino Oscillation Experiment) [19], respectively. In addition, the solar neutrino data from SNO (Sudbury Neutrino Observatory) [20] show indications of $\nu_{e}$ transformation. In the last years it has become increasingly clear, that the SM will have to be extended in order to explain, or at least, accommodate these new experimental results.

\subsubsection{Local Symmetry Groups and the SM Lagrangian}

In Quantum Electrodynamics (QED) [11] the correspondence between the symmetry of the underlying gauge theory and the physical interaction is easy to see. QED is based on the Abelian symmetry group $\mathrm{U}(1)$, known as $\mathrm{U}(1)_{E M}$. There is only one generator, the electric charge $Q_{f}$, which couples the fermion fields to the gauge field, $A_{\mu}$, identified with the photon. Photon self-interactions are non-existent at tree-level, since the photon does not carry electric charge, which is reflected by the choice of an Abelian symmetry group as the basis for the mathematical description of electromagnetic interactions.

Analogously, the theory of strong interactions, Quantum Chromodynamics (QCD) is based on the special unitary group $\mathrm{SU}(3)_{C}$, with $C$ denoting colour. Each quark flavour forms an $\mathrm{SU}(3)_{C}$ triplet, composed of red, green and blue eigenstates in a 3-dimensional colour-charge space. However, contrary to the photon, the eight generators of $\mathrm{SU}(3)_{C}$, the gluons, carry colour charge and thus the group has to be non-Abelian to account for the gluon self-interactions (Yang-Mills theory). This, and the fact, that the strong coupling constant $\alpha_{S}=g_{s}^{2} /(4 \pi) \gg \alpha_{E M}$ leads to a much richer structure of QCD compared to QED. It is assumed that, due to the confining properties of the strong interaction [10], quarks and gluons only exist bound into hadrons, which are colourless systems of either three quarks ( $q q q$, baryons) or one quark and one anti-quark ( $q \bar{q}$, mesons). Glueballs, states consisting solely of gluons, or bound states consisting of more than three quarks or a quark and an anti-quark may also exist. Comparing QCD with the weak interaction and QED, one would expect, that the former has an infinite range due to its massless field quanta. In reality, however, its reach is limited to roughly the perimeter of a nucleon, which is also related to the phenomenon of confinement. Colourless objects (pions, kaons) must first be formed from the quarks before the strong interaction can be mediated across larger distances.

When it was first proposed to unify electromagnetic and weak interactions, in the early 1960s, only charged weak interactions (Charged Currents, $C C$ ) were known. The symmetry group SU(2) is the smallest Lie group providing three generators for the three interactions to be described, two charged weak interactions and one neutral electromagnetic interaction. Unfortunately $\mathrm{SU}(2)$ alone led to difficulties because the photon, mediating electromagnetic interactions, does not distinguish between different chiralities of the fermion fields, contrary to what was observed for the charged weak interactions. Glashow, Salam and Weinberg proposed to describe electroweak interactions using the direct product of two symmetry groups, $\mathrm{SU}(2)_{L} \otimes \mathrm{U}(1)_{Y}$ as new gauge group [5]. Although $\mathrm{U}(1)_{Y}$ is mathematically the same group as $\mathrm{U}(1)_{E M}$ of $\mathrm{QED}$, the physical meaning is different. The new larger gauge group, now made up by four generators, the three weak isospin operators $\vec{T}=\left(T_{1}, T_{2}, T_{3}\right)$ of $\mathrm{SU}(2)_{L}$ and the weak hypercharge operator $Y_{w}$ of $\mathrm{U}(1)_{Y}$, predicted an additional weak interaction, the so-called Neutral Current. For more than 10 years, the new theory was not fully accepted, because of the non-observation of Neutral Currents and a theoretical difficulty due to the non-Abelian structure of the gauge group, since it was believed, that non-Abelian gauge theories were not renormalisable. Finally, in 1971, Veltman 
and 't Hooft proved the renormalisability of Yang-Mills (non-Abelian) gauge theories [12] and shortly afterwards, in 1973, Neutral Current interactions were observed using the Gargamelle bubble-chamber [21] at CERN ${ }^{3}$.

Thus the SM is based on the gauge group $\mathrm{SU}(3)_{C} \otimes \mathrm{SU}(2)_{L} \otimes \mathrm{U}(1)_{Y}$. All gauge fields with their respective quantum numbers and $\mathrm{SU}(2)_{L}$ and $\mathrm{SU}(3)_{C}$ representations are given in Table 1.2.

\begin{tabular}{llccc}
\hline \hline Field name & Boson fields & $\mathrm{Q} / \mathrm{e}$ & $T_{3}$ & $\mathrm{SU}(3)_{C}, \mathrm{SU}(2)_{L}, \mathrm{U}(1)_{Y}$ \\
\hline \hline $\mathrm{U}(1)$ gauge field & $B$ & 0 & 0 & $(\mathbf{1}, \mathbf{1}, 0)$ \\
$\mathrm{SU}(2)$ gauge fields & $W^{1}, W^{2}, W^{3}$ & $(+1,-1,0)$ & $(+1,-1,0)$ & $(\mathbf{1}, \mathbf{3}, 0)$ \\
$\mathrm{SU}(3)$ gauge fields & $G^{1}, G^{2}, \ldots G^{8}$ & 0 & 0 & $(\mathbf{8}, \mathbf{1}, 0)$ \\
\hline \hline
\end{tabular}

Table 1.2: The boson fields of the Standard Model. Similar to the down-type quarks fields, the electroweak gauge fields of $S U(2)_{L} \otimes U(1)_{Y}$ mix to form mass eigenstates.

Mathematically, all the particles of the SM, fermions and bosons and their interactions are described by a Langrangian density comprising four different contributions:

$$
\mathcal{L}_{S M}=\mathcal{L}_{\text {Fermions }}+\mathcal{L}_{\text {Yang-Mills }}+\mathcal{L}_{\text {Higgs }}+\mathcal{L}_{\text {Yukawa }}
$$

Here the fermion Lagrangian, $\mathcal{L}_{\text {Fermions }}$, contains terms describing the kinetic energy of the fermions, as well as their interactions with the gauge bosons entering through the covariant derivative given by Equation (1.10) below, (compare Eq. (1.4) in section 1.1).

$$
\begin{aligned}
& \mathcal{L}_{\text {Fermions }}=\sum_{\psi_{L}} \bar{\psi}_{L} i \gamma^{\mu} \mathcal{D}_{\mu} \psi_{L}+\sum_{\psi_{R}} \bar{\psi}_{R} i \gamma^{\mu} \mathcal{D}_{\mu} \psi_{R}
\end{aligned}
$$

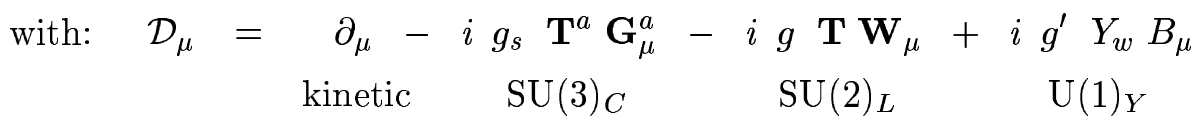

The first term of the covariant derivative corresponds to the kinetic term, while the second term represents the coupling of the quarks to the colour charge operator $\mathbf{T}^{a}$ of $\mathrm{SU}(3)_{C}$ with the coupling strength $g_{s}=2 \sqrt{\pi \cdot \alpha_{S}}$, where $\alpha_{S}$ is the strong coupling constant. The last two terms describe the electroweak interactions of the fermions, specifying their coupling to the $\mathrm{SU}(2)_{L}$ weak isospin operator $\mathbf{T}=\left(T_{1}, T_{2}, T_{3}\right)$ with the strength $g$ and the coupling to the weak hypercharge operator $Y_{w}$ with the strength $g^{\prime}$. The matrix representations of the $\mathrm{SU}(2)_{L}$ and $\mathrm{SU}(3)_{C}$ generators are the Pauli matrices $\sigma_{i}$ and the Gell-Mann matrices $\lambda_{a}$, respectively.

The Yang-Mills Lagrangian describes the kinetic energies of the various gauge fields and the self-interactions of the corresponding bosons within each non-Abelian group

$$
\begin{aligned}
& \mathcal{L}_{\text {Yang-Mills }}=-\frac{1}{4}\left[\mathbf{G}_{a}^{\mu \nu} \mathbf{G}_{\mu \nu}^{a}+\mathbf{W}^{\mu \nu} \mathbf{W}_{\mu \nu}+B^{\mu \nu} B_{\mu \nu}\right], \\
& \begin{array}{lll}
\mathrm{SU}(3)_{C} & \mathrm{SU}(2)_{L} & \mathrm{U}(1)_{Y}
\end{array}
\end{aligned}
$$

\footnotetext{
${ }^{3}$ Originally the acronym CERN meant Conseil Européen pour la Recherche Nucléaire, but nowadays the laboratory is referred to as the European Laboratory for Particle Physics.
} 
with the following expressions for the field strength tensors, which predict the existence of trilinear and quadrilinear couplings.

$$
\begin{array}{rlr}
\mathbf{G}_{\mu \nu}^{a} & =\partial_{\mu} \mathbf{G}_{\nu}^{a}-\partial_{\nu} \mathbf{G}_{\mu}^{a}-g_{s} f_{a b c} \mathbf{G}_{\mu}^{b} \mathbf{G}_{\nu}^{c} & \mathrm{SU}(3)_{C} \\
\mathbf{W}_{\mu \nu} & =\partial_{\mu} \mathbf{W}_{\nu}-\partial_{\nu} \mathbf{W}_{\mu}-g \mathbf{W}_{\mu} \times \mathbf{W}_{\nu} & \mathrm{SU}(2)_{L} \\
B_{\mu \nu} & =\partial_{\mu} B_{\nu}-\partial_{\nu} B_{\mu} & \mathrm{U}(1)_{Y}
\end{array}
$$

The physical, observable boson fields $W^{ \pm}, Z^{0}$ and $\gamma$ are obtained by a mixing of the basic $\mathrm{SU}(2)_{L} \otimes \mathrm{U}(1)_{Y}$ generators. This specific choice of linear combinations takes into account, that the neutral electroweak bosons, $A_{\mu}$ (photon) and $Z_{\mu}$ ( $Z^{0}$ boson) couple to all fermions, while the charged weak gauge bosons $W_{\mu}^{ \pm}$only couple to the left-chiral fermions, i.e. to particles with non-zero isospin $\mathbf{T} \neq 0$.

$$
\begin{aligned}
W_{\mu}^{ \pm} & =\frac{1}{\sqrt{2}}\left(W_{\mu}^{1} \mp i W_{\mu}^{2}\right) \\
A_{\mu} & =B_{\mu} \cos \theta_{w}+W_{\mu}^{3} \sin \theta_{w} \\
Z_{\mu} & =-B_{\mu} \sin \theta_{w}+W_{\mu}^{3} \cos \theta_{w} .
\end{aligned}
$$

A direct consequence of requiring the electromagnetic field $A_{\mu}$ to couple with the strength $e$ to the charged leptons, but not to the neutrinos, is the connection between the weak coupling constants $g, g^{\prime}$ and the electric charge $e$, expressed in terms of the weak mixing angle $\theta_{w}$

$$
g \sin \theta_{w}=g^{\prime} \cos \theta_{w}=e .
$$

Compared to the simple vectorial coupling of the photon, the couplings of the massive electroweak bosons $W^{ \pm}$and $Z^{0}$ to fermions are slightly more complicated, since they consist of two different types, a vectorial and an axial-vectorial part. For the charged weak bosons this results in a pure V-A structure, independent of the fermion's charge (Eq. (1.15)), while the coupling of the $Z^{0}$ boson depends on the charge of the fermion it couples to:

$$
\begin{aligned}
& -i \frac{g}{\sqrt{2}} \quad \gamma^{\mu} \quad \frac{1}{2}\left(1-\gamma^{5}\right) \\
& -i \frac{g}{\cos \theta_{w}} \gamma^{\mu} \frac{1}{2}\left(g_{V}-g_{A} \gamma^{5}\right), \quad \text { with: }\left\{\begin{array}{l}
g_{V}=T_{3}-2 Q_{f} \cdot \sin ^{2} \theta_{w} \\
g_{A}=T_{3}
\end{array}\right.
\end{aligned}
$$

All vertex factors, i.e. the couplings of the gauge boson to the fermions, including those of the photon and the gluons, are summarised in Table 1.3 in section 1.2.3, where the mass eigenstates of the gauge bosons are listed.

For the description of the SM-particles and all their interactions the above ingredients would be sufficient. Problems arise when the observed masses of the fermions and the massive vector bosons $W^{ \pm}$and $Z^{0}$ ought to be included in the theoretical framework. Mass terms for spin- $1 / 2$ particles (the fermions) have the form $m \bar{\psi} \psi=m\left(\bar{\psi}_{R} \psi_{L}+\bar{\psi}_{L} \psi_{R}\right)$, which does not transform as a $\mathrm{SU}(2)_{L}$ singlet as is required to retain the gauge invariance of the Lagrangian. On the other hand, mass terms for the vector bosons $W_{\mu}^{ \pm}$and $Z^{0}$ take the form $m^{2} W_{\mu} W^{\mu}$, which is not invariant under gauge transformations of the field $W_{\mu}$, hence, both such types of mass terms cannot be part of a $\mathrm{SU}(3)_{C} \otimes \mathrm{SU}(2)_{L} \otimes \mathrm{U}(1)_{Y}$ invariant Lagrangian. An elegant solution is provided by the Higgs mechanism, named after P.W. Higgs, who invented this mechanism of mass generation in non-Abelian gauge theories [6], based on earlier work of Nambu, Goldstone and in particular Anderson [22]. 


\subsubsection{The Higgs Mechanism}

The main characteristics of the Higgs mechanism are the spontaneous symmetry breaking and the subsequent generation of gauge boson masses. In case of the SM, this is achieved by introducing a characteristic potential with a degenerate ground state, corresponding to a $\mathrm{SU}(2)_{L} \otimes \mathrm{U}(1)_{Y}$ rotation symmetry, into the SM Lagrangian, see also Figure 1.1. Choosing one specific state as vacuum expectation value amounts to breaking this initial symmetry.

The mass terms of the gauge bosons are contained in the Higgs-Lagrangian $\mathcal{L}_{\text {Higgs }}$ mentioned above, while the Yukawa Lagrangian $\mathcal{L}_{\text {Yukawa }}$ contains mass terms for the SM-fermions, which are introduced by so-called »Yukawa couplings « of the fermions to the new scalar Higgs field $H(x)$. The Lagrangian density of Equation (1.8) remains invariant if an additional contribution of the form

$$
\mathcal{L}_{\text {Higgs }}=\left(\mathcal{D}_{\mu} \Phi\right)^{\dagger} \mathcal{D}^{\mu} \Phi-V(\Phi)
$$

is added. The field $\Phi(x)$ is a two-component complex scalar field forming a weak isospin doublet with weak hypercharge $Y_{w}=1$ :

$$
\Phi(x)=\left(\begin{array}{c}
\phi^{+} \\
\phi^{0}
\end{array}\right)=\frac{1}{\sqrt{2}}\left(\begin{array}{c}
\varphi_{1}+i \varphi_{2} \\
\varphi_{3}+i \varphi_{4}
\end{array}\right)
$$

and the $\mathrm{SU}(2)_{L}$ invariant potential is of the form:

$$
V(\Phi)=\mu^{2} \Phi^{\dagger} \Phi+\lambda\left(\Phi^{\dagger} \Phi\right)^{2}
$$

with real parameters $\mu$ and $\lambda$. For stability reasons $\lambda$ has to be positive, such that $V \rightarrow \infty$ for large values of the $\varphi_{i}$, but the mass parameter $\mu^{2}$ may still be negative. Due to the invariance of the Higgs potential under $\mathrm{SU}(2)_{L}$ transformations, for $\mu^{2}<0$, a whole family of non-trivial minima exists:

$$
\Phi^{\dagger} \Phi=\frac{1}{2}\left(\varphi_{1}^{2}+\varphi_{2}^{2}+\varphi_{3}^{2}+\varphi_{4}^{2}\right)=-\frac{\mu^{2}}{2 \lambda} .
$$

The initial $\mathrm{SU}(2)_{L}$ symmetry is broken spontaneously as soon as a specific minimum is chosen as the vacuum

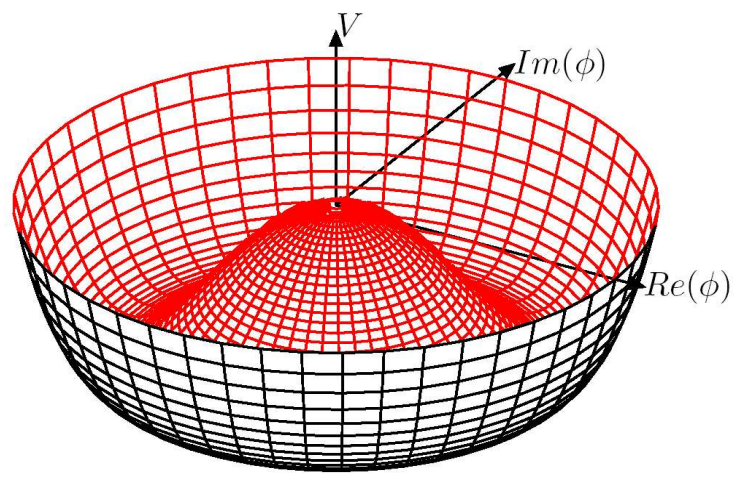

Figure 1.1: The Higgs potential. ground state. Local gauge invariance allows to choose the ground state such that the charged upper component is eliminated and only a neutral scalar field remains. These considerations lead to the choice $\varphi_{1}=\varphi_{2}=\varphi_{4}=0$ and $\varphi_{3}=v$, resulting in the following expression for the remaining complex scalar field $\Phi(x)$, with corresponding vacuum expectation value (VEV) $\langle 0|\Phi| 0\rangle=v=\sqrt{\frac{-\mu^{2}}{\lambda}}:$

$$
\Phi(x)=\frac{1}{\sqrt{2}}\left(\begin{array}{c}
0 \\
v+H(x)
\end{array}\right) .
$$

The remaining real field $H(x)$ is identified with the one physical Higgs boson, while three of the originally four degrees of freedom (Nambu-Goldstone bosons [22]) reappear only later in the process of mass generation, where they form the longitudinal degrees of freedom of the electroweak gauge bosons $W_{\mu}^{ \pm}$and $Z_{\mu}$. Substituting the above representation of the Higgs 
field into the Higgs Lagrangian (Eq. (1.17)) and expanding $\mathbf{T} \mathbf{W}_{\mu}$ of the covariant derivative (Eq. (1.10)) in a spherical basis ${ }^{4}$ leads to the following Lagrangian density:

$$
\begin{aligned}
\mathcal{L}_{\text {Higgs }}= & \left(\frac{1}{2} g\right)^{2} W^{-\mu} W_{\mu}^{+}(v+H)^{2}+\frac{1}{8}\left(g W_{3}^{\mu}-g^{\prime} B^{\mu}\right)\left(g W_{\mu}^{3}-g^{\prime} B_{\mu}\right)(v+H)^{2} \\
& +\frac{1}{2}\left(\partial^{\mu} H\right)\left(\partial_{\mu} H\right)-\frac{\mu^{2}}{2}(v+H)^{2}-\frac{\lambda}{4}(v+H)^{4} .
\end{aligned}
$$

The first and second terms contain the mass terms of the electroweak gauge bosons, $W_{\mu}^{ \pm}$and $Z_{\mu}$ (terms $\propto v^{2}$ ) and terms predicting tri- and quadrilinear couplings of the gauge bosons to the new scalar Higgs boson (terms $\propto v H$, or $H^{2}$ in the first line of $\mathcal{L}_{\text {Higgs }}$ ).

Using the relations (1.13) to (1.14), the physical gauge fields are identified and the coefficients in front of the terms $\propto v^{2}$ can now be used to infer their masses, leading to:

$$
M_{W}^{2}=\frac{1}{4} v^{2} g^{2} \quad M_{Z}^{2}=\frac{1}{4} v^{2}\left(g^{2}+g^{\prime 2}\right) \quad M_{\gamma}^{2}=0 .
$$

Although the absolute masses of the $W^{ \pm}$and $Z^{0}$ bosons cannot be predicted, the above terms allow to establish an important relation between the masses of the spin-1 gauge bosons:

$$
\frac{M_{W}}{M_{Z}}=\frac{g}{\sqrt{g^{2}+g^{\prime 2}}}=\cos \theta_{w} .
$$

Independent measurements of all three observables, $M_{W}, M_{Z}$ (Table 1.3) and the weak mixing angle $\sin ^{2} \theta_{w}$ allow for a stringent test of this theoretically predicted relation in the SM [23].

Since $\Phi$ is invariant under transformations of the sub group $\mathrm{U}(1)_{E M}$, the photon field $A_{\mu}$, which is proportional to the orthogonal combination $g B_{\mu}+g^{\prime} W_{\mu}^{3}$, does not couple to the Higgs field, hence the photon remains massless as required. Table 1.3 provides an overview of the mass eigenstates of all SM gauge bosons, their observed masses (or upper bounds in case of the photon and the gluons), the interactions they mediate and their vertex factors, i.e. their coupling strengths to the fermions, respectively.

\begin{tabular}{lccc}
\hline \hline Boson name & Interaction & Observed mass & Vertex factors \\
\hline \hline photon $\gamma,\left(A_{\mu}\right)$ & electromagnetic & $<6 \cdot 10^{-17} \mathrm{eV}$ & $-i e Q_{f} \gamma^{\mu}$ \\
$Z^{0}$ boson, $\left(Z_{\mu}\right)$ & electroweak & $91.1876 \pm 0.0021 \mathrm{GeV}$ & $-i \frac{g}{\cos \theta_{w}} \gamma^{\mu} \frac{1}{2}\left(g_{V}-g_{A} \gamma^{5}\right)$ \\
$W^{ \pm}$bosons,,$\left(W_{\mu}^{ \pm}\right)$ & weak & $80.425 \pm 0.038 \mathrm{GeV}$ & $-i \frac{g}{\sqrt{2}} \gamma^{\mu} \frac{1}{2}\left(1-\gamma^{5}\right)$ \\
gluons $g,\left(G_{\mu}^{a}\right)$ & strong & 0 (theo. value) & $-i g_{s} \frac{\lambda^{\alpha}}{2} \gamma^{\mu}$ \\
\hline \hline
\end{tabular}

Table 1.3: The gauge boson fields of the Standard Model represented in their mass eigenstates. Three $S U(2)_{L}$ degrees of freedom of the Higgs field are absorbed by the three electroweak gauge bosons $W^{+}$, $W^{-}$and $Z^{0}$, which thus acquire their masses (from [23]). Only the ratio of the $W$ and $Z$ boson masses, $M_{W} / M_{Z}=\cos \theta_{w}$ is theoretically predictable, not the absolute values.

The remaining terms in the second line of $\mathcal{L}_{\text {Higgs }}$ (Eq. (1.22)) govern the dynamics of the physical, scalar Higgs boson, its kinetic energy and its self-couplings (trilinear and quadrilinear couplings are predicted by terms tri- or quadrilinear in $H$ ). Finally, the mass of the Higgs boson itself

\footnotetext{
${ }^{4} \mathbf{T} \mathbf{W}_{\mu}=\sqrt{2}\left(T^{+} W_{\mu}^{+}+T^{-} W_{\mu}^{-}\right)+T^{3} W_{\mu}^{3}$, where $T^{ \pm}=T_{1} \pm i T_{2}$ represent the weak isospin operators.
} 
must be of the form $\frac{1}{2} m^{2} H$, since it is a scalar spin-0 particle. Thus, collecting all terms, where only the field $H^{2}$ appears, leads to $M_{H}=\sqrt{2 \lambda} v$. Unfortunately the mass of the Higgs boson can neither be predicted since both parameters of the potential, $\lambda$ and $\mu$ are free parameters of the theory. Only the vacuum expectation value can be estimated by comparing Fermi's currentcurrent theory [24] of the $\beta$-decay with its electroweak formulation in the limit of negligible momentum transfer $\left(q^{2} \ll M_{W}^{2}\right.$, including the dependence of $M_{W}$ on $\left.v\right)$, which leads to:

$$
\begin{aligned}
\frac{G_{F}}{\sqrt{2}}=\frac{g^{2}}{8 M_{W}^{2}} & =\frac{1}{2 v^{2}} \longrightarrow v^{2}=\frac{1}{\sqrt{2} G_{F}} \cong(246 \mathrm{GeV})^{2} \\
& \text { resulting in a VEV: } \frac{v}{\sqrt{2}} \cong 174 \mathrm{GeV} .
\end{aligned}
$$

Here, $G_{F}=1.16637(1) \cdot 10^{-5} \mathrm{GeV}^{-2}[25]$ is the Fermi constant, which has been measured very precisely in muon decays.

With the introduction of the Higgs field not only the weak gauge bosons acquire masses, but also the fermions interacting with the Higgs field. Mass terms for spin- $1 / 2$ particles are of the form $m_{f} \bar{f} f$, they are introduced via Yukawa couplings of the fermions to the $\mathrm{SU}(2)_{L}$ Higgs doublet. The Yukawa Lagrangian thus reads:

$$
\mathcal{L}_{\text {Yukawa }}=-g_{f}\left[\bar{L} \Phi R+\bar{R} \Phi^{\dagger} L\right]=-\frac{1}{\sqrt{2}} g_{f} v \bar{f} f-\frac{1}{\sqrt{2}} g_{f} \bar{f} f H
$$

with $L, R$ representing left-chiral doublets and right-chiral singlets under $\mathrm{SU}(2)_{L} \otimes \mathrm{U}(1)_{Y}$ transformations, respectively. The first term describes the fermion's mass $m_{f}=g_{f} v / \sqrt{2}$, while the second term describes the fermion's coupling to the Higgs boson with a coupling strength proportional to the fermion's mass, $g_{f} / \sqrt{2}$. Since the coupling constants, $g_{f}$, are not predicted theoretically or related to each other, they may differ for every fermion. This makes the fermion masses, in addition to the mass of the Higgs boson itself, free parameters of the theory.

In summary, the Lagrangian of the SM, after the breaking of the electroweak $\mathrm{SU}(2)_{L} \otimes \mathrm{U}(1)_{Y}$ symmetry $(E W S B)$ - as introduced in the last section - can be written as follows

$$
\begin{aligned}
\mathcal{L}_{S M}= & +\bar{L} \gamma^{\mu}\left(\partial_{\mu}-i g_{s} \mathbf{T}^{a} \mathbf{G}_{\mu}^{a}-i g \mathbf{T} \mathbf{W}_{\mu}+i g^{\prime} Y_{w} B_{\mu}\right) L \\
& +\bar{R} \gamma^{\mu}\left(\partial_{\mu}-i g_{s} \mathbf{T}^{a} \mathbf{G}_{\mu}^{a}+i g^{\prime} Y_{w} B_{\mu}\right) R \\
& -\frac{1}{4}\left[\mathbf{G}_{a}^{\mu \nu} \mathbf{G}_{\mu \nu}^{a}+\mathbf{W}^{\mu \nu} \mathbf{W}_{\mu \nu}+B^{\mu \nu} B_{\mu \nu}\right] \\
& +\left|\left(\partial_{\mu}-i g_{s} \mathbf{T}^{a} \mathbf{G}_{\mu}^{a}-i g \mathbf{T} \mathbf{W}_{\mu}+i g^{\prime} Y_{w} B_{\mu}\right) \Phi\right|^{2}-V(\Phi) \\
& -\frac{1}{\sqrt{2}} g_{f} v \bar{f} f-\frac{1}{\sqrt{2}} g_{f} \bar{f} f H .
\end{aligned}
$$

The first two lines of the Lagrangian describe the fermion dynamics, i.e. their kinetic energy and interactions with the SM gauge bosons. All fermions thus interact with the gluons of QCD and the neutral electroweak bosons, $\gamma$ and $Z^{0}$ (ii); left-chiral isospin doublets additionally interact with the charged weak bosons $W^{ \pm}$(i). In the third line (iii) the dynamics of the gauge bosons are described by their respective field strength tensors. The fourth line (iv) contains the mass terms of the electroweak bosons and the Higgs boson and furthermore describes their interactions, as 
well as the Higgs self-interactions. Finally, the first term in the last line (v), holds the fermion mass terms $\left(m_{f}\right)$, while the second and last term governs the interactions of fermions and the scalar Higgs boson.

\subsubsection{Open Questions and Limitations}

As was already mentioned earlier, the SM can only be a low energy effective theory, because the fourth fundamental force, gravity, is not included, since so far no consistent quantum field theory of gravity has been found. Fortunately it is possible to simply neglect gravitational interactions in the SM due to the weakness of the gravitational force, which is about 40 orders of magnitude smaller than the electromagnetic force. This works well at relatively low energies, but ultimately, at the latest at the Planck scale $\left(M_{P}=\sqrt{\frac{\hbar c}{G_{N}}} \approx 1.2 \cdot 10^{19} \mathrm{GeV}\right)$, where quantum gravitational effects become important, gravity has to be included into the description.

Another weakness of the SM is the so-called naturalness problem [26], referring to the large difference between the electroweak and the Planck scale $\left(M_{P} / M_{E W} \approx \mathcal{O}\left(10^{17}\right)\right)$, for which the SM provides no explanation. The naturalness problem is connected to yet another difficulty of the SM, known as the hierarchy problem, which is a synonym for the high sensitivity of the Higgs potential to quantum corrections. The effective Higgs boson mass is expected to be of the order of $100 \mathrm{GeV}$, as suggested by fits to the electroweak precision data [27] and the lower limit of $M_{H}>114.4 \mathrm{GeV}$ from direct searches performed at the Large Electron Positron collider, LEP [28]. Like all masses in the SM, this effective value, $M_{H}$ comprises the Higgs pole mass plus radiative corrections of virtual effects from all particles coupling directly or indirectly to the Higgs field. These corrections, which are quadratically divergent in the relevant energy scale, enter into the calculation via fermionic loops and bosonic loops from scalar (spin-0) particles, as shown in Figure 1.2 for one-loop corrections.

(a)

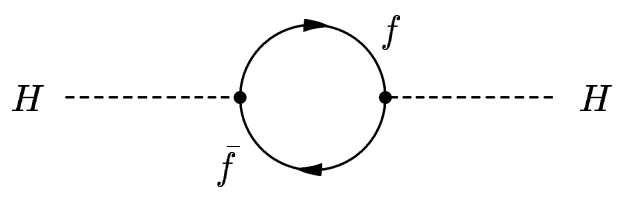

$H, f$ coupling: $-\lambda_{f} H \bar{f} f$ (b)

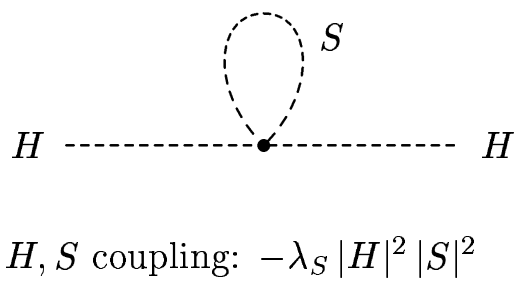

Figure 1.2: Corrections to the Higgs boson mass from (a) fermions, (b) scalar particles.

Assuming the SM to be valid up to a high energy scale, $\Lambda_{U V}$, the bosonic loops would lead to a Higgs pole mass of the order of the ultraviolet cutoff $\left(M_{H}^{\text {pole }} \approx \mathcal{O}\left(\Lambda_{U V}\right)\right.$. Although fermionic loops may compensate this huge pole mass, such that the effective value is of the order of the electroweak scale again $\left(M_{H} \approx \mathcal{O}(100 \mathrm{GeV})\right)$, this would require an enormous fine tuning with an accuracy of approximately $10^{-34}$.

The »running « of the electroweak and strong coupling constants, $g, g^{\prime}$ and $g_{s}$, suggests their unification at a higher energy scale. However, when extrapolating their measured values to higher energy scales, the inverse of the couplings do approach each other, with $1 / g^{\prime}$ decreasing and $1 / g$ and $1 / g_{s}$ increasing with increasing energy, but they do not meet at a single point.

This specific problem of coupling unification is addressed by many of the currently considered Grand Unified Theories (GUTs), most of which assume a supersymmetric extension of the SM 
as their low energy limit. A comparison of the situation in the SM with the one in a phenomenological supersymmetric model (MSSM) is presented in Figure 1.3 of section 1.3.1.

Massive neutrinos are also not described by the SM, although it is clear, that neutrinos do possess mass. Recent data indicate the existence of neutrino flavour transformations, consistent with at least two non-zero mass differences $[18,19,20]$. Nevertheless, the nature of their masses is still entirely unclear, which is partly due to the smallness of their masses. In addition, it is also unknown, if neutrinos are Dirac $(\bar{\nu} \neq \nu)$ or Majorana particles $(\bar{\nu} \equiv \nu)$. Future neutrino oscillation experiments may be able to measure the mass differences $\left(\Delta m_{1}\right.$ and $\left.\Delta m_{2}\right)$ very precisely, yet the only known process to distinguish Dirac from Majorana neutrinos is neutrinoless double $\beta$-decay $(0 \nu \beta \beta)$ [29], and [30]. This process can only occur, if the neutrino is its own antiparticle, so that a neutrino emitted by one nucleus is immediately absorbed by another nucleus.

The SM does not provide any explanation of dark matter or dark energy. These two components are part of the Cosmological Standard Model (sometimes also abbreviated as $\Lambda C D M$, for $\Lambda$ Cold Dark Matter) whose parameters have been measured by the COBE satellite (COsmic Background Explorer) [31] and recently more precisely by the WMAP experiment (Wilkinson Microwave Anisotropy Probe) [32]. Among other results concerning the age or expansion rate of the universe, the best fit to the data from measurements of anisotropies in the microwave background radiation at high angular resolution suggests that the universe is made up of only $4 \%$ of ordinary, baryonic matter, followed by about $23 \%$ of dark matter and more than $70 \%$ of dark energy, which corresponds to the cosmological constant $\Lambda$ in Einstein's field equations of General Relativity. Einstein had originally introduced this constant term to allow for a static universe - this was, what he later called his »biggest blunder «- and after the discovery of the expansion of the universe ${ }^{5}$ he completely removed $\Lambda$ from his equations.

Since the accelerating expansion rate of the universe would require a non-zero vacuum energy, the $\Lambda$ term was reintroduced into the field equations. Unfortunately the measured value of $\Lambda$ is more than 30 orders of magnitude lower than the value predicted by the SM according to quantum fluctuations alone [33].

At last it should be mentioned, that the predictive power of the SM is somewhat lessened by the large number of free parameters. The 6 quark flavours and 3 charged leptons lead to 13 parameters for the fermion sector alone, 9 fermion masses, 3 mixing angles and one CP-violating phase $\left(V_{C K M}\right)$. In case the neutrinos are not assumed to be massless, this number increases to 20 , since the 3 neutrino masses and their probable mixing angles and CP-phases, corresponding to the Maki-Nakagawa-Sakata matrix ( $V_{M N S}$ ) [34] need to be accounted for, as well. Furthermore, the strength of the three gauge couplings $g, g^{\prime}$ and $g_{s}=2 \sqrt{\pi} \alpha_{S}$, as well as the parameter $\theta_{Q C D}$, describing the $\mathrm{CP}$ violation in the strong interaction, need to be considered. In addition, the Higgs boson mass and the field's non-vanishing vacuum expectation value are also free parameters of the theory. Thus the SM depends on, at least, 19 arbitrary parameters, or, alternatively, 26 parameters, if massive neutrinos are considered.

Some of the above problems and questions are addressed by supersymmetric extensions of the Standard Model, of which the general aspects and two phenomenological models will be described in the following section.

\footnotetext{
${ }^{5}$ In 1929 E. Hubble and M.L. Humason formulated their empirical Redshift Distance Law of galaxies (now known as Hubble's Law), which is consistent with the solution of Einstein's equations of General Relativity, for an homogeneous, isotropic and expanding space.
} 


\subsection{Introduction to Supersymmetry}

First proposed about 30 years ago, in the early 1970-ies, by Y.A. Golfand, E.P. Likhtman, D.V. Volkov and V.P. Akulov [35], Supersymmetry (SUSY) is one of the many extensions of the Standard Model, designed to overcome some of its limitations. Supersymmetry postulates the existence of a new particle, a so-called superpartner, for each fundamental SM-particle, differing only by half a unit in spin, but otherwise sharing the same quantum numbers. Although, at first sight, supersymmetry seems to complicate things by essentially doubling the particle content of the model, it nevertheless promises to solve at least some of the numerous theoretical and phenomenological problems of the SM, which have been discussed in the last paragraph.

Starting with a short motivation of supersymmetry, this section serves as a general introduction into supersymmetry. After an overview of the particle content and the nomenclature of the new supersymmetric particles, the underlying theoretical formalism and some of the basic concepts are discussed, followed by a description of two exemplary phenomenological models: the minimal Supergravity (mSUGRA) model and the Minimal Supersymmetric Standard Model (MSSM), both of which are used to interpret the results of this search in the final chapter. This section is primarily based on references [36, 37, 38, 39].

\subsubsection{Motivating Supersymmetry}

Although it is clear from the non-observation of any supersymmetric particles, that supersymmetry itself must be broken, there are still powerful arguments favouring supersymmetric extensions of the SM. Most of these arguments require SUSY-particle masses of the order of $\mathcal{O}(100 \mathrm{GeV}-1 \mathrm{TeV})$, which is even more desirable from an experimental point of view, since these energy regimes are testable in part already at the Fermilab Tevatron or in the near future at the Large Hadron Collider ( $L H C$ ) or the International Linear Collider (ILC).

One of the strongest arguments for low scale supersymmetry, is the elegant avoidance of the Higgs potential's disturbing sensitivity to quantum corrections, which is achieved by the mere assumption of a symmetry relating fermions and bosons. One-loop corrections to the squared Higgs boson mass, $\Delta m_{H}^{2}$, as shown in Figure 1.2, are proportional to:

$$
\begin{array}{lll}
\text { fermion loops: } & \Delta m_{H}^{2} \propto\left|\lambda_{f}\right|^{2}\left[-2 \Lambda_{U V}^{2}+6 m_{f}^{2} \ln \left(\frac{\Lambda_{U V}}{m_{f}}\right)+\cdots\right] \\
\text { boson loops: } & \Delta m_{H}^{2} \propto \lambda_{S}\left[+\Lambda_{U V}^{2}-2 m_{S}^{2} \ln \left(\frac{\Lambda_{U V}}{m_{S}}\right)+\cdots\right] .
\end{array}
$$

These terms already suggest a relation between fermions and bosons due to the relative minus sign of their respective higher order contributions to $m_{H}^{2}$ [38], [40] and [41]. The introduction of two complex scalar partners for each SM-fermion, corresponding to their left- and right-chiral states, with $\lambda \equiv \lambda_{S}=\left|\lambda_{f}\right|^{2}$ yields a systematic cancellation [42] of all of these higher order contributions of bosonic or fermionic origin, such that the Higgs potential is stabilized against radiative corrections. For one SM-particle this correction to the squared Higgs boson mass reads:

$$
\Delta m_{H}^{2} \approx \lambda^{2}\left(m_{f}^{2}-m_{S}^{2}\right) .
$$

So far, none of the predicted SUSY-particles has been observed experimentally, which leads to the conclusion that SUSY must be broken, so that the masses of SM-particles and their superpartners 
differ. As can be seen from Equation (1.29) the argument of small corrections to the Higgs boson mass weakens, if the mass differences between SM-particles and their superpartners become too large. This is, why relatively »light « superpartners with masses in the $1 \mathrm{TeV}$ range are preferred.

Another quite intriguing argument is the integration of gravity in the framework of quantized gauge theories, which is possible in some supersymmetric models, i.e. Supergravity (SUGRA) models. In these models, the SUSY-Lagrangian is required to remain invariant under local transformations of supersymmetry, which naturally leads to the postulate of a graviton field, corresponding to a spin-3/2 fermion, the gravitino. The SM-partner of this spin-3/2 particle would then be the spin-2 graviton, as the gauge boson of gravity. Thus the required local invariance under SUSY transformation, not only »allows «, but »necessitates « the inclusion of gravity. In paragraph 1.3.5 the $»$ minimal « Supergravity (mSUGRA) model, i.e. the one with minimal particle content, will be discussed in more detail.

The possibility of gauge coupling unification at an energy scale of roughly $10^{16} \mathrm{GeV}$ in supersymmetric models (see Figure 1.3) nourishes the hope that the electromagnetic, weak and strong interactions only seem to be different at the electroweak scale $(\approx 100 \mathrm{GeV})$, but, in reality, are only different manifestations of a single overarching force. So-called Grand Unified Theories (GUTs) attempt to construct one Quantum Field Theory (QFT), with one »unified « gauge coupling to describe this »one force« and thus, all known interactions.
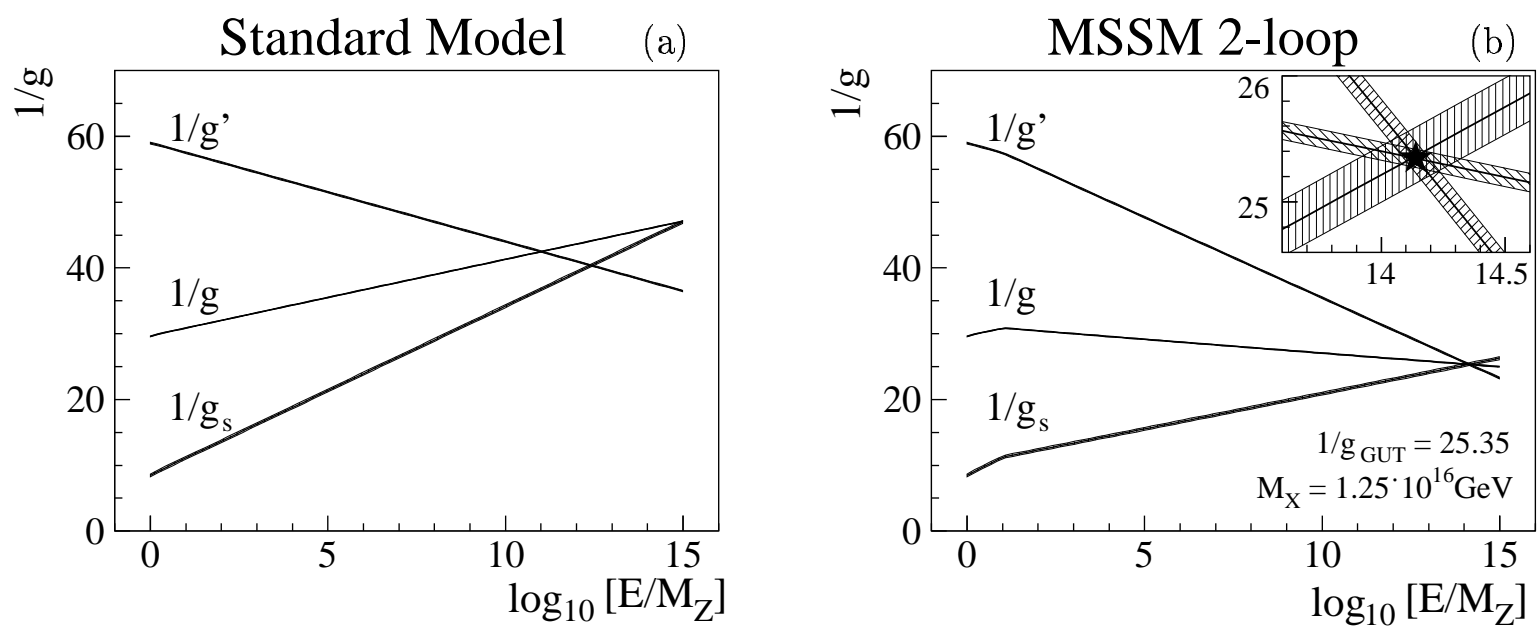

Figure 1.3: The scale dependence of the inverse gauge couplings as a function of the logarithm of energy in the SM (a) and the MSSM including threshold corrections of SUSY particles (b). In case of the MSSM the unification of couplings can be achieved. (This figure was extracted from [43]).

Moreover SUSY models with conserved R-parity (see section 1.3.6) require the lightest supersymmetric particle (LSP) to be stable. This would - at least for a neutral LSP - provide a good candidate for cold dark matter [44], which may also explain the great interest in SUSY models with conserved R-parity, though a more general model allows for small R-parity violating $\left(\not \mathbb{R}_{p}\right)$ terms, too. These terms must be small, since, although not theoretically forbidden, their existence is severely constrained by the non-observation of proton decay [45]. 


\subsubsection{The Particle Content in Supersymmetric Models}

As has already been mentioned, supersymmetry introduces a corresponding superpartner for each SM-particle differing only by half a unit in spin, and, thus, effectively doubling the particle content of the SM. All particles of the MSSM (Minimal Supersymmetric SM, see section 1.3.5) are represented in supermultiplets in Table 1.4. Similar to the familiar multiplets of the SM, there exist two different types: supermultiplets of chiral fields $(\boldsymbol{\Phi})$ and of gauge fields $(\boldsymbol{V})$.

The names of the SUSY-particles are derived from the SM-particles' names. A SUSY-partner of an ordinary fermion, e.g. lepton/quark, is called sfermion, or $\operatorname{slepton}(\widetilde{\ell}) / \operatorname{squark}(\widetilde{q})$, respectively. The same convention is used for the single particle names, e.g.: electron $\rightarrow$ selectron $(\widetilde{e})$, topquark $\rightarrow$ top-squark $(\widetilde{t})$, though the squarks are also often referred to as: stop, or sbottom. Each sfermion is also labelled with an index $L$ or $R$ to indicate to which of the two chiral states of the SM-particle $\left(\psi_{R}, \psi_{L}\right)$ the spin-0 SUSY-scalar belongs.

Supersymmetric spin-1/2 partners of the gauge bosons $\left(W_{1,2,3}, B\right.$ and $\left.G\right)$ are known as winos $\left(\widetilde{W}_{1,2,3}\right)$, binos $(\widetilde{B})$ and gluinos $(\widetilde{g})$, but there is no one-to-one correspondence between the mass eigenstates of the SM gauge bosons $\left(W^{ \pm}, \gamma, Z^{0}\right)$ and those of the SUSY-partners. Although names and symbols like, e.g. photino $(\widetilde{\gamma})$, zino $(\widetilde{Z})$, are sometimes used, no SUSY-particles, neither symmetry, nor mass eigenstates, are associated with them. Mass eigenstates of the $\mathrm{SU}(2)_{L}$ and $\mathrm{U}(1)_{Y}$ bosinos are formed after EWSB and SUSY-breaking (see section 1.3.4), by mixing of the respective symmetry eigenstates. Thus, winos, binos and the fermionic SUSY-partners of the Higgs bosons, the higgsinos, mix to form so-called charginos $\left(\widetilde{\chi}_{1,2}^{ \pm}\right)$and neutralinos $\left(\widetilde{\chi}_{1,2,3,4}^{0}\right)$, where increasing indices refer to increasing mass.

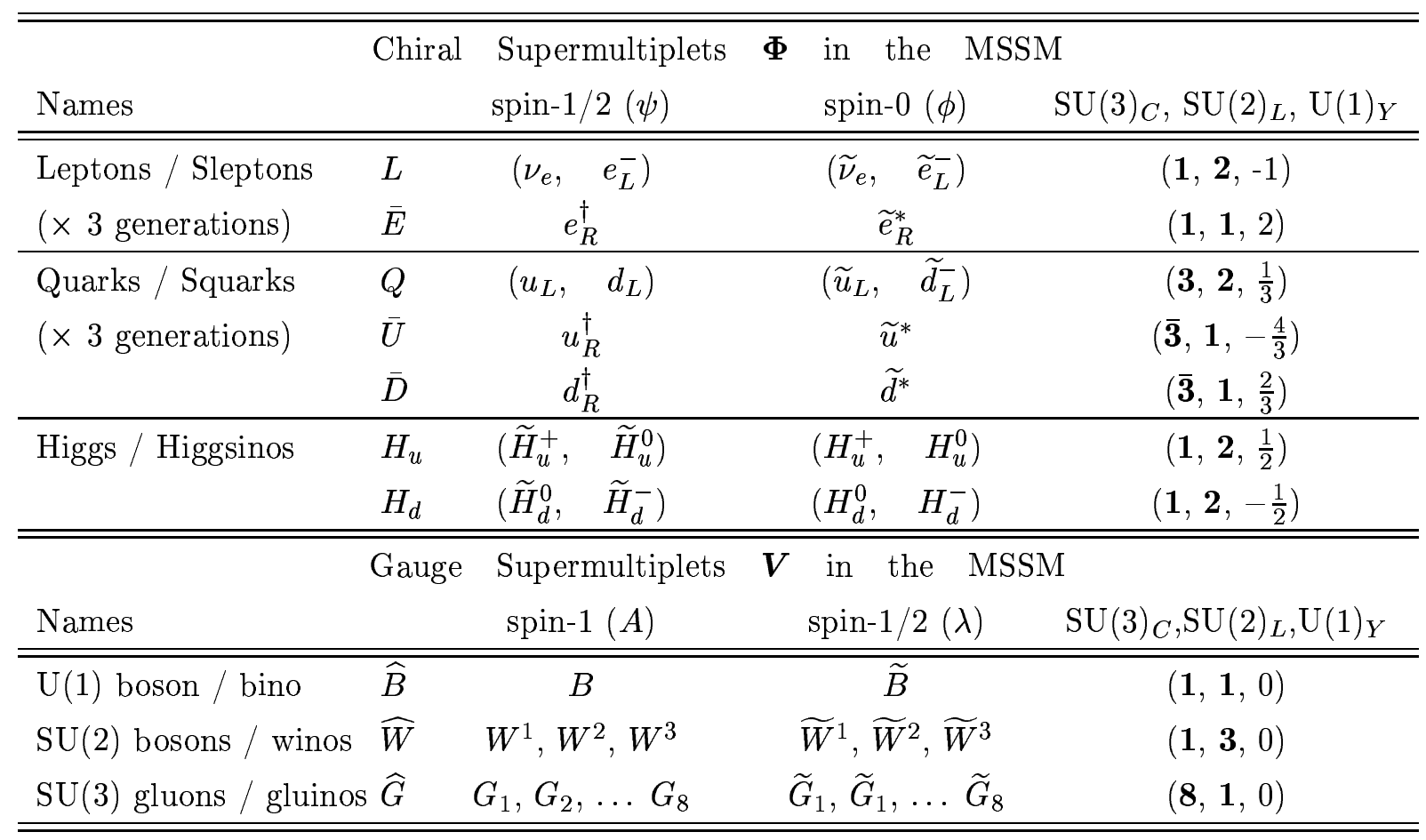

Table 1.4: The chiral and gauge supermultiplets of the MSSM. One generation of (s)fermions and the Higgs boson / higgsino doublets are presented as chiral supermultiplets. The convention of using left-chiral Weyl spinors leads to the appearance of the conjugates for the right-chiral quarks and leptons in the chiral supermultiplets. 
At least two Higgs supermultiplets are needed, because one Higgs doublet with its associated higgsinos is not sufficient to avoid triangle gauge anomalies, which in turn would invalidate the gauge symmetry of the SUSY-Lagrangian. These dangerous anomalies are absent in the SM due to the already existing fermions, but they would appear in a supersymmetric theory because of the fermionic superpartners of the scalar Higgs doublet, the higgsinos. Since the latter must be a weak

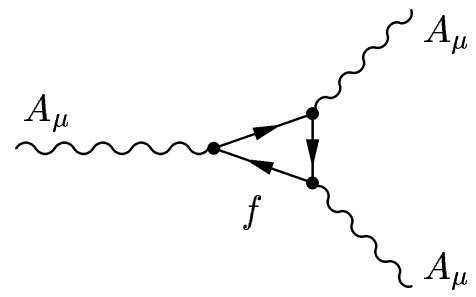

Figure 1.4: Triangle anomaly. isospin doublet, with weak hypercharge being either +1 or -1 , they will in either case make a non-zero contribution to the traces $\operatorname{Tr}\left[Y^{3}\right]=\operatorname{Tr}\left[T_{3}^{2} Y\right]$, which should give zero for a gauge anomaly free theory. To circumvent this problem and to ensure masses for up- and down-type quarks and leptons after EWSB a second Higgs doublet is introduced, with opposite hypercharge of its corresponding higgsino weak iso-doublet.

\subsubsection{The Theoretical Formalism}

Supersymmetry is, unlike the gauge symmetries discussed in section 1.2 , no internal, but a space-time symmetry able to transform a bosonic state $|S\rangle$ into a fermionic state $|F\rangle$ and vice versa

$$
\mathcal{Q}|B\rangle=|F\rangle, \quad \text { and } \quad \mathcal{Q}|F\rangle=|B\rangle .
$$

The Operator $\mathcal{Q}$ is thus an anticommuting complex spinor, whose hermitian conjugate, $\overline{\mathcal{Q}}$, is also a symmetry generator. Analogously to the SM, Supersymmetry is based on the Poincaré algebra (more exactly on an extension of this algebra [46]) defined on the four-dimensional Minkowski spacetime. The SUSY-Lagrangian, as well as the SM-Lagrangian, is invariant under global Poincaré transformations, i.e. under translations $\left(\mathcal{P}^{\mu}\right)$ and rotations and Lorentz boosts $\left(\mathcal{J}^{\mu \nu}\right)$, which satisfy the following relations:

$$
\begin{aligned}
{\left[\mathcal{P}^{\mu}, \mathcal{P}^{\nu}\right] } & =0 \\
{\left[\mathcal{J}^{\mu \nu}, \mathcal{J}^{\alpha \beta}\right] } & =i\left(\mathcal{J}^{\mu \alpha} g^{\nu \beta}+\mathcal{J}^{\nu \beta} g^{\mu \alpha}-\mathcal{J}^{\mu \beta} g^{\nu \alpha}-\mathcal{J}^{\nu \alpha} g^{\mu \beta}\right) \\
{\left[\mathcal{J}^{\mu \nu}, \mathcal{P}^{\alpha}\right] } & =i\left(\mathcal{P}^{\nu} g^{\mu \alpha}-\mathcal{P}^{\mu} g^{\nu \alpha}\right),
\end{aligned}
$$

where $g_{\mu \nu}=\operatorname{diag}(1,-1,-1,-1)$ is the metric tensor. In addition to the above equations, the following commutators relate the supersymmetric and the Poincaré generators

$$
\begin{aligned}
{\left[\mathcal{Q}_{a}, \mathcal{P}_{\mu}\right]=\left[\overline{\mathcal{Q}}_{a}, \mathcal{P}_{\mu}\right] } & =0 \\
{\left[\mathcal{J}_{\mu \nu}, \mathcal{Q}_{a}\right]=\left[\mathcal{J}_{\mu \nu}, \overline{\mathcal{Q}}_{a}\right] } & =-i\left(\sigma_{\mu \nu}\right)_{a}^{b} \mathcal{Q}_{b}
\end{aligned}
$$

while the following anti-commutators, where $[A, B]_{-}=A B+B A$, define the relations among the fermionic supersymmetry generators, $\mathcal{Q}$ and $\overline{\mathcal{Q}}$, themselves:

$$
\begin{aligned}
{\left[\mathcal{Q}_{a}, \mathcal{Q}_{b}\right]_{-}=} & {\left[\overline{\mathcal{Q}}_{a}, \overline{\mathcal{Q}}_{b}\right]_{-}=} \\
& {\left[\mathcal{Q}_{a}, \overline{\mathcal{Q}}_{b}\right]_{-}=2\left(\sigma^{\mu}\right)_{a b} \mathcal{P}_{\mu}, }
\end{aligned}
$$

where $\sigma_{\mu \nu}$ is a $2 \times 2$ matrix given by $\sigma_{\mu \nu}=\frac{1}{4}\left(\bar{\sigma}^{\mu} \sigma^{\nu}-\bar{\sigma}^{\nu} \sigma^{\mu}\right)$, with $\sigma^{\mu}=\left(\sigma^{0}, \sigma^{i}\right)$ and $\sigma^{i}$ are the Pauli Spin matrices. Since supersymmetry is a spacetime symmetry, independent of the internal symmetries, all its generators commute with those of the internal symmetries. It follows that 
all particles of the same supermultiplet have the same quantum numbers, like electric charge, weak isospin and hypercharge, colour, lepton and baryon numbers, etc. As can be seen from Equations (1.32), the operator $\mathcal{P}^{2}=\mathcal{P}_{\mu} \mathcal{P}^{\mu}$, which corresponds to (mass) ${ }^{2}$, commutes with the supersymmetric operators so that all particles in a supermultiplet should also have the same mass. At this very point it becomes clear, that supersymmetry cannot be an exact symmetry, but must be broken at a higher energy scale.

The number of supersymmetry generators, i.e. pairs of $\mathcal{Q}, \overline{\mathcal{Q}}$, define the number of supersymmetries, $N$, since there may be more than just one distinct pair of generators. However, for phenomenologically viable, »ordinary « supersymmetry, one usually considers only the case $N=1$, because $N>1$ would require more than four dimensions to allow for chiral fermions and parity violation as observed in the SM.

\section{A SUSY Lagrangian for a free chiral supermultiplet}

Starting from the simplest possible Lagrangian of a single two-component Weyl fermion $(\psi)$ and its corresponding scalar superpartner $(\phi)$, the basic concepts of SUSY transformations and the role of additional scalar fields $F$ are discussed. The Lagrangian $\mathcal{L}_{\text {free }}^{W Z}$ of a single massless, non-interacting particle, first introduced by J. Wess and B. Zumino [47], reads:

$$
\mathcal{L}_{\text {free }}^{W Z}=\mathcal{L}_{\text {scalar }}+\mathcal{L}_{\text {fermion }}=-\partial^{\mu} \phi^{*} \partial_{\mu} \phi-i \psi^{\dagger} \bar{\sigma}^{\mu} \partial_{\mu} \psi
$$

A simple SUSY transformation, changing the scalar field $\phi$ into some fermionic field $\psi_{a}$, requires a slightly more complicated transformation of the fermion field, such that the action $S=\int d^{4} x \mathcal{L}$, remains invariant under supersymmetry transformations.

$$
\begin{array}{rlrl}
\text { scalar } \rightarrow \text { fermion } & \text { fermion } & \rightarrow \text { scalar } \\
\delta \phi & =\epsilon \psi & \delta \psi_{\alpha} & =i\left(\sigma^{\mu} \epsilon^{\dagger}\right)_{\alpha} \partial_{\mu} \phi \\
\text { h.c. } \delta \phi^{*} & =\epsilon \psi^{\dagger} & \text { h.c. } \delta \psi_{\alpha}^{\dagger} & =i\left(\epsilon \sigma^{\mu}\right)_{\alpha} \partial_{\mu} \phi^{*}
\end{array}
$$

where $\epsilon$ is an infinitesimal, anti-commuting, Weyl object parameterising the SUSY transformations. This leads to the following transformed scalar and fermionic parts of the free Lagrangian:

$$
\begin{aligned}
\delta \mathcal{L}_{\text {scalar }}= & -\epsilon \partial^{\mu} \psi \partial_{\mu} \phi^{*}-\epsilon^{\dagger} \partial^{\mu} \psi^{\dagger} \partial_{\mu} \phi \\
\delta \mathcal{L}_{\text {fermion }}= & +\epsilon \partial^{\mu} \psi \partial_{\mu} \phi^{*}+\epsilon^{\dagger} \partial^{\mu} \psi^{\dagger} \partial_{\mu} \phi \\
& -\partial_{\mu}\left(\epsilon \sigma^{\nu} \bar{\sigma}^{\mu} \psi \partial_{\nu} \phi^{*}+\epsilon \psi \partial^{\mu} \phi^{*}+\epsilon^{\dagger} \psi^{\dagger} \partial^{\mu} \phi\right) .
\end{aligned}
$$

As can be seen, the first two terms of $\delta \mathcal{L}_{\text {fermion }}$ cancel against the transformed scalar terms and the remaining terms, $-\partial_{\mu}(\ldots)$, do not contribute to the equations of motion, which are obtained from minimising the action $S$ :

$$
\delta S^{W Z}=\int d^{4} x\left(\delta \mathcal{L}_{\text {scalar }}+\delta \mathcal{L}_{\text {fermion }}\right)=0 .
$$

To ensure that the free Lagrangian and the chosen transformations really serve as a supersymmetric theory, the commutator of any two transformations must also be symmetric. Applying the commutator $\left[\delta_{\epsilon_{1}}, \delta_{\epsilon_{2}}\right]$ to a scalar field $\phi$, and to a fermion field $\psi$, results in:

$$
\begin{aligned}
{\left[\delta_{\epsilon_{1}}, \delta_{\epsilon_{2}}\right] \phi } & =i\left(\epsilon_{2} \sigma^{\mu} \epsilon_{1}^{\dagger}-\epsilon_{1} \sigma^{\mu} \epsilon_{2}^{\dagger}\right) \partial_{\mu} \phi \\
{\left[\delta_{\epsilon_{1}}, \delta_{\epsilon_{2}}\right] \psi_{\alpha} } & =i\left(\epsilon_{2} \sigma^{\mu} \epsilon_{1}^{\dagger}-\epsilon_{1} \sigma^{\mu} \epsilon_{2}^{\dagger}\right) \partial_{\mu} \psi_{\alpha}-i \epsilon_{2 \alpha} \epsilon_{1}^{\dagger} \bar{\sigma}^{\mu} \partial_{\mu} \psi+i \epsilon_{1 \alpha} \epsilon_{2}^{\dagger} \bar{\sigma}^{\mu} \partial_{\mu} \psi
\end{aligned}
$$


where the operator $i \partial_{\mu}$ is a spacetime operator, corresponding to the generator of spacetime translations, $\mathcal{P}_{\mu}$, of Equation (1.33). Obviously, the results of the commutator applied to a fermion field (Eq. 1.40) and to a scalar field (Eq. 1.39), are only symmetric, if the »classical « equations of motion $\bar{\sigma}^{\mu} \partial \psi=0$, are satisfied and the last two terms thus vanish. This special case is referred to as a theory being valid $\gg$ on-shell $«$, contrary to the $»$ off-shell « case, which implies that a theory is also valid quantum mechanically.

Since supersymmetry should be valid off-shell as well as on-shell (closed algebra), another complex, scalar field has to be introduced. This auxiliary field $F$, whose Lagrangian density is simply $\mathcal{L}_{\text {aux }}=F^{*} F$ allows to close the supersymmetry algebra, but does not generate any kinetic terms. To achieve a cancellation between the additional, off-shell terms of $\mathcal{L}_{\text {fermion }}$, the auxiliary field is transformed into a multiple of the equation of motion for the fermion field $\psi$ :

$$
\left.\begin{array}{rl}
\delta F & =i \epsilon^{\dagger} \bar{\sigma}^{\mu} \partial_{\mu} \psi \\
\text { h.c. } \quad \delta F^{*} & =-i \partial_{\mu} \psi^{\dagger} \bar{\sigma}^{\mu} \epsilon
\end{array}\right\} \delta \mathcal{L}_{a u x}=i \epsilon^{\dagger} \bar{\sigma}^{\mu} \partial_{\mu} \psi F^{*}-i \partial_{\mu} \psi^{\dagger} \bar{\sigma}^{\mu} \epsilon F
$$

This requires to modify the fermion field transformations, too, by adding the terms $\epsilon_{\alpha} F$ and $\epsilon_{\alpha}^{\dagger} F^{*}$ to the respective transformations $\gg$ fermion $\rightarrow$ scalar « given in Equation (1.35). These modified SUSY-transformations then lead to additional contributions to $\delta \mathcal{L}_{\text {fermion }}$, which cancel with the corresponding contributions of $\delta F$, except for a total derivative which is not relevant for the action or the derived equations of motion. All in all the commutator of the modified, free Lagrangian $\mathcal{L}_{\text {free }}^{W Z}=\mathcal{L}_{\text {scalar }}+\mathcal{L}_{\text {fermion }}+\mathcal{L}_{\text {aux }}$ can be written as follows:

$$
\left[\delta_{\epsilon_{1}}, \delta_{\epsilon_{2}}\right] X=i\left(\epsilon_{2} \sigma^{\mu} \epsilon_{1}^{\dagger}-\epsilon_{1} \sigma^{\mu} \epsilon_{2}^{\dagger}\right) \partial_{\mu} X,
$$

where $X$ stands for any of the fields $\phi, \psi, F$, or their respective conjugates. The introduction of the auxiliary field $F$ can also be understood from a physics (and not only a mathematical) point of view: effectively the complex, two-component Weyl fermion $\psi$ has four real degrees of freedom, while the complex scalar field $\phi$ has only two. On-shell the degrees of freedom of the scalar particles match the two spin-polarisation of the fermionic states, but off-shell the scalar field lacks two degrees of freedom. Thus, in order to adjust the bosonic and fermionic degrees of freedom also off-shell, an auxiliary complex, scalar field $F$, had to be introduced. These three fields and their conjugates now comprise a so-called chiral supermultiplet.

\section{A SUSY Lagrangian for a free, massless gauge supermultiplet}

The next ingredient to be considered, on the way to a complete SUSY-Lagrangian are gauge bosons and their respective superpartners. Again a non-interacting, massless gauge boson, $A_{\mu}^{a}$, with its fermionic partner, $\lambda^{a}$, are chosen for the gauge field Lagrangian:

$$
\mathcal{L}_{\text {gauge }}=-\frac{1}{4} \mathcal{F}_{\mu \nu}^{a} \mathcal{F}^{\mu \nu a}-i \lambda^{\dagger a} \bar{\sigma}^{\mu} \mathcal{D}_{\mu} \lambda^{a}+\frac{1}{2} D^{a} D^{a}
$$

where $\mathcal{D}_{\mu}$ and $\mathcal{F}_{\mu \nu}^{a}$ represent the usual covariant derivative and field strength tensor as introduced in Equations (1.4) and (1.6), respectively. Especially the covariant derivative $\mathcal{D}_{\mu}$ is required to ensure local gauge invariance. The index $a$ runs over the adjoint representation of the relevant gauge group, i.e. $a=1, \ldots 8$ for $\mathrm{SU}(3)_{C}$, or $a=1,2,3$ for $\mathrm{SU}(2)_{L}$ and $a=1$ for $\mathrm{U}(1)_{Y}$.

In analogy to the scalar, auxiliary field $F$, a bosonic, auxiliary field, usually named $D^{a}$, is needed in the gauge Lagrangian to close the algebra off-shell. Once again, this is due to the different number of bosonic and fermionic degrees of freedom. While the off-shell gaugino $\left(\lambda^{a}\right)$ exhibits 
four real degrees of freedom, the off-shell boson $\left(A_{\mu}^{a}\right)$ has only three real degrees of freedom. Like the scalar field $F$, the bosonic field $D^{a}$ has no associated kinetic term, which leads to its cancellation on-shell. The corresponding SUSY transformations for all the fields of a gauge supermultiplet $\left(A_{\mu}^{a}, \lambda^{a}, D^{a}\right)$ are:

$$
\begin{aligned}
\delta A_{\mu}^{a} & =-\frac{1}{\sqrt{2}}\left(\epsilon^{\dagger} \bar{\sigma}_{\mu} \lambda^{a}+\lambda^{\dagger a} \bar{\sigma}_{\mu} \epsilon\right) \\
\delta \lambda_{\alpha}^{a} & =-\frac{i}{2 \sqrt{2}}\left(\sigma^{\mu} \bar{\sigma}^{\nu} \epsilon \lambda^{a}\right)_{\alpha} \mathcal{F}_{\mu \nu}^{a}+\frac{1}{\sqrt{2}} \epsilon_{\alpha} D^{a} \\
\delta D^{a} & =-\frac{i}{\sqrt{2}}\left(\epsilon^{\dagger} \bar{\sigma}^{\mu} \mathcal{D}_{\mu} \lambda^{a}-\mathcal{D}_{\mu} \lambda^{\dagger a} \bar{\sigma}^{\mu} \epsilon\right) .
\end{aligned}
$$

Using these equations, the commutator of two infinitesimal SUSY transformations, applied to any of the gauge-covariant fields $X=\mathcal{F}_{\mu \nu}^{a}, \lambda^{a}, \lambda^{\dagger a}$, or $D^{a}$ thus reads:

$$
\left[\delta_{\epsilon_{1}}, \delta_{\epsilon_{2}}\right] X=i\left(\epsilon_{2} \sigma^{\mu} \epsilon_{1}^{\dagger}-\epsilon_{1} \sigma^{\mu} \epsilon_{2}^{\dagger}\right) \mathcal{D}_{\mu} X
$$

This ensures, that the supersymmetry algebra as defined in Equation (1.33) is realised also on any gauge-invariant combinations of fields in the gauge supermultiplets $\left(A_{\mu}^{a}, \lambda^{a}, D\right)$.

\section{Adding interactions to the free chiral and gauge Lagrangians}

Since the world of fundamental particles does not consist of free, non-interacting, massless particles, any phenomenological viable theory must also describe interactions between the different particles. First the interactions between fermions and their scalar superpartners, i.e. among chiral supermultiplets, are discussed and subsequently interactions between chiral and gauge fields are included. According to [38] it can be shown, that the most general, renormalisable interactions of chiral superfields can be written as:

$$
\mathcal{L}_{\text {int }}^{\text {chiral }}=-\frac{1}{2} W^{i j} \psi_{i} \psi_{j}+W^{i} F_{i}+c . c,
$$

where $W^{i j}$ and $W^{i}$ are functions of the scalar fields $\phi_{i}$ and $\phi^{* i}$, with dimensions (mass) or, at most, (mass) ${ }^{2}$. Since the free Lagrangian itself is already invariant under SUSY transformations, certain conditions need to be imposed on the $W^{i j}$ and $W^{i}$ to also guarantee the invariance of $\mathcal{L}_{\text {int }}^{\text {chiral }}$ under these transformations.

$$
\begin{aligned}
\delta \mathcal{L}_{\text {int }}^{\text {chiral }}= & -\frac{1}{2} \frac{\delta W^{i j}}{\delta \phi_{k}}\left(\epsilon \psi_{k}\right)\left(\psi_{i} \psi_{j}\right)-\frac{1}{2} \frac{\delta W^{i j}}{\delta \phi^{* k}}\left(\epsilon^{\dagger} \psi_{\dagger k}\right)\left(\psi_{i} \psi_{j}\right)+\text { h.c. } \\
& -i W^{i j} \partial_{\mu} \phi_{j} \psi_{i} \sigma^{\mu} \epsilon^{\dagger}-i W^{i} \partial_{\mu} \psi_{i} \sigma^{\mu} \epsilon^{\dagger}+\text { h.c. }
\end{aligned}
$$

Here the first line (i), permits constraining $W^{i j}$, since the first term can only vanish if $\delta W^{i j} / \delta \phi_{k}$ is totally symmetric under the exchange of the indices $i, j, k$ (Fierz identity), and the second term will only not contribute, if $W^{i j}$ does not depend on $\phi^{* k}$, which entails that the whole term vanishes due to the derivative with respect to $\phi^{* k}$. Both these arguments lead to

$$
W^{i j}=m^{i j}+y^{i j k} \phi_{k},
$$

where $m^{i j}$ can be identified as a symmetric mass matrix for the fermion fields, since the relevant term appears together with $\psi_{i} \psi_{j}$. Similarly $y^{i j k}$ can be understood as a trilinear Yukawa coupling 
of a scalar field $\phi_{k}$ and two fermionic fields $\psi_{i} \psi_{j}$. Further it is desirable that the terms in line (ii) of Equation (1.47) become a total derivative and thus irrelevant for the action $S$ or the equations of motion. This is only realised, if the $W^{i}$ can be expressed as

$$
\begin{aligned}
W^{i} & =m^{i j} \phi_{j}+\frac{1}{2} y^{i j k} \phi_{j} \phi_{k}, \\
\text { from: } W^{i j} \partial_{\mu} \phi_{j} & =\frac{\delta^{2}}{\delta \phi_{i} \delta \phi_{j}} W_{S U S Y}=\partial_{\mu}\left(\frac{\delta W_{S U S Y}}{\delta \phi_{i}}\right)=\partial_{\mu} W^{i},
\end{aligned}
$$

where a so-called superpotential, $W_{S U S Y}$, has been introduced. This superpotential is solely a function of the complex, scalar fields, as required by the above considerations of $W^{i j}$ and $W^{i}$

$$
W_{S U S Y}=\frac{1}{2} m^{i j} \phi_{i} \phi_{j}+\frac{1}{6} y^{i j k} \phi_{i} \phi_{j} \phi_{k},
$$

Now only terms linear in the auxiliary fields $F_{i}$, or $F^{* i}$ remain in the transformed Lagrangian $\delta \mathcal{L}_{\text {int }}^{\text {chiral }}$. All these terms cancel, if the classical equations of motion, e.g. $\frac{\partial \mathcal{L}}{\partial F^{i}}=F^{* i}+W^{i}=0$ (and h.c.) are implied, because all auxiliary fields can be replaced by the scalar fields $W_{i}^{*}$ and $W^{i}$, leading to the single term $W^{i} W_{i}^{*}$, which constitutes the scalar potential $V\left(\phi, \phi^{*}\right)$.

So, the Lagrangian of massive, interacting chiral supermultiplets follows from the combination of the free Wess-Zumino Lagrangian of Equation (1.34), (extended to describe a collection of free particles $\psi_{i}$ and $\left.\phi_{i}\right)$, and the Lagrangian of interactions to:

$$
\begin{aligned}
\mathcal{L}_{\text {chiral }}= & -\partial^{\mu} \phi^{* i} \partial_{\mu} \phi_{i}-i \psi^{\dagger i} \bar{\sigma}^{\mu} \partial_{\mu} \psi_{i} \\
& -\frac{1}{2}\left(W^{i j} \psi_{i} \psi_{j}+W^{* i j} \psi^{\dagger i} \psi^{\dagger j}\right)-W^{i} W_{i}^{*}
\end{aligned}
$$

where the index $i$ runs over all flavour and gauge degrees of freedom and is summed over, if repeated, according to Einstein's sum rule. Line (i) describes the kinetic energy of all the fermions and their corresponding scalar superpartners and line (ii) represents the mass terms and trilinear couplings in terms of the scalar fields $W^{i j}$ and $W^{i}$.

The only missing ingredient for a complete SUSY-Lagrangian is the inclusion of gauge interactions, i.e. interactions coupling the fields in the chiral supermultiplets to those of the gauge supermultiplets. Since all necessary steps have already been mentioned, this is now relatively straightforward. All partial derivatives $\partial_{\mu}$ in the chiral Lagrangian of Equation (1.51) have to be replaced by gauge-covariant derivatives $\mathcal{D}_{\mu}$

$$
\begin{aligned}
\partial_{\mu} \phi_{i} & \rightarrow \mathcal{D}_{\mu} \phi_{i}=\partial_{\mu} \phi_{i}+i g A_{\mu}^{a}\left(T^{a} \phi\right)_{i} \\
\partial_{\mu} \phi^{* i} & \rightarrow \mathcal{D}_{\mu} \phi^{* i}=\partial_{\mu} \phi^{* i}-i g A_{\mu}^{a}\left(\phi^{*} T^{a}\right)^{i} \\
\partial_{\mu} \psi_{i} & \rightarrow \mathcal{D}_{\mu} \psi_{i}=\partial_{\mu} \psi_{i}+i g A_{\mu}^{a}\left(T^{a} \psi\right)_{i}
\end{aligned}
$$

In a next step, all interaction terms, involving $D^{a}$ and gaugino fields, that are not already included in either the chiral or the gauge Lagrangian (Eq. (1.43)) have to be considered. Three possible, renormalisable interaction terms exist

$$
\left(\phi^{*} T^{a} \psi\right) \lambda^{a}, \quad \lambda^{\dagger a}\left(\psi^{\dagger} T^{a} \phi\right), \quad \text { and } \quad\left(\phi^{*} T^{a} \phi\right) \lambda^{a},
$$

where the $T^{a}$ are the generators of the relevant gauge group, e.g. for the $\mathrm{SU}(2)_{L}$ of the $\mathrm{SM}, T^{a}$ would be the weak isospin operator $\mathbf{T}=\left(T^{1}, T^{2}, T^{3}\right)$ introduced in the covariant derivative of 
the SM in Equation (1.10). These terms are added to the chiral and gauge parts of the total Lagrangian $\mathcal{L}_{S U S Y}$, which is then required to be real and to remain invariant under SUSY transformations. This is achieved, if the SUSY transformation laws of the chiral fields are modified accordingly, which means, that they cannot contain partial derivatives neither. Additionally an extra term needs to be introduced into the transformation of $\delta F_{i}$ :

$$
\begin{aligned}
\delta \phi_{i} & =\epsilon \psi_{i} \\
\delta \psi_{\alpha} & =i\left(\sigma^{\mu} \epsilon^{\dagger}\right)_{\alpha} \mathcal{D}_{\mu} \phi_{i}+\epsilon_{\alpha} F_{i} \\
\delta F_{i} & =i \epsilon^{\dagger} \bar{\sigma}^{\mu} \mathcal{D}_{\mu} \psi_{i}+\sqrt{2} g\left(T^{a} \phi\right)_{i} \epsilon^{\dagger} \lambda^{\dagger a}
\end{aligned}
$$

Using the new, modified transformation laws allows to infer the coefficients of the extra interaction terms in Equation (1.53). Assembling now all the different pieces of the previous paragraphs into one, i.e. combining the chiral Lagrangian of Equation (1.51) with the gauge Lagrangian of Equation (1.43), and replacing all partial with covariant derivatives, the final SUSY-Lagrangian emerges:

$$
\begin{aligned}
\mathcal{L}_{S U S Y}= & -\mathcal{D}^{\mu} \phi^{* i} \mathcal{D}_{\mu} \phi_{i}-i \psi^{\dagger i} \bar{\sigma}^{\mu} \mathcal{D}_{\mu} \psi_{i} \\
& -\frac{1}{2} m^{i j} \psi_{i} \psi_{j}-\frac{1}{2} m_{i j}^{*} \psi^{\dagger i} \psi^{\dagger j}-M_{i k}^{*} M^{k j} \phi^{* i} \phi_{j} \\
& -\frac{1}{2} y^{i j k} \phi_{i} \psi_{j} \psi_{k}-\frac{1}{2} y_{i j k}^{*} \phi^{* i} \psi^{\dagger j} \psi^{\dagger k} \\
& -\frac{1}{2} M^{i n} y_{j k n}^{*} \phi_{i} \phi^{* j} \phi^{* k}-\frac{1}{2} M_{i n}^{*} y^{j k n} \phi^{* i} \phi_{j} \phi_{k}-\frac{1}{4} y^{i j n} y_{k l n}^{*} \phi_{i} \phi_{j} \phi^{* k} \phi^{* l} \\
& -\frac{1}{4} \mathcal{F}_{\mu \nu}^{a} \mathcal{F}^{\mu \nu a}-i \lambda^{\dagger a} \bar{\sigma}^{\mu} \mathcal{D}_{\mu} \lambda^{a} \\
& -\sqrt{2} g\left[\left(\phi^{*} T^{a} \psi\right) \lambda^{a}+\lambda^{\dagger a}\left(\psi^{\dagger} T^{a} \phi\right)\right] \\
& +g\left(\phi^{*} T^{a} \phi\right) D^{a}+\frac{1}{2} D^{a} D^{a} .
\end{aligned}
$$

The first line (i) of $\mathcal{L}_{S U S Y}$ contains the kinetic terms of all fermions/sfermions and the higgsinos/Higgs bosons, respectively. If the chiral supermultiplets are no gauge singlets, interactions between the fermions/scalars and the vector gauge bosons arise also from the kinetic terms due to the covariant derivatives and modified SUSY transformations, (Fig. 1.5). Mass terms for fermionic and scalar fields appear in the second line (ii). The next two lines describe all possible trilinear interactions of two fermion fields and one scalar field (iii) and between scalar fields alone (iv). The last term of line (iv) even describes a quadrilinear interaction, where the coupling strength is proportional to Yukawa couplings (Fig. 1.6). In line (v) the gauge bosons' dynamics, including their self-interactions are expressed in terms of the field strength tensors $\mathcal{F}_{\mu \nu}^{a}$, whereas the interactions between gauge bosons and gauginos are governed by the second term (Fig. 1.7). Line (vi) describes a completely new kind of trilinear interactions involving gaugino, fermionic and scalar fields (Fig. 1.8, left). Finally, line (vii) holds another quadrilinear interaction of four scalar fields, (Fig. 1.8, right). Contrary to the one described by the last term of line (iv), this new quartic interaction is determined by the respective gauge coupling (due to $T^{a}$ ) and not by Yukawa couplings. 


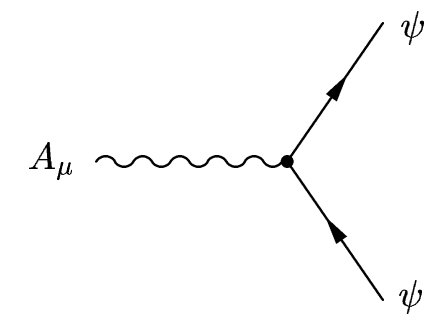

$g\left[A_{\mu}^{a} \psi^{\dagger} \bar{\sigma}^{\mu}\left(T^{a} \psi\right)+\right.$ h.c. $]$

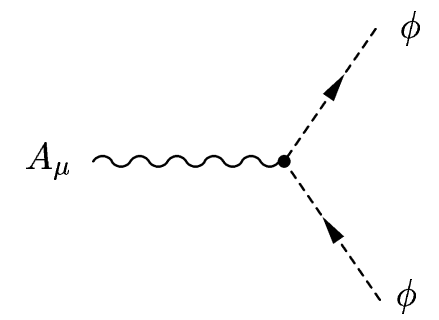

$g\left[A^{a \mu}\left(T^{a} \phi\right) \partial_{\mu} \phi^{*}+\right.$ h.c. $]$

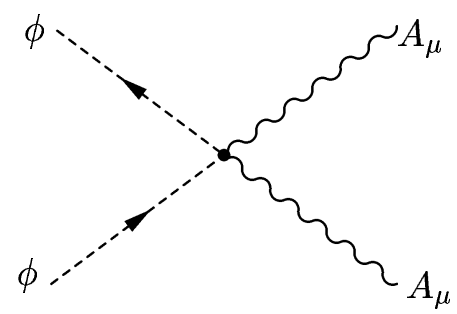

$g^{2} A^{a \mu} A_{\mu}^{b}\left(T^{a} \phi\right)\left(\phi T^{b}\right)$

Figure 1.5: Interactions between gauge bosons $\left(A_{\mu}\right)$ and fermionic or scalar fields of the chiral supermultiplets, i.e. between fermions, or higgsinos $(\psi)$ and sfermions or Higgs bosons $(\phi)$.

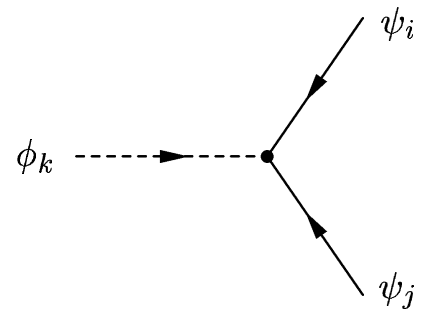

$y^{i j k} \phi_{k} \psi_{i} \psi_{j} \quad+$ h.c.

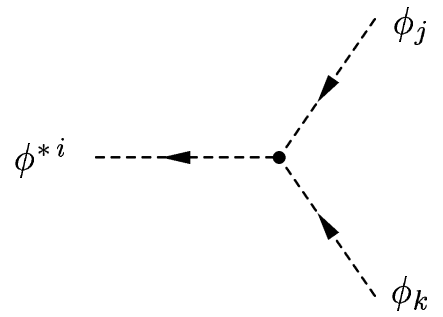

$M_{i n}^{*} y^{j k n} \phi^{* i} \phi_{k}+$ h.c.

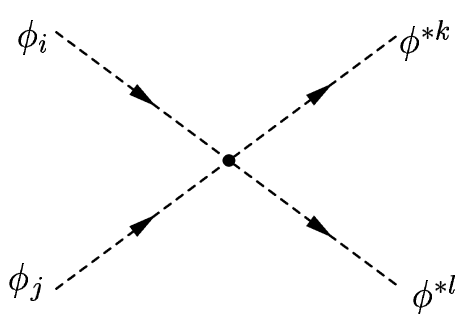

$y^{j k n} y_{k l n}^{*} \phi_{i} \phi_{j} \phi^{* k} \phi^{* l}$

Figure 1.6: Trilinear interactions between fermions and scalars, or of scalar fields alone; the rightmost diagram depicts a quadrilinear interaction of four scalar fields. To obtain the correct Feynman diagrams of the conjugate versions of the first two terms, all chiralities (arrows) have to be switched.
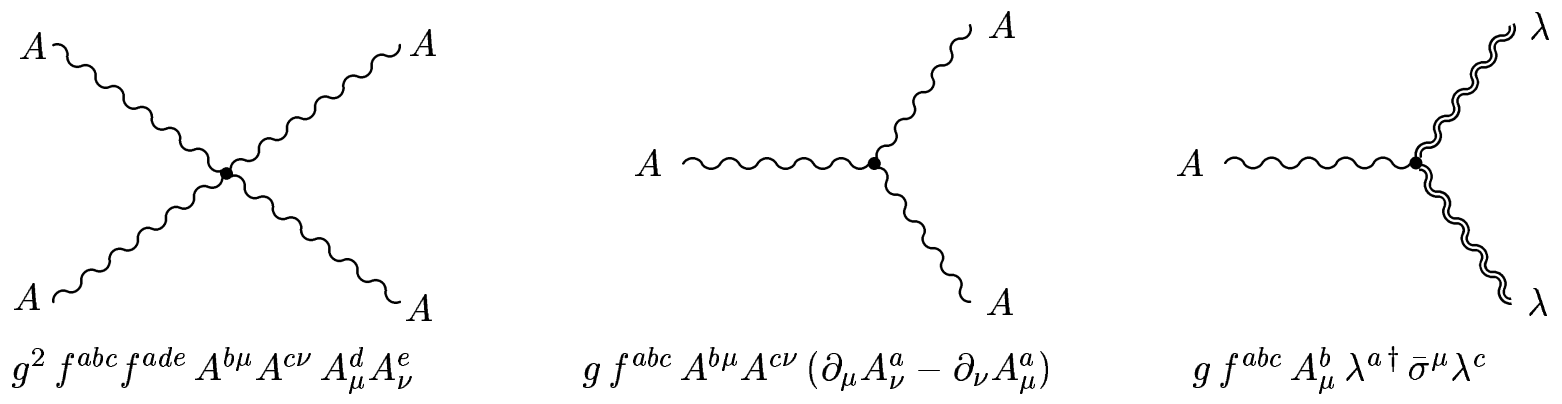

$g f^{a b c} A^{b \mu} A^{c \nu}\left(\partial_{\mu} A_{\nu}^{a}-\partial_{\nu} A_{\mu}^{a}\right)$

$g f^{a b c} A_{\mu}^{b} \lambda^{a \dagger} \bar{\sigma}^{\mu} \lambda^{c}$

Figure 1.7: Quadri- and trilinear interactions among gauge bosons (A) from the $F_{\mu \nu} F^{\mu \nu}$-term and trilinear interactions of gauge bosons and gauginos $(\lambda)$ from $\lambda^{\dagger a} \bar{\sigma}^{\mu} \mathcal{D}_{\mu} \lambda^{a}$, second term in line (v), Eq. (1.55).

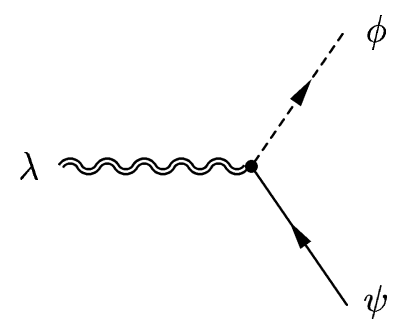

$g\left[\left(\phi^{*} T^{a} \psi\right) \lambda^{a}+\right.$ h.c. $]$

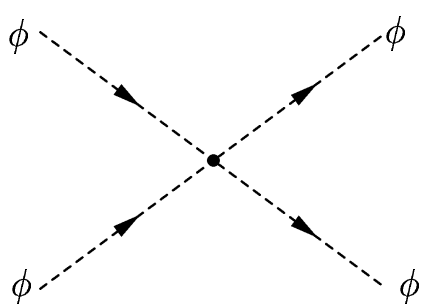

$g^{2}\left(\phi^{*} T^{a} \phi\right)^{2}$

Figure 1.8: Additional interaction, connecting a chiral scalar and fermion field $(\phi, \psi)$ to a gaugino $(\lambda)$ of a vectorial supermultiplet (left). This type of interactions may be interpreted as a »supersymmetrisation « of the first two diagrams in Fig. (1.5). Another quartic interaction of four scalar fields (right), where the coupling strength is determined by the gauge coupling $(g)$. 


\subsubsection{SUSY Breaking and the Higgs Sector}

Despite numerous attempts to introduce spontaneous supersymmetry breaking analogously to EWSB in the SM, which is achieved by a non-zero VEV of the Higgs field, no convincing mechanism has been found so far. The two most well-known proposals are probably those by FayetIliopoulos [48] and O'Raifeartaigh [49], but both their models suffered from severe phenomenological difficulties. The predicted scalar partners were too light with masses even below the SM-fermion masses.

Apart from their varying ability for predictions, all of the proposed mechanisms to spontaneously break supersymmetry and thus generate masses for the SUSY-particles, require extensions of the MSSM including new particles and interactions at very high energy scales. Since there is no consensus as to how this should be done, it is easier to parametrise the possible effects of SUSY breaking and introduce all of the soft breaking terms explicitly into the SUSY-Lagrangian, which amounts to simply ignoring the question of »how SUSY is actually broken «. These terms are called soft, because they should not re-introduce quadratic divergences into the higher order corrections to the Higgs boson mass [50]. They must also be renormalisable and invariant under gauge transformations, but they need not be invariant under the SUSY transformations defined in Equations (1.44) and (1.54). The »solution « to deal with supersymmetry breaking is, to assume that the breaking itself takes place in a so-called hidden sector, which is completely (or almost completely) independent of the visible sector of the chiral and gauge supermultiplets. Two main proposals exist:

GMSB: meaning Gauge Mediated Supersymmetry Breaking, where the soft breaking terms arise from loop diagrams involving so-called messenger particles, which not only couple to the SUSY breaking VEV,$\langle F\rangle$, in the hidden sector, but likewise - because they also possess $\mathrm{SU}(3)_{C} \otimes \mathrm{SU}(2)_{L} \otimes \mathrm{U}(1)_{Y}$ interactions - to the the normal SUSY-particles of the visible sector. In this scenario the scale of SUSY breaking can be estimated by:

$$
m_{\text {soft }} \sim \frac{\langle F\rangle}{M_{\text {mess }}} \rightarrow \sqrt{\langle F\rangle} \sim 10^{4}-10^{5} \mathrm{GeV},
$$

where $m_{\text {soft }} \approx 1 \mathrm{TeV}$ is the characteristic mass scale of the soft breaking terms, $\langle F\rangle$ is the VEV of the hidden sector field responsible for SUSY breaking and $M_{\text {mess }}$ is the mass scale of the messenger fields, which should be roughly equal to $\sqrt{\langle F\rangle}$. Gauge mediation with messenger particles, whose masses are well below the Planck scale $\left(M_{\text {mess }} \ll M_{P}\right)$, generate an extremely light gravitino, $\mathcal{O}(\mathrm{eV}-\mathrm{keV})$, which would become the LSP. Hence, collider phenomenology assuming GMSB scenarios, is characterised by NLSP-decays into the gravitino.

SUGRA: where the hidden sector communicates with the visible sector through gravitational interactions [51], implying the successful inclusion of gravity into the supersymmetric gauge field theory, which also gives rise to the model's popular name »supergravity «. In SUGRA scenarios the scale of SUSY breaking is approximately $10^{11} \mathrm{GeV}$, which can be understood in terms of:

$$
m_{\text {soft }} \sim \frac{\langle F\rangle}{M_{P}} \rightarrow \sqrt{\langle F\rangle} \sim 10^{11} \mathrm{GeV},
$$

with $m_{\text {soft }}$ and $\langle F\rangle$ as defined above and $M_{P}$ being the Planck scale of $\approx 10^{19} \mathrm{GeV}$. The gravitino $(\widetilde{G})$ which needs to be introduced in local supersymmetry to retain the invariance 
of the Lagrangian under local SUSY transformations, may thus be identified as »gauge « particle of local SUSY transformations, similar to the gluons, which are the gauge particles of the $\mathrm{SU}(3)_{C}$ transformations. As long as SUSY is unbroken, the spin-2 graviton and its superpartner, the spin- $3 / 2$ gravitino are both massless and thus possess only two spin helicity states. According to the Nambu-Goldstone theorem [22], the spontaneous breaking of a global symmetry always generates a massless particle with the same quantum numbers as the broken symmetry operator. In the case of supersymmetry, the broken symmetry operator is the complex spinor $\mathcal{Q}$, which results in the Nambu-Goldstone mode being a massless, neutral Weyl fermion, the goldstino. In local supersymmetry, the longitudinal components of the goldstino are then absorbed by the gravitino, which thus acquires a mass. This is also called Super-Higgs mechanism, since it is entirely analogous to the Higgs mechanism of the SM responsible for EWSB and the masses of the gauge bosons. One of the advantages of (minimal) SUGRA is the explanation of EWSB as a consequence of radiative corrections from the evolution of masses and couplings from the GUT scale $\left(M_{G U T}\right)$ to the electroweak scale $\left(M_{E W}\right)$ via the Renormalisation Group Equations (RGE), which is known as radiative EWSB. In contrast to GMSB-scenarios, gravity mediated SUSY breaking yields gravitino masses of the order of $\mathcal{O}(100 \mathrm{GeV})$. Even if the gravitino is the LSP, it will not play a significant role in collider physics, since it interacts with the coupling strength of gravity. The NLSP will simply appear to be stable over usual detector distances. In cosmology, however, a gravitino-LSP could be of substantial importance.

Fortunately, the exact breaking mechanism is largely irrelevant for the resulting phenomenology and only the flavour-blind interactions, actually mediating the breaking, are important, as is the associated scale of SUSY breaking (GMSB versus SUGRA scenarios). So, in general the soft breaking terms are parametrised in the following way:

$$
\mathcal{L}_{\text {soft }}^{\text {generic }}=-\frac{1}{2} M_{\lambda} \lambda^{a} \lambda^{a}-\left(m^{2}\right)_{j}^{i} \phi^{* j} \phi_{i}-\frac{1}{2} m^{i j} \phi_{i} \phi_{j}-\frac{1}{6} A^{i j k} \phi_{i} \phi_{j} \phi_{k}+\text { h.c. }
$$

where the first term holds the gaugino mass terms, the second and third contain mass terms for scalar particles, i.e. for sfermions and Higgs bosons, respectively, and the fourth term defines trilinear scalar coupling terms. Assuming now the minimal particle content of the supersymmetric extension of the SM, the notation introduced in Table 1.4 of section 1.3 .2 allows to write:

$$
\begin{aligned}
\mathcal{L}_{\text {soft }}= & -\frac{1}{2}\left(M_{3} \widetilde{g} \widetilde{g}+M_{2} \widetilde{W} \widetilde{W}+M_{1} \widetilde{B} \widetilde{B}\right)+\text { h.c. } \\
& -\widetilde{Q}^{\dagger} \mathbf{m}_{\mathbf{Q}}^{2} \widetilde{Q}-\widetilde{L}^{\dagger} \mathbf{m}_{\mathbf{L}}^{2} \widetilde{L}-\widetilde{\bar{U}} \mathbf{m}_{\overline{\mathbf{U}}}^{\mathbf{2}} \widetilde{\bar{U}}^{\dagger}-\widetilde{\bar{D}} \mathbf{m}_{\overline{\mathbf{D}}}^{\mathbf{2}} \widetilde{\bar{D}}^{\dagger}-\widetilde{\bar{E}} \mathbf{m}_{\overline{\mathbf{E}}}^{\mathbf{2}} \widetilde{\bar{E}}^{\dagger} \\
& -m_{H_{u}}^{2} H_{u}^{*} H_{u}-m_{H_{d}}^{2} H_{d}^{*} H_{d}-\left(m_{12}^{2} H_{u} H_{d}+h . c .\right) \\
& -\left(\widetilde{\bar{U}} \mathbf{A}_{\mathbf{u}} \widetilde{Q} H_{u}-\widetilde{\bar{D}} \mathbf{A}_{\mathbf{d}} \widetilde{Q} H_{d}-\widetilde{\bar{E}} \mathbf{A}_{\mathbf{e}} \widetilde{L} H_{d}\right)+\text { h.c. }
\end{aligned}
$$

The first line (i) of $\mathcal{L}_{\text {soft }}$ contains the gluino $\left(M_{3}\right)$, wino $\left(M_{2}\right)$ and bino $\left(M_{1}\right)$ mass terms, while in the second line (ii) the mass terms of the squarks and sleptons are defined and line (iii) holds the respective mass terms of the two (up- and down-type) Higgs bosons. Here appears also the only bilinear term $m_{12}^{2} H_{u} H_{d}$. Each one of the $\mathbf{m}_{\mathbf{I}}^{\mathbf{2}}$-matrices for the sfermions is a $3 \times 3$ matrix in generation space, as are the trilinear coupling matrices, $\mathbf{A}_{\mathbf{i}}$, governing the interactions between sleptons/squarks and the Higgs bosons $H_{u}$ and $H_{d}$, in line (iv). As can be seen from the above 
terms, this Lagrangian really breaks supersymmetry, since all of the terms only involve scalars, or gauginos, but not their respective superpartners, i.e. fermions (Higgs bosons) or gauge bosons.

Now, since SUSY-breaking has been arranged for with (radiative) EWSB following from it, the Higgs sector, or more exactly, the conditions for a successful breaking of the electroweak symmetry $\mathrm{SU}(2)_{L} \otimes \mathrm{U}(1)_{Y}$ are briefly discussed. In unbroken SUSY, the relevant part of the scalar Higgs potential is given by

$$
W_{H}^{u n b r o k e n}=\mu^{2}\left(H_{u}^{2}+H_{d}^{2}\right)+\frac{1}{8}\left(g^{2}+g^{\prime} 2\right)\left(H_{u}^{2}-H_{d}^{2}\right) .
$$

With this kind of scalar Higgs potential, EWSB cannot take place, because of $W_{H} \geq 0$, which leads to both Higgs field components equal zero $\left(H_{u}=H_{d}=0\right)$. Contrary to this, in broken SUSY, new terms are introduced into the Higgs scalar potential. These are the mass terms $m_{H_{u}}$, $m_{H_{d}}$ and the bilinear coupling $m_{12}$

$$
\begin{aligned}
W_{H}= & \left(|\mu|^{2}+m_{H_{u}}^{2}\right)\left|H_{u}^{0}\right|^{2}+\left(|\mu|^{2}+m_{H_{d}}^{2}\right)\left|H_{d}^{0}\right|^{2}-\left(m_{12}^{2} H_{u}^{0} H_{d}^{0}+h . c .\right) \\
& +\frac{1}{8}\left(g^{2}+g^{\prime} 2\right)\left(H_{u}^{2}-H_{d}^{2}\right) .
\end{aligned}
$$

In combination with the $m_{12}^{2}$ term, there is one linear combination of $H_{u}^{0}$ and $H_{d}^{0}$, that can have a negative (mass) $)^{2}$ term, which is expressed by the inequality

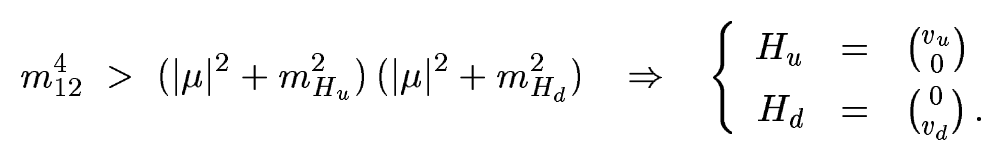

Only if this inequality is satisfied, has the SUSY Higgs potential a SM-like shape (mexican hat as in Fig. 1.1), otherwise the minimum will be stable with $H_{u}=H_{d}=0$ so that no EWSB can occur. Similar to Equation (1.23), there exists a relation between $v_{u}, v_{d}$ and $M_{Z}$, the precisely measured mass of the $Z^{0}$ boson:

$$
v_{u}^{2}+v_{d}^{2}=v_{S M}^{2}=\frac{2 \cdot M_{Z}^{2}}{g^{2}+g^{\prime 2}} \approx(174 \mathrm{GeV})^{2},
$$

Usually the ratio of the two VEVs of the Higgs fields is written as $\tan \beta=\frac{v_{d}}{v_{u}}$. Requiring the minimum of the Higgs potential $\frac{\partial W_{H}}{\partial H_{u}^{0}}=\frac{\partial W_{H}}{\partial H_{d}^{0}}=0$ to satisfy Equation (1.63) leads to the following two conditions, which allow to eliminate two of the lagrangian parameters, namely $m_{12}^{2}$ and $|\mu|$

$$
\begin{aligned}
|\mu|^{2}+m_{H_{u}}^{2} & =m_{12}^{2} \cot \beta+\left(\frac{M_{Z}^{2}}{2}\right) \cos (2 \beta) \\
|\mu|^{2}+m_{H_{d}}^{2} & =m_{12}^{2} \tan \beta-\left(\frac{M_{Z}^{2}}{2}\right) \cos (2 \beta) .
\end{aligned}
$$

The Higgs scalar fields consist of two complex scalar $\mathrm{SU}(2)_{L}$-doublets, which corresponds to eight real degrees of freedom. After EWSB, three of the eight degrees of freedom are »eaten « by the $W^{ \pm}$and $Z^{0}$ bosons, and become their longitudinal spin polarisation modes, while five real degrees of freedom and thus five real Higgs bosons remain. At tree-level, or leading order (LO), the mass eigenstates are given by

$$
\begin{aligned}
M_{A^{0}}^{2} & =m_{H_{u}}^{2}+m_{H_{d}}^{2}+2 \mu^{2}=\frac{2 m_{12}^{2}}{\sin (2 \beta)} \equiv M_{A}^{2} \quad \text { (MSSM parameter) } \\
M_{H^{ \pm}}^{2} & =M_{A}^{2}+M_{W}^{2} \\
M_{h^{0}, H^{0}}^{2} & =\frac{1}{2}\left[M_{A}^{2}+M_{Z}^{2} \mp \sqrt{\left(M_{A}^{2}+M_{Z}^{2}\right)^{2}-4 M_{Z}^{2} M_{A}^{2} \cos ^{2}(2 \beta)}\right]
\end{aligned}
$$


These mixed mass eigenstates are: one CP-odd pseudoscalar $A^{0}$, two charged $H^{+}, H^{-}$and two CP-even neutral scalars $H^{0}$ and $h^{0}$, where the capital letter denotes the heavier of the two CP-even states. The Higgs mass spectrum is thus determined by only two parameters, which are chosen to be the mass of the pseudoscalar, $M_{A}$ and the ratio of the two VEVs, $\tan \beta$. The Higgs mass equations given above are only valid in leading order, the real Higgs boson masses receive substantial radiative corrections from SM- and SUSY-particles. Typically, the dominant contribution comes from the loops of the most massive particle and its superpartner, i.e. from top-stop loops [52] and increases with $m_{t}^{4}$ and logarithmically with $M_{S U S Y}$, the approximate scale of the superpartner masses.

\subsubsection{Phenomenological SUSY Models}

So far, the theoretical models were generic supersymmetry models, but in what follows only minimal extensions of the Standard Model will be considered. The particle content of such, SM-based, SUSY-models is the one previously defined in Table 1.4 of section 1.3.2. The aim of this subsection is to derive two models, that allow phenomenological predictions at low energies, e.g. at a mass scale below $1 \mathrm{TeV}$.

Unfortunately the soft terms of $\mathcal{L}_{\text {soft }}$ introduce more than 100 additional parameters into any generic SUSY Lagrangian. To reduce this huge number of free parameters to a manageable number (phenomenology-wise), a set of simplifying assumptions - some arising from experimental constraints - is applied. Most of the soft terms are already severely constrained, because they involve CP-violation or flavour mixing to an extent ruled out by experiment [38]. Two of the strongest constraints on FCNC-terms (Flavour Changing Neutral Currents) come from the nonobservation of $\mu \rightarrow e \gamma$ and from the neutral Kaon system $\left(K^{0} \leftrightarrow \bar{K}^{0}\right)$. The former, with an upper limit of $1.2 \times 10^{-11}$ [23] on the $\mathrm{BR}(\mu \rightarrow e \gamma)$ severely restricts the off-diagonal elements of the slepton mass matrices $\mathbf{m}_{\mathbf{L}}^{\mathbf{2}}$ and $\mathbf{m}_{\mathbf{E}}^{\mathbf{2}}$ [53], whereas the latter (Kaon system) constrains the mixing of the first and second generation squarks and corresponding CP-violating complex phases [54].

All of these dangerous FCNC- and CP-violating effects contradicting experimental observations are readily avoided, if the so-called soft breaking universality is assumed. This means that all of the soft breaking parameters are required to be real, the sfermion $(\widetilde{\ell}, \widetilde{q})$ mass matrices are assumed to be diagonal in generation space [56] and the trilinear coupling matrices $\mathbf{A}_{\mathbf{i}}$ proportional to the corresponding Yukawa couplings at the GUT scale. Under these conditions 22 free parameters remain:

- 3 gaugino masses: $M_{1}$ (bino), $M_{2}$ (winos) and $M_{3}$ (gluinos)

- 5 sfermion masses of the first two generations $\left(m_{\widetilde{e}_{L}}, m_{\widetilde{e}_{R}}, m_{\widetilde{u}_{L}}, m_{\widetilde{u}_{R}}, m_{\widetilde{d}_{R}}\right)$

- 5 sfermion masses of the third generation $\quad\left(m_{\widetilde{\tau}_{1}}, m_{\widetilde{\tau}_{2}}, m_{\widetilde{t}_{1}}, m_{\widetilde{t}_{2}}, m_{\widetilde{b}_{R}}\right)$

- the mass of the pseudoscalar Higgs boson $M_{A}$

- the mass mixing parameter of the Higgs doublets $\mu$

- the ratio of the two Higgs VEVs: $\tan \beta=v_{d} / v_{u} \quad\left(\right.$ or $\left.\tan \beta=v_{2} / v_{1}\right)$

- 6 trilinear coupling matrices $\left(A_{e}, A_{u}, A_{d}, A_{\tau}, A_{t}, A_{b}\right)$ 
After SUSY-breaking, all SUSY-particles acquire masses as a consequence of either the newly introduced direct mass terms, or couplings to the up- or down-type Higgs fields. Due to the subsequent breaking of the electroweak symmetry, fields with different $\mathrm{SU}(2)_{L} \otimes \mathrm{U}(1)_{Y}$, but equal $\mathrm{SU}(3)_{C}$ and $\mathrm{U}(1)_{E M}$ quantum numbers can mix. If the mass matrices are expressed in terms of the $\mathrm{SU}(2)_{L} \otimes \mathrm{U}(1)_{Y}$ eigenstates, the slepton, as well as the squark mass matrices all contain off-diagonal elements proportional to the masses of their respective SM-partners. Since they are negligible in case of the first two fermion generations, this leads to quasi-degenerate masses for the first and second generation sleptons and squarks. Only the superpartners of leftand rightchiral fermions of the third generation mix, due to the relatively large Yukawa $\left(y_{\tau}, y_{t}\right.$, $\left.y_{b}\right)$ and soft $\left(\mathbf{A}_{\tau}, \mathbf{A}_{\mathbf{t}}, \mathbf{A}_{\mathbf{b}}\right)$ couplings, e.g. stau leptons: $\widetilde{\tau}_{R}, \widetilde{\tau}_{L} \rightarrow \widetilde{\tau}_{1}, \widetilde{\tau}_{2}$.

Mixing is not only possible in the sfermion sector, but also in the gaugino/higgsino sector. Here, neutral higgsinos $\left(\widetilde{H}_{u}^{0}, \widetilde{H}_{d}^{0}\right)$ and gauginos $\left(\widetilde{B}, \widetilde{W}^{3}\right)$ mix to neutral mass eigenstates, the neutralinos and likewise the charged higgsinos $\left(\widetilde{H}_{u}^{+}, \widetilde{H}_{d}^{-}\right)$and gauginos $\left(\widetilde{W}^{1}, \widetilde{W}^{2}\right)$ mix to the charged counterparts, the charginos, as given below in their respective eigenstate bases.

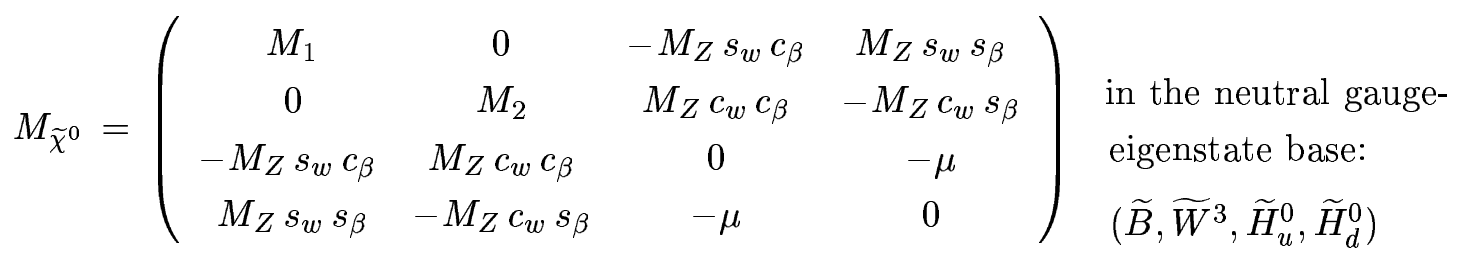

$$
\begin{aligned}
& M_{\widetilde{\chi}^{ \pm}}=\left(\begin{array}{cccc}
0 & 0 & M_{2} & \sqrt{2} M_{W} s_{\beta} \\
0 & 0 & \sqrt{2} M_{W} c_{\beta} & \mu \\
M_{2} & \sqrt{2} M_{W} s_{\beta} & 0 & 0 \\
\sqrt{2} M_{W} c_{\beta} & \mu & 0 & 0
\end{array}\right) \begin{array}{l}
\text { in the charged gauge- } \\
\text { eigenstate base: } \\
\left(\widetilde{W}+, \widetilde{H}_{u}^{+}, \widetilde{W}^{-}, \widetilde{H}_{d}^{-}\right)
\end{array}
\end{aligned}
$$

with the abbreviations $s_{w}=\sin \theta_{w}, c_{w}=\cos \theta_{w}$, where $\theta_{w}$ is the weak mixing angle (see Eq. (1.13) and (1.14)) and $s_{\beta}=\sin \beta, c_{\beta}=\cos \beta$, where $\tan \beta=v_{d} / v_{u}$ is the ratio of the Higgs fields' VEVs. The observable mass eigenstates, four neutralinos $\left(\widetilde{\chi}_{1}^{0}, \widetilde{\chi}_{2}^{0}, \widetilde{\chi}_{3}^{0}, \widetilde{\chi}_{4}^{0}\right)$ and two charginos $\left(\widetilde{\chi}_{1}^{ \pm}, \widetilde{\chi}_{2}^{ \pm}\right)$, follow from diagonalising the above matrices, where increasing indices denote larger mass. As can be seen from the matrices, the masses of neutralinos and charginos only depend on the supersymmetric parameters $M_{1}, M_{2}, \mu$ and $\tan \beta$. If the Higgs mixing parameter, $\mu$, is larger than the $\mathrm{U}(1)$ and $\mathrm{SU}(2)$ gaugino mass parameters $\left(M_{1}\right.$ and $M_{2}$, respectively), the $\widetilde{W}-\widetilde{H}$ mass matrices are almost diagonal. Consequently the lightest neutralino eigenstates are dominated by wino and bino components and the lightest chargino is almost a pure wino [57], which is why this region is referred to as the gaugino region. In the opposite case, where $\mu \ll M_{1,2}$ and the lightest neutralinos are rather higgsino-like, the region is called the higgsino region.

The gluinos cannot mix with the other gauginos (higgsinos), since they do not possess the same $\mathrm{SU}(3)_{C}$ and $\mathrm{U}(1)_{E M}$ quantum numbers as the latter. Gluinos carry - in analogy to their SMpartners, the gluons - colour charge; their mass terms are given directly by the soft breaking term $\left(-\frac{1}{2} M_{3} \widetilde{g} \widetilde{g}\right)$ from line (i) in Equation (1.59).

To further reduce the number of free parameters in phenomenological models, supersymmetry has to be embedded into more fundamental GUT models [55], which allow further assumptions regarding the particle masses and their respective couplings. 


\section{The Minimal Supergravity model - mSUGRA}

Due to the apparent unification of couplings at a higher energy scale (see Fig. 1.3), e.g. the GUT scale at $\approx 10^{16} \mathrm{GeV}$, further assumptions are made, leading to the following parameters in mSUGRA:

- the gaugino masses $M_{1}, M_{2}$ and $M_{3}$ are unified $\rightarrow$ common gaugino mass: $m_{1 / 2}$

- all sfermion and Higgs boson masses are unified $\rightarrow$ common scalar mass: $m_{0}$ (Higgs boson masses: $m_{H_{u}}^{2}=m_{1}^{2}-\mu^{2}$ and $m_{H_{d}}^{2}=m_{2}^{2}-\mu^{2}$ see also Figure 1.9)

- all trilinear couplings are unified into one $\rightarrow$ common trilinear coupling: $A_{0}$

- the ratio of Higgs fields' VEVs $\rightarrow \tan \beta \quad$ (as before)

- the sign of the Higgs mixing parameter $\rightarrow \operatorname{Sgn}(\mu)$

The inclusion of the Higgs boson masses into the common scalar mass $m_{0}$ allows to express the mass of the pseudoscalar Higgs $M_{A}^{2}$ and the Higgs mixing parameter $\mu^{2}$ in terms of $\tan \beta$ and $m_{0}$, so that only the sign of $\mu$ is left free. The effective masses and couplings at the SUSY $(\approx 1 \mathrm{TeV})$ or the electroweak scale $(\approx 100 \mathrm{GeV})$ are then $\gg$ only « a consequence of radiative corrections taken into account when running the RGEs from the GUT scale down to the electroweak scale. Figure 1.9 from [58] illustrates the evolution of masses.

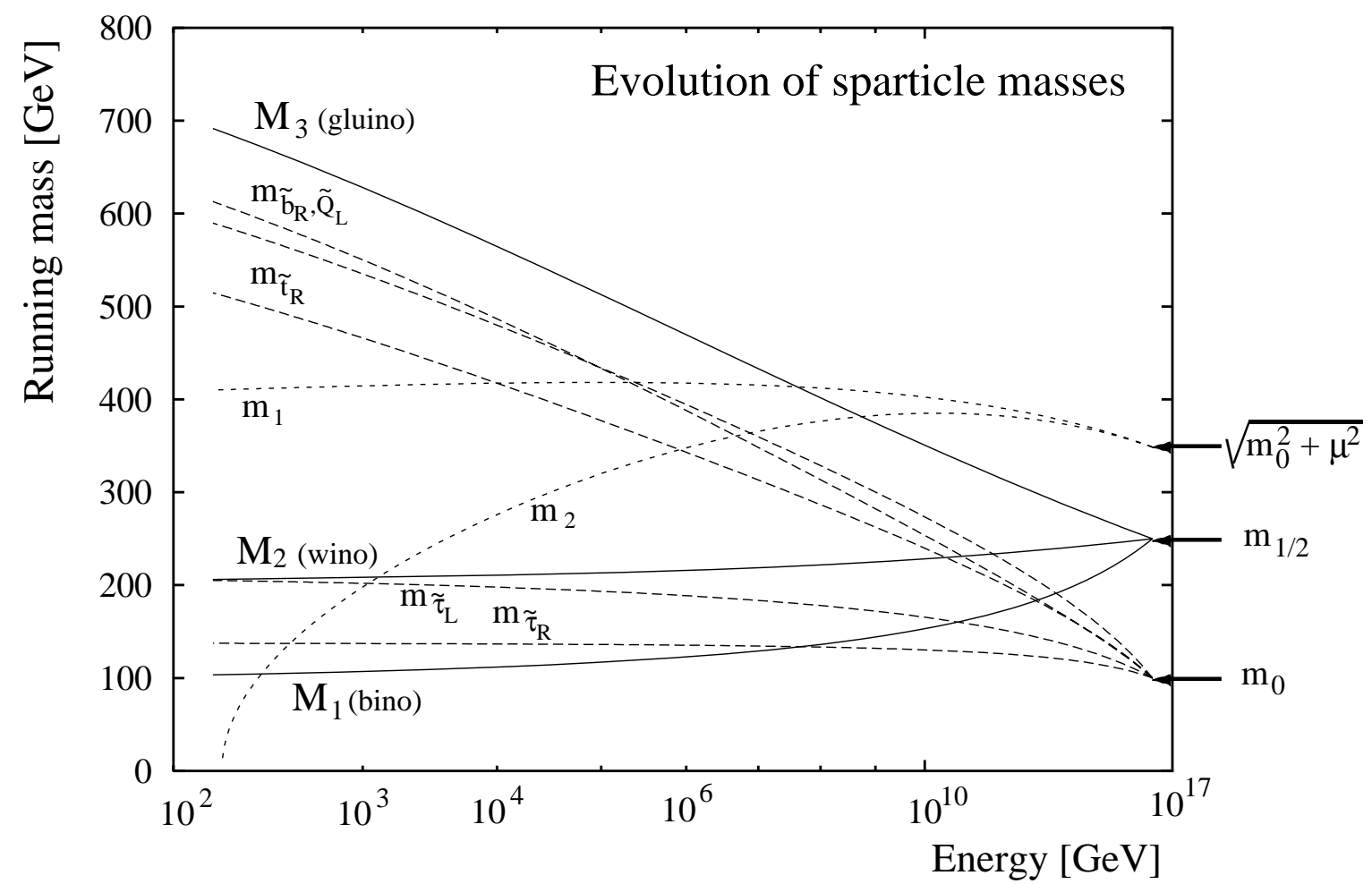

Figure 1.9: Typical evolution of sparticle masses in a SUGRA scenario, due to the running of the Renormalisation Group Equations (RGE) from the GUT scale down to the electroweak scale, (This figure was extracted from [58] and has been edited).

As mentioned already, the sparticle masses at the weak scale are obtained from the RGEs. Simple relations can be derived for the gaugino masses and couplings, since the three ratios 
$M_{i} / g_{i}^{2}(i=1,2,3)$ should be independent of the renormalisation group scale (RG-scale), except for small second order corrections. Hence, in the hypothesis that the gauge couplings unify at a higher energy scale, the GUT scale, the following equation must be valid at any RG-scale below the unification scale $\left(Q<Q_{G U T}\right)$, where $Q_{G U T}$ is the GUT, or input scale.

$$
M_{i}(Q)=\frac{g_{i}^{2}(Q)}{g_{i}^{2}\left(Q_{G U T}\right)} \cdot M_{i}(G U T) \quad \Longrightarrow \quad \frac{M_{1}}{g_{1}^{2}} \approx \frac{M_{2}}{g_{2}^{2}} \approx \frac{M_{3}}{g_{3}^{2}} \approx \frac{m_{1 / 2}}{g_{G U T}^{2}}
$$

In case of mSUGRA models, where only the sign of $\mu$ is still undetermined, the above relations expressed in terms of the weak mixing angle, are further constrained:

$$
\begin{aligned}
& M_{1} \approx \frac{5}{3} \tan ^{2} \theta_{w} M_{2} \quad\left(\approx 0.5 M_{2}\right) \\
& M_{3} \approx \frac{\alpha_{S}}{\alpha} \sin ^{2} \theta_{w} M_{2} \quad\left(\approx 3.5 M_{2}\right)
\end{aligned}
$$

Using the approximate $Z$-pole values for the electroweak and strong coupling constants $\alpha \approx 1 / 128$ and $\alpha_{S} \approx 0.118$, as well as for the weak mixing angle $\sin ^{2} \theta_{w}=0.2312$, the above relations lead to $M_{1}: M_{2}: M_{3} \approx 1: 2: 7$. Consequently, the gluino is expected to be heavier than the two lighter neutralinos and the lightest chargino. Since, for most of the parameter space, $\mu^{2}$ is larger than $m_{1 / 2}$ (corresponding to the lightest neutralinos and charginos being rather gaugino-like), the following relations between these lighter neutralinos/charginos and the gluino masses can be derived:

$$
2 m\left(\widetilde{\chi}_{1}^{0}\right) \approx m\left(\widetilde{\chi}_{2}^{0}\right) \approx m\left(\widetilde{\chi}_{1}^{ \pm}\right) \approx \frac{1}{3} m(\widetilde{g}) .
$$

The sfermion masses are related in a similar way, so that one can get a rough idea of the typical mass spectrum in mSUGRA scenarios, as given in Figure 1.10.

$$
m_{\widetilde{\tau}_{1}} \lesssim m_{\widetilde{\ell}_{R}}<m_{\widetilde{\ell}_{L}} \ll m_{\widetilde{t}_{1}} \lesssim m_{\widetilde{q}_{R}}<m_{\widetilde{q}_{L}}
$$

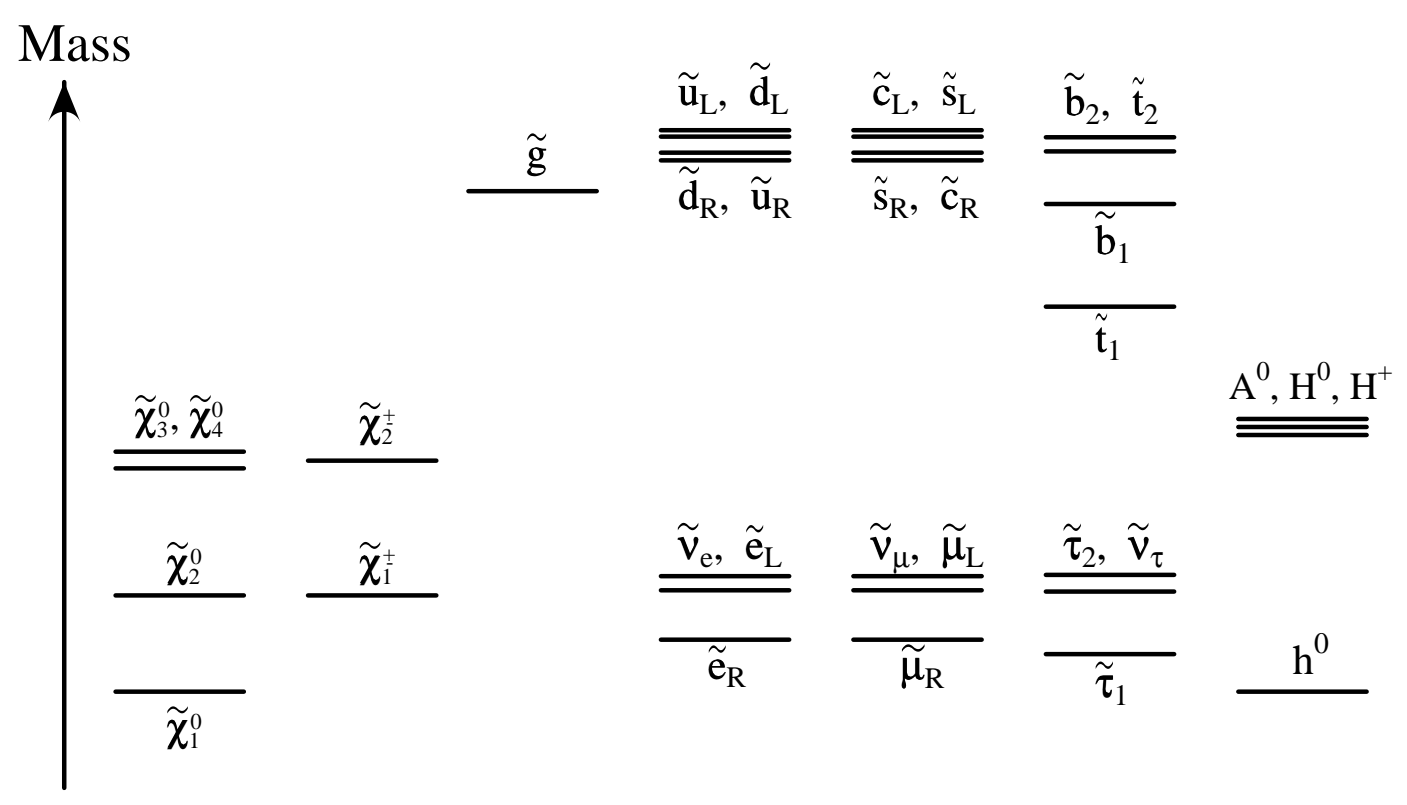

Figure 1.10: Schematic mass spectrum of all supersymmetric particles and Higgs bosons in mSUGRA or MSSM scenarios with unified gaugino masses, scalar masses and trilinear couplings. (taken from [38]) 


\section{The Minimal Supersymmetric Standard Model - MSSM}

Another phenomenological model, similar to the previously discussed mSUGRA model, is the MSSM. This model is based on essentially the same assumptions, like a common gaugino mass $\left(m_{1 / 2}\right)$, a common scalar mass $\left(m_{0}\right)$ and a common trilinear coupling parameter $\left(A_{0}\right)$. The main difference between mSUGRA and the MSSM considered here, is the exclusion of the Higgs masses $\left(M_{H_{u}}, M_{H_{d}}\right)$ from the common scalar mass in the latter. This leads to two more continuous free parameters, namely the mass of the pseudoscalar Higgs boson $\left(M_{A}\right)$ and the mixing parameter of the Higgs doublets $\mu$, both of which are calculable from $\tan \beta$ and $m_{0}$ in mSUGRA, leaving only the sign of $\mu$ free. The ratio of the Higgs field VEVs, $\tan \beta$, is defined as in mSUGRA, such that the MSSM is made up of six continuous free parameters: $m_{1 / 2}, m_{0}, A_{0}, \tan \beta$ and additionally also $M_{A}$ and $\mu$.

However, the specific phenomenological MSSM, in which the results of this search for supersymmetry ought to be interpreted, is different in that the previously assumed unification of couplings at the GUT scale is relaxed. This means, Equation (1.67) relating $M_{1}$ and $M_{2}$ is not expected to be valid anymore, such that the neutralino and chargino masses are not related to each other. This allows to cover a larger parameter space and set more independent lower limits on the masses of the lightest chargino and neutralino states. Although relation (1.68) is still assumed to hold, this model is referred to as no-GUT MSSM in what follows. (Due to the much higher gluino mass $\left(M_{3}\right)$, relation (1.68) has no considerable influence on neither the topology, nor the kinematics of the class of supersymmetric events studied here.)

\begin{tabular}{|c|c|c|c|c|}
\hline Particle Names & Spin & Gauge Eigenstates & Mass Eigenstates & $R_{p}$ \\
\hline Higgs bosons & 0 & $\begin{array}{cccc}H_{u}^{+} & H_{u}^{0} & H_{d}^{0} & H_{d}^{-} \\
\end{array}$ & $\begin{array}{llll}A^{0} & H^{ \pm} & H^{0} & h^{0} \\
\end{array}$ & +1 \\
\hline Sleptons & 0 & $\begin{array}{ccc}\widetilde{e}_{L} & \widetilde{e}_{R} & \widetilde{\nu}_{e} \\
\widetilde{\mu}_{L} & \widetilde{\mu}_{R} & \widetilde{\nu}_{\mu} \\
\widetilde{\tau}_{L} & \widetilde{\tau}_{R} & \widetilde{\nu}_{\tau}\end{array}$ & \begin{tabular}{cc}
\multicolumn{2}{c}{ same } \\
\multicolumn{2}{c}{ same } \\
$\widetilde{\tau}_{1} \quad \widetilde{\tau}_{2} \quad \widetilde{\nu}_{\tau}$
\end{tabular} & -1 \\
\hline Squarks & 0 & $\begin{array}{cccc}\widetilde{u}_{L} & \widetilde{u}_{R} & \widetilde{d}_{L} & \widetilde{d}_{R} \\
\widetilde{c}_{L} & \widetilde{c}_{R} & \widetilde{s}_{L} & \widetilde{s}_{R} \\
\widetilde{t}_{L} & \widetilde{t}_{R} & \widetilde{b}_{L} & \widetilde{b}_{R} \\
\end{array}$ & \begin{tabular}{cccc}
\multicolumn{5}{c}{ same } \\
\multicolumn{4}{c}{ same } \\
$\tilde{t}_{1}$ & $\widetilde{t}_{2}$ & $\widetilde{b}_{1}$ & $\widetilde{b}_{2}$
\end{tabular} & -1 \\
\hline $\begin{array}{l}\text { Neutralinos } \\
\text { Charginos }\end{array}$ & $\begin{array}{l}1 / 2 \\
1 / 2\end{array}$ & $\begin{array}{cccc}\widetilde{B}^{0} & \widetilde{W}^{0} & \widetilde{H}_{u}^{0} & \widetilde{H}_{d}^{0} \\
\widetilde{W}^{ \pm} & \widetilde{H}_{u}^{+} & \widetilde{H}_{d}^{-}\end{array}$ & $\begin{array}{cccc}\widetilde{\chi}_{1}^{0} & \widetilde{\chi}_{2}^{0} & \widetilde{\chi}_{3}^{0} & \widetilde{\chi}_{4}^{0} \\
& \widetilde{\chi}_{1}^{ \pm} & \widetilde{\chi}_{2}^{ \pm} & \end{array}$ & $\begin{array}{l}-1 \\
-1\end{array}$ \\
\hline $\begin{array}{l}\text { Gluinos } \\
\text { (Gravitino }\end{array}$ & $\begin{array}{l}1 / 2 \\
3 / 2\end{array}$ & $\begin{array}{c}\widetilde{g}_{1}, \widetilde{g}_{2}, \ldots \widetilde{g}_{8} \\
\widetilde{G}\end{array}$ & $\begin{array}{l}\text { same } \\
\text { same }\end{array}$ & $\begin{array}{l}-1 \\
-1)\end{array}$ \\
\hline
\end{tabular}

Table 1.5: Overview of the »undiscovered « particles in the mSUGRA and MSSM models. The mass eigenstates are shown in comparison with the symmetry eigenstates. ( ${ }^{\ddagger}$ only in SUGRA scenarios)

Independent of the specific model, the (minimal) particle content in terms of the physical states is the same in either mSUGRA, or the MSSM. For the not yet discovered particles, it is given in Table 1.5, where the mass eigenstates are related to the gauge eigenstates. In the last column, the R-parity quantum number is denoted. As will be explained more thoroughly in the next section, this number allows to distinguish SM and SUSY-particles. The earlier distinction between chiral 
and gauge supermultiplets as in Table 1.4 is no longer possible, because of the mixing of chiral higgsinos and gauginos into neutralino and chargino mass eigenstates.

\subsubsection{R-parity and Its Consequences}

Now, with the introduction of R-parity $\left(R_{p}\right)$ - or rather its violation [59] - another complication is added to the overall picture... To start with, each particle, SM-particle or SUSY-particle, is characterized by an R-parity quantum number defined as $R_{p}=(-1)^{3(B-L)+2 S}$, with $B$ being the baryon, $L$ the lepton and $S$ the spin quantum number. Hence, SM-particles have $R_{p}=+1$ and supersymmetric particles $R_{p}=-1$, see also Table 1.5 above. R-parity in supersymmetry refers to a discrete symmetry which follows from the conservation of lepton-number $(L)$ and baryon-number $(B)$. The most general R-parity conserving superpotential can be written as:

$$
W_{R_{p}}=\bar{U} \mathbf{y}_{\mathbf{u}} Q H_{u}-\bar{D} \mathbf{y}_{\mathbf{d}} Q H_{d}-\bar{E} \mathbf{y}_{\mathbf{e}} L H_{d}+\mu H_{u} H_{d} .
$$

Since the notation is the same as in Table 1.4 of section 1.3 .2 , the superfields $Q, L, H_{u}$ and $H_{d}$ are the usual weak-isospin $\left(\mathrm{SU}(2)_{L}\right)$ doublets, while $\bar{U}, \bar{D}$ and $\bar{E}$ are $\mathrm{SU}(2)_{L}$-singlet superfields. The bold face matrices $\mathbf{y}_{\mathbf{u}}, \mathbf{y}_{\mathbf{d}}$ and $\mathbf{y}_{\mathbf{e}}$ are the $3 \times 3$ Yukawa matrices in generation space and the last term $\mu H_{u} H_{d}$ mixes the up- and down-type Higgs fields. Lepton and baryon numbers are assigned to the chiral supermultiplets in the following way:

\begin{tabular}{crrccccc} 
& $L_{i}$ & $\bar{E}_{i}$ & $Q_{i}$ & $\bar{U}_{i}$ & $\bar{D}_{i}$ & $H_{u}$ & $H_{d}$ \\
\hline \hline$L:$ & +1 & -1 & 0 & 0 & 0 & 0 & 0 \\
$B:$ & 0 & 0 & $+1 / 3$ & $-1 / 3$ & $-1 / 3$ & 0 & 0
\end{tabular}

In models with exact R-parity, every interaction vertex must contain an even number of $R_{p^{-}}$ negative particles, i.e. SUSY-particles. Furthermore, the lightest supersymmetric particle (LSP) is absolutely stable; due to its negative $R_{p}$ quantum number, it cannot decay into SM-particles. On the other hand, all heavier SUSY-particles, once produced, will sooner or later cascade decay into the lightest $R_{p}$-negative particle, the LSP. For this reason, an electrically neutral LSP would make a perfect candidate for dark matter required by cosmology.

However, neither $B$-, nor $L$-conservation is required by gauge or any other invariance. As has been pointed out, minimal SUSY requires the presence of two Higgs SU(2)-doublets, $H_{u}$ and $H_{d}$. Since $H_{d}$ has exactly the same gauge quantum numbers as the lepton doublet superfields $L_{i}$, the latter can replace the former in the $R_{p}$-conserving Yukawa potential $W_{R_{p}}$ of Equation (1.71) and the gauge invariance of the Lagrangian is retained. Another gauge invariant term, breaking R-parity, is the baryon number violating term $U_{i} D_{j} \bar{D}_{k}$ and no theoretical consideration prevents the introduction of Yukawa interactions involving three $\mathrm{SU}(2)$-singlet fermion superfields. All of these $\not_{p}$-terms are parametrized in the superpotential $W_{\mathbb{R}_{p}}[60]$ and drafted in Figure 1.11.

$$
W_{\not R_{p}}=\underbrace{\frac{1}{2} \lambda_{i j k} L_{i} L_{j} \bar{E}_{k}+\lambda_{i j k}^{\prime} L_{i} Q_{j} \bar{D}_{k}+\mu_{i} L_{i} H_{u}}_{\Delta L=1}+\underbrace{\frac{1}{2} \lambda_{i j k}^{\prime \prime} \bar{U}_{i} \bar{D}_{j} \bar{D}_{k}}_{\Delta B=1}
$$

These additional terms in the superpotential not only allow single sparticle production, (resonant production), but also the decay of SUSY-particles into ordinary SM-particles. The coupling strengths are given by the Yukawa couplings $\lambda, \lambda^{\prime}$ and $\lambda^{\prime \prime}$, where $\lambda_{i j k}$ is antisymmetric under the 
exchange of the first two $\mathrm{SU}(2)$ indices, while $\lambda_{i j k}^{\prime \prime}$ is antisymmetric under the exchange of the last two. Since the $L$-violating, bilinear term, $\mu_{i} L_{i} H_{u}$, mixing lepton and Higgs superfields, is usually rotated to zero, to eliminate direct bilinear $R_{p}$-violation [61], a total of 45 new parameters $(L L \bar{E}+L Q \bar{D}+U \bar{D} \bar{D}=9+27+9)$ is introduced into the superpotential.
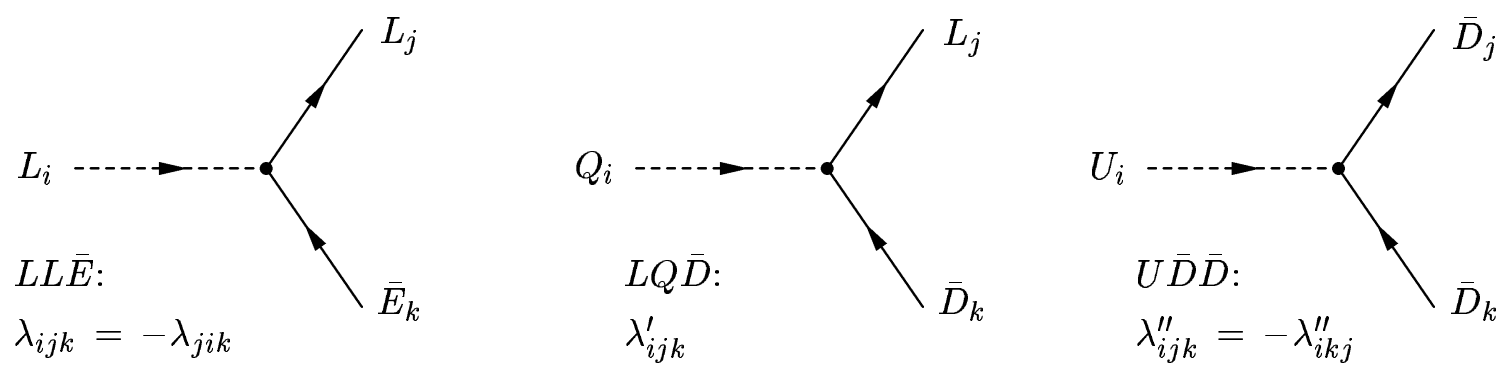

Figure 1.11: Interactions (LO) associated with trilinear $\not R_{p}$-terms. The Yukawa couplings $\lambda$, $\lambda^{\prime}$ and $\lambda^{\prime \prime}$ give the strength of the interaction, while $L$ and $\bar{E}$ denote the weak isospin doublet and singlet (s)leptonfields and $Q$ and $\bar{U}, \bar{D}$ denote the respective doublet and singlet (s)quark eigenstates.

Originally, R-parity was introduced in 1978 by G.R. Farrar and P. Fayet [62] to account for the experimental limits on $B$ - and $L$-violation, especially the non-observation of proton decay. However, it was questioned even earlier that the latter would require an absolute conservation of baryon number [63]. The lower limit on the proton lifetime is $10^{32}$ to $10^{33}$ years, depending on the assumed decay mode. It allows to set stringent limits only on the product $\left(\lambda^{\prime} \cdot \lambda^{\prime \prime}\right)$ of $B$ - and $L$-violating couplings [64], see also Figure 1.12 (a). Hence, it is sufficient to require only one type of trilinear $\boldsymbol{R}_{p}$-coupling to be present at a time to adequately protect the proton. The resonant production of sleptons at hadron colliders requires a coupling of the $L Q \bar{D}$-type,

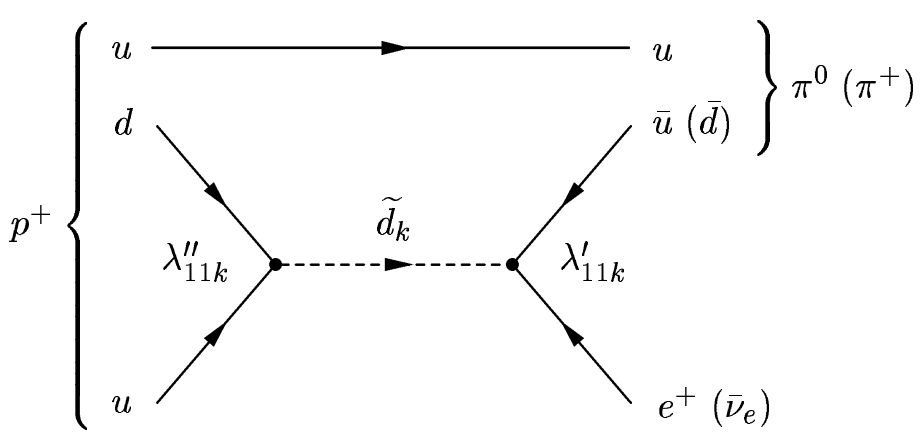

(a)

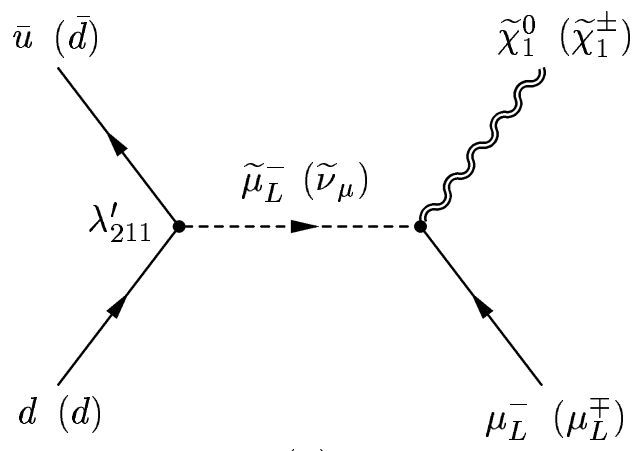

(b)

Figure 1.12: $R$-parity violating processes: (a) proton decay is possible, if $L Q \bar{D}$ and $U \bar{D} \bar{D}$-couplings are present at the same time and (b) resonant slepton production via the $L Q \bar{D}$-coupling $\lambda_{211}^{\prime}$.

of which an example is depicted in Figure 1.12 (b) for the $\lambda_{211}^{\prime}$ coupling. This coupling allows the production of second generation sleptons, i.e. smuon $(\widetilde{\mu})$ or muon sneutrino $\left(\widetilde{\nu}_{\mu}\right)$; for a more detailed description of this process and an analysis of the resulting final states, see [65]. Various other diagrams involving one or more $\lambda, \lambda^{\prime}$, or $\lambda^{\prime \prime}$ couplings are possible. Apart from the stringent limits on the simultaneous presence of $L Q \bar{D}$ - and $U \bar{D} \bar{D}$-operators from proton decay, other significant limits are extracted from measurements of rare decays, like, e.g. $K^{+} \rightarrow \pi^{+} \nu \bar{\nu}$, or $B_{d, s}^{0} \rightarrow e^{+} e^{-}\left(\mu^{+} \mu^{-}\right)$, or $b \rightarrow s \gamma$. Similarly, neutrinoless double $\beta$-decay $(0 \nu \beta \beta)$ offers the possibility to constrain single $L Q \bar{D}$-couplings or products of this type of couplings. Different 
couplings, primarily of the $L L \bar{E}$, or the $L Q \bar{D}$-type can be constrained by measurements of the mixing in the neutral Kaon system $\left(K^{0} \leftrightarrow \bar{K}^{0}\right)$, or the $B_{d}\left(B_{s}\right)$-meson systems $\left(B_{d}^{0} \leftrightarrow \bar{B}_{d}^{0}\right.$, or $\left.B_{s}^{0} \leftrightarrow \bar{B}_{s}^{0}\right)$. Precise measurements of the electron electric dipole moment, where $L L \bar{E}$-couplings alone, or $L L \bar{E}$ - together with $L Q \bar{D}$-couplings can contribute to higher order corrections, also allow to set bounds on the strength of these couplings or products of them. For a detailed review of $\not R_{p}$-SUSY and a complete compilation of (nearly) all possible combinations of couplings and the corresponding upper bounds see [61]. Similar, but somewhat shorter reviews include [66] and [67]. Another general motivation for investigating $\not R_{p}$-models lies in their inherent ability to explain small neutrino masses through the mixing of neutrinos with neutralinos as is detailed in references [68] and [69].

In the following, the so-called $\not R_{p}$ weak limit [70] will be adopted, where the $\not R_{p}$-couplings are considerably smaller than the gauge couplings. Additionally, the single coupling dominance hypothesis [59], [61] is employed, which essentially means that only one coupling has a significant influence, while all other $\boldsymbol{R}_{p}$-couplings are assumed to be either zero, or negligibly small.

\subsubsection{Gaugino Pair Production and $\not R_{p}$-decay of the LSP}

In this thesis, a search for multilepton final states in $p \bar{p}$ annihilation is performed. Final states, with at least two muons and one additional charged lepton, either an electron or a muon, are interpreted in the hypothesis of $\not R_{p}$-supersymmetry with the $L L \bar{E}$-coupling $\lambda_{122}$ dominating all other couplings [57]. Since $L L \bar{E}$-couplings connect only (s)lepton, but no (s)quark fields, resonant sparticle production is not possible at a $p \bar{p}$-collider as the Tevatron (see section 2.2). The production process thus conserves R-parity and, because the squarks and gluinos are assumed to be heavier than neutralinos and charginos, the dominant production process is gaugino pair production [71], [72]. In the gaugino region, where the gauginos contain mostly wino or bino components, the production proceeds via the electroweak bosons (Fig. 1.13 (a)), while in the opposite case - in the higgsino region - the gaugino couplings to squarks are larger, so that they are predominantly produced via t-channel virtual squark exchange as shown in Figure 1.13 (b).
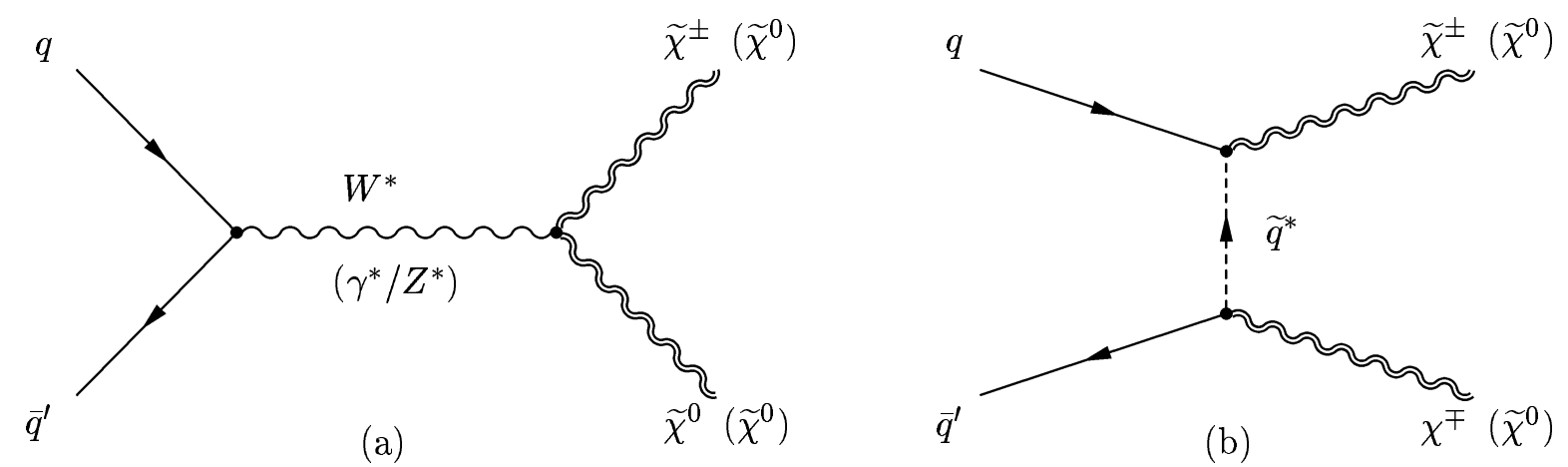

Figure 1.13: $R$-parity conserving gaugino pair production via (a) s-channel gauge boson exchange or (b) t-channel squark exchange.

Due to the assumption of the $\not R_{p}$ weak limit, the heavier gauginos $\left(\widetilde{\chi}^{ \pm}{ }_{2}\right.$ and $\left.\widetilde{\chi}^{0}{ }_{2,3,4}\right)$ usually cascade decay into the lightest SUSY-particle (LSP), which is assumed to be always the lightest neutralino $\left(\widetilde{\chi}_{1}^{0}\right)$. Since this lightest SUSY-particle cannot decay into any other sparticles anymore, it must decay into SM-particles via the one $\mathbb{R}_{p}$-coupling, that was chosen not to be 
negligibly small, i.e. $\lambda_{122}$. The Feynman diagrams of all possible LSP-decays via $\lambda_{122}$ are given in Figure 1.14. Direct decays of the heavier gaugino mass eigenstates into SM-particles are also possible, though suppressed due to the smallness of the $\not R_{p}$-couplings compared to the gauge couplings.
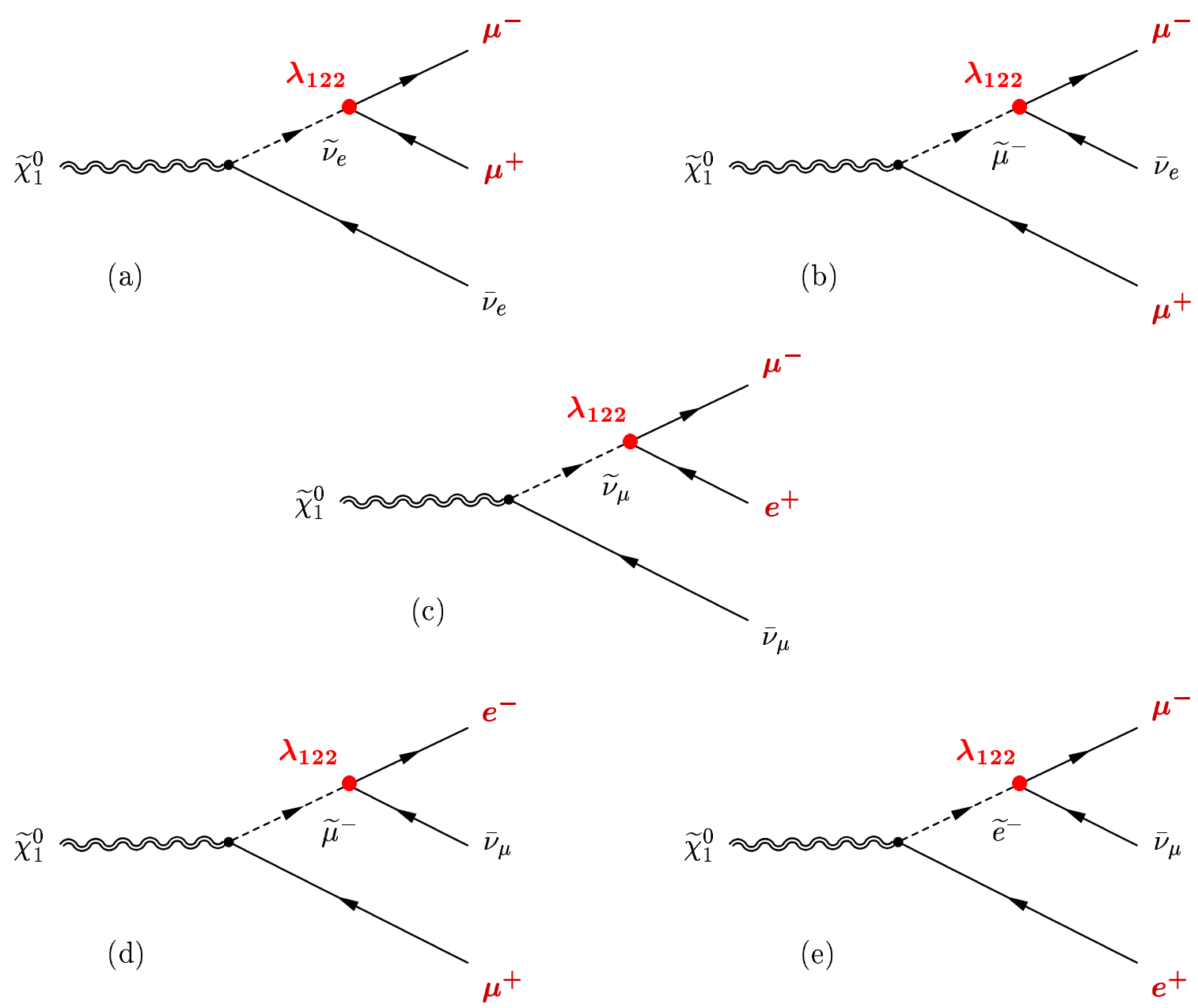

Figure 1.14: $R$-parity violating decays of the lightest neutralino (LSP) via the $L L \bar{E}$-coupling $\lambda_{122}$. In the processes $(a, b)$ two muons are produced, while $(c, d, e)$ lead to an electron-muon final state.

The heavy neutralinos or charginos usually cascade decay ( $R_{p}$-conserving) into the LSP. If they are sufficiently heavy, their decays into lighter sparticles are accompanied by either an electroweak gauge boson or one of the five Higgs bosons $h, H, H^{ \pm}$or $A$, see Figure 1.15 (a). In case the decay with associated bosons is kinematically not possible, they decay predominantly into a fermion/anti-fermion pair and a lighter gaugino (three body decay) as illustrated in Figure 1.15 (b). The heavy gaugino mass eigenstates may also decay directly into SM particles via the $\not_{p}$-coupling $\lambda_{122}$. Since the coupling is small, these direct decays are negligible for $\widetilde{\chi}_{3}^{0}, \widetilde{\chi}_{4}^{0}$, and the two charginos, but can account for up to $30 \%$ of the branching ratio of the second lightest neutralino, $\widetilde{\chi}_{2}^{0}$, in regions with low gaugino mass parameter $m_{1 / 2}$. The direct decays of heavier neutralinos proceed in exactly the same way as for the LSP, leading to final states with $\mu \mu \nu_{e}$ or $\mu e \nu_{\mu}$. For completeness, the Feynman diagrams of the chargino direct $\not R_{p}$-decays via $\lambda_{122}$ are presented in Figure 1.16. The resulting final states are $\mu \mu e$ (50\%), $e \nu_{\mu} \nu_{\mu}(25 \%)$, and $\mu \nu_{\mu} \nu_{e}(25 \%)$. 


$$
\left.\begin{array}{rl}
\widetilde{\chi}_{p}^{ \pm} & \rightarrow \widetilde{\chi}_{n}^{ \pm}+Z^{0}(h, H, A) \\
& \rightarrow \widetilde{\chi}_{n}^{0}+W^{ \pm}\left(H^{ \pm}\right) \\
\widetilde{\chi}_{p}^{0} & \rightarrow \widetilde{\chi}_{n}^{ \pm}+W^{\mp}\left(H^{\mp}\right) \\
& \rightarrow \widetilde{\chi}_{n}^{0}+Z^{0}(h, H, A)
\end{array}\right\} p>n
$$

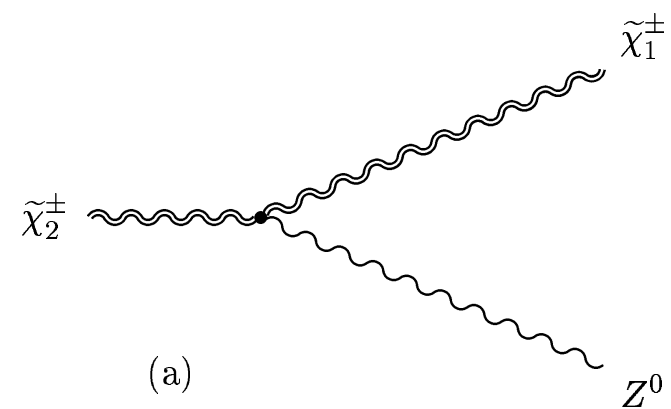

$$
\left.\begin{array}{rl}
\widetilde{\chi}_{p}^{ \pm} & \rightarrow \widetilde{\chi}_{n}^{ \pm}+f \bar{f} \\
& \rightarrow \widetilde{\chi}_{n}^{0}+\ell^{ \pm} \nu(\bar{\nu}) \\
\widetilde{\chi}_{p}^{0} & \rightarrow \widetilde{\chi}_{n}^{0}+f \bar{f} \\
& \rightarrow \widetilde{\chi}_{n}^{ \pm}+\ell^{\mp} \bar{\nu}(\nu)
\end{array}\right\} p>n
$$

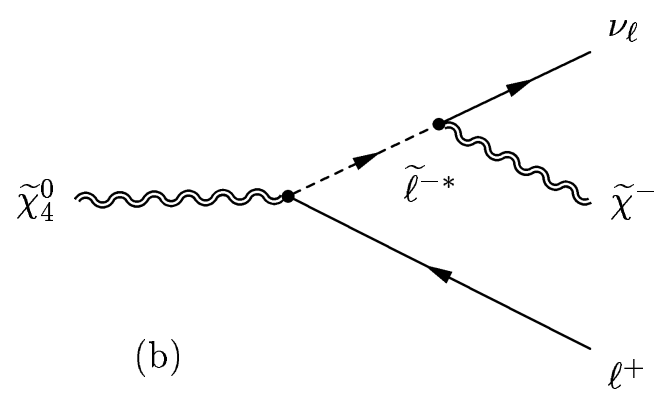

Figure 1.15: $R$-parity conserving cascade decays of heavier chargino and neutralino mass eigenstates. In (a) the decay is accompanied by a gauge or Higgs boson; in (b) the gauginos decay into a fermion/antifermion pair and a lighter gaugino, respectively.
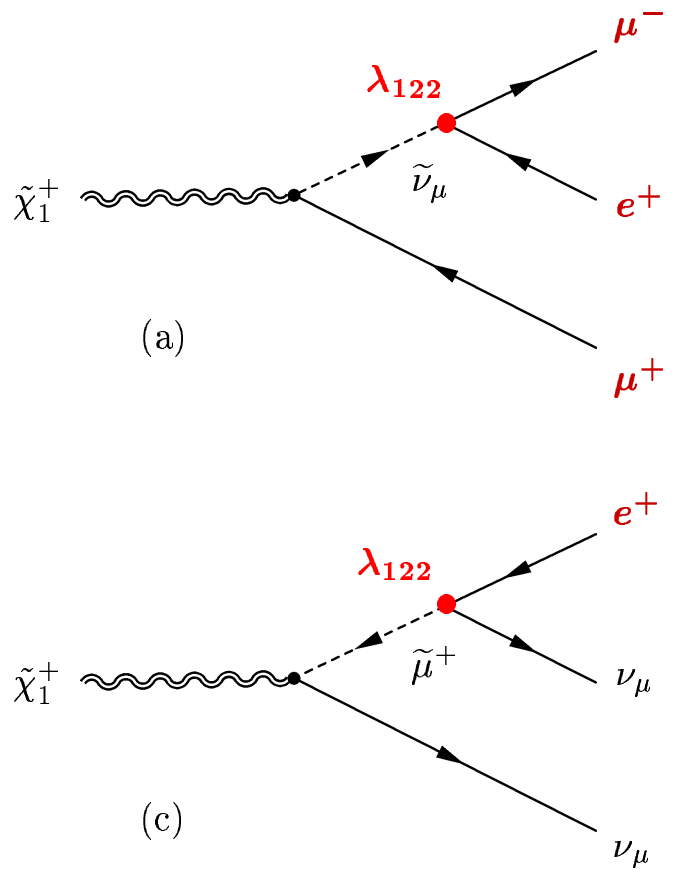
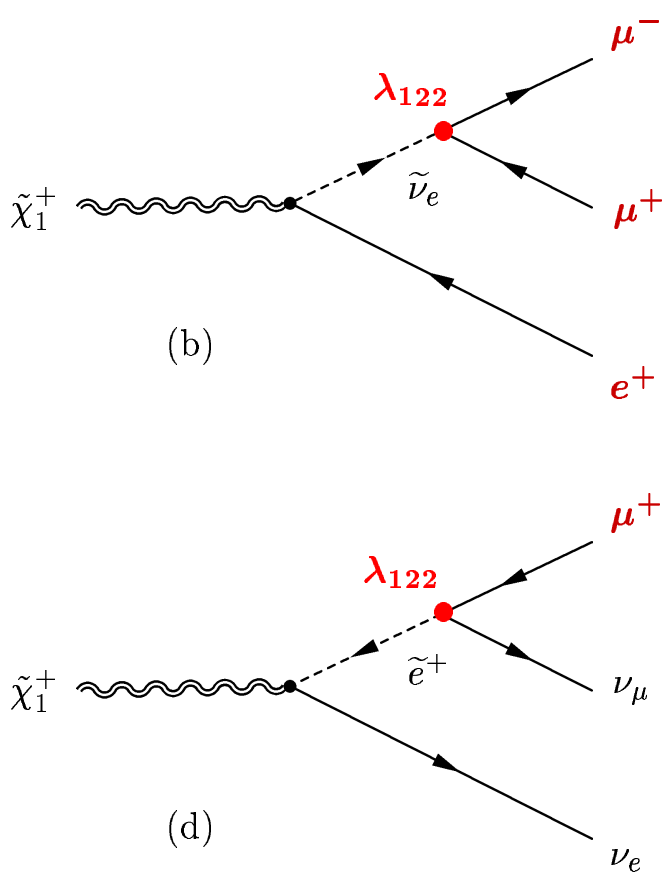

Figure 1.16: Direct $\not R_{p}$-decays of the lightest chargino via the $L L \bar{E}$-coupling $\lambda_{122}$. The processes $(a, b)$ produce three charged leptons ( $\mu \mu e)$ and processes $(c, d)$ lead to a final state with two neutrinos and either and electron or a muon. The charge conjugate processes are not shown explicitely.

With the above assumption of gaugino pair production, and a negligible contribution of chargino direct $\not R_{p}$-decays, always two neutralinos (mostly the LSPs) decay R-parity violating via $\lambda_{122}$. This leads to at least four charged leptons in the final state, which are easily identified by combining any two of the Feynman diagrams of Figure 1.14 into three different multilepton signatures: $\mu \mu \mu \mu+X, \mu \mu \mu e+X$ and $\mu \mu e e+X$. Although $X$ may be comprised of hadrons only, 
it is likely that even more charged leptons are present, depending on the preceding cascade of the heavier gauginos and on the actual SUSY mass spectrum.

However, since the $\widetilde{\chi}_{1}^{0}$ can be very light, with a mass as small as $\approx 30 \mathrm{GeV}$ (in case of the no-GUT MSSM, see section 1.3.5), its decay products, the leptons in the final state may have only small transverse momentum $\left(p_{\mathrm{T}}\right)$ and hence, are difficult to detect. For this reason, only three charged leptons are required to be identified and the search is optimised with respect to the two topologies $\mu \mu \mu$ and $\mu \mu e$. Although $\mu e e$ is a possible combination of three lepton flavours from the $\lambda_{122}$-coupling, (i.e. if one of the expected two muons is not detected), this one is not considered here, since it is already covered by two other searches for multilepton final states from $\not R_{p}$-SUSY scenarios. Both of which have also been developed within the D $\varnothing$ collaboration over the last three years [73] and [74]. The former primarily investigates decays involving the $L L \bar{E}$-coupling $\lambda_{121}$, which lead to the lepton flavour combinations eeee, eеe $\mu$ and ee $\mu \mu$; similarly to those of the $\lambda_{122}$-coupling considered in the present analysis. The latter search focuses on the topology ee $\tau$ (with hadronically decaying tau leptons) of yet another $L L \bar{E}$-coupling, namely $\lambda_{133}$. Both analysis are able to recover potential inefficiencies, especially with respect to the $\mu e e$ topology of the present analysis, and vice versa. This is precisely the reason, why the results of all three analyses are combined for each one of the three couplings $\lambda_{121}, \lambda_{122}$ and $\lambda_{133}$, see chapter 4 .

\subsubsection{Indirect Bounds On $\not R_{p}$ Couplings}

In this last theoretical section the existing limits and their origins are reviewed. As $\not R_{p}$ interactions can contribute to various processes, also at low energies through the exchange of virtual SUSYparticles, bounds on $\not_{p}$-couplings can be derived from many measurements provided the data agree with the SM predictions. Since this thesis is based on a non-vanishing $\lambda_{122}$ coupling, and the two other already mentioned analyses from [73], and [74] involve the couplings $\lambda_{121}$ and $\lambda_{133}$, respectively, the constraints on operators of the $L L \bar{E}$-type are discussed in more detail.

The most stringent bounds on the $L L \bar{E}$-couplings come from either Charged Current universality, or the indirect limits on the masses of neutrinos. Charged (Neutral) Current universality is nothing extraordinarily new, but just refer to the universality of the fermions' couplings to the $W(Z)$ bosons as predicted by the SM. Hence, precision tests, such as measurements of the electroweak observables $M_{Z}$ and $\sin ^{2} \theta_{w}$, the lifetime of the muon $\left(G_{F}\right)$ or the CKM-matrix elements allow to constrain only single $L L \bar{E}$-couplings very well [66], [75]. The operator $L_{1} L_{2} \bar{E}_{k}$
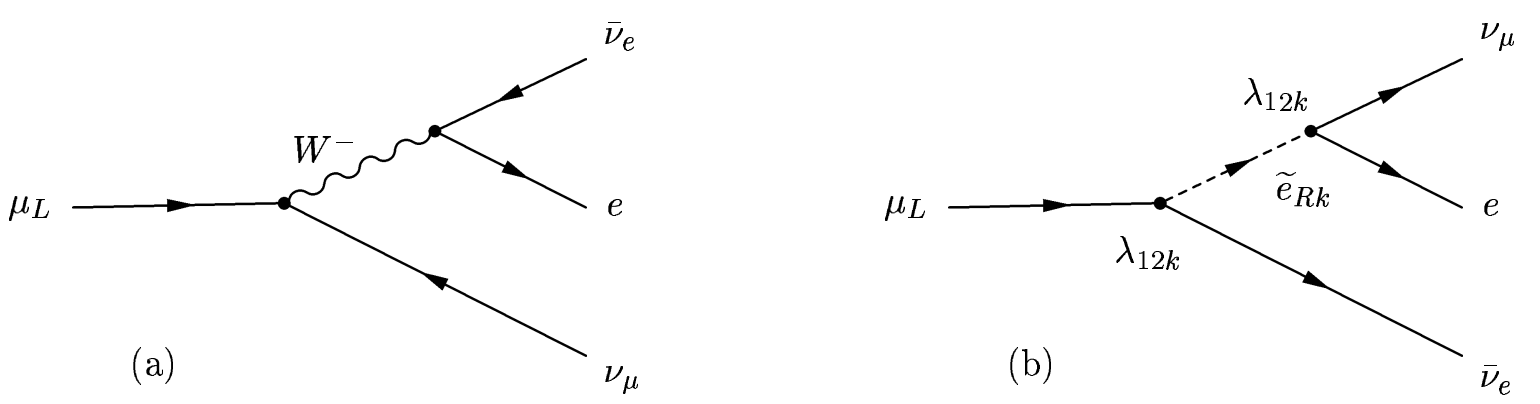

Figure 1.17: The muon decay contributions to the Fermi constant $G_{F}$ from (a) SM interactions and (b) $\not_{p}$-interactions, involving the operator $L_{1} L_{2} \bar{E}_{k}$, i.e. coupling constants $\lambda_{12 k}$. 
leads, for example, to additional contributions in the decay of muons, as shown in Figure 1.17, and thus, also changes the Fermi coupling constant $G_{F}$, which is determined from precision measurements of exactly this decay. The tree-level effective constant in the presence of an $L_{1} L_{2} \bar{E}_{k}$ operator reads:

$$
G_{F}=\frac{\sqrt{2} g^{2}}{8 M_{W}^{2}}+\frac{\sqrt{2}}{8 m_{\widetilde{e}_{R k}}^{2}}\left|\lambda_{12 k}\right|^{2} .
$$

However, due to large radiative corrections, the tree-level relation for $G_{F}$ cannot be used to derive conservative constraints on any $\lambda_{12 k}$ coupling. A theoretical analysis using two different renormalisation schemes is given in [61]. From an experimental point of view, one measures the ratio $\left(R_{\tau \mu}\right)$ of the rates of tau-decay and muon-decay and the comparison of the measured value with the SM-expectation then leads to an upper bound on the $R_{p^{-}}$-coupling $\lambda_{12 k}$ [75].

$$
R_{\tau \mu}^{\text {meas }}=\frac{\Gamma(\tau \rightarrow \mu \nu \bar{\nu})}{\Gamma(\mu \rightarrow e \nu \bar{\nu})} \leftrightarrow R_{\tau \mu}^{S M} \quad \Rightarrow \quad \lambda_{12 k}<0.07\left(\frac{m_{\widetilde{e}_{R}}}{100 \mathrm{GeV}}\right)
$$

The measurement of $R_{\tau}$, the ratio of tau-decay rates $\left(\tau \rightarrow e \bar{\nu}_{e} \nu_{\tau}\right.$ and $\left.\tau \rightarrow \mu \bar{\nu}_{\mu} \nu_{\tau}\right)$ analogously allows to extract upper bounds on the $\lambda_{13 k}\left(\lambda_{23 k}\right)$ couplings, which correspond to the $L_{1} L_{3} \bar{E}_{k}$ $\left(L_{2} L_{3} \bar{E}_{k}\right)$ operators. Although this method already yields tight upper limits on the strengths of possible $\lambda_{12 k}$ and $\lambda_{13 k}$ couplings, precision measurements of the CKM-matrix elements, in particular $V_{u d}$ allow to extract even more restrictive bounds on $\lambda_{12 k}$, although processes in the quark sector always involve also a coupling of the $L Q \bar{D}$, or the $U \bar{D} \bar{D}$-type [76].

Indirect limits on neutrino masses provide another possibility to constrain $L L \bar{E}$ couplings with the last two $\mathrm{SU}(2)_{L}$ indices being identical. This is explained by a possible slepton-lepton loop in higher order corrections to the electron neutrino mass [61]. In case of the two couplings $\lambda_{122}$ and $\lambda_{133}$, that are of interest here, the bounds from the electron neutrino mass limit are the most restrictive ones. Figure 1.18 shows the corresponding Feynman graphs to illustrate how R-parity violating interactions can contribute to the electron neutrino mass. All indirect upper bounds on the relevant $L L \bar{E}$-couplings are summarised in Table 1.6.

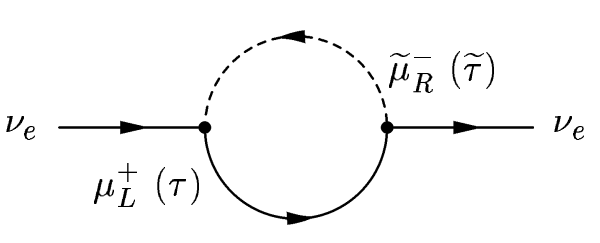

Figure 1.18: Higher order contributions to the neutrino mass $m\left(\nu_{e}\right)$ through $R$-parity violating interactions via either $\lambda_{122}$ or $\lambda_{133}$.

\begin{tabular}{lclc}
\hline \hline$R_{p}$-operator & Coupling(s) & \multicolumn{1}{c}{ Indirect Bounds } & Process \\
\hline \hline$L_{1} L_{2} \bar{E}_{k}$ & $\lambda_{121}, \lambda_{122}$ & $<0.05(0.07) \frac{m\left(\widetilde{e}_{R k}\right)}{100 \mathrm{GeV}}$ & $V_{u d}\left(R_{\tau \mu}\right)$ \\
$L_{1} L_{3} \bar{E}_{k}$ & $\lambda_{133}$ & $<0.07 \frac{m\left(\widetilde{e}_{R k}\right)}{100 \mathrm{GeV}}$ & $R_{\tau}$ \\
\hline$L_{i} L_{2} \bar{E}_{2}$ & $\lambda_{122}$ & $<0.027 \sqrt{m\left(\widetilde{\mu}_{R}\right) / 100 \mathrm{GeV}}$ & $m\left(\nu_{e}\right)<1 \mathrm{eV}$ \\
$L_{i} L_{3} \bar{E}_{3}$ & $\lambda_{133}$ & $<0.0016 \sqrt{m\left(\widetilde{\tau}_{R}\right) / 100 \mathrm{GeV}}$ & $m\left(\nu_{e}\right)<1 \mathrm{eV}$ \\
\hline \hline
\end{tabular}

Table 1.6: Indirect bounds on single $\not R_{p}$-couplings of the $L L \bar{E}$-type at the $2 \sigma$-level. Only couplings relevant for this analysis (and the combination with [73], and [74]) are shown. 



\section{Chapter 2}

\section{The Experimental Setup}

An experiment is a question which science poses to Nature, and a measurement is the recording of Nature's answer.

Max Planck (German Physicist, 1858-1947)

In order to search for new particles, or - more precisely - for new phenomena in particle physics, experimentalists need, firstly, a powerful accelerator and, secondly, a large and very good detector to identify and measure all the particles produced in a collision. In this case, the accelerator is the $p \bar{p}$ collider Tevatron, at the Fermi National Accelerator Laboratory, (Fermilab in what follows) near Chicago in the USA, and the DØ detector serves as the experimentalists' eye.

However, before giving a more detailed description of the accelerator and the detector, the first section of this chapter serves as an introduction to some general aspects of $p \bar{p}$ collisions and gives definitions of the most important terms in hadron collider physics. The second section contains a description of the different components of Fermilab's accelerator chain [77, 78] and the third section focuses on the $\mathrm{D} \emptyset$ detector and its various sub-systems [79, 80, 81]. All photographs are taken from reference [82].

\subsection{General Aspects of $p \bar{p}$ Collisions}

A common term used in collider physics is the luminosity $\mathcal{L}$, relating the instantaneous interaction rate at which a specific type of event occurs $(R)$ and the cross section $(\sigma)$ for the considered process:

$$
R=\frac{d N}{d t}=\mathcal{L} \cdot \sigma
$$

The instantaneous luminosity $\mathcal{L}$ is also a measure for the intensity of the two beams and their mutual penetration. It is therefore useful to express the luminosity in terms of machine parameters:

$$
\mathcal{L}=f \cdot \frac{N_{b} n_{p} n_{\bar{p}}}{2 \pi\left(\sigma_{p}^{2}+\sigma_{\bar{p}}^{2}\right)} \cdot F\left(\frac{\sigma_{l}}{\beta^{*}}\right)
$$

where $f$ denotes the revolution frequency of the bunches ( $N_{b}$ in either beam), $n_{p}\left(n_{\bar{p}}\right)$ are the number of protons (antiprotons) per bunch, and $A_{e f f}=2 \pi\left(\sigma_{p}^{2}+\sigma_{\bar{p}}^{2}\right)$ is the effective area of the reaction with $\sigma_{p}\left(\sigma_{\bar{p}}\right)$ being the widths of the Gaussian shape of the $p(\bar{p})$ bunches, respectively. 
An additional form factor $F$, dependent upon the bunch length $\left(\sigma_{l}\right)$ and the amplitude function at the interaction point $\left(\beta^{*}\right)$, corrects for the bunch shape. The beta function is a measure of the beams width and is tuned such that the luminosity is high at the collision points in the accelerator, where experiments are located. This ensures high event rates and allows experimentalists to precisely measure the properties of rarely produced particles. The instantaneous luminosity is measured in $\mathrm{cm}^{-2} \mathrm{~s}^{-1}$. Integration over time of the above equation (2.1) for the rate $R$, yields the number of events $N$ for a specific process

$$
N=\int \mathcal{L} d t \cdot \sigma
$$

The quantity $\int \mathcal{L} d t$ is referred to as integrated luminosity and usually denotes the amount of data collected by a high-energy experiment. Since cross sections are measured in $\mathrm{cm}^{2}$, or rather in pico barn ${ }^{1}$ ( $10^{-12}$ barn) or even femto barn $\left(10^{-15}\right.$ barn $)$, the usual dimension of the integrated luminosity are inverse barns. The number of events produced $(N)$ by a process with the cross section $1 \mathrm{pb}(1 \mathrm{fb})$ can be denoted by the equivalent integrated luminosity of $N \mathrm{pb}^{-1}\left(N \mathrm{fb}^{-1}\right)$.

A major difference between an electron positron collider, like LEP (Large Electron Positron collider) at CERN, and a hadron collider, like the $p \bar{p}$ collider Tevatron at Fermilab, is the fact that the former collides elementary, point-like particles (at least as far as we know today) and the latter collides rather complex particles (protons and antiprotons), made up of various partons (quarks and gluons). This poses an additional problem in the description of the collision process, since the initial momenta of the partons are not known. In case of an electron positron collider, the centre-of-mass energy $\sqrt{s}$ relates directly to the momentum, or more exactly to the momentum transfer $(Q)$, of the colliding particles, whereas the connection between $\sqrt{s}$ and $Q^{2}$ is more complicated in hadron collisions.

Usually a proton is pictured as consisting of only three quarks $(u, u, d)$, but in reality the proton is a much more complex object, where the usual three quarks only appear as a somewhat more pronounced feature, the so-called »valence« quarks. Apart from that, a »sea of quarks and gluons exists within the proton. Differing numbers of quarks (and/or antiquarks) of any of the six quark flavours and a number of gluons $g$ comprise this sea and are continuously created and annihilated.

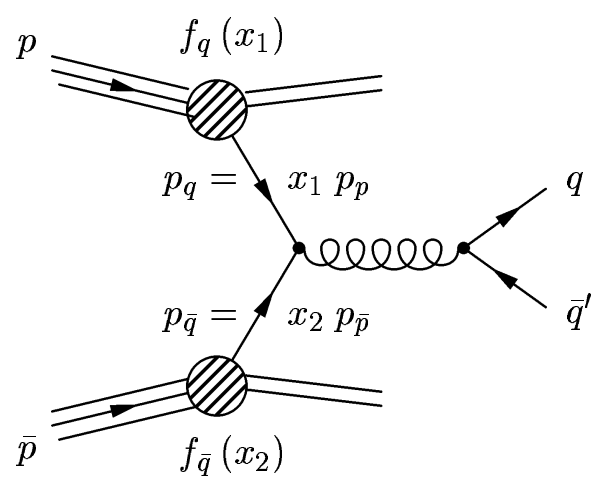

Figure 2.1: Feynman diagram of the proton-antiproton reaction $p \bar{p} \rightarrow 2$ jets.

Each of these constituents, or partons, may carry a different fraction $x$ (also called Bjørken $x$ [83]) of the total momentum $p$ of the proton, see Figure 2.1. Thus the available centre-of-mass energy of the quark-(anti)quark collision is given by $\sqrt{\hat{s}}=\sqrt{x_{1} x_{2} s}$, where $x_{1}$ and $x_{2}$ denote the fraction of momentum carried by the two colliding partons inside the (anti-)proton. However, to be able to theoretically calculate properties of hadronic collisions and to describe them properly in Monte Carlo simulations, these momentum fractions need to be known. Although it is - according to Quantum Mechanics - impossible to know the exact state a parton is in, e.g. its precise position and momentum, it fortunately is possible to define the probability to find a certain parton in a certain state. This probability approach, known under the name parton density function (PDF) will be described in the following.

\footnotetext{
${ }^{1}$ One barn corresponds to $10^{-24} \mathrm{~cm}^{2}$ and is the usual dimension for cross section measurements.
} 


\subsubsection{Parton Density Functions (PDF)}

Parton density functions $\left(f_{\alpha}\right.$, with $\alpha=q, \bar{q}, g$ ) are parametrisations of parton states inside particles such as the proton or antiproton. They are experimentally determined with high accuracy and are a measure for the probability to observe a parton (quark or gluon) with a certain momentum fraction in the intervall $[x, x+d x]$ of the (anti-)proton momentum $p$. The sum of all the different parton momenta must equal the (anti-)proton momentum:

$$
\sum_{\alpha} \int_{0}^{1} x \cdot f_{\alpha}(x) d x=1 .
$$

The leading order cross section for a proton-antiproton interaction as illustrated in Figure 2.1 can then be calculated using $Q^{2}=\left(p_{q}+p_{\bar{q}}\right)^{2}$. The differential cross section for the hard scatter follows as:

$$
\frac{d \sigma}{d Q^{2}}(p \bar{p} \rightarrow X) \propto \sum_{q} \int d x_{1} \int d x_{2} f_{q}\left(x_{1}\right) f_{\bar{q}}\left(x_{2}\right) \frac{d \hat{\sigma}}{d Q^{2}}
$$

where $\frac{d \hat{\sigma}}{d Q^{2}}$ is the differential cross section of the sub-process $q \bar{q}^{\prime} \rightarrow 2$ jets. A summation over all partons of a proton leads to the structure functions $F_{1}(x)$ and $F_{2}(x)$ used in the description of deep inelastic scattering:

$$
2 x F_{1}(x)=F_{2}(x)=x \cdot \sum_{q} e_{q}^{2} \cdot f_{q}(x) .
$$

This description, however, is not complete since the gluons inside a proton or antiproton have to be taken into account as well. A quark with the momentum fraction $x$, participating in the hard scatter may stem, for example, from a gluon with a momentum fraction $y$, which annihilated into a quark-antiquark pair. Hence, additional contributions from sub-processes such as $g \rightarrow q \bar{q}$, $q \rightarrow q g$, or $g \rightarrow g g$ need to be considered, which result in a modification of the structure functions of the proton

$$
\frac{F_{2}\left(x, Q^{2}\right)}{x}=\sum_{q} e_{q}^{2} \cdot\left(f_{q}(x)+\Delta q\left(x, Q^{2}\right)\right)
$$

Although it is possible to calculate the $Q^{2}$-dependence, i.e. the evolution of $\Delta q\left(x, Q^{2}\right)$, the absolute scale of the correction can only be determined experimentally. The complete evolution of quark and gluon densities in the proton are given by a set of differential equations, the so-called DGLAP ${ }^{2}$ equations [3, 84]:

$$
\frac{d}{d \ln Q^{2}}\left(\begin{array}{c}
q_{i}\left(x, Q^{2}\right) \\
g\left(x, Q^{2}\right)
\end{array}\right)=\frac{\alpha_{s}\left(Q^{2}\right)}{q \pi} \sum_{j} \int_{x}^{1} \frac{d y}{y} \cdot\left(\begin{array}{cc}
P_{q_{i} q_{j}}(z) & P_{q_{i} g}(z) \\
P_{g q_{j}}(z) & P_{q q}(z)
\end{array}\right)\left(\begin{array}{c}
q_{j}\left(y, Q^{2}\right) \\
g\left(y, Q^{2}\right)
\end{array}\right)
$$

with $z=\frac{x}{y}$ and $q_{i}\left(x, Q^{2}\right), g\left(x, Q^{2}\right)$ being the different parton density functions $f_{\alpha}$ for the quarks and gluons. The factors $P_{\alpha \beta}$, with $\alpha, \beta$ being either a quark or a gluon, denote the probability to observe a parton $\alpha$ with a certain momentum fraction $x$, that originated from another parton $\beta$ with a momentum fraction $y$.

Experimental data at high energies, mostly from the two HERA-experiments H1 and ZEUS ${ }^{3}$, provide valuable knowledge of the proton structure functions $F_{2}$, necessary for all experiments with

\footnotetext{
${ }^{2}$ DGLAP: derived from the names of the main authors: Dokshitzer, Gribov, Lipatov, Altarelli, and Parisi.

${ }^{3} \mathrm{H} 1$ and ZEUS (greek: Search to Elucidate the Underlying Symmetry) are two multi-purpose detectors at the Hadron-Elektron Ring Anlage (HERA) located at the Deutsches Elektronen-Synchrotron (DESY) in Hamburg.
} 
protons or antiprotons in the initial state. The phase space covered by cross section measurements of inclusive deep inelastic scattering at HERA ranges from small Bjørken $x$ at low momentum transfer $\left(Q^{2}\right)$ to large $x$ at $Q^{2}$ values larger than the squared masses of the electroweak gauge bosons. Figure 2.2 shows an H1/ZEUS compilation of measurements of the proton structure function $F_{2}\left(x, Q^{2}\right)$ over a tremendous phase space region: (a) in dependence of $Q^{2}$ for different values of the momentum fraction $x$ and (b) in dependence of $x$ for $Q^{2}=7 \mathrm{GeV}$.

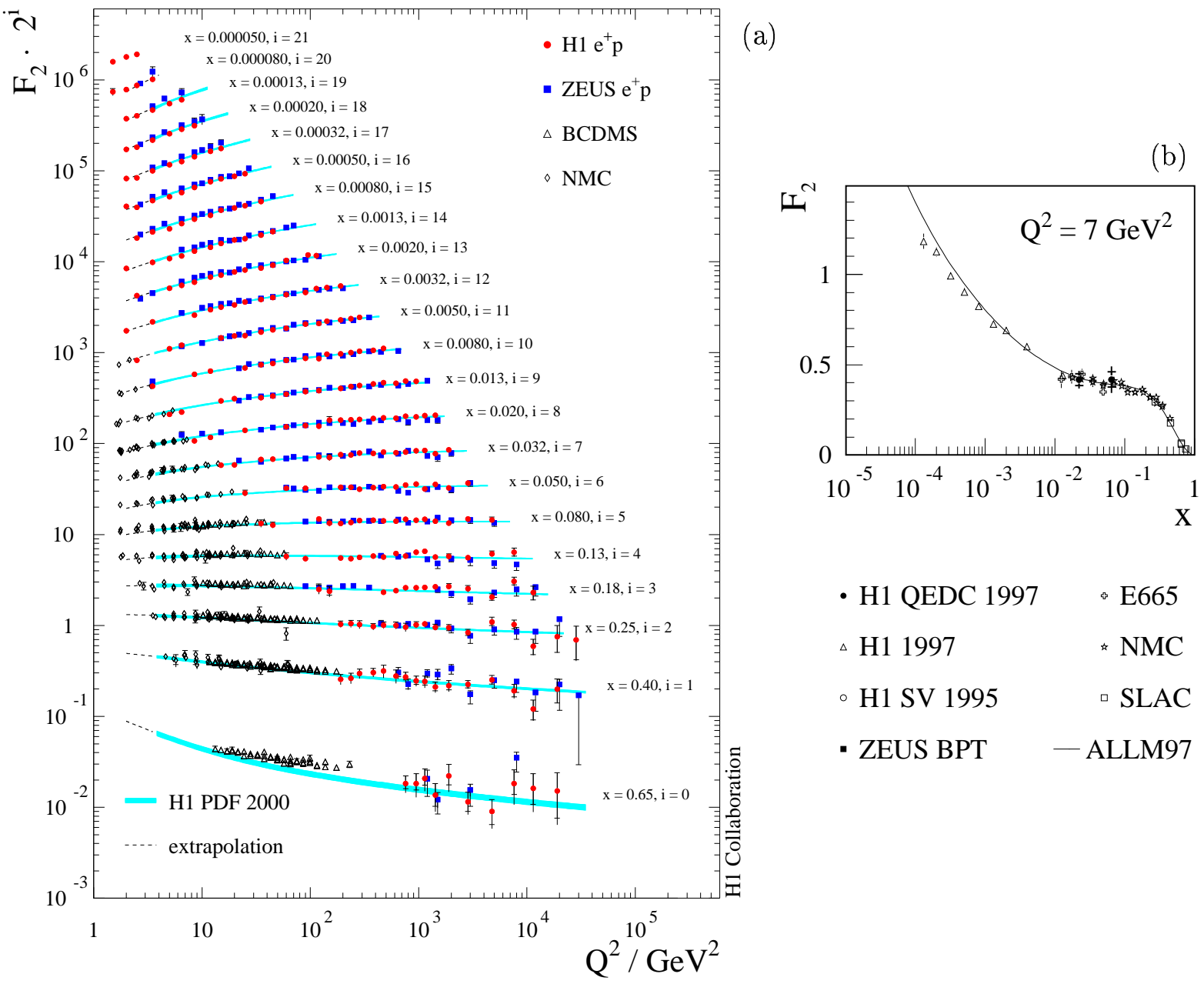

Figure 2.2: Measurements of the proton structure function $F_{2}\left(x, Q^{2}\right)$ in dependence of (a) the momentum transfer squared $\left(Q^{2}\right)$ for different $x$ and $(b)$ the momentum fraction $x$ for a fixed momentum transfer squared of $Q^{2}=7 \mathrm{GeV}$. The scaling violation, i.e. the $F_{2}$-dependence on $Q^{2}$ for small values of the Bjørken $x$, is clearly visible. (Compiled by the H1 and ZEUS collaborations [85, 86] and including measurements of other (fixed target) experiments.)

In Figure 2.3, the parton distribution functions $f(x)$ for valence quarks, sea quarks and gluons, as extracted from H1 [85] and ZEUS [86] data, are compared to those obtained from a fit using the CTEQ6.1M [87] set of PDFs. This set, provided by the CTEQ ${ }^{4}$ [88] collaboration, contains PDF parametrisations calculated at next-to-leading order (NLO). Further details on the measurement of $F_{2}(x)$ and the extraction of PDFs from $\mathrm{H} 1$ and ZEUS data can be found in [85, 86, 89, 90, 91].

${ }^{4}$ CTEQ: Coordinated Theoretical-Experimental Project on QCD 
Figure 2.3: Distributions of the $u$ and $d$ valence quark, sea quark, and gluon densities at a momentum transfer squared of $Q^{2}=10 \mathrm{GeV}^{2}$ as extracted from the H1 PDF 2000 fit (yellow/red) and the ZEUS$S N L O Q C D$ fit (green/black), respectively. The fits from the two HERA experiments are also compared to the distributions obtained from a fit with the recent CTEQ6.1M [87] set of PDFs (blue), which makes use of next-to-leading order PDFs.

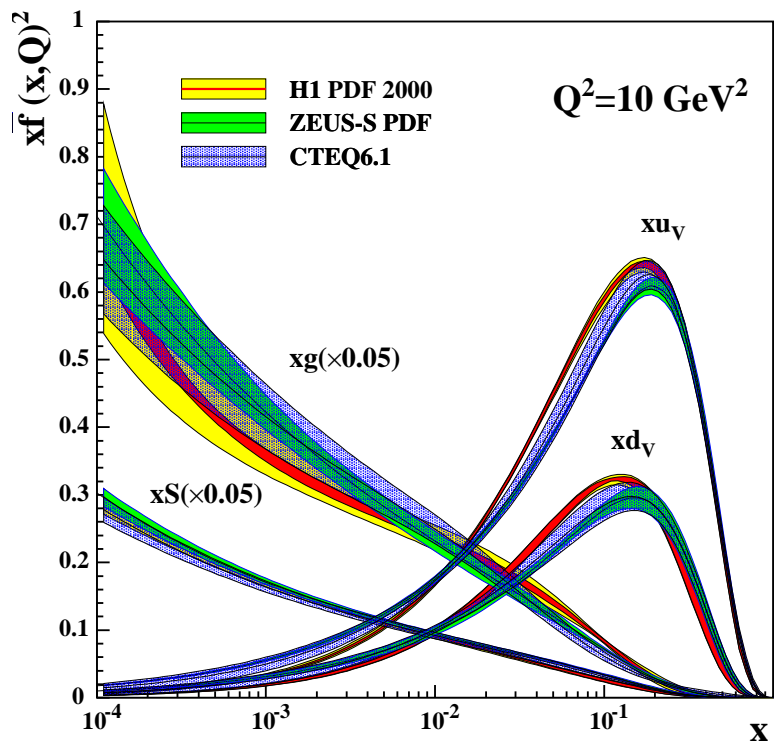

\subsubsection{Hadronisation and Additional Partons}

The quarks and gluons produced in the hard process do not exist as free (coloured) particles, but form colourless states, called hadrons. These are generally split into two subgroups: baryons consisting of three quarks or antiquarks ( $q q q$, or $\bar{q} \bar{q} \bar{q}$ ) and mesons, made up of a quark and an antiquark $(q \bar{q})$. The actual process, how quarks, antiquarks, or gluons hadronise cannot be calculated perturbatively and has to be modeled. Similar to the parton density functions parametrising the state of partons inside particles, hadronisation functions exist, which parametrise the $»$ real « processes by which a parton $»$ transforms « into a hadron. More exactly, the hadronisation functions $D_{q}^{h}(x)$, determine the probability with which a quark $q$ becomes part of a hadron $h$ carrying the quark's momentum fraction $x$.

Although it seems complicated enough to deal with the partons instead of the particles and use two different parametrisations to describe the occurring processes, there are even more complications in real life. In addition to the products of the hard scattering process, more partons may be produced by initial or final state radiation (ISR, FSR), where partons (quarks or gluons) may be emitted before or after the hard interaction. These extra partons also need to be taken into account during the hadronisation process.

\subsubsection{The Underlying Event, Multiple Interactions, and Pile-Up}

The underlying event is a collective term for all particles from a single $p \bar{p}$ interaction that do not originate from the outgoing partons of the hard interaction (including final state radiation). In other words, particles stemming from multiple parton interactions, like interactions of the spectator quarks, which are also called beam-beam remnants being the breakup of the proton and antiproton, or from initial state radiation. These inelastic interactions are mostly soft, characterised by low momentum transfers, but they nevertheless can leave a signature in the detector system. 
Another complication is the possibility of multiple particle interactions per bunch crossing (i.e. more than one $p \bar{p}$ interaction). The occurrence of overlap events is - as every event rate proportional to the instantaneous luminosity. To estimate the average number of additional interactions per beam crossing $\bar{N}_{\text {inel }}$, Equation (2.1) has to be modified by the number of particle bunches per beam and the time it takes for one such bunch to complete a single revolution. The Tevatron with a circumference of $L \approx 6.3 \mathrm{~km}$, a revolution time of $T_{b}=\frac{L}{c} \approx 2.1 \cdot 10^{-5} \mathrm{~s}$ and proton and antiproton beams with $N_{b}=36$ bunches each, is used as an example.

$$
\bar{N}_{\text {inel }}=\frac{\mathcal{L} \cdot \sigma_{\text {inel }} \cdot T_{b}}{N_{b}} \Rightarrow \text { with } \quad \mathcal{L}=20 \cdot 10^{30} \mathrm{~cm}^{-2} \mathrm{~s}^{-1} \rightarrow \overline{\mathrm{N}}_{\text {inel }} \approx 0.8
$$

Doubling the average instantaneous luminosity, not only leads to higher rates of the desired events, but always also doubles the average number of overlap events, or multiple interactions. The real difficulty, however, is the enormous cross section for this type of events. It is approximately $\sigma_{\text {inel }} \approx 70 \mathrm{mb}$, which is at least three orders of magnitude above any cross sections for interesting processes, such as diboson, or top-antitop $(t \bar{t})$ production. Figure 2.4 provides an overview of cross section curves for several SM processes in $p \bar{p}$ collisions. The two cross section curves for the production of a SM Higgs boson with a mass of $M_{H}=150 \mathrm{GeV}$, and alternatively a mass of $M_{H}=500 \mathrm{GeV}$, are also included. All cross sections are shown as a function of the centre-of-mass energy, with the CM-energies of the Tevatron and the future LHC indicated by the dashed lines.

Another complication is added by a detector effect, called pile-up. It denotes remnants from previous collisions, either from the hard interaction, from the underlying event, or from multiple interactions. These remnants might be residual charge (energy) depositions in the calorimeter, that have not yet been read out or faded away.

\subsubsection{Common Variables and Definitions}

Apart from the luminosity $\mathcal{L}$, the characteristic quantity of a collider is the centre-of-mass energy $\sqrt{s}$. It is invariant under Lorentz transformations and represents the maximum available energy for the production of particles from colliding beams. Assuming a »head-on « collision of two identical particles, the centre-of-mass energy is given by

$$
\sqrt{s}=\sqrt{\left(E_{1}+E_{2}\right)^{2}-\left(\vec{p}_{1}+\vec{p}_{2}\right)^{2}} .
$$

In collider physics, the corresponding centre-of-mass reference system is widely used, since in this coordinate system the common center of mass of the particles involved in a hard interaction remains at rest, which is usually not the case in the laboratory system. Also, only particles with a rest mass $m_{0}$ of at most the centre-of-mass energy $E=m_{0} c^{2}=\sqrt{s}$ can be produced. The rest mass of a particle is referred to as the invariant mass $\left(M_{i n v}\right)$. Assuming the new particle decays into a certain number $n$ of known particles, one can calculate the invariant mass of this system of $n$ particles as

$$
M_{i n v}=\sqrt{\left(E_{1}+E_{2}+\cdots+E_{n}\right)^{2}-\left(\vec{p}_{1}+\vec{p}_{2}+\cdots+\vec{p}_{n}\right)^{2}}
$$

For example, an up to then unknown resonance $(p e a k)$ in the invariant mass distribution of these $n$ particles would reveal the new particle. 


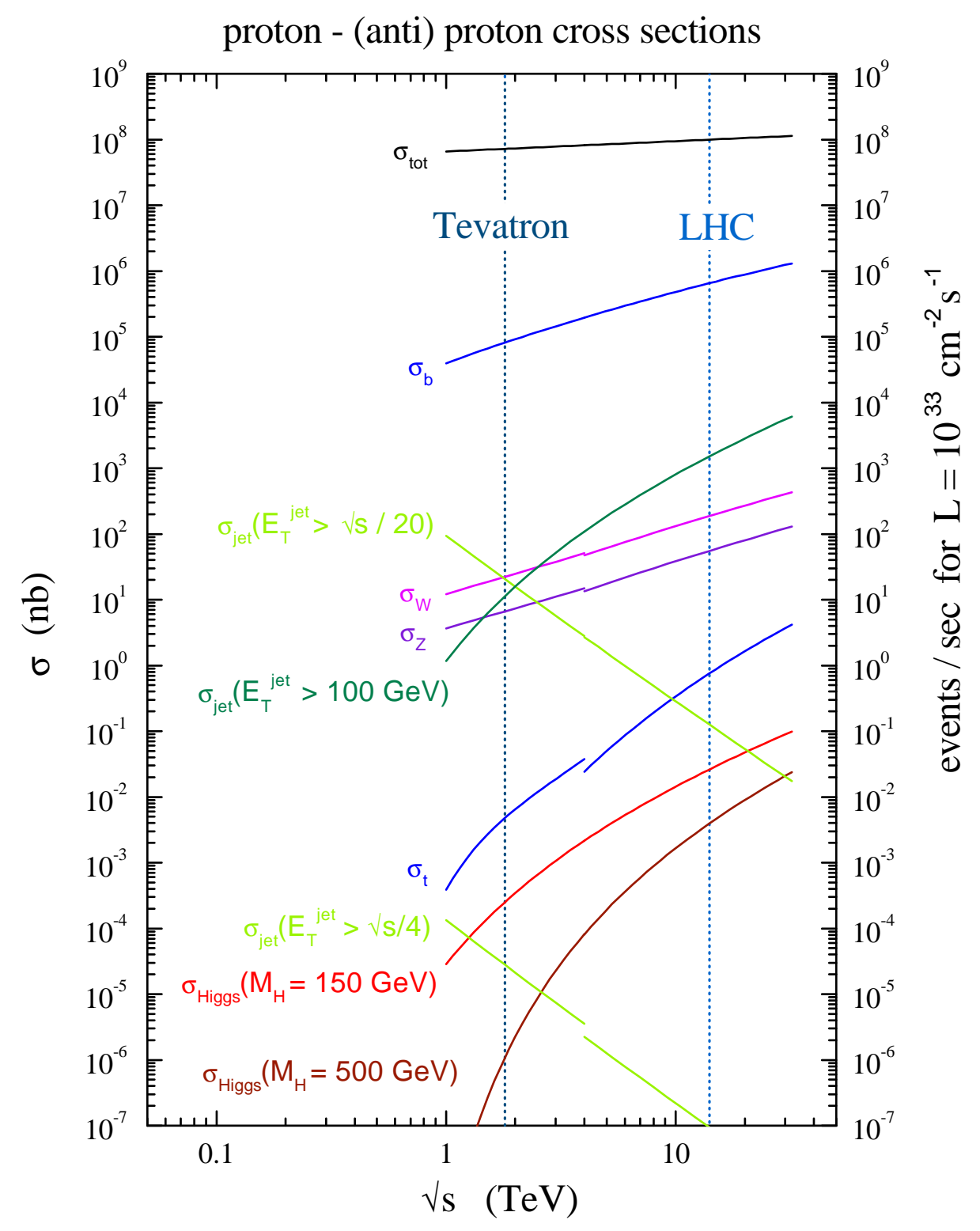

Figure 2.4: Overview of the cross sections for several processes as a function of the centre-of-mass energy. The total $p \bar{p}(p p)$ cross section $\left(\sigma_{t o t}, t o p\right)$ is orders of magnitude higher than cross sections for interesting but rare processes, like, e.g. $t \bar{t}$ or $W$ production. Predicted cross sections for the production of a Higgs boson of $M_{H}=150 \mathrm{GeV}$ and $M_{H}=500 \mathrm{GeV}$ are also indicated. It is approximately of the order of 1-100 fb at the Tevatron centre-of-mass energy of $\sqrt{s} \approx 1.96 \mathrm{TeV}$. The CM-energy of about $14 \mathrm{TeV}$ of the future LHC is also indicated. In general, the cross sections for new physics processes, like SUSY particle production, especially chargino/neutralino pair production, are of the order of $1 \mathrm{fb}$ to $1 \mathrm{pb}$, i.e. similar to the indicated production cross section for a Higgs boson of $M_{H}=150 \mathrm{GeV}$. (This figure was taken from [92] and has been edited.)

\section{Coordinate System}

In a right-handed cartesian coordinate system, the $z$-axis is defined to be parallel to the beam axis, while $x$ and $y$ define the transverse plane, perpendicular to the beam axis. In case of the 
$\mathrm{D} \varnothing$ experiment, the utilised coordinate system has its origin at the nominal interaction point in the geometric center of the $\mathrm{D} \varnothing$ detector. The $x$ and $y$ axes are chosen to point away from the center of the Tevatron ring and upwards, respectively, while the $z$ axis is parallel to the beam line and agrees with the direction of the proton beam. The usage of polar coordinates $(r, \varphi, \eta)$, however, is much more common. Here, $\varphi$ is the azimuthal angle and the pseudorapidity $\eta$ is defined as

$$
\eta=-\ln \left(\tan \frac{\theta}{2}\right)
$$

where $\theta$ is the polar angle. For detector related measurements the polar angle $\left(\theta_{\text {det }}, \eta_{\text {det }}\right)$ is measured from the geometric center of the detector $(x, y, z)=(0,0,0)$ and with respect to the positive $z$-axis. On the other hand, for physics measurements, the origin is usually changed to coincide with the interaction vertex, so that the polar angle is measured from $(x, y, z)=\left(0,0, v_{z}\right) \neq(0,0,0)$. With this latter definition, the origin might of course change from one event to the next. The pseudorapidity $\eta$ (or $\eta_{\text {det }}$ ) is used because it is invariant under Lorentz transformations, whereas the polar angle $\theta$ is not and changes due to a relativistic Lorentz boost along the $z$-axis. The azimuthal angle $\varphi$ is Lorentz invariant due to its definition in the transverse plane.

\section{Quantities in the transverse plane}

Another commonly used variable is the transverse momentum $p_{\mathrm{T}}$, or the transverse energy $E_{\mathrm{T}}$, of particles. Due to the unknown longitudinal component of the colliding partons, the momentum fraction $x$ as defined in 2.1.1, a meaningful »momentum « balance can only be established for the two components $p_{x}$ and $p_{y}$, in the transverse plane perpendicular to the beam axis. The same holds for the transverse energy, or for this reason the missing transverse energy $\not_{T}$, which is not really missing energy, but due to particles, which escaped the detector, i.e. neutrinos or SUSY-neutralinos that interact only very weakly with any material. However, not the complete »missing « energy can be inferred, but only the transverse component:

$$
E_{\mathrm{T}}=\left|\left(\sum_{i=1}^{\text {all }} \vec{p}_{T}\right)_{F S}-\left(\sum_{j=1}^{\text {all }} \vec{p}_{T}\right)_{I S}\right| \quad \text { with: } \quad p_{\mathrm{T}}=\sqrt{p_{x}^{2}+p_{y}^{2}},
$$

where $I S / F S$ denote the initial/final state, respectively. The incoming particles, do not have significant momentum components other than in the $z$ direction, so that the second term, the sum of transverse momenta of the incoming particles vanishes.

\section{Particle Distances}

To correctly describe the spacial distance between two objects in the detector (energy depositions or tracks) it needs to be defined in a Lorentz invariant way. The angular distance in the transverse plane, given by the difference between the azimuthal angles of the two objects $\Delta \varphi=\varphi_{1}-\varphi_{2}$ is Lorentz invariant. Similarly, a Lorentz invariant spacial distance is commonly defined by:

$$
\mathcal{R}=\sqrt{(\Delta \varphi)^{2}+(\Delta \eta)^{2}},
$$

where $\varphi$ is again the azimuthal angle and $\eta$ the pseudorapidity. 


\subsection{The Accelerator Chain at Fermilab}

The Tevatron is a proton-antiproton $(p \bar{p})$ collider, located at the Fermilab site about $80 \mathrm{~km}$ west of Chicago in the USA. Figure 2.5 provides an aerial overview of the $27.5 \mathrm{~km}^{2}$ large Fermilab area with the Tevatron and the Main Injector as the most prominent features, respectively.

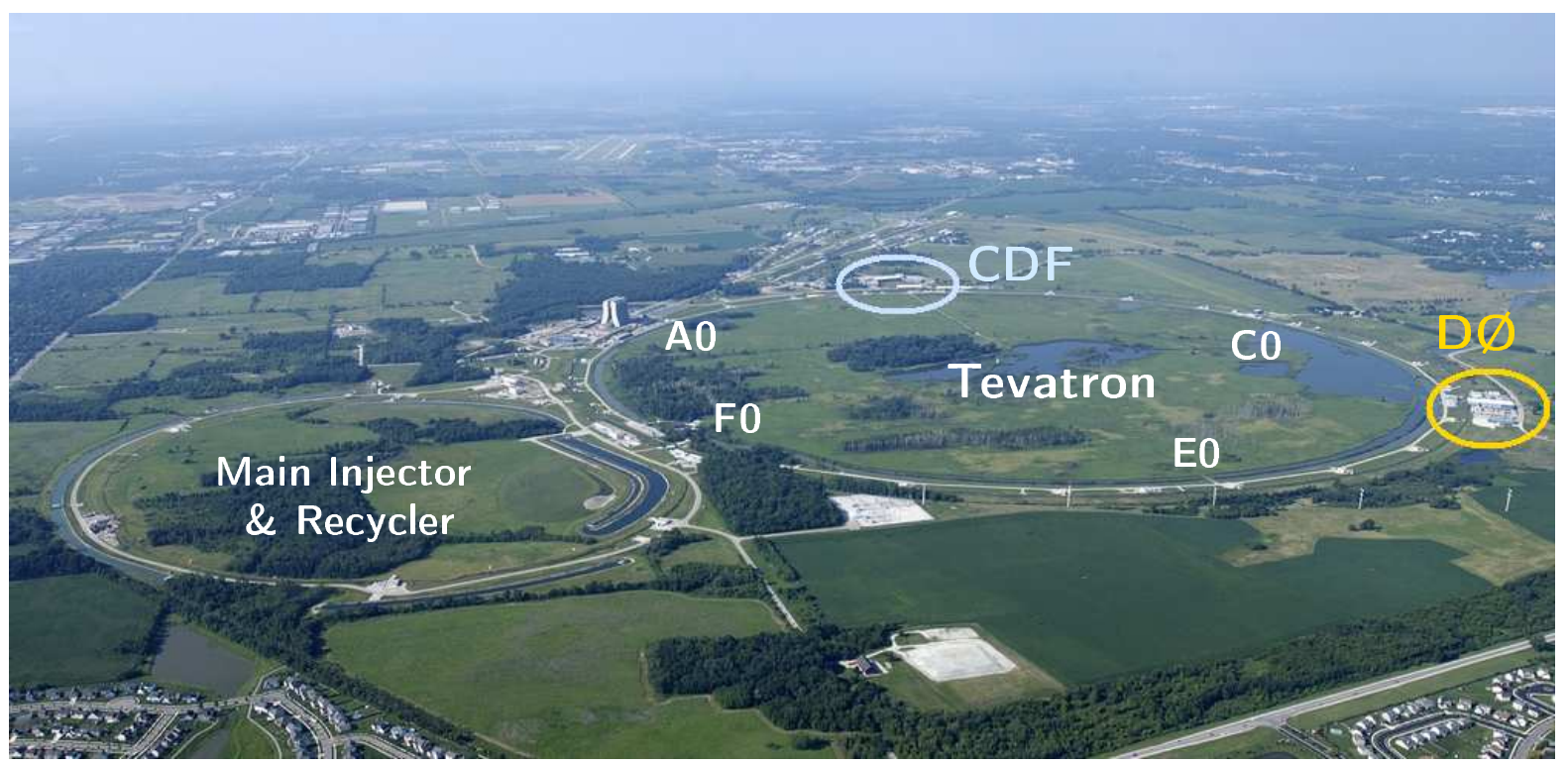

Figure 2.5: Aerial view [82] of the Fermilab site, featuring the Tevatron (middle-right) and the Main Injector (left). The main office building, to the left of the Tevatron collider is called »Wilson Hall . It is located near section $A 0$ of the Tevatron, while the CDF experiment (highlighted in light-blue) resides at the collision point B0, and the DØ experiment (highlighted in orange) is located at D0, as its name already implies. Section C0, between the two multipurpose detectors CDF and DØ was to become the site of the BTeV experiment, which was canceled in February 2005. Section F0 holds the radio frequency system (RF-system) powering the Tevatron's accelerating cavities.

The Tevatron circumference is roughly $6.3 \mathrm{~km}$ and two oppositely directed beams of protons and antiprotons, accelerated to $980 \mathrm{GeV}$ each ${ }^{5}$, are brought to collision to produce high energy reactions at two designated points in the accelerator. One such point is B0, the site of the $\mathrm{CDF}$ experiment, the other collision point is $\mathrm{D} 0$, the location of the $\mathrm{D} \varnothing$ experiment. Since both particle beams have an energy of roughly $980 \mathrm{GeV}$, the centre-of-mass energy of the Tevatron is $\sqrt{s}=1.96 \mathrm{TeV}$ in its second phase of data-taking, the so-called Run II. This seemingly huge amount of energy corresponds to $»$ only $« \approx 3.14 \cdot 10^{-7} \mathrm{~J}$, which is approximately the kinetic energy of an 0.2 gram ant, crawling at a speed of $5.6 \mathrm{~cm} / \mathrm{s}$. This tiny amount of energy only becomes enormous if one takes into account that it is released in the collision of partons (quarks and/or gluons), whose size is less than $1 \cdot 10^{-18} \mathrm{~m}$, i.e. more than 100 million times smaller than the distance between two crests of a wave of visible light [93]. This also explains why experimental particle physics and high energy physics are used as synonyms, although it is not the energy itself that is high but only the energy density. To resolve anything at such tiny distances and thus to

\footnotetext{
${ }^{5}$ One electronvolt $\left(1 \mathrm{eV}=1.602 \cdot 10^{-19} \mathrm{~J}\right)$ is the energy gained by an electron accelerated through a potential difference of $1 \mathrm{~V}$, whereas the two beams of the Tevatron are accelerated through a potential difference of some 980 billion Volts.
} 
test physics there, one needs probes of extremely short wavelengths and this is precisely what is achieved by accelerating matter (antimatter) to extremely high energies.

After the shutdown of the Large Electron Positron (LEP) collider at CERN at the end of 2000, the hunt for the Higgs boson, or particles predicted by Beyond the $S M$ theories, passed from CERN to Fermilab. Currently the successor of LEP, the LHC (Large Hadron Collider), is being constructed at CERN. The LHC will be a proton-proton collider with a centre-of-mass energy of $\sqrt{s}=14 \mathrm{TeV}$. However, since the construction and commissioning of the accelerator and its associated detectors will not be completed before 2007 and data-taking is scheduled to start at the end of 2007, beginning of 2008 at the earliest, the Tevatron will be the world's most energetic particle collider until then. After a very successful Run I, from 1992 to 1996, during which the last and heaviest quark, the top quark had been discovered, a five year shutdown followed. During this period not only the Tevatron, but Fermilab's whole accelerator chain was upgraded, as were the two experiments, CDF (Collider Detector Facility) and DØ. In early 2001, the Tevatron finally resumed operation at a roughly $9 \%$ higher centre-of-mass energy compared to Run I. The number of bunches of protons and antiprotons in the Tevatron had been increased as well (from previously 6 to 36 ) and the beam intensities are many times higher than the Run I intensities. This led to a considerably higher instantaneous luminosity, with peak luminosities of $1.6-1.7 \cdot 10^{32} \mathrm{~cm}^{-2} \mathrm{~s}^{-1}$ achieved at the end of 2005 , beginning of 2006 . Table 2.1 provides an overview of some of the parameters of the Tevatron and the Main Injector, while Figure 2.6 shows the average peak luminosity (a) and the intergrated luminosity (b) up to February 2006.

\begin{tabular}{lc}
\hline \hline Parameter & Value \\
\hline \hline Circumference: Tevatron (Main Injector) & $6.28(3.32) \mathrm{km}$ \\
Max. beam energy: Tevatron (Main Injector) & $980(150) \mathrm{GeV}$ \\
\hline Bunches per beam $(p \times \bar{p})$ & $36 \times 36$ \\
Bunch spacing & $396 \mathrm{~ns}$ \\
Protons per bunch $\left(n_{p}\right) /$ transverse emittance $\left(\varepsilon_{p}\right)$ & $23.0 \cdot 10^{10} / 20 \pi \mathrm{mm}-\mathrm{mrad}$ \\
Antiprotons per bunch $\left(n_{\bar{p}}\right) /$ transverse emittance $\left(\varepsilon_{\bar{p}}\right)$ & $5.8 \cdot 10^{10} / 15 \pi \mathrm{mm}-\mathrm{mrad}$ \\
$\quad$ The emittance is a measure of the distribution of transverse positions and angles of the beam particles. \\
Bunch length (rms) & $0.37 \mathrm{~m}$ \\
Interactions per crossing (average) & 1.7 \\
Antiproton production rate & $15.0 \cdot 10^{10} \bar{p} / \mathrm{h}$ \\
\hline Average peak luminosity: in 2002 & $2.0 \cdot 10^{31} \mathrm{~cm}^{-2} \mathrm{~s}^{-1}$ \\
& in 2003 \\
$\quad$ in 2004 & $3.2 \cdot 10^{31} \mathrm{~cm}^{-2} \mathrm{~s}^{-1}$ \\
& $5.8 \cdot 10^{31} \mathrm{~cm}^{-2} \mathrm{~s}^{-1}$ \\
\hline \hline
\end{tabular}

Table 2.1: Some of the most important parameters of the Tevatron (and the Main Injector) and, in the bottom part, the average peak luminosities from 2002 until 2005.

Figure 2.7 presents a schematic overview of the different accelerator systems, most of which are briefly described in the following, while Figure 2.8 is a close-up aerial overview of section A0 and thus of the »smaller « machines at Fermilab: linear accelerator, Booster and Antiproton Source. 


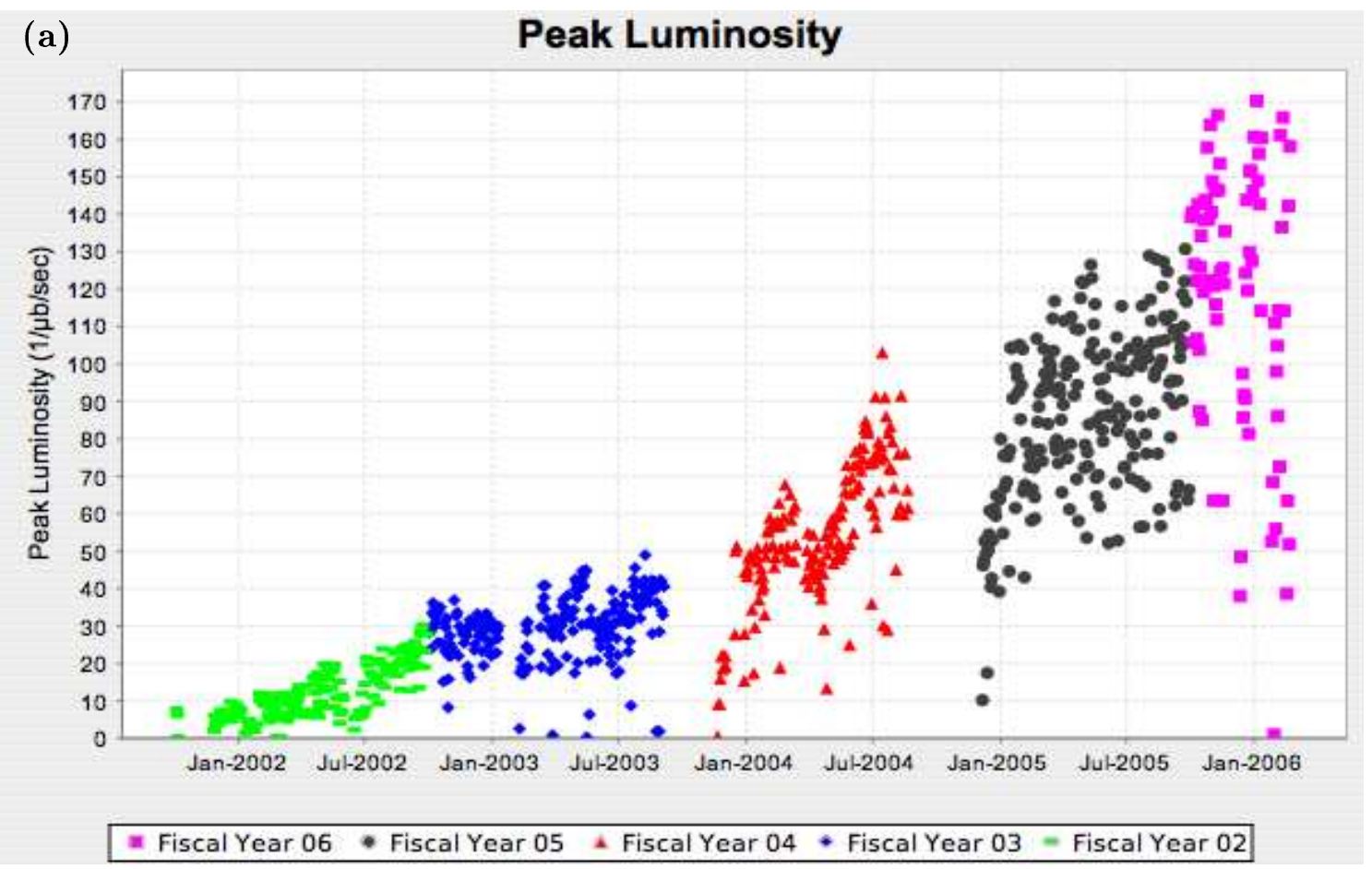

(b)

\section{Integrated Luminosity}

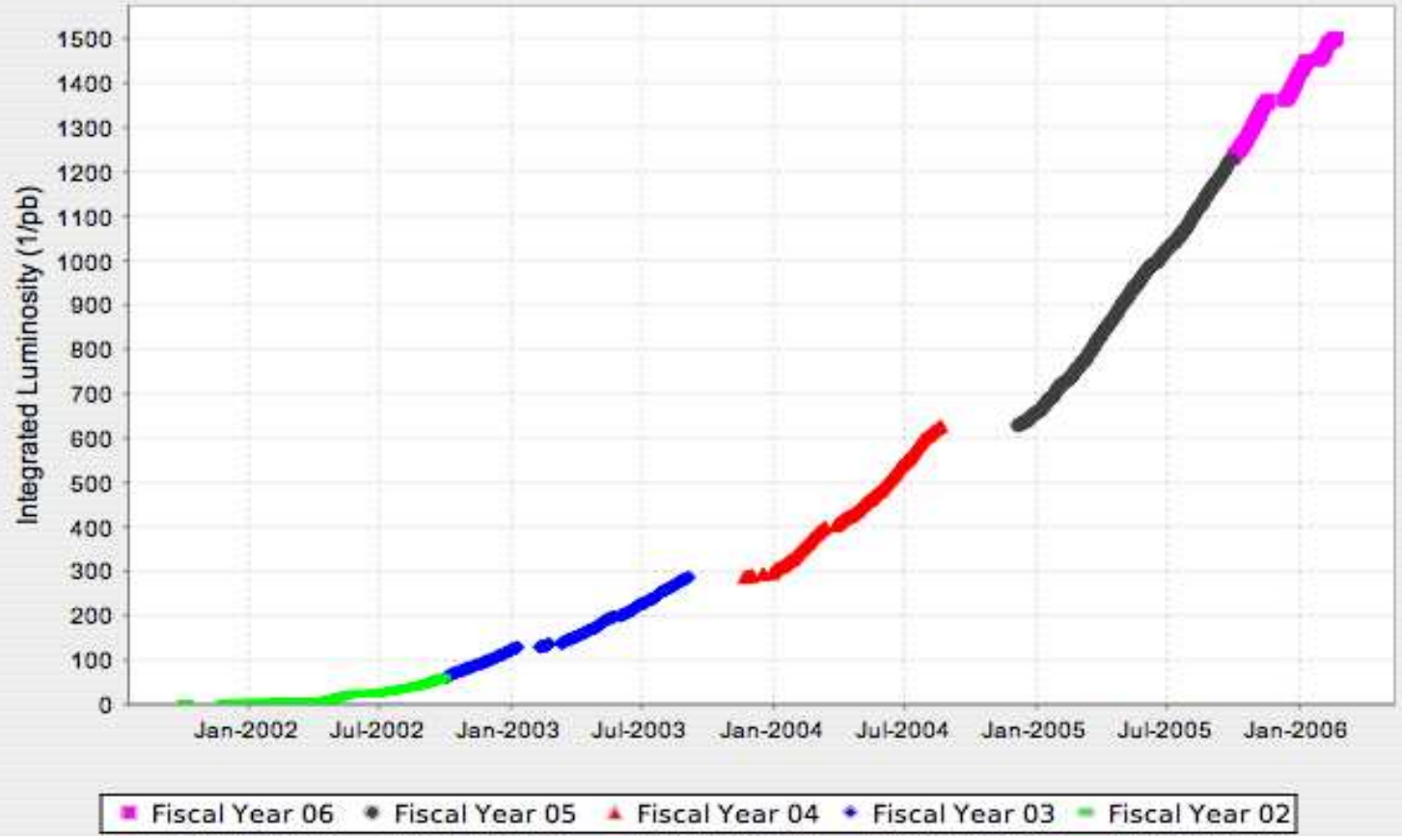

Figure 2.6: (a) The development of the "peak « luminosity (i.e. the luminosity at the beginning of a new store at the Tevatron) with time; from fall 2001 until January 2006. (b) The integrated luminosity over the same time period. The different colours indicate so-called $»$ Fiscal Years «, starting in October. 


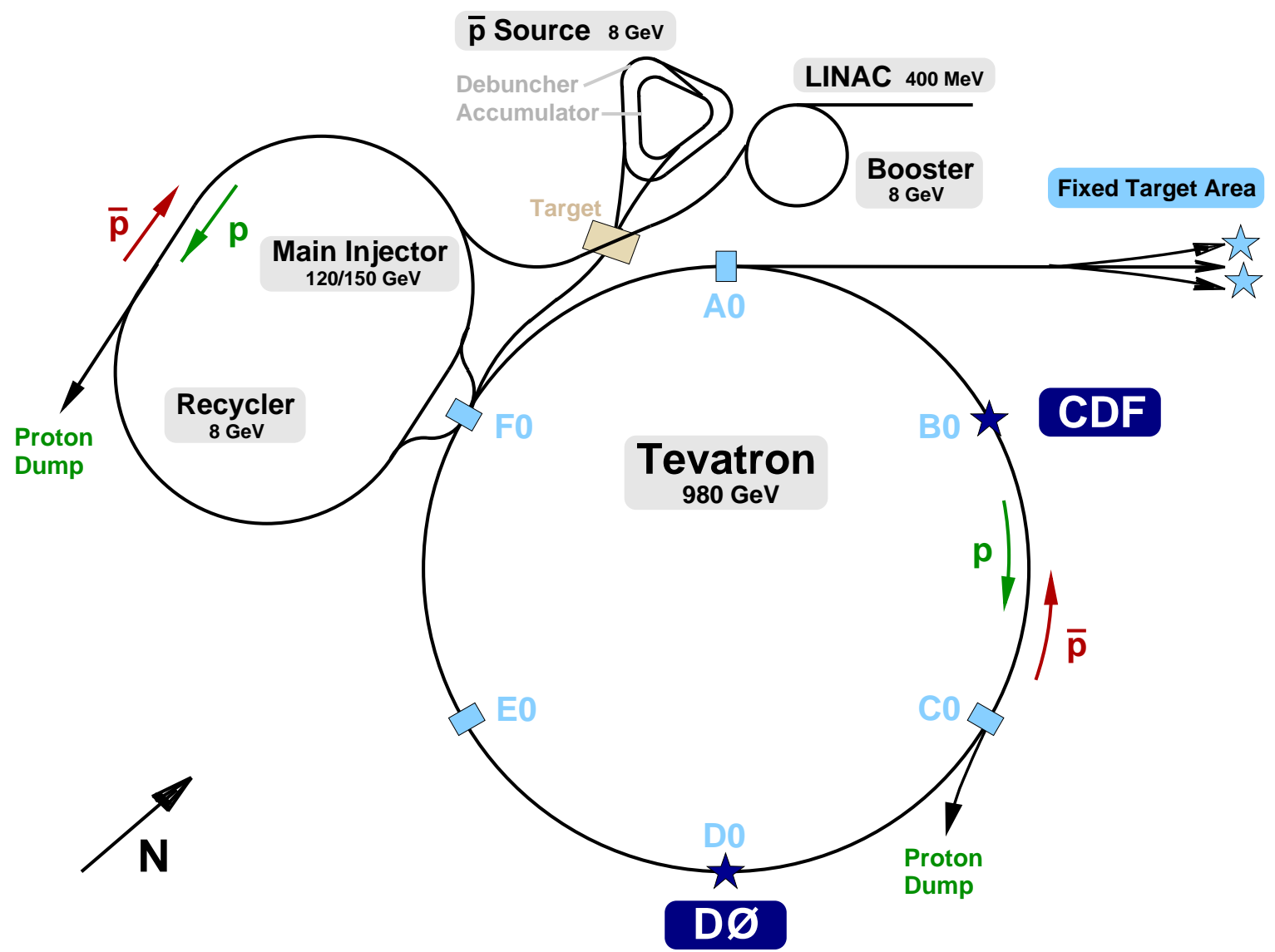

Figure 2.7: Schematic overview of Fermilab's accelerator chain. Protons start as negative hydrogen ions from a Cockcroft-Walton generator and are then successively accelerated by the LINAC, the Booster ( $H^{-}$ions become protons), the Main Injector and the Tevatron. Antiprotons, however, are first produced by firing $120 \mathrm{GeV}$ protons onto a target right before the antiproton facility, consisting of the Debuncher and the Accumulator. When enough antiprotons have been accumulated, they are also transferred to the Main Injector, accelerated to $150 \mathrm{GeV}$ and injected into the Tevatron. Here, the proton and antiproton beams are finally accelerated to $980 \mathrm{GeV}$, corresponding to a centre-of-mass energy of $\sqrt{s}=1.96 \mathrm{TeV}$.

Figure 2.8: Aerial overview of the smaller accelerators at Fermilab, which are at the beginning of the chain. The LINAC, just right of the main office building (Wilson Hall), the Booster synchrotron (middle) and in the background the Antiproton Source, with its characteristic triangular shape.

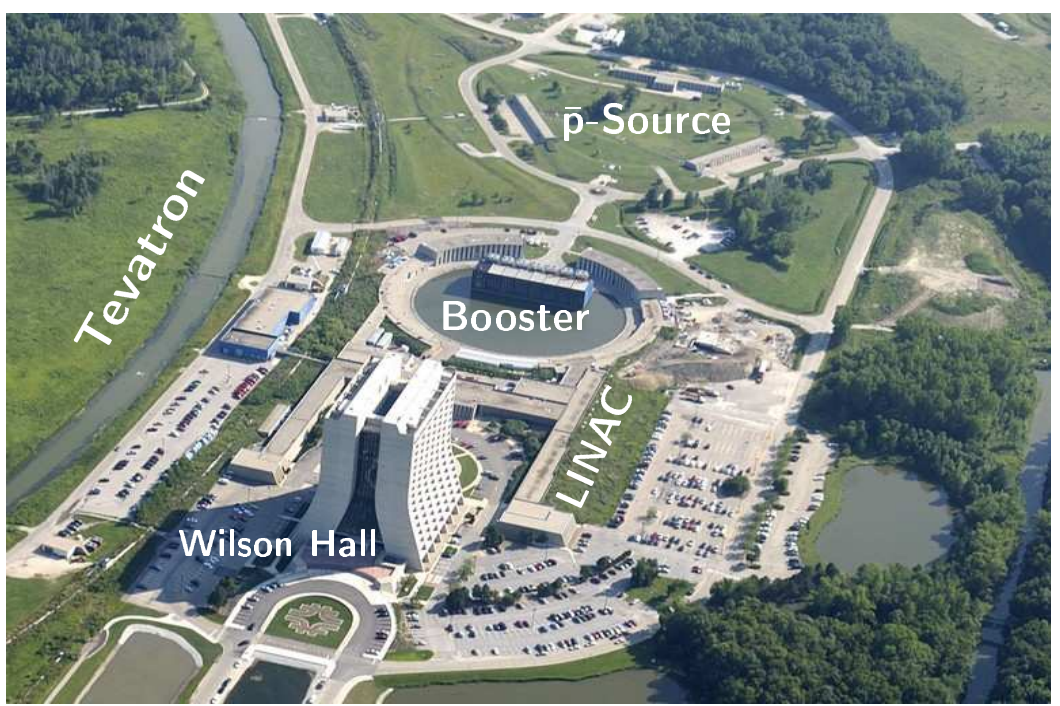




\section{Pre-Accelerator (Cockcroft-Walton)}

The high energetic particle beams of the Tevatron start from a small bottle of pure hydrogen, located inside an electrostatic Cockcroft-Walton pre-accelerator, see Figure 2.9 (a). A magnetron creates negative hydrogen ions $\left(H^{-}\right)$, which then travel through a potential difference of $750 \mathrm{kV}$ along the accelerating column of the Cockcroft-Walton generator. The necessary high DC voltage of the latter is produced from a circuit of rectifiers and capacitors to which a relatively low AC voltage is applied. Figure 2.9 (b) illustrates this principle of voltage multiplication for a simple four stage cascade voltage multiplier.

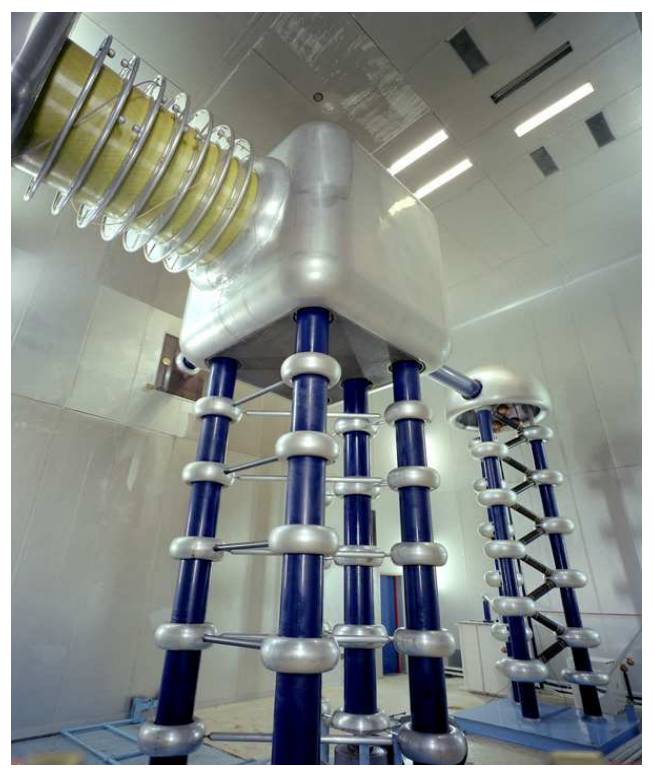

(a)

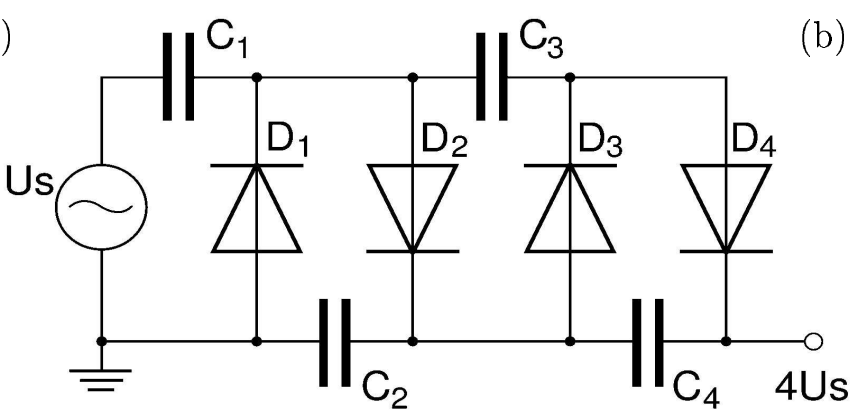

Figure 2.9: The Fermilab accelerator chain starts with a Cockcroft-Walton generator (left), accelerating $H^{-}$ions to $750 \mathrm{keV}$. The necessary high DC voltage is produced from a circuit of rectifiers and capacitors to which a low $A C$ voltage is applied as shown schematically above for a four stage cascade voltage multiplier.

\section{Linear Accelerator (LINAC)}

In a next step, the $H^{-}$ions are accelerated in a two-stage linear accelerator. The first stage consists of five drift-tube cavities and takes the $H^{-}$ions to $116 \mathrm{MeV}$, while the second stage consists of seven side-coupled cavity modules accelerating the beam to $400 \mathrm{MeV}$. In the first part, acceleration is achieved by coupling an oscillating electric field (radio frequency $(\mathrm{RF})$, $f=201.25 \mathrm{MHz}$ ) into the LINAC cavities, which are cylindrical, electrically-resonant steel tanks, that are clad with copper on the inside. The length of the drift-tubes inside the cavities is chosen such that the electric field points in the accelerating direction, when the hydrogen ions traverse the gap between two drift-tubes, but are protected from the phase change of the sinusoidal varying electric field, when it points in the backward direction and would exert a decelerating force on the particle bunches. During this time the $H^{-}$bunches are shielded in the interior of the copper drift-tubes, where they drift in the absence of electric fields. Hence, the $H^{-}$ions will always see a positive gradient and get a boost in energy and velocity with every gap crossed.

However, the $\mathrm{H}^{-}$ions have to be fed into the RF-cavities in a bunched form and at the correct time to enable them to complete the acceleration cycle. This poses another problem, since the pre-accelerator outputs a continuous supply of $H^{-}$ions, so that this $H^{-}$beam has to pass through a so-called electrostatic Chopper. This device »chops« out $42 \mu$ s long pulses of the continuous $H^{-}$beam at the frequency the LINAC uses. Each of these pulses goes through a Buncher, which slows down particles arriving early in the RF-cycle with a decelerating voltage, and vice 
(a)

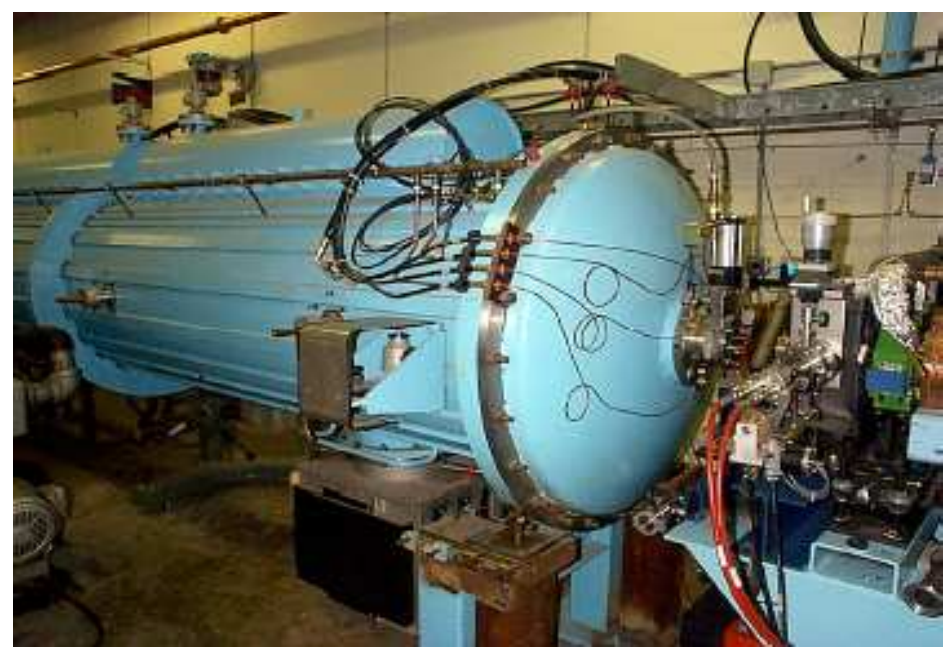

(c)

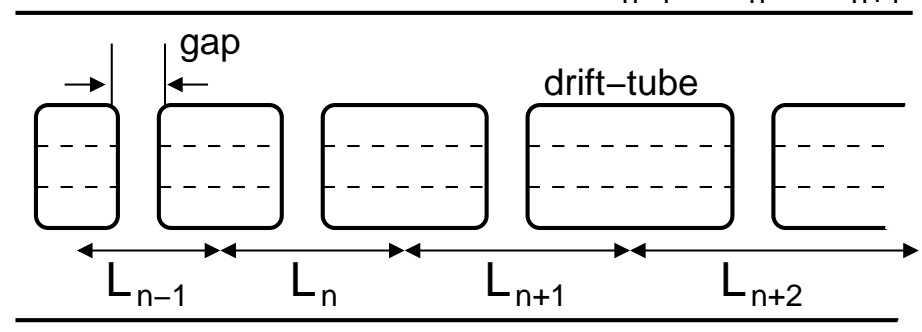

(b)

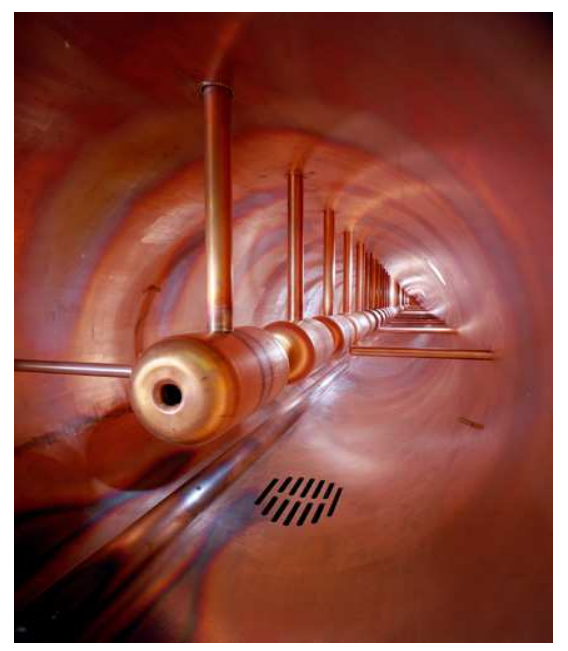

Figure 2.10: The first stage LINAC drift-tube cavities accelerate the $H^{-}$ ions to about $116 \mathrm{MeV}$. One of the water-cooled steel tanks is shown from outside (a), and inside (b), with the copper drift-tubes clearly visible. The principle of acceleration is schematically illustrated in (c).

versa speeding up those arriving later, thus creating a bunch ${ }^{6}$. These bunched beams, where the $H^{-}$ions occupy smaller phase space areas as in the previous pulse are then transferred to the LINAC, where they have to fit in so-called buckets ${ }^{7}$ of phase space. These are defined, stable regions in the longitudinal phase space, where a beam may be captured and accelerated. Ideally, the $\mathrm{RF}$-system is timed such that the bunches arrive synchronized with the radio frequency. The bunch center should coincide with the crest of the RF-wave, so the $H^{-}$ions essentially ride on the crest of an electromagnetic wave.

The second stage, side-coupled cavity modules operate at $805 \mathrm{MHz}$, where each module has 16 accelerating and 15 coupling cells and is powered by its own RF-source, which amounts to seven $12 \mathrm{MW}, 805 \mathrm{MHz}$ Klystron RF power supplies. The accelerating gradient for each module is about $7.5 \mathrm{MV} / \mathrm{m}$, which is three times that of the drift-tube cavities in the first stage of the LINAC. Additionally the $2^{\text {nd }}$ stage, side-coupled cavities allow the particle bunches to be accelerated over the whole length of each cavity and not only in the gaps in between. This is, because each individual cell is a separate accelerating cavity coupled to other cells in the module. Thus, one module is not only one cavity (as the drift-tube cavities), but rather several separate cavities powered by the same RF-source by coupling. When a bunch enters the first cell, the field is in the accelerating direction. As the bunch travels through and into the next cavity, the fields shift phases, such that when the bunch enters the second cell, the field there is now in the accelerating direction, while the field in the first cell is in the decelerating direction. Since there

\footnotetext{
${ }^{6} \mathrm{~A}$ bunch is a group of particles characterised by a large momentum and small time (or phase) spread.

${ }^{7}$ A bucket's width gives the maximum phase (or timing) error a particle may have, while still being able to complete the whole acceleration cycle. Correspondingly, a bucket's height is a measure for the momentum error.
} 


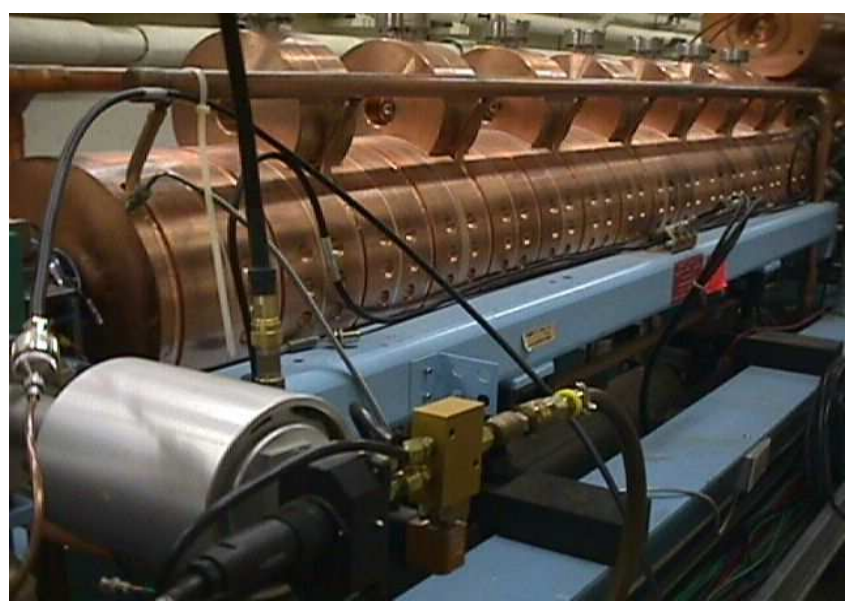

(a)

(b)

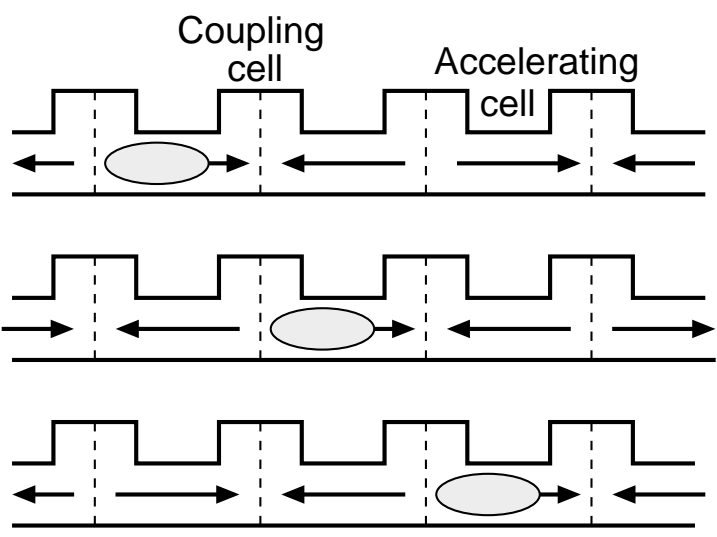

Figure 2.11: In the high energy section of the LINAC, side-coupled cavities (a) accelerate the $H^{-}$ion bunches to $400 \mathrm{MeV}$. In (b) the principle of a coupled accelerator is depicted; see text for details.

is no beam present in the first cell to see this decelerating field, no particles are decelerated. The field shape in the side-coupled cavities is optimized by so-called nose cones, which create stronger and more concentrated fields, that accelerate the bunched beams more efficiently than the uniform fields across the gaps in the drift-tube part. From the LINAC, the negative hydrogen ions can go to any of three destinations: two are dump lines that allow for measurements of either the momentum spread or the transverse emittance, and the third line goes to the Booster.

\section{Booster}

The Booster is the first circular accelerator, a rapid cycling synchrotron, where protons are accelerated from $400 \mathrm{MeV}$ to $8 \mathrm{GeV}$. It consists of a series of magnets arranged around a circle of about $475 \mathrm{~m}$ circumference. More exactly, the Booster is made up of 24 identical cells, referred to as the magnetic lattice. Each cell consists of two horizontally focusing quadrupole magnets (F-magnet), two horizontally defocusing quadrupole magnets (D-magnet), one long (OO) and one short (O) straight drift section, such that $» F O F D O O D \ll$ is the repetitive lattice. Contrary to the magnets in the following Main Injector, all Booster magnets are so-called combined function magnets, which bend the beam and focus it either horizontally or vertically (horizontal defocusing). The usage of alternating gradients to keep the proton bunches tightly constrained inside
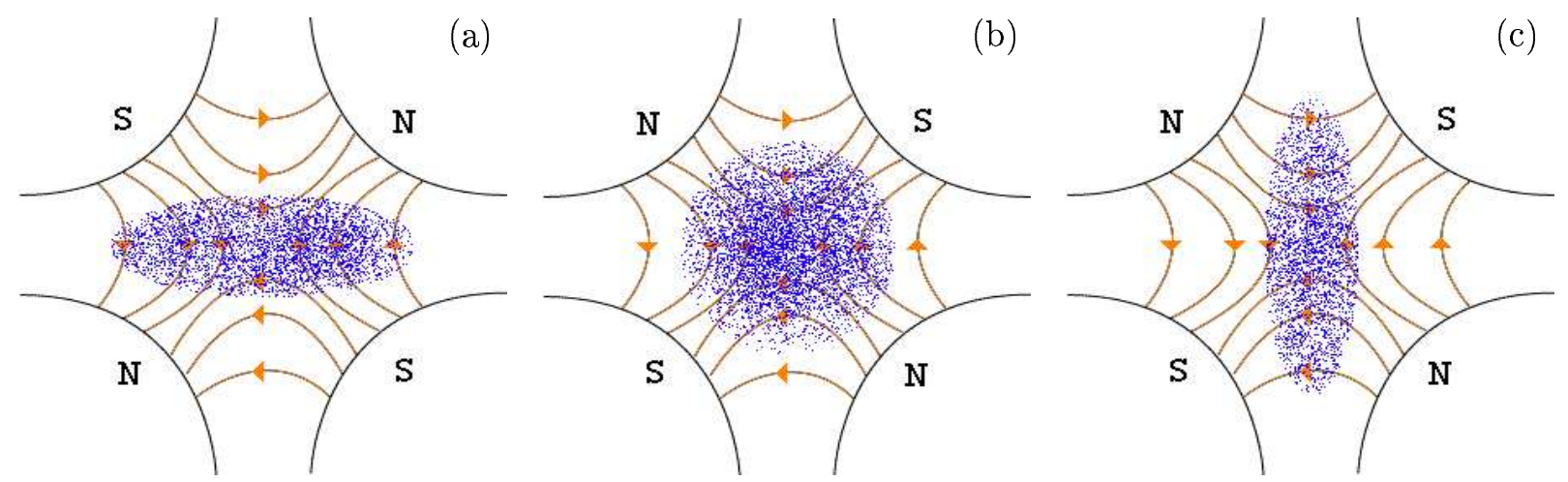

Figure 2.12: Illustration of strong focusing via quadrupole magnets with alternating focal planes, i.e. horizontally focusing / vertically defocusing and vice versa. 
the vacuum chamber of the beam pipe is usually referred to as strong focusing (Figure 2.12) and is very similar to alternating concave and convex optical lenses.

Compared to the Booster's circumference of only $2.2 \mu \mathrm{s}$, the LINAC pulses are rather long $(40 \mu \mathrm{s})$. This means, that the LINAC beam has to be injected continuously into the Booster (multi-turn injection) without kicking out the protons that are already inside the Booster. This is the reason, why negative hydrogen ions instead of protons are accelerated up to the Booster. The $H^{-}$beam, due to its opposite charge, can be »merged « into an existing proton beam, right where the $H^{-}$ions enter the Booster. Before reaching the next magnet, though, the merged beam of protons and hydrogen ions is passed through a thin carbon foil, where the weakly bound electrons are stripped of the $\mathrm{H}^{-}$ions to leave bare protons. Since the foil is very thin, the highenergetic protons are not affected at all. An additional advantage of the multi-turn injection system is the increased beam intensity in the Booster. The Pre-accelerator, the LINAC and the Booster together comprise what is known as the proton source.

\section{Main Injector / (Recycler)}

From the Booster, the $8 \mathrm{GeV}$ protons are transferred to the Main Injector, another synchrotron with a circumference of approximately $3.3 \mathrm{~km}$, i.e. slightly more than half the circumference of the Tevatron and about seven times the one of the Booster. The tunnel of the Main Injector actually houses two separate rings (Figure 2.13): the Main Injector (lower ring), which can perform a multitude of operations and the Recycler, a storage ring for $8 \mathrm{GeV}$ antiprotons (upper ring). The »rings « are not really circular, but rather made up of arcs and straight sections. Contrary to the Booster magnets, the Main Injector uses separate large dipole magnets populating the ring segments to bend the beam, while special focusing / defocusing quadrupole magnets are utilised to keep the beam restricted to the beam pipe; the resulting repetitive lattice is $\gg$ FODO «. The straight sections in between are used for a variety of specialized functions, usually involving beam transfer. The Main Injector has 18 accelerating cavities and can accelerate beam as fast as every
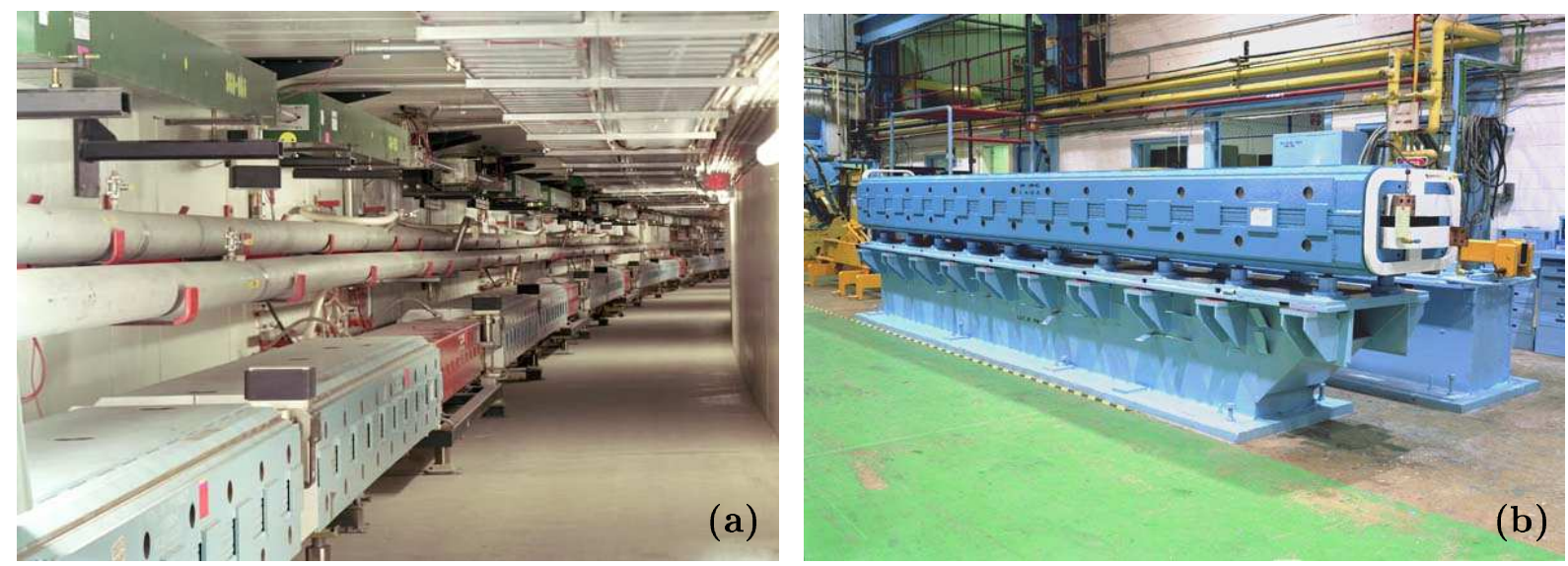

Figure 2.13: (a) A section of the tunnel housing the Main Injector (bottom ring, blue dipole / red quadrupole magnets) and the Recycler (upper ring, green magnets). (b) One of the $6 \mathrm{~m}$ long (blue) dipole electromagnets used in the Main Injector before installation.

$2.2 \mu \mathrm{s}$. As already mentioned, the beam does not form a continuous stream, but is bunched. Since the Main Injector is much larger than the Booster, 84 bunches are required to fill all of the available RF slots (buckets) around the circumference of the Main Injector; the total sum of the bunches, i.e. all available buckets, constitutes a batch. 
Although there are several different modes the Main Injector can be operated in, only the collider mode will be described here, since this is the one that generates the two colliding beams of protons and antiprotons in the Tevatron. The Main Injector accepts $8 \mathrm{GeV}$ protons from the Booster, or $8 \mathrm{GeV}$ antiprotons from the Antiproton Source and accelerates the $8 \mathrm{GeV}$ protons to either $120 \mathrm{GeV}$, or $150 \mathrm{GeV}$, depending on their destination. If the Main Injector is used to inject protons (antiprotons) into the Tevatron, its final beam energy is $150 \mathrm{GeV}$; if it is used to supply protons for the further production of antiprotons, the final energy is $120 \mathrm{GeV}$. Additionally, the $150 \mathrm{GeV}$ proton and antiproton bunches delivered to the Tevatron must be »superbunches «, i.e. more intense than any individual bunch that can be accelerated by the Booster. To meet this requirement, the Main Injector coalesces 7-11 Booster bunches into one superbunch, before transferring them to the Tevatron.

\section{Antiproton Source: Target, Debuncher and Accumulator}

Before actually introducing the Recycler and what it does, the Antiproton Source [94] is described in more detail. A bottleneck in the operation of a proton-antiproton collider is the time needed to accumulate the required number of antiprotons, this is why the performance of the antiproton production facility greatly affects the quality and the duration of stores in the Tevatron. Antiproton production is inherently inefficient; for every million protons on target, typically only about 10 to 20 antiprotons can be captured and stored. Fermilab's Antiproton Source is comprised of a target station, two triangular shaped »rings « called the Debuncher and the Accumulator and the transport lines between those rings and the Main Injector.

Antiprotons are created at the target station by firing $120 \mathrm{GeV}$ protons from the Main Injector onto a nickel/copper target $(\approx 1.3 \mathrm{~cm}$ thick) encased in a titanium sheath to hold it together. Downstream of the target, negative secondaries are focused with a lithium collection lens and are momentum selected $(8 \mathrm{GeV})$ by a pulsed magnet. Off-momentum and/or positively charged particles are absorbed in a beam dump. Most of the non- $\bar{p}$ secondaries decay away during their travel to the Debuncher and another large part is lost during the first few turns in the Debuncher. Only a tiny fraction of antiprotons with the appropriate energy survive and are actually debunched and cooled, before they are sent on to the Accumulator.

\section{The Debuncher}

The Debuncher captures the antiprotons coming off the target and the Accumulator stores (or accumulates) them. Both, the Debuncher and the Accumulator are synchrotrons with a mean radius of approximately $90 \mathrm{~m}$ and are divided into six sectors. The Debuncher has a simple $»$ FODO « lattice, with a total of 19 quadrupole and 11 dipole magnets (see also Figure 2.16). The Accumulator, however, uses a much more complicated lattice structure, since special arrangements of quadrupoles are needed towards the three straight sections in order to create regions with low or high dispersion. In addition to dipoles and quadrupoles, both machines use various other magnetic devices, e.g. sextupole (octupole) magnets, as higher order correction elements. This multitude of systems and devices also explains the peculiar triangular shape, chosen to accommodate the necessary, up to $16 \mathrm{~m}$ long, straight sections.

After capturing the $8 \mathrm{GeV}$ antiprotons from the target, the Debuncher allows to exchange their small time with their large momentum spread $\left(\frac{\Delta p}{p}\right)$ via bunch rotation and subsequent adiabatic debunching. Bunch rotation is an RF manipulation, in which RF cavities are phased such that particles at the leading edge of a bunch (higher momentum particles) are decelerated, while the trailing particles are accelerated, whereby the momentum range of the particles in a bunch is 


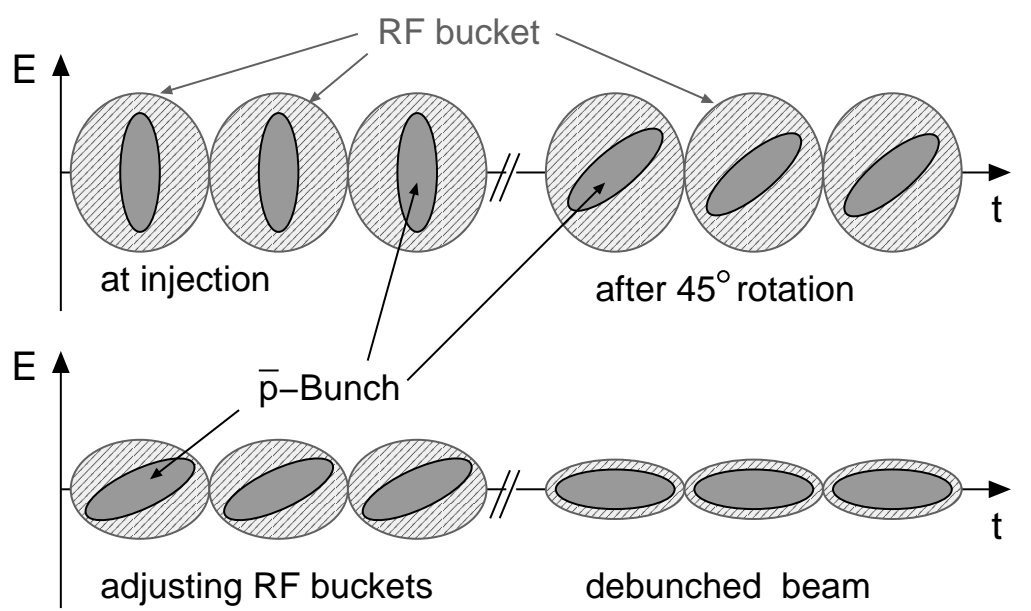

Figure 2.14: Schematic illustration of bunch rotation and subsequent adiabatic debunching of the antiproton bunches arriving at the Debuncher. Essentially, the large momentum spread is exchanged with the small time spread, which results in a debunched beam, characterised by a small energy (momentum) range of the particles.

reduced. In a second step, the beam is adiabatically (slowly) debunched over about $60 \mathrm{~ms}$ by lowering the RF voltage accordingly. As illustrated schematically in Figure 2.14, the antiprotons are injected into mismatched (much larger) RF buckets in the Debuncher and thus rotate in these large buckets. After a short time, when the bunches have rotated about $45^{\circ}$ in phase space, the $\mathrm{RF}$ voltage is rapidly decreased to match the large buckets to the smaller, rotated bunches. Then the RF voltage is adiabatically decreased, during which the bunches rotate another $45^{\circ}$. Two additional beam-cooling ${ }^{8}$ systems act on the beam to further increase the particle density. Both are transverse systems, reducing the horizontal and vertical emittances of the beam (its physical size) by betatron cooling based on the method of stochastic cooling ${ }^{9}$, which was developed by S. van der Meer in the late 1970 s precisely to increase the accumulation of antiprotons for their usage in the $p \bar{p}$ collider SPS (Super Proton Synchrotron) at CERN. In 1984, S. van der Meer was awarded the Nobel Price in Physics for this work, together with C. Rubbia, who led one of the experimental collaborations that discovered the $W$ and $Z$ bosons. Stochastic cooling [95] consists in picking up an error signal from the circulating antiprotons on one side of the ring,

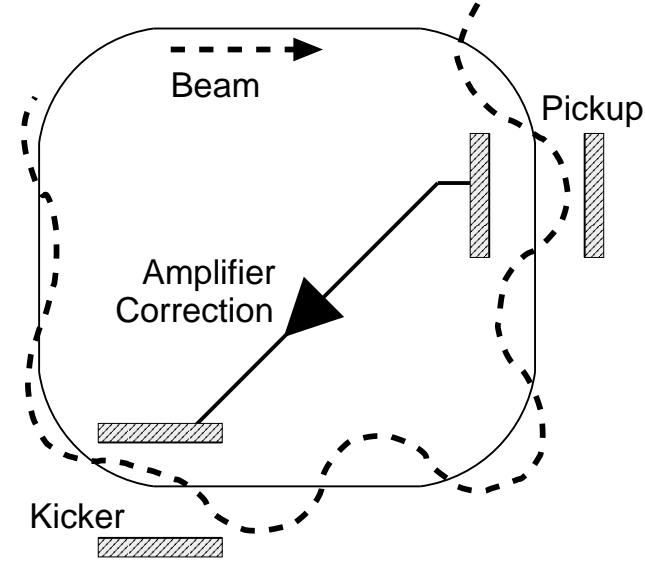

Figure 2.15: Illustration of betatron stochastic cooling to reduce the transverse beam size (horizontal and vertical emittance). amplifying it, calculating the appropriate correction and applying this correction to the antiproton beam on the other side of the ring. As shown in Figure 2.15, the pickup and kicker are designed such that a particle passing the pickup at the peak of its oscillation will cross the kicker with zero position error, but with an angular deviation proportional to its displacement at the pickup. If the betatron phasing and the kicker response were perfect, the trajectory of the particle would be corrected to that of the ideal, central orbit. After debunching and stochastic cooling, the horizontal and vertical transverse emittances are reduced from approximately $20 \pi \mathrm{mm}$-mrad

\footnotetext{
${ }^{8}$ Initially, the particles in the beam occupy a wide range of energies and behave very randomly, which is considered $»$ hot « in analogy to gases. The final, more coherent beam, where particle movements perpendicular to the beam propagation are much smaller, is referred to as »cold « beam.
}

${ }^{9}$ The name is due to the stochastic nature of the beam, where particles move at random w.r.t one another. 
to less than $7 \pi \mathrm{mm}$-mrad, which makes it much easier to transfer the antiprotons from the Debuncher to the Accumulator, which has a very limited momentum aperture at injection.

\section{The Accumulator}

Just before the next pulse arrives from the target the antiprotons are extracted from the Debuncher and injected into the Accumulator, see Figure 2.16. The Accumulator storage »ring « collects successive pulses of antiprotons from the Debuncher over the course of several hours or even days. A process known as momentum stacking is used to accumulate (or stack) antiprotons. When a beam pulse from the Debuncher is newly injected, the Accumulator RF system is used to decelerate the beam initially. The lowered energy results in the particles circulating in an orbit of smaller radius. They eventually get onto the so-called stack tail, where the antiprotons can be captured by the stack tail momentum cooling system, which is based on stochastic cooling, similar to the systems used in the Debuncher. The stack tail momentum cooling system slowly sweeps the beam away from the edge of the tail and decelerates it towards the densest portion of the stack, the core. This process takes about 30 minutes, after which the core cooling systems, i.e. up to six additional, stochastic cooling systems act on the beam. Two momentum cooling systems control the momentum spread and keep the antiprotons from hitting the low momentum aperture, and two betatron cooling systems (one horizontal and one vertical) keep the beam emittances minimized, at approximately $2 \pi \mathrm{mm}-\mathrm{mrad}$ in both planes.
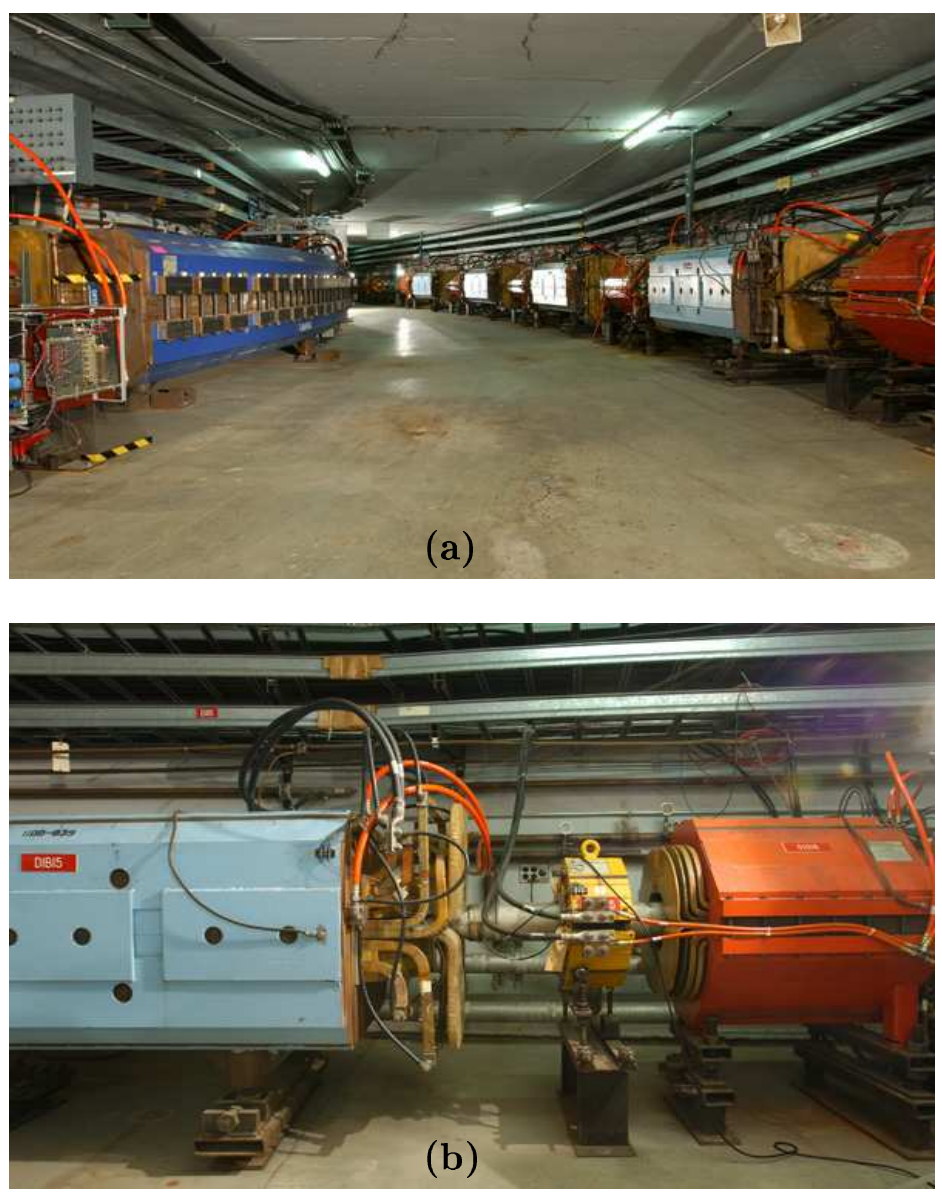

(c)

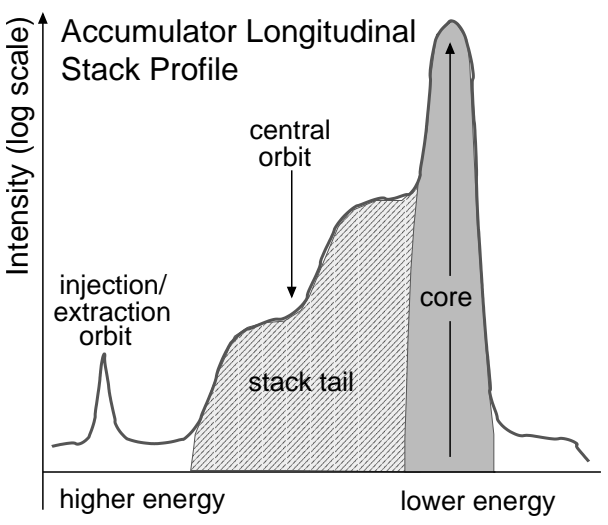

Figure 2.16: (a) The Accumulator (inner ring) and the Debuncher (outer ring) share the same tunnel. (b) Close-up view of two Debuncher magnets: dipoles are blue, quadrupoles red. From both photographs the FODO lattice of the Debuncher, with dipole magnets in the drift spaces $(O)$ is clearly visible. (c) Schematic illustration of the momentum stack profile in the Accumulator, with the core at low energy / momentum and the stack tail extending towards higher particle energies.

Before the advent of the Recycler, this process continued for hours or days with the stack growing in size until the maximum accumulator intensity was reached or the Tevatron needed to be refilled. 
Now, with the Recycler operational, the antiprotons are transferred to the Main Injector and from there to the Recycler, as soon as the maximum beam intensity in the Accumulator is reached. The antiprotons are then stored in the Recycler, so that a new stack of antiprotons can be built using the Debuncher and Accumulator. For the transfer of antiprotons to the Main Injector, a special RF system is used, whose frequency corresponds to the revolution frequency of the dense antiproton beam in the core. The RF voltage is slowly increased and a portion of the beam is adiabatically captured into four buckets and is then slowly moved through the stack and accelerated onto the extraction orbit. Exactly four RF buckets are used because the transfer is accomplished by means of a bucket to bucket (synchronous) transfer, requiring that the bunch structure of the extracted antiprotons is compatible with the Main Injector RF bucket structure.

Although neither the Debuncher, nor the Accumulator accelerate the beam (at least not in the common sense), both machines are crucial for the production of antiprotons at a sufficiently high rate, simply because it takes hours to build up a suitable stack to use for colliding beams and thus to efficiently operate the Tevatron.

\section{Recycler}

The Recycler, which resides in the same tunnel as the Main Injector (see Figure 2.13, upper ring), is used to store antiprotons from the Accumulator to allow for a higher production and stack rate in the latter. The Recycler has no special features except for the usage of mostly permanent magnets, contrary to the electromagnets utilised in the other accelerators and storage rings at Fermilab. Until the fall of 2005, the Recycler only used stochastic cooling as described in the context of the Debuncher and Accumulator functionality. Since then a second type of cooling, so-called electron cooling, is additionally used in the Recycler and enables it to store even more antiprotons at a higher beam density.

Electron cooling [96] is based on the exchange of energy in elastic collisions between a beam of $»$ cold « electrons and the »hot « antiproton beam. The electrons interact with the antiprotons, cooling the beam and reducing the spread in longitudinal momentum: antiprotons travelling too fast are slowed down when bumping into electrons, and slow antiprotons are sped up as they are hit by faster electrons.

At Fermilab, a $20 \mathrm{~m}$ long section of the Recycler is used for electron cooling. A continuous »cold « beam of $4.3 \mathrm{MeV}$ electrons, where the electrons have approximately the same velocity as the average velocity of the antiprotons, mixes with the »hot $\ll 8 \mathrm{GeV}$ antiproton beam. As a result a smaller beam size and higher particle density of the antiproton beam is obtained. With the successful implementation of electron cooling, it could be demonstrated in summer 2005 that the increased number of antiprotons stored in the Recycler, not only leads to a higher antiproton production rate in the Accumulator, but in turn also increases the luminosity of the Tevatron collider [97]. Momentarily, a typical number of stored antiprotons is of the order of $2 \cdot 10^{12}$; the design goal is to store and cool up to $6 \cdot 10^{12}$ antiprotons in the Recycler.

\section{Tevatron}

The Tevatron is the largest of the Fermilab accelerators, with a circumference of approximately $6.3 \mathrm{~km}$. Like the other circular accelerators encountered so far, it also is a synchrotron and is not a perfect circle neither. The $\gg$ ring $«$ is divided into six sections labeled A through F. Each sector starts with a section called the zero location, which are long straight sections with specialized 
functions. A0 contains the proton/antiproton colliding beam abort kickers, at B0 and D0 the collider detectors CDF and $\mathrm{D} \varnothing$ are located, respectively, $\mathrm{C} 0$ holds another proton abort line, E0 contains the so-called scrapers (to remove circulating protons, but not antiprotons, after the end of a collider store), and F0, finally, contains the eight accelerating RF cavities, as well as the transfer lines to and from the Main Injector. In colliding beams mode the RF cavities operate in two groups: the first group (cavities $1,3,5,7$ ) accelerates antiprotons and the second group (cavities 2, 4, 6, 8) accelerates protons.

The magnetic lattice of the Tevatron is the same as for the Debuncher and the Main Injector; alternating horizontally and vertically focusing quadrupoles with drift spaces in between (FODO). Each drift space is actually occupied by four of the large $6.4 \mathrm{~m}$ long beam bending dipole magnets. Contrary to all other accelerators operated at Fermilab, the Tevatron uses cryogenically cooled superconducting magnets to bend and focus the high-energetic proton and antiproton beams. The magnets are made of a niobium/titanium alloy, cooled to $4.2 \mathrm{~K}$ by liquid single-phase Helium to ensure the superconductivity of the material. Due to the superconducting magnets, higher field strengths are possible, at the cost of an extensive cryogenic system and a sophisticated magnet protection system.

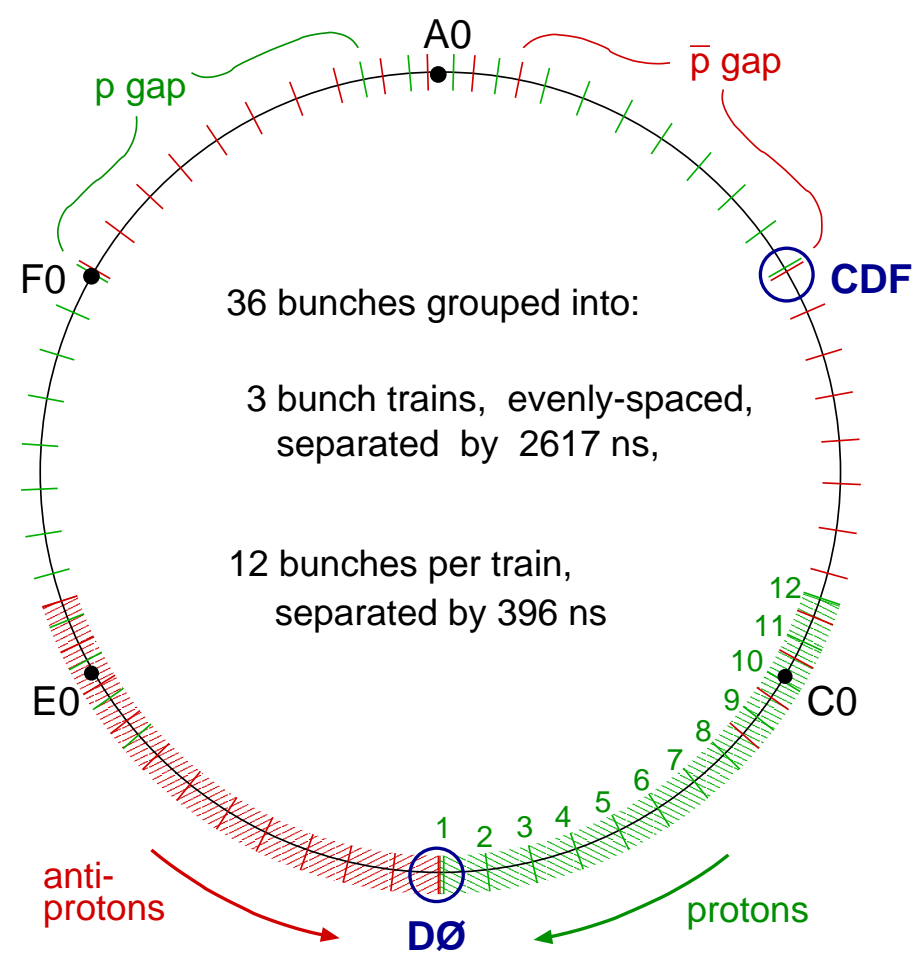

Figure 2.17: Schematic illustration of the bunches and bunch trains circulating in the Tevatron, with protons circulating clockwise, antiprotons counter-clockwise. The three large bunch trains with 12 bunches $(p, \bar{p})$ each are separated by $2.617 \mu \mathrm{s}$ and the bunches in a train are spaced 396 ns apart. The gaps in between the $p(\bar{p})$ bunch trains are either used for synchronisation or beam abortion.

In colliding beams mode, the Tevatron is loaded with $150 \mathrm{GeV}$ protons and antiprotons, which are ramped to $980 \mathrm{GeV}$ and are then brought to collision at two points in the ring, B0 and D0, which are the nominal interaction points within the CDF and DØ detectors, respectively. Filling (or loading) the Tevatron with $150 \mathrm{GeV}$ protons and antiproton is done as follows: As soon as a sufficiently large antiproton stack has been built, the transfers to the Main Injector and subsequently to the Tevatron are initiated. First, the necessary number of protons is loaded into 
the Tevatron. Protons are accelerated through the complete proton chain, the Pre-accelerator, the LINAC and the Booster. Only about 7-10 proton bunches (of the 84 available) are extracted from the Booster, accelerated to $150 \mathrm{GeV}$, coalesced into one superbunch and then injected into the Tevatron. This procedure is repeated 36 times in a row until 36 coalesced superbunches are circulating clockwise in the Tevatron. In a next step, four groups of about 7-10 antiproton bunches from the Recycler (or the Accumulator) are transferred to the Main Injector, accelerated to $150 \mathrm{GeV}$ and coalesced into four superbunches. After extraction to the Tevatron, this process is repeated nine times, resulting in 36 antiproton superbunches circulating counter-clockwise in the Tevatron. The 36 proton and antiproton superbunches are grouped into three $\gg$ bunch trains «, that are evenly distributed in the Tevatron ring, see Figure 2.17. Given the even spacing of the bunch trains of about $2.6 \mu \mathrm{s}$, and the $396 \mathrm{~ns}$ separation of the twelve bunches in one train, a bunch crossing takes place approximately every $580 \mathrm{~ns}$ on average. The longer gaps between the trains allow to abort the proton or antiproton beam in case of potential danger to either the superconducting Tevatron magnets or the two large detectors.

Upon completion of the filling process, the Main Injector and Antiproton Source return to antiproton production and stacking, respectively, and the Tevatron ramps to $980 \mathrm{GeV}$ with all of the coalesced proton and antiproton bunches. As soon as a stable situation is established in which the two counter-rotating beams produce proton-antiproton collisions, the two experiments start taking data. This situation is known as a $(36 \times 36)$ store and it usually lasts for about 20-30 hours, depending on the initial luminosity. When the luminosity decreases too much to be useful - or, in a less favourable scenario, if a component failure causes the beams to be lost the store is ended and the Tevatron prepared for new beam.
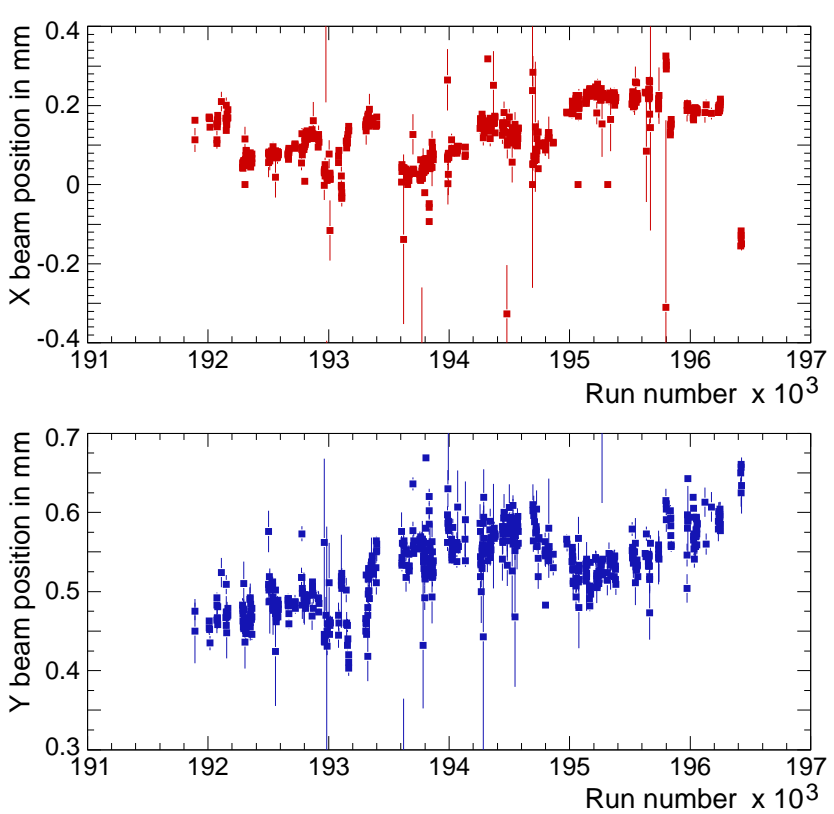

Figure 2.18: Beam positions in the transverse plane as a function of the data-taking period.
Finally, the beam profile at the $\mathrm{D} \varnothing$ interaction point is shortly described. The region in which interactions can take place has a spread in $z$ direction of approximately $28 \mathrm{~cm}$, but is much smaller in the transverse plane $\left(\sigma_{x} \approx 25 \mu \mathrm{m}, \sigma_{y} \approx 20 \mu \mathrm{m}\right)$, thus forming an elongated ellipsoid.

Figure 2.18 illustrates the spread of the beamspot in the transverse plane over time, where the variations are of the order of 250 $300 \mu \mathrm{m}$. If these store-to-store variations exceed approximately $100 \mu \mathrm{m}$, they have to be taken into account in the programming of the fiber track trigger (CTT).

In the present analysis, the $z$ component of the primary vertex is required to lie within $\pm 60 \mathrm{~cm}$, relative to the nominal interaction point at $z=0$, see also chapter 4 . This corresponds to roughly two sigma of the spread of the interaction region in the $z$ direction and ensures that the tracks lie within the fiducial volume of the inner tracking detectors, so that reliable tracking can be guaranteed. 


\subsection{The DØ Detector}

Most detectors of colliding beam experiments follow the same general »onion skin « layout as illustrated in Figure 2.19 [98]. In the innermost region, closest to the interaction point, is the tracking system, designed to measure the trajectories of passing particles in three dimensions. Usually, the tracking system is immersed in a magnetic field, which additionally permits to determine the momentum of charged particles via their bending radius.

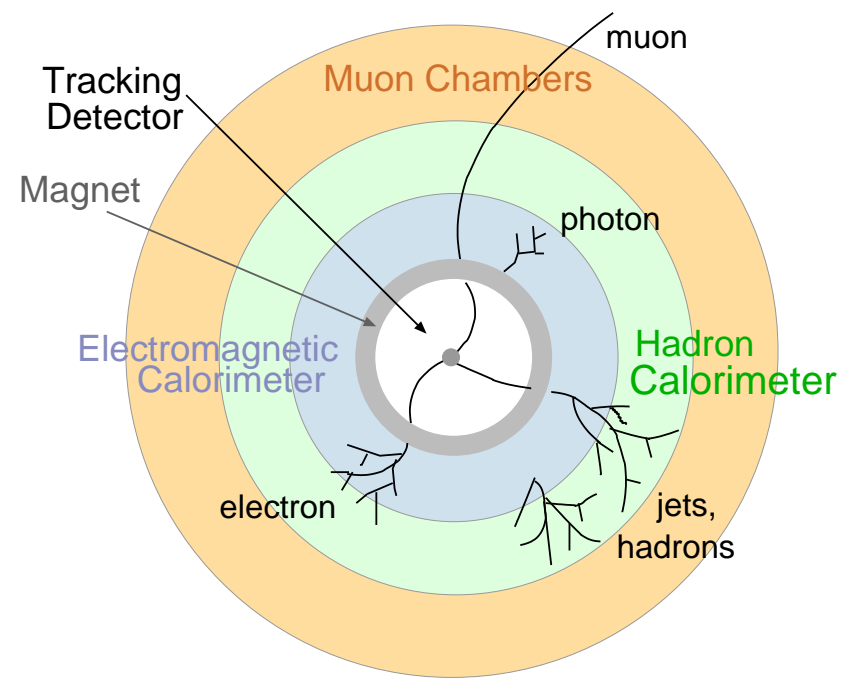

Figure 2.19: Schematic view of the $»$ onion skin « layout of a usual colliding beams detector in the $(r, \varphi)$-plane, with the beam axis going into the paper. The different detection systems are wrapped around the interaction region as concentric cylinders. Tracking detectors with very little material constitute the innermost detection systems, followed by the denser calorimeters and muon chambers.

A calorimeter constitutes the next layer and allows to measure the energy This device is typically $»$ thick « enough to absorb the energy of all incident particles (charged and neutral) with the exception of only weakly interacting particles, such as muons or neutrinos. In contrast to the thick calorimeter, tracking detectors contain as little material as possible to minimize their effect on the particle trajectory and the loss of particles prior to the calorimeter. The outermost layer consists of the muon system, which is usually made of tracking detectors interspersed with layers of denser material to absorb energy. The presence of neutrinos, the only Standard Model particles that are usually not detected in collider experiments, can, however, be inferred from a momentum imbalance in the total measured momentum perpendicular to the beam, i.e. the transverse momentum $\left(p_{\mathrm{T}}\right)$ or the transverse energy $\left(E_{\mathrm{T}}\right)$.

The $\mathrm{D} \varnothing$ detector, one of the two large multipurpose detectors located at the Fermilab Tevatron, follows this general layout as can be seen from Figure 2.20. Like the accelerators, the detector was upgraded in the five year period from 1996 to 2001, to take advantage of the higher centreof-mass energy and luminosity the Tevatron is now able to provide. During the first phase of data taking, Run I, the DØ experiment did not have a magnetic field in the inner detector region. This, and the fact that radiation damage was evident there, are the main reasons why the old tracking system was completely replaced. Other major improvements include a new forward muon system, the addition of a forward proton detector (FPD) for diffractive physics and a considerable upgrade of the trigger and readout electronics, as well as the data acquisition (DAQ) system, which became necessary due to the large reduction in bunch spacing, from $3.5 \mu \mathrm{s}$ (Run I) to 396 ns (Run II). In the following the subdetectors and their interplay are briefly described (stand: September 2005). 


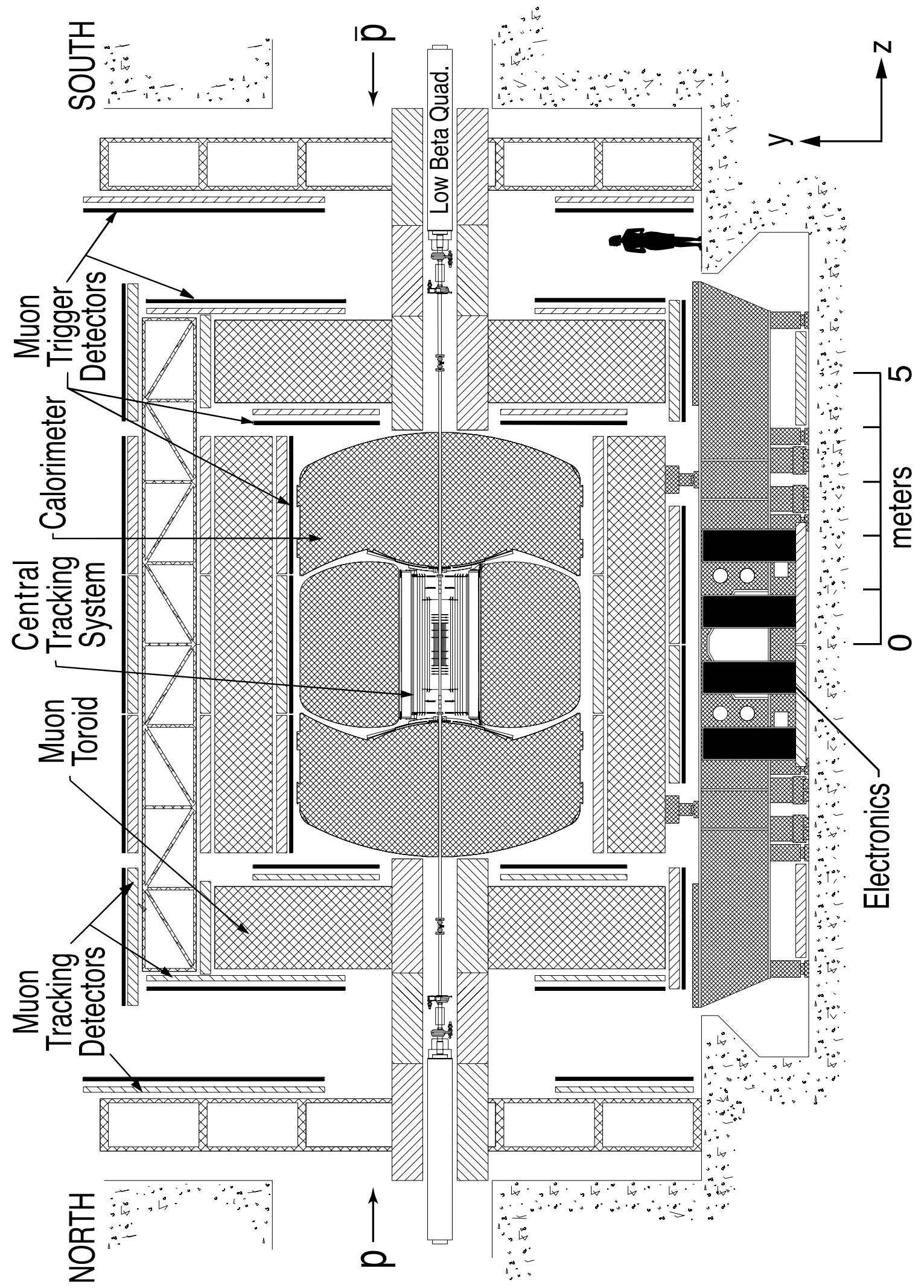

Figure 2.20: Longitudinal cross section of the DØ detector after the upgrade for the Run II. The coordinate system is defined with $x$ pointing away from the center of the Tevatron and $y$ upwards. The $z$-axis is parallel to the beam axis and corresponds to the direction of the protons (taken from [80]). 


\subsubsection{The Central Tracking Systems}

In the very center of the detector lies the $2.37 \mathrm{~m}$ long beam pipe, whose outer radius is about $1.9 \mathrm{~cm}$. The thin pipe with a wall thickness of approximately $0.51 \mathrm{~mm}$ is made of beryllium and is surrounded by the tracking system, whose innermost components have a radius of $2.6 \mathrm{~cm}$. The complete system consists of a silicon microstrip vertex tracker (SMT) in the innermost region, and a scintillating fiber tracker (CFT), both of which are located within a $2 \mathrm{~T}$ axial magnetic field of a solenoidal magnet as shown in Figure 2.21.

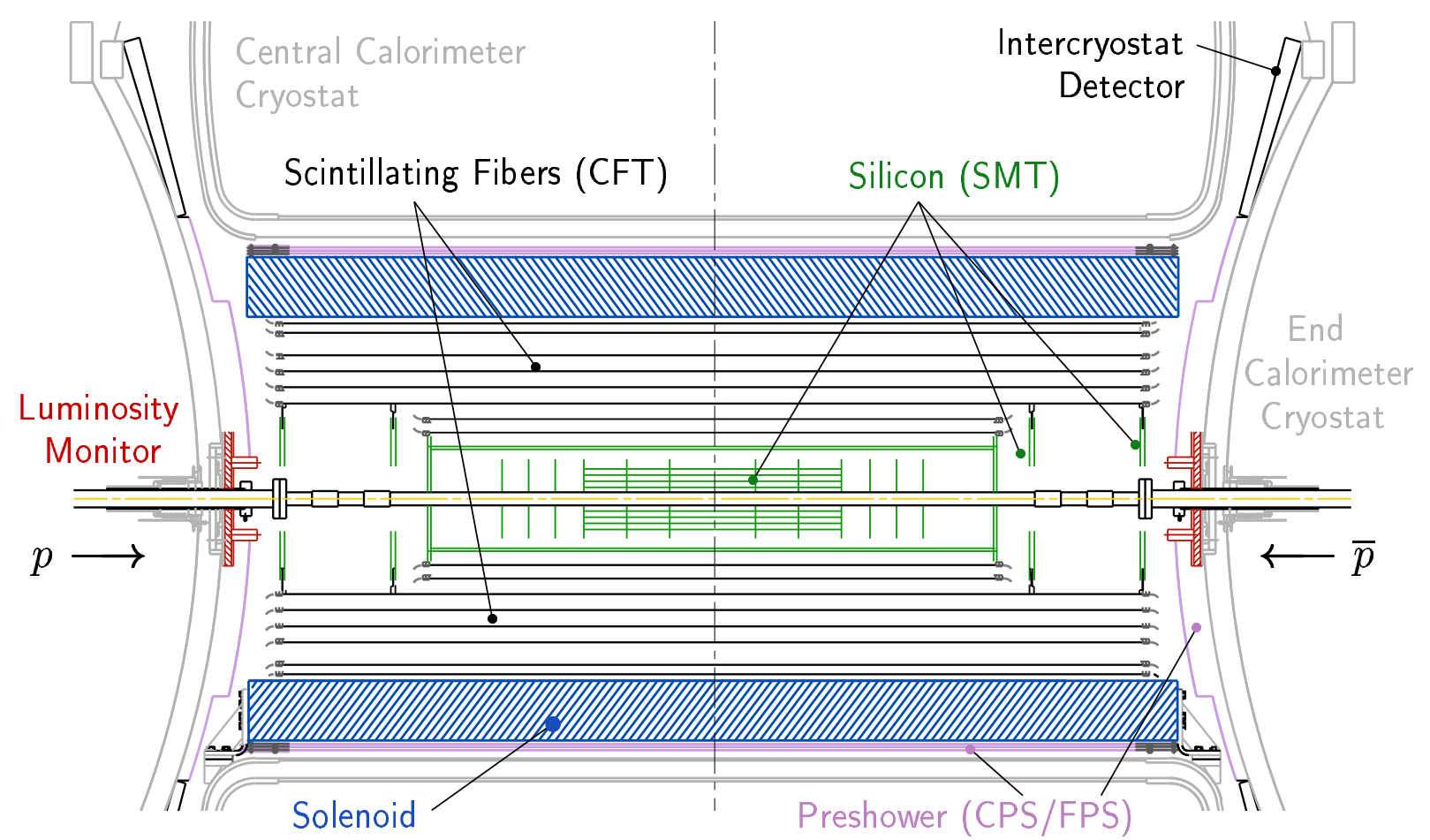

Figure 2.21: Schematic illustration of the inner tracking system in the $(y, z)$-plane. Depicted are the two tracking detectors, SMT (green) and CFT (black), located inside the 2 T solenoid (blue hatched). For completeness also shown are: the two luminosity monitors (dark red) at high $|z|$ (section 2.3.4), the central and forward preshower detectors (light violet), and the four intercryostat detectors (ICD, black).

The two tracking detectors allow for a precise location of the primary interaction vertex with a resolution of less than $15 \mu \mathrm{m}$ in the the $(r, \varphi)$-plane for particles with a transverse momentum of at least $10 \mathrm{GeV}$ at $|\eta|=0$; along the beam pipe in $z$ direction, the resolution is approximately $35 \mu \mathrm{m}$. Given the high resolution of the vertex position and the strong magnetic field, precise measurements of lepton transverse momenta $\left(p_{\mathrm{T}}\right)$ are possible. Calorimeter measurements of jet transverse energies $\left(E_{\mathrm{T}}\right)$ and missing transverse energy $\left(\not_{T}\right)$ are also improved due to the possibility to calculate cluster centroids much more precisely. In contrast to Run I, this also allows to calibrate the electromagnetic calorimeter using $E / p$ for electrons, where $E$ is measured in the calorimeter and $p$ in the tracking detectors. The SMT, as well as the CFT provide information to the trigger system. Since the scintillating fibers can be read out very quickly, the CFT provides a fast and continuous readout of discriminator signals to the first level trigger (Level 1). Upon a Level 1 accept, the tracking information is sent on to Level 2, where also SMT tracking information is handled. The SMT is mainly used to trigger on displaced vertices 
from $b$-quark decays. Finally, upon a Level 2 accept all information is sent to the Level 3 trigger, which receives further CFT readout, based on the CFT's digitised analog signals in addition to the sole discriminator information available to Level 1 and Level 2. (For a detailed description of the $\mathrm{D} \varnothing$ trigger and data acquisition (DAQ) systems, see chapter 2.4.)

\section{Silicon Microstrip Vertex Detector: SMT}

The SMT detector covers a pseudorapidity range of $-4.0<\eta<+4.0$, which roughly corresponds to the full calorimeter coverage. Since the interaction region is quite large, about $25 \mathrm{~cm}$ in the $z$-direction, i.e. along the beam pipe, the SMT was designed from two different types of modules, barrels and disks, so that the tracks are in general perpendicular to the detector surfaces over the complete $\eta$-range. Six barrel modules, intespersed with three disk modules, occupy the central region, while an assembly of different disk modules is used in the forward region, see Figure 2.22.

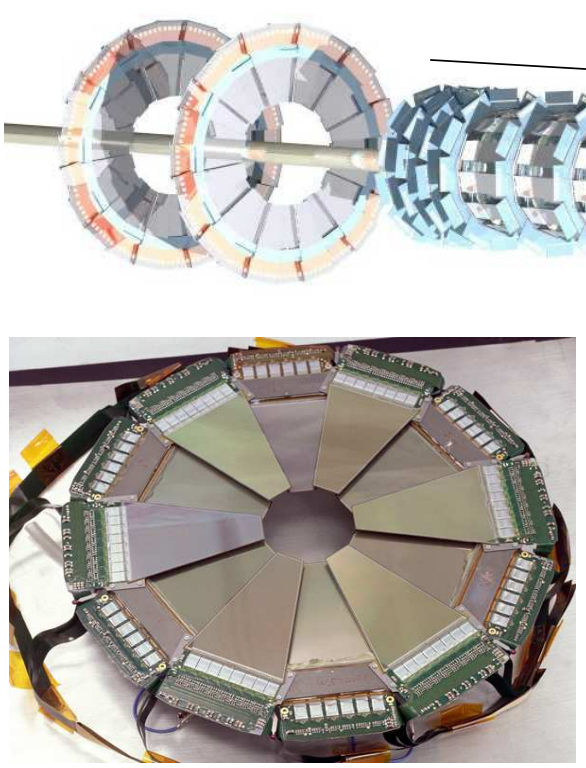

$1.2 \mathrm{~m}$

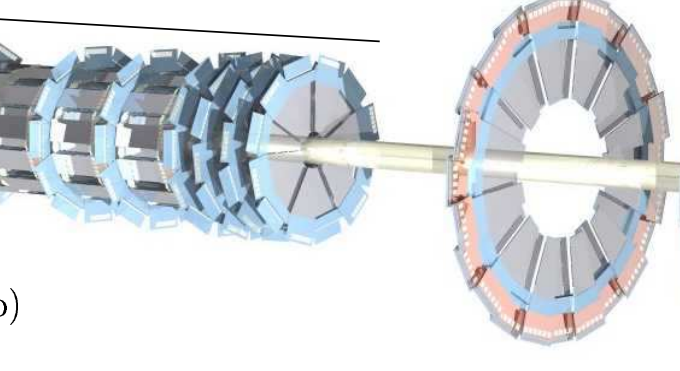

(b)

Figure 2.22: (a) Isometric drawing of the SMT detector, with barrel/disk assemblies in the central region and a pure $F$ and $H$-disk design in the far forward regions, at $|z| \approx 43 \mathrm{~cm}$. (b) A photograph of a spare F-disk component with 12 doublesided silicon sensors (wedges) mounted back-to-back on the support structure.

Barrel modules are located at $|z|=6.2,19.0,31.8 \mathrm{~cm}$, measured from the middle of each barrel. The central disks, or F-disks, terminate each barrel on the high $|z|$ side and three more F-disks follow on either side of the barrel/disk assembly in shorter intervals of about $5 \mathrm{~cm}$. The F-disks are thus located at $|z|=12.5,25.3,38.2 \mathrm{~cm}$, and at $|z|=43.1,48.1$, and $53.1 \mathrm{~cm}$. In the far forward regions $(|z|>100 \mathrm{~cm})$, two large-diameter H-disks are installed. Their centers are located at $|z|=100.4$, and $121.0 \mathrm{~cm}$, respectively.

Each barrel module is made of four layers, which in turn hold the silicon modules installed on socalled »ladders «. Layers 1 and 3 consist of 12 ladders each and layers 2 and 4 of 24, giving a total of 432 silicon modules in the barrel modules alone. All four layers have axial strips, measuring the $(r, \varphi)$-coordinate, while layers 1 and 3 use small angle stereo sensors, tilted by $2^{\circ}$ w.r.t. the axial strips, and layers 2 and 4 use full $90^{\circ}$ stereo sensors to allow for a 3D-vertex reconstruction. The F-disks (H-disks) are made up of 12 (24) double-sided silicon sensors, so-called wedges, each of which consists of two single-sided »half« wedges mounted back-to-back on each disk. Each side of a full wedge, (i.e. down- or upstream), is read out separately. There are 144 full F-wedges and 96 full H-wedges, which - together with the 432 barrel sensors - results in a grand total of 912 readout modules with 792,576 channels. Depending on the location of the interaction vertex and the direction of flight in between four to eight hits are expected in the SMT. 


\section{Central Scintillating Fiber Detector: CFT}

The scintillating fibers of the CFT are arranged into doublet layers $(2 \times 128$ fibers per layer $)$ and mounted on eight concentric support cylinders, occupying the radial space from $20 \mathrm{~cm}$ to $52 \mathrm{~cm}$ from the beam axis, see Figure 2.23. While the six outer cylinders are $2.52 \mathrm{~m} \mathrm{long}$, the two innermost CFT cylinders are only $1.66 \mathrm{~m}$ long to accommodate the four forward H-disks of the SMT detector, see Figure 2.21 for comparison. The outermost cylinder covers a pseudorapidity range of up to $|\eta| \approx 1.7$. Two doublet layers with different orientations are supported by one cylinder. One layer, called $z$-layer, is aligned along the direction of the beam axis, the second layer is slightly tilted with respect to this axis. Layers, where the fibers are oriented at a stereo angle of $\varphi=+3^{\circ}$ are called $u$-layers, those where the angle is negative, $\varphi=-3^{\circ}$, are denoted $v$ layers. An alternating fiber doublet orientation $(z u-z v)$ is used to provide the best possible track coordinate measurement of the passing particles. Hence, starting from the innermost cylinder, this results in the following pattern: $z u-z v-z u-z v-z u-z v-z u-z v$.

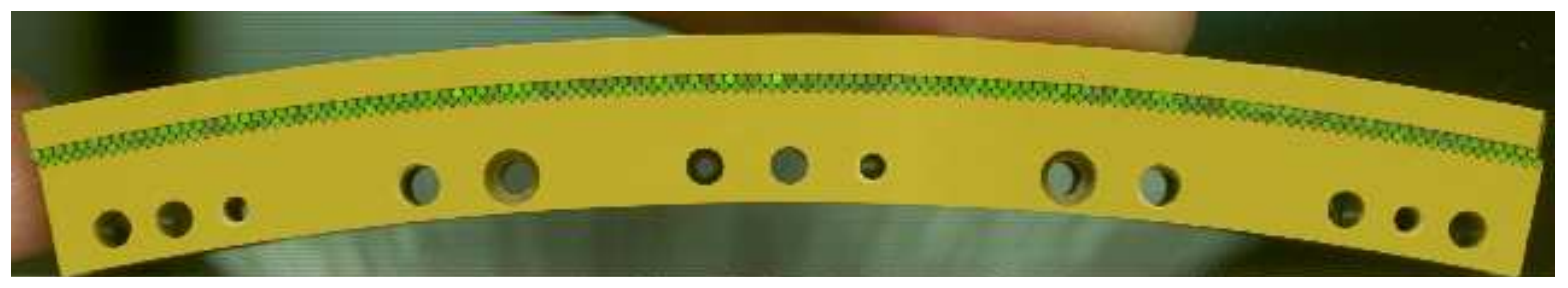

Figure 2.23: CFT doublet layer, consisting of $2 \times 128$ scintillating fibers glued to plastic grooves, which in turn are mounted on the support cylinders.

Each fiber has a diameter of $835 \mu \mathrm{m}$ and is in between $1.66 \mathrm{~m}$ and $2.52 \mathrm{~m}$ long. Together with the clear waveguide fibers of the same diameter and a length varying from $7.8 \mathrm{~m}$ to $11.9 \mathrm{~m} \mathrm{a}$ total of $200 \mathrm{~km}$ of scintillating and $800 \mathrm{~km}$ of clear fibers are used in the CFT detector. Due to the small fiber diameter, the CFT has an inherent resolution of approximately $200 \mu \mathrm{m}$, if the actual location of each individual fiber is known with a precision no less than $50 \mu \mathrm{m}$. Further details about scintillating fiber detectors can be found in [99].
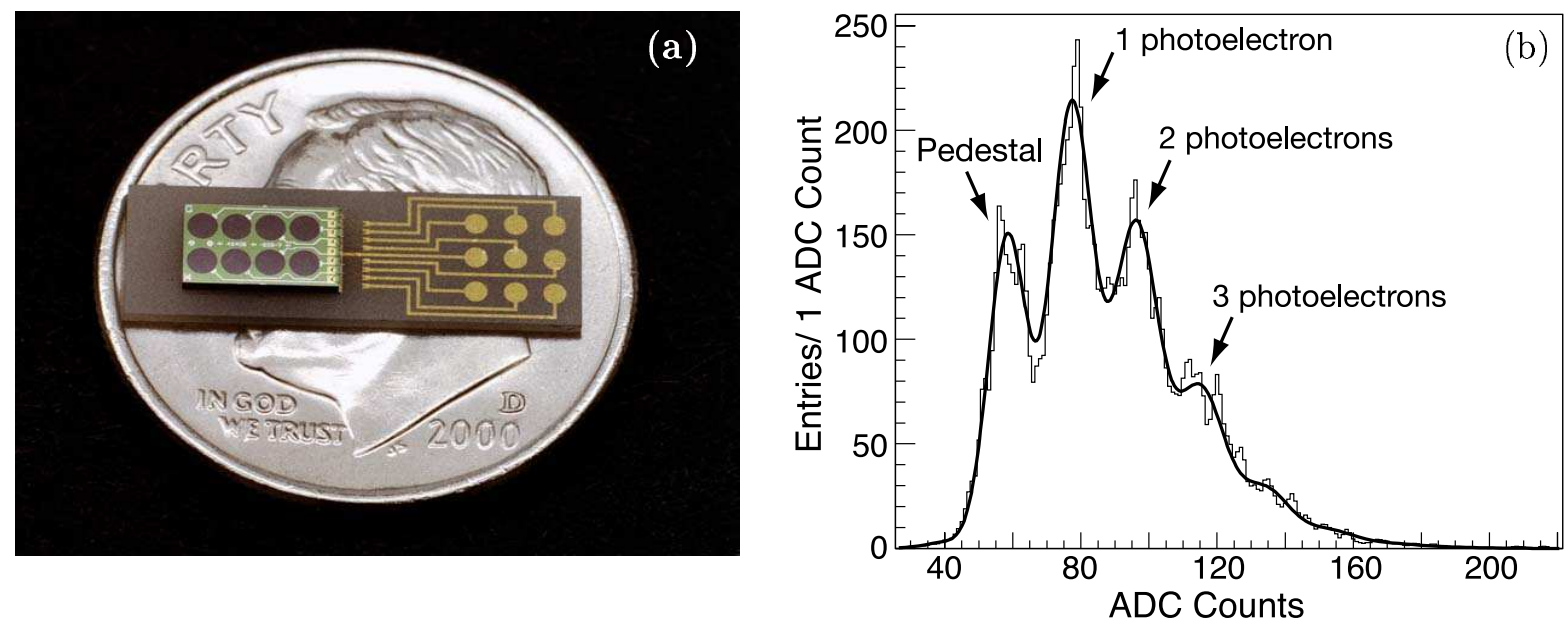

Figure 2.24: (a) Photograph of a VLPC on a dime to provide a feeling for the smallness of this device. Clearly visible are the eight individual silicon pixels (1 $\mathrm{mm}$ diameter) on the left and the contact pads and electronic circuits on the right. (b) Typical LED calibration spectrum of a single VLPC for an axial CFT fiber. The data is shown as a histogram and the solid line corresponds to the fit. 
The scintillating fibers are read out via clear fiber waveguides carrying the scintillation light to visible light photon counters (VLPCs), which transform the light into electrical signals. Since all readout electronics are located about $6 \mathrm{~m}$ below the central calorimeter cryostat of the detector, highly effective photodetectors became necessary, because of the long way and the small diameter (hence only few photons) of the scintillating fibers. The VLPCs, which are impurity-band silicon avalanche photodetectors, not only satisfy this requirement, but even allow the detection of single photons [100]. The only drawback is that they have to be operated at $9 \mathrm{~K}$, but they provide a fast response and a very high quantum efficiency $(\approx 75 \%)$ and are, additionally, capable of functioning in a high background environment. Figure 2.24 shows (a) one of the tiny VLPCs on a dime and (b) a typical LED spectrum obtained with a single VLPC for an axial CFT fiber.

The alignment of SMT and CFT is better than $10 \mu \mathrm{m}$, leading to a track resolution of roughly $20 \mu \mathrm{m}$ as can be seen in Figure 2.25 (a,b), showing axial residual distributions using tracks with a minimum transverse momentum of $3 \mathrm{GeV}$. The residuals are calculated as the distance between one SMT hit and a fit of all SMT and CFT hits excluding the hit in question. A simulation of the resolution assuming perfect alignment yields about $16 \mu \mathrm{m}$, compared to the actually achieved $20.2 \mu \mathrm{m}$. For the transverse momentum of charged particles traveling through the entire tracking system, this translates into a resolution of:

$$
\frac{\Delta p_{\mathrm{T}}}{\mathrm{GeV}}=0.002 \cdot\left(\frac{p_{\mathrm{T}}}{\mathrm{GeV}}\right)^{2} \Longrightarrow \quad \begin{aligned}
& p_{\mathrm{T}}=50 \mathrm{GeV} \\
& p_{\mathrm{T}}=100 \mathrm{GeV} \quad \rightarrow \Delta p_{\mathrm{T}}=5 \mathrm{GeV}(10 \%) \\
& p_{\mathrm{T}}=20 \mathrm{GeV}(20 \%)
\end{aligned}
$$

Figure 2.25 (c) provides a schematic overview of the arrangement of CFT and central preshower (CPS) detector, although it should be noted that the CPS is farther away from the CFT layers than shown in the illustration.

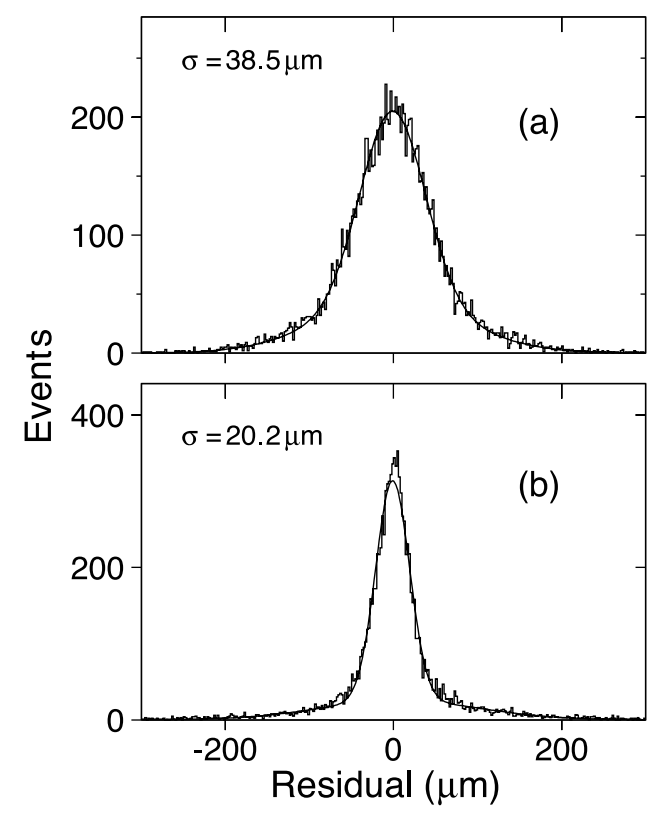

Figure 2.25: Axial residual distributions using tracks with $p_{\mathrm{T}}>3 \mathrm{GeV}$ are shown (a) upon initial installation and (b) after software alignment of the SMT and CFT barrel detectors. (c) Schematic view of a $4.5^{\circ}$ CFT/CPS sector.

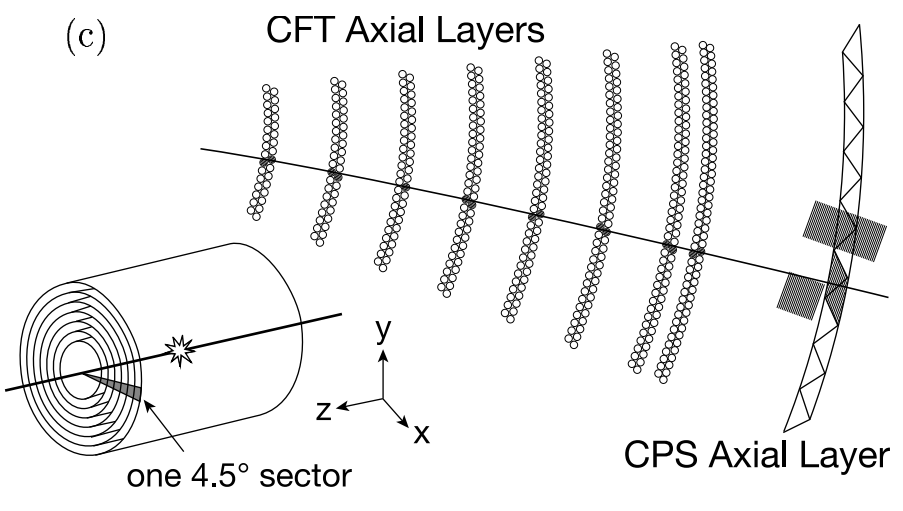

The CFT provides discriminator signals from the axial doublet layers to the Level 1 trigger for a fast hardware trigger based on the number of track candidates above certain $p_{\mathrm{T}}$ thresholds (minimum threshold of $1.5 \mathrm{GeV}$ ). These Level 1 track candidates are also used by the Level 2 trigger; only Level 3 makes use of the full CFT readout information. 


\section{Solenoidal Magnet}

The complete inner tracking system as described previously is immersed in a $2 \mathrm{~T}$ axial magnetic field of a superconducting solenoidal magnet [101]. The solenoid consists of two layers of superconductor to ensure the needed linear current density. To achieve a highly uniform field inside the magnet bore, a narrower conductor is used at the ends of the coil allowing for a higher current density there. Both types of superconductors are made of $\mathrm{Cu}: \mathrm{NbTi}$ in the ratio 1:34:1 and stabilised with pure aluminum. Although the solenoid provides a high central field, it neverless is rather thin in terms of radiation lengths. The whole magnet, coil and support cylinder, only amounts to $\approx 0.87 X_{0}$ for particles at normal incidence $(|\eta|=0)$. The complete tracking system, including the solenoid thus presents only a total of about two radiation lengths of material for particles at normal incidence, increasing to about four radiation lengths at large angles $(|\eta|>2)$. The operating current of the solenoid is $4.75 \mathrm{kA}$, its cooldown time is less than 40 hours and the time needed to energize it is about 15 minutes. The complete system was designed to safely and reliably operate over a twenty-year lifetime, to withstand up to 150 »warm-up cool-down « cycles, about 2500 energisation cycles and at least 400 fast dumps.

\section{Central and Forward Preshower Detectors: CPS / FPS}

The preshower detectors have been installed primarily to enhance the spatial matching between tracks and calorimeter towers. They function as a small calorimeter (hence the name preshower) and also as tracking detectors. Due to their fast energy and position measurements, the preshower information is included in the Level 1 trigger. The central preshower detectors (CPS) are located in between the solenoid and the central calorimeter and cover the region $|\eta|<1.3$, while the forward preshower detector (FPS), attached to the inner faces of the end-cap calorimeter cryostats cover a pseudorapidity range of $1.5<|\eta|<2.5$. Both detectors (CPS and FPS) are made from triangular strips of scintillator, which are interleaved to avoid dead regions. Depending on the region where the strips are being employed (central/forward), the layout geometry as shown in Figure 2.26 differs slightly.

(a)
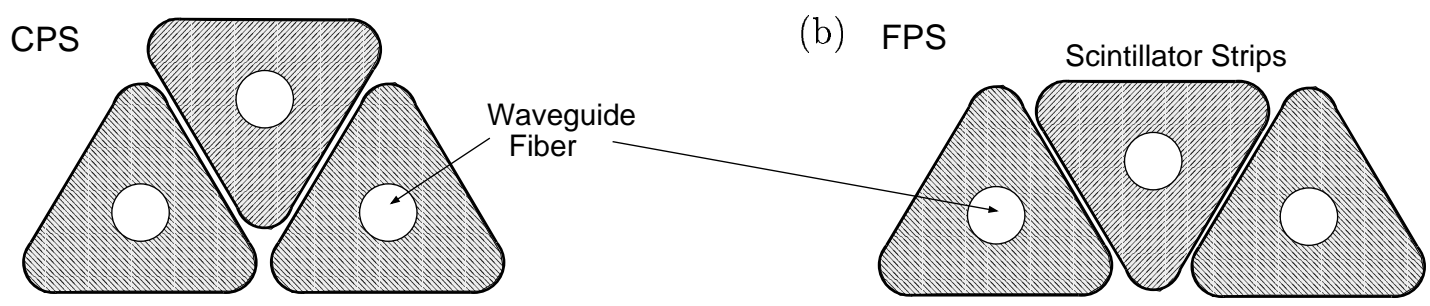

Figure 2.26: Layout geometry of the CPS and FPS triangular scintillating strips: (a) in the CPS employed in the central region and (b) in the FPS for the forward detector regions.

The preshower detectors and the CFT share the same readout technology via VLPCs. Already the CFT alone requires 76,800 individual channels of readout, which, combined with another 22,564 channels for the central and forward preshower detectors, amounts to roughly 100,000 VLPC readout channels. The design or functionality of the CPS/FPS is not further described here, since both detectors have not yet been in use for most of the data-taking period considered in this work. A more detailed description can be found in [102] for the CPS and in [103] for the FPS detectors. 


\subsubsection{The Liquid Argon Calorimeter}

The $\mathrm{D} \varnothing$ calorimeter was designed to provide precise energy measurements of electrons, photons and hadronic jets and to aid in their respective identification. It also allows the measurement of the transverse energy imbalance in events and can detect muons as minimum ionizing particles (MIPs).

The calorimeter is a nearly compensating sampling calorimeter with uranium absorbers immersed in liquid argon, which serves as the active medium. It is not fully compensating, but the $e / \pi$ response ratio is close to one, ranging from about 1.11 at $10 \mathrm{GeV}$ to about 1.04 at $150 \mathrm{GeV}$. The calorimeter is divided into three parts: the barrel, or central calorimeter (CC) covers the region up to $|\eta| \lesssim 1$ and two end calorimeters (EC) in the forward regions at the north and south ends of the detector (ECN/ECS) extend the coverage up to pseudorapidities of $|\eta| \approx 4$. Each calorimeter part is enclosed by a separate cryogenic vacuum vessel as can be seen from Figure 2.27. In order

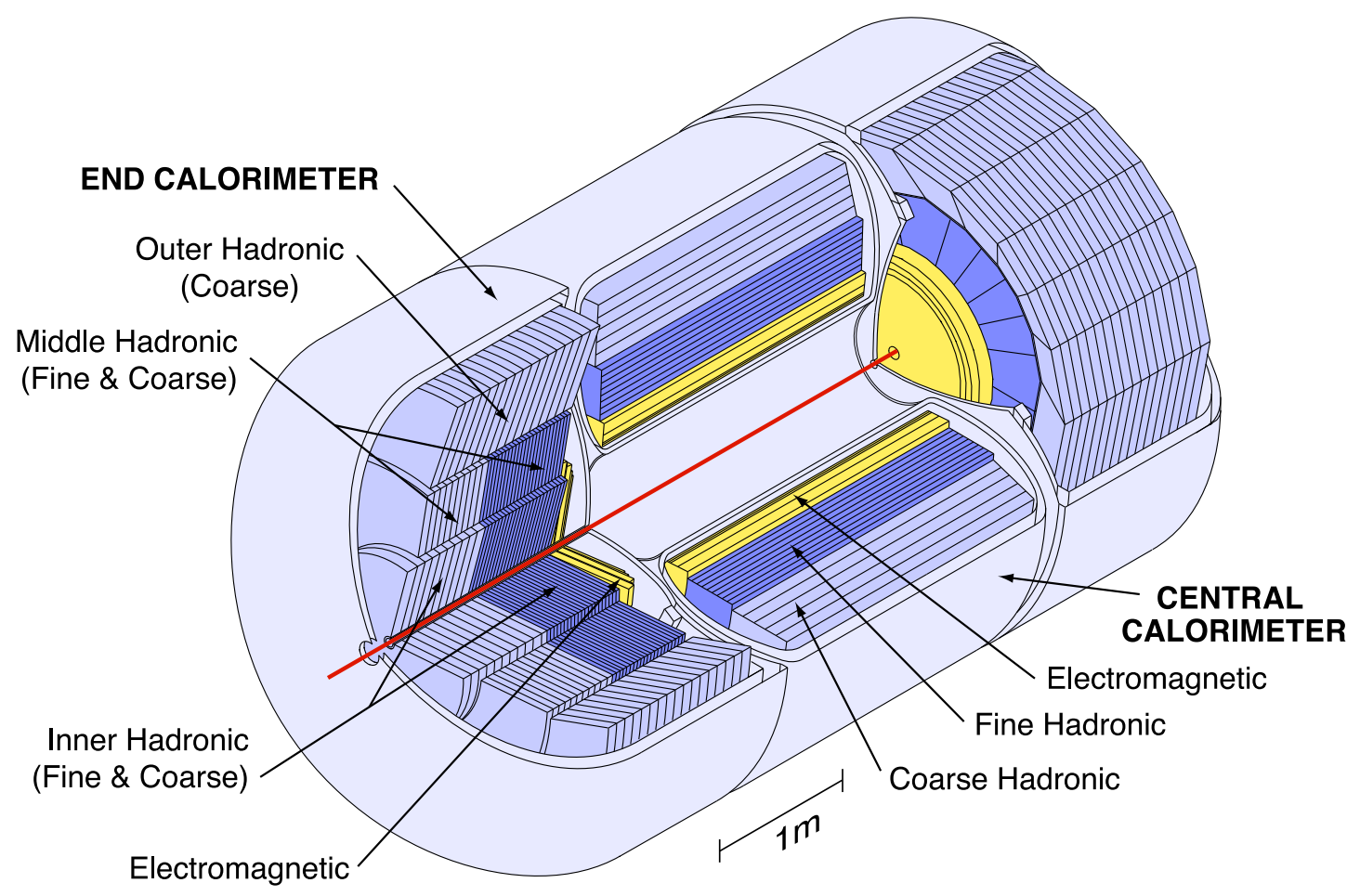

Figure 2.27: Isometric view of the central and the two endcap calorimeters. Each calorimeter is contained in its own cryostat, which maintains the temperature at approximately $90^{\circ} \mathrm{K}$.

to provide hermetic coverage over the large range of pseudorapidities and because of the separate vacuum vessels for the three calorimeters a fourth component, the intercryostat detector (ICD) has been installed, located in between the central and end-cap cryostats. The ICD will be briefly described after the liquid argon calorimeters.

Each calorimeter is divided into three different sections, the electromagnetic calorimeter, the fine hadronic and the coarse hadronic calorimeter. Closest to the interaction point is the finely segmented electromagnetic section, followed by the fine and then the coarse hadronic section. While all three calorimeters use liquid argon as active medium, different absorber plates (type and thickness) are used. For the electromagnetic section thin plates (3 to $4 \mathrm{~mm}$ ) of nearly pure depleted uranium are used, whereas the fine hadronic sections contain $6 \mathrm{~mm}$ thick plates made 
of uranium-niobium (2\%) alloy. The coarse hadronic section do not contain any uranium, but use instead thick $(46.5 \mathrm{~mm})$ plates of copper (CC region) or stainless steel (EC region).

All three calorimeters function in the same way. Particles traversing the calorimeter loose their energy gradually by interacting with the different materials (showering). Electrons, photons, and, in part, tau leptons produce electromagnetic showers, which are usually confined to a few layers in the first, electromagnetic section of the calorimeter. Hadronic particles interact primarily with the nuclei of the absorber materials and produce large hadronic showers, called (hadronic) jets. Either shower leads to an ionisation of the liquid argon. To collect the resulting charge, the electrons (ions are usually too slow to be considered) are drifted towards copper plates. The necessary electric field in the liquid argon gaps between the absorber plates is established by grounding the latter and connecting the resistive surfaces of the charge collecting signal boards to a high positive voltage (typically $\approx 2 \mathrm{kV}$ ). As a result, the electrons drift in about $450 \mathrm{~ns}$ across the $2.3 \mathrm{~mm}$ gap. A simple schematic view of a calorimeter unit cell is given in Figure 2.28.

Figure 2.28: Illustration of a calorimeter unit cell. Electrons drift in an electric field across the liquid argon gaps to be collected at the signal boards. The dimensions are given in $\mathrm{mm}$ and are typical for the electromagnetic section of the calorimeter; the absorber plates are thicker in the fine and coarse hadronic sections.

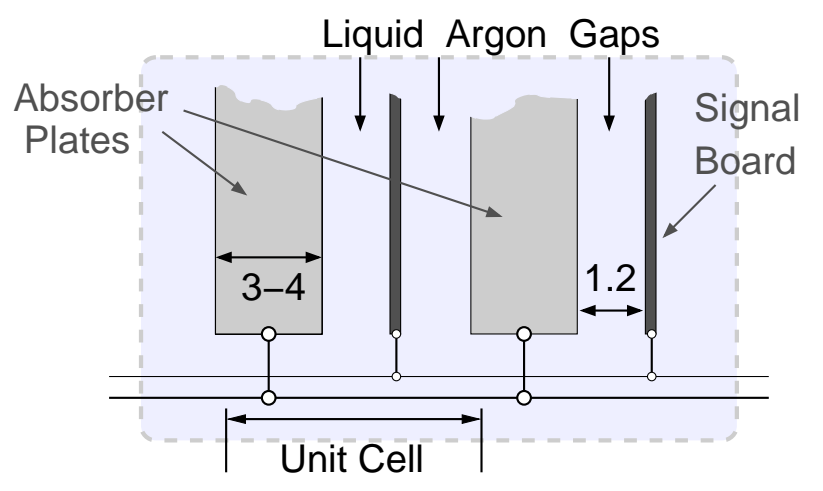

Several calorimeter cells at approximately the same $\eta$ and $\varphi$ are ganged together in depth to form a single readout cell. These readout cells form semi-projective towers, whose imaginary tips would meet at the nominal interaction point in the geometric center of the detector at $r=z=0$, see also Figure 2.29.

The tower dimensions roughly correspond to $\Delta \eta \times \Delta \varphi=0.1 \times 0.1$, which reflects the transverse sizes of particle showers. Electromagnetic showers are about 1-2 cm wide in the transverse plane, while hadronic showers are mostly a factor of 10 wider, i.e. about $10-20 \mathrm{~cm}$. In the electromagnetic section there are four separate layers (EM1-4) of different thickness, corresponding to 1.4, 2.0, 6.8 , and $9.8 X_{0}$ in the central region and to 1.6, 2.6, 7.9, and $9.3 X_{0}$ in the forward region. Detector components in front of the calorimeters as seen from the interaction region (SMT, CFT, the solenoidal magnet and the preshower detectors) account for another approximately $4.0 X_{0}$ in the central region for particles at normal incidence $(|\eta|=0)$, and for slightly more, $4.4 X_{0}$, in the EC region at $|\eta|=2$. The third electromagnetic layer is segmented twice as fine as the other layers, because electromagnetic showers, originating from particles of intermediate energies $(\approx 30-100 \mathrm{GeV})$ develop their maximum there.

The fine hadronic section of the central calorimeter is subdivided into three layers (FH1-3), which are $1.3,1.0$, and $0.8 \lambda_{A}$ thick, where $\lambda_{A}$ is the hadronic interaction length. The coarse hadronic section consists of a single module $(\mathrm{CH})$ of roughly $3.2 \lambda_{A}$. Although the fine (coarse) hadronic calorimeters in the forward regions are subdivided into two (three) cylindrical modules, the general layout is the same as for the CC hadronic sections. Similarly to these, the fine 


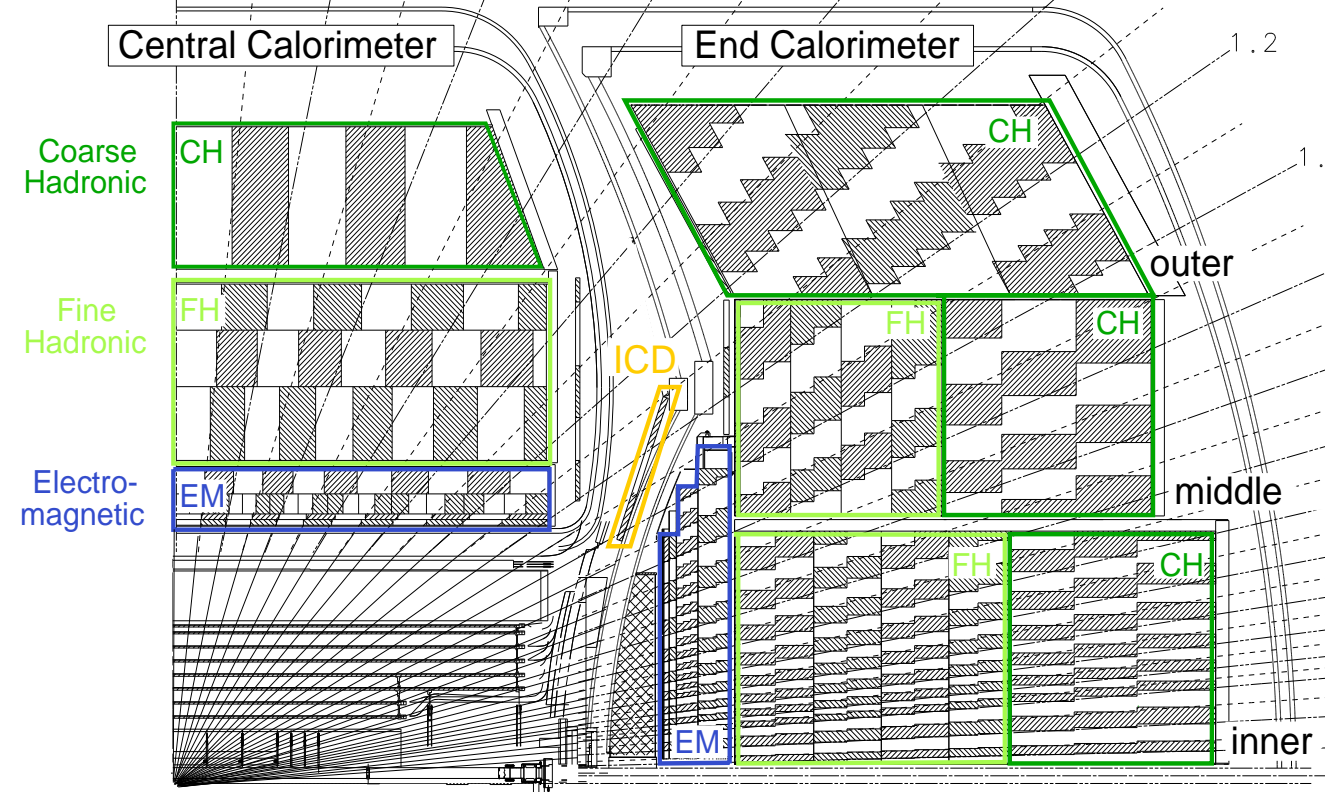

Figure 2.29: Schematic illustration of one quadrant of the calorimeters showing the semi-projective tower geometry. The shading indicates which groups of cells are ganged together for signal readout.

hadronic end calorimeters are divided into 4 layers of approximately $1.0 \lambda_{A}$ and the coarse hadronic part consists of a single readout cell of about $4.0 \lambda_{A}$. At $\eta=0$ (CC region), the hadronic and electromagnetic layers of the calorimeter account for a total hadronic interaction length of $7.2 \lambda_{A}$, while it is $10.3 \lambda_{A}$ at the smallest angle in the EC region. Table 2.2 provides an overview of the dimension and thickness of the different calorimeter sections.

\begin{tabular}{lclccc}
\hline \hline Layer & $d_{C C} / d_{E C}$ & Layer & $d_{C C} / d_{E C}$ & Layer & $d_{C C} / d_{E C}$ \\
\hline EM1 & $1.4 / 1.6 X_{0}$ & FH1 & $1.3 / 1.1 \lambda_{A}$ & & - \\
EM2 & $2.0 / 2.6 X_{0}$ & FH2 & $1.0 / 1.1 \lambda_{A}$ & & - \\
EM3 & $6.8 / 7.9 X_{0}$ & FH3 & $0.8 / 1.1 \lambda_{A}$ & & - \\
EM4 & $9.8 / 9.3 X_{0}$ & FH4 & $-/ 1.1 \lambda_{A}$ & & - \\
EM (all) & $\mathbf{2 0 . 0} / \mathbf{2 1 . 4} \boldsymbol{X}_{\mathbf{0}}$ & FH (all) & $\mathbf{3 . 1} / \mathbf{4 . 4} \boldsymbol{\lambda}_{\boldsymbol{A}}$ & $\mathbf{C H}$ & $\mathbf{3 . 2} / \mathbf{4 . 1} \boldsymbol{\lambda}_{\boldsymbol{A}}$ \\
\hline \hline
\end{tabular}

Table 2.2: Overview of dimensions and thickness of the different calorimeter layers in the central region $(C C)$ at $\eta=0$ and in the forward region $(E C)$ at $\eta \approx 2$. Electromagnetic, as well as hadronic readout cells (calorimeter towers) are segmented in $\Delta \eta \times \Delta \varphi=0.1 \times 0.1$, with the exception of the third electromagnetic layer $(0.05 \times 0.05)$, and the very forward region of $|\eta|>3.2$ with a coarser granularity.

The calorimeters themselves have not been changed since Run I, so that the most accurate description can still be found in [79]. However, the complete readout electronics have been exchanged to maintain the good signal-to-noise ratio from Run I, in a much more challenging environment with higher luminosity and a much higher bunch crossing rate; the frequency is a factor of 3.8 higher compared to Run I. In order to achieve this goal, two entirely new readout components have been installed for Run II: charge preamplifiers on top of the cryostats and baseline subtractor (BLS) boards below, where access is significantly easier. In a first stage the 
signals from single calorimeter cells are transported to a feedthrough port, which is the interface between the cold and warm region of the calorimeter cryostats, and on to the charge preamplifiers located as close as possible to the feedthrough ports on top of the vacuum vessels. From there, the amplified analog signals are transported to the BLS boards below for signal shaping, removal of low frequency noise or pile-up, and storage. Finally, if the Level 1 trigger decision is made after about $4 \mu \mathrm{s}$ and the event is accepted, the analog precision signals from the BLS boards are transmitted to analog/digital converters and from there they enter the data acquisition system, so that the precision readout from the calorimeter can be used for the Level 3 trigger decision. In addition, the trigger makes use of faster shaped analog sums of the calorimeter signals, so-called trigger towers of $\Delta \eta \times \Delta \varphi=0.2 \times 0.2$, which are picked off to provide inputs to the trigger Levels 1 and 2. Coarse hadronic sections do not contribute to any trigger decisions.

The energy resolution of the calorimeters (electromagnetic and hadronic) can be parametrised as follows:

$$
\left(\frac{\Delta E}{E}\right)^{2}=\frac{N^{2}}{(E / \mathrm{GeV})^{2}}+\frac{S^{2}}{E / \mathrm{GeV}}+C^{2}
$$

The first term $\Delta E / \mathrm{GeV}=N$ describes a fixed contribution, attributable to electronic noise and independent of the energy of the incident particle, the second term $\Delta E / \mathrm{GeV}=S \cdot \sqrt{E / \mathrm{GeV}}$ is of statistical origin and describes fluctuations in the energy depositions and the third term, finally, $\Delta E / \mathrm{GeV}=C \cdot(E / \mathrm{GeV})$ is due to systematic uncertainties in the energy calibration of the calorimeters. The first term dominates at extremely low energies, where the particle energy is close to the invariably present electronic noise of a few hundred $\mathrm{MeV}$ and the last, systematic term becomes dominant at high energies, e.g. in the electromagnetic calorimeter for particles of roughly $E_{\mathrm{T}} \approx 40 \mathrm{GeV}$. The different parametrisation constants have been measured separately for the electromagnetic and hadronic sections, resulting in:

\begin{tabular}{lcc}
\hline \hline $\begin{array}{l}\text { Incident particle } \\
\text { of energy } E\end{array}$ & Electromagnetic $\Delta E$ & Hadronic $\Delta E$ \\
\hline $50 \mathrm{GeV}$ & $N=0.2, S=0.23, C=0.004$ & $N=5.0, \quad S=1.0, C=0.1$ \\
$100 \mathrm{GeV}$ & $\Delta E=2.6 \mathrm{GeV}(5.2 \%)$ & $\Delta E=10.0 \mathrm{GeV}(20 \%)$ \\
\hline \hline
\end{tabular}

Table 2.3: Overview of the different resolution parameters in the electromagnetic and hadronic sections of the calorimeter. All parameters are taken from [104].

\section{Intercryostat Detector: ICD}

The location of the ICD with respect to the liquid argon calorimeters can be seen in Figure 2.29. It is attached to the exterior surfaces of the two EC cryostats and provides scintillator sampling in the region $1.1<|\eta|<1.4$. A series of scintillating tiles enclosed in light-tight aluminum boxes is used as active material. Each tile is about $1.3 \mathrm{~cm}$ thick and divided into 12 subtiles, of which each covers an area of approximately $\Delta \eta \times \Delta \varphi=0.1 \times 0.1$ as the cells of the liquid argon calorimeters.

The subtiles are read out via wavelength shifting fibers, which carry the signals to photomultiplier tubes housed in special electronics »drawers « below the ICD and the calorimeter cryostats, in an area where the residual magnetic field is low. 


\subsubsection{The Muon System}

The muon system is divided into separate systems, the central system based on proportional drift tubes (PDTs) covers a pseudorapidity region up to $|\eta| \leq 1$ and the forward system, which uses mini drift tubes (MDTs) extends the coverage up to $|\eta| \leq 2$. The complete Run I forward muon system [79] was removed during the five year shutdown before Run II and replaced by the new MDTs and trigger scintillation counters. For certification studies of the muon resolution, comparing data with simulated Monte Carlo data, see reference [105].

\section{The Toroidal Magnets}

One very prominent feature of the $\mathrm{D} \emptyset$ muon system are the toroidal magnets, which account for about $65 \%$ of the total weight $(\approx 5000$ tons $)$ of the detector. They were already present during Run I, allowing for a good momentum measurement in the muon system, since this was not possible with the central tracking detectors due to the lack of a magnetic field (the solenoid was installed between Run I and Run II). The toroidal magnets, illustrated in Figure 2.30 (Fig. 2.20), are divided into a central, $109 \mathrm{~cm}$ thick, square »annulus « and two end toroids, which are $\approx 156 \mathrm{~cm}$ thick plates with a $183 \mathrm{~cm}$ square hole in the middle centered about the beam pipe. The inner surface of the central toroid is at a distance of $318 \mathrm{~cm}$ from the beam line.

(a)

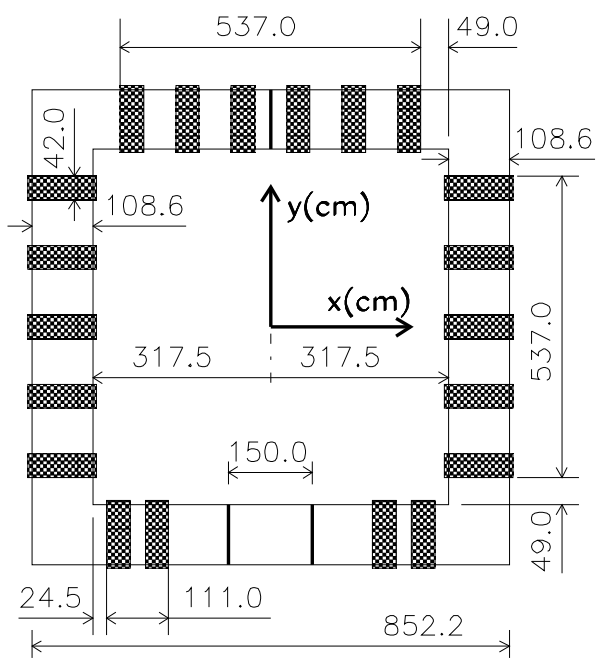

(b)
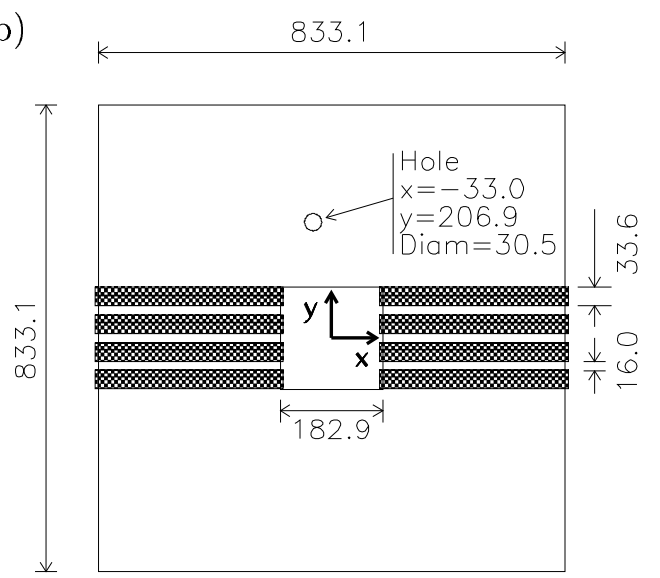

Figure 2.30: Technical'drawing of the toroidal magnets. (a) The central toroid, build from the two $C$-shaped pieces and the central beam, indicated by the thick lines, uses twenty coils of ten turns, five coils on either side, four at the bottom and six at the top. (b) One of the two end toroids with eight winding of eight turns each (four on either side). The hole in the upper part of the end toroids is a remainder of the Run I accelerator bypass of the former »Main Ring».

It is constructed from three parts: one center-bottom section, a $150 \mathrm{~cm}$ wide (and about $7.6 \mathrm{~m}$ long) beam, fixed to the detector platform, provides a base for the calorimeter vessels and the central tracking system, and two C-shaped sections, movable perpendicular to the center beam, facilitate the access to the inner detector parts (Fig. 2.30). The two end toroids are located at about $4.5 \mathrm{~m} \leq|z| \leq 6.1 \mathrm{~m}$ from the center of the detector. Both magnet coils are operated in series, at a current of $1500 \mathrm{~A}$, which is about $40 \%$ lower than during Run I, but still enables a stand-alone momentum measurement. The primary muon momentum measurement is now done with the central tracking system of the SMT and CFT detectors. While the momentum 
measurement of the muon system still improves the resolution for high momentum muons $\left(p_{\mathrm{T}}>150 \mathrm{GeV}\right)$, the lower operating current and magnetic field in the toroids allow for a low- $p_{\mathrm{T}}$ cutoff in the Level 1 muon triggers and a cleaner matching of muon track segments with the inner tracks measured with the tracking detectors. The polarity of the toroids is reversed regularly (approximately every two weeks) between stores to avoid a bias. Similarly, the polarity of the solenoid in the central tracking system is also alternated periodically, ensuring that the detector takes approximately the same amount of data in each of the four possible configurations of the magnetic field.

\section{The Proprotional Drift Tubes in the Central Region}

The central region PDTs are arranged in three layers: layer A is located inside and layers B and $\mathrm{C}$ outside of the toroids. The B/C-layer PDTs and the bottom A-layer PDTs consist of three planes of drift cells, while the remaining A-layer PDTs are made of four planes. Due to an uninstrumented region in the bottom part of the detector, only about $55 \%$ of the central region is covered by three layers of PDTs, but close to $90 \%$ are covered by at least two layers.

The drift tubes are made of rectangular aluminum tubes and are quite large, compared to the MDTs, typical sizes are about $2.8 \times 5.6 \mathrm{~m}^{2}$. A drift chamber is usually 24 cells wide and contains either 72 or 96 cells, with each cell being $10.1 \mathrm{~cm}$ wide. At the center of each cell is the anode wire, which, together with Vernier cathode pads located above and below it, provides information on the hit position along the wire. The PDT chambers are mounted such that the anode wires are oriented along the primary B-field direction to enable accurate measurements of the bend coordinate. Two wires within a plane are always ganged

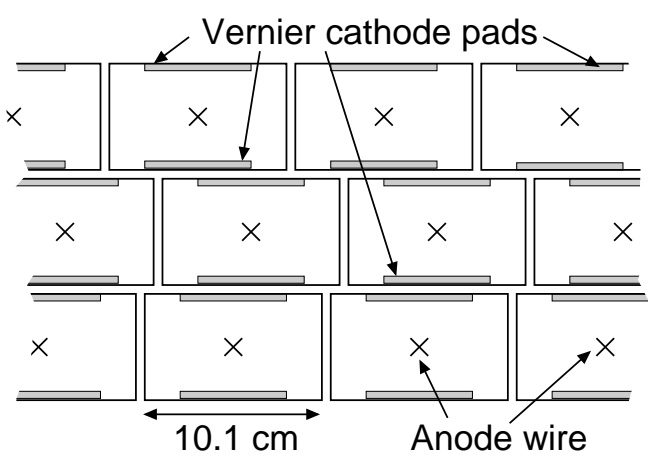

Figure 2.31: Schematic illustration of the cell configuration in a PDT chamber. together at one end of each drift chamber and are read out at the opposite end. As can be seen from Figure 2.31 the typical three-plane PDTs are setup with a relative transverse offset between the planes, so that the left-right drift-time ambiguity can be resolved.

The drift gas used in the central muon wire chambers is $\mathrm{Ar}-\mathrm{CH}_{4}-\mathrm{CF}_{4}$ with a ratio of $84 \%$ argon (Ar), $8 \%$ methane $\left(\mathrm{CH}_{4}\right)$, and $8 \%$ tetrafluoride $\left(\mathrm{CF}_{4}\right)$ and the operating high voltage is $2.3 \mathrm{kV}$ for the cathode pads and $4.7 \mathrm{kV}$ for the wires. This configuration leads to a drift velocity of approximately $10 \mathrm{~cm} / \mu \mathrm{s}$ over a maximum drift time of about $500 \mathrm{~ns}$. For filtering and removal of contaminants the gas is circulated through the drift chambers of the muon system at a rate of 2 liters per minute, corresponding to an average exchange rate of roughly 3 volumes per day.

For each hit in the PDTs, the electron drift time, the charge deposition on the Vernier pads, and the difference in the arrival times $(\Delta T)$ of the signal pulses on two of the combined wires are recorded. The hit position along the wire is determined from a combination of the charge deposition and the arrival time difference of the signal pulses. Using the method of charge division, the pad signal resolution is about $5 \mathrm{~mm}$, and the drift distance can be determined with a resolution of approximately $1 \mathrm{~mm}$. The resolution of the arrival time measurement $\Delta T$ largely depends on where the muon passes through the drift cell, since the accuracy of the measurement degrades with increasing signal propagation delay. If the muon passes on the far side of the 
readout electronics, the resolution is about $10 \mathrm{~cm}$. Since both signal pulses travel roughly equal distances (about one wire length) and arrive at about the same time, the resulting time difference measurement is accurate. On the other hand, if the muon passes the cell on the near side (w.r.t. the readout), one signal pulse arrives almost instantly and the other has to travel two wire lengths. Due to this longer propagation time, the dispersion in the signal is increased and thus leads to a degradation of the resolution, which is only about $50 \mathrm{~cm}$ in this case.

(a)

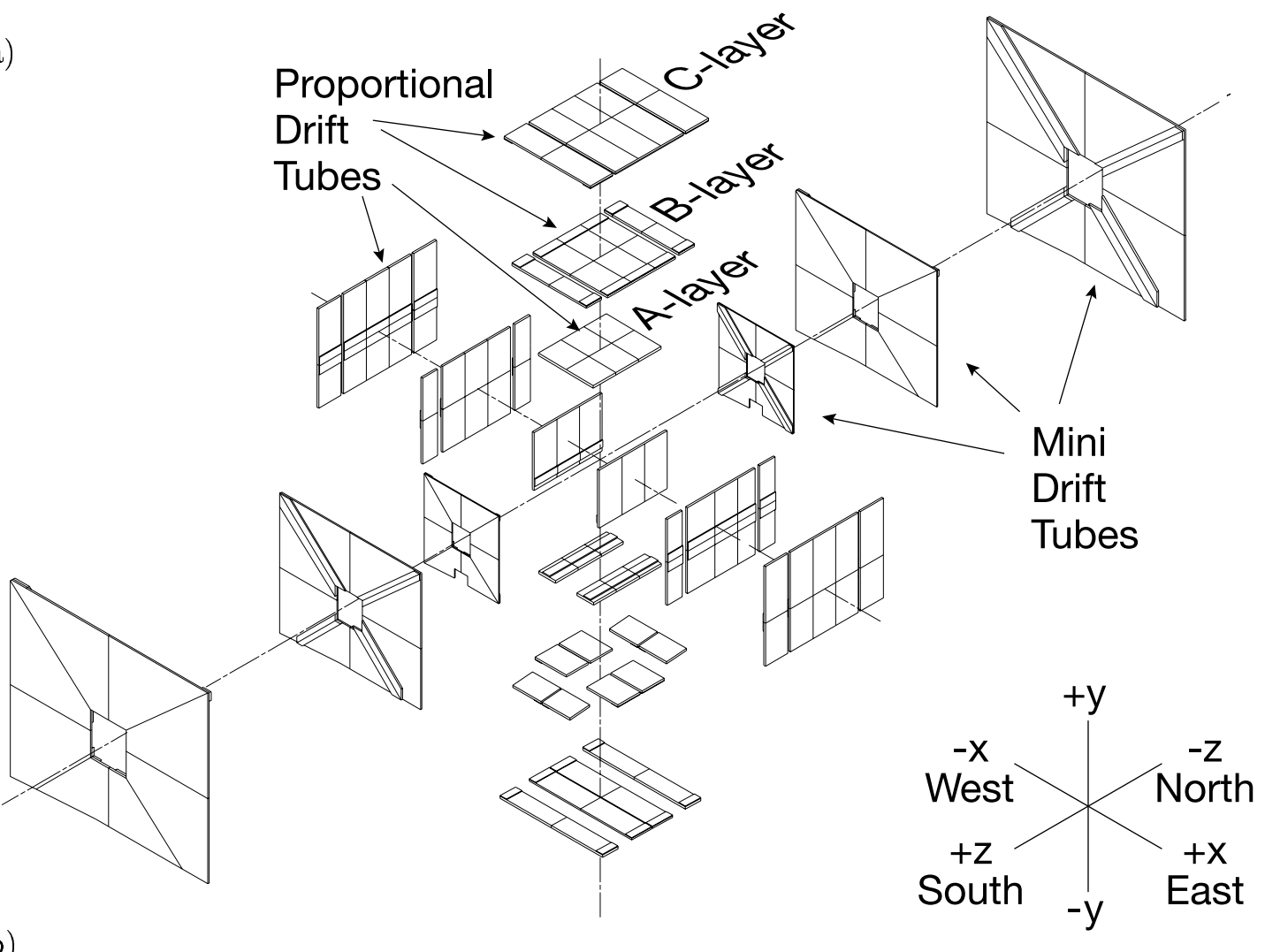

(b)

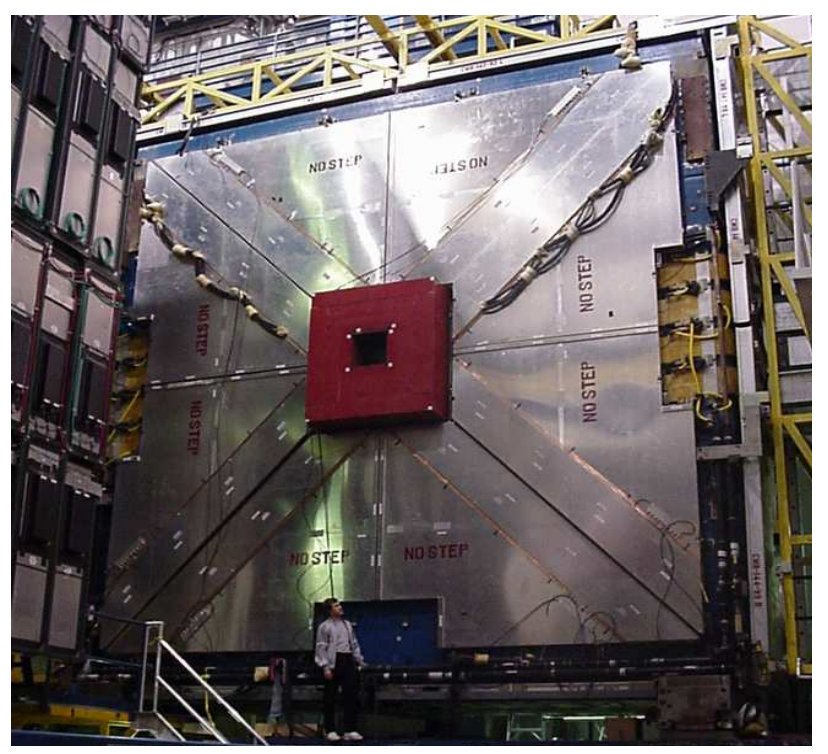

(c)

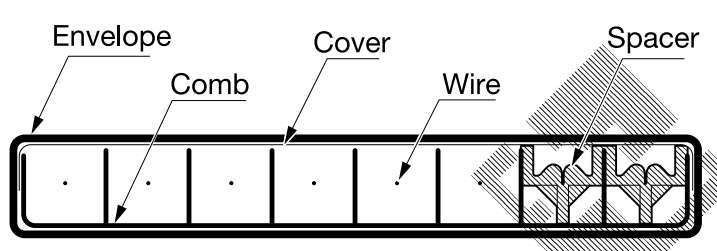

Figure 2.32: (a) Exploded view of the muon wire chambers: the central proportional drift tubes (PDTs) and the forward mini drift tubes (MDTs). (b) The photograph of the Alayer MDTs was taken right before $»$ roll-in $«$ (from [106]). Clearly visible are the eight MDT octants of the A-layer. (c) Schematic illustration of a single $M D T$ tube.

An exploded view of the muon wire chambers, central PDTs and forward MDTs, can be found in Figure 2.32 (a). The wire chambers are used for precision measurements of the coordinates 
and, in part, for triggering, while the scintillation counters are mainly used for triggering. Both detectors contribute to the background rejection. The wire chambers allow to reduce the background from random hits due to hot wires or even single hot chambers by measuring track segments over two or three layers of the muon system and the scintillation detectors provide timing information for the reduction of out-of-time background like cosmic muons.

\section{The Mini Drift Tubes in the Forward Regions}

The mini drift tubes (MDTs) in the forward region are a major part of the Run II detector upgrade. They were chosen to replace the Run I small angle muon system (SAMUS), since their electron drift time is much smaller, keeping in mind the higher bunch crossing rate in Run II. Nevertheless, the MDTs still provide a good coordinate resolution. Additionally, they are more radiation hard, which is related to the utilized material, but in part also due to the finer segmentation and thus lower occupancy.

Similarly to the PDTs, the MDTs are arranged in three layers (A, B, and C), where layer A is again inside the end toroids and layers $\mathrm{B}$ and $\mathrm{C}$ are on the outside. As can be seen from Figure $2.32(\mathrm{a}, \mathrm{b})$, each layer is divided into octants and made up of three planes (B/C-layers) or four planes (A-layer). A single mini drift tube is made of extruded aluminum combs and consists of eight cells, covering an area of $9.4 \times 9.4 \mathrm{~mm}^{2}$, see Figure 2.32 (c) for details. In contrast to the rather slow drift gas mixture used in the PDT system, the MDT system uses tetrafluoromethane $\left(\mathrm{CF}_{4}-\mathrm{CH}_{4}\right.$, with $90 \%$ tetrafluor and $10 \%$ methane). This mixture is fast, non-flammable and exhibits no radiation aging, which is very important in the high radiation forward regions. As a result, the drift time for tracks incident at $90^{\circ}$ is at most $40 \mathrm{~ns}$ and for tracks inclined at $45^{\circ}$ it is about $60 \mathrm{~ns}$. The operating high voltage scheme used for the MDTs is also different from the one for the PDTs, a negative high voltage of $-3.2 \mathrm{kV}$ is applied to the cathode, while the anode wires are grounded at the amplifier to which they are connected.

The limiting factor in the coordinate resolution of the MDTs is the measurement of the signal arrival time, which is measured with an accuracy of $18.8 \mathrm{~ns}$ w.r.t. the beam crossing. This is due to the fact that each amplifier-discriminator board contains 32 channels and outputs logical differential signals that are send to the digitising electronics. This solution was chosen to reduce the cost of the otherwise large number of about 50,000 time-to-digital converters. Drift times are also recorded for the MDT system, although, contrary to the arrival times, they are not used for triggering, but serve only as input to the data acquisition system (section 2.4).

Multiple scattering in the toroids represents the limiting factor for the momentum resolution of the forward muon spectrometer. The digitisation in time intervals of $18.8 \mathrm{~ns}$ leads to a factor of two worse coordinate resolution of the MDT system $(\approx 0.7 \mathrm{~mm}$ per hit $)$, compared to the resolution achieved in test beam measurements. This translates into an overall momentum resolution of approximately $20 \%$ for muon momenta below $40 \mathrm{GeV}$ and using the forward muon system in a stand-alone mode. The resolution for particle momenta up to $100 \mathrm{GeV}$ is determined by the central tracking detectors, but for higher momenta the muon system can improve the momentum resolution. Particularly important is the forward muon spectrometer for tracks at pseudorapidities $|\eta|>1.6$, since these will not traverse all layers of the central fiber tracker anymore. Particle momenta of about $50 \mathrm{GeV}$ are measured with an accuracy of $20 \%$ (central region), for those in $1.6<|\eta|<2.0$ the resolution is approximately $35 \%$. 


\section{Central Region Scintillators: Cosmic Cap/Bottom and A- $\boldsymbol{\varphi}$ Counters}

The central scintillation counters are installed on the top, sides and bottom of the central Clayer PDTs (called cosmic cap and bottom counters) and on the inside of the central A-layer (called A- $\varphi$ counters). In Figure 2.33 (a-c) an exploded view of the scintillation counters and two photographs of the central and forward C-layer muon scintillation detectors are provided, similarly as for the wire chambers in Fig. 2.32. All scintillation counters are mainly used for triggering purposes and to discriminate against cosmic ray background or backscatter from the forward regions.

(a)

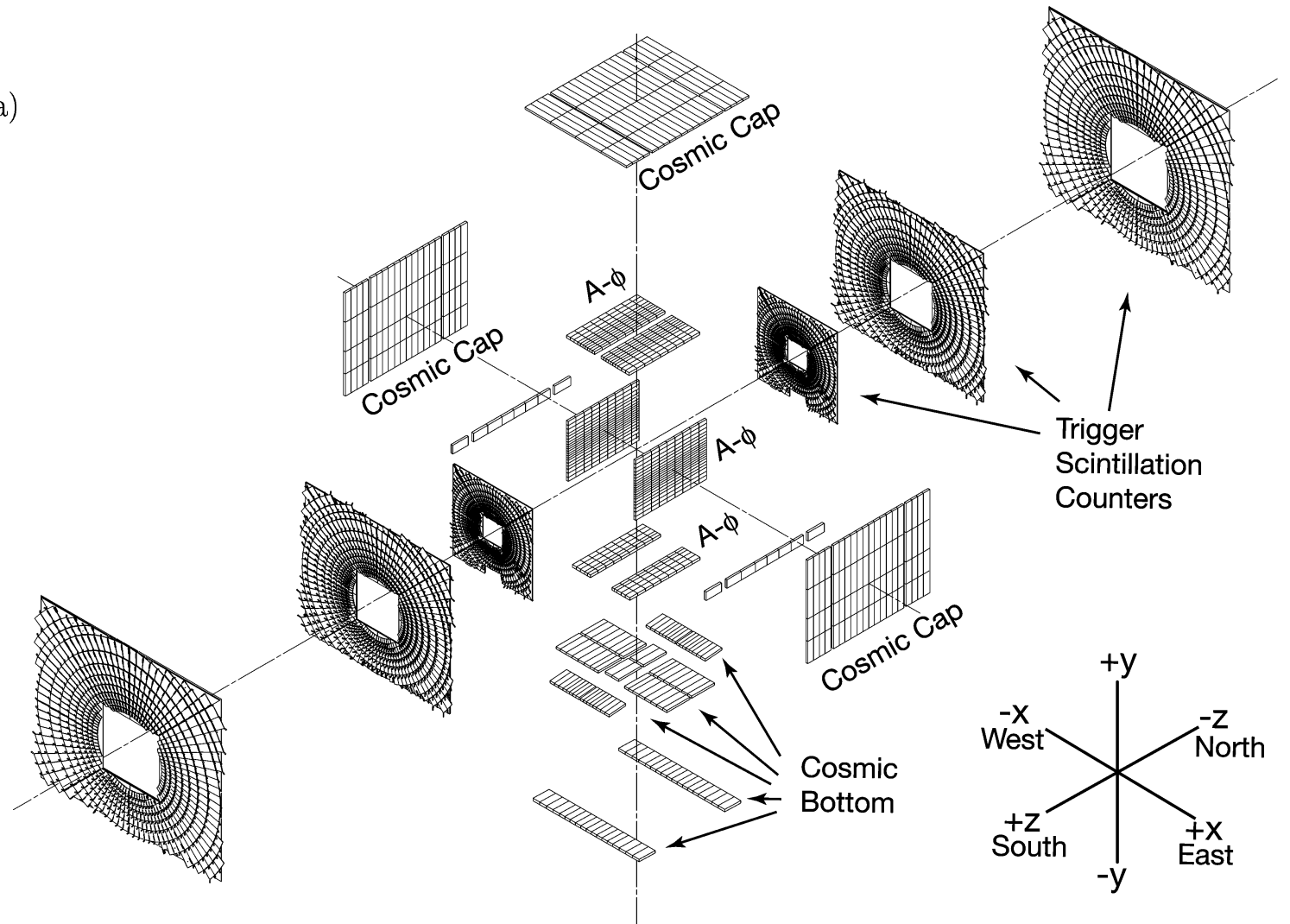

(b)

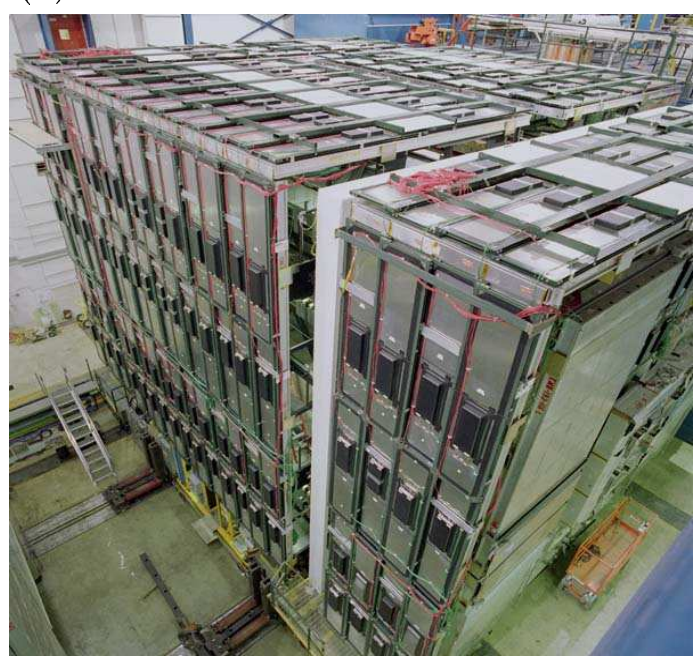

(c)

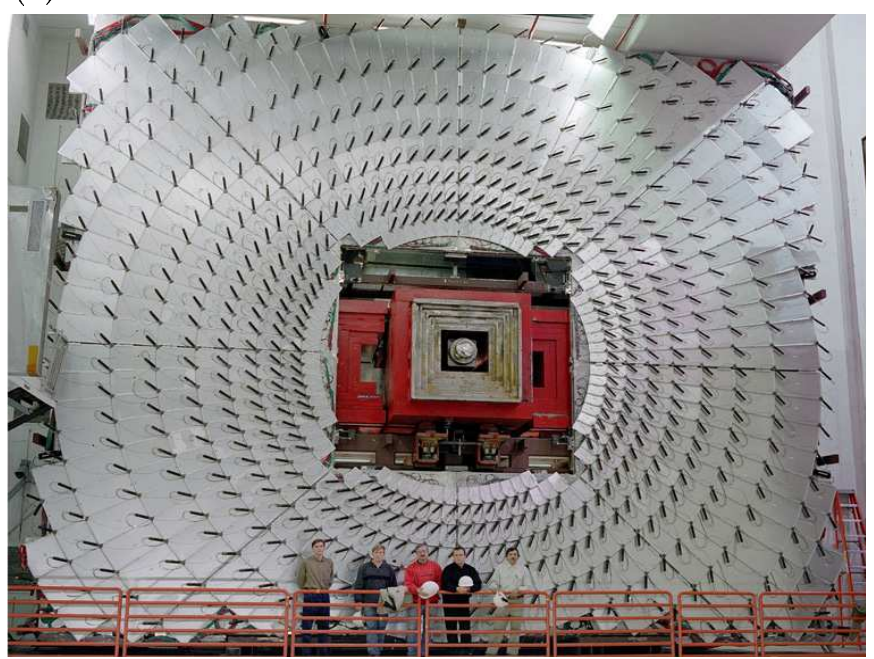

Figure 2.33: (a) Exploded view of the muon scintillation detectors: the central top, cosmic cap and bottom counters, and the A-layer A- $\varphi$ counters, as well as the forward counters. (b) Central C-layer and (c) forward muon scintillation counters photographed with the detector in rolled out position. 
Although all scintillation counters are made from Bicron 404A scintillator and are $1.3 \mathrm{~cm}$ thick, their overall design depends on the location. The 240 central cosmic cap and 132 cosmic bottom counters are rather similar rectangular counters, $63.5 \mathrm{~cm}$ wide and in between $207 \mathrm{~cm}$ and $287 \mathrm{~cm}$ long. The cosmic cap counters, at the top and the sides of the $\mathrm{D} \varnothing$ detector are positioned with their width along the $z$ direction and their length along $\varphi$. The wavelength shifting fibers glued to the scintillators are read out via two 10 or 12-stage photomultiplier tubes (PMTs) mounted directly on the counters. The light yield varies depending on the distance from the phototubes and on the location of the hit w.r.t. the scintillator area. For hits near the PMTs, typically 30 photoelectrons are produced per phototube and for hits close to the distant corners this number drops to about 18 photoelectrons per PMT.

Apart from minor improvements in the placement and arrangement of the wavelength shifting fibers, the cosmic bottom counters differ only in one aspect from the cosmic cap counters. Contrary to the cap counters, the bottom counters are positioned with their width along $\varphi$ and their length along the $z$ direction. This orientation leads to a better matching in the $\varphi$ corrdinate for track segments measured with the muon system and tracks measured with the central tracking system, especially the CFT trigger. The counters are about as long as their respective PDTs are wide and the counter width is approximately $4.5^{\circ}$ in $\varphi$ to match the CFT trigger segmentation. The nearly constant width in $\varphi$ is achieved by using three different widths of scintillators: 40.0 , 47.0 , and $56.8 \mathrm{~cm}$, where the narrowest counters are placed at the center of the detector sides and the widest at the corners, respectively. Although Figure 2.34 actually shows an end view of the A- $\varphi$ counter arrangement, the general layout of the differently sized scintillators also applies to the cosmic cap and bottom counters.

The 630 A- $\varphi$ counters, placed on the inside of the A-layer PDTs, are the scintillation counters closest to the interaction region (directly after the calorimeter) and thus provide a very good possibility to trigger on, identify muons, and to reject out-of-time backscatter. For high- $p_{\mathrm{T}}$ single muon triggers and low- $p_{\mathrm{T}}$ dimuon trigger, the in-time hits on the scintillation counters are matched with tracks measured in the CFT on trigger Level 1 already. The A- $\varphi$ counters are also the only detector element to provide a precise time stamp for low- $p_{\mathrm{T}}$ muons, that cannot penetrate the iron toroids and thus do not reach the B or C-layer counters.

As can be seen from Figure 2.34, the segmentation of the A- $\varphi$ counters in $\varphi$ is nearly constant with each scintillator covering about $4.5^{\circ}$ in $\varphi$ similar to the cosmic cap and bottom counters. This segmentation was again chosen to match the CFT trigger segmentation and is implemented by usage of three differently sized scintillators of $23.1,27.5$, and $36.7 \mathrm{~cm}$ width. The are placed analogously to the C-layer cosmic cap and bottom counters, i.e. from the narrowest at the center towards the widest at the corners of each detector side.

Since the A- $\varphi$ counters, which are the innermost scintillation counters of the muon system, have to operate in a residual magnetic field of about $20-35 \mathrm{mT}$, due to the toroidal and solenoidal magnets, they are enclosed in magnetic shields. These shields have a diameter of $48 \mathrm{~mm}$ and are made of 1.2 -mm-thick $\mu$-metal ${ }^{10}$ and 6 -mm-thick soft iron ${ }^{11}$. The shields reduce the perturbing effects of the residual magnetic field on the phototube gain to less than $10 \%$.

\footnotetext{
${ }^{10}$ Nickel-iron alloy (77\% nickel, $15 \%$ iron, plus copper and molybdenum) with a high magnetic permeability, $\mu$. The material is very effective at screening static or low-frequency magnetic fields.

${ }^{11}$ Soft iron loses its magnetism as soon as the current stops; it is said to form a temporary magnet.
} 


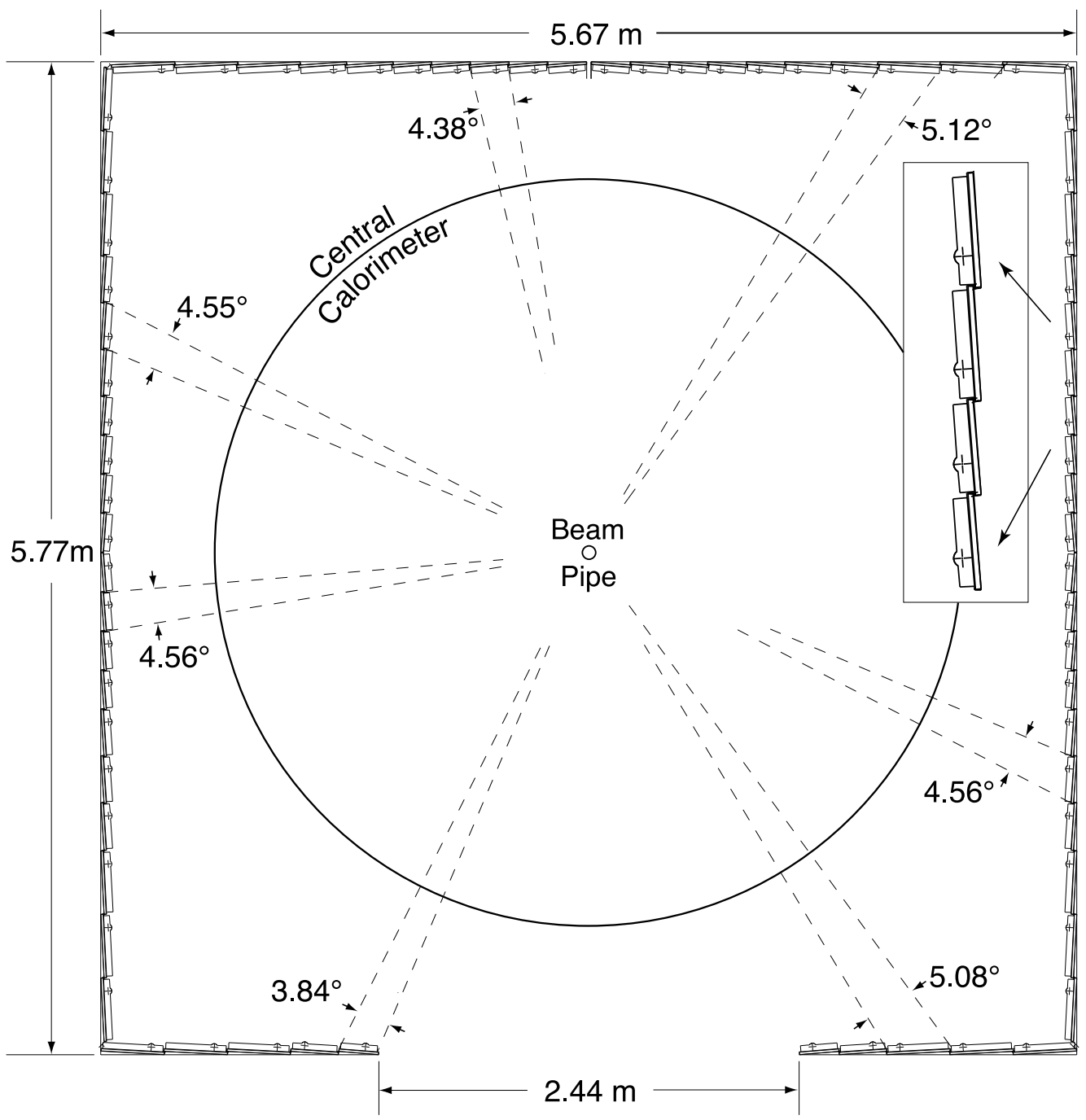

Figure 2.34: Schematic end view of the layout of the A- $\varphi$ scintillation counters. A nearly constant azimuthal coverage (indicated by the dashed lines for seven counters) is achieved by the usage of three different sizes of scintillation counters. The inlet shows an enlarged view of four such counters.

The timing resolution of the A- $\varphi$ counters is approximately $2 \mathrm{~ns}$ and varies due to photoelectron statistics, variations in the $z$ position of the interaction vertex, and variations of the amplitude and hit position along the length of the scintillation counter. On average a muon signal produces about 50-60 photoelectrons per A- $\varphi$ PMT with small variations of $\pm 7 \%$ depending on the location of the signal, close to the center or the ends of the counter.

\section{Forward Region Scintillation Counters}

The forward scintillation counters are located on the inside of the forward A-layer MDTs and the outside of the B/C-layer MDTs (see Fig. 2.33). They are usually referred to as forward or pixel counters. In analogy to the forward wire chambers, the three layers of scintillation counters are divided into octants, each containing 96 counters. As for the central counters, the $\varphi$-segmentation is $4.5^{\circ}$ and matches the CFT trigger sectors, as can be seen in Figure 2.25 (c). The largest counters cover an area of about $60 \times 110 \mathrm{~cm}^{2}$ and are placed in the C-layer, which is the outermost layer. A typical intermediate counter size is $24 \times 34 \mathrm{~cm}^{2}$ and the smallest counters are only about $17 \times 24 \mathrm{~cm}^{2}$. Since nearly 5000 counters were needed for the forward muon system, 
the counter design had to be optimised w.r.t. the cost, while still providing a good time resolution and amplitude uniformity over the length of a counter to ensure a high detection efficiency and good background rejection. The quantum efficiency of the photomultipliers is about $15 \%$ for photons of $500 \mathrm{~nm}$ wavelength and their gain is approximately $10^{6}$.

As the central A- $\varphi$ counters on the inside of the A-layer PDT, the forward counters are located in regions with residual magnetic fields, so that the phototubes need to be shielded. For the Alayer forward counters, the used magnetic shields are identical to those used for the A- $\varphi$ counters, because the highest fringe fields of up to about $30 \mathrm{mT}$ are expected there. For the remaining forward counters, located on the outside of the B/C-layer MDTs, the magnetic shields are made of only 3-mm-thick soft iron instead of 6 -mm-thick, the thickness of the $\mu$-metal remains constant at $1.2 \mathrm{~mm}$ and the diameter is also unchanged. The residual effect is less than $10 \%$ for magnetic fields up to $35 \mathrm{mT}$ (shields with 6 -mm-thick soft iron) and thus comparable to the reduction achieved for the A- $\varphi$ counters.

The time resolution is better than $1 \mathrm{~ns}$ and the detection efficiency is very close to $100 \%$ for all counter sizes. In tests with cosmic muons the average number of photoelectrons was found to be about 60 for the largest counters and roughly thrice as many for the smallest counters. The signals are read out via wavelength shifting bars attached to the PMTs, amplified and then sent on to the Level 1 trigger system. After digitisation in the scintillator front-end time-to-digital converters (TDCs), with 1.03 ns time bins, the amplitude and time information is passed to the Level 2 trigger and data acquisition systems.

\section{Additional Shielding}

Background interactions producing hits in the muon layers can contribute to the early aging of the muon system. During the upgrade for Run II, additional shielding was installed in the forward regions close to the beam pipe with the goal to reduce all three possible sources significantly:

- Scattered proton/antiproton fragments interacting with the calorimeter or beam pipe can lead to background in the central and forward A-layer elements;

- Proton/antiproton fragments interacting with the Tevatron magnets or other beamline elements can produce hits in the B/C-layers of the forward muon system;

- Beam halo interactions from the Tevatron tunnel can lead to background in all layers of the forward muon system.

The shielding consists of three rectangular pieces with layers of iron $(41 \mathrm{~cm})$, polyethylene $(15 \mathrm{~cm})$, and lead $(5 \mathrm{~cm})$ enclosed in steel structures. It surrounds the beam pipe and starts at either of the two end calorimeter cryostats, goes through the end toroids, and extends out to the wall of the $\mathrm{D} \emptyset$ collision hall (see Fig. 2.20 and Fig. 2.33 (c)). Iron was chosen as the electromagnetic and hadronic absorber due to its relatively short radiation and interaction length of $X_{0} \approx 1.8 \mathrm{~cm}$ and $\lambda_{A} \approx 16.8 \mathrm{~cm}$, respectively and its rather moderate cost. Lead serves as gamma ray absorber and polyethylene is a good absorber of neutrons, because of its high hydrogene content.

With the added shielding, the goal to reduce any non-collision background has been accomplished, so that, together with the usage of radiation-harder detector components, i.e. the MDTs in the forward regions, a long-term, reliable operation of the muon system is ensured. 


\subsubsection{The Luminosity System}

This system allows to determine the Tevatron luminosity at the D $\varnothing$ interaction region, measurements of the beam halo rates (proton and antiproton beam) and a fast determination of the $z$ coordinate of the hard interaction vertex. Two dedicated luminosity monitors are installed at either side of the $\mathrm{D} \varnothing$ detector, at $z=135 \mathrm{~cm}$ from the geometric center in front of the endcap calorimeters, see also Figure 2.21. Both these devices consist of two arrays of twenty-four wedgelike plastic scintillation counters, that are read out via photomultiplier tubes (see Figure 2.35). The counters are $15 \mathrm{~cm}$ long and cover an area of $2.7<|\eta|<4.4$ in pseudorapidity.

(a)

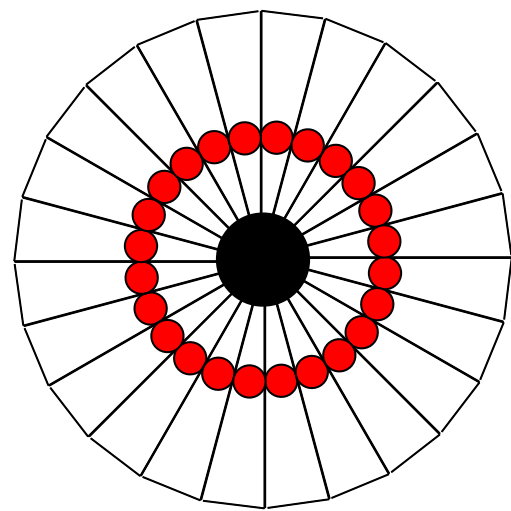

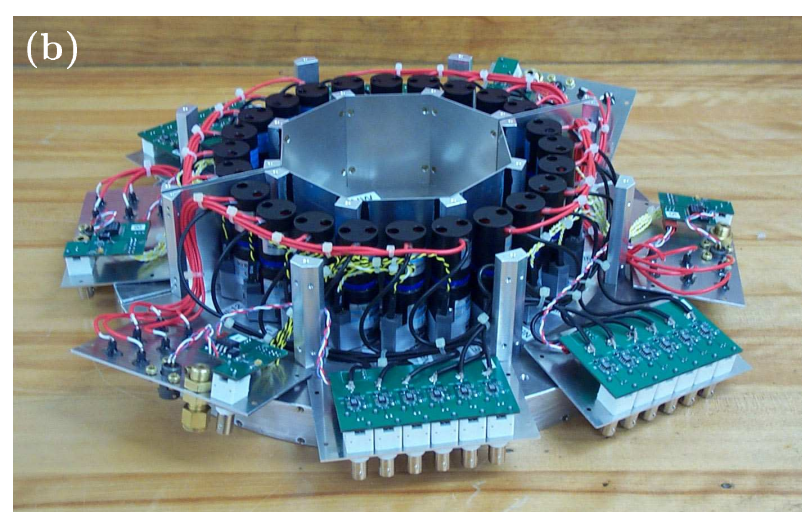

Figure 2.35: (a) Schematic view of one of the luminosity monitors. The filled (red) circles indicate the locations of the photomultiplier tubes. (b) The inside of a luminosity monitor before installation.

The rate of coincidence of the two monitors is used to determine the luminosity $\mathcal{L}$ from the average number of inelastic $p \bar{p}$ interactions per beam crossing $\bar{N}_{\text {inel }}$ (compare Eq. (2.9))

$$
\mathcal{L}=\frac{f \cdot \bar{N}_{\text {inel }}}{\sigma_{\text {inel }}},
$$

where $f$ is the beam crossing frequency and $\sigma_{\text {inel }}$ the effective cross section of the luminosity monitors. The latter takes acceptances and efficiencies of the monitors into account. Usually $\bar{N}_{\text {inel }}$ is greater than one, which underlines the importance of correctly taking into account multiple $p \bar{p}$ interactions in a single crossing. This is achieved by counting the fraction of beam crossings with no collisions at all and using Poisson statistics to infer the average number of inelastic collisions $\bar{N}_{\text {inel }}$. One difficulty of this approach is the necessity to distinguish $\gg$ real $« p \bar{p}$ interactions from beam-halo background interactions. To ensure sufficient background reduction, precise time-of-flight measurements of particles at small angles w.r.t. the beam axis are made. The resolution of the scintillation counters is about 200-300 ps, where the dominant contribution is due to the variation of the length of the light paths of particles hitting different locations on the counters. Under the assumption, that the incident particles on the luminosity monitors come from a real $p \bar{p}$ interaction, the $z$ position of the vertex is estimated via the time-of-flight difference: $z_{V}=\frac{c}{2}\left(t_{-}-t_{+}\right)$, with $t_{-/+}$being the time-of-flight measured for particles striking the luminosity monitor at $z=+/-135 \mathrm{~cm}$. To ensure the selection of beam-beam collisions and not beam-halo or other background interactions, $z_{V}$ is required to be below $\left|z_{V}\right|=100 \mathrm{~cm}$.

The luminosity is measured in units of luminosity blocks, which are short time periods of $60 \mathrm{~s}$ or less, and each luminosity block is indexed by a luminosity block number (LBN). The luminosity 
block number increases monotonically throughout the data-taking period and is incremented under various circumstances (see section 2.4). The period of one luminosity block is chosen short enough, so that the instantaneous luminosity is effectively constant over the time of one luminosity block, ensuring that the uncertainties introduced by these time $»$ slices « remain negligible.

\subsubsection{The Forward Proton Detector: FPD}

The forward proton detector (FPD) [107] measures protons and antiprotons scattered at small angles of the order of $\mathcal{O}(1 \mathrm{mrad})$. These particles do not hit any other parts of the $\mathrm{D} \emptyset$ detector and it is rather difficult to measure/trigger these diffractive events. In Run I, diffractive events could only be tagged using rapidity gaps, regions of the calorimeter without particles.

(a)

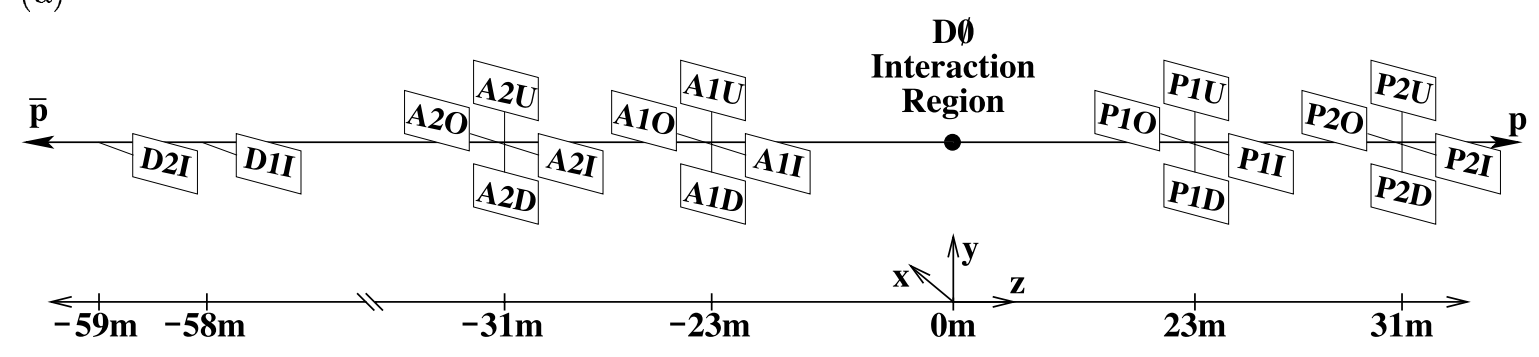

(b)

Figure 2.36: (a) Overview of the general layout of the FPD position detectors at various distances from the main $D \varnothing$ detector. (b) One FPD castle installed in the Tevatron tunnel.

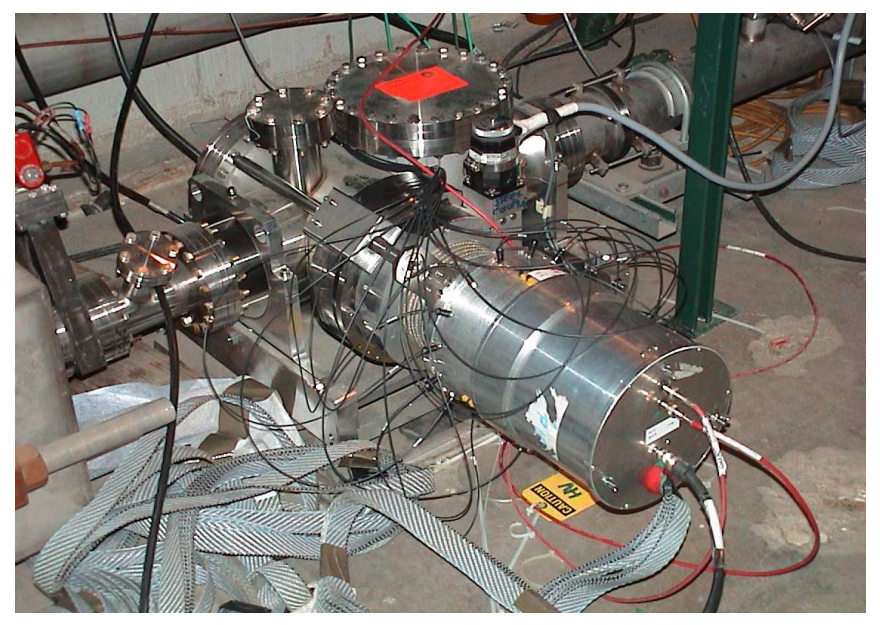

Since the particles are scattered at such small angles, the detector consists of a series of momentum spectrometers spread along the beam line as shown in Figure 2.36. Accelerator magnets are used together with position detectors operating only a few millimeters away from the beam axis. Due to this small distance to the beams the FPD position detectors have to be moved out of the beam line during injection of protons or antiprotons into the Tevatron. The position detectors are located in remotely controlled, stainless steel containers (Roman Pots) [108] allowing for operation outside the ultrahigh vacuum of the accelerator but still close to the beam line. In total, there are 18 Roman Pots arranged in steel chambers (castles) at six different locations, or distances of the main $\mathrm{D} \varnothing$ detector (see Fig. 2.36).

Before moving on to the trigger and data acquisition systems, two photographs of the entire DØ detector in open configuration at the end of the Run II upgrade period are presented. 
(a)

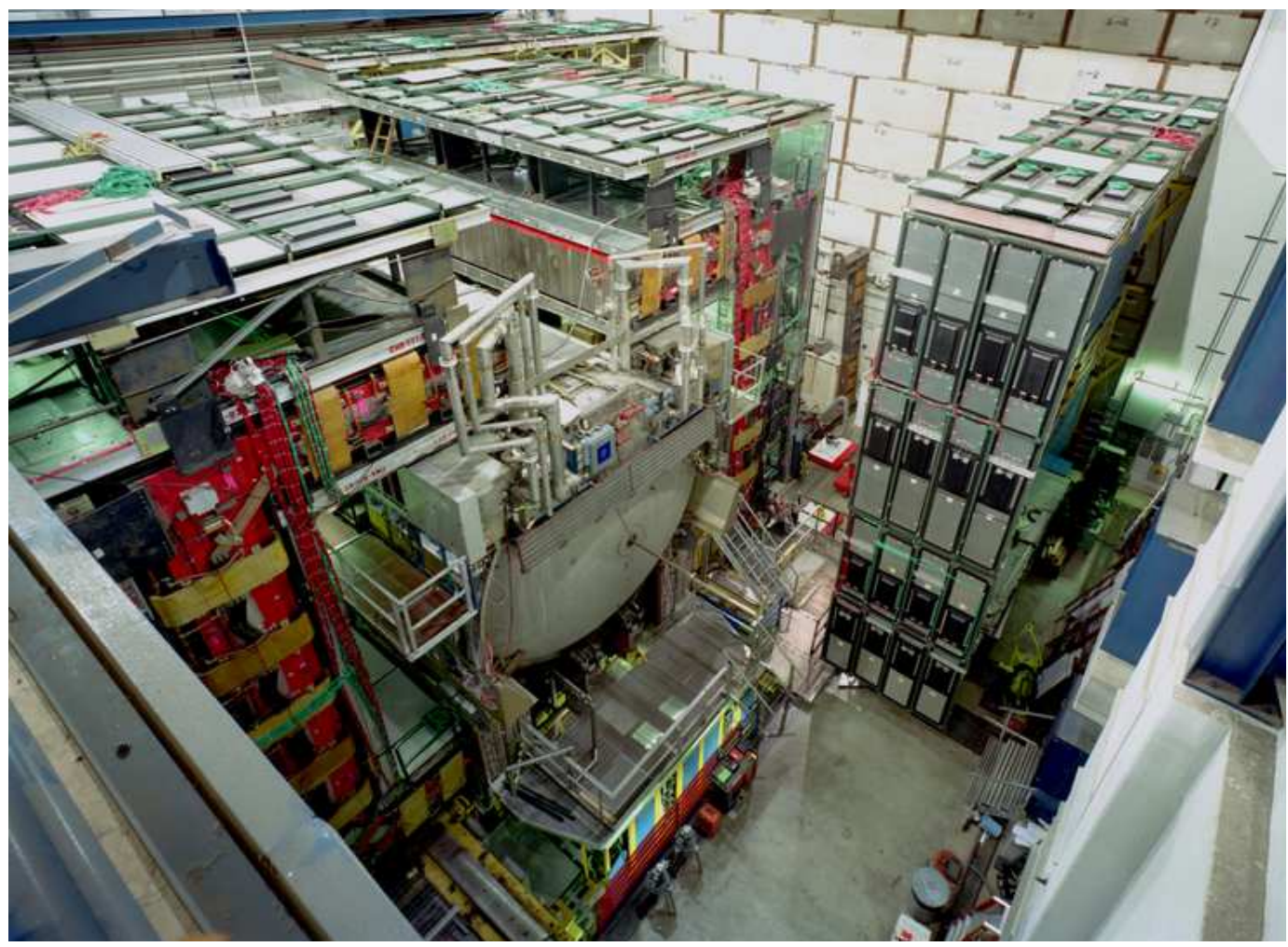

(b)

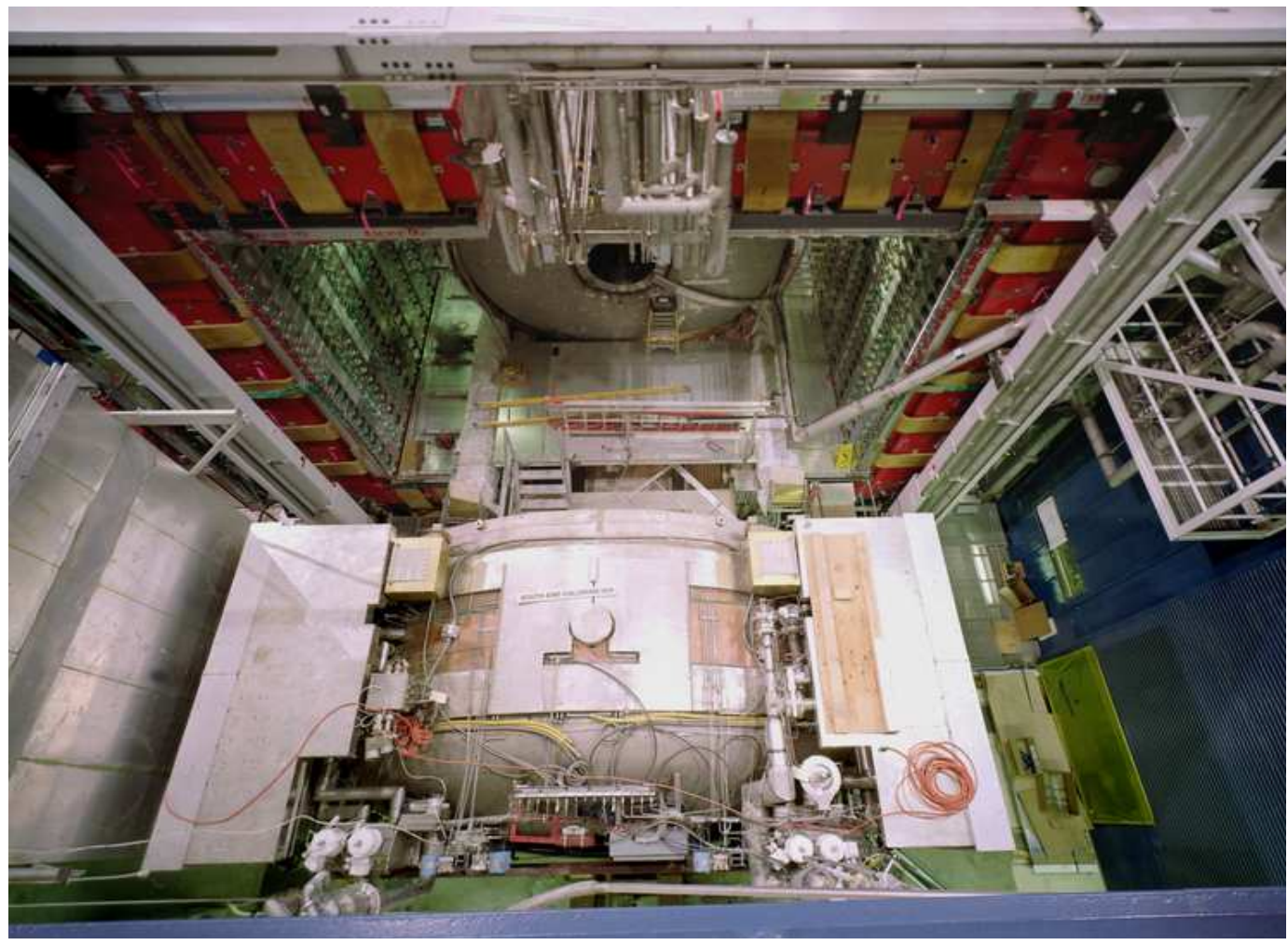

Figure 2.37: (a) The large forward part with the outer C-layer scintillators is moved away (upper right side), so that the end calorimeter and the central toroids (red with yellow-brown windings) are visible. The central toroid is also moved apart laterally as can be seen at the space above the calorimeter cryostat. (b) Clearly visible are the central and south end calorimeter cryostats, the central toroids and on the inside the $A-\varphi$ scintillation counters of the muon system. 


\subsection{The DØ Trigger and Data Acquisition System}

Due to the increased luminosity and high bunch crossing (high interaction) rate of the upgraded Tevatron, the trigger and data acquisition systems [109] needed to be enhanced, too. Since it is not possible to write all of the detected events to tape, or to even read out the entire detector with its huge number of readout channels every $396 \mathrm{~ns}$, a real-time, three-stage trigger system is used to select only interesting events for storage and thus for further physics analyses. Figure 2.38 provides a simplified overview of the closely related $\mathrm{D} \emptyset$ trigger and data acquisition systems.

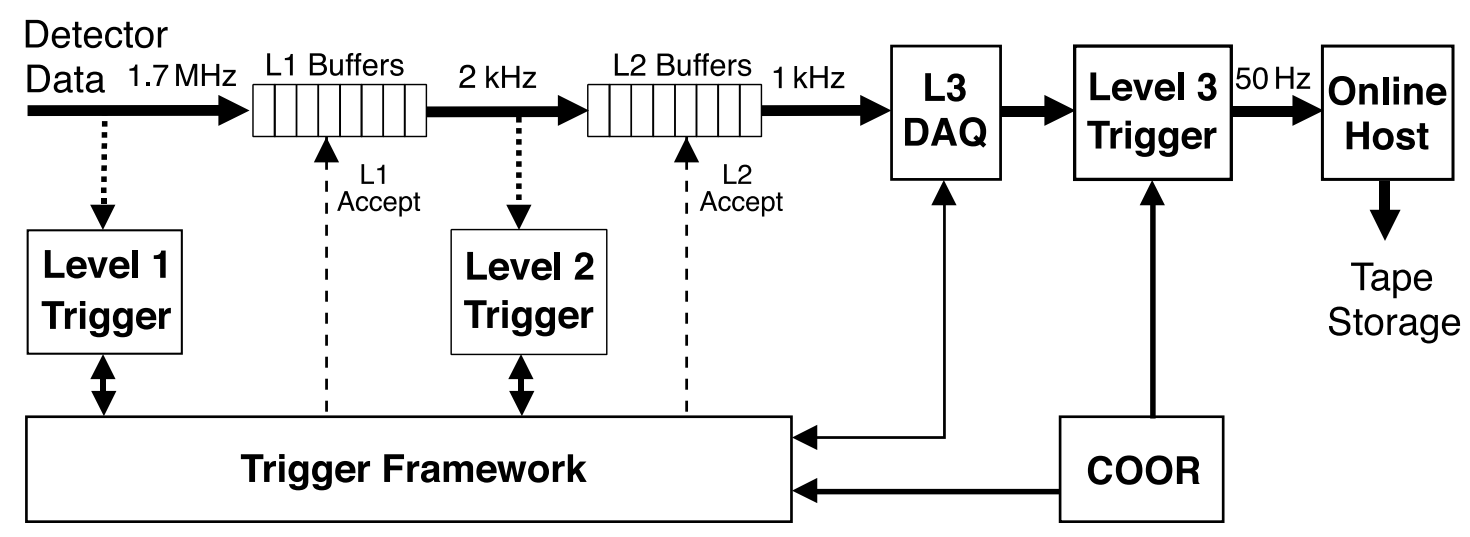

Figure 2.38: Overview of the integrated $D \varnothing$ trigger and data acquisition systems.

The data rate at Level 1 (L1), the interaction rate, is about 1.7 million events per second $(1.7 \mathrm{MHz}$ ). This huge rate has to be reduced to about $50 \mathrm{~Hz}$ (i.e. 50 events per second), because this is the limit with which the events can be written to tape.

The first stage of the $D \emptyset$ trigger system consists of a collection of hardware trigger elements, belonging to specific detector subsystems: the calorimeter (L1CAL), the combined fiber tracker and preshower detectors (L1CTT), the muon system (L1MUO), the forward proton detector (L1FPD) and the luminosity system. All of these provide digitised information to the trigger framework (TFW). As can be seen from Figure 2.38, all information of a specific bunch crossing is pipelined, i.e. stored in FIFO (first-in-first-out) buffers, which serve to minimise the experiment's deadtime. All subsystems must send information, which is to be taken into account in the L1 decision, to the TFW within $3.5 \mu$ s from the corresponding bunch crossing. The trigger framework uses fast algorithms implemented in field programmable gate arrays (FPGAs) to test up to 128 specific Level 1 trigger terms, whose logical »or « determine, whether a given event is accepted and passed on to Level 2 (L2). With a latency of $3.5 \mu$ s the TFW can issue a L1 decision every $396 \mathrm{~ns}$; this is referred to as timing-in. In case the L1 decision is positive and the event accepted and passed on to Level 2, about $10 \mu$ s are needed to read out the entire silicon tracker (SMT), whose information is used in the second trigger level, along with information of the central fiber and preshower detectors. The calorimeter precision readout for Level 3 (L3) takes longer but is only started upon an L2 accept. The first trigger level reduces the event rate to roughly $2 \mathrm{kHz}$ (L1 accept rate). All 128 trigger terms and other resources are programmed from COOR via text-based commands and interpreted by the trigger control computer, which in turn configures the trigger framework [110]. The second stage, L2, comprises custom-built VME (VERSA Module Eurocard) electronics and embedded microprocessors (again associated 
with the different subsystems) to provide further information to a global processor, the so-called L2Global. This specialised device uses individual objects, as well as object correlations in physics signatures and across different detector subsystems to construct the final L2 decision. The L2 system reduces the $2 \mathrm{kHz}$ accept rate of $\mathrm{L} 1$ further by approximately a factor of two, resulting in an $\mathrm{L} 2$ accept rate of $1 \mathrm{kHz}$. (Originally, Level 2 was designed to handle input rates of up to $10 \mathrm{kHz}$.) A block diagram of the first and second trigger stage is given in Figure 2.39. The functional principle and the different abbreviations are explained throughout the text.

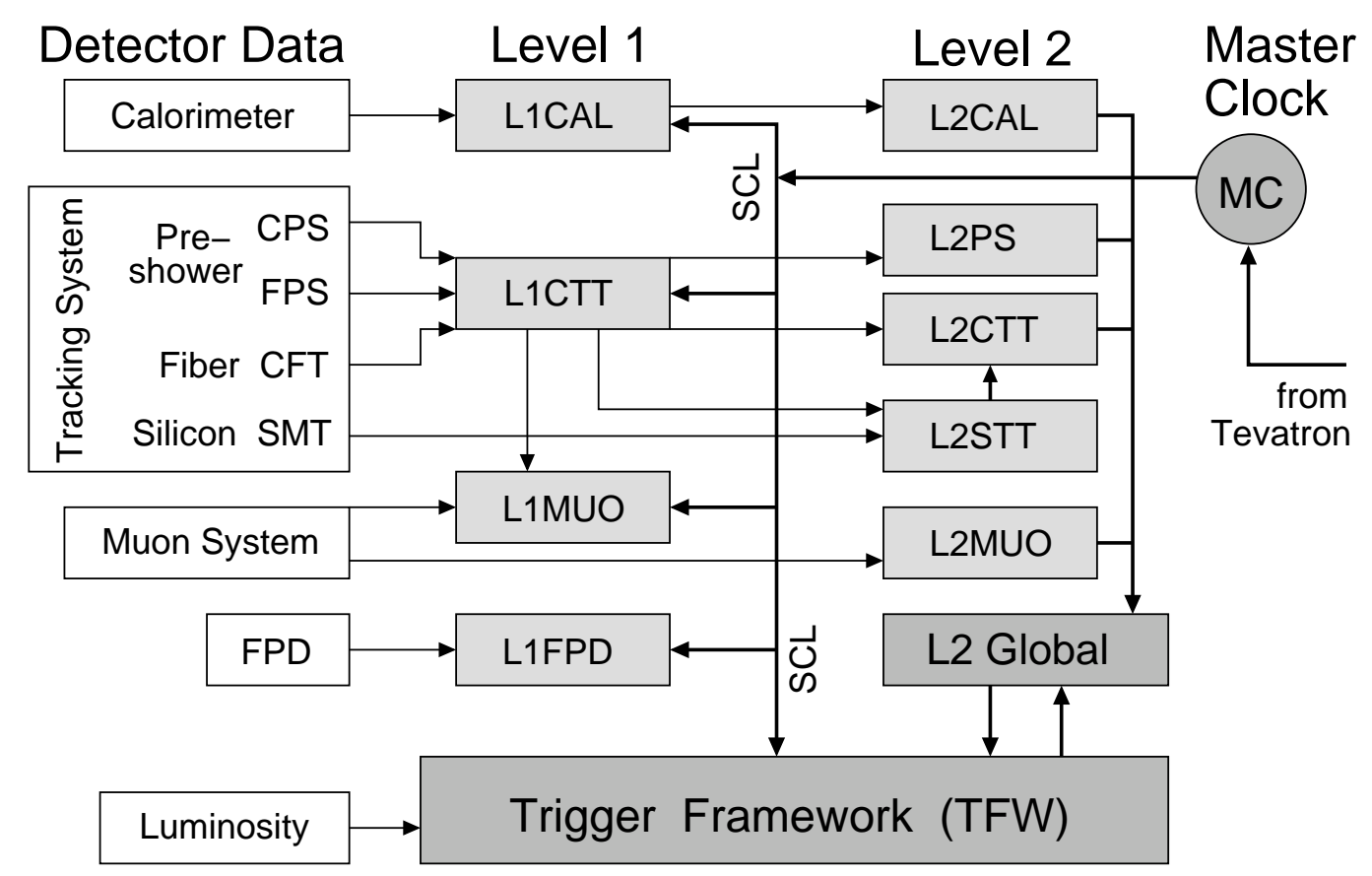

Figure 2.39: Block diagram of the L1 and L2 trigger systems. The L1 and L2 trigger decisions are communicated to the front-end boards by the trigger framework (TFW) via the SCL, which also distributes the master clock timing signals to all of the 128 geographic sectors of the DØ detector to ensure synchronisation with the Tevatron bunch crossings.

Candidate events passed by L1 and L2 are sent on to a farm of specialised Level 3 (L3) microprocessors, where sophisticated algorithms further reduce the event rate to about $50 \mathrm{~Hz}$, so that it can be written to tape. This fully programmable software trigger uses complete, i.e. fully reconstructed, physics objects and relationships between these objects (variables like invariant masses, rapidities or $\Delta \varphi$-separation between two objects) and in this way allows to enrich the physics samples, while still providing a large reduction in the rate. Only the events passing all three triggers stages, i.e. about 50 events per second, are recorded to disk and latter written to tape. As can be seen from Figure 2.38, events awaiting an L2 decision or transfer to the L3 farm are also pipelined, similar to the information gathered by the first level trigger elements. Dead time due to full L2 FIFOs and/or an L3-busy signal is very rare. An L3-busy signal is issued, if all L3 processors are working on event data (or data logging) and are unable to receive another event from Level 2. The majority of the trigger dead time arises from the approximately $10 \mu \mathrm{s}$ needed for the SMT readout.

The central coordination package (COOR) controls and coordinates all aspects of triggering and data acquisition (DAQ). It interacts directly with the supervising systems for all three trigger 
levels, which are the trigger framework (for L1 and L2) and the DAQ-supervisor (for L3). Upon an L2 accept, the data acquisition system ensures the data flow of the fully digitised events from the L2 FIFO buffers to the L3 farm nodes. This system, including the mutual interplay between COOR and the DAQ supervising systems is described in section 2.4.3.

\subsubsection{The Level 1 Trigger}

This trigger stage is based entirely on hardware and firmware. It examines every bunch crossing and decides if the event is worth to be looked at in more detail by the second and third level triggers. For triggering and data acquisition purposes the detector is divided into subsections, which are served by a single serial command link (SCL). Typical geographic sectors are for example the front-ends of the detector subsystems. The trigger framework, besides receiving triggering information from all 128 geographic sectors and determining if an event passes Level 1 or if it is rejected, also sends an L1 accept (followed by an L2 accept or reject) to all of these sectors via the SCL, which is the only fast communication line between the front-end boards and the TFW. Additionally, it allows to prescale certain triggers too copious to pass events without rate reduction and provides scalars to count the triggers and monitor live crossings, which are those bunch crossings, where all L1 trigger elements are idle and able to fire. The master clock (MC) provides the timing signals necessary to synchronise all trigger and readout electronics with the Tevatron bunch crossing periodicity.

As illustrated in Figure 2.39, the central track trigger (L1CTT) is connected to the scintillatorbased tracking detectors: the central fiber tracker (CFT) and the central and forward preshower detectors (CPS, FPS), described in section 2.3.1. The L1CTT comprises three subsystems, the CFT/CPS axial, the CPS stereo and the FPS systems. While all of these subsystems provide information on tracks and/or preshower clusters for the L2/L3 readout, only the first two participate in the L1 trigger decision. Fast discriminator data is used by the L1CTT to reconstruct charged particle trajectories and provide information for the L1 trigger terms. The more detailed data, stored by the L1CTT electronics, are also used as seeds by other trigger systems. Lists of seed tracks are sent to the L1MUO and L2STT systems, while track and cluster information are sent to the L2CTT and L2PS preprocessors.

The calorimeter Level 1 trigger system (L1CAL) is connected to the calorimeter readout and looks for energy deposition patterns exceeding programmable thresholds on transverse energy deposits. The inputs are electromagnetic and hadronic trigger tower energies summed in depth (one layer) and transverse coordinates $(\Delta \eta \times \Delta \varphi=0.2 \times 0.2)$. To enable a fast trigger decision on L1 and L2, the finer segmented precision readout of $\Delta \eta \times \Delta \varphi=0.1 \times 0.1$ is only used in the third trigger level. The fast analog signals are converted to transverse energies on input, have the pedestals subtracted and the energy scales adjusted. In case of electromagnetic trigger terms (for electrons and photons) only the transverse energies of the 1280 electromagnetic towers are examined, while for hadronic trigger terms (jets), the sums of the electromagnetic and hadronic tower energies $(1280 \mathrm{EM}+\mathrm{H})$ are compared to the programmed thresholds.

The Level 1 muon system (L1MUO) uses hits from the muon wire chambers, as well as from the muon scintillation counters and seed tracks from the L1CTT to look for muon-like patterns. It is divided into three subsystems, the central, north and south (forward) regions, each of which is further divided into octants to reflect the geometry of the PDT and MDT wire chambers. Two types of trigger elements, scintillator triggers and wire triggers, are used for each region. The for- 
mer matches scintillator hits with central tracks (CFT/CPS information) and the latter matches single scintillator-confirmed track stubs of one muon wire layer with the two or three other muon layers, depending on the region. The L1MUO makes use of FPGAs for the combinatorial logic of about 60,000 muon channels and up to $480 \mathrm{~L} 1 \mathrm{CTT}$ seed tracks per bunch crossing.

The FPD trigger element (L1FPD), connected to the forward proton detector, searches for coincidences between the hit signals of both position detectors in any of the nine FPD spectrometers (see Fig. 2.36). For single diffractive triggers, events are selected in which at least one of the outgoing beam particles left the interaction region intact. Analogously, coincidences between FPD spectrometers on both sides of the $\mathrm{D} \varnothing$ interaction region are used for the elastic diffractive trigger terms to trigger on events in which both of the outgoing particles are still intact.

\subsubsection{The Level 2 Trigger}

As mentioned above, the second trigger stage not only triggers on individual objects, such as tracks, muon track segments or calorimeter clusters, but in a global stage (L2Global) also on correlations between these object. To this end, the Level 2 preprocessors (called L2beta processors) collect data from the L1 trigger system and from the detector front-end boards, combine the data into physics objects (i.e. muons, EM-objects, like electrons or photons and jets) and also across detector subsystems to examine event-wide correlations in all of these objects. Based on the Level 1 decision and additional script-controlled L2 trigger criteria the L2Global processor selects events, which are then tagged for full readout and send to Level 3 for further analysis.

The L2STT performs pattern recognition in the SMT data, while the L2CTT uses track list inputs from the L1CTT and from L2STT, which in turn receives information from L1CTT and the SMT barrel front-ends. Each list corresponds to a different $\varphi$-region of the L1 track trigger system. The lists are concatenated and sorted according to the transverse momentum $\left(p_{\mathrm{T}}\right)$, while also being refined using additional information from the $\mathrm{L} 2$ readout, which was not available at Level 1. Two azimuthal angles are determined for each track: $\varphi_{0}$, w.r.t. the beam axis and $\varphi_{E M 3}$ w.r.t. the third, finer segmented calorimeter EM-layer, which differs from $\varphi_{0}$ due to the bending of tracks in the solenoidal magnetic field. Different isolation criteria are also evaluated for each track to enhance the trigger capabilities for tau-leptons [74]. The final, $p_{\mathrm{T}}$-sorted list of tracks is sent to L2Global to form part of the L2 trigger decision.

The L2PS treats the central and forward preshower detectors separately. CPS cluster centroids are compared to provide transverse coordinates $(\eta$ and $\varphi)$ for clusters matching in three layers. The presence or absence of CFT trigger tracks associated with either CPS axial or stereo clusters is tagged and the resulting transverse coordinates are binned to match the calorimeter trigger towers of $\Delta \eta \times \Delta \varphi=0.2 \times 0.2$. Similar functionality is provided by the FPS, which is, in addition, the only source of forward tracking available before the L3 software trigger.

At L2 (L2CAL), the calorimeter trigger towers match the cell geometry of $\Delta \eta \times \Delta \varphi=0.1 \times 0.1$, which means that each processor uses $E_{\mathrm{T}}$ data from 2560 trigger towers. There are two algorithms, the electron/photon and the jet algorithm. The first one starts from an $E_{\mathrm{T}}$-ordered list of EM towers with $E_{\mathrm{T}}>1 \mathrm{GeV}$ and combines, for each predefined seed tower, the largest of the neighboring towers with it to form an EM-cluster. Of these clusters, the EM energy fraction of the leading and next-to-leading trigger towers and the amount of total $E_{\mathrm{T}}$ in a $3 \times 3$ tower array around the seed tower are used for background reduction. The final list of electron/photon candidates is sent to L2Global, where certain trigger criteria are applied as previously defined by 
the trigger menu (or trigger list). The second algorithm for jet identification first clusters $5 \times 5$ groups of calorimeter trigger towers centered around a seed tower, with at least $E_{\mathrm{T}}>2 \mathrm{GeV}$. The highest $E_{\mathrm{T}}$ jet of overlapping candidates is kept and the $E_{\mathrm{T}}$-sorted lists are reported to L2Global, which applies predefined trigger requirements to the jets.

The L2MUO preprocessors use more precise timing information and calibration [111] for improving the quality of the muon candidates found at Level 1. The input consists of the L1MUO output and data from about 150 front-end modules of the muon system, PDT/MDT wire chamber readout, as well as timing information from the scintillation counters. In order to keep the total execution time of the algorithms independent of the actual number of hits in the wire chambers or scintillation counters, eighty DSPs (digital signal processors) are used in parallel. Each DSP is used to find muon track segments in a small detector region, and the L2beta processor builds integrated muon candidates from the stubs found by the DSPs. Every L2 muon candidate contains the track $p_{\mathrm{T}}$ measurement and transverse coordinates $(\eta, \varphi)$, as well as different quality and timing information.

In the L2Global processor the information of the different L2 worker processors is gathered and the current trigger criteria applied. A separate L2 script is used for each L2 trigger condition, which has to be satisfied in order for the L2 trigger to fire. Each script is, in turn, associated with a specific L1 bit, allowing the L2Global worker to check which L1 bit fired to decide which L2 script(s) it has to run on the data. Depending on the specific L1 trigger decision mask prepared by the trigger framework for each accepted event, the L2Global worker executes the necessary L2 scripts, makes a trigger decision and returns this decision to the TFW. The exact trigger requirements are specified in the trigger list that can be changed (i.e. downloaded to the TFW and to L2Global) as frequently as every new run ${ }^{12}$. The L2 scripts are built from different filters, relying on tools, to provide flexible functionality. The tools apply selection criteria to the various preprocessor objects, decide which of these objects should become global L2 objects, and build a specific L2Global object type of these. In addition, the tools can correlate different preprocessor objects: for example an EM-object from L2CAL and a track object from L2CTT/L2PS, referring to the same electron candidate. After the tools finish processing and produce a list of L2Global objects, the filters impose the current trigger requirements on the objects and generate a new list of objects. The final decision is made by the L2 script, which simply looks if the minimum number of objects satisfying the trigger conditions was found in the event.

\subsubsection{The Level 3 Trigger and Data Acquisition}

The Level 3 trigger is a high-level, fully programmable software trigger, intertwined with the $\mathrm{D} \emptyset$ data acquisition system; both are referred to as L3DAQ in what follows. Controlled via the COOR package, as the L1 and L2 systems, L3DAQ handles the transport and distribution of the readout data from the detector to available computing nodes of the L3 filter farm. The L3 farm consists of 274 computing nodes, running a Linux operating system and using the TCP/IP protocoll for communication and data transfers. Single board computers (SBCs) in each VME

\footnotetext{
${ }^{12} \mathrm{~A}$ run is a variable period of data-taking within a store, typically in between two to four hours. It is characterised by the use of the same trigger list, defining a certain set of trigger conditions and prescale factors.
} 
crate read out the different VME modules and send the data to one or more of the L3 farm nodes as specified by the Routing Master (RM). Up to $63 \mathrm{VME}$ crates may be read out per event. On each farm node runs an event builder process (EVB), that collates the various fragments into complete events and places them into shared memory buffers, where the L3 tools and filtering processes can work on the events. Since the EVB process has to know whether a certain event is complete or not, i.e. if it should receive fragments from 20, 40 or all 63 crates, it receives an expected-crate list from the Routing Master. In return, the EVB routinely informs the RM of the number of available buffers. In case there are too few free buffers, the RM can instruct the trigger framework to disable triggers, allowing the farm nodes to catch up with the processing of events. If the event is accepted by the third level trigger, the event data is sent to the online host for storage and/or distribution to various online monitoring applications. The combined data rate is approximately $10 \mathrm{MB} / \mathrm{s}$, which corresponds to a $50 \mathrm{~Hz} \mathrm{~L} 3$ accept rate of events with a typical size of $200 \mathrm{kB}$. Figure 2.40 provides an overview of the information and data flow through the L3DAQ system.

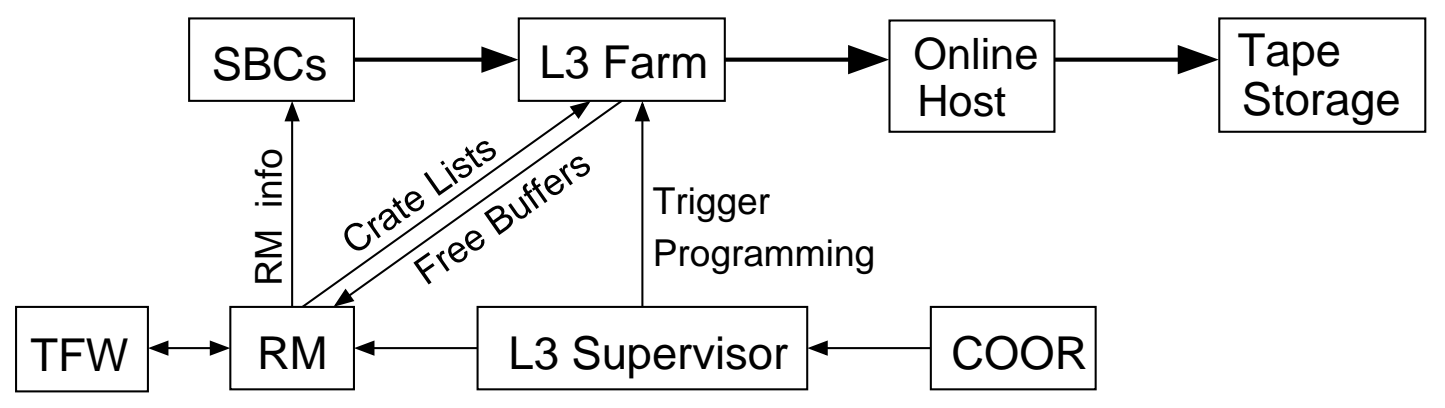

Figure 2.40: Schematic view of the information and data flow through the L3DAQ system. The different abbreviations are explained throughout the text.

The L3 trigger performs a fast event reconstruction, so that its decision to reject or accept a given event is based on the complete physics objects and relationships between them. In principle an arbitrary number of L3 trigger conditions may be defined in the trigger list. The limiting factor, however, is the execution time for testing each event sent to L3. The trigger list is a menu of various L1, L2 and L3 trigger requirements with programmable parameters. It is downloaded to the TFW, L2Global and the L3 Supervisor usually at the beginning of a store and might be changed as frequently as for every run. Analogously to L2, tools and filters perform the main work: unpacking raw data, locating hits or track segments, forming clusters, applying calibrations and reconstructing physics objects: electrons/photons, muons, taus, jets, vertices and missing transverse energy, $\not_{T}$. The L3 terms of the trigger list govern the input of programmable algorithm parameters (reference sets, containing precise definitions of the different physics objects) to the tools. The algorithms implemented in the L3 tools are similar to the offline algorithms, though they are not identical, due to a trade-off between execution time and the actually performed reconstruction and/or correlation of physics objects by the online algorithms. On average, the processing time per event is about $300 \mathrm{~ms}$.

The distribution of event data to the different filters and tools is handled by a special interface of the L3 framework, the ScriptRunner. At the beginning of each run, ScriptRunner initiates the parsing of tools and filter scripts. During data-taking, it processes any errors and handles the output of the tools and filters, sends filtered data to the datalogger, and stores monitoring information and error messages, which are reported at the end of each run and upon request. 
Four different tools are available for physics object reconstruction and correlation of objects: the L3 tracking tool, the L3 jet and electron tool, the L3 muon tool, and the L3 $E_{T}$ tool. In addition, higher level filters are used to find relationships between physics object candidates and to select/trigger on, e.g. multiparticle (multijet) invariant masses, or the scalar sum of the transverse energies of all jet candidates $\left(H_{T}\right.$-filter). A general feature of all L3 tools and filters is their ability to correlate data or object candidates of different detector subsystems and thus use all available information for a certain physics object. The L3 muon tool for example uses scintillator hits and track segments of all three layers of the muon system to find muon tracks in three dimensions, and additionally it can also call subordinate tools, making use of the inner tracking system and the calorimeter. In this way, the L3 muon tool can match tracks measured with the inner tracking detectors to muon tracks measured with the muon system, and use vertex constraints from the L3 tracking tool to further improve the momentum resolution of L3 muon candidates. The matching of muon tracks to paths of minimum ionizing particles in the calorimeter enables L3 to distinguish between isolated and non-isolated muons. For further details on the algorithms implemented in the L3 tools, the reader is referred to [80].

All tools cache their results to expedite possible multiple calls within the same event and, upon accepting the event, write the L3 object parameters to the corresponding event data block. The Level 3 trigger reduces the L2 output rate of $1 \mathrm{kHz}$ to $50 \mathrm{~Hz}$ for recording to disk and later storage on tape.

The architecture of the online system and, separately, the flow of the event data from the L3 filter farm to its final repository on tape is illustrated schematically in Figure 2.41. The
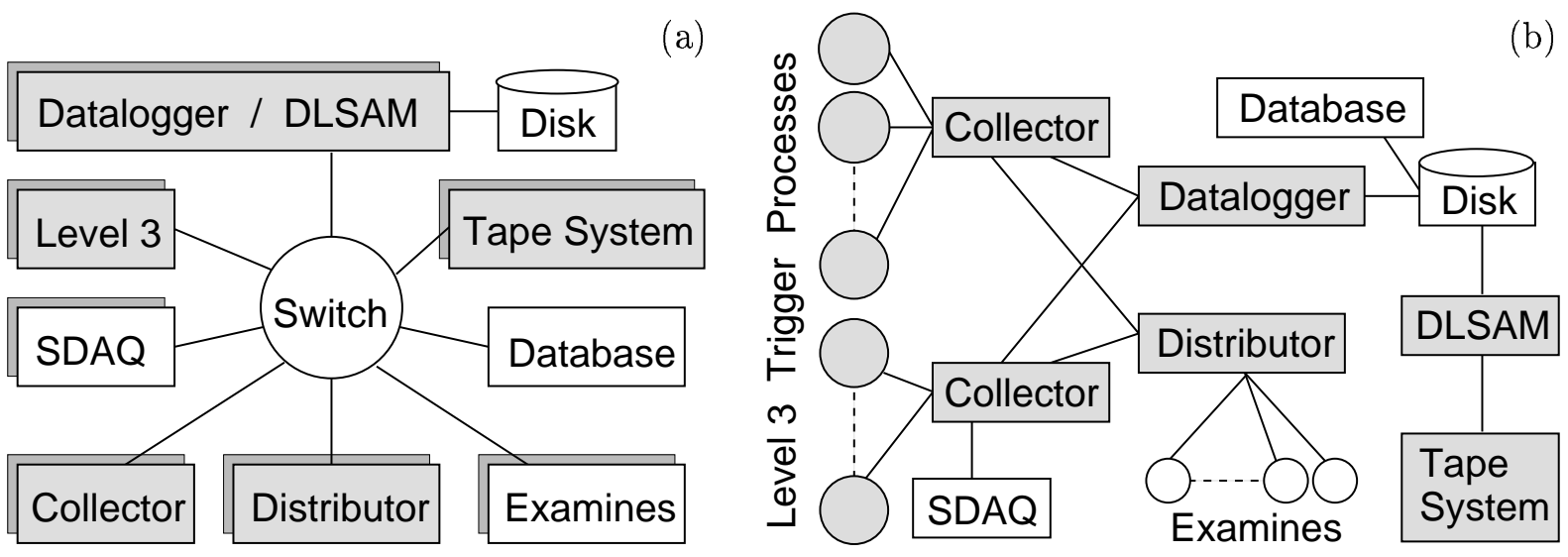

Figure 2.41: Schematic illustrations of (a) the physical architecture and (b) the data flow of the online host system. The darker shades in (a) indicate the use of many computing nodes for one process.

online host system (see Fig. 2.41 (a)) comprises the L3DAQ, various monitoring applications (Examines), the SDAQ system (secondary DAQ processes for calibration and/or commissioning) and interfaces to the Fermilab mass storage system ENSTORE [112]. The entire online system is built around a single, fast ethernet switch. Upon accepting an event, the data is sent from the L3 nodes to collector processes, directing the event data to the datalogger and a part of it additionally to the distributor. The first one saves the data to a disk array for buffering and generates metadata information in file format to facilitate the storage in the SAM (Sequential Access via Metadata) database [113]. The DLSAM (datalogger SAM) processes represent the interface to the SAM/ENSTORE mass storage facility. They monitor the buffer disk array at $\mathrm{D} \emptyset$ and take care that the data are sent regularly to the final repository, a robotic tape system, 
located in the Fermilab Feynman Computing Center, about $3 \mathrm{~km}$ away from the D $\varnothing$ detector. The second process, the distributor provides event data in near real-time to various online analysis and monitoring applications, the Examine processes.

\section{The Luminosity System}

The luminosity data acquisition system, referred to as LDAQ, is a stand-alone system running on the online computer cluster. The system is designed to collect, measure, verify and monitor the luminosity delivered to the $\mathrm{D} \emptyset$ experiment and that is used by physics analyses. It not only connects to the DØ trigger and DAQ control systems: COOR, the trigger framework (section 2.4.1), and L3DAQ, ScriptRunner and datalogger (section 2.4.3), but also delivers information to the accelerator control system (ACNET). The data from many different sources are correlated, loaded into a database and used for luminosity calculations.

As mentioned already in section 2.3.4, the luminosity is measured in units of indexed luminosity blocks of $60 \mathrm{~s}$ duration or less. The indices (LBNs) increase monotonically throughout Run II and are incremented upon store transitions, run transitions, or using special commands, and normally every 60 seconds. To simplify the luminosity calculation, different Level 1 triggers are grouped together, so that they share the same dead time. The luminosity is determined separately for each of these trigger groups, taking into account the instantaneous luminosity and the usually different trigger dead-times between different groups. Further details on the D luminosity measurement can be found in reference [114].

Figures 2.42 and 2.43 on the following two pages show two stores. The first one (Figure 2.42) shows store number 4400, taken September 24-25, 2005, which lasted about 26 hours, as an example for a good data-taking efficiency and very few problems. The initial instantaneous luminosity (peak luminosity) was $116 \cdot 10^{30} \mathrm{~cm}^{2} \mathrm{~s}^{-1}$. The luminosity profile is given by the dashed pink line, and the L1/L2/L3 accept rates are shown as solid black/blue/red lines, respectively. Also indicated are the L1/L2-busy rates (dashed grey and brownish lines), where the L1-busy rate amounts to about 4-5\%. Cyan coloured spikes correspond to short periods of L3 disables, usually requested by the routing master when the L3 farm has run out of buffering capacity. Clearly visible are also the beginning and ending of runs, marked by numbers and characterised by the steps in the trigger rates, which then start falling again exponentially with decreasing luminosity. The second example (Figure 2.43) shows store number 3980, taken February 13-15, 2005. The initial instantaneous luminosity, about $40 \%$ lower than in the previous example, is $71 \cdot 10^{30} \mathrm{~cm}^{2} \mathrm{~s}^{-1}$, and the store lasted for approximately 36 hours. As can be seen, the first three runs suffered from considerable problems, indicated by the large number of cyan-coloured spikes (L3 disables) due to a wrong version of the reconstruction code running on the L3 farm.

\subsubsection{Online Monitoring of the Detector and Data Quality}

\section{Online Detector Monitoring}

The DØ experiment uses extended EPICS (Experimental Physics and Industrial Control System) [115] for monitoring and slow control purposes. The EPICS system has been adapted and extended to meet the special needs of the D $\varnothing$ detector controls [116]. The support for new device types was extended and the significant event system (SES) for global event reporting, as well as a centralised relational database was added to the set of EPICS software building blocks. The SES 


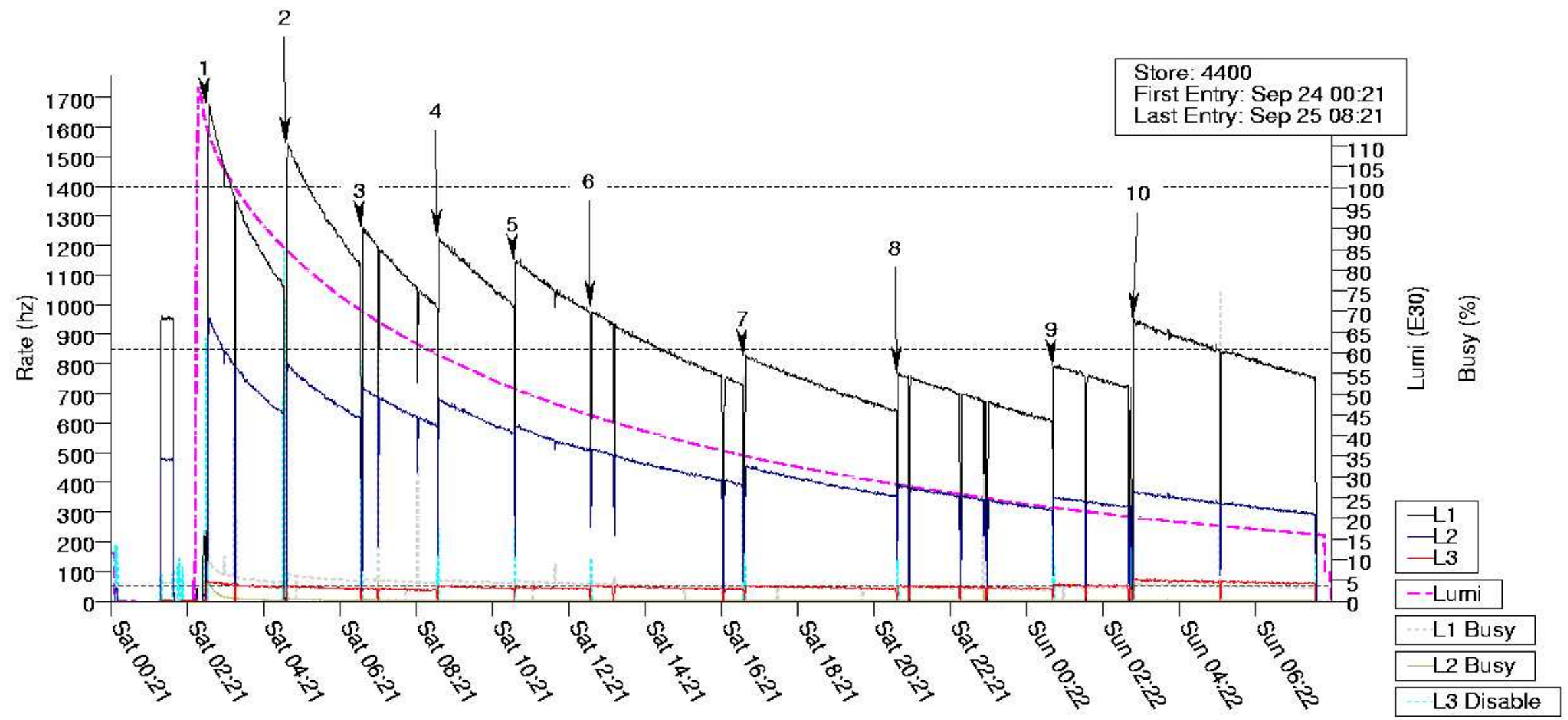

Figure 2.42: The luminosity profile (dashed pink line) and the L1/L2/L3 accept rates (solid black/blue/red lines, respectively) for the physics store number 4400, taken September 24-25, 2005. The whole store lasted about 26 hours and the initial instantaneous luminosity was $116 \cdot 10^{30} \mathrm{~cm}^{2} \mathrm{~s}^{-1}$. At high instantaneous luminosities a run (indexed by numbers) is usually ended after about two hours; later in the store, when the instantaneous luminosity has decreased and triggers need not be prescaled by large factors, runs last for about four hours. Between the »run end « and »run start« commands, new trigger prescale factors are usually downloaded to the trigger framework. The prescales are adjusted to the continually decreasing instantaneous luminosity as can clearly be seen towards the end of the store, where the L1 and L2 accept rates increase again, although the luminosity is significantly reduced compared to the beginning of the store. 




Figure 2.43: Physics store number 3980, taken February 13-15, 2005 is shown, where considerable problems were observed during the first three data-taking runs. The initial instantaneous luminosity was $71 \cdot 10^{30} \mathrm{~cm}^{2} \mathrm{~s}^{-1}$. For the meaning of the different lines and colours, see the caption of Figure 2.42. The large number of L3 disables (cyan coloured spikes) during the first three runs was due to a wrong executable version of the reconstruction code running on all nodes of the L3 trigger filtering farm. 
system keeps track of the overall alarm state of the detector and if it receives alarm messages, compromising the data quality, it automatically pauses the current data-taking run.

Another vital point in the successful operation of the $\mathrm{D} \emptyset$ detector is a good knowledge of the accelerator status. To this end, a gateway based on the XML-RPC protocol provides a fast and reliable messaging connection between the $\mathrm{D} \emptyset$ control system and the Accelerator Control NETwork (ACNET). D $\varnothing$ receives information on the beam status, the luminosity and other critical accelerator parameters and in turn sends cryogenic and magnet data, the luminosity as measured with the DØ luminosity monitors and FPD data (roman pot positions) to ACNET.

\section{Online Monitoring of the Data Quality}

Several different applications run in the $\mathrm{D} \emptyset$ control room and are continuously provided with recent data by the distributor (see section 2.4.3). Apart from monitoring applications specifically designed for each detector subsystem, e.g. the silicon or fiber tracker, the calorimeter, or the muon system, there are also monitoring applications for the trigger rates, the available buffer space of the L3 farm nodes, an event display showing recent events in different detector views and also a global monitoring tool [117], which serves to monitor various distributions of physics variables. This last tool allows monitoring of nearly all detector subsystems by providing plots of low-level variables, e.g. hit distributions, occupancies, and efficiencies of planes and layers of the muon system, but also high-level physics objects, such as invariant masses or jet multiplicity. An example of some low-level muon variables is given in Figure 2.44. A yellow (red) frame around a specific distribution indicates discrepancies between the current data and reference data sets used for comparison. The deviations are measured using two different methods: a simple bin-by-bin method (max.Diff.), and via Kolmogorov-Smirnov tests (KS-prob.).
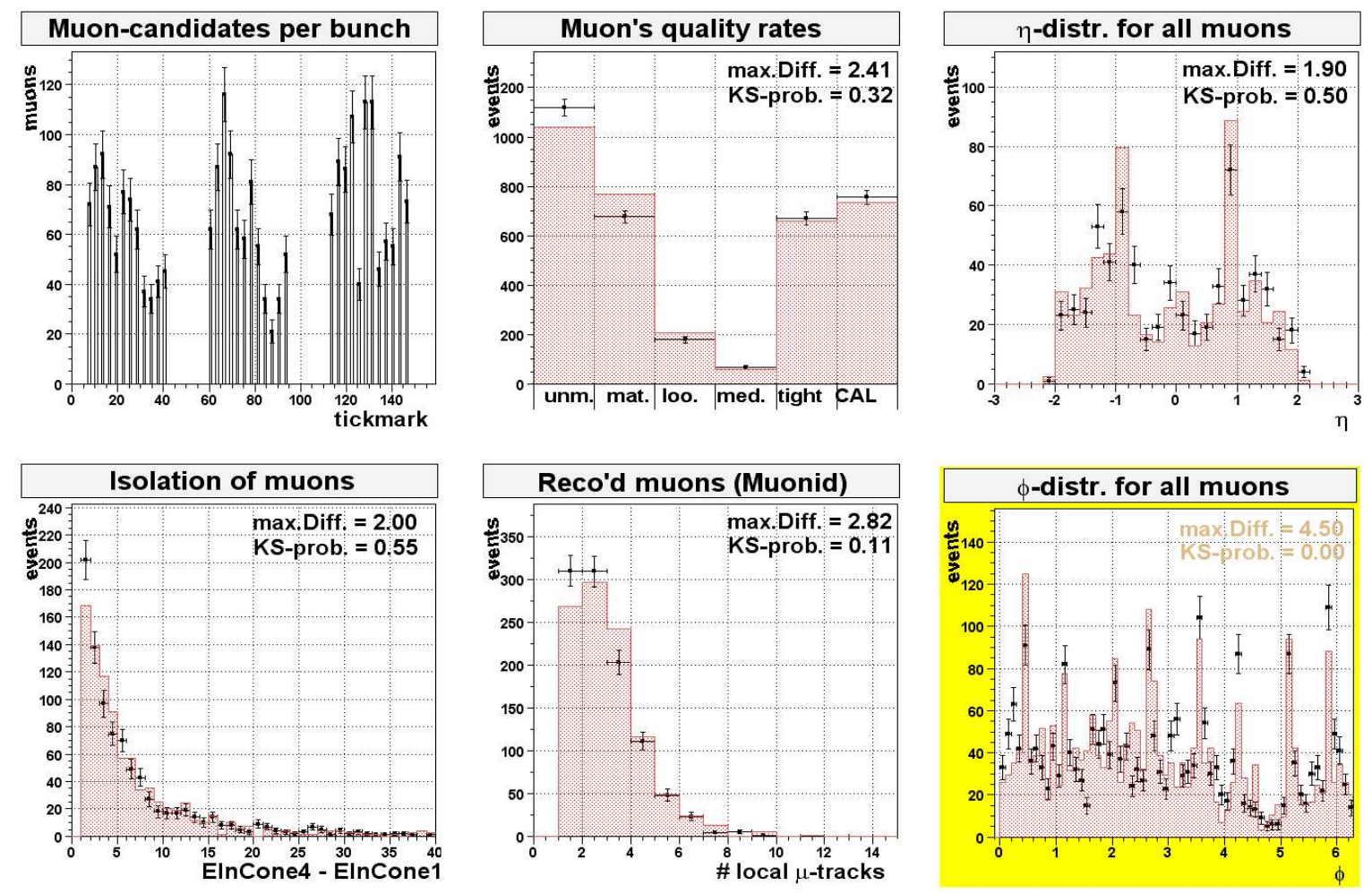

Figure 2.44: Global Monitoring: low-level muon variables. The muon quality rates in the upper middle plot refer to different hit patterns required for muon types of different quality. 


\subsection{Data Simulation, Handling, and Storage}

In Run II, the $\mathrm{D} \emptyset$ collaboration decided to use the $\mathrm{C}++$ programming language as the official software development language and to wrap Run I Fortran software and/or programs from other sources in $\mathrm{C}++$ code. One of the main uses of the $\mathrm{C}++$ wrappers is for Monte Carlo (MC) event generation/simulation programs. Besides, $\mathrm{D} \emptyset$ uses an event data model (EDM) that consists of a library of $\mathrm{C}++$ classes and templates, facilitating the implementation of new reconstruction and analysis software. The main feature of this model is an event container class managing all data associated with a single bunch crossing: the raw detector output, the trigger related results and data from different offline reconstruction algorithms, as well as the parameters used for each algorithm. The additional storage of configuration parameters allows for multiple instances of the same algorithm in different configurations. For example, an iterative seed-based cone algorithm, known as the midpoint cone algorithm (see section 4.3.2), is used to identify jets in the calorimeter, and using multiple instances allows to run it with different cone-radii, while still being able to distinguish the results.

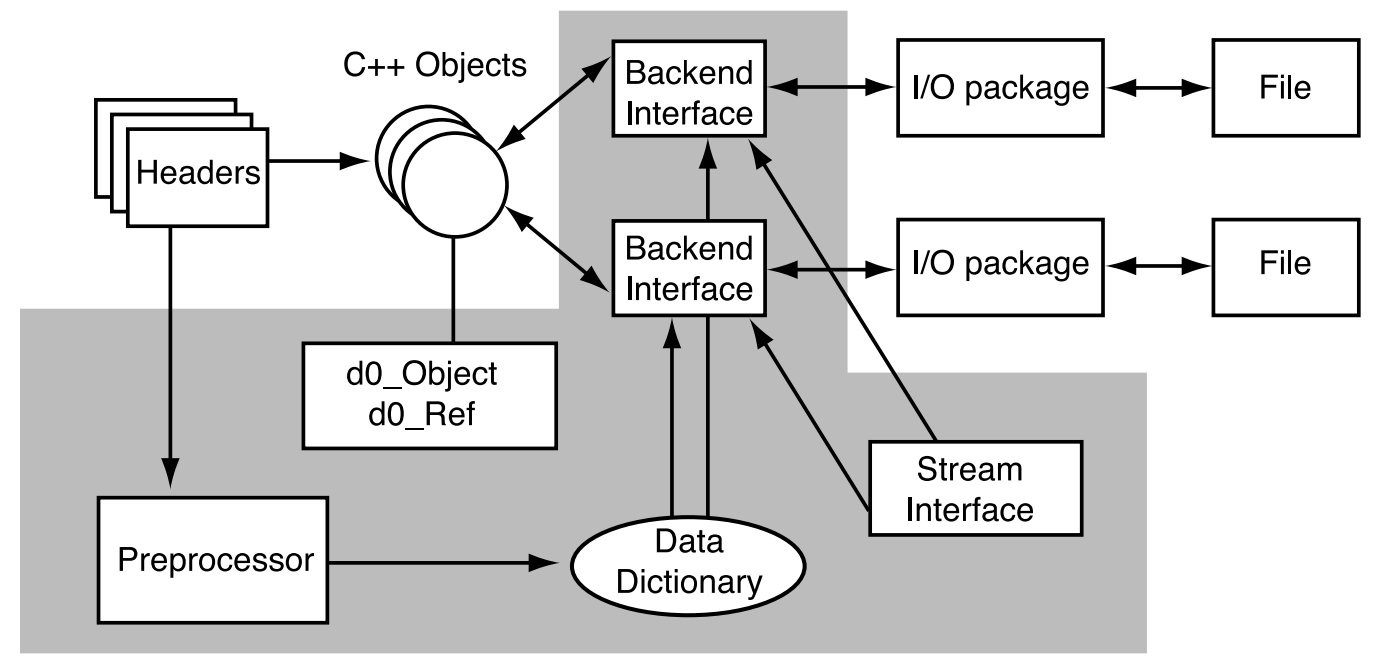

Figure 2.45: The DØ software structure. The shaded area shows the components that belong to DØOM, the $\gg D \varnothing$ Object Model « [118]. As mentioned, all DØ software, comprising packages made up of one or more Headers and $C++$ Objects, needs to be written according to a common standard to comply with the EDM. The coding conventions are discussed in more detail in [119].

The DØ Object Model, DØOM [118], (see the shaded area in Figure 2.45) handles all C++ Objects and defines which of them are to be used persistently. The layout of the $\mathrm{C}++$ classes is described by a dictionary maintained by DØOM. This dictionary is generated by a preprocessor, based on the CINT C/C ++ interpreter [120], also used by the ROOT system, and can be queried at run-time. The independence of user code on the external input/output (I/O) packages that actually handle the translation between the $\mathrm{C}++$ objects and the persistent format, allows to add new formats or change existing ones without having to change the reconstruction code. Additionally $\mathrm{D} \varnothing \mathrm{OM}$ handles the adding and deleting of data members without explicit user intervention, allowing for intercommunication between different objects.

Although a number of different data formats is used within D $\varnothing$, all of them are compatible with the EDM model. The following formats are used: raw data, data summary tape (DST), thumbnail 
(TMB) [121], ROOT-trees [122] and recently the so-called common analysis format (CAF) [123], based on the ROOT-tree format, has been designed and is in use since mid 2005 .

Since the reconstruction software is continuously developed and improved, the collaboration decided that all $D \emptyset$ data would be reconstructed with the same version of the reconstruction software from time to time. This is one reason why all raw data, i.e. unreconstructed data, without energy or other calibrations applied, is also kept on tape. The thumbnail format was developed in 2001/2002, because the older data summary tape format became too difficult to handle due to the large event size $(\approx 250 \mathrm{kB})$ and the huge amount of data anticipated from the higher luminosity. Originally, in the DST format, all information of the reconstructed physics objects was stored and also raw data information, which lead to the large event size. In contrast to this, the thumbnail format mostly contains information on physics objects and no longer raw data information. The TMBs contain the data (reconstructed detector or simulated Monte Carlo data) in a compressed form and all events are labeled with specific tags, for example »dielectron «, or »dimuon « to save the time for unpacking certain events when processing the TMB files. Trigger information, which is only available for the real detector data, is stored in an uncompressed form. The complete event size is around $25 \mathrm{kB}$, about a factor of ten smaller than the DST format.

All data, simulated Monte Carlo and real data, are stored in the SAM database, allowing for easy access from around the world. Apart from the tape storage in the Fermilab Computing Center, or, in case of reconstructed Monte Carlo data, which is also produced and stored at different computing facilities in other countries (e.g. France, Great Britain, or Germany), the most frequently used data is also kept cached on disk servers. Users communicate with the SAM system via metadata commands and SAM handles everything that is not directly visible to the user: data transfer to and from the tape storage facilities, allocation and monitoring of computing resources and cache space, bookkeeping at the user process level, etc. While the bookkeeping functions are provided by an external database, all other functionality is implemented in the SAM software. The concept of SAM is similar, though not yet as sophisticated and as distributed as the concepts of grid-computing developed for the LHC-era, on which the LHC-experiments will heavily rely for their enormous data storage and processing needs.

The present analysis uses data in TMB format as starting point and, although it is possible to write analysis code for TMB data, this method is deemed ineffective, since compilation and debugging times are rather long. Therefore the TMB data is transformed into a private analysis format, based on the ROOT-tree format, to make use of the more convenient and flexible possibilities of this format.

Simulated data used in the present analysis has been generated with the Monte Carlo event generators PYTHIA [124] for background processes and SUSYGEN [125] for SUSY signal processes. The MC generators produce 4-vectors of particles that need to be traced through the detector and have all effects simulated. D $\emptyset$ uses two separate programs to simulate how generated particles would look like as seen by the detector. The first program, D Øgstar (DØGeant Simulation of the Total Apparatus Response) [126] is based on GEANT [127] and simulates the particle trajectories, energy depositions, and all interactions with the detector material. The second program, D $\emptyset$ sim [128] uses D $\emptyset$ gstar output as input and simulates the detector response, i.e. electrical pulses of different lengths and amplitudes and thus provides the digitisation of the simulated data. Analog and electronic noise from the detector and readout, as well as detector 
inefficiencies are taken into account in the simulations. Also considered are effects due to pileup and the underlying event. Multiple interactions are simulated by adding so-called minimum bias events to the generated physics events. Minimum bias events are simulated events of $p \bar{p}$ scattering (elastic or inelastic) that the detector would have registered and where at least one of the luminosity monitors would have fired. These events are generated with PyTHIA, using the $\mathrm{CDF} »$ Tune $\mathrm{A}$ « [129] obtained from CDF measurements of jet evolution and the underlying event during Run I of the Tevatron.

As can be seen from Figure 2.46, simulated MC data (*.sim files) and raw detector data (*.raw files) are both reconstructed with the $\mathrm{D} \varnothing$ reconstruction code, D $\varnothing$ reco [130]. DØreco comprises reconstruction algorithms for vertexing, track-finding and clustering to the point of using the primary objects (vertices, tracks, clusters) to reconstruct high-level physics objects, such as electrons, muons, jets, $\not_{T}$, secondary vertices, and use these in turn to identify candidates for heavy quarks or tau decays for example. As mentioned, so far all data is re-reconstructed from time to time, usually every one to two years, after a major upgrade of the reconstruction software, which is also called production release. All data used in this analysis has been re-reconstructed with release p14.06.01, containing many improvements and serving as a major production release for the following one and a half years.

Before the reconstructed TMB data are converted into ROOT format, the thumbnails are skimmed ${ }^{13}$, which means that the events are sorted into various samples (skims) [131] according to their physics objects content: e.g. »single electron skim «, »single muon skim «, »dimuon skim «, »jet skim «, etc. A single event can be part of more than one skim, although each event should only appear once per skim. In this analysis the »dimuon skim « is used and its TMB data is transformed into the more convenient and flexible ROOT format using the top_analyze package [132], derived from an example of the $D \varnothing$ framework program $D \varnothing$ ChunkAnalyze. Apart from the format conversion, the framework package also runs additional algorithms and applies further corrections to the data that are not yet included in the main reconstruction code. These are, for example, jet energy scale corrections, advanced b-tagging algorithms, a more sophisticated noise rejection and also corrections of the efficiency and resolution of jets and $\not_{T}$. The top_analyze package was developed within the Top Group [133].

At the next stage (user level), the ROOT-trees are preselected, corresponding to the preselection sample of this analysis, and written to new ROOT-trees, as indicated by the ReFill program. In addition, the TriggerEfficiency package [134] is included, which serves to estimate the effect of trigger (in)efficiencies on the preselected Monte Carlo event samples. A detailed overview of the procedure is given in section 4.3.1.

Unnormalisable luminosity blocks and physics runs, where one of the necessary detector subsystems was not fully functional, are removed at this stage, as are events with noisy calorimeter cells or other detector related problems. In the end, the output ROOT-trees from ReFill only contain dimuon events, triggered by at least one of a set of dimuon triggers, corresponding to a definite luminosity (see again section 4.3.1, details about the applied procedure can be found in [134]). The luminosity is separately calculated, using a tool named runrange_luminosity [135]. Besides the luminosity to which a certain data sample corresponds, it also outputs a list of

\footnotetext{
${ }^{13}$ to skim: to remove the best or most easily obtainable contents from something; to (read, study) examine sth. superficially and rapidly for the chief ideas or the plot; having the cream removed by skimming.
} 


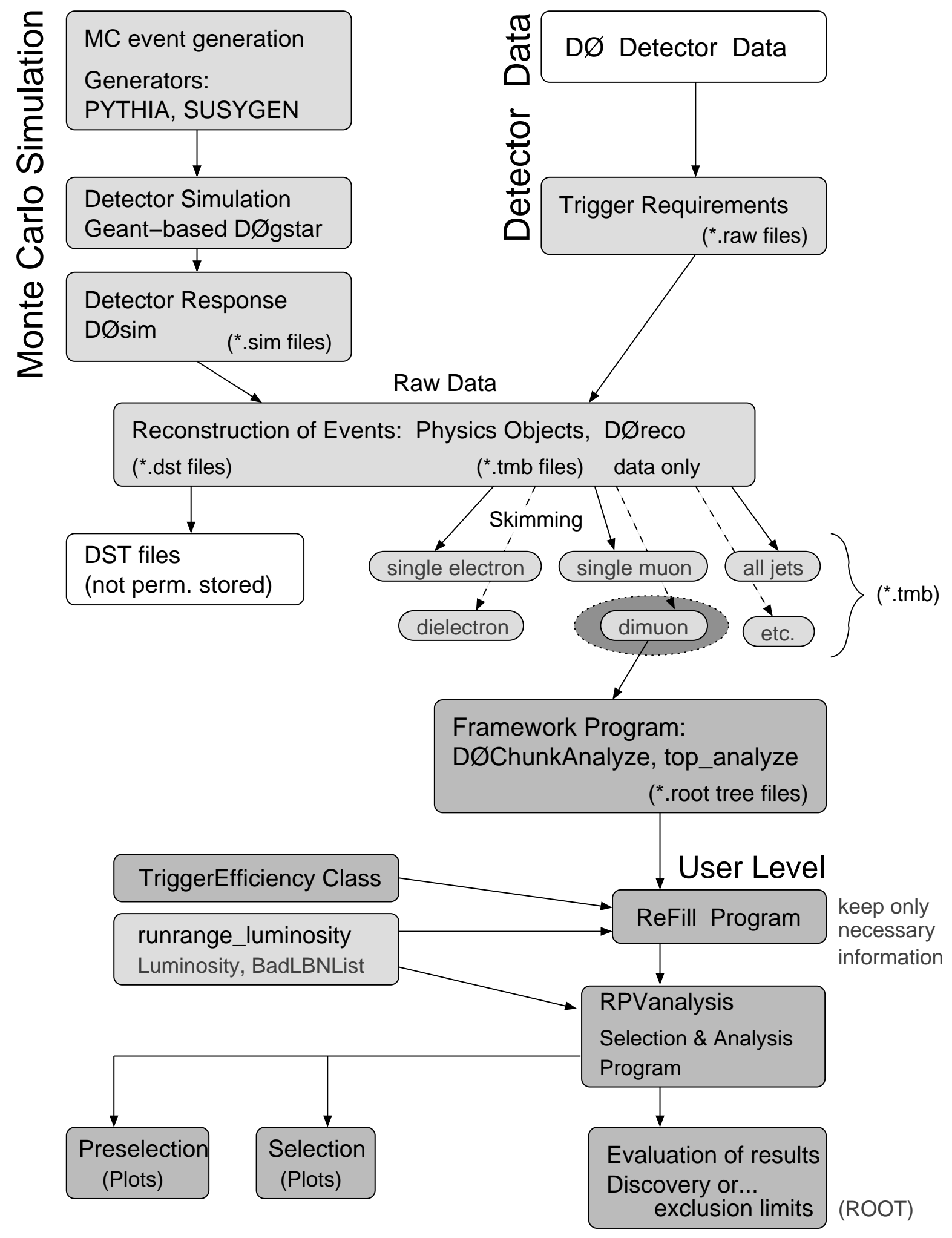

Figure 2.46: Analysis chain for DØ detector data and Monte Carlo simulated data. Software packages shaded light-grey are maintained centrally, whereas darker-grey shaded programs/packages are either maintained by analysis subgroups (e.g. the Top Group responsible for the top_analyze package [132], or the Top Trigger Group maintaining the TriggerEfficiency class [134]) or single users. Files in DST format can also be converted into ROOT-trees using a framework package like top_analyze, but since this feature is not utilised here, this branch is not pursued any further. 
unusable luminosity block numbers (BadLBNList), that have to be removed from the analysis data sample. Per event only about 75 high-level variables are kept, among these are the reconstructed dimuon invariant mass, jet energy scale corrected $\not_{T}$, different angular separations between various leptons, and leptons and $\mathbb{F}_{T}$, and event weights due to trigger parametrisations of the TriggerEfficiency class.

Following the ReFill program, the final analysis program RPVanalysis, which has been developed to search for events compatible with $\not R_{p}$-SUSY (see chapter 4 , especially sections 4.1 and 4.5 ), is run on the remaining events (data and MC samples). At this level, the MC events are weighted according to the equivalent luminosity, making again use of the outputs of the runrange_luminosity program. Control distributions are generated, and the resulting numbers of events of data and expected MC background events (with their respective statistical and systematic uncertainties) after each successive application of selection criteria are saved.

The last program serves to evaluate the results. It decides if the observation of an excess of events in the data is compatible with a signal from $\not R_{p}$-SUSY, or it calculates the expected (observed) cross section upper limits at $95 \%$ confidence level (CL) in case no deviation is found. Further details will be given later in chapter 5, mainly in subsections 5.1.3 and 5.1.4. 


\section{Chapter 3}

\section{Phenomenology of $\not p_{p}$-SUSY and Signal MC Simulation Studies}

Tug on anything at all, and you'll find it connected to everything else in the universe.

John Muir (Environmentalist, 1838-1914)

This chapter provides a short overview of $\not_{p}$-SUSY phenomenology at the Tevatron Collider. The production of gauginos and the usage of $K$-factors are treated in sections one and two, while section three provides an overview of the decays and branching ratios of the second lightest neutralino and the lightest chargino. Section four, finally, summarises generator level studies of signal Monte Carlo samples. All signal samples are produced with the event generator SusYgen [125], which is interfaced with a separate program, Suspect [136], for the evolution of masses and couplings through the renormalisation group equations. Signal properties, such as production cross sections, masses, and branching ratios, and also kinematic variables of the resulting leptons, are studied in detail.

\subsection{Gaugino Pair Production at the Tevatron}

Only one of the $\not_{p}$-couplings is assumed to be non-zero, while all others are either zero or negligibly small (single coupling dominance hypothesis [59], [61], see also section 1.3.6). In particular, the $L L \bar{E}$ coupling $\lambda_{122}$ is assumed to be dominant in this analysis. As a consequence, SUSY particles can only be produced in pairs at a $p \bar{p}$ collider like the Tevatron, since there are no leptons in the initial state which would be required for a production of single sparticles.

Within the domain of the explored SUSY parameter space, pair production of gauginos is the dominant process for large values of $m_{0}$. At small $m_{0}$, i.e. $m_{0} \leq 100 \mathrm{GeV}$ and low values of $m_{1 / 2}\left(m_{1 / 2} \approx 160\right.$ to $\left.260 \mathrm{GeV}\right)$, pair production of sleptons is of the same order of magnitude as gaugino pair production, i.e. 2.0 to $0.1 \mathrm{pb}$. The cross sections of squark and gluino production ( $\widetilde{q} \widetilde{q}$, $\widetilde{q} \widetilde{g}$, and $\widetilde{g} \widetilde{g}$ ) also increase, but are still about an order of magnitude smaller than the inclusive gaugino production cross section. In this analysis, only the pair production of gauginos and gaugino-gluino production is considered and all results relate to this production cross section. 
Pair production of other sparticles, e.g. of sleptons or squarks is not taken into account, even though this would enlarge the cross section and thus also the possibility for decays into leptons in the region of small $m_{0}$ values.

From the gaugino pair production processes, $\widetilde{\chi}_{1}^{ \pm} \widetilde{\chi}_{2}^{0}$ and $\widetilde{\chi}_{1}^{ \pm} \widetilde{\chi}_{1}^{\mp}$ production dominate. While pairwise production of heavier gauginos is also possible, the cross sections of these production processes are about two orders of magnitude below the ones for $\widetilde{\chi}_{1}^{ \pm} \widetilde{\chi}_{2}^{0}$ and $\widetilde{\chi}_{1}^{ \pm} \widetilde{\chi}_{1}^{\mp}$ production. Figure 3.1 illustrates the different leading-order cross sections of gaugino pair production (chargino-neutralino, chargino-chargino and neutralino-neutralino) and gaugino-gluino production for two different values of the common sfermion mass $m_{0}$ : $(\mathrm{a}, \mathrm{b})$ for $m_{0}=100 \mathrm{GeV}$ and $(\mathrm{c}, \mathrm{d})$ for $m_{0}=1000 \mathrm{GeV}$ and for both signs of the Higgs mixing parameter $(\mathrm{a}, \mathrm{c})$ for $\mu<0$, and (b,d) for $\mu>0$. The cross section of associated gaugino-gluino production is at least one order of magnitude below the inclusive cross section for gaugino production for $m_{0}=100$ and $\mu<0$ (a), while this difference increases to nearly three orders of magnitude for $m_{0}=1000$ and $\mu>0$ (d). In general, all cross sections decrease with increasing gaugino mass parameter $m_{1 / 2}$, which is expected since the masses of the gauginos increase with $m_{1 / 2}$.

The dependence on the value of $A_{0}$, which governs the mixing of the third generation sfermions, is very weak. When $A_{0}$ is varied from $-1000 \mathrm{GeV}$ to $+1000 \mathrm{GeV}$, the gaugino and slepton masses change only slightly with the largest variations in the masses of staus and the top and bottom squarks. The production cross section for gaugino pair production changes only by about $10 \%$ to $15 \%$. Hence the value of the trilinear coupling is fixed at the GUT scale to $A_{0}=0$ and not varied in what follows. The masses and cross sections also vary slightly with $\tan \beta$, the ratio of the vacuum expectation values of the two Higgs fields. The lightest neutralino, for example, is up to $3 \mathrm{GeV}$ lighter for $\tan \beta=20$ compared to $\tan \beta=5$, while the mass of the lightest chargino even decreases by up to $15 \mathrm{GeV}$ (in case of negative Higgs mixing parameter $\mu$ ). On the other hand, the production cross sections increase by roughly $25-30 \%$, when $\tan \beta$ increases from $\tan \beta=5$ to $\tan \beta=20$, with exceptions at $m_{0}=250 \mathrm{GeV}$ and $1000 \mathrm{GeV}$ for positive $\mu$. There, the production cross sections decrease by about $15-20 \%$ which can be seen in Tables A.1 and A.2 in the appendix. These differences directly translate into higher (resp. lower) cross section limits and the corresponding lower mass bounds.

In case heavier gaugino mass eigenstates are produced, these cascade decay into the lightest supersymmetric particle (LSP), since the $\not R_{p}$-coupling of SM particles to SUSY particles is much smaller than the gauge couplings of SUSY particles. For the mSUGRA signal Monte Carlo simulations the $\not R_{p}$-coupling is chosen to be $\lambda_{122}=0.001$, while 0.01 is used for the production of the MSSM signal samples. Both values are clearly below the current upper bound of $\lambda_{122}<0.027 \sqrt{m\left(\widetilde{\mu}_{R}\right) / 100 \mathrm{GeV}}$ which is derived from the upper bound on the electron neutrino mass $m\left(\nu_{e}\right)<1 \mathrm{eV}$ (see also section 1.3.8).

The strength of the $\lambda$ coupling anyhow only influences the lifetime (decay length) of the LSP, $c \tau\left(\widetilde{\chi}_{1}^{0}\right)$, which can be expressed in terms of its mass, $m\left(\widetilde{\chi}_{1}^{0}\right)$, the sfermion mass, $m_{\tilde{f}}$, and the coupling strength, $\lambda_{i j k}[137]$ :

$$
c \tau\left(\widetilde{\chi}_{1}^{0}\right)[\mathrm{cm}] \approx 0.3 \mathrm{~cm} \cdot\left(\frac{m(\widetilde{f})}{100 \mathrm{GeV}}\right)^{4} \cdot\left(\frac{1 \mathrm{GeV}}{m\left(\widetilde{\chi}_{1}^{0}\right)}\right)^{5} \cdot \frac{1}{\lambda_{i j k}^{2}} .
$$

Within the mSUGRA model, a neutralino lower mass bound of $m\left(\widetilde{\chi}_{1}^{0}\right)>52 \mathrm{GeV}$ can be derived from the lower bound on the chargino mass $m\left(\widetilde{\chi}_{1}^{ \pm}\right)>103.5 \mathrm{GeV}$, which results from searches for 

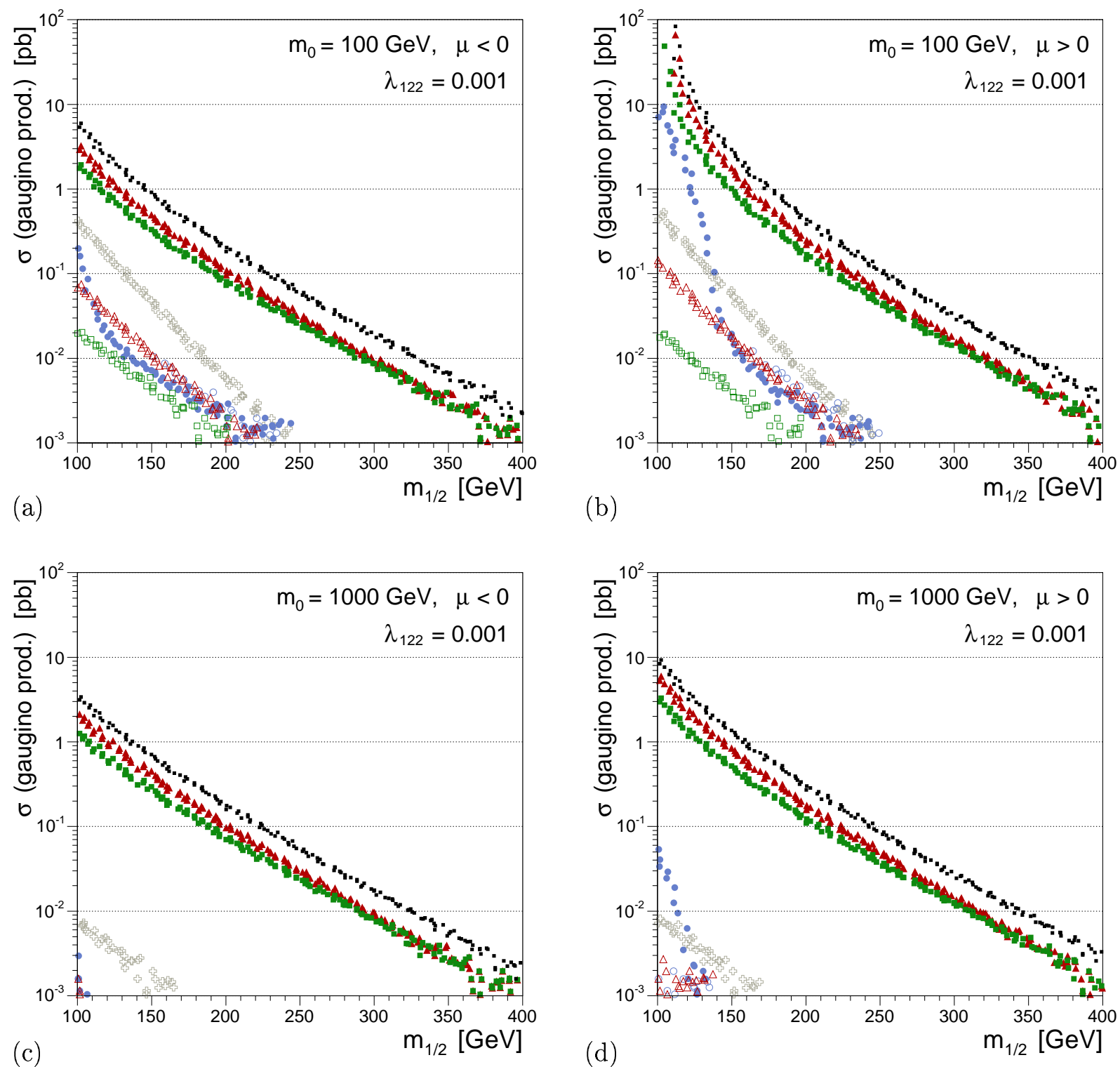

- Gaugino pair production

\ $\tilde{\chi}_{1}^{ \pm} \tilde{\chi}_{1}^{0}+\tilde{\chi}_{1}^{ \pm} \tilde{\chi}_{2}^{0}$

$\Delta \tilde{\chi}_{2}^{ \pm} \tilde{\chi}_{1}^{0}+\tilde{\chi}_{2}^{ \pm} \tilde{\chi}_{2}^{0}+\tilde{\chi}_{1,2}^{ \pm} \tilde{\chi}_{3,4}^{0}$

- $\tilde{\chi}_{1}^{ \pm} \tilde{\chi}_{1}^{ \pm}$

ㅁ $\tilde{\chi}_{1}^{ \pm} \tilde{\chi}_{2}^{ \pm}+\tilde{\chi}_{2}^{ \pm} \tilde{\chi}_{2}^{ \pm}$

- $\tilde{\chi}_{1}^{0} \tilde{\chi}_{1}^{0}+\tilde{\chi}_{1}^{0} \tilde{\chi}_{2}^{0}$

- $\tilde{\chi}_{2}^{0} \tilde{\chi}_{2}^{0}+\tilde{\chi}_{1,2,3,4}^{0} \tilde{\chi}_{3,4}^{0}$

$\tilde{\chi}_{1}^{0} \tilde{\mathrm{g}}+\tilde{\chi}_{1}^{ \pm} \tilde{\mathrm{g}}$
Figure 3.1: Gaugino pair and gaugino-gluino production cross sections at LO (SUSYGEN) as a function of the gaugino mass parameter $m_{1 / 2}$ for negative $(a, c)$ and positive $(b, d)$ Higgs mixing parameter $\mu$.

The cross sections for chargino-neutralino production, chargino pair and neutralino pair production, and chargino/neutralino-gluino production are shown separately, as are the production cross sections of light and heavy gaugino mass eigenstates. All cross sections decrease with increasing $m_{1 / 2}$, which is due to the increasing gaugino masses governed by $m_{1 / 2}$. The remaining mSUGRA parameters are set to $\tan \beta=5$ and $A_{0}=0$. 
$\not R_{p}$-SUSY performed by the four LEP experiments [138]. In analogy to the present analysis, the LEP searches were also performed assuming only one dominant $L L \bar{E}$ coupling with the coupling strength chosen such, that the LSP lifetime was negligible. In case of the $\lambda_{122}$ coupling, the then valid upper limit on the coupling strength was $\lambda_{122}<0.049 \sqrt{m\left(\widetilde{\mu}_{R}\right) / 100 \mathrm{GeV}}$, for further details the reader is referred to [138], [139]. As shown in the latter reference, the obtained results are valid for all values of $\tan \beta \geq 5$, while the lower bound on the neutralino mass decreases to approximately $30 \mathrm{GeV}$ for lower values of $\tan \beta$. Table 3.1 presents a few examples for extreme values of $m\left(\widetilde{\chi}_{1}^{0}\right)$ and $m_{0}$, which corresponds to the common sfermion mass $m(\widetilde{f})$. Since Equation (3.1) is only an approximation, the SUSYGEN generated values for the mass parameters $m_{0}, m_{1 / 2}$, the neutralino mass, and its respective decay length are given in Table 3.1.

\begin{tabular}{|c|c|c|c|c|c|c|c|}
\hline \multicolumn{4}{|c|}{ 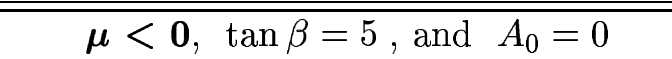 } & \multicolumn{4}{|c|}{ 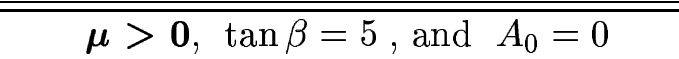 } \\
\hline $\begin{array}{c}m_{0} \\
{[\mathrm{GeV}]}\end{array}$ & $\begin{array}{r}m_{1 / 2} \\
{[\mathrm{GeV}]}\end{array}$ & $\begin{array}{c}m\left(\widetilde{\chi}_{1}^{0}\right) \\
{[\mathrm{GeV}]}\end{array}$ & $\begin{array}{c}c \tau\left(\widetilde{\chi}_{1}^{0}\right) \\
{[\mathrm{mm}]}\end{array}$ & $\begin{array}{c}m_{0} \\
{[\mathrm{GeV}]}\end{array}$ & $\begin{array}{r}m_{1 / 2} \\
{[\mathrm{GeV}]}\end{array}$ & $\begin{array}{c}m\left(\widetilde{\chi}_{1}^{0}\right) \\
{[\mathrm{GeV}]}\end{array}$ & $\begin{array}{c}c \tau\left(\widetilde{\chi}_{1}^{0}\right) \\
{[\mathrm{mm}]}\end{array}$ \\
\hline 100 & 150 & 61.0 & 0.0044 & 100 & 190 & 69.8 & 0.0030 \\
\hline 100 & 230 & 93.8 & 0.0008 & 100 & 220 & 83.2 & 0.0015 \\
\hline 100 & 330 & 135.3 & 0.0002 & 100 & 340 & 135.1 & 0.0002 \\
\hline 250 & 140 & 57.9 & 0.1180 & 250 & 160 & 57.2 & 0.1355 \\
\hline 250 & 220 & 90.6 & 0.0142 & 250 & 220 & 84.3 & 0.0209 \\
\hline 250 & 280 & 115.4 & 0.0047 & 250 & 280 & 110.3 & 0.0061 \\
\hline 1000 & 230 & 98.2 & 1.8577 & 1000 & 220 & 88.8 & 3.0990 \\
\hline 1000 & 270 & 114.9 & 0.8497 & 1000 & 260 & 105.9 & 1.2888 \\
\hline 1000 & 330 & 140.4 & 0.3152 & 1000 & 340 & 140.2 & 0.3202 \\
\hline
\end{tabular}

Table 3.1: The decay length of the lightest neutralino, $c \tau\left(\widetilde{\chi}_{1}^{0}\right)$, as a function of its mass, $m\left(\widetilde{\chi}_{1}^{0}\right)$, and the common sfermion mass, $m_{0}$ for negative $\mu$ (left-hand side) and positive $\mu$ (right-hand side). The gaugino mass parameter $m_{1 / 2}$ is also listed.

On the other hand, within the no-GUT MSSM scenario, there is no lower bound on the neutralino mass, since it is impossible - due to the non-existing GUT relation between $M_{1}$ and $M_{2}$ - to extract a bound on the neutralino mass from an existing bound on the chargino mass. As a result, the neutralino mass is unconstrained in this scenario and could even lie below $10 \mathrm{GeV}$. However, neutralino masses below $30 \mathrm{GeV}$ are not studied due to the combined effect of the coupling strength and the $\widetilde{\chi}_{1}^{0}$ (LSP) lifetime. To be able to rely on the tracking with the DØ detector, a cutoff is introduced at a decay length of $1 \mathrm{~cm}$. Neutralinos with longer lifetimes, i.e. $c \tau\left(\widetilde{\chi}_{1}^{0}\right)>1 \mathrm{~cm}$, are treated as stable particles in the SUSYGEN event generator and are passed on to the detector simulation software. Since this latter can only handle SM particles, and no SUSY or other exotic particles, it can thus not take care of the neutralino decays neither. As a result, the neutralinos are stable and escape detection, which is why the present analysis is insensitive to neutralino masses below $30 \mathrm{GeV}$. 


\subsection{Next-to-leading Order Signal Cross Sections}

Since SusYgen cannot calculate cross sections of SUSY processes at next-to-leading order (NLO), the separate program GAUGINOs [140] is used to calculate the cross section for gaugino pair production at leading order (LO) and at next-to-leading order. From these two, the ratio (or $K$-factor) is calculated as:

$$
K \text {-factor }=\sigma_{N L O}^{\text {Gauginos }} / \sigma_{L O}^{\text {Gauginos }},
$$

with which the Susygen LO cross section is then multiplied. For all mSUGRA and MSSM signal points, produced with the SUSYGEN event generator, the appropriate $K$-factor is calculated separately using Gauginos. This procedure was chosen, because Susygen and Gauginos do not use the same set of renormalisation group equations and hence produce different LO cross sections, so that the GaUginos NLO cross section could not be used directly. The $K$-factors vary between 1.27 for low values of the gaugino mass parameter $m_{1 / 2}$ to 1.13 for high values of $m_{1 / 2}$, but are rather stable w.r.t. the mSUGRA parameters $m_{0}$ and $\mu$. Figure 3.2 shows the $K$-factors for (a) $m_{0}=100 \mathrm{GeV}$ and (b) $m_{0}=1000 \mathrm{GeV}$ and both signs of $\mu$. The relative systematic uncertainty on the $K$-factor is approximately $3 \%$, which is due to the calculation of the LO and NLO cross sections with GaUginos.
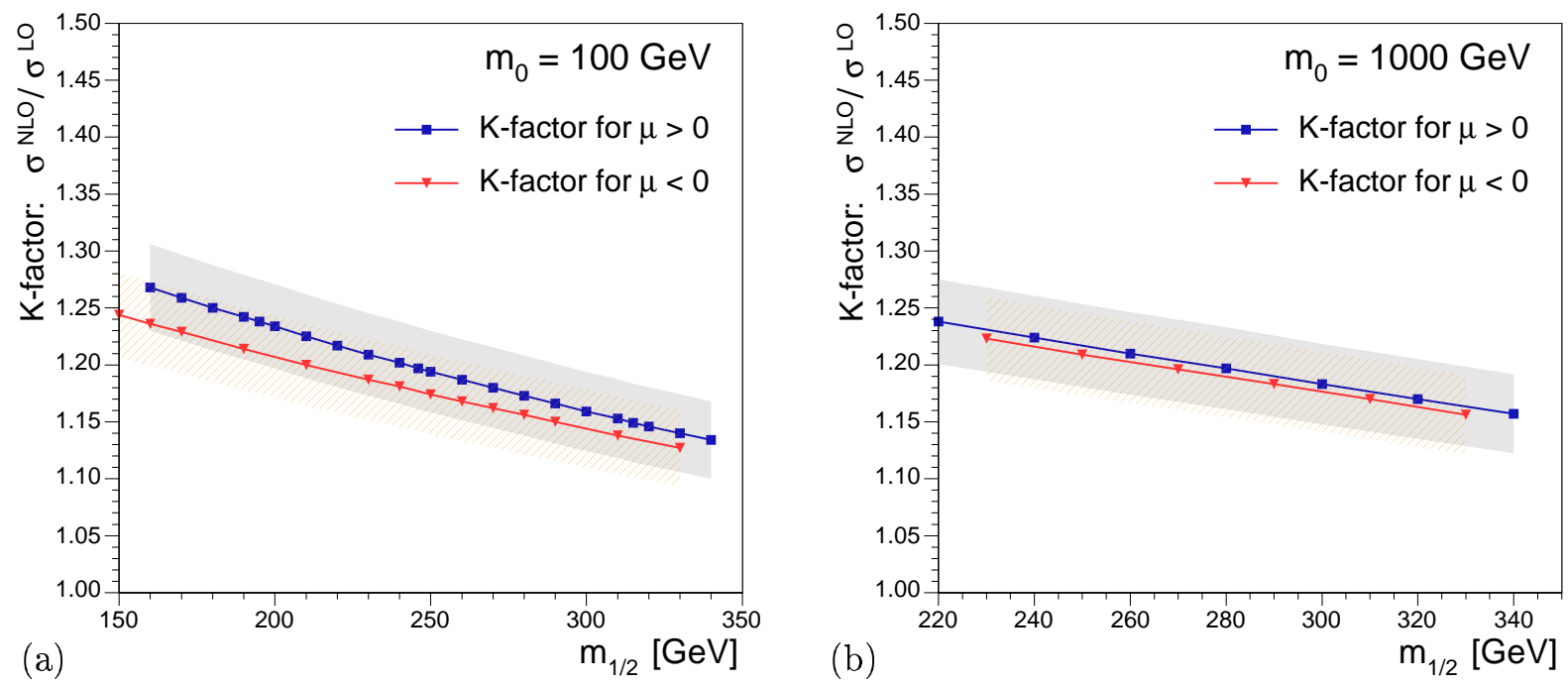

Figure 3.2: The $\sigma^{N L O} / \sigma^{L O} K$-factors as calculated with GAUGINOS as a function of the gaugino mass parameter $m_{1 / 2}$ and for (a) $m_{0}=100 \mathrm{GeV}$ and (b) $m_{0}=1000 \mathrm{GeV}$ and for both signs of the Higgs mixing parameter $\mu$. The remaining mSUGRA parameters are chosen as $\tan \beta=5$ and $A_{0}=0$.

The possible effect of the choice of the renormalisation and factorisation scales on the NLO signal cross section is studied by varying both scales independently by a factor of two. A systematic uncertainty of $5 \%$ on the $K$-factor is estimated from comparing GaUGinos LO and NLO cross sections calculated for a renormalisation scale $\mu_{r}$ and a factorisation scale $\mu_{f}$ with those calculated for $2 \cdot \mu_{r, f}$, as well as for $\frac{1}{2} \cdot \mu_{r, f}$. 


\subsection{Cascade Decays and Branching Ratios of $\widetilde{\chi}_{2}^{0}$ and $\widetilde{\chi}_{1}^{ \pm}$}

The neutralino LSP decays into two charged leptons and one neutrino, with the lepton flavours depending on the $\not R_{p}$-coupling $\lambda_{i j k}$. In particular, for $\lambda_{122}$, the charged leptons are either muons or electrons. Final states with at least four charged and two neutral leptons are expected from the $\not R_{p}$-decays of the two LSPs alone. Besides these four, additional charged leptons may be produced in the cascade decays, for example via intermediate $W$ or $Z$ boson exchange.

To facilitate the discussion of certain regions in the SUSY parameter space, the following figures provide an overview of the masses of SUSY particles and the branching ratios of the second lightest neutralino and the lightest chargino as a function of the gaugino mass parameter $m_{1 / 2}$. Figure 3.3 shows the sparticle masses for (a) $m_{0}=100 \mathrm{GeV}$ and (b) $m_{0}=250 \mathrm{GeV}$ for positive Higgs mixing parameter $\mu$, and for (c) $m_{0}=100 \mathrm{GeV}$ and negative $\mu$. Although all intersections
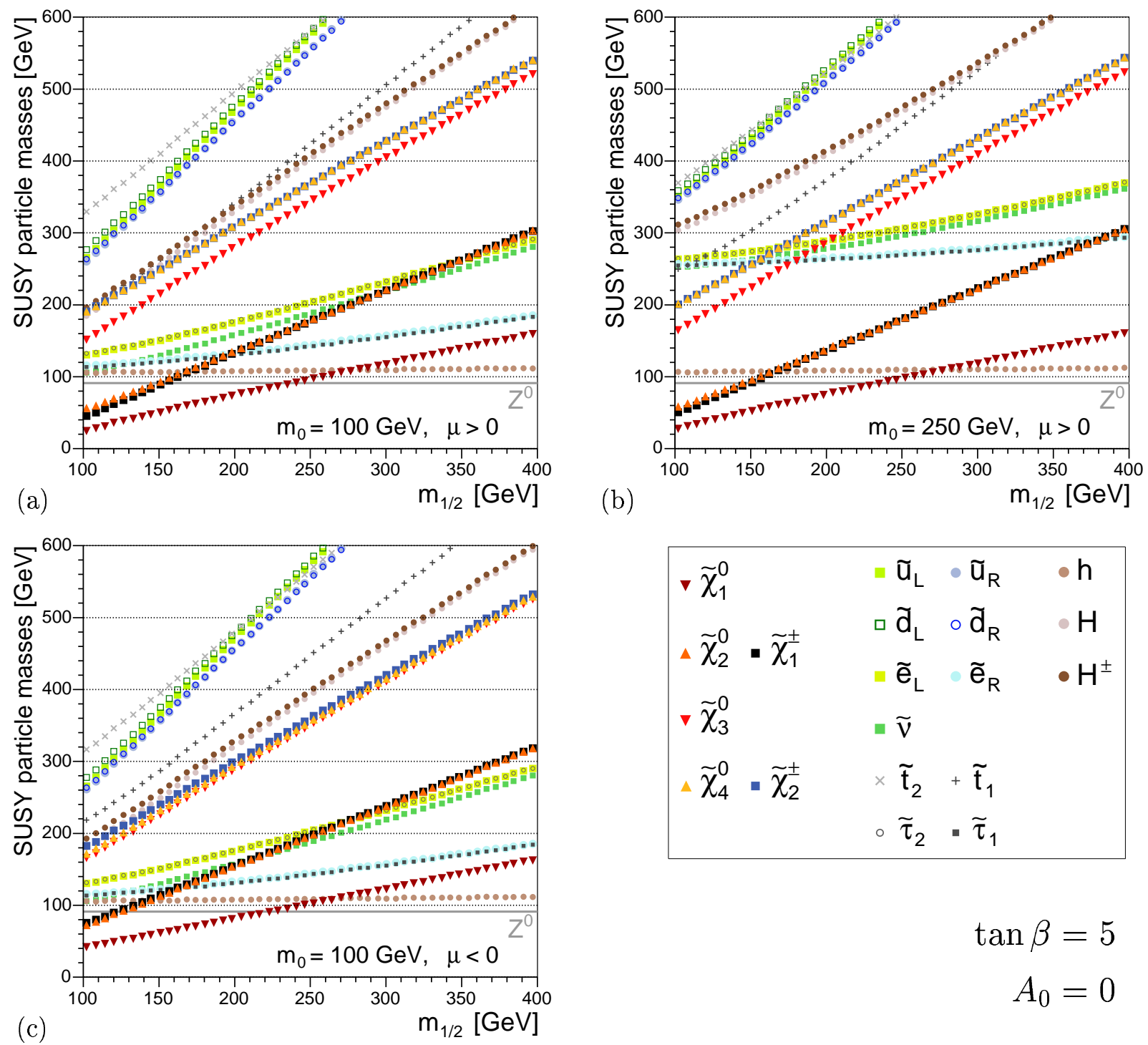

$$
\begin{aligned}
\tan \beta & =5 \\
A_{0} & =0
\end{aligned}
$$

Figure 3.3: SUSY particle masses as a function of $m_{1 / 2}$ for positive $\mu$ and $m_{0}=100 \mathrm{GeV}(a), m_{0}=$ $250 \mathrm{GeV}$ (b), and (c) for negative $\mu$ and $m_{0}=100 \mathrm{GeV}$. While $\mu$ has only a small influence on the gaugino masses, the increase of the common scalar mass, $m_{0}$, causes large differences in the sfermion and Higgs boson masses, compare Figure 1.9. 
between the slepton and the gaugino masses are shifted towards lower values of $m_{1 / 2}$, (due to the slightly larger gaugino masses), the overall behaviour of the sparticle masses in dependence of $m_{1 / 2}$ is the same for positive and negative Higgs mixing parameter.

Of interest are especially the light sleptons, i.e. the left- and right-handed superpartners of the SM leptons, $\widetilde{\ell}_{R}, \widetilde{\ell}_{L}$, and $\widetilde{\nu}$, (denoted $\widetilde{e}_{R}, \widetilde{e}_{L}, \widetilde{\tau}_{1}, \widetilde{\tau}_{2}$, and $\widetilde{\nu}$ in the legend of Figure 3.3 ) and the lightest, supersymmetric Higgs boson, the CP-even $h$. As can be seen from the figure, not only the masses of the lightest chargino and the second lightest neutralino (the second chargino and the fourth neutralino) are quasi-degenerate, but also the following eigenstates are quasi mass-degenerate: the right-handed and the left-handed sleptons, respectively, and the left-handed (right-handed) squarks, with the exception of the $\widetilde{t}_{1,2}$ and $\widetilde{b}_{1,2}$ mass eigenstates which are somewhat special due to the mixing in the third generation. The masses of the bottom squarks are not shown separately, since they lie between those of the lighter and heavier top squark masses (dark and light grey crosses) and very close to the up- and down-type squark masses, so that a differentiation would become very difficult.

The characteristics of the sparticle mass curves will not be discussed separately, but in combination with the following four figures of branching ratios of the second lightest neutralino $\widetilde{\chi}_{2}^{0}$ and the lightest chargino $\widetilde{\chi}_{1}^{ \pm}$, which are the most abundantly produced gauginos. Figures 3.4 and 3.5 provide an overview of these branching ratios for positive Higgs mixing parameter $\mu$, and Figures 3.6 and 3.7 for negative $\mu$. The left-hand side figures (a,c,e) always show the second lightest neutralino branching ratios, while the right-hand side figures $(\mathrm{b}, \mathrm{d}, \mathrm{f})$ show the branching ratios of the lightest chargino.

In general, three different decay scenarios can be distinguished:

Two-body decay: into a sfermion-fermion pair, if the decay is kinematically allowed. The sfermion then decays further into a fermion and usually the LSP.

Three-body decay: into a lighter gaugino mass eigenstate and a pair of fermions via on- or off-shell gauge $(W, Z)$ or Higgs bosons or a virtual sfermion-fermion pair, of which the sfermion decays again into the LSP and an ordinary fermion.

Direct decay: into SM particles. Via the $\not R_{p}$-coupling $\lambda_{122}$, the neutralinos decay in equal shares into $\mu \mu \nu_{e}$ and $\mu e \nu_{\mu}$, while direct chargino decays lead to final states composed of $\mu \mu e(50 \%), \mu \nu_{\mu} \nu_{e}(25 \%)$ and $e \nu_{\mu} \nu_{\mu}(25 \%)$. However, since the $\mathbb{R}_{p}$-coupling is small, direct decays of heavier gaugino mass eigenstates $\left(\widetilde{\chi}_{3}^{0}, \widetilde{\chi}_{4}^{0}\right.$, and both charginos) are negligible.

In case there is a light sfermion with a mass below the masses of the two nearly mass degenerate gauginos, $\widetilde{\chi}_{2}^{0}$ and $\widetilde{\chi}_{1}^{ \pm}$, these two may decay via two-body decay, producing the light sfermion and its associated SM fermion, see also Figure 3.3. In general, for low values of the common scalar mass $m_{0}$, the lighter mass eigenstate of the third generation sleptons and in particular the superpartners of the right-handed first (and second) generation leptons are candidates for the next-to-lightest supersymmetric particle (NLSP), and thus candidates for a two-body decay of the $\widetilde{\chi}_{2}^{0}$ and/or the $\widetilde{\chi}_{1}^{ \pm}$.

Whenever kinematically possible, the $\widetilde{\chi}_{2}^{0}$ decays more likely into left-handed sleptons than for example into the $\widetilde{\tau}_{1}$, which is mostly right-handed. This is due to the fact that the $\widetilde{\chi}_{2}^{0}$ is gauginolike, with a large wino component and rather small bino and higgsino components (see also 

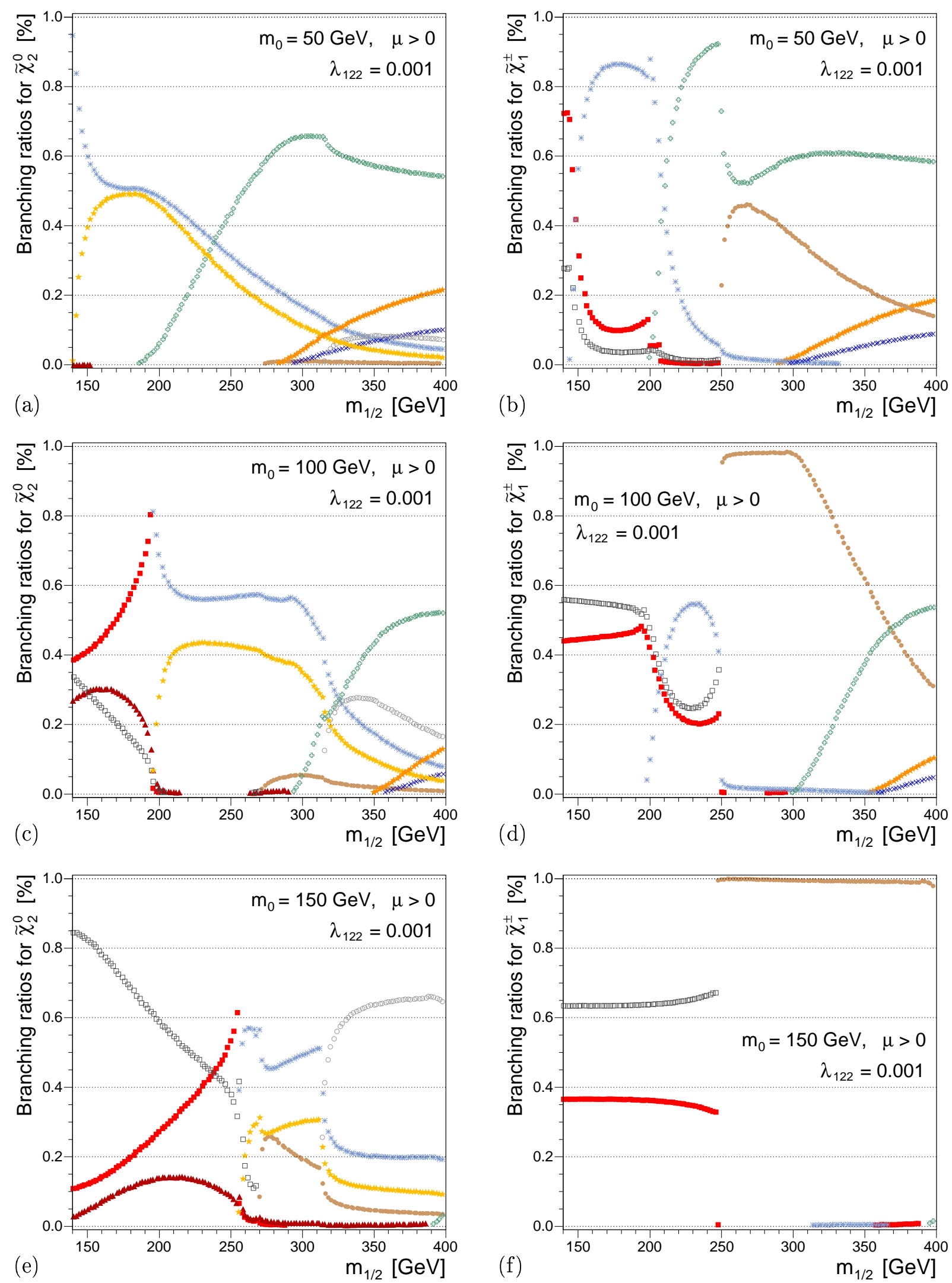

Figure 3.4: The branching ratios of the second lightest neutralino $(a, c, e)$ and the lightest chargino $(b, d, f)$ as a function of $m_{1 / 2}$ for positive Higgs mixing parameter $\mu, \tan \beta=5, A_{0}=0$, and $m_{0}$ as indicated. The BRs into electrons and muons are degraded in a narrow corridor region for $m_{0}=100 \mathrm{GeV}$ and $m_{1 / 2}=170-220 \mathrm{GeV}$, where the dominant decays are $\widetilde{\chi}_{2}^{0} \rightarrow \widetilde{\tau}_{1} \tau$ and $\widetilde{\chi}_{1}^{ \pm} \rightarrow \widetilde{\tau}_{1} \nu_{\tau}$. Decays with a branching ratio above 0.5\% (threshold) are listed. (See legend in Fig. 3.5.) 

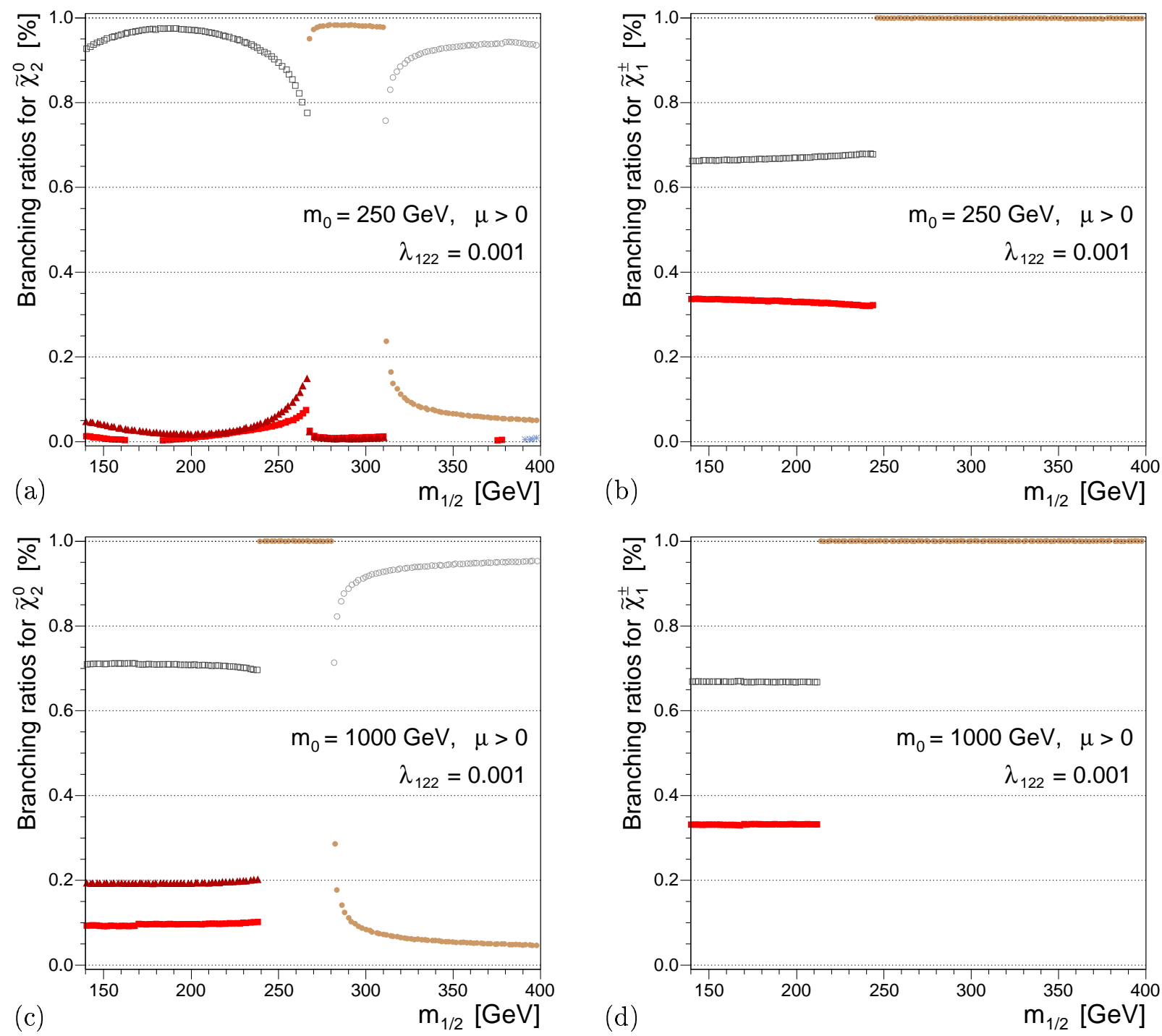

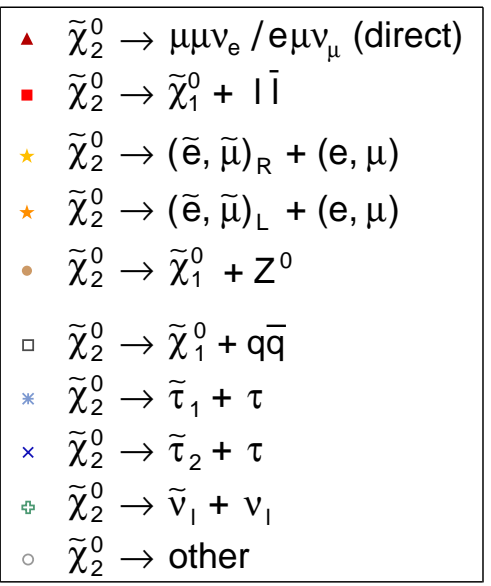

\begin{tabular}{|c|}
\hline $\begin{array}{l}\tilde{\chi}_{1}^{ \pm} \rightarrow \tilde{\chi}_{1}^{0}+\mid v_{1} \\
\tilde{\chi}_{1}^{ \pm} \rightarrow(\widetilde{e}, \tilde{\mu})_{L}+\left(v_{e}, v_{\mu}\right) \\
\tilde{\chi}_{1}^{ \pm} \rightarrow \tilde{\chi}_{1}^{0}+W^{ \pm} \\
\tilde{\chi}_{1}^{ \pm} \rightarrow \tilde{\chi}_{1}^{0}+q q^{\prime} \\
\tilde{\chi}_{1}^{ \pm} \rightarrow \tilde{\tau}_{1}+v_{\tau} \\
\tilde{\chi}_{1}^{ \pm} \rightarrow \tilde{\tau}_{2}+v_{\tau}^{-} \\
\tilde{\chi}_{1}^{ \pm} \rightarrow \tilde{v}_{i}+I_{i}\end{array}$ \\
\hline
\end{tabular}

Figure 3.5: Continuation of Fig. 3.4: the branching ratios of the second lightest neutralino (a,c) and the lightest chargino $(b, d)$ as a function of $m_{1 / 2}$ for positive Higgs mixing parameter $\mu, \tan \beta=5, A_{0}=0$, and $m_{0}$ as indicated. At intermediate and high values of $m_{0}$ and for $m_{1 / 2}>200 \mathrm{GeV}$ the two most important decay channels are $\widetilde{\chi}_{1}^{ \pm} \rightarrow \widetilde{\chi}_{1}^{0} W$ and, in a constrained $m_{1 / 2}$ region, $\widetilde{\chi}_{2}^{0} \rightarrow \widetilde{\chi}_{1}^{0}+Z$. 

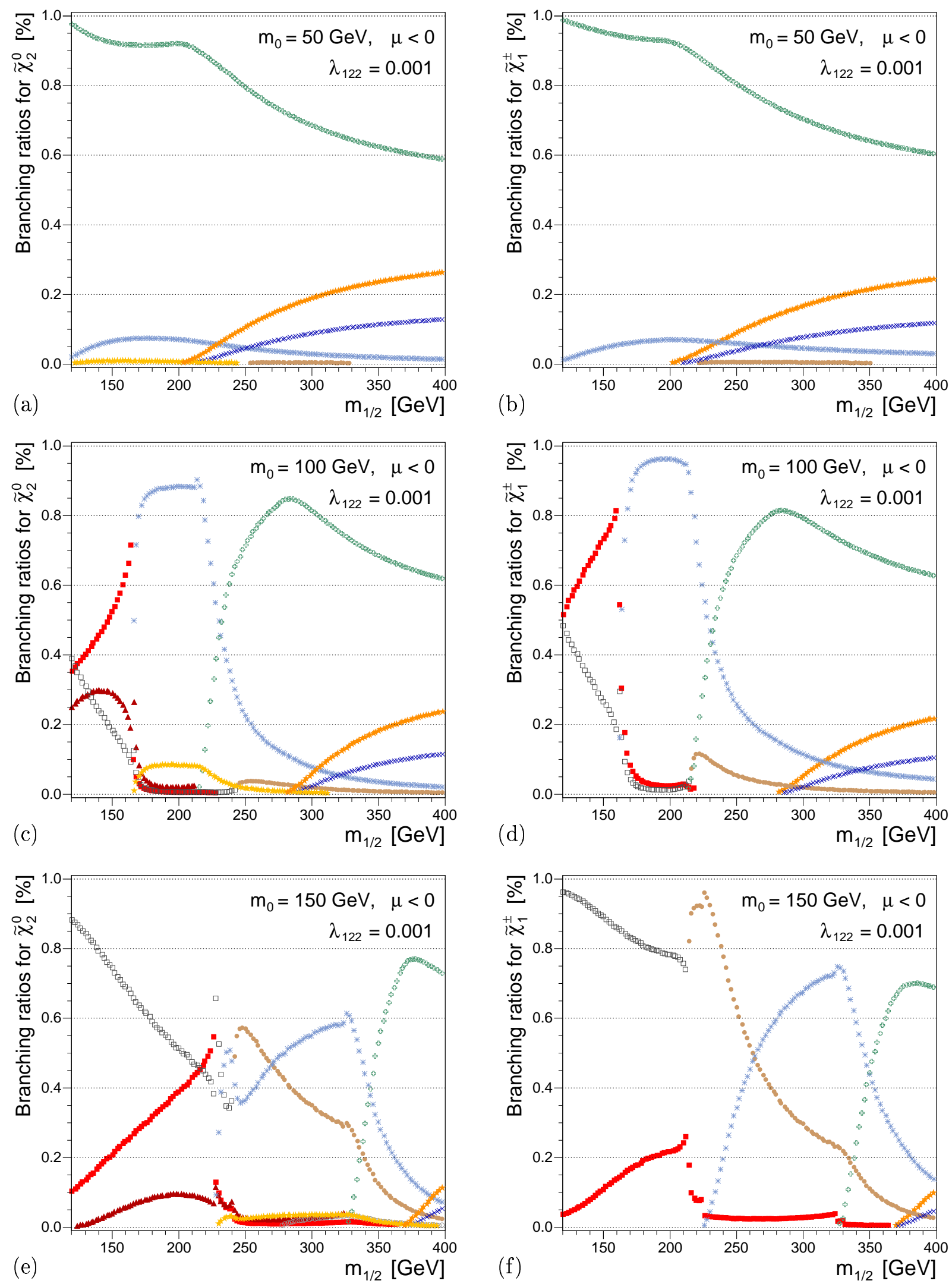

Figure 3.6: The branching ratios of the second lightest neutralino (a,c,e) and the lightest chargino $(b, d, f)$ as a function of $m_{1 / 2}$ for negative $\mu, \tan \beta=5, A_{0}=0$, and $m_{0}$ as indicated. In analogy to the case for $\mu>0$, the corridor region with degraded BRs into electrons and muons lies at $m_{1 / 2}=170-220 \mathrm{GeV}$ for $m_{0}=100 \mathrm{GeV} ;$ for $\mu<0$ the dominant decays are $\widetilde{\chi}_{2}^{0} \rightarrow \widetilde{\tau}_{1} \tau$ and $\widetilde{\chi}_{1}^{ \pm} \rightarrow \widetilde{\tau}_{1} \nu_{\tau}$. 

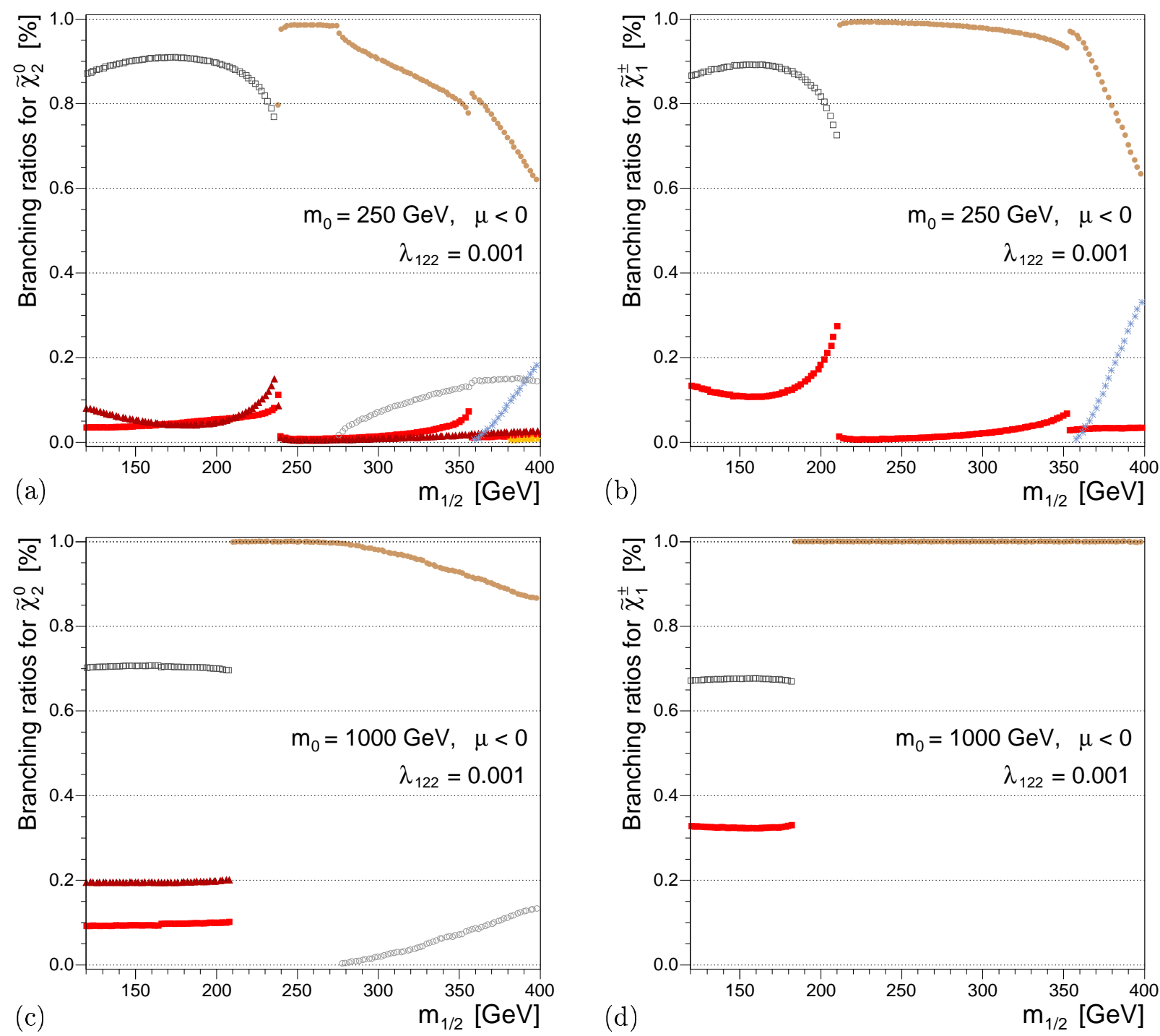

\begin{tabular}{|c|}
\hline $\begin{array}{ll}\Delta & \tilde{\chi}_{2}^{0} \rightarrow \mu \mu v_{\mathrm{e}} / \mathrm{e} \mu v_{\mu} \text { (direct) } \\
& \tilde{\chi}_{2}^{0} \rightarrow \tilde{\chi}_{1}^{0}+\mathrm{I} \overline{1} \\
\star & \tilde{\chi}_{2}^{0} \rightarrow(\widetilde{\mathrm{e}}, \tilde{\mu})_{\mathrm{R}}+(\mathrm{e}, \mu) \\
\star & \tilde{\chi}_{2}^{0} \rightarrow(\widetilde{\mathrm{e}}, \tilde{\mu})_{\mathrm{L}}+(\mathrm{e}, \mu) \\
& \tilde{\chi}_{2}^{0} \rightarrow \tilde{\chi}_{1}^{0}+Z^{0} \\
& \tilde{\chi}_{2}^{0} \rightarrow \tilde{\chi}_{1}^{0}+\mathrm{q} \bar{q} \\
* & \tilde{\chi}_{2}^{0} \rightarrow \tilde{\tau}_{1}+\tau \\
\times & \tilde{\chi}_{2}^{0} \rightarrow \tilde{\tau}_{2}+\tau \\
* & \tilde{\chi}_{2}^{0} \rightarrow \widetilde{v}_{1}+v_{1} \\
& \tilde{\chi}_{2}^{0} \rightarrow \text { other } \\
\end{array}$ \\
\hline
\end{tabular}

\begin{tabular}{|ll|}
\hline - & $\tilde{\chi}_{1}^{ \pm} \rightarrow \tilde{\chi}_{1}^{0}+\mid v_{\mid}$ \\
$\star$ & $\tilde{\chi}_{1}^{ \pm} \rightarrow(\widetilde{\mathrm{e}}, \tilde{\mu})_{\mathrm{L}}+\left(v_{\mathrm{e}}, v_{\mu}\right)$ \\
$*$ & $\tilde{\chi}_{1}^{ \pm} \rightarrow \tilde{\chi}_{1}^{0}+\mathrm{W}^{ \pm}$ \\
$\square$ & $\tilde{\chi}_{1}^{ \pm} \rightarrow \tilde{\chi}_{1}^{0}+\mathrm{qq}$ \\
$*$ & $\tilde{\chi}_{1}^{ \pm} \rightarrow \tilde{\tau}_{1}+v_{\tau}$ \\
$\star$ & $\tilde{\chi}_{1}^{ \pm} \rightarrow \tilde{\tau}_{2}+v_{\tau}^{-}$ \\
$\Rightarrow$ & $\tilde{\chi}_{1}^{ \pm} \rightarrow \tilde{v}_{\mathrm{i}}+\mathrm{I}_{\mathrm{i}}$ \\
\hline
\end{tabular}

Figure 3.7: Continuation of Fig. 3.6: the branching ratios of the second lightest neutralino $(a, c)$ and the lightest chargino $(b, d)$ as a function of $m_{1 / 2}$ for negative Higgs mixing parameter $\mu, \tan \beta=5, A_{0}=0$, and $m_{0}$ as indicated. Again, as for $\mu>0$, the important decay channels at intermediate and high $m_{0}$ are $\widetilde{\chi}_{1}^{ \pm} \rightarrow \widetilde{\chi}_{1}^{0} W$ and $\widetilde{\chi}_{2}^{0} \rightarrow \widetilde{\chi}_{1}^{0}+Z$. 
section 1.3.5). However, since the left-handed sleptons are usually heavier than the right-handed ones, this coupling effect is not visible as clearly for the $\widetilde{\chi}_{2}^{0}$ as for the $\widetilde{\chi}_{1}^{ \pm}$. This latter is mostly wino-like and thus couples almost exclusively to left-handed particles, which is clearly visible in Figures 3.4 and $3.6(\mathrm{~b}, \mathrm{~d}, \mathrm{f})$. The lightest chargino only decays into $\widetilde{\tau}_{1}+\nu_{\tau}$ as long as other decay channels are kinematically not allowed, compare also Figure $3.3(\mathrm{a}, \mathrm{c})$ for low values of the common scalar mass $m_{0}$. As soon as the channels $\widetilde{\chi}_{1}^{ \pm} \rightarrow \widetilde{\nu}_{i}+\ell_{i}$ and/or $\widetilde{\chi}_{1}^{ \pm} \rightarrow \widetilde{\chi}_{1}^{0}+W^{ \pm}$open (especially Figures $3.4(\mathrm{~b}, \mathrm{~d})$ and $3.6(\mathrm{~d}, \mathrm{f})$ ), the $\widetilde{\chi}_{1}^{ \pm}$branching ratio into $\widetilde{\tau}_{1}+\nu_{\tau}$ drops to nearly zero, while the first two decay channels account for nearly $100 \%$ of the total decay width of the lightest chargino.

Two-body decays are only important for small values of the common sfermion mass $m_{0}$, i.e. for values of $m_{0}<250 \mathrm{GeV}$. Above this value, the only relevant decay channels of the second lightest neutralino are:

- for small values of $m_{1 / 2}$ (i.e. $m_{1 / 2} \approx 140-250 \mathrm{GeV}$ ), the decays $\widetilde{\chi}_{2}^{0} \rightarrow \widetilde{\chi}_{1}^{0}+q \bar{q}, \widetilde{\chi}_{2}^{0} \rightarrow \widetilde{\chi}_{1}^{0}+\ell \bar{\ell}$, and the direct $\not R_{p}$-decays account for nearly $100 \%$ of the total decay width;

- for intermediate values of $m_{1 / 2}$ (i.e. $m_{1 / 2}=250-300 \mathrm{GeV}, \mu>0$ ), the decays $\widetilde{\chi}_{2}^{0} \rightarrow \widetilde{\chi}_{1}^{0}+Z$ account for about $100 \%$ of the total width, with a slight dependence on $m_{0}$;

- for large values of $m_{1 / 2}$ (i.e. $m_{1 / 2}>300 \mathrm{GeV}, \mu>0$ ), the decays involving neutral or charged Higgs bosons $\left(\widetilde{\chi}_{2}^{0} \rightarrow \widetilde{\chi}_{1}^{0}+h / H\right.$ and $\left.\widetilde{\chi}_{2}^{0} \rightarrow \widetilde{\chi}_{1}^{ \pm}+H^{\mp}\right)$ dominate, see also Figures 3.5 and 3.7 (a,c), where these decays are labeled as $\widetilde{\chi}_{2}^{0} \rightarrow$ other. (An additional, though small component are decays involving photons, via loop-diagrams, e.g. $\widetilde{\chi}_{2}^{0} \rightarrow \widetilde{\chi}_{1}^{0}+\gamma$.)

Even fewer possibilities exist for the lightest chargino, with only three relevant decay channels:

- for $\mu>0$ and up to $m_{1 / 2} \approx 250 \mathrm{GeV}(210 \mathrm{GeV})$ for $m_{0}=250 \mathrm{GeV}(1000 \mathrm{GeV})$, respectively, the two decay channels $\widetilde{\chi}_{1}^{ \pm} \rightarrow \widetilde{\chi}_{1}^{0}+q q^{\prime}(\approx 65 \%), \widetilde{\chi}_{1}^{ \pm} \rightarrow \widetilde{\chi}_{1}^{0}+\ell \nu(\approx 35 \%)$ dominate;

- for $\mu>0$ and $m_{1 / 2}$ values above $250 \mathrm{GeV}(210 \mathrm{GeV})$ for $m_{0}=250 \mathrm{GeV}(1000 \mathrm{GeV})$, the third decay channel, $\widetilde{\chi}_{1}^{ \pm} \rightarrow \widetilde{\chi}_{1}^{0}+W^{ \pm}$, opens and accounts for nearly $100 \%$ of the total $\widetilde{\chi}_{1}^{ \pm}$ decay width. For negative $\mu$, the situation is very similar, except that the $W$-decay channel opens even earlier, at $220 \mathrm{GeV}(180 \mathrm{GeV})$ for $m_{0}=250 \mathrm{GeV}(1000 \mathrm{GeV})$, respectively.

In addition, there is a special »corridor region« for $m_{0}=100 \mathrm{GeV}$, where neither the second lightest neutralino, nor the lightest chargino decay such that a significant number of additional electrons or muons are produced.

- For $\mu>0$, this region is located between $m_{1 / 2}=200-250 \mathrm{GeV}$, which corresponds to $m\left(\widetilde{\chi}_{1}^{ \pm}\right) \approx 135-175 \mathrm{GeV}$, and $m\left(\widetilde{\chi}_{1}^{0}\right) \approx 74-96 \mathrm{GeV}$;

- for $\mu<0$ it lies between $m_{1 / 2}=170-220 \mathrm{GeV}$, corresponding to $m\left(\widetilde{\chi}_{1}^{ \pm}\right) \approx 130-170 \mathrm{GeV}$, and $m\left(\widetilde{\chi}_{1}^{0}\right) \approx 70-90 \mathrm{GeV}$.

The dominant decays in both cases are $\widetilde{\chi}_{2}^{0} \rightarrow \widetilde{\tau}_{1}+\tau$ and $\widetilde{\chi}_{1}^{ \pm} \rightarrow \widetilde{\tau}_{1}+\nu_{\tau}$, and, although electrons and muons can result from (s)tau decays, their momenta are usually not large enough for them to be detected with a high efficiency. For $\mu>0$, the decay $\widetilde{\chi}_{2}^{0} \rightarrow(\widetilde{e}, \widetilde{\mu})_{R}+(e, \mu)$ accounts for about $40 \%$ of the total decay width of the second lightest neutralino, nevertheless this is not sufficient to prevent the signal selection efficiency from decreasing significantly within this region, (see also Chapter 5 and Tables C.1 and C.2 for positive and negative $\mu$, respectively). A similar region 
exists in case of $m_{0}=50 \mathrm{GeV}, \mu>0$, though it is slightly shifted to lower values of $m_{1 / 2}$ and not as pronounced as for $m_{0}=100 \mathrm{GeV}$.

In the adjacent region $m_{1 / 2} \approx 250-350 \mathrm{GeV}$, corresponding to $m\left(\widetilde{\chi}_{1}^{ \pm}\right) \approx 180-260 \mathrm{GeV}$, and $m\left(\widetilde{\chi}_{1}^{0}\right) \approx 96-140 \mathrm{GeV}$, it becomes possible to produce the $W$ boson on-shell, which is why the dominant decay of the lightest chargino is $\tilde{\chi}_{1}^{ \pm} \rightarrow \widetilde{\chi}_{1}^{0}+W^{ \pm}$. Due to the fact that about $20 \%$ of the time, the $W$ boson contributes another charged lepton to the final state, enlarging the probability to detect/reconstruct three charged leptons, a large increase in the selection efficiency is expected. In addition, the channel $\widetilde{\chi}_{1}^{ \pm} \rightarrow \widetilde{\nu}_{i} \ell_{i}$ opens at $m_{1 / 2} \approx 300 \mathrm{GeV}$, which corresponds to $m\left(\widetilde{\chi}_{1}^{ \pm}\right) \approx 220 \mathrm{GeV}$ and $m\left(\widetilde{\chi}_{1}^{0}\right) \approx 118 \mathrm{GeV}$, while the decay $\widetilde{\chi}_{1}^{ \pm} \rightarrow(\widetilde{e}, \widetilde{\mu})+\left(\nu_{e}, \nu_{\mu}\right)$ becomes possible at $m_{1 / 2} \approx 350 \mathrm{GeV}\left(m\left(\widetilde{\chi}_{1}^{ \pm}\right) \approx 260 \mathrm{GeV}\right.$ and $\left.m\left(\widetilde{\chi}_{1}^{0}\right) \approx 140 \mathrm{GeV}\right)$. Mainly the opening of the former of these two decay channels, is the reason why a large signal selection efficiency is observed for $m_{0}=100 \mathrm{GeV}, \mu>0$ and relatively large values of $m_{1 / 2}$.

\section{Multiplicities of Additional Charged Leptons}

The possibility to detect and reconstruct electrons or muons is correlated with their respective multiplicities in the final state. Figure 3.8 summarises the discussion of the decay channels and branching ratios by illustrating the different final state multiplicities of $\gg$ additional charged leptons from the cascade decays (not from the LSP decays) for (a,b) electrons and muons, and $(\mathrm{c}, \mathrm{d})$ tau leptons for both signs of the Higgs mixing parameter: (a,c) $\mu<0$ and (b,d) $\mu>0$, as well as for various values of $m_{0}$ (see legend for details). Electrons and muons from tau decays are included, but - as mentioned - their transverse momenta are usually not large enough for them to be efficiently detected and reconstructed.

The multiplicities of electrons and muons are the same within statistical fluctuations, which is explained by the mass degeneracy of the first and second generation sfermions and because of lepton universality, i.e. the couplings of the $W(Z)$ bosons to leptons are equal for all charged leptons. From Figure 3.8 it also becomes clear, why it is advantageous to combine the three different searches: where the electron and muon multiplicity decreases the multiplicity of additional tau leptons increases and vice versa. Therefore it is possible that even the ee $\tau$ analysis, where no final state muons are expected from the LSP-decays alone, may recover small inefficiencies of the present analysis due to the occurrence of additional charged leptons from the cascade decays of heavier gauginos. Electron and muon multiplicities are high in the region $m_{1 / 2} \approx 250-350 \mathrm{GeV}$ for positive $\mu$ and $m_{0}=100 \mathrm{GeV}$, in agreement with the previous discussion of the branching ratios for the lightest chargino. As a consequence, the signal selection efficiency is also high, so that the achieved low upper cross section limits are easily explained (see also chapter 5 ). 

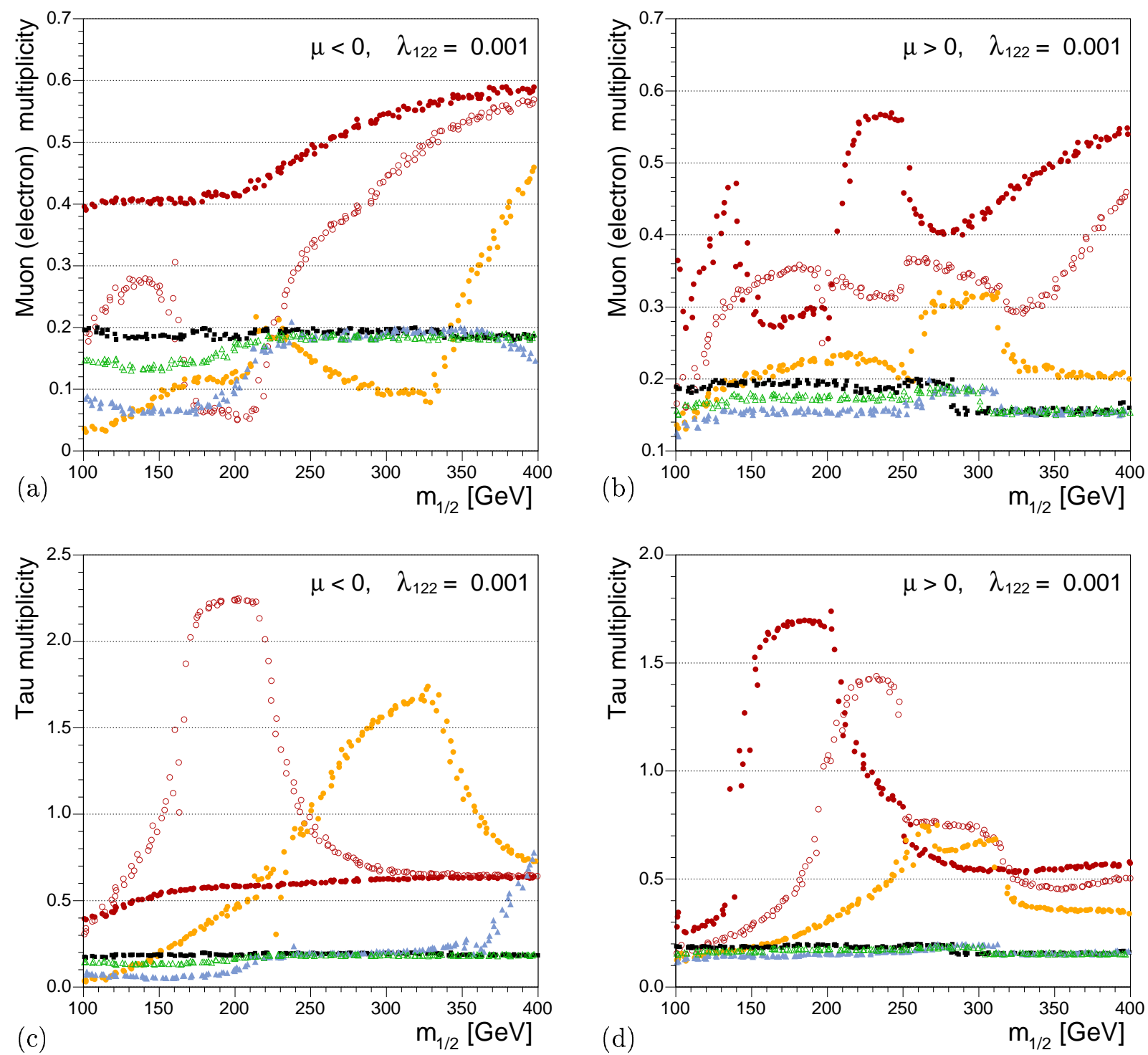

$$
\begin{aligned}
& \text { - } \mathrm{m}_{0}= 50 \mathrm{GeV} \\
& \text { - } \mathrm{m}_{0}=100 \mathrm{GeV} \\
& \text { - } \mathrm{m}_{0}=150 \mathrm{GeV}
\end{aligned}
$$$$
\begin{array}{ll}
\triangle & \mathrm{m}_{0}=250 \mathrm{GeV} \\
\Delta & \mathrm{m}_{0}=500 \mathrm{GeV} \\
\text { - } & \mathrm{m}_{0}=1000 \mathrm{GeV}
\end{array}
$$

$$
\begin{array}{r}
\tan \beta=5 \\
A_{0}=0
\end{array}
$$

Figure 3.8: The multiplicity of additional charged leptons from the cascade decays of the second lightest neutralino $\left(\widetilde{\chi}_{2}^{0}\right)$ and the lightest chargino $\left(\widetilde{\chi}_{1}^{ \pm}\right)$as a function of $m_{1 / 2}$ for negative $(a, c)$ and positive $(b, d)$ Higgs mixing parameter $\mu$ and various values of $m_{0}$. All mSUGRA parameters are given in the figure. Electrons and muons originating from tau decays are included in the above multiplicity curves, but due to their small transverse momenta, they are only rarely detected. 


\subsection{Kinematic Properties of the Produced Electrons and Muons}

A number of different kinematic variables has been studied; among them angular distributions of single muons and electrons, and of pairs and triplets of these two types of leptons. However, only the transverse momentum distributions of electrons and muons are found to be useful in characterising the SUSY signals w.r.t. the simulated SM background. Angular distributions of single charged leptons (or pairs, triplets thereof), suffer from the mostly independent distribution of the leptons produced in the cascade decays and/or the two LSPs.

A reconstruction of the mass of one or two gauginos from the two types of charged leptons and the missing transverse energy has also been attempted, but has been unsuccessful. This is due to the many possibilities of cascade decays for the produced gauginos and also because at least two neutrinos are produced in the LSP decays. The rather large probability for the production of even more neutrinos in the cascade decays makes it effectively impossible to reconstruct the correct gaugino mass from the momentum of the charged decay leptons and the measured missing transverse energy.

Fortunately, the fact that many charged leptons are produced, facilitates the background reduction and, hence, the detection of this type of SUSY signals. One useful observation is that the transverse momenta of the electrons and muons hardly depend on the three mSUGRA parameters $\mu, \tan \beta$, and $A_{0}$. The following Figures 3.9, 3.10 and 3.11 illustrate the influence of the remaining two mass parameters on the electron and muon transverse momenta; i.e. the common sfermion mass, $m_{0}$, and the common gaugino mass, $m_{1 / 2}$. The lines and arrows indicate the analysis requirements on the transverse momenta of the leptons: $p_{\mathrm{T}}(\ell 1)>15 \mathrm{GeV}$, $p_{\mathrm{T}}(\ell 2)>8 \mathrm{GeV}$, and $p_{\mathrm{T}}(\ell 3)>5 \mathrm{GeV}$. However, the $p_{\mathrm{T}}$ criteria that are finally used for the different types of leptons, i.e. electrons or muons differ slightly and are explained in more detail in Chapter 4.

While the events are not weighted according to the signal cross section and data luminosity, the geometric acceptance, trigger efficiency and the efficiencies of various quality criteria (see also Chapter 4) are included in the distributions of the reconstructed electron and muon transverse momenta on the right-hand sides of the figures $(b, c, d)$. In this way, the combined effect of the acceptance and efficiency induced reduction of the signal events can be observed without the complication of the cross sections that decrease rapidly with $m_{1 / 2}$.

Comparing the generated electron and muon transverse momenta $(\mathrm{a}, \mathrm{c}, \mathrm{e})$ with the reconstructed momenta (b,d,f), one general observation is the increasing detection / reconstruction efficiency as a function of $m_{1 / 2}$. This is mainly due to the fact, that all lepton transverse momenta increase due to the larger masses of the gauginos. On the one hand, this leads to a better detection efficiency for the low- $p_{\mathrm{T}}$ electrons and muons (the third and optional fourth charged lepton) and on the other hand, the lepton $p_{\mathrm{T}}$ requirements do not reduce the signal events as much as for small values of $m_{1 / 2}$. For large values of $m_{0}$, the third (and fourth) charged lepton is reconstructed less often, resulting in a large reduction of the corresponding $p_{\mathrm{T}}$ distribution. This is the reason why the $p_{\mathrm{T}}$ distribution of the third (fourth) reconstructed electron/muon appears to be very different compared to the distribution on generator level as can clearly be observed in Figure $3.10(\mathrm{~b}, \mathrm{~d}, \mathrm{f})$ and Figure $3.11(\mathrm{~b}, \mathrm{~d})$. 

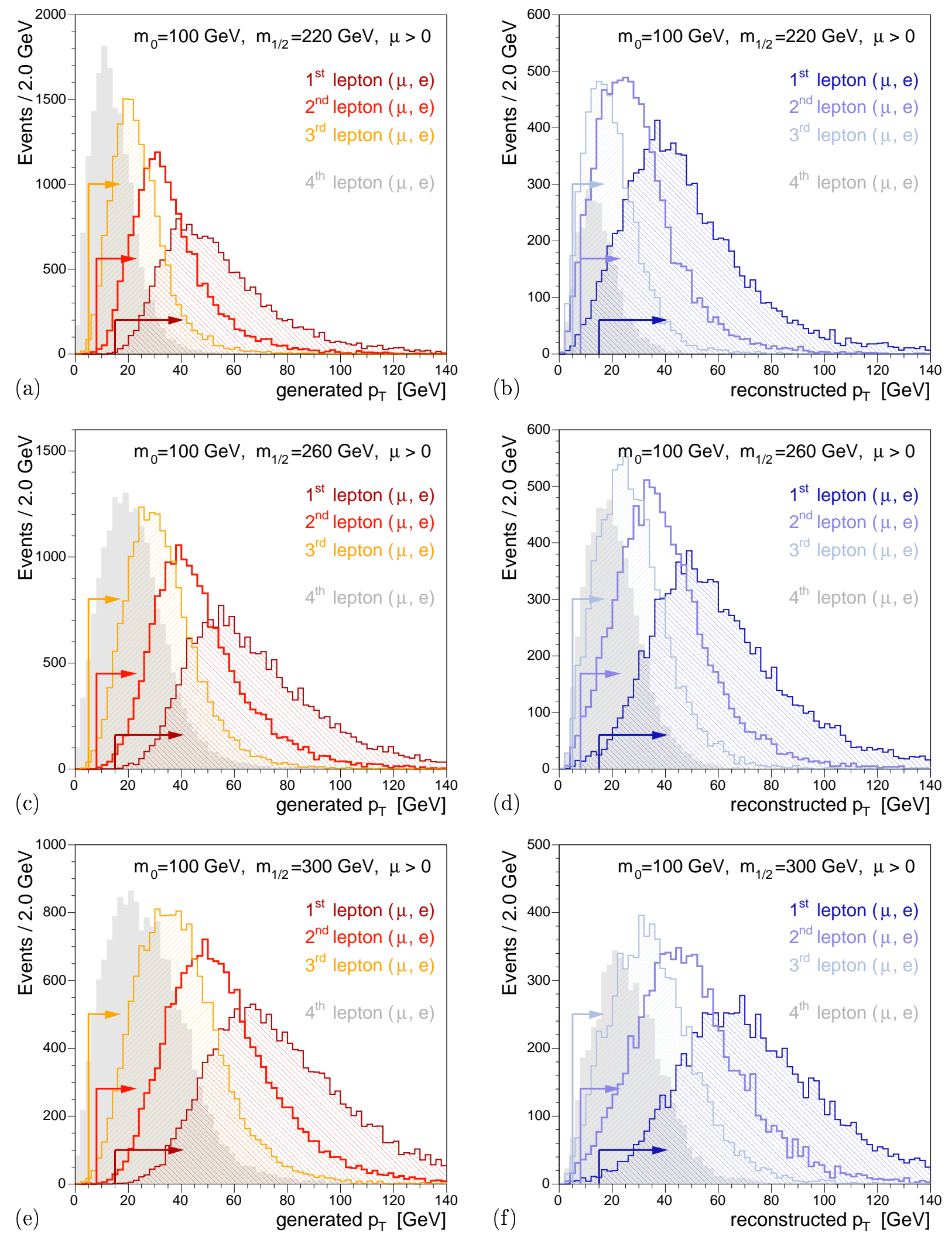

Figure 3.9: Comparison of generator level $(a, c, e)$ and detector level $(b, d, f)$ electron and muon transverse momenta for $m_{0}=100 \mathrm{GeV}, \mu>0, \tan \beta=5$, and $A_{0}=0$. Due to the second lightest neutralino and the lightest chargino BRs into leptons (see Fig. 3.5), the detection and reconstruction efficiency for electrons/muons increases dramatically above $m_{1 / 2} \geq 250 \mathrm{GeV}$, due to the decays via the gauge bosons $W$ and $Z$, and, especially at $m_{0}=100 \mathrm{GeV}$ also $\widetilde{\chi}_{2}^{0} \rightarrow(\widetilde{e}, \widetilde{\mu})_{R}+(e, \mu)$. 
3.4 Kinematic Properties of the Produced Electrons and Muons
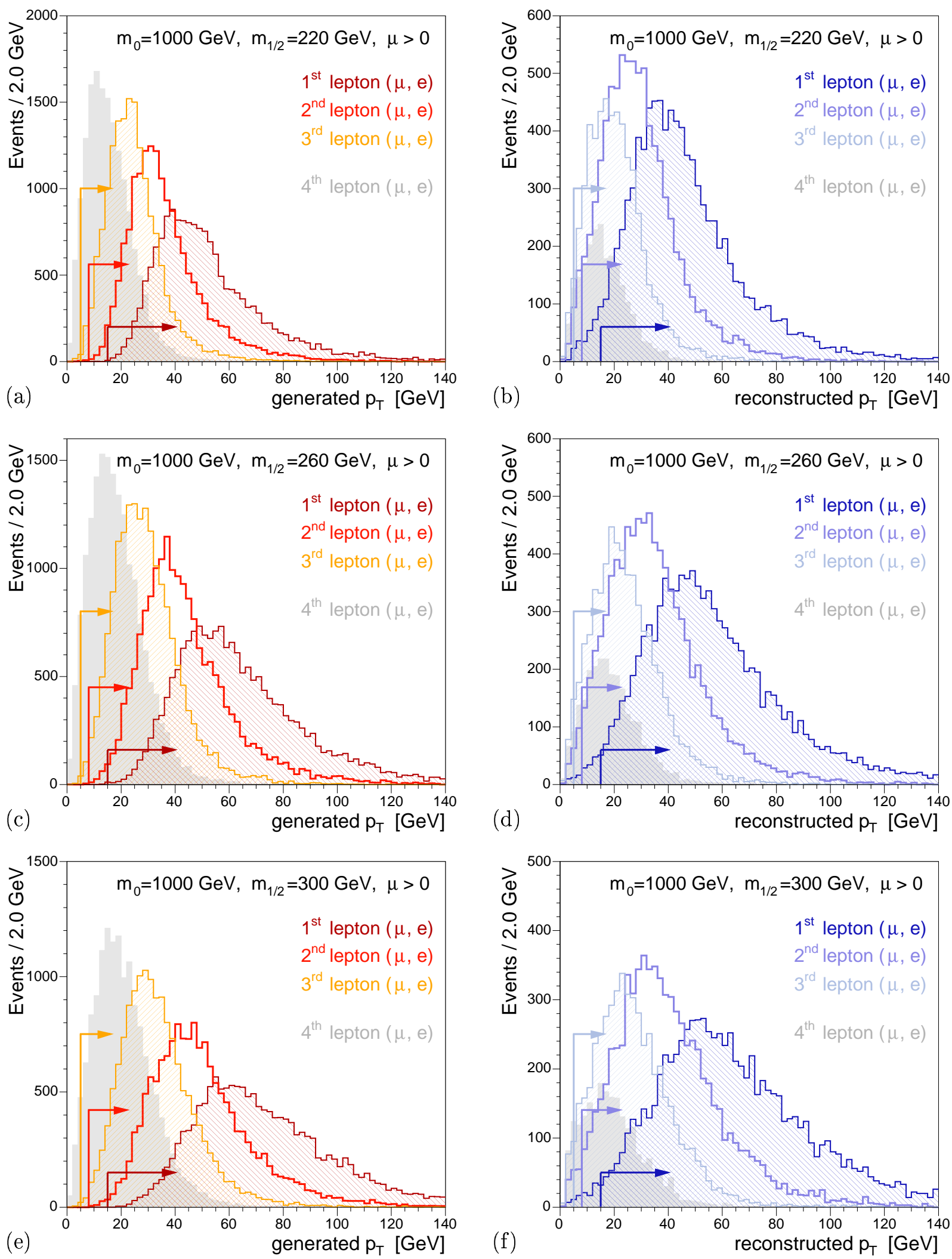

Figure 3.10: Comparison of generator level $(a, c, e)$ and detector level $(b, d, f)$ lepton $(e, \mu)$ transverse momenta for $m_{1 / 2}=300 \mathrm{GeV}, \mu>0, \tan \beta=5$, and $A_{0}=0$. Similar observations for $m_{0}=1000 \mathrm{GeV}$ compared to $m_{0}=100 \mathrm{GeV}$, (250 GeV) show that the dependence of the lepton transverse momenta on the value of $m_{0}$ is rather small. For large values of $m_{0}$, the third (fourth) electron/muon is reconstructed less often, resulting in a large reduction of the corresponding $p_{\mathrm{T}}$ distribution. 
However, since the cross section decreases with increasing gaugino masses, i.e. with increasing gaugino mass parameter $m_{1 / 2}$, the effect of increased detection/reconstruction efficiency is more or less canceled by the decreasing cross section. As a consequence the upper cross section limit is expected to be nearly flat as a function of $m_{1 / 2}$ and $m_{0}$. The only deviation is expected in the »corridor region $\ll$ from $m_{1 / 2}=200-250 \mathrm{GeV}$ for $m_{0}=100 \mathrm{GeV}$.

The requirements on the transverse momenta of the electrons and muons are chosen w.r.t. the $p_{\mathrm{T}}$ distributions for low and intermediate values of $m_{1 / 2}$, see especially Figures $3.10(\mathrm{~b}, \mathrm{~d})$ and 3.11 , where the $p_{\mathrm{T}}$ distribution of the fourth lepton is hardly visible anymore and even the third lepton is only detected or reconstructed with a rather small probability.
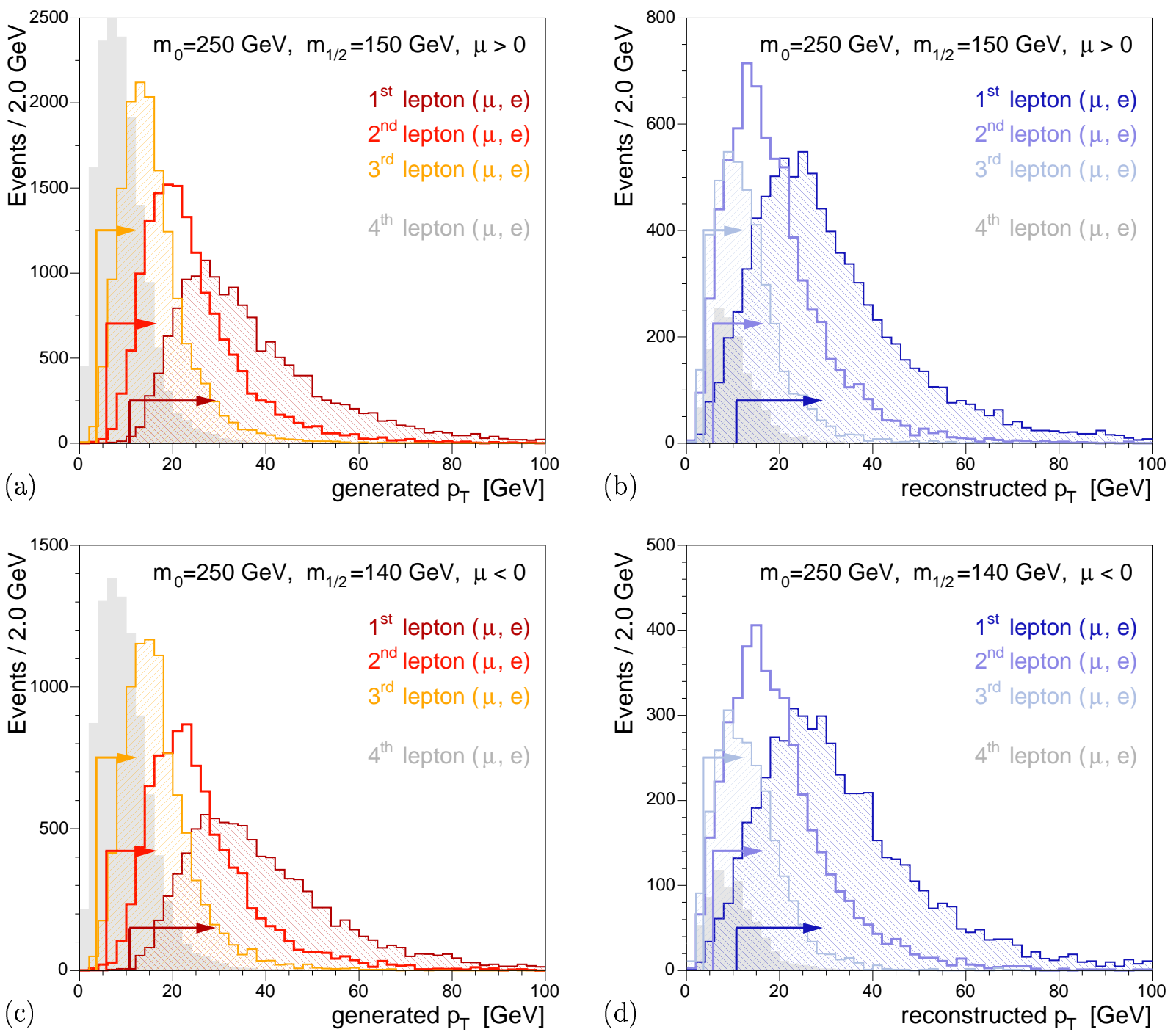

Figure 3.11: Comparison of generator level $(a, c)$ and detector level $(b, d)$ lepton $(e, \mu)$ transverse momenta for $m_{0}=250 \mathrm{GeV}, m_{1 / 2}=150 \mathrm{GeV}(140 \mathrm{GeV})$ for $\mu>0(\mu<0)$, respectively. The remaining parameters are: $\tan \beta=5$, and $A_{0}=0$. The probability to detect and reconstruct the third charged lepton is rather small for low values of $m_{1 / 2}$, and the $p_{\mathrm{T}}$ distribution of the fourth lepton is almost not visible anymore. 

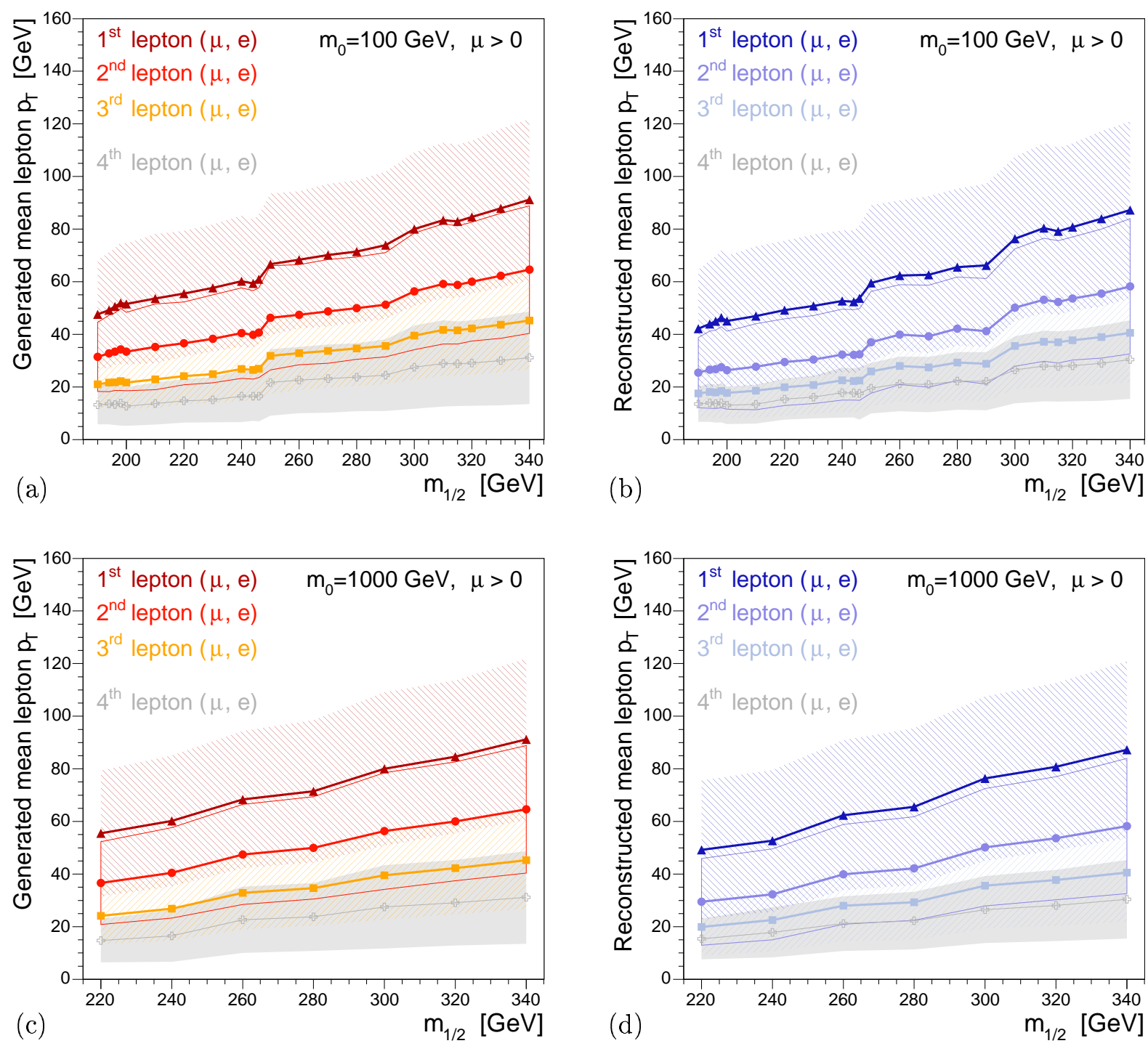

Figure 3.12: The mean lepton $(e, \mu)$ transverse momentum as a function of the gaugino mass parameter $m_{1 / 2}$ for positive Higgs mixing parameter $\mu$ : (a,c) as generated with the SUSYGEN event generator and $(b, d)$ after the complete reconstruction and including acceptance and efficiency effects. (a,b) for $m_{0}=$ $100 \mathrm{GeV}$ and $(c, d)$ for $m_{0}=1000 \mathrm{GeV}$, respectively. The spread, or root mean square, of the mean lepton $p_{\mathrm{T}}$ is indicated as the hatched/shaded regions for the $1^{\text {st }}, 3^{\text {rd }}$, and optional $4^{\text {th }}$ lepton.

The increase in the mean generated and reconstructed lepton $p_{\mathrm{T}}$ with increasing $m_{1 / 2}$ is very similar for $\mu>0$ and $\mu<0$. Hence, this is only illustrated for positive Higgs mixing parameter $\mu$. Figure 3.12 shows the mean and spread (root mean square) of the $p_{\mathrm{T}}$ distributions of the first, second, third, and optional fourth lepton at generator level (a,c) and after the reconstruction $(\mathrm{b}, \mathrm{d})$ for $m_{0}$ values of $(\mathrm{a}, \mathrm{b}) m_{0}=100 \mathrm{GeV}$ and $(\mathrm{c}, \mathrm{d}) m_{0}=1000 \mathrm{GeV}$, respectively.

Two more, general observations are that the reconstructed $p_{\mathrm{T}}$ distributions are wider and slightly shifted towards lower $p_{\mathrm{T}}$ values compared to the distributions of the generated transverse momenta. 



\title{
Chapter 4
}

\section{The Event Selection}

\author{
In physics, you don't have to go around making trouble for yourself \\ - nature does it for you.
}

Frank Wilczek (American Physicist, *1951)

This chapter discusses all necessary ingredients for the selection and analysis of trilepton events: The first section presents the utilised data sample and trigger requirements, as well as the corresponding integrated luminosity. In section two, background and signal MC samples are described, including the generation and simulation of signal samples. In the third section, the determination of trigger efficiencies and their application to the simulated data is discussed and the quality of the MC simulations is studied. This is done by measuring various efficiencies separately in data and MC samples, determining correction factors, and applying them to the simulated data. The fourth section deals with the estimation of multijet background from data to avoid the associated large theoretical uncertainties. In addition, using data prevents difficulties with the generation/simulation of huge amounts of QCD events.

In the fifth section the selection criteria for the »dilepton « control sample are discussed. The development of two-dimensional cuts is presented in detail, since they enable a better background rejection, while keeping a high signal efficiency. The optimisation procedure and the $\gg$ trilepton « selection criteria are also described in section five. The sixth section serves to discuss the systematic uncertainties, and section seven presents the results of this analysis.

\subsection{Data Sample and Luminosity}

In the present analysis data collected with the $\mathrm{D} \emptyset$ detector between April 2002 and August 2004 are used. The analysis is performed on PASS 1 data, reconstructed with version p14.06.01 of $\mathrm{D} \emptyset$ reco. These data, about 1 billion events, would require an enormous amount of disk and cache space ( $\approx 250 \mathrm{kB}$ per event $\rightarrow 250 \mathrm{~TB}$ ). Therefore a preselected subsample is used; the $2 \mathrm{MU}$-skim provided by the Common Sample Group (CSG) [141]. It contains only events with at least two reconstructed muons, but without requirements on their transverse momenta. As pointed out in the previous chapter on $\not_{p}$-SUSY phenomenology, the expected and thus sought-after signature is $\mu \mu \ell$, with $\ell=e$, or $\mu$, justifying the restriction to use only the $2 \mathrm{MU}$-skim as basis for the entire analysis. 
Since the corresponding total integrated luminosity of a certain data sample can only be determined correctly for a given trigger, or group of triggers, a set of unprescaled dimuon triggers is defined. The luminosity for this set is calculated, using the program runrange_luminosity, provided by the D $\varnothing$ Luminosity Group [135] (see also Fig. 2.46, in section 2.5). This program takes as input three lists: a BadRunsList, a BadLBNList, and a list with the chosen triggers and corresponding run-ranges, i.e. the physics runs taken with a trigger list version in which the specified trigger was defined. The BadRunsList is defined using the Run Quality Database [142] and contains all runs graded either $»$ bad « or »special « for any one of the following subsystems: SMT, CFT, the calorimeter, and the muon system. Bad runs are runs for which either the hardware was not completely functional or where the triggers were not normalisable in terms of recorded luminosity. Special runs refer to data taken under special conditions, e.g. muon cosmic runs, detector calibrations, tests of new hard- or software, including studies and rate tests for the development of new triggers. The initial BadLBNList contains information of several lists provided by the JetMET group [143] to account for calorimeter problems, influencing jet and $\boldsymbol{\not}_{T}$ calibration and, additionally, all LBNs for which the specified dimuon triggers were not functional. The runrange_luminosity program then takes care of issues related with the readout, data acquisition, and reconstruction, where data losses might occur, or more luminosity blocks be flagged unnormalisable due to other circumstances. Apart from calculating the luminosity for each pair of trigger and specified run-range, the program also outputs a list with bad LBNs, built from the initial BadLBNList, all LBNs corresponding to the runs contained in the BadRunsList, and additional LBNs found to be unnormalisable at run-time.

Data corresponding to a bad LBN (or a bad run) is not used for the luminosity determination and consequently has to be removed from the analysis sample, which is done by vetoing events corresponding to an LBN from the final list of bad LBNs output by runrange_luminosity.

The set of dimuon triggers and run-ranges specified for this analysis are summarised in Table 4.1, along with the corresponding luminosities. The entire data set corresponds to a total integrated luminosity of $\int \mathcal{L} d t=360 \pm 23 \mathrm{pb}^{-1}$. Figure 4.1 shows the visible cross section for dimuon events as preselected in the $2 \mathrm{MU}$-skim and serves as a consistency check of the data and luminosity from different run-ranges.

\begin{tabular}{lrrrr}
\hline \hline Trigger Name & TL & Run-range & L3 & Luminosity \\
\hline \hline 2MU_A_L2MO & $\leq v 10$ & $151817-174895$ & - & $50.5 \mathrm{pb}^{-1}$ \\
2MU_A_L2MO_TRK10 & $v 11$ & $174896-178721$ & $p_{\mathrm{T}}($ track $)>10 \mathrm{GeV}$ & $56.3 \mathrm{pb}^{-1}$ \\
2MU_A_L2MO_L3L15 & $v 11$ & $174896-178721$ & $p_{\mathrm{T}}(\mu)>15 \mathrm{GeV}$ & $56.3 \mathrm{pb}^{-1}$ \\
2MU_A_L2MO_TRK5 & $v 12$ & $178097-194566$ & $p_{\mathrm{T}}($ track $)>5 \mathrm{GeV}$ & $208.0 \mathrm{pb}^{-1}$ \\
2MU_A_L2MO_L3L6 & $v 12$ & $178097-194566$ & $p_{\mathrm{T}}(\mu)>6 \mathrm{GeV}$ & $208.0 \mathrm{pb}^{-1}$ \\
DMU1_TK5 & $v 13$ & $194567-196584$ & $p_{\mathrm{T}}($ track $)>5 \mathrm{GeV}$ & $46.5 \mathrm{pb}^{-1}$ \\
DMU1_LM6 & $v 13$ & $194567-196584$ & $p_{\mathrm{T}}(\mu)>6 \mathrm{GeV}$ & $46.5 \mathrm{pb}^{-1}$ \\
\hline The total integrated luminosity of the dimuon data set is: & $\int \mathcal{L} d t=361.3 \pm 23.5 \mathrm{pb}^{-1}$ \\
\hline \hline
\end{tabular}

Table 4.1: Summary of the utilised dimuon triggers (छ trigger list versions), as well as the run-ranges during which the triggers were valid and the corresponding luminosity as output by runrange_luminosity. All dimuon triggers share the same L1 and L2 conditions, see text for details. 


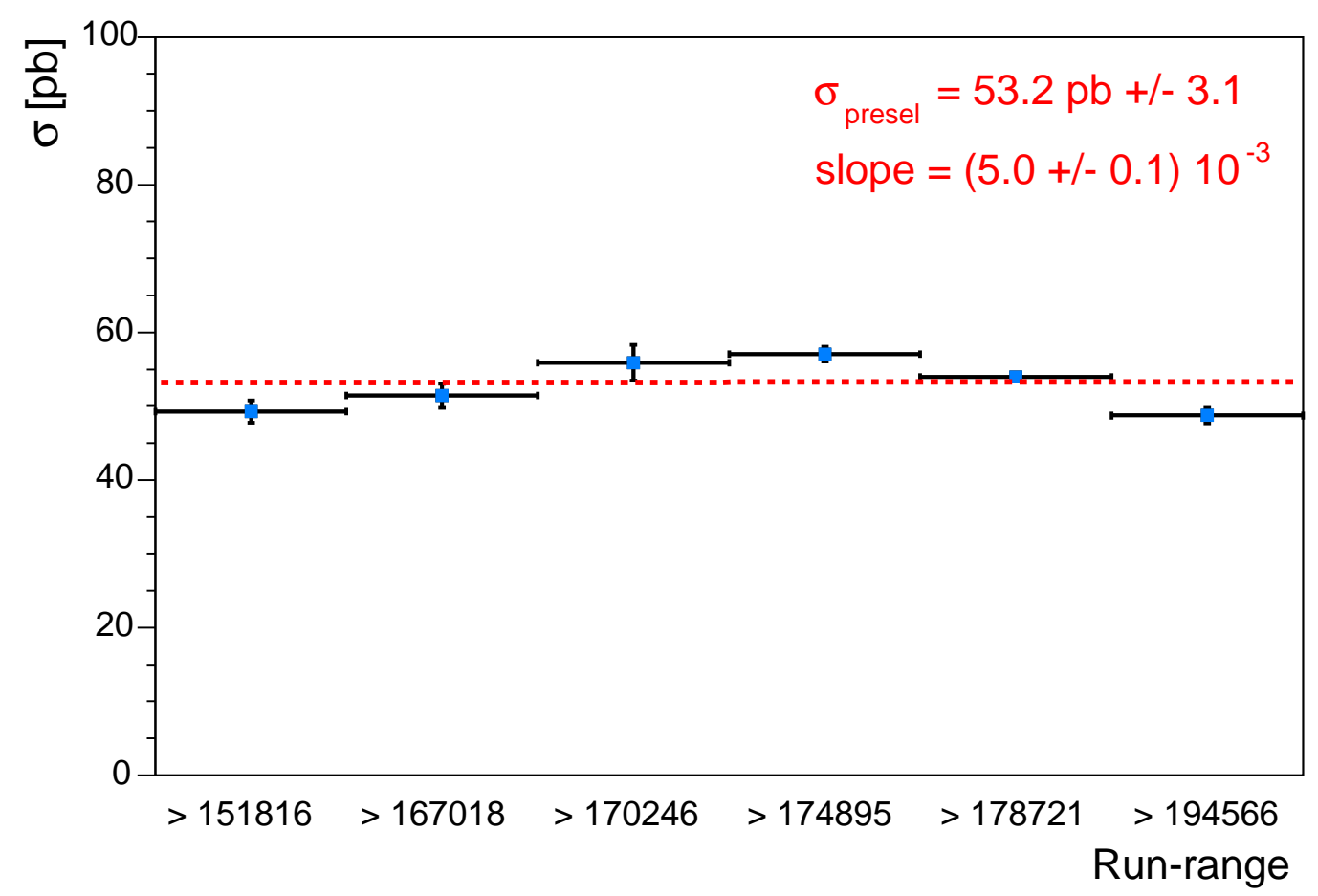

Figure 4.1: Data and luminosity consistency check: Shown is the number of events per run-range divided by the corresponding luminosity, i.e. the approximate production cross section of the dimuon events preselected in the $2 M U$-skim.

All dimuon triggers are based on muon scintillator hits at Level 1, a muon quality requirement at Level 2 and different criteria at Level 3 (see Table 4.1). The L1 and L2 requirements are the same for all of the utilised dimuon triggers. The L1 condition, abbreviated mu2ptxatxx, requires two muons (mu2), without a specific $p_{\mathrm{T}}$ threshold (ptx), anywhere in the detector (a), with tight scintillator hits ( $\mathrm{t})$, but no wire hits ( $\mathrm{xx}$ ). The L2 condition, abbreviated MUON (1, med), requires a certain quality, i.e. hit pattern, but again no $p_{\mathrm{T}}$ threshold. Muons are classified according to the follwing hit patterns in the local muon system [105]:

- $\quad \geq 1$ A layer scintillator hit

- $\geq 2$ A layer wire hits
- $\quad \geq 1$ BC layer scintillator hit

- $\quad \geq 2$ BC layer wire hits

Since in this analysis only muons with a matched central ${ }^{1}$ track are considered, the classification of muon qualities is also only given for track-matched muons. A »loose« muon must satisfy at least two of the above requirements and have at least one scintillator hit, either in layer A or BC. »Medium « muons must fulfill at least three of the above requirements unless they are located in the bottom part of the detector $(|\eta|<1.6)$ in which case they only need to satisfy either the two A, or BC-layer conditions. Low momentum muons, i.e. muons whose probability to reach the BC-layer is less than $70 \%$, also qualify as »medium « muons if they satisfy both A-layer requirements. Muons satisfying all requirements and where additionally a local fit from the muon system towards the central tracking detectors converged are called »tight the local fit did not converge, the fit from the tracking detectors to the muon system (central fit) is used to determine if a muon is matched to a central track or not.

\footnotetext{
${ }^{1}$ The terms central, or inner in association with tracks always refer to tracks measured with the inner tracking detectors (SMT/CFT), contrary to the term local, which refers to a muon measured with the muon-system alone.
} 
For dimuon triggers defined in early trigger lists ( $v 10$ and below) used for instantaneous luminosities below $35-40 \cdot 10^{30} \mathrm{~cm}^{2} \mathrm{~s}^{-1}$, no Level 3 muon criteria were employed. However, to allow for unprescaled dimuon triggers at higher instantaneous luminosities, the trigger criteria needed to change. Later versions of dimuon triggers thus also employ Level 3 requirements. Either a central track above a certain $p_{\mathrm{T}}$ threshold is required, or a $p_{\mathrm{T}}$ requirement is imposed on a local muon, which is reflected in the last part of the trigger names, e.g. _L3L15 for a $15 \mathrm{GeV}$ local muon in trigger list $v 11$.

The thumbnail files of the 2MU skim are processed with the top_analyze package [132] (version $\gg$ Ipanema «) to apply the certified object identification and reconstruction criteria and standardised corrections for electromagnetic objects (EM-objects: electrons, photons), muons, jets and $\not_{T}$. In addition, a vertex constraint is applied to tracks measured exclusively with the CFTdetector and the two leading muons are required to pass low $p_{\mathrm{T}}$-thresholds to reduce the sample size. The output files are in ROOT tree format, as indicated in Figure 2.46 of section 2.5.

In a next step, these files are processed with the ReFill program, where a further preselection is applied and the data events are required to have fired at least one of the set of dimuon triggers. Duplicate events that may have been introduced during the skimming process are removed, as are events whose event and LBN numbers match exactly with a pair of the BadLBNList, generated by the runrange_luminosity program. So in the end, events contained in the output ROOT files, only contain clean dimuon events corresponding to a definite luminosity. Although some variables, like the invariant mass of the two leading muons, angular or spatial distances between various objects are calculated and stored in the output files, the overall number of variables per event is drastically reduced by keeping only variables that are of interest for the further analysis.

In a last step the complete analysis program is run on the remaining events. A control sample of dimuon events is created and analysed and the trilepton selection is applied. A similar, but separate program is used to optimise the selection criteria w.r.t. the signal selection efficiency times signal purity, $\varepsilon_{S} \times \frac{S}{S+B}$, where $S(B)$ stand for signal and background expectation, respectively. The output are the numbers of observed events in data and expected Monte Carlo events, including their statistical and systematic uncertainties, as well as selection efficiencies.

\subsection{Monte Carlo Simulations}

The simulated data samples can be split into two different types: those generated via known processes representing the backgrounds for this analysis, and those generated via SUSY signal processes. The former are mostly generated using PYTHIA [124], while the latter have been produced with Susygen [125]. The Susygen event generator was originally developed to simulate the production of supersymmetric events in electron-positron collisions, for example, at LEP. Over the last six years SusYgen has continually been extended, first to describe lepton-hadron collisions (HERA) and finally also hadron-hadron collisions as produced at the Tevatron and the future LHC.

\subsubsection{Monte Carlo Simulations of Standard Model Processes}

All SM background Monte Carlo simulations are official D $\emptyset$ MC production, requested by different physics groups and produced centrally, either at Fermilab, or at remote production sites. 
The samples are available from the DØ MC production WEB-page [144]. The MC samples used in this analysis are generated with PYTHIA, versions: 6.201 and 6.202 [124], with the exception of the $t \bar{t}$ sample, which has been generated with AlPGEN [145]. All MC samples were produced with CTEQ5L parton density functions (PDFs) [146], except for some $Z / \gamma^{*}$ samples, where statistics were not sufficient. In these cases, MC samples produced with CTEQ4L PDFs [147] have also been taken into account. A comparison of the samples generated with two different versions of PDFs (CTEQ4L \& CTEQ5L) can be found in Figure $4.2(\mathrm{a}, \mathrm{b})$. The dimuon invariant mass after full simulation and reconstruction is shown in (a) and the resulting ratio between both samples in (b). All considered SM processes, as well as the cross section $\sigma_{M C} \times B R$, the equivalent integrated luminosity $\left(\mathcal{L}_{M C}\right)$ and the number of generated events are listed in Table 4.2.

\begin{tabular}{|c|c|c|c|c|}
\hline Process $(M[\mathrm{GeV}])$ & $\sigma_{M C}^{L O} \times B R[\mathrm{pb}]$ & $\mathrm{K}$-fac. $\left(\frac{N N L O}{L O}\right) \pm \mathrm{PDF}$ & $\mathcal{L}_{M C}\left[\mathrm{pb}^{-1}\right]$ & $N_{\text {gen }}$ \\
\hline$Z / \gamma^{*} \rightarrow \mu \mu(5-15)$ & 3558.0 & $1.29 \pm 0.06$ & $\approx 47$ & 219,250 \\
\hline$Z / \gamma^{*} \rightarrow \mu \mu(15-60)$ & 337.6 & $(1.29, . .1 .36) \pm 0.05$ & $\approx 770$ & 343,250 \\
\hline$Z / \gamma^{*} \rightarrow \mu \mu(60-130)$ & 183.0 & $(1.36, . .1 .39) \pm 0.04$ & $\approx 1,830$ & 460,000 \\
\hline$Z / \gamma^{*} \rightarrow \mu \mu(130-250)$ & 1.370 & $1.39 \pm 0.04$ & $\approx 5,250$ & 10,000 \\
\hline$Z / \gamma^{*} \rightarrow \mu \mu(250-500)$ & 0.115 & $(1.39, . .1 .36) \pm 0.04$ & $\approx 116,570$ & 18,500 \\
\hline$Z / \gamma^{*} \rightarrow \mu \mu(>500)$ & $4.7 \cdot 10^{-3}$ & $(1.36, . .1 .33) \pm 0.06$ & $\approx 1,502,800$ & 9,500 \\
\hline$Z / \gamma^{*} \rightarrow \tau \tau(15-60)$ & 337.6 & $(1.29, . .1 .36) \pm 0.05$ & $\approx 1,210$ & 544,563 \\
\hline$Z / \gamma^{*} \rightarrow \tau \tau(60-130)$ & 183.0 & $(1.36, . .1 .39) \pm 0.04$ & $\approx 2,590$ & 655,000 \\
\hline$Z / \gamma^{*} \rightarrow \tau \tau(130-250)$ & 1.370 & $1.39 \pm 0.04$ & $\approx 54,610$ & 104,000 \\
\hline$Z / \gamma^{*} \rightarrow \tau \tau(250-500)$ & 0.115 & $(1.39, . .1 .36) \pm 0.04$ & $\approx 69,570$ & 11,000 \\
\hline$Z / \gamma^{*} \rightarrow \tau \tau(>500)$ & $4.7 \cdot 10^{-3}$ & $(1.36, . .1 .33) \pm 0.06$ & $\approx 1,542,400$ & 9,750 \\
\hline Process & \multicolumn{2}{|c|}{$\overline{\sigma_{M C}^{N L O} \times B R[\mathrm{pb}]}$} & $\mathcal{L}_{M C}\left[\mathrm{pb}^{-1}\right]$ & $N_{\text {gen }}$ \\
\hline$W W$ inclusive & \multicolumn{2}{|c|}{ 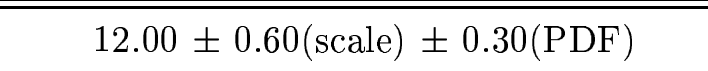 } & $\approx 4,170$ & 50,000 \\
\hline$W Z$ inclusive & \multicolumn{2}{|c|}{$3.68 \pm 0.22($ scale $) \pm 0.12(\mathrm{PDF})$} & $\approx 14,400$ & 53,000 \\
\hline$Z Z$ inclusive & \multicolumn{2}{|c|}{$1.42 \pm 0.06($ scale $) \pm 0.05(\mathrm{PDF})$} & $\approx 37,680$ & 53,500 \\
\hline$t \bar{t} \rightarrow \ell \ell+X$ & \multicolumn{2}{|c|}{$0.69 \pm 0.12$} & $\approx 13,040$ & 9,000 \\
\hline$\Upsilon(1 s) \rightarrow \mu \mu$ & \multicolumn{2}{|c|}{$48 \pm 14\left(\sigma^{L O}\right)$} & $\approx 708$ & 34,000 \\
\hline$\Upsilon(2 s) \rightarrow \mu \mu$ & \multicolumn{2}{|c|}{$49 \pm 15\left(\sigma^{L O}\right)$} & $\approx 612$ & 30,000 \\
\hline
\end{tabular}

Table 4.2: The Monte Carlo simulations of all considered Standard Model background processes, with the corresponding cross section, the equivalent luminosity and the number of generated events. For the $Z / \gamma^{*}$ samples, which are produced in different mass intervals, the corresponding $Z / \gamma^{*}$-mass is given in parenthesis right after the name. The cross sections of the $Z / \gamma^{*}$ processes are given at leading order (PYтніA) and are multiplied by the corresponding mass-dependent $K$-factor [148] in the analysis.

For the $Z / \gamma^{*}$ processes only leading order (LO) cross sections from PyTHIA are given, since the corresponding scale factors depend on the generated dimuon invariant mass of the virtual $Z$ boson or photon. These so-called $K$-factors [148] take into account higher order effects and have recently been calculated up to next-to-next-to-leading order (NNLO) [149]. The next-to- 


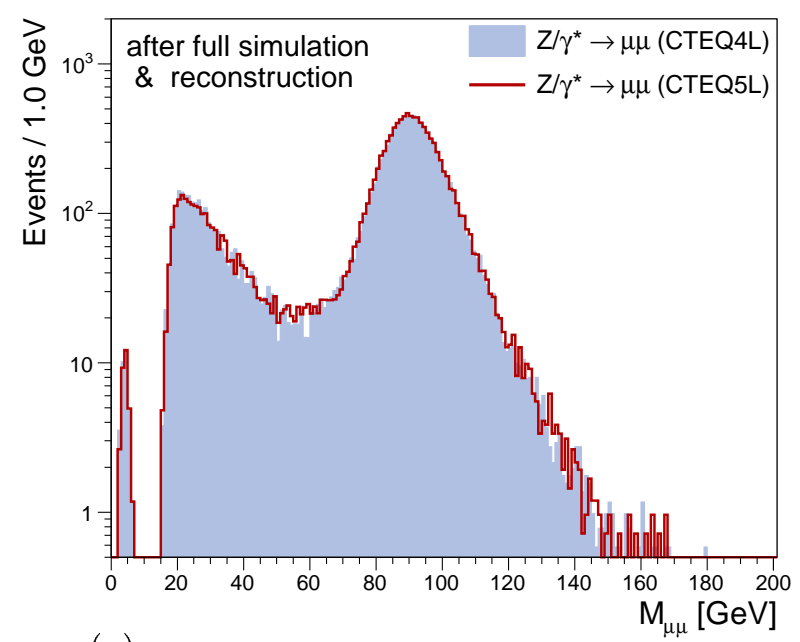

(a)

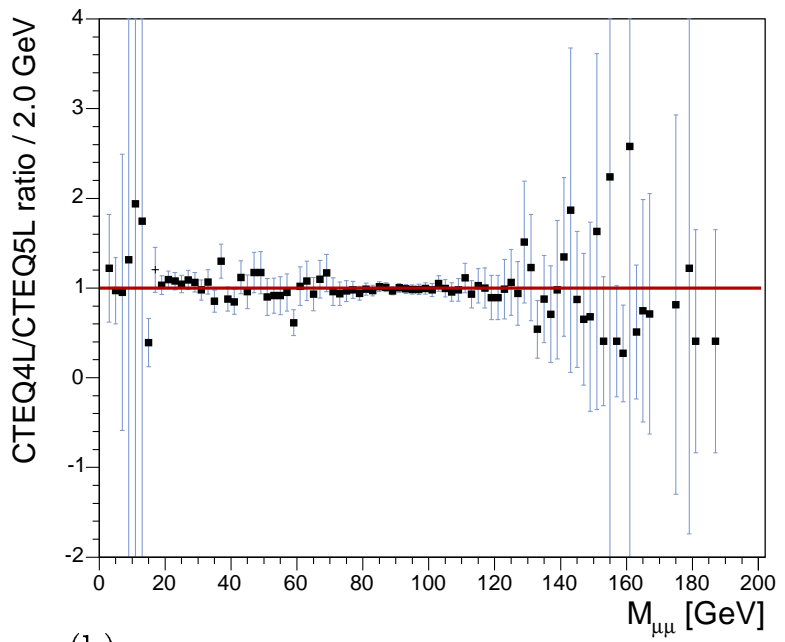

(b)

Figure 4.2: Comparison of $Z / \gamma^{*}$ samples, produced with two different PDF versions: CTEQ4L and CTEQ5L. From the dimuon invariant mass distribution in (a) the resulting ratio, displayed in (b) is calculated. The difference between both samples lies well within the statistical uncertainties.

leading order (NLO) cross sections for diboson production are taken from [150] and the NLO cross section for $t \bar{t}$-production can be found in reference [151], where the $18 \%$ error includes the uncertainty due to the measurement of the top mass. The $\Upsilon$ cross sections have been adjusted to match the dimuon invariant mass distribution after the dimuon preselection and the error is conservatively estimated to be $\approx 30 \%$ to account for the limited statistics and systematic uncertainties in the production mechanism. The process $W \rightarrow \mu \nu$ has also been studied, but was found to be negligible despite its large production cross section $(\approx 2700 \mathrm{pb})$. A final state with at least two muons above certain $p_{\mathrm{T}}$-thresholds (see section 4.5.1) and another additional electron or muon would only be possible, if at least two jets were produced in association with the muon and neutrino and both would have to be misidentified as muons or as a muon and an electron. This probability is too small to seriously consider $W \rightarrow \mu \nu$ as a background to trilepton production.

Another source of background for the considered $\mu \mu \ell(\ell=e$, or $\mu)$ signature are multijet events, where the jets are either produced via the strong interaction, or in association with a gauge boson. This type of background is estimated from data to avoid the associated large theoretical uncertainties and the time-consuming generation and simulation of huge amounts of QCD events, necessary to achieve a reasonable equivalent MC luminosity of $\mathcal{L}_{M C} \geq 2 \times \mathcal{L}_{\text {data }}$. The estimation of a multijet background sample will be explained in section 4.4, after the discussion of the reconstruction of various physics objects and identification variables, which are necessary for the creation of an adequate multijet sample from data.

\subsubsection{Monte Carlo Simulations of SUSY Signal Processes}

All signal Monte Carlo samples are generated with the SusYgEn event generator (version 3) [125], interfaced with the separate program SUSPECT [136] for the evolution of masses and couplings from the renormalization group equations (RGE). For the generation CTEQ5L PDF's [146] are used, and all samples are run through the full detector simulation (D Øgstar and DØsim, using version p14.05.02). The events are combined with an average of 0.8 minimum bias events, corre- 
sponding to an average instantaneous luminosity of about $20 \cdot 10^{30} \mathrm{~cm}^{-2} \mathrm{~s}^{-1}$, and reconstructed with DØreco (version p14.06.01) [130].

The results are interpreted in two different SUSY models: an mSUGRA scenario with the usual approximate mass relation $2 \cdot m\left(\widetilde{\chi}_{1}^{0}\right) \approx m\left(\widetilde{\chi}_{2}^{0}\right) \approx m\left(\widetilde{\chi}_{1}^{ \pm}\right)$and in the no-GUT MSSM, defined in section 1.3.5. In the mSUGRA scenario [?], the following sets of parameters are studied:

\begin{tabular}{lrrr}
\hline \hline $\operatorname{sgn}(\mu)$ & $\tan \beta$ & \multicolumn{1}{c}{$m_{0}[\mathrm{GeV}]$} & \multicolumn{1}{c}{$m_{1 / 2}[\mathrm{GeV}]$} \\
\hline \hline & 5 & $50,100,250,500,750,1000$ & $200-320 \quad$ (or: $190-340)$ \\
$\mu>0$ & 20 & $100,500,1000$ & $240-320$ \\
\hline & 5 & $50,100,250,500,750,1000$ & $230-310$ (or: $150-330)$ \\
$\mu<0$ & 20 & $100,500,1000$ & $230-310$ \\
\hline \hline
\end{tabular}

Table 4.3: The mSUGRA parameter sets investigated in this analysis, the universal trilinear coupling $A_{0}$ is not varied and set to $A_{0}=0$. The analysis is optimised for the $\tan \beta=5$ signal points. For details about the choices of parameters, the reader is referred to the main text.

The universal trilinear coupling $\left(A_{0}\right)$ has only a small influence on the gaugino pair production cross section [73] and is therefore set to zero for all generated points as in a previous D $\varnothing$ Run I analysis [152]. Searches for supersymmetric Higgs bosons at LEP [153] imply that $\tan \beta \leq 2$ is excluded $\left(\tan \beta=v_{d} / v_{u}\right.$, ratio of the two neutral Higgs VEVs). Since the cross section for gaugino pair production increases with increasing $\tan \beta$ due to decreasing gaugino masses, a value of $\tan \beta=5$ (close to the LEP limit) is chosen for most of the signal samples. Some signal samples are also generated for $\tan \beta=20$. Both signs of the Higgs mixing parameter $(\mu)$ are considered and different values of the universal scalar mass $\left(m_{0}\right)$ are studied. At low $m_{0}$, the stau $\left(\widetilde{\tau}_{1}\right)$ can be lighter than the second lightest neutralino $\left(\widetilde{\chi}_{2}^{0}\right)$ and the lightest chargino $\left(\widetilde{\chi}_{1}^{ \pm}\right)$, leading to a larger number of final states with taus [74]. By contrast, a high value of $m_{0}$ prevents complex cascade decays involving sleptons. A scan in steps of $10-20 \mathrm{GeV}$ in the common gaugino mass $\left(m_{1 / 2}\right)$ is performed.

Between 7,000 and 10,000 events are produced per mSUGRA point. All generated mSUGRA signal points are summarised in Table A.1 for $\mu>0$ and in Table A.2 for $\mu<0$ in the appendix. Listed are the relevant parameters $\left(m_{0}, m_{1 / 2}\right)$, the masses of the lightest neutralino and chargino $\left(\widetilde{\chi}_{1}^{0}, \widetilde{\chi}_{1}^{ \pm}\right)$, the LO cross sections for the two dominant production processes: $\widetilde{\chi}_{1}^{ \pm} \widetilde{\chi}_{1}^{\mp}(C C)$ and $\widetilde{\chi}_{2}^{0} \widetilde{\chi}_{1}^{ \pm}(N C)$, as well as the appropriate $K$-factor, the total cross section at next-to-leading order, the equivalent luminosity and the number of generated events. All masses and LO cross sections ( $C C$ and $N C$ production) are taken from the Monte Carlo event generator SusYGEN. However, the total NLO cross section is obtained by multiplying the SusYGEN LO cross section with the appropriate NLO/LO $K$-factor, computed with the program GAUGiNos [140] via Equation (3.2) in section 3.2. The LO cross sections calculated with SUSYGEN are always between 10-40\% below those calculated with PYTHIA. Within a large region of the considered parameter space, the LO cross sections of SusYgen and Gauginos fluctuate around each other within less than $10 \%$, see also [73]. The largest difference, however, is observed for $m_{0}=1000 \mathrm{GeV}$ and positive Higgs mixing parameter $\mu$, where the SusYgen LO cross sections lie about $20 \%$ below the Gauginos LO cross sections. Hence, the results obtained w.r.t. the Susygen cross sections, albeit multiplied with a GAUGinos NLO/LO $K$-factor, will be rather conservative. 
As discussed in section 3.1 , the value of the $\not R_{p}$-coupling is chosen to be $\lambda_{122}=0.001$ for all mSUGRA points. Its strength only influences the lifetime of the lightest neutralino, which is assumed to be the LSP. To be able to rely on the tracking algorithms used in the D $\emptyset$ reconstruction software, the coupling strength was chosen such that the neutralino lifetime never exceeds $c \cdot \tau\left(\widetilde{\chi}_{1}^{0}\right)=1 \mathrm{~cm}$ and the leptons from its decay can be matched to the primary vertex of the event. Neither the event topology, nor the mass spectrum or the decay branching ratios depend on the $\lambda_{122}$ coupling strength. The selection is optimised for some representative mSUGRA signal points (see the small crosses ( $x$ ) in Tables A.1 and A.2 in the appendix).

However, the same selection is also used in case of the second SUSY scenario, the no-GUT MSSM as introduced in section 1.3.5, since the basic properties of SUSY events do not change significantly in going from the mSUGRA to the no-GUT MSSM model. The only fundamental visible difference is the dependence of the signal selection efficiency on the $\lambda_{122}$ coupling strength at very low neutralino masses $(\approx 30 \mathrm{GeV})$ in the no-GUT MSSM scenario. A similar dependence would also be observable in the mSUGRA model, but in this case neutralino masses below $\approx 52 \mathrm{GeV}$ have already been excluded by the four LEP experiments [138]. This interdependence between the coupling strength and the selection efficiency at low neutralino masses is due to a combined effect of the decay length (lifetime) of the LSP and the chosen value of the $\lambda_{122}$ coupling, see also section 3.3, where this is discussed in more detail. For this reason, the coupling strength is increased to $\lambda_{122}=0.01$ for the signal points generated in the framework of the no-GUT MSSM model. As can be seen from Table 1.6 in section 1.3.8, this value lies still well below the current upper limit on the $\mathbb{R}_{p}$-coupling $\lambda_{122}$, which is determined to be $\lambda_{122}<0.027$ for a smuon mass of $m\left(\widetilde{\mu}_{R}\right)=100 \mathrm{GeV}$. Even if the slepton mass is chosen to be $m\left(\widetilde{\mu}_{R}\right)=30 \mathrm{GeV}$, the limit of $\lambda_{122}<0.027 \cdot \sqrt{0.3} \approx 0.015$ is respected. In Table 4.4, the dependence of the LSP decay length $\left(\propto\right.$ lifetime: $\tau\left(\widetilde{\chi}_{1}^{0}\right)$ ) on the LSP mass is shown for a coupling strength of $\lambda_{122}=0.01$.

\begin{tabular}{ccccccc}
\hline \hline$M_{1}$ & $M_{2}$ & $M_{3}$ & $m\left(\widetilde{\chi}_{1}^{0}\right)$ & $m\left(\widetilde{\chi}_{1}^{ \pm}\right)$ & $\tau\left(\widetilde{\chi}_{1}^{0}\right)[\mathrm{cm}]$ & $\sigma_{N L O}[\mathrm{pb}]$ \\
\hline \hline 10.8 & 103.3 & 373.4 & 10.0 & 100.2 & 165.5 & 3.128 \\
10.8 & 153.5 & 554.6 & 10.0 & 149.9 & 165.1 & 0.493 \\
20.8 & 103.5 & 373.9 & 20.0 & 100.3 & 5.14 & 3.096 \\
20.8 & 153.5 & 554.6 & 20.0 & 149.9 & 5.13 & 0.492 \\
20.8 & 204.0 & 737.0 & 20.0 & 200.0 & 5.12 & 0.102 \\
\hline 30.8 & 103.5 & 373.9 & 30.0 & 100.3 & 0.676 & 2.922 \\
30.8 & 128.3 & 463.5 & 30.0 & 124.9 & 0.677 & 1.165 \\
40.9 & 118.5 & 428.1 & 40.1 & 115.2 & 0.159 & 1.705 \\
40.9 & 153.5 & 554.6 & 40.1 & 149.9 & 0.158 & 0.491 \\
40.9 & 214.0 & 773.2 & 40.0 & 210.0 & 0.160 & 0.077 \\
50.9 & 108.2 & 390.9 & 50.0 & 105.0 & 0.0526 & 2.556 \\
51.2 & 223.5 & 807.5 & 50.3 & 219.4 & 0.0508 & 0.058 \\
\hline \hline
\end{tabular}

Table 4.4: The dependence of the decay length of the lightest neutralino (LSP) on its mass for a coupling strength of $\lambda_{122}=0.01$. The remaining MSSM parameters are: $\tan \beta=5, \mu=1 \mathrm{TeV}, A_{0}=0$, and $m_{A}=400 \mathrm{GeV}$. As can be seen from the above decay lengths for the same values of $m\left(\widetilde{\chi}_{1}^{0}\right)\left(M_{1}\right)$, but varying $m\left(\widetilde{\chi}_{1}^{ \pm}\right)\left(M_{2}\right)$, the mass parameters $M_{2}\left(M_{3}\right)$ governing the masses of the lightest chargino (gluino, $\left.S U(3)_{C}\right)$ are largely irrelevant for the decay length of the LSP. 
The ratio of the VEVs of the two neutral Higgs fields is again chosen as $\tan \beta=5$ for the same reasons as explained previously in case of the mSUGRA scenario. The Higgs mixing parameter is set to $\mu=1 \mathrm{TeV}$, so that the heavier gauginos $\left(\widetilde{\chi}_{3}^{0}, \widetilde{\chi}_{4}^{0}\right.$, and $\left.\widetilde{\chi}_{2}^{ \pm}\right)$are only rarely produced and the dominant production processes are the same as in the mSUGRA scenarios: $\widetilde{\chi}_{1}^{ \pm} \widetilde{\chi}_{1}^{\mp}$ and $\widetilde{\chi}_{2}^{0} \widetilde{\chi}_{1}^{ \pm}$. Nevertheless it has been checked, that this setting does not single out special cases. All sfermion masses, i.e. the squark and slepton masses are also set to $1 \mathrm{TeV}$, to ensure that on-shell sfermions are hardly produced and do not interfere in the cascade decays of heavier gauginos into the LSP. The trilinear coupling $A_{0}$ and the the mass of the supersymmetric pseudoscalar Higgs boson $m_{A}$ are set to $A_{0}=0$, and $m_{A}=400 \mathrm{GeV}$, respectively. The two mass parameters $M_{1}$ and $M_{2}$ for the gaugino partners of the $\mathrm{U}(1)_{Y}$ and $\mathrm{SU}(2)_{L}$ gauge bosons are varied and, since the GUT-relation between $M_{1}$ and $M_{2}$ :

$$
M_{1}=\frac{5}{3} \cdot \tan ^{2} \theta_{w} \cdot M_{2},
$$

is assumed not to be valid anymore, these two parameters can be varied independently of each other. Technically, this is achieved by multiplying the right-hand side of Equation 4.1 with a variable parameter $(R S)$ upon generation with Susygen.

Various MSSM signal points are generated with about 4,000 to 7,000 events per point. Tables B.2, B.1 and B.3 in the appendix list the corresponding MSSM model parameters for all generated MSSM signal samples: the value of the Higgs mixing parameter $\mu$, the mass parameters and masses of the $\mathrm{U}(1)_{Y}$ and $\mathrm{SU}(2)_{L}$ gauge bosons: $M_{1}$ and $M_{2}$, which respectively govern the masses of the lightest neutralino $\left(m\left(\widetilde{\chi}_{1}^{0}\right)\right)$ and the lightest chargino $\left(m\left(\widetilde{\chi}_{1}^{ \pm}\right)\right)$, and the $R S$-parameter, allowing the independent variation of the latter two parameters $\left(M_{1}\right.$ and $\left.M_{2}\right)$. Also given are the LO cross sections of the dominant $C C$ and $N C$ pair production processes $\left(\widetilde{\chi}_{1}^{ \pm} \widetilde{\chi}_{1}^{\mp}\right.$ and $\left.\widetilde{\chi}_{2}^{0} \widetilde{\chi}_{1}^{ \pm}\right)$, the NLO/LO $K$-factor, again computed with GaUGinos [140], the resulting NLO total cross section, the equivalent luminosity $\left(\mathcal{L}_{M C}\right)$ and the number of generated events. As for the mSUGRA scenario, various parameter sets are also investigated in case of the considered no-GUT MSSM model (see Table 4.5). In addition, a complete scan of the $\left(\widetilde{\chi}_{1}^{0}, \widetilde{\chi}_{1}^{ \pm}\right)$-mass plane is performed for

\begin{tabular}{crrrr}
\hline \hline$\mu[\mathrm{TeV}]$ & $M_{1}[\mathrm{GeV}]$ & $m\left(\widetilde{\chi}_{1}^{0}\right)[\mathrm{GeV}]$ & $M_{2}[\mathrm{GeV}]$ & $m\left(\widetilde{\chi}_{1}^{ \pm}\right)[\mathrm{GeV}]$ \\
\hline \hline+1 & $\approx 50.8-267.5$ & $50.0-103.5$ & 106.7 (fixed) & 103.5 (fixed) \\
+1 & $\approx 35.8-225.0$ & $35.0-200.0$ & 204.0 (fixed) & 200.0 (fixed) \\
+1 & $\approx 28.7-229.7$ & $27.9-224.0$ & 229.1 (fixed) & 225.0 (fixed) \\
-1 & 81 (fixed) & 80 (fixed) & $\approx 103-335$ & $100-330$ \\
+1 & 100 (fixed) & 100 (fixed) & $\approx 139-239$ & $141-240$ \\
\hline \hline
\end{tabular}

Table 4.5: The studied no-GUT MSSM parameter sets: the ratio of the Higgs field VEVs is not varied and fixed at $\tan \beta=5$. The universal trilinear coupling is set to $A_{0}=0$ and the mass of the pseudoscalar Higgs boson is set to $400 \mathrm{GeV}$. In addition all sfermion masses are set to $m(\widetilde{f})=1 \mathrm{TeV}$.

$\mu=1 \mathrm{TeV}$, with $M_{1}$ and $M_{2}$ varied independently. The boundaries of the scan are approximately given by: $m\left(\widetilde{\chi}_{1}^{0} / \widetilde{\chi}_{1}^{ \pm}\right)=30 / 100 \mathrm{GeV}$, and $m\left(\widetilde{\chi}_{1}^{0} / \widetilde{\chi}_{1}^{ \pm}\right)=218 / 240 \mathrm{GeV}$. This scan enables the derivation of mass bounds for the lightest neutralino and chargino, although the two masses are not connected via a simple GUT-relation as in an mSUGRA scenario. 


\subsection{Trigger Efficiency and Object Identification}

All physics objects, i.e. electrons, muons, taus, jets, $\not_{T}$, vertices and tracks are already identified and selected as such during the skimming process, see Figure 2.46 in section 2.5. Upon conversion of the thumbnail files into ROOT tree format via the framework program top_analyze, a number of standardised and certified corrections are applied to these objects, most notably the jet energy scale (JES) correction [154] and corrections to EM-objects, muons and $\not_{T}$, that take into account different resolutions in the $\gg$ real « data and the simulated Monte Carlo data. These latter are sometimes also referred to as smearing, which can be applied to different variables, e.g. the momentum, transverse momentum $\left(p_{\mathrm{T}}\right)$, transverse energy $\left(E_{\mathrm{T}}\right)$, or in case of angular resolution also to the $\eta, \varphi$ variables of the respective objects. The criteria and algorithms used for the primary identification and also for the corrections are developed by the respective object-ID groups of the DØ experiment, e.g. the EMID [104], the MuonID [105], and the combined JetMET groups $\left(\mathrm{MET}: \not_{T}\right)$. Upon each major new release of the main reconstruction code DØreco, the identification criteria are refined and the algorithms optimised, so that standardised and certified code is used by all analysis groups.

With the exception of the trigger efficiencies, described in the following subsection, all efficiencies and acceptances are taken from the full detector simulation. Therefore the simulation is carefully checked with real data and - where necessary - correction factors are determined, which are applied to the Monte Carlo simulated data.

\subsubsection{The Dimuon Trigger Efficiency and Application to MC}

To correctly reproduce the trigger efficiency for Monte Carlo data, one first has to determine this efficiency for the relevant triggers. This has been done for the TriggerEfficiency class [134] according to the prescription detailed in [156], which is briefly described in the following.

There are five trigger objects, that can fire a trigger and lead to the recording of a given event: an electron, a muon, a jet, a track, or missing transverse energy $\left(\not_{T}\right)$. The event is recorded if the requirements on all three trigger levels are satisfied, which can also be written in terms of probabilities:

$$
P_{\text {trigger }}=P(L 1) \times P(L 2 \mid L 1) \times P(L 3 \mid L 1 L 2),
$$

which means the overall trigger efficiency is the product of the probability for the event to pass L1, multiplied by the probability to pass L2, given that it has already passed L1, and multiplied by the probability to also pass L3 given that it has already passed L1 and L2.

Two general, simplifying assumptions are made:

(i) the probability of an object to fire a certain trigger is independent of other objects being present in the event or not $\longrightarrow$ the probabilities for different objects factorise, e.g.: $P(\mu$, jet $)=P(\mu) \times P(j e t)$ for a combined muon and jet trigger;

(ii) the probability of one type of object to fire a trigger is independent of the number of objects of this type $\longrightarrow$ the probabilities for same type objects also factorise, e.g.: $P\left(\mu_{1}, \mu_{2}\right)=P\left(\mu_{1}\right) \times P\left(\mu_{2}\right)$ for a dimuon trigger. 
These assumptions are valid separately for each of the three trigger levels. Hence, the probability for an event with $N$ reconstructed muons to pass a trigger that requires at least one muon, can be formulated as one minus the probability for none of the muons to pass:

$$
P(\geq 1 \mu, N)=1-P(0 \mid N)=1-\prod_{i=1}^{N} P\left(\overline{\mu_{i}}\right)=1-\prod_{i=1}^{N}\left(1-P\left(\mu_{i}\right)\right),
$$

where $P\left(\overline{\mu_{i}}\right)=\left(1-P\left(\mu_{i}\right)\right)$ denotes the probability that a muon does not satisfy the specified trigger criteria. If the trigger requires at least two muons, not only the probability for none of the muons passing the trigger conditions need to be subtracted, but also the probability for only one muon satisfying the conditions, which leads to:

$$
\begin{aligned}
P(>1 \mu, N) & =1-P(0 \mid N)-P(1 \mid N) \\
& =1-\prod_{i=0}^{N}\left(1-P\left(\mu_{i}\right)\right)-\sum_{i=0}^{N} P\left(\mu_{i}\right) \prod_{j=0, j \neq i}^{N}\left(1-P\left(\mu_{j}\right)\right) .
\end{aligned}
$$

According to this prescription, the trigger efficiencies are measured separately for each object type, each trigger and each trigger level, i.e. $P(L 1, o b j), P(L 2 \mid L 1, o b j)$, and $P(L 3 \mid L 1 L 2, o b j)$. They are then parametrised in dependence of $p_{\mathrm{T}}, \eta_{\text {det }}$, and $\varphi_{\text {det }}: P(o b j)=P\left(p_{\mathrm{T}}, \eta_{\text {det }}, \varphi_{\text {det }}\right)$. Besides that, all trigger efficiencies are determined on data samples collected with independent triggers, which ensures that the parametrised trigger efficiencies, being applied to the Monte Carlo events later on, do not generate a bias w.r.t. the preselected 2MU data sample.

$$
P_{\text {dimuon }}=P(L 1) \times P(L 2 \mid L 1) \times P(L 3 \mid L 1, L 2)=f_{L 1}^{2} \cdot\left(1-\left(1-f_{L 2}\right)^{2}\right) \cdot f_{L 3},
$$

where $f_{L 1}, f_{L 2}$, and $f_{L 3}$ are the parametrisations of the probabilities for a muon to pass the L1, L2, and L3 trigger conditions, respectively. The L1 condition appears squared in Eq. (4.5), since the dimuon triggers all require two muons to pass the mu1ptxatxx requirement of Level 1. On the other hand, only one from two L1-muons is required to be of »medium « quality on L2, which gives the factor $\left(1-\left(1-f_{L 2}\right)^{2}\right)$. The functions implemented in the TriggerEfficiency class are given in Equations (4.6) and (4.7), where it is assumed that the dependence on the pseudorapidity $\left(\eta_{\text {det }}\right)$ and on the transverse momentum factorise $f\left(\eta_{\text {det }}, p_{\mathrm{T}}\right) \approx f\left(\eta_{\text {det }}\right) \cdot f\left(p_{\mathrm{T}}\right)$ :

$$
\begin{gathered}
f_{L 1}\left(\eta_{d e t}\right)=f_{L 2}\left(\eta_{d e t}\right)=A_{3}+A_{0} \cdot e^{-A_{1}\left(\eta_{\text {det }}^{2}-A_{2}\right)} \sin \left(\eta_{\text {det }}^{2}-A_{2}\right) \\
f_{L 1}\left(p_{\mathrm{T}}\right)=f_{L 2}\left(p_{\mathrm{T}}\right)=1-\operatorname{erf}\left(-\frac{1}{4} \cdot \frac{\left(p_{\mathrm{T}}-A_{4}\right)^{2}}{A_{5}^{2}}\right)
\end{gathered}
$$

where $\operatorname{erf}(b)=\frac{2}{\sqrt{\pi}} \int_{0}^{b} e^{-x^{2}} d x$ denotes the Gauss error function. The L3 trigger conditions were found to be $100 \%$ efficient. As can be seen from the two examples in Figure 4.3, the parametrisation of the $p_{\mathrm{T}}$-dependence is different for each trigger list, whereas the parametrisation of the $\eta_{d e t^{-} \text {-dependence is the same (within uncertainties) for all trigger lists. The latter only differs for }}$ the Level 1 and Level 2 trigger criteria, for which the parameters are summarised in Table 4.6. Further details can be found in references [134, 156].

The program takes into account information $\left(E, p_{\mathrm{T}}\right.$ or $\left(E_{\mathrm{T}}\right), \eta_{\text {det }}$ and $\left.\varphi\right)$ of all physical objects of an event, i.e. of EM-objects, muons, jets and missing transverse energy, and calculates the probability for this simulated event to fire one (or more) of the specified triggers. This probability 


\begin{tabular}{lcccc}
\hline \hline & $A_{0}$ & $A_{1}$ & $A_{2}$ & $A_{3}$ \\
\hline \hline L1: mu1ptxatxx & $-0.8 \pm 0.2$ & $2.8 \pm 0.5$ & $0.1 \pm 0.1$ & $0.99 \pm 0.01$ \\
L2: MUON(1,med) & $0 \pm 0$ & $8.7 \pm 7.0$ & $1.779 \pm 0.006$ & $0.981 \pm 0.007$ \\
\hline \hline
\end{tabular}

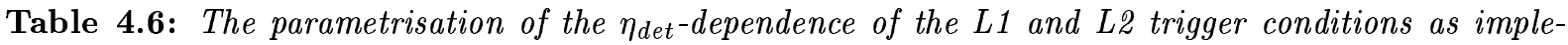
mented in the TriggerEfficiency package [134]. The parameters $A_{4}$ and $A_{5}$, describing the $p_{\mathrm{T}}$-dependence are not listed here, since they not only differ for L1 and L2, but also from one trigger list to another.
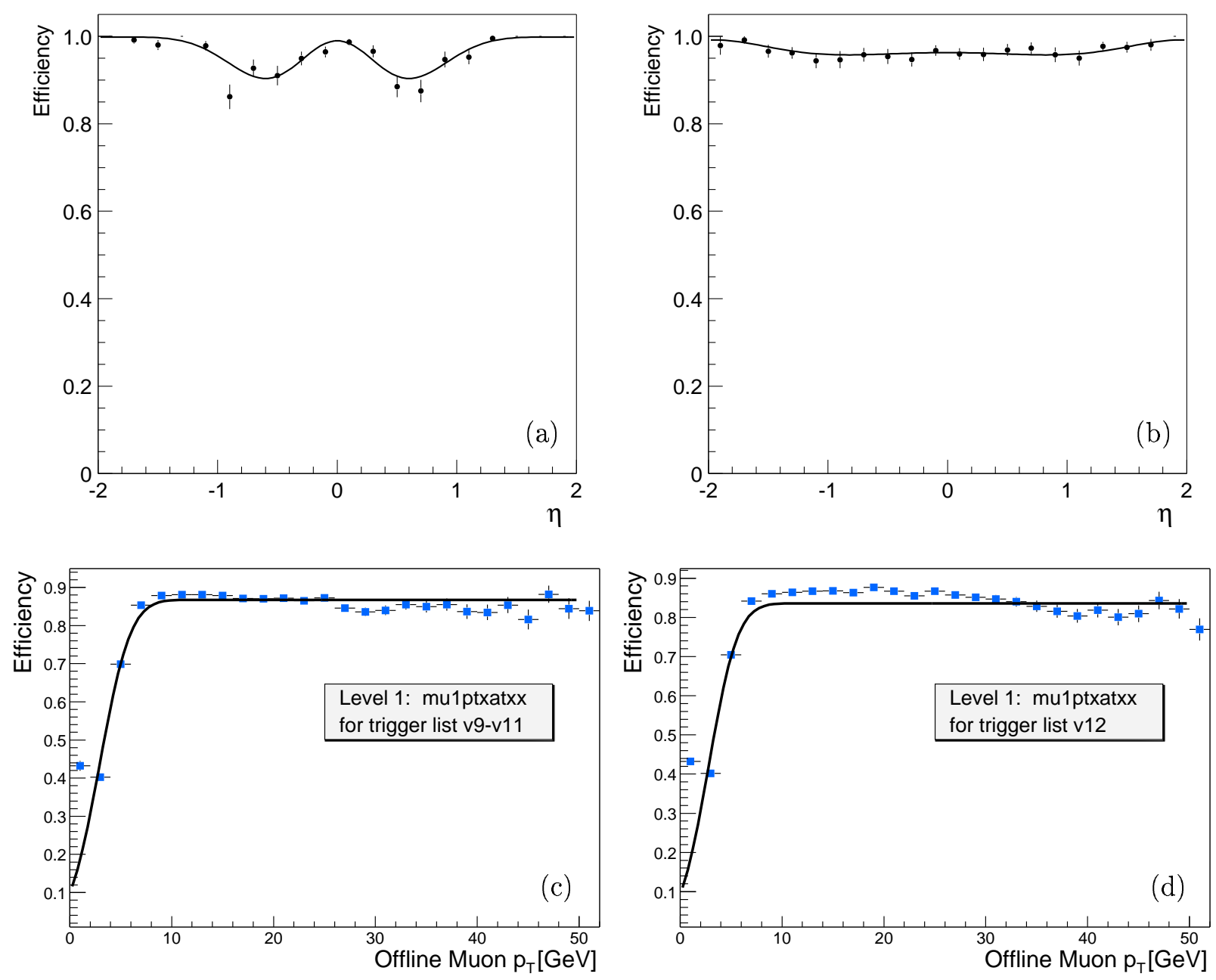

Figure 4.3: Measurements of the L1 and L2 muon trigger efficiencies: $(a, b)$ in dependence of the pseudorapidity $\eta_{\text {det }}$ for the L1 (a) and the L2 (b) condition. (c,d) Two examples of trigger turn-on curves as a function of the muon transverse momentum for triggers valid in trigger lists v9-11 (c) and v12 (d). [The plots are taken from the documentation of the TriggerEfficiency class [134].]

is then used to weight the Monte Carlo event accordingly. All simulated events, SM background and signal events, are treated in the same way. On average the weights are $\approx 0.94 \pm 0.03$ for signal events and $\approx 0.88 \pm 0.08$ for background events, though these weights largely depend on the considered process.

The only drawback in utilising the TriggerEfficiency package is the fact, that there is no parametrisation of the L2 dimuon trigger criterion for muons with a transverse momentum below $15 \mathrm{GeV}$. Fortunately, however, the L2 condition MUON (1, med), only requires one muon, not two muons of 
an event to pass. Hence, it also enters only once in the determination of the probability for an event to fire a dimuon trigger. This leads to the rather high $p_{\mathrm{T}}$ requirement of $15 \mathrm{GeV}$ in the subsequent event selection, in cases where the leading lepton is a muon.

\subsubsection{Object Identification and Efficiencies}

As mentioned, the physics and detector simulation is carefully checked by comparing real data with simulated Monte Carlo data and determining correction factors in case the agreement is not satisfying. Since in this analysis, the primary focus is on muons, of which at least two are required in the final state, the efficiencies to identify and reconstruct muons will be discussed in detail, as are efficiencies of some basic muon properties, such as isolation or quality criteria. For other efficiencies and correction factors, e.g. electron or tau identification / reconstruction efficiencies, the reader is referred to different analyses and the $\mathrm{D} \emptyset$ documentation.

The muon efficiencies are determined using the common $»$ tag and probe « method [155]. Since no background subtraction is performed, a clean sample of muons is required. Usually $Z / \gamma^{*} \rightarrow \mu \mu$ events are selected from data, since the production and decay of $Z$ bosons is a well-understood and well-measured process. In addition, the signature of $Z / \gamma^{*} \rightarrow \mu \mu$ events is very clean and only little background from other processes is expected, which predestines this type of events for efficiency studies, allowing for a meaningful comparison of simulated MC data and real data.

The tag and probe method now consists in tagging one muon if it satisfies tight selection or quality criteria and probing the other muon for the quantity of interest, e.g. isolation or the existence of a track that matches the probe muon. If the second muon also satisfies the tag criteria, the roles of the two muons are interchanged and the process repeated. The efficiency is then given by the number of events, in which the probe was successful, divided by the number of events where a muon could be tagged, independent of the outcome of the probe and considering each tag as a separate event:

$$
\varepsilon=\frac{2(t t)+(t p)}{2(t t)+(t p)+(t f)}
$$

where $t, p, f$ denote tag, pass, and fail, respectively, for a muon satisfying either the tag criteria, or the probe criteria, or failing both sets of criteria. The distinction between $t$ and $p$ is due to the fact, that the requirements for the probe muon are usually softer than the criteria used to tag a muon. In case the tag and probe criteria are the same, the terms $(t p)$ have to be omitted from the above equation.

The efficiency is measured separately for data and for a $Z / \gamma^{*}$ Monte Carlo sample. In case of the recorded data, another difficulty arises when determining efficiencies: any efficiency could be biased, if the event had been recorded due to exactly the property in question. For example, if there was a special muon trigger for isolated muons and one would not require at least one independent trigger, the entire sample would be biased towards more isolated muons, because the probe muon could have fired this trigger. It would have exactly the opposite effect, i.e. a bias towards less isolated muons in the sample, if one instead required that the probe muon had not fired this special trigger. The only other alternative to an entirely independent trigger having fired and thus led to the recording of the event, would be to match the tag muon to the trigger requirement, that is the same or very similar to the property being tested. 
In the following the first alternative - independent triggers - is mostly used. Only in case of the trackfinding / -matching efficiency, which provides information on how well a muon (muon segment) can be matched to an inner track, the second method is used. For this efficiency measurement the tag muon can be matched to a L3 track object.

\section{Muon Identification}

Muons are identified based on hits in the central/forward muon drift chambers and scintillators within $\left|\eta_{\text {det }}\right|<2.0$. Track segments are fitted first across one layer, and in a second step these segments are interpolated in between two or all three layers of the muon system, to form a local muon candidate. While the scintillator hits are primarily used for triggering purposes (especially at Level 1), the timing information also provides an excellent possibility for the rejection of out-of-time background like cosmic muons. Additionally, a matching inner track (i.e. measured with the SMT/CFT detectors) and/or a matching path of a minimum ionising particle in the calorimeter provide the possibilities to define effective isolation criteria for muons.

In this analysis, each muon candidate is required to satisfy the following criteria: the object must be of $\gg$ loose « quality (see section 4.1) and in-time to reject cosmic muons, i.e. the times measured in the A and BC-layer scintillators should not exceed $\pm 10 \mathrm{~ns}\left(\left|t_{A-\text { layer }}\right|<10 \mathrm{~ns}\right.$ and $\left.\left|t_{B C-\text { layer }}\right|<10 \mathrm{~ns}\right)$. Its transverse momentum must be larger than $4 \mathrm{GeV}$, and hits are required in either the BC-layer, or all three layers of the muon system. A matched central track, consisting of at least 8 hits in the CFT-detector is also required. Candidates, called $»$ high quality « muons (the difference between normal/loose muons and high quality muons will become important during the selection) must additionally satisfy the following requirements:

- Hits in all layers of the muon system (in the A, B, and C-layer);

- Isolation (tracker): $\sum p_{\mathrm{T}}^{t r}<2.5 \mathrm{GeV}$ and $\sum p_{\mathrm{T}}^{t r} / p_{\mathrm{T}}(\mu)<6 \%$, where $p_{T}^{t r}$ is summed within an $\mathcal{R}=0.5$ cone around the muon, excluding the muon track itself, see Figure 4.4 (a);

- Isolation (calorimeter): $E_{\mathrm{T}}^{\text {halo }}<2.5 \mathrm{GeV}$ and $E_{\mathrm{T}}^{\text {halo }} / p_{\mathrm{T}}(\mu)<8 \%$, where $E_{\mathrm{T}}^{\text {halo }}$ is defined as the energy deposited in a hollow cone around the direction of the muon track (with inner/outer cone radii: $\mathcal{R}_{i}=0.1 / \mathcal{R}_{o}=0.4$ ), see also Figure $4.4(\mathrm{~b})$;

- »Medium« quality (denotes a certain hit pattern in the muon system, see section 4.1).

(a)

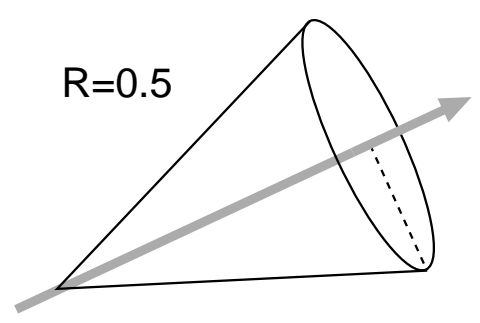

(b)

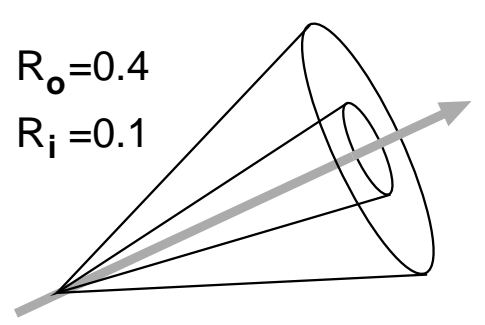

Figure 4.4: Schematic illustration of the muon isolation (a) in tracks and (b) within the calorimeter.

Since the transverse momentum resolution of the muon and the tracking system is worse than the one expected from Monte Carlo simulations, the $p_{\mathrm{T}}$ of each muon candidate in $\mathrm{MC}$ events is smeared. The smearing corresponds to an artificial broadening of the $p_{\mathrm{T}}$ distribution in $Z / \gamma^{*} \rightarrow \mu \mu \mathrm{MC}$ events until the $\mathrm{MC}$ distribution matches the one measured in $Z / \gamma^{*} \rightarrow \mu \mu$ 
data. Assuming a gaussian distribution of the transverse momentum, this is achieved by applying a gaussian function to the $p_{\mathrm{T}}$ of each muon candidate [132]:

$$
\frac{1}{p_{T}^{\prime}}=\frac{1}{\alpha \cdot p_{T}}+\operatorname{Gauss}(0, \sigma), \quad \begin{aligned}
& \alpha=0.9936, \sigma=0.0024, \text { for }|\eta|<1.62 \\
& \alpha=0.9731, \sigma=0.0036, \text { for }|\eta|>1.62
\end{aligned}
$$

The $»$ loose « muon reconstruction efficiency and the »medium « efficiency are determined on the MU_RECO_EFF skim of the top group, while the isolation and the trackfinding/-matching efficiencies are determined on the 2MU skim of the common samples group, which is also used for the analysis itself. This choice of samples is due to the requirement that events on which the identification / reconstruction efficiency for muons, and the efficiency for a muon to be of »medium « quality are tested, should not be selected on the grounds of two already identified muons per event. The MU_RECD_EFF skim contains only events events with at least one reconstructed muon of loose quality and $p_{\mathrm{T}}>15 \mathrm{GeV}$, and at least two tracks with $p_{\mathrm{T}}>15 \mathrm{GeV}$. Additionally the muon must be isolated according to the above mentioned criteria and its spatial distance to any jet must be at least $\Delta \mathcal{R}(\mu$, jet $)>0.5$.

To measure the loose muon reconstruction efficiency, the tag muon is required to be of medium quality, matched to a central track, isolated in tracks and calorimeter, in-time to reject cosmics, and its transverse momentum must be above $p_{T}>15 \mathrm{GeV}$. The tag muon is also required to fulfill $|d c a|<16 \mathrm{~mm}$, where $d c a$ denotes the distance of closest approach of the fitted track to the primary vertex. The event must either be triggered by a single muon trigger where the tag muon is required to match the trigger object, or a calorimeter trigger, i.e. EM-shower or jet triggers, must have fired. The probe object must consist of a well reconstructed track with more than 8 CFT hits, more than one SMT hit, $\chi^{2}<4$, and a $|d c a|<16 \mathrm{~mm}$, and the matched track must be isolated in the tracking detectors. In addition, in order to be able to compare to a $Z / \gamma^{*}$ Monte Carlo sample, the probe objects' transverse momentum has to be larger than $20 \mathrm{GeV}$, it has to be back-to-back to the tag muon $(\Delta \varphi>2.8)$, and the reconstructed invariant mass of the tag muon and the probe object is required to lie between $71 \mathrm{GeV}$ and $111 \mathrm{GeV}$. The measured reconstruction efficiency for loose muons as a function of $\eta, p_{\mathrm{T}}$, and the invariant mass is shown in Fig. 4.5. It is reasonably well described by the simulation and the slight dependence on the pseudorapidity $\eta$ is parametrised in the corresponding $\mathrm{MC}$ correction factor $f_{\text {reco }}^{\mu}$ as follows: for all muons in $|\eta|<2.1$

$$
f_{\text {reco }}^{\mu}(\eta)=+0.956+0.002 \eta+0.032 \eta^{2}-0.000 \eta^{3}-0.003 \eta^{4}
$$

Since »medium « quality muons are required in the analysis, the correct description of this quality criterion was also studied. To measure the efficiency for a muon to be of medium quality, the muon is required to match a central track $(|d c a|<16 \mathrm{~mm})$, be isolated in tracks and in the calorimeter, have a transverse momentum of more than $15 \mathrm{GeV}$, and be of $»$ loose« quality. The muon is then tested for the »medium « requirement. Fig. 4.6 shows a good agreement between the measured and the simulated medium muon efficiency as a function of $\varphi, \eta$ and $p_{\mathrm{T}}$.

The track-finding and -matching efficiency is measured requiring the tag muon to be a medium muon matched to a central track, isolated in the tracker as well as in the calorimeter, in-time to reject cosmics and with a $p_{\mathrm{T}}$ of more than $15 \mathrm{GeV}$. The event is allowed to be triggered by either calorimeter triggers, a single or dimuon trigger without track requirement, or a single or 
dimuon trigger with track requirement, where the tag muon is matched to the L3-track object. The probe muon must have hits in either all layers or at least in the post-toroid BC-layer, its transverse momentum measured exclusively with the muon system must exceed $p_{T}>10 \mathrm{GeV}$, $\chi_{\text {local }}^{2}<5$ (a measure for the reconstructed segments to belong to one muon candidate) and the distance to a jet must be at least $\Delta \mathcal{R}>0.5$. Tag and probe muon must be of opposite charge, back-to-back in $\varphi(\Delta \varphi>2.8)$ and the reconstructed invariant dimuon mass must lie in a $20 \mathrm{GeV}$ interval around the $Z^{0}$ mass to be comparable to a $Z / \gamma^{*}$ Monte Carlo sample. The measured track-finding and -matching efficiency is reasonably well described by the simulation and is shown as a function of $\varphi, p_{\mathrm{T}}$ and the invariant dimuon mass in Fig. 4.7.

In the event selection applied later on, high quality muons are required to be isolated in tracks and within the calorimeter according to the criteria defined above. Since so far no isolation requirements are implemented in any muon trigger, the event is allowed to be triggered by any trigger including single and dimuon triggers. The tag and probe muons are required to satisfy the same criteria as the tag muon in case of the medium muon efficiency measurement, including the medium quality requirement, but with the exception of the isolation criteria in case of the probe muon. The isolation criteria are of special importance for the estimation of the multijet background in the following section 4.4, where they constitute the primary variables to decide wether a given data event is to be treated as a potential multijet background event for this analysis or not.

Although high efficiencies are desirable, since they increase the discovery potential, or allow to set very stringent limits in case no signal is found, they are of no use, if the efficiencies measured separately for data and Monte Carlo events do not match. Meaningful results, either a discovery or improved exclusion limits, can only be achieved by proving a good understanding of the detector by means of MC simulations. As can be seen from the following pages, all correction factors are small, of the order of a few percent at most, thus proving the required good understanding of the $\mathrm{D} \varnothing$ detector. Table 4.7 provides an overview of the average efficiencies in data and Monte Carlo and the resulting "per muon « correction factors. The uncertainties of the correction factors are determined by requiring that $68 \%$ of the points, including the statistical errors, lie within the blue shaded bands in the following Figures 4.5, 4.6, 4.7, and 4.8.

\begin{tabular}{lccc}
\hline \hline Criterion tested & $\varepsilon_{\text {data }}[\%]$ & $\varepsilon_{M C}[\%]$ & $f_{\text {corr }}^{\mu}$ for MC \\
\hline \hline Identification/reconstruction (»loose $\ll)$ & 91.8 & 93.7 & $0.979 \pm 0.024$ \\
Muon quality $»$ medium $«$ & 97.1 & 97.5 & $0.999 \pm 0.021$ \\
Trackfinding/-matching & 97.2 & 95.3 & $1.020 \pm 0.013$ \\
Isolation (tracker \& calorimeter) & 94.6 & 96.1 & $0.986 \pm 0.016$ \\
\hline \hline
\end{tabular}

Table 4.7: The average efficiencies for muon identification / reconstruction, quality, isolation and trackmatching, together with the resulting Monte Carlo correction factors and the respective uncertainties.

The orange shaded vertical bands, indicate regions (or bins), where the statistics in either data or $\mathrm{MC}$ are too small to calculate the efficiency in a reliable way. On the left-hand side of each figure (a,c,e), the efficiencies measured separately for data and $\mathrm{MC}$ events are shown as a function of different variables, e.g. $\eta, p_{\mathrm{T}}$, or the dimuon invariant mass. The corresponding correction 
factors, that will be applied to the MC events in the analysis, are shown on the right-hand sides $(\mathrm{b}, \mathrm{d}, \mathrm{f})$, respectively.

The light-blue coloured texts provide the results from two independent methods used to extract the correction factors: in the first method, each efficiency bin for data is divided by the corresponding efficiency bin for $\mathrm{MC}$ and the resulting factors $f_{\text {corr }}=\varepsilon^{\text {data }} / \varepsilon^{M C}$ are shown on the right-hand sides of the figures. These factors are then fitted and the result is given by the first light-blue coloured line xpos $=\ldots$ (Fit), while the width of the fit can be found in the last line (width). The fit values for each efficiency differ from variable to variable since the weighting depends on the chosen variable.

In a second method, the overall efficiency for data is calculated and divided by the overall MC efficiency. The result is shown in the second light-blue coloured line xpos $=\ldots$ (1 bin). The final correction factor per muon and criterion $f_{\text {corr }}^{\mu}$ is then obtained by forming the weighted average of all four factors (three variables plus the result of the 1-bin-method).

With the exception of a slight $\eta$-dependence of the correction factor of the muon reconstruction efficiency, all correction factors are flat in $\eta, \varphi, p_{\mathrm{T}}$, (and the dimuon invariant mass $M_{\mu \mu}$ ). Hence, the constant factors, as given in Table 4.7 are applied to the MC simulations. 

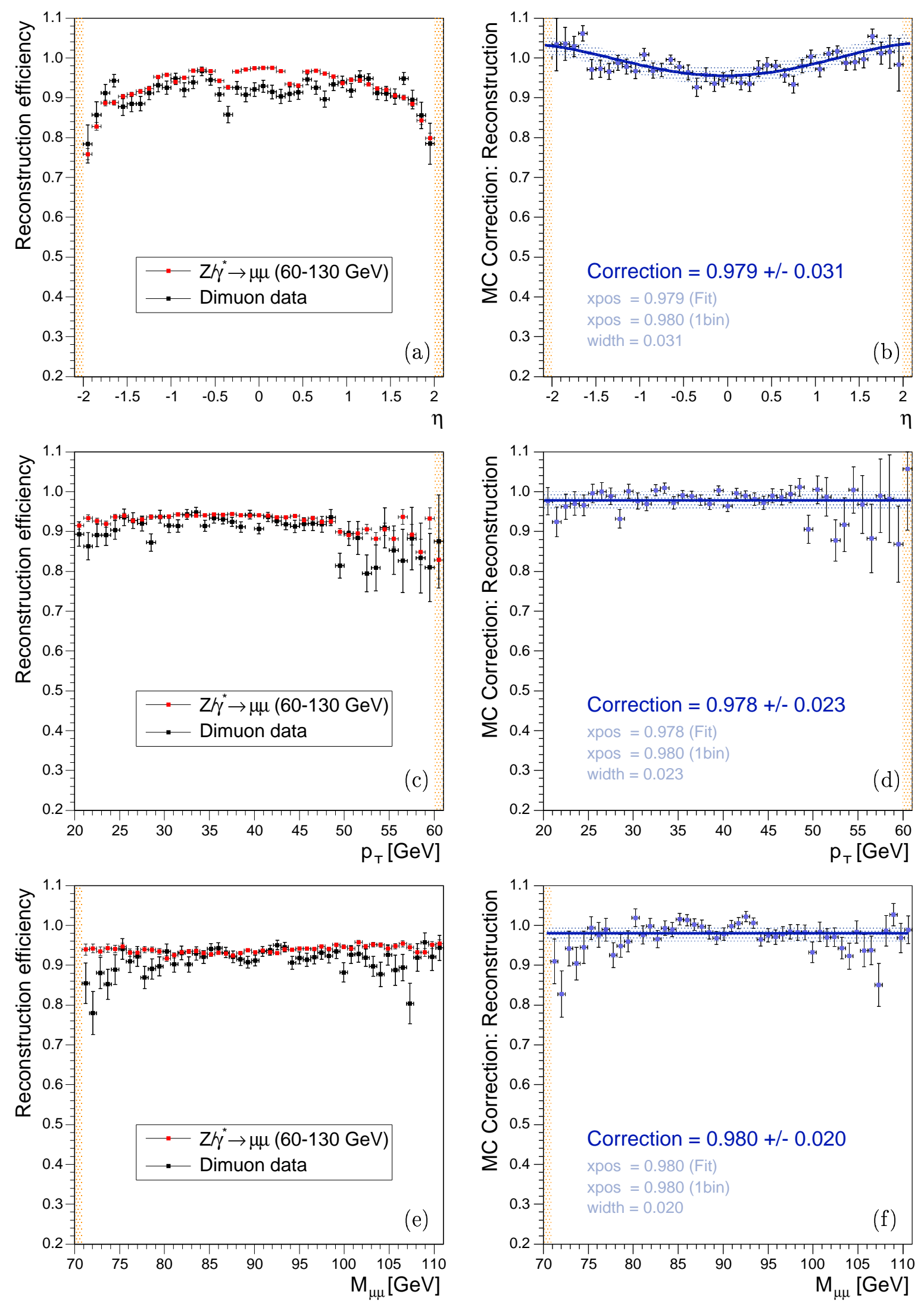

Figure 4.5: The loose muon reconstruction efficiency as a function of $\eta, p_{\mathrm{T}}$, and the dimuon invariant mass $M_{\mu \mu}$ for data and $Z / \gamma^{*}$ Monte Carlo (a,c,e). Also shown is the resulting correction factor for the Monte Carlo $(b, d, f)$. See text for the meaning of the horizontal blue band and the orange shaded regions. 

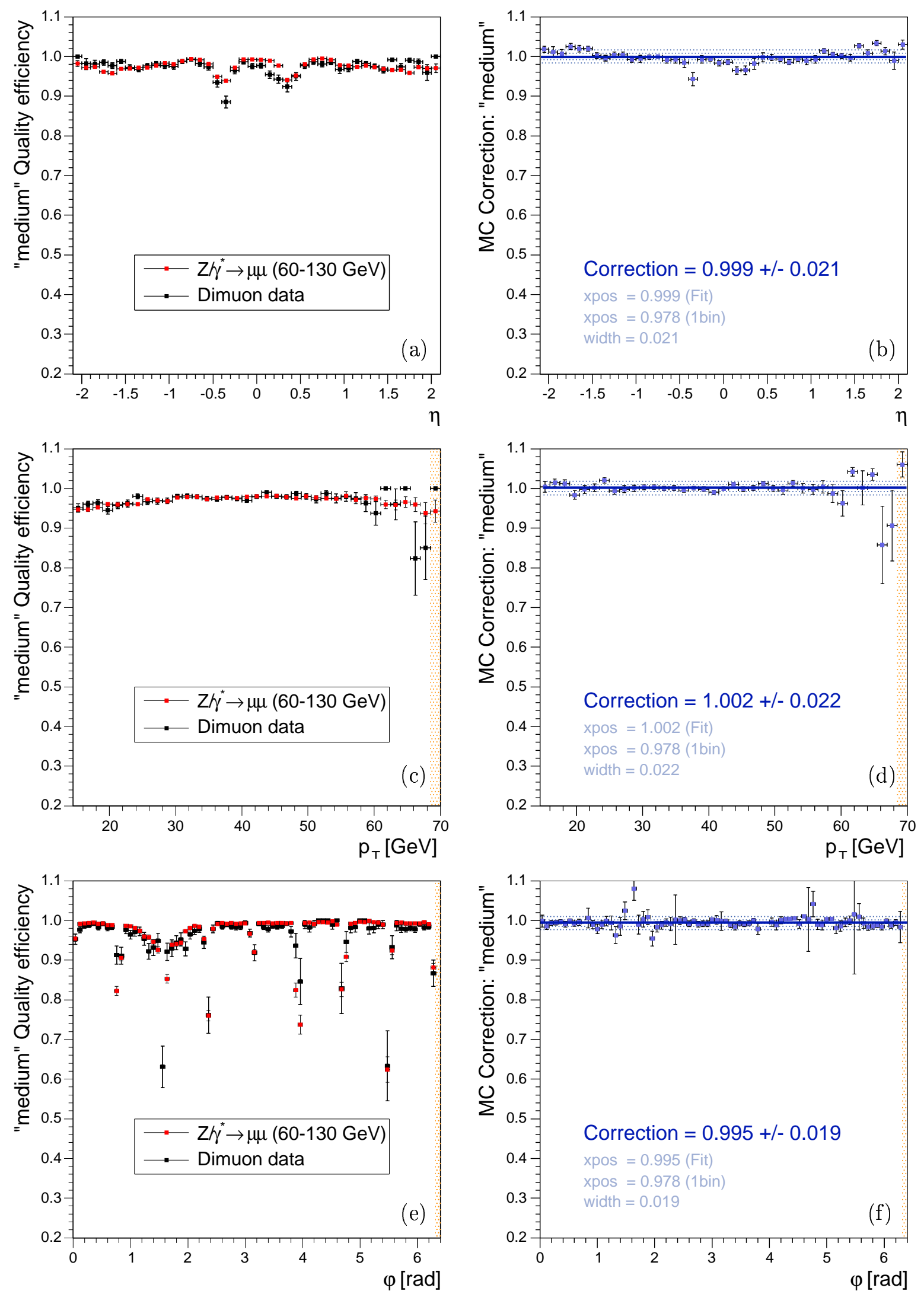

Figure 4.6: The efficiency with which a muon is identified to be at least of »medium « quality as a function of $\eta, p_{\mathrm{T}}$ and $\varphi$ for data and $Z / \gamma^{*}$ Monte Carlo (a,c,e), and the resulting $M C$ correction factors in $(b, d, f)$. See text for the meaning of the horizontal blue band and the orange shaded regions. 

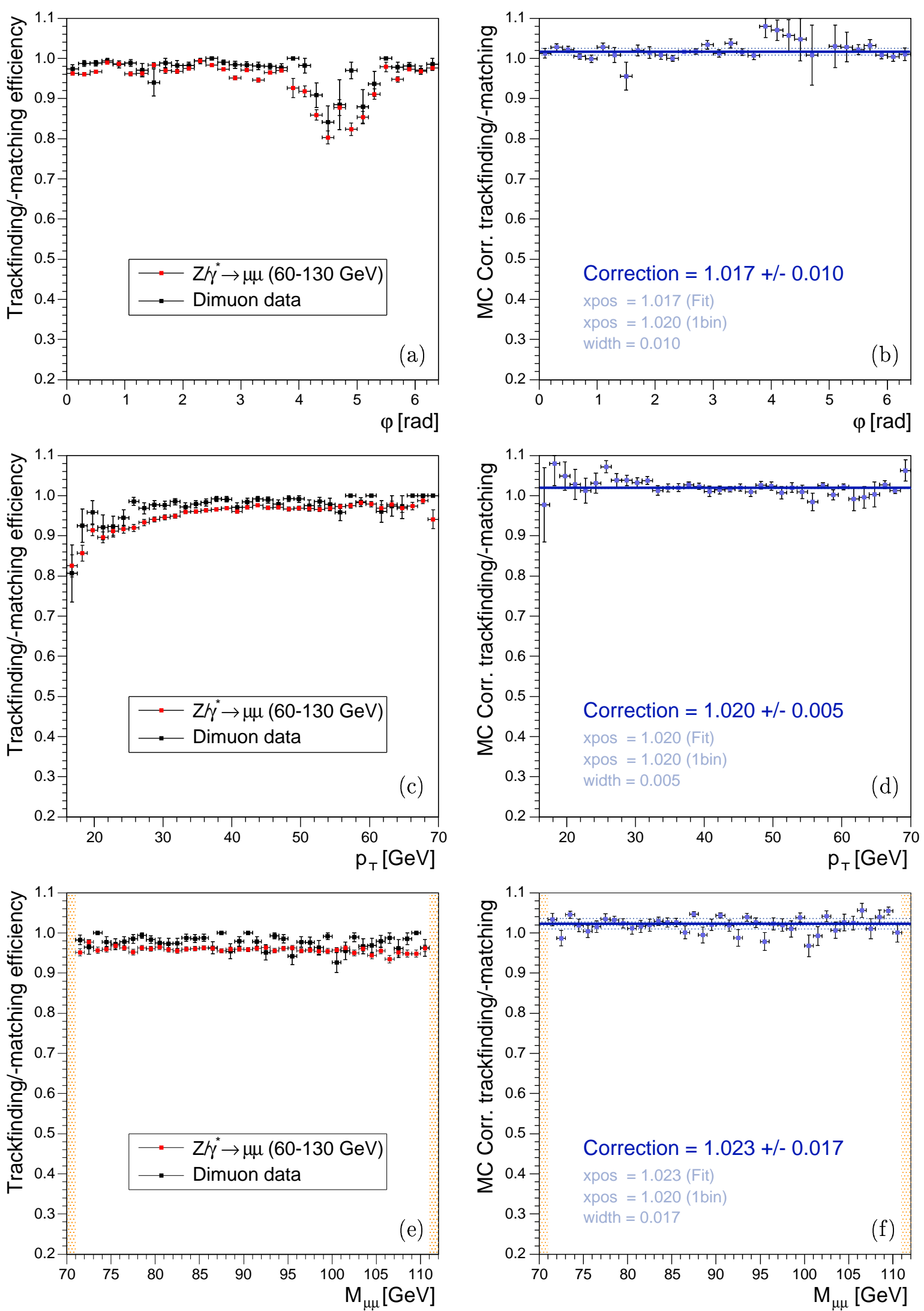

Figure 4.7: The efficiency of the trackmatch requirement for »medium « quality muons as a function of $\varphi, p_{\mathrm{T}}$ and the dimuon invariant mass $M_{\mu \mu}$ for data and $Z / \gamma^{*}$ Monte Carlo (a,c,e) and the resulting $M C$ correction factors $(b, d, f)$. The blue bands and orange shaded regions are explained in the text. 

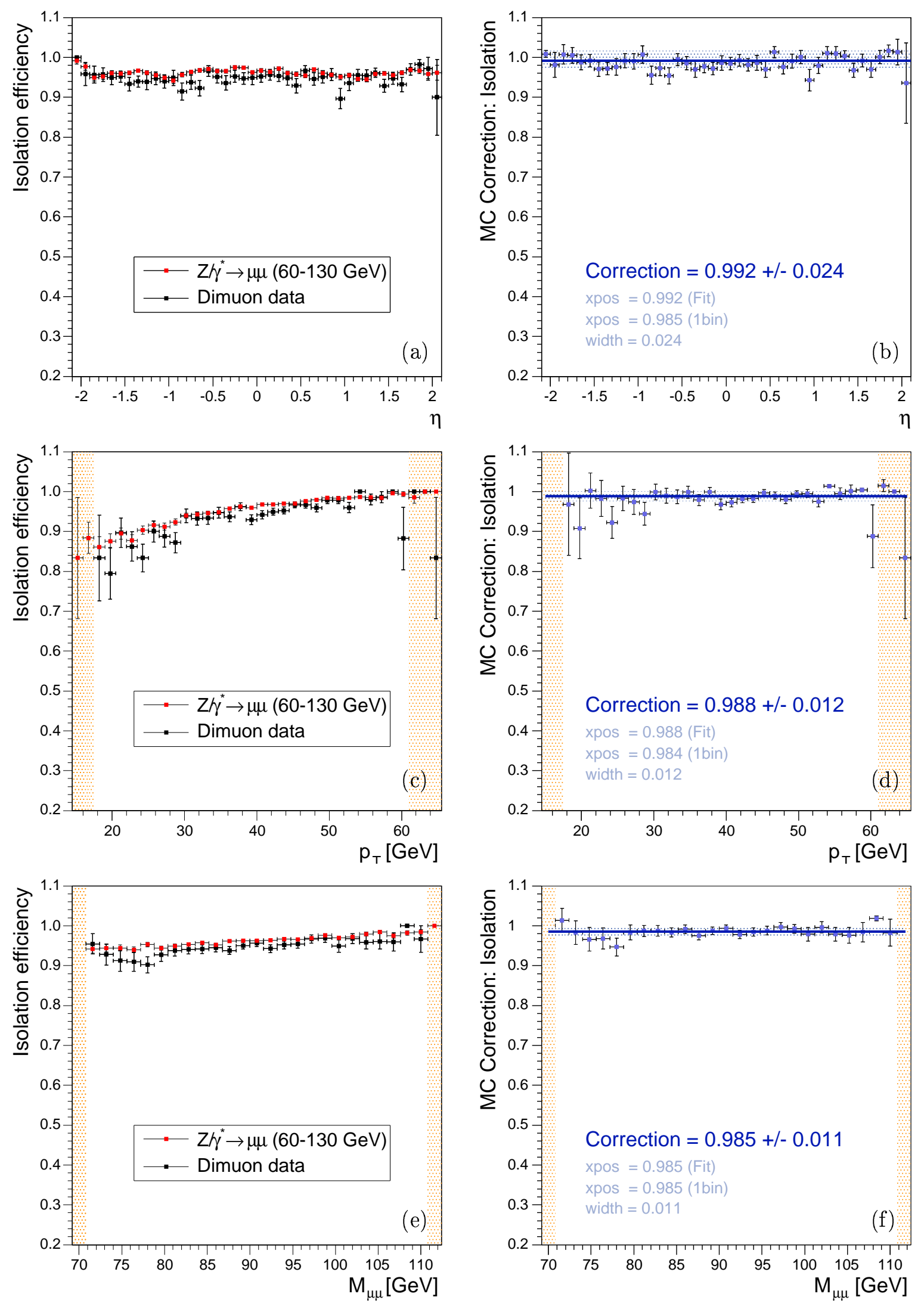

Figure 4.8: The per muon isolation efficiency for »medium « quality muons as a function of $\eta, p_{\mathrm{T}}$, and the dimuon invariant mass $M_{\mu \mu}$ for data and $Z / \gamma^{*}$ Monte Carlo $(a, c, e)$ and the resulting correction factor $\varepsilon^{d a t a} / \varepsilon^{M C}(b, d, f)$. The horizontal blue bands and the orange shaded bins are explained in the text. 


\section{Identification of Electromagnetic \& Hadronic Calorimeter Showers}

Before discussing the identification and differentiation of electrons, (photons), tau leptons, jets and $\not_{T}$, all of which manifest themselves as different formations of energy depositions in the calorimeter (resp. their absence in case of $\not_{T}$ ), the algorithm used to reconstruct any calorimeter objects is briefly described. Electrons, positrons and photons produce rather narrow particle showers in the first few layers of the calorimeter (i.e. in the electromagnetic section), which is why the resulting showers are also called EM-clusters, or EM-objects. Contrary to these constrained EM-clusters, a quark or gluon causes a spray of new particles, that is usually more extended in both, the longitudinal and transverse directions, and is referred to as (hadronic) jet.

The objective of such an algorithm is to find and identify electromagnetic and hadronic energy clusters, so that the measured energy depositions can be related to the original partons. Ideally, the jet-finding and clustering should be collinear and infrared safe, which means that the solutions, i.e. the number, energy and position of reconstructed calorimeter showers should not depend on the presence or absence of any soft or collinear radiation in the event (see Figures 4.9 and $4.10(\mathrm{a}, \mathrm{b})$ for details). Furthermore the ideal algorithm should find the same solutions independent of longitudinal boosts, that are common at hadron colliders like the Tevatron. The algorithm should not show a strong dependence on detector segmentation (i.e. calorimeter cell type and size), energy response and resolution, and any dependence on the instantaneous luminosity (e.g. additional multiple hard scatterings) should be low. Finally, the algorithm should make efficient use of computer resources and enable a reliable calibration of the kinematic properties of the final jets or EM-objects.

As in Run I of the Tevatron, DØ makes use of a seed-based cone algorithm to find and identify calorimeter showers. The initial starting points for trial jet-cones are referred to as seeds and the cone shape closely resembles the naive picture of a particle jet. In an ideal world, the energy depositions of all particles, belonging to a certain EM-object or jet, would lie within the reconstructed (jet)-cone of the calorimeter shower. The cone axis would coincide with the shower axis, which is given by the $E_{\mathrm{T}}$-weighted centroid of all energy depositions within the specified cone radius. In real life, however, numerous difficulties arise from this naive picture of cone-shaped particle jets.
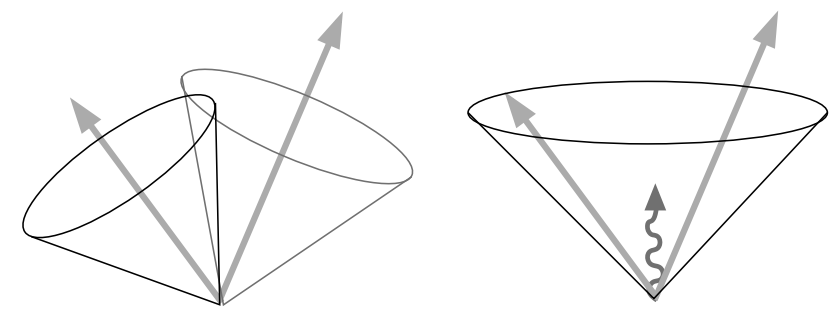

Figure 4.9: Illustration of infrared sensitivity: the algorithm finds different jets, depending on the presence/absence of soft radiation in the event.
First and foremost, seed-based algorithms are usually neither infrared, nor collinear safe. Soft (infrared) sensitivity is related to an unspecified behaviour of the splitting \& merging of preclusters as shown in Figure 4.9. This can result in different energies, positions and/or angles of reconstructed jets/EM-objects and even the number of identified showers can differ, depending on the presence or absence of soft radiation in the event. Collinear sensitivity, on the other hand, is related to the dependence of the jet-finding and clustering on the seed $E_{\mathrm{T}}$-threshold as illustrated in Figure 4.10 (a) and also on the possible $E_{\mathrm{T}}$-ordering of energy depositions, shown in Figure 4.10 (b). The dependence on the $E_{\mathrm{T}}$-threshold will disappear, if 
the jets are of sufficiently large $E_{\mathrm{T}}$, so that a splitting of the seed energy between different detector towers does not affect the jet-finding anymore. However, a possible dependence on the $E_{\mathrm{T}}$-ordering of seeds or preclusters does not vanish with increasing energy. Instead, the algorithm will most likely relate different jets with the same energy depositions, depending on wether collinear radiation is present or not.

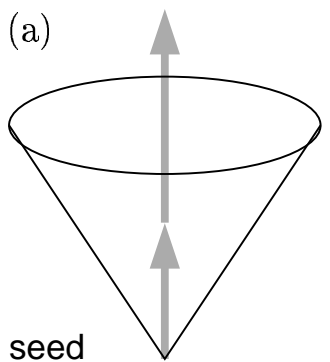

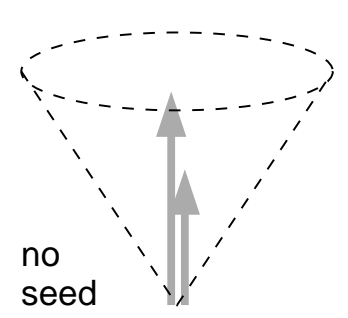

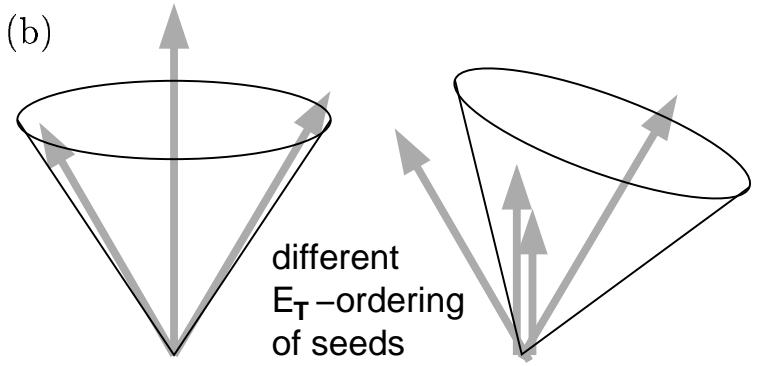

Figure 4.10: Illustration of collinear sensitivity of a seed-based cone algorithm. (a) dependence on the $E_{\mathrm{T}}$-threshold: the left-hand side configuration with narrow energy depositions produces a seed tower, while the right-hand side configuration fails to do so, because the energy is split among different calorimeter towers. (b) possible dependence on the $E_{\mathrm{T}}$-ordering of seed towers: for the left-hand side configuration, where the largest energy deposition is the middle one, the algorithm finds one jet with three partons. In the situation on the right, where the middle deposition is split due to collinearly emitted partons, the rightmost tower has the largest $E_{\mathrm{T}}$ and is evaluated first. This leads to two jets: one, containing the rightmost and the middle partons, and a second one formed solely by the leftmost parton.

These problems are avoided by seedless algorithms, which are collinear and infrared safe, but require a much larger computing time. This option is not feasible in $\mathrm{D} \emptyset$ as a general purpose jet reconstruction algorithm, since all calorimeter towers in the $(\eta, \varphi)$-space would be treated as seeds. For the D $\varnothing$ calorimeter coverage of $|\eta|<3.0$ and $0<\varphi<2 \pi$ with a segmentation of $\eta \times \varphi=0.1 \times 0.1$, this would lead to $\approx 4000$ seed towers. In case of a seeded algorithm, only towers that exceed a certain $E_{\mathrm{T}}$-threshold are considered as seeds, which greatly reduces the necessary computing time.

The algorithm used at D $\varnothing$ in Run II of the Tevatron is the Improved Legacy Cone Algorithm (ILCA), described in detail in [157]. While this is still a seed-based cone algorithm, based on the simple cone algorithm (JetClu) used during Run I [158], the insertion of midpoints essentially removes its sensitivity to soft radiation (Fig. 4.9) and largely reduces its collinear sensitivity.

The idea of the addition of extra seeds, the midpoints, is to approximate a seedless algorithm. This is based on theoretical QCD calculations at fixed order N, showing that only $2^{N}-1$ possible positions exist for stable cones, e.g. for all positions of individual partons $\left(p_{i}\right)$, all pairs of partons $\left(p_{i}+p_{j}\right)$, all combinations of three partons $\left(p_{i}+p_{j}+p_{k}\right)$, and so on. Considering the energy depositions in the calorimeter as the original partons, one can in principle argue along the line of a parton level algorithm: the positions of pairs of energy depositions, triplets thereof, etc. serve as additional starting positions for trial clustering cones in this modified seed-based algorithm. Not all midpoints calculated in this way have to be considered as seed towers, since widely separated seeds cannot be clustered anyway. Thus, it is sufficient to only consider those, lying within a distance of $\Delta \mathcal{R}<2 \cdot \mathcal{R}_{\text {cone }}$.

The algorithm starts with a trial cone of specified radius $\mathcal{R}$ centered about a seed calorimeter tower of at least $E_{\mathrm{T}}>1 \mathrm{GeV}$, or about one of the additional midpoints. It then calculates the 
$E_{\mathrm{T}}$-weighted centroid of all particles within this cone. This new cluster axis is then used as the center for the next trial cone. The cone center is allowed to »drift « around freely during the iteration procedure until one of the following three possibilities occurs:

- a »stable« solution is found, i.e. the centroid of all energy depositions within the cone is aligned with the geometric cone axis within less than $\Delta \mathcal{R} \leq 0.001$;

- the resulting $E_{\mathrm{T}}$ is less than half the minimum jet (EM-object) transverse energy, with $E_{\mathrm{T}}^{j e t}=8 \mathrm{GeV}$ (within $\mathcal{R}=0.5$ ) and $E_{\mathrm{T}}^{e m}=1.5 \mathrm{GeV}$ (within $\mathcal{R}=0.2$ ), respectively ${ }^{2}$;

- the number of iterations exceeds 50, which is an arbitrary cutoff to avoid an infinite (or very large number) of cycles.

Unfortunately, however, it is quite common, that stable cones share one or more energy depositions (overlapping cones). While not all calorimeter towers in the final state need to be assigned to a cluster, a tower should not belong to more than one final jet or EM-object. Hence, another step is introduced between the stable cones (or clusters) and the final jets/EM-objects, where overlapping cones are either merged or the shared energy depositions split unambiguously between the two clusters. In $\mathrm{D} \varnothing$ the splitting/merging fraction is fixed at $f=50 \%$. This means that cones whose shared energy is larger than $50 \%$ of the energy in the lower energetic cluster are merged, while in the opposite case, the shared calorimeter towers are assigned to the cluster that is closer in $\mathcal{R}$. An overview of the specifications of the ILCA algorithm as used in DØ is given in Table 4.8 .

\begin{tabular}{lcc}
\hline \hline Parameter & EM-objects & Jets \\
\hline \hline Cone size $R=\sqrt{(\Delta \eta)^{2}+(\Delta \varphi)^{2}}$ & 0.2 & 0.5 \\
Seed $E_{\mathrm{T}}$-threshold & $1 \mathrm{GeV}$ & $1.0 \mathrm{GeV}$ \\
Splitting \& merging fraction $f$ & $50 \%$ & $50 \%$ \\
Final object $E_{\mathrm{T}}$-threshold & $1.5 \mathrm{GeV}$ & $8 \mathrm{GeV}$ \\
\hline \hline
\end{tabular}

Table 4.8: Specifications of the Improved Legacy Cone Algorithm (ILCA), which is used for jet-finding \& cone-clustering in DØ during the Tevatron Run II.

While the reconstruction of jets and EM-objects relies primarily on the calorimeter, tracking information can be used in addition to enable the special tagging of heavy-quark jets, i.e. $b / c$ jets. Since these jets originate from the decay of mesons, containing $b(c)$ quarks, they can often be matched to the decay-vertex of the respective meson, which is distinct from the primary interaction vertex and thus also referred to as a secondary vertex.

\footnotetext{
${ }^{2}$ Due to the differences of electromagnetic and hadronic showers, the algorithm is used with two different cone sizes: a smaller radius of $\mathcal{R}=0.2$ is employed to find EM-clusters, while a larger radius of $\mathcal{R}=0.5$ serves to identify hadronic jets.
} 


\section{Electron Identification}

Each EM-object in an event must satisfy the following criteria to be considered as electron:

- $e m f>0.9$, where $e m f$ is the energy fraction of the shower deposited in the electromagnetic layers of the calorimeter versus the total energy; i.e. the fraction of electromagnetic energy of a shower should exceed $90 \%$;

- $i s o=\frac{E_{0.4}^{t o t}-E_{0.2}^{e m}}{E_{0.2}^{e m}}<0.2$, where $E_{0.4}^{t o t}$ denotes the total energy and $E_{0.2}^{e m}$ the electromagnetic energy deposited in a cone with the respective radius of $\mathcal{R}=0.4\left(E^{t o t}\right)$ and $\mathcal{R}=0.2\left(E^{e m}\right)$; less than $20 \%$ of the shower energy may be deposited outside a cone of $\mathcal{R}=0.2$ or outside the electromagnetic section of the calorimeter;

- the calorimeter shower must be matched to a central track and lie within $\left|\eta_{\text {det }}\right|<3$, i.e. either the central or forward region of the calorimeter (excluding the ICD regions);

- the shower shape should be compatible with an electron, which is achieved by requiring: $H M x 7<40$ and an electron likelihood of lhood $>0.2$; (see text for details.)

- the transverse momentum of the electron is required to exceed $p_{\mathrm{T}}>5 \mathrm{GeV}$.

The $H$-matrix defines the longitudinal and lateral shape of an electromagnetic shower and is used to discriminate between electrons/photons and calorimeter objects of hadronic origin [159]. It is the inverse of a covariance matrix of seven (eight) correlated variables, of which the variable $H M x 7(H M x 8)$ forms a kind of $\chi^{2}$-test in which the measured shape of a candidate shower is compared to Monte Carlo simulations. The following seven variables are used as input to the covariance matrix:

- the energies deposited in the four layers of the electromagnetic section of the calorimeter ( $E M 1, E M 2, E M 3, E M 4)$ provide information on the longitudinal shower shape;

- the total electromagnetic energy $\left(E^{e m}\right)$ is included to account for the dependence of the fractional layer energies on the shower energy;

- the $z$ position of the primary vertex;

- the transverse width of the shower as a function of $\varphi$;

(In case of $H M x 8$, the transverse shower width versus $z$ is used additionally. It is omitted from the 7 -variable $H$-matrix, since it is not well described by the MC simulations.)

The covariance matrix $M$ is then determined using Monte Carlo data. For two variables $x_{i}, x_{j}$, it is calculated as:

$$
M_{i j}=\frac{1}{N} \sum_{n=1}^{N}\left(x_{i}^{n}-\bar{x}_{i}\right)\left(x_{j}^{n}-\bar{x}_{j}\right),
$$

where $x_{i}^{n}, x_{j}^{n}$ denote the observed values and $\bar{x}_{i}, \bar{x}_{j}$ the respective average values. Using the inverse of the covariance matrix $H_{i j}=M_{i j}^{-1}$, one can calculate the following parameter:

$$
H M x 7=\sum_{i, j}\left(x_{i}^{d a t a}-\bar{x}_{i}^{M C}\right) H_{i j}\left(x_{j}^{d a t a}-\bar{x}_{j}^{M C}\right),
$$

and thus measure, if the shape of a certain shower is consistent with the shape of an electromagnetic shower. Although the distribution of $H M x 7$ does not follow a true $\chi^{2}$ distribution 
because usually the observables $x_{i}$ do not follow a Gaussian distribution, it is nevertheless a useful variable to distinguish electromagnetic from hadronic showers. Showers that are rather electron/photon-like tend to have low $H M x 7$-values compared to showers of hadronic origin, that accumulate at high values of $H M x 7$. For further details on the $H$-matrix and the respective input variables see [160]. In this analysis a shower is referred to as electromagnetic shower, or - in case of an associated track - as an electron, if the $H$-matrix criterion of $H M x 7<40$ is satisfied.

The above mentioned electron likelihood (lhood) is designed to discriminate between electrons and hadronic calorimeter objects, as well as photons. It comprises seven variables:

- the fraction of electromagnetic energy: emf;

- the shower shape variable: $H M x 7$;

- the distance of closest approach of the associated track: $d c a_{t r}$;

- a $\chi^{2}$-method to spatially match a candidate track with the considered calorimeter cluster in the $(\eta, \varphi)$-coordinates only: $\chi_{\text {spatial }}^{2}$ probability;

- the ratio of the calorimeter $E_{\mathrm{T}}$ and the $p_{\mathrm{T}}$ of the associated track: $E_{\mathrm{T}} / p_{\mathrm{T}}$;

- the number of tracks in a cone of $\mathcal{R}=0.05$ around the candidate track;

- the $p_{\mathrm{T}}$-sum of all tracks in a cone of $\mathcal{R}=0.4$ around the candidate track.

The electromagnetic fraction emf, the shower shape variable $H M x 7$, and the distance of closest approach $d c a$ have already been discussed. The spatial trackmatch probability is a $\chi^{2}$-value to test wether the track and cluster positions match. The $\chi_{\text {spatial }}^{2}$ is defined as:

$$
\chi_{\text {spatial }}^{2}=\left(\frac{\Delta \varphi}{\sigma_{\varphi}}\right)^{2}+\left(\frac{\Delta z}{\sigma_{z}}\right)^{2},
$$

where $\Delta \varphi$ and $\Delta z$ are the differences between the track coordinates $\varphi_{t r}\left(z_{t r}\right)$, extrapolated into the finely segmented third EM-layer and the coordinates of the electromagnetic cluster $\varphi_{e m}$ $\left(z_{e m}\right)$ in the same layer. The respective uncertainties are given by $\sigma_{\varphi}$ and $\sigma_{z}$. In addition to this spatial matching between track and calorimeter object, the next criterion $E_{\mathrm{T}} / p_{\mathrm{T}}$ is used to further refine the method and also compare the transverse energy of the calorimeter shower and the transverse momentum of the spatially associated track. However, for the further analysis of electromagnetic objects (but also for hadronic objects, i.e. jets), the transverse energy measured with the calorimeter is used.

The following two variables in the electron likelihood: the »number of tracks in a cone of $\mathcal{R}=0.05 \ll$ and the $» p_{\mathrm{T}}$-sum of all tracks in a cone of $\mathcal{R}=0.4$ « are isolation variables. The former serves to reduce the background from photon conversions $\left(\gamma \rightarrow e^{+} e^{-}\right)$, while the latter is designed to distinguish single isolated electrons from electrons that are part of hadronic jets with a naturally higher track multiplicity.

Similar to the smearing of the $p_{\mathrm{T}}$-resolution of muons, the transverse energy of electromagnetic objects is smeared to account for a worse resolution in data and possible noise effects not described by the standard Monte Carlo simulation:

$$
E_{\mathrm{T}}^{\prime}=\operatorname{Gauss}(0, \sigma) \cdot E_{\mathrm{T}}, \quad \text { with: } \sigma=0.0047 .
$$


The identification efficiency for electrons and the efficiencies to pass or fail the quality criteria described above, have been studied in detail for a complementary search for $\not R_{p}$-SUSY in eel final states $[1,73]$. The efficiency measurements for electrons and the extraction of the corresponding Monte Carlo correction are discussed there. Figure 4.11 shows this efficiency of the electron quality criteria for data and MC events $(\mathrm{a}, \mathrm{c})$ and the corresponding $\mathrm{MC}$ correction $(\mathrm{b}, \mathrm{d})$ versus $p_{\mathrm{T}}$ of the probe electron, separately for the central $(\mathrm{a}, \mathrm{b})$ and the forward $(\mathrm{c}, \mathrm{d})$ regions of the calorimeter. The MC correction is parametrised by polynomials of variable degree in $p_{\mathrm{T}}$, to account for the $\eta$-dependence, where the obtained fit values are reproduced in Table 4.9.
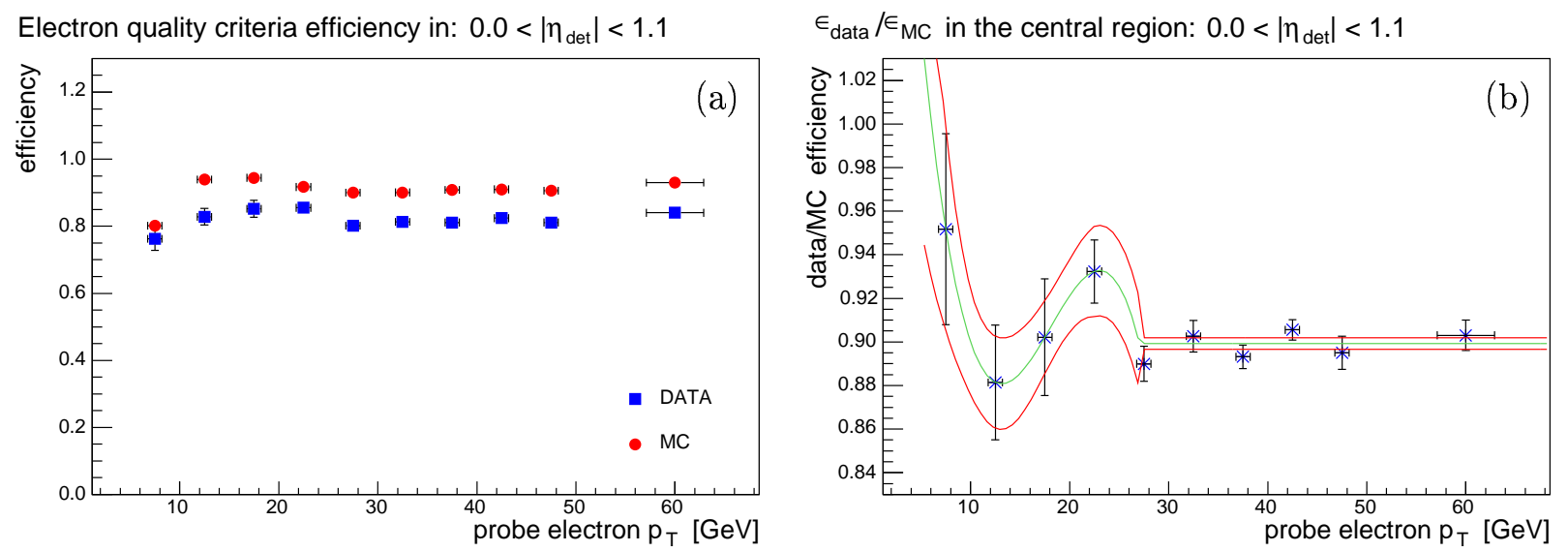

Electron quality criteria efficiency in: $1.5<\left|\eta_{\text {det }}\right|<2.5$
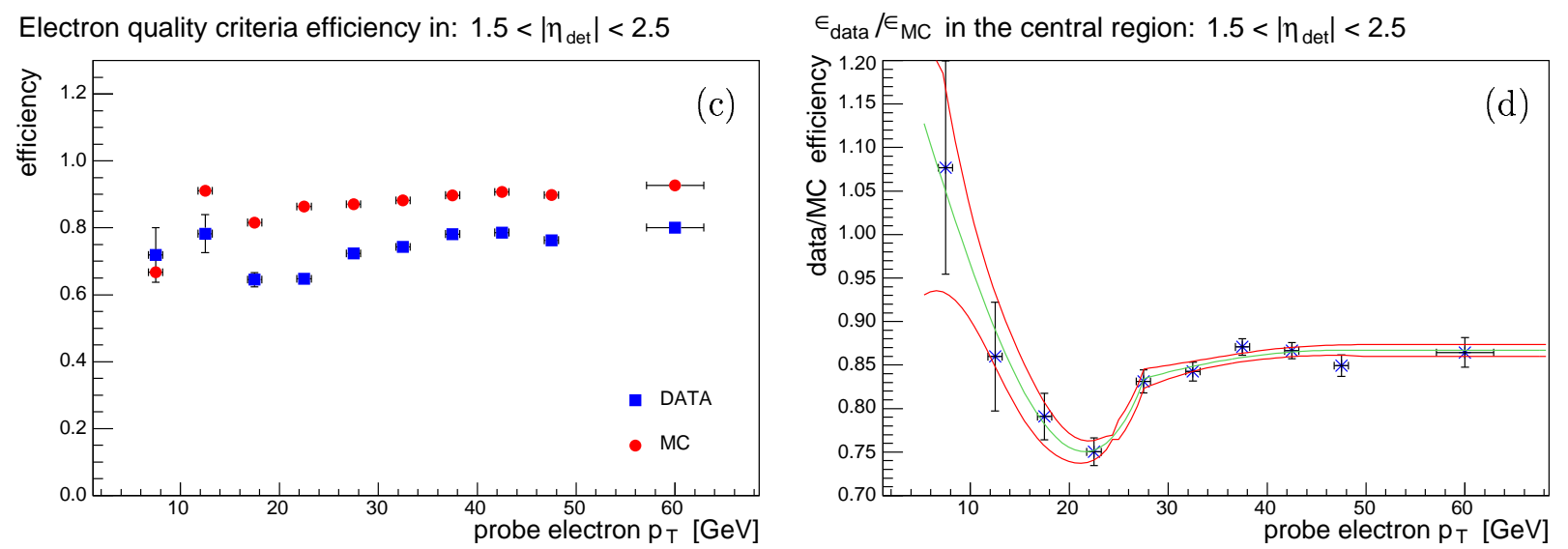

Figure 4.11: The efficiency of the electron quality criteria for data and $M C$ simulations (a,c) and the corresponding $M C$ corrections $(b, d)$ are shown versus the $p_{\mathrm{T}}$ of the probe electron, separately for the central $(a, b)$ and the forward $(c, d)$ region of the calorimeter. (taken from [73])

\begin{tabular}{lccccc}
\hline \hline Calorimeter region & $a_{0}$ & $a_{1}$ & $a_{2}$ & $a_{3}$ & $p_{\mathrm{T}}$ region \\
\hline \hline central (CC) & +1.406 & -0.0987 & $+5.89 \cdot 10^{-3}$ & $-1.09 \cdot 10^{-4}$ & $p_{\mathrm{T}}<27 \mathrm{GeV}$ \\
$\left|\eta_{\text {det }}\right|<1.1$ & +0.899 & - & - & - & $p_{\mathrm{T}} \geq 27 \mathrm{GeV}$ \\
\hline forward (EC) & +1.319 & -0.0346 & $-4.50 \cdot 10^{-4}$ & $+3.87 \cdot 10^{-5}$ & $p_{\mathrm{T}}<27.5 \mathrm{GeV}$ \\
$\left|\eta_{\text {det }}\right|<1.1$ & +0.684 & $+7.65 \cdot 10^{-3}$ & $-8.01 \cdot 10^{-5}$ & - & $p_{\mathrm{T}}<50 \mathrm{GeV}$ \\
& +0.867 & - & - & - & $p_{\mathrm{T}} \geq 50 \mathrm{GeV}$ \\
\hline \hline
\end{tabular}

Table 4.9: Fit parameters for the $p_{\mathrm{T}}$ and $\eta$ dependent $M C$ efficiency correction of the electron quality criteria given by: $f_{q u a l}^{e}\left(p_{\mathrm{T}}, \eta\right)=a_{0}+a_{1} p_{\mathrm{T}}+a_{2} p_{\mathrm{T}}^{2}+a_{3} p_{\mathrm{T}}^{3} . \quad$ (taken from [73]) 


\section{Jet Identification}

As detailed before, all jets are reconstructed from energy depositions in the calorimeter via a seed-based cone algorithm with a cone radius of $\mathcal{R}=0.5$ and a jet $E_{\mathrm{T}}$-threshold of $8 \mathrm{GeV}$. To distinguish good jets from bad or fake jets further quality criteria need to be satisfied. Bad or fake jets might stem from, e.g. EM-objects that have been misidentified as hadronic jets, or real jets originating from noisy calorimeter readout cells/towers. All of the following jet requirements are standard certified quality criteria developed by the JetID-group [161]:

- the electromagnetic fraction of jets $(E M F)$ should lie between $5 \%$ and $95 \%$ to reduce fake jets from calorimeter noise;

- the coarse hadronic fraction $(C H F)$ is required to be less than $40 \%$ of the total energy deposited in the calorimeter, which takes into account the elevated noise level in this coarsely granulated section of the calorimeter;

- the so-called hot fraction, which is the ratio of the highest to the second highest cell energy, must be smaller than $H o t F<10$, to effectively reduce bad jets clustered from hot cells;

- the number of calorimeter towers, containing at least $90 \%$ of the jet energy must be larger than one to reduce noise jets: $n 90>1$;

- a Level 1 trigger confirmation of the offline reconstructed transverse energy of a jet is required to minimise the impact of calorimeter data from periods, in which the precision readout of the calorimeter had been multiplied with the wrong amplifier gain(1 or 8$)$ :

$$
\frac{L 1 \text { set }}{E_{\mathrm{T}}^{\text {jet }} \cdot(1-C H F)}>0.4 \text { (in CC/EC regions), }>0.2 \text { (in the ICD region), }
$$

where L1set denotes the scalar sum of the transverse energies of the L1 trigger towers inside the respective $\mathrm{L} 1$ jet cone.

- a reconstructed jet is discarded if an EM-object, i.e. an electron or photon with a transverse momentum of at least $p_{\mathrm{T}}>15 \mathrm{GeV}$, lies within $\Delta \mathcal{R}<0.5$ of the hadronic jet.

Since the reconstruction efficiency for jets is different in data and MC simulations, correction factors have to be determined to match the MC efficiency to the one measured in the data. Similar to the correction factors for the electron quality criteria, the correction factors to the jet reconstruction also depend on the jet $p_{\mathrm{T}}$ and the $\eta$ region $(\mathrm{CC}, \mathrm{EC}, \mathrm{ICD})$. The factors are parametrised by Equation (4.15), and are applied during the processing with the framework program top_analyze [132], which in turn uses the package JetCorr (version 5.3) provided by the DØ Jet Energy Scale (JES) group [154].

$$
f_{\text {reco }}^{\text {jet }}\left(p_{\mathrm{T}}\right)=A_{2} \cdot\left(\frac{1}{2}+\frac{1}{\sqrt{\pi}} \int_{0}^{b} e^{-x^{2}} d x\right) \text { with: } \quad b=\frac{p_{\mathrm{T}}-A_{0}}{A_{1} \sqrt{p_{\mathrm{T}}}}
$$

The parameters were obtained in a study of $Z+$ jet and $\gamma+$ jet events [154] and are reproduced in Table 4.10 for completeness.

Another correction to the reconstructed jet energy is due to the mismatch between the measured jet energies $E_{\text {jet }}^{\text {meas }}$ and the original particle jet energies $E_{\text {jet }}^{\text {part }}$. A matching between both these quantities is necessary to derive any conclusions about the hard interaction at particle, rather 


\begin{tabular}{lrcc}
\hline \hline Calorimeter region & \multicolumn{1}{c}{$A_{0}$} & $A_{1}$ & $A_{2}$ \\
\hline \hline $\mathrm{CC}$ & $9.8 \pm 2.7$ & $3.4 \pm 0.7$ & $1.01 \pm 0.01$ \\
$\mathrm{EC}$ & $13.5 \pm 3.3$ & $2.2 \pm 1.0$ & $0.98 \pm 0.02$ \\
$\mathrm{ICD}$ & $15.0 \pm 2.3$ & $2.0 \pm 0.7$ & $0.93 \pm 0.02$ \\
\hline \hline
\end{tabular}

Table 4.10: Parameters used in the calculation of the $M C$ correction factors to the jet reconstruction efficiency, according to Eq. (4.15); taken from [132].

than detector level. This is achieved by calibrating the jet energy scale (JES), which is done via the so-called JES correction parametrised by:

$$
E_{\text {jet }}^{\text {part }}=\frac{E_{\text {jet }}^{\text {meas }}-E_{\text {off }}}{R_{\text {jet }} \times S_{\text {cone }}}
$$

where the parameters $E_{\text {off }}, R_{\text {jet }}$ and $S_{\text {cone }}$ have the following meaning [162]:

$\boldsymbol{E}_{\boldsymbol{o f f}}$ : denotes an energy offset not associated with the hard scatter. Excess energy due to, e.g. noise (radioactivity in U-absorbers, readout electronics), pile-up, underlying events, etc. has to be subtracted from the reconstructed jet energy. The offset is measured in zero and minimum bias events as a function of the luminosity, the cone size $\mathcal{R}_{\text {cone }}$ and $\eta$. (zero bias: bunch crossing, clock; minimum bias: coincidence of both luminosity monitors)

$\boldsymbol{R}_{\text {jet }}$ : parametrises the different calorimeter responses to particle jets. It is determined from the $E_{\mathrm{T}}$ balance in $\gamma+$ jet events, where the parton and the photon are back-to-back. The precise knowledge of the EM-energy scale from $Z \rightarrow e e$ data, allows to infer the particle energy depending on the cone size $\mathcal{R}_{\text {cone }}$, the measured jet energy $E_{\text {jet }}^{\text {meas }}$, and $\eta$.

$\boldsymbol{S}_{\text {cone }}$ : is a measure for the out-of-cone showering: particles of the original particle jet that belong to the shower can scatter out of the jet cone, while non-shower particles can scatter into it. Measurements of the energy flow as a function of the distance from the jet axis yield parametrisations of $S_{\text {cone }}$ again as a function of $\mathcal{R}_{\text {cone }}, E_{\text {jet }}^{\text {meas }}$, and $\eta$.

In addition to the differing detector and particle jet energies, the jet energies measured in $\mathrm{MC}$ events do not correctly describe the jet energies observed in data. Therefore, different corrections need to be applied to the measured detector jets and the MC simulated jets, which can also be observed in Figure 4.12 (JES correction for data) and Figure 4.13 (JES correction for MC events). The total systematic uncertainty of the JES correction is given by the statistical and systematic uncertainties of the corrections to data and MC events added in quadrature:

$$
\sigma_{J E S}=\sqrt{\left(\sigma_{\text {stat }}^{\text {data }}\right)^{2}+\left(\sigma_{\text {stat }}^{M C}\right)^{2}+\left(\sigma_{\text {sys }}^{\text {data }}\right)^{2}+\left(\sigma_{\text {sys }}^{M C}\right)^{2}} .
$$

The parametrisations of the JES correction and its associated total uncertainty for detector and MC data are also contained in the JetCorr package and are thus applied via the top_analyze framework program, as recommended by the JES group [154].

The jet energy resolution $\sigma\left(E_{\mathrm{T}}\right)$ is determined for every jet in an $\mathrm{MC}$ event according to the following equation, (compare: Eq. (2.16) for the calorimeter transverse energy resolution in section 2.3.2):

$$
\frac{\sigma\left(p_{T}\right)}{p_{T}}=\sqrt{\frac{N^{2}}{p_{T}^{2}}+\frac{S^{2}}{p_{T}}+C^{2}} .
$$



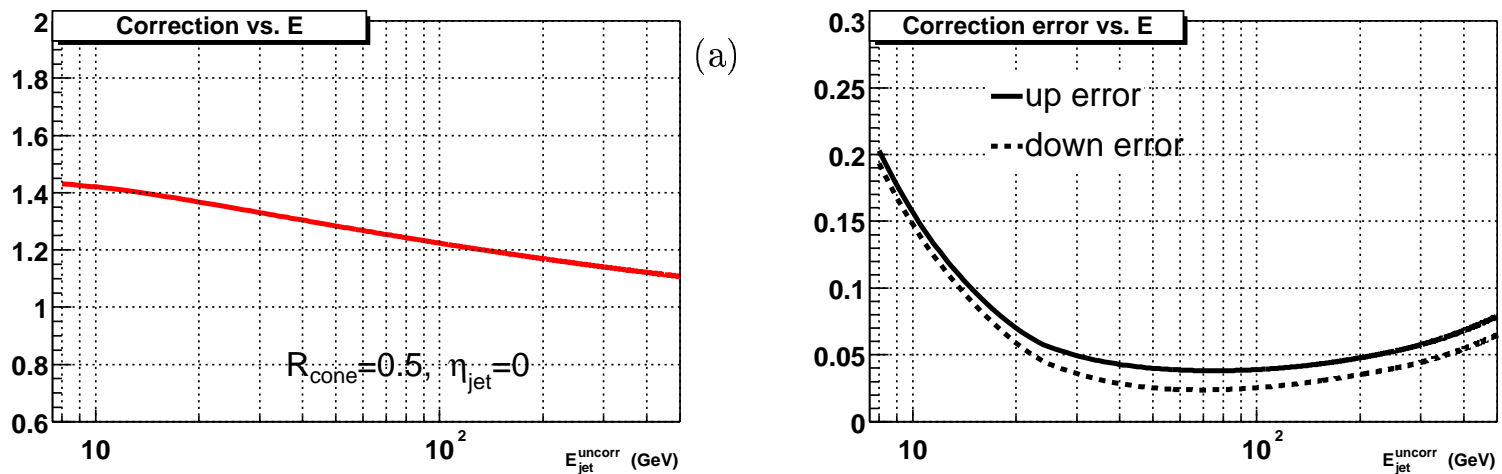

(b)
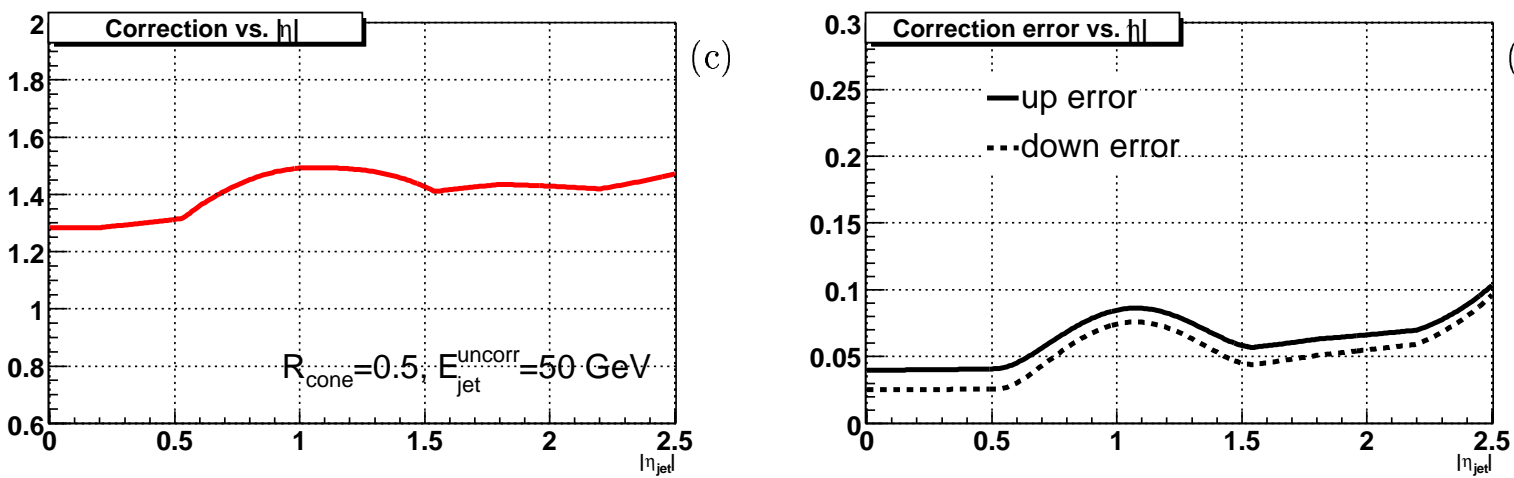

(d)

Figure 4.12: The jet energy scale correction $(a, c)$ and its total uncertainty $(b, d)$ for data events versus the measured energy of the uncorrected, reconstructed jet $(a, b)$ and versus the pseudorapidity $(c, d)$.

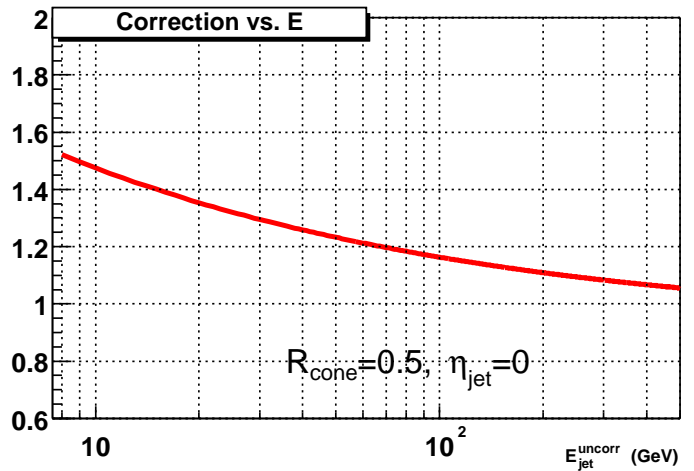

(a)
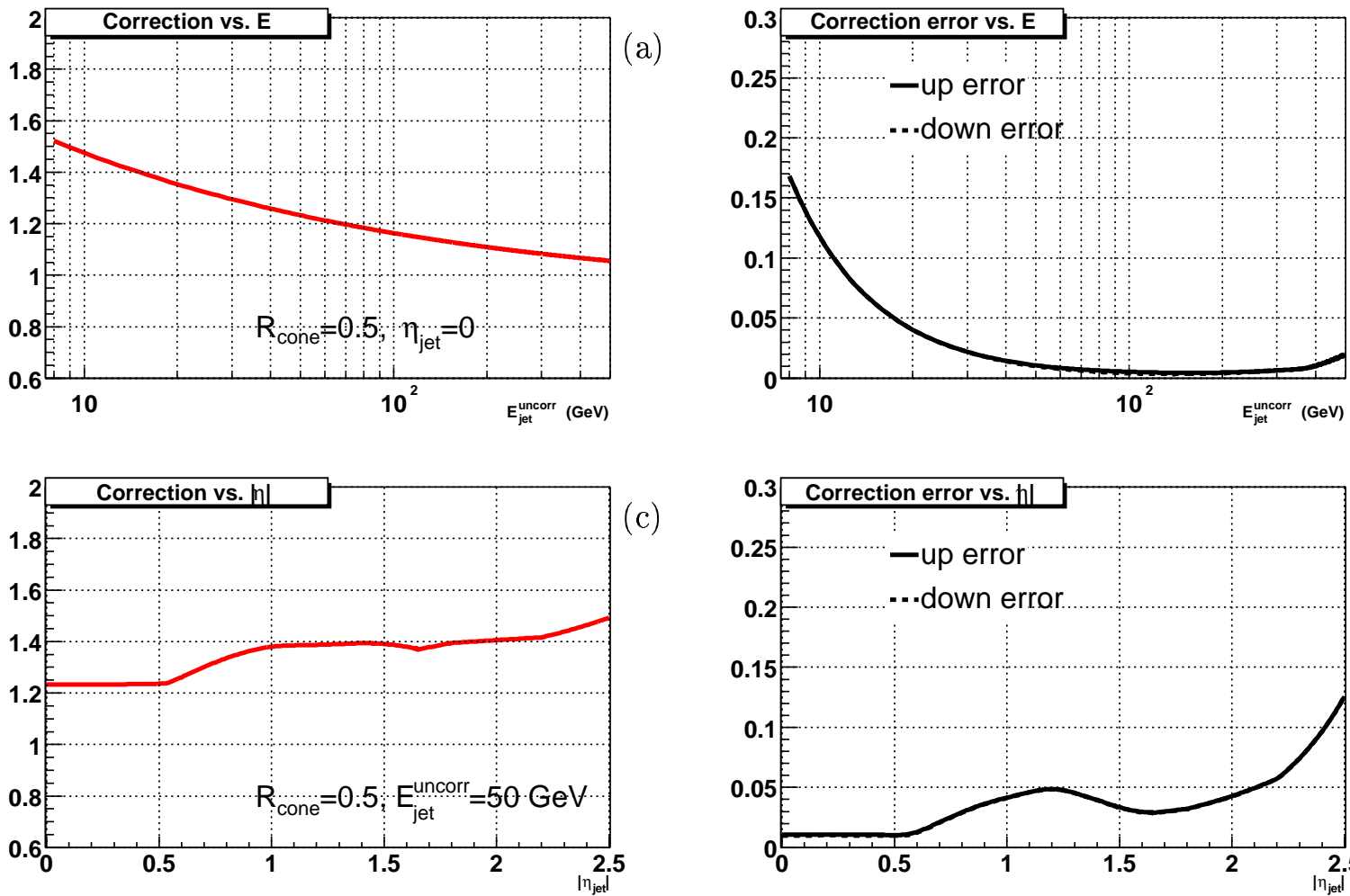

(b)

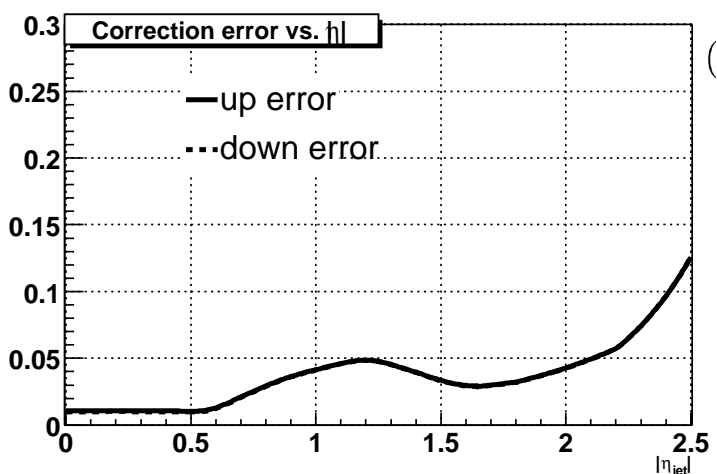

(d)

Figure 4.13: The jet energy scale correction and corresponding total uncertainty for simulated data. 
As was already mentioned, the terms denote energy-independent noise $(N)$, sampling fluctuations $(S)$, and systematic uncertainties due to the energy calibration of the calorimeter $(C)$. All contributions are measured in dijet events for data, as well as for Monte Carlo events, separately for several $\eta$ regions. The resolution $\sigma\left(E_{\mathrm{T}}\right)$ is then calculated for the obtained data and MC coefficients, and only if $\sigma_{M C}\left(E_{\mathrm{T}}\right) \neq \sigma_{\text {data }}\left(E_{\mathrm{T}}\right)$, the momentum and energy resolution of a jet are smeared with a gaussian function of width:

$$
\sigma^{\text {smear }}=\sqrt{\left(\sigma_{\text {data }}\right)^{2}-\left(\sigma_{M C}\right)^{2}} .
$$

Details on the measurements of the three different components and on the determination of the respective energy and momentum resolutions can be found in [163].

\section{The Calculation of the Missing Transverse Energy $\left(\mathbb{F}_{T}\right)$}

Particles that do not (or only very rarely) interact with matter, cannot be detected directly. Nevertheless, it is possible to obtain some information about these particles from the energy/momentum imbalance of all measured particles in an event. Since the longitudinal components of the colliding particle momenta are unknown at a hadron collider (see section 2.1), and the transverse momentum is approximately zero before the collision, momentum conservation implies that the sum of transverse momenta of all particles after the collision should also be zero, i.e. $\sum_{i} \vec{p}_{T, i}=\overrightarrow{0}$. However, in the case, that not all particles have been detected and due to detector resolution, there will be a transverse momentum/energy imbalance, which is nothing else than the definition of the missing transverse energy:

$$
\overrightarrow{\ddot{E}}_{T}=-\sum_{i} \vec{E}_{T, i}
$$

The missing transverse energy is defined as the negative vectorial sum of all calorimeter cells with a transverse energy of at least $100 \mathrm{MeV}$, with the exception of cells in the coarse hadronic layer. The latter cells are only taken into account if they are part of good reconstructed jets that satisfy all quality criteria defined in the previous section. This $r a w \not_{T}$ is subsequently corrected for the jet energy scale and the electromagnetic energy scale $\left(\rightarrow \mathbb{F}_{T}^{j e s}\right)$ and in a last step for the energy carried away by muons $\left(\rightarrow \not_{T}^{\mu}\right)$. Since muons are minimum ionising particles [23], they deposit only about 2 to $3 \mathrm{GeV}$ in the calorimeter, no matter how energetic they are (up to $p_{\mathrm{T}}(\mu) \approx 500 \mathrm{GeV}$ ). Muons, whose energy is added to the missing transverse energy must have hits in the pre- and post-toroid layers (»medium « quality) and be matched to a central track.

While the missing transverse energy itself is not separately smeared, the corrections and additional smearing applied to all previously discussed objects is taken into account for the $\mathbb{F}_{T}$ calculation. As can be seen from Figure 4.14, the distribution of $\not_{T}^{\text {jes }}$ at preselection level (see section 4.5.1) shows a good agreement between data and the background expected from SM processes. If not explicitly stated, the correction due to reconstructed muons is not taken into account, since MC simulations do not describe this variable very well, see Figure 4.15. The observed mismatch between $\mathrm{MC}$ simulations and data for high values of the fully corrected missing transverse energy is likely due to the fact that the transverse momentum resolution of high- $p_{\mathrm{T}}$ muons is not measured very precisely. This, in turn can produce $»$ fake $« \not_{T}$, such that a subsequent $\gg$ correction $«$ due to the (mismeasured) muon- $p_{\mathrm{T}}$ would cause an even greater damage.

Missing transverse energy is also expected from the SUSY signal, due to the production of at least two neutrinos from the decay of the two lightest neutralinos. Additional neutrinos might be produced in the cascade decays of the pair produced heavier neutralinos and charginos. Hence, $\not_{T}$ it another main observable to distinguish events from signal processes from those produced from SM reactions and will be used in the selection of $\mu \mu \ell$ trilepton events. 


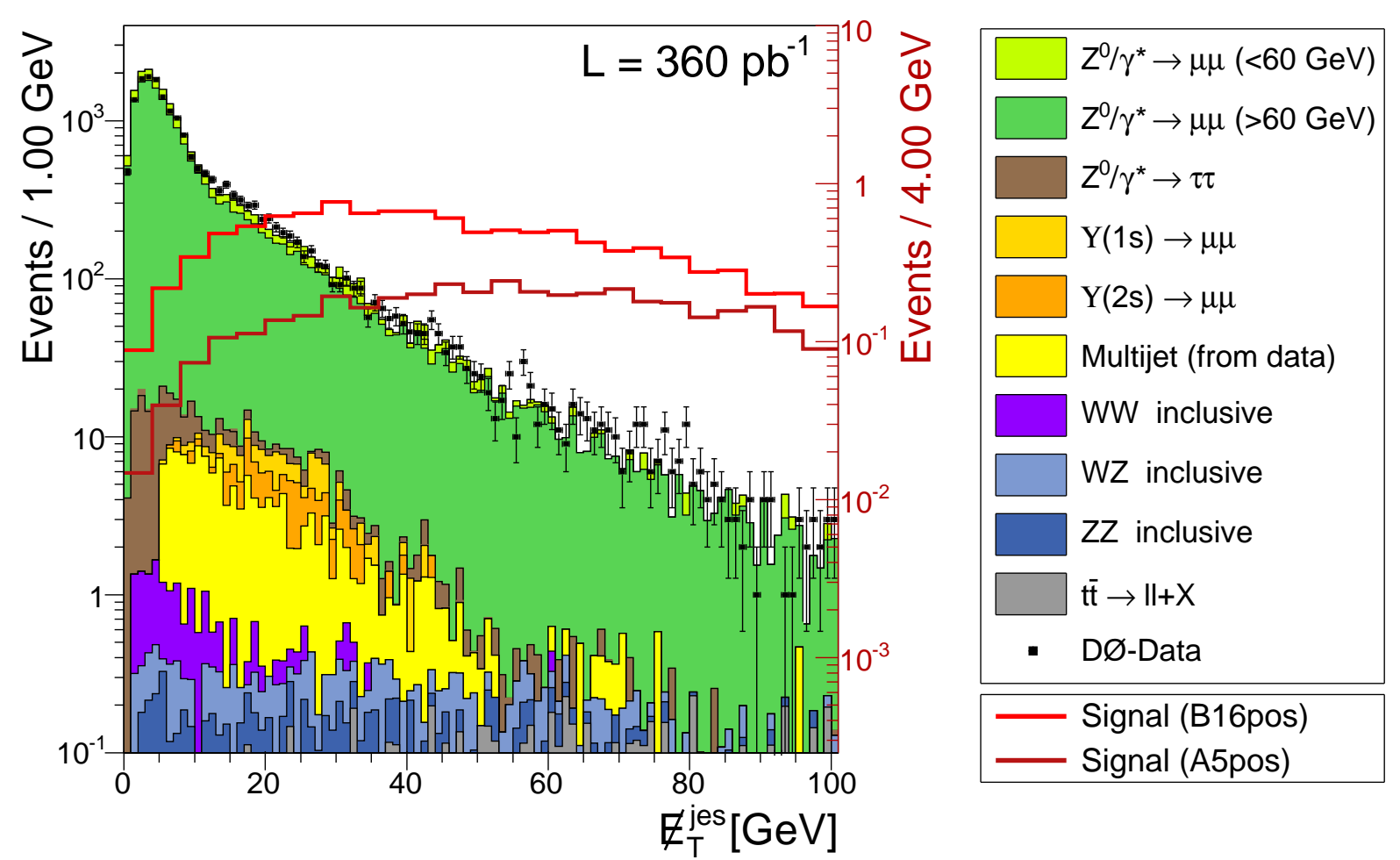

Figure 4.14: The distribution of the missing transverse energy $\left(\mathbb{Z}_{T}^{j e s}\right)$ after the preselection of two high-quality muons (or: a good electron, a good muon and another »loose« muon), see section 4.5. Corrections for the jet energy scale (JES) [154] and the electromagnetic energy scale are applied. Two signal distributions scaled by a factor of 400 are overlaid (red/darkred solid lines, right scale): $\mathcal{B} 16$ pos corresponds to an mSUGRA point with low scalar mass $m_{0}=100 \mathrm{GeV}$, while $\mathcal{A} 5$ pos has $m_{0}=1000 \mathrm{GeV}$.

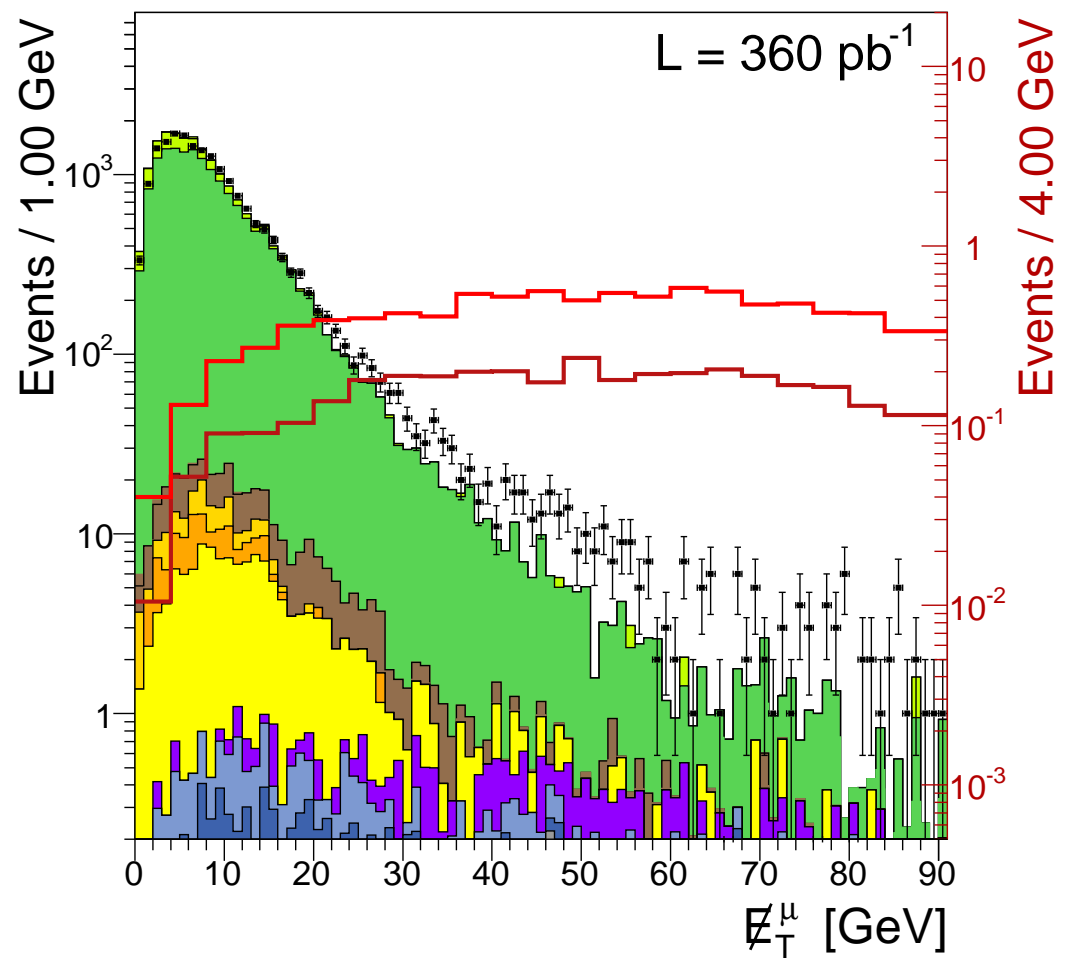

Figure 4.15: The distribution of the missing transverse energy $\left(\mathcal{H}_{T}^{\mu}\right)$ after the preselection, see section 4.5 . Again, two signal distributions scaled by a factor of 400 are overlaid, see the legend of Fig. 4.14. All corrections are applied: JES, EMscale and the $E_{\mathrm{T}}$-correction due to reconstructed muons. The mismatch between $M C$ simulations and data for high values of the fully corrected missing transverse energy is likely due to the fact, that high-p $p_{\mathrm{T}}$ muons are not properly simulated. 


\section{Identification of Tau Leptons}

While tau leptons are not needed for this analysis, they are part of the final state and thus a vital ingredient in the complementary analysis of eer trilepton events, which is described in detail in $[2,74]$. Leptonically decaying tau leptons are detected either as electrons or as muons and no additional criteria are needed. Identifying hadronically decaying tau leptons $\left(\tau_{\text {had }}\right)$ is more challenging. Due to the similarity to hadronic jets, a number of criteria is necessary to distinguish hadronic tau decays and QCD jets, originating from quarks or gluons, from each other. There are, however, a few differences:

- the particle multiplicity in hadronic tau decays is less than for QCD jets, which can be seen from the number of 1-prong and 3-prong tau decays: about $77 \%$ of hadronically decaying tau leptons produce only one charged hadron (one track), e.g. $\tau_{\text {had }} \rightarrow \pi^{-} \pi^{0} \nu_{\tau}$ and approximately $23 \%$ produce three charged hadrons (three tracks), e.g. $\tau_{\text {had }} \rightarrow \pi^{-} \pi^{+} \pi^{-} \pi^{0} \nu_{\tau}$; Decays with five or more tracks are very rare, below $1 \%$.

- hadronic tau jets are usually more collimated than jets from multijet processes (for the same jet energy), which is related to the much smaller particle multiplicity;

- the multiplicity of neutral hadrons from tau decays is even smaller: one or more neutral pions (or other light mesons) are produced in only about $22 \%$ of hadronic tau decays

Taus are identified in two steps: a jet-like algorithm, called taureco is employed to restrict a $(\eta, \varphi)$-region in the detector, containing a narrow, isolated hadronic jet with a specific ratio of electromagnetic to hadronic energy. For this potential tau candidate, the algorithm additionally calculates a number of characteristic variables, which are subsequently used in up to three neural networks to determine if the candidate can be identified as a tau lepton and, if so, what type it corresponds to, Three different types of hadronic tau decays are distinguished in DØ [164]:

$\tau$-type 1: 1-prong tau, e.g. with one track associated with a calorimeter cluster and without an electromagnetic sub-cluster ( $\pi$-like $\tau_{\text {had }}$ decay);

$\boldsymbol{\tau}$-type 2: 1-prong tau, e.g. with one track associated with a calorimeter cluster and with an electromagnetic subcluster ( $\rho$-like $\tau_{\text {had }}$ decay);

$\tau$-type 3: 3-prong tau, e.g. with three or two tracks (one undetected) associated with a calorimeter cluster. The masses of the tracks must be smaller than the one calculated for the tau candidate. (primarily $\tau \rightarrow \pi^{-} \pi^{+} \pi^{-}\left(n \geq 0 \pi^{0}\right) \nu_{\tau}$ )

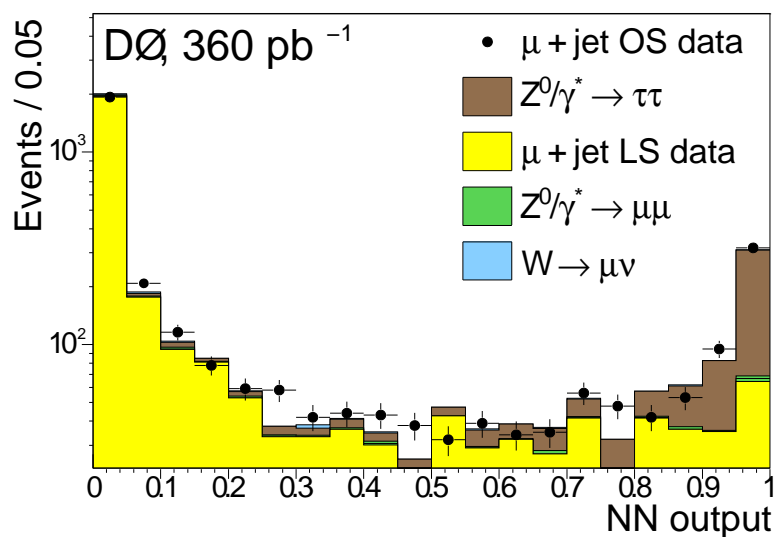

Figure 4.16: The neural network output for a loose $Z \rightarrow \tau \tau \rightarrow \tau_{\text {had }} \mu$ selection is used as identification criterion for $\tau$ leptons [165].

A possible contamination from muons misidentified as taus is removed by taking the shower shape of the hadronic cluster into account.

Figure 4.16 shows the neural network output for a loose $Z \rightarrow \tau \tau \rightarrow \tau_{\text {had }} \mu$ selection, which is used as an identification criterion for taus in the eer analysis (developed primarily to study the $\boldsymbol{R}_{p^{-}}$ coupling $\lambda_{133}$ ). The $\mu+$ jet opposite-sign data sample (OS) represents the control sample, while the $\mu+$ jet like-sign data sample (LS) is used to model the multijet background. The different contributions are scaled to the control sample by fitting the $E_{T}$ spectrum of the tau candidate. 


\subsection{Estimation of the Multijet Background from Data}

Multijet background from QCD, but also from electroweak production with initial or final state gluon radiation is difficult to simulate. The dominant source of background events are semileptonic events from heavy flavour production, i.e. $b \bar{b}$ and $c \bar{c}$ events, since these heavy flavour jets can contain one or more muons from the semileptonic decay of $B(D)$-mesons. Unfortunately this type of events is especially difficult to model due to large theoretical uncertainties and several contributing QCD processes. Hence, using data to estimate the contribution of multijet background to the selected final state, not only avoids these theoretical uncertainties, but also the time-consuming generation/simulation of huge amounts of multijet events. Two different methods for the estimation of the multijet contribution to the dimuon sample have been studied:

first method: modeling of a like-sign dimuon sample with a sample of »loosely « isolated unlikesign dimuon events, see Table 4.11 for details;

second method: modeling of the multijet background with a sample of dimuon events, enriched with $b \bar{b}(c \bar{c})$ events due to the additional requirement of a loose b-tag. To ensure an orthogonal sample (w.r.t. the dimuon analysis sample), the more energetic muon must be less isolated than required in the dimuon analysis (Table 4.12).

The first method is described in detail in references $[166,167]$, thus only a brief summary is given here. A sample of like-sign muon pairs with special isolation requirements is chosen in order to not use the phase space region where the signal is expected to lie. This sample will contain essentially events from semileptonic $b$ and $c$-decays, which is also the dominant QCD contribution in the unlike-sign sample. For most other SM processes (like $Z / \gamma^{*}, W W$ or $\Upsilon$ production) events with two unlike-sign leptons are expected. The main differences between the two samples (like-sign and unlike-sign) are the decay chains and the production rates. Most of the $b$ and $c$-decays arise from direct production via gluon-gluon fusion and flavor excitation [168], while contributions from resonances, like $J / \psi$ or $\Upsilon$, are small compared to the inclusive production.

Events with two like-sign muons of loose quality are selected from the analysis sample. This likesign dimuon sample is then split again into two subsamples: events where both muons satisfy the tight isolation criteria (referred to as iso-sample) and a second subsample containing events where one of the two muons is tightly isolated, and the other muon is nearly isolated. The nearly isolated muon is defined by softened isolation criteria in order to obtain a kinematically similar sample to the isolated selection. If one muon already satisfies one of the soft isolation criteria (either the calorimeter or the track isolation) then the other muon may meet the soft or the tight criterion; see Table 4.11, providing an overview of the tight and soft isolation criteria.

\begin{tabular}{lcc}
\hline \hline Isolation Criterion & Standard isolation criteria & First method: soft isolation \\
\hline \hline Tracks: & $\sum p_{\mathrm{T}}^{\text {tr }}<2.5 \mathrm{GeV} \& \sum p_{\mathrm{T}}^{\text {tr }} / p_{\mathrm{T}}^{\mu}<6 \%$ & $2.5 \mathrm{GeV}<\sum p_{\mathrm{T}}^{\text {tr }}<7 \mathrm{GeV}$ \\
Calorimeter: & $E_{\mathrm{T}}^{\text {alo }}<2.5 \mathrm{GeV} \& E_{\mathrm{T}}^{\text {alo }} / p_{\mathrm{T}}^{\mu}<8 \%$ & $2.5 \mathrm{GeV}<E_{\mathrm{T}}^{\text {halo }}<7 \mathrm{GeV}$ \\
\hline \hline
\end{tabular}

Table 4.11: Overview of the different isolation criteria, used with the first method to estimate the contribution of the multijet background to the expectation of the SM background.

The sample of nearly isolated muon pairs is chosen, because any possible signal contamination is expected to be negligible, since muons from the signal are expected to be isolated. Nevertheless, a correlation is expected between the isolation requirements and the $p_{\mathrm{T}}$-spectra of the muons, which can be explained in the following way: lower $p_{\mathrm{T}}$ muons are more likely to get $\gg$ kicked away « from their $b$-jet and are thus more isolated than higher $p_{\mathrm{T}}$ muons for which this kicking is 
not as effective. Indeed, the ratio $R=$ iso/nearly, calculated from the number of isolated dimuon events versus the transverse momentum of the most energetic muon and the number of nearly isolated dimuon events versus the $p_{\mathrm{T}}$ of the nearly isolated muon as shown in Figure 4.17 (a) shows a clear dependence on the $p_{\mathrm{T}}$-spectra/isolation criteria of the muons. The ratio $R$ is thus used to weight the $p_{\mathrm{T}}$-spectrum of the nearly-sample, so that it resembles the $p_{\mathrm{T}}$-spectrum of the iso-sample. In a last step the reweighed sample of nearly isolated dimuon events is scaled to the data after subtracting all other expected backgrounds.
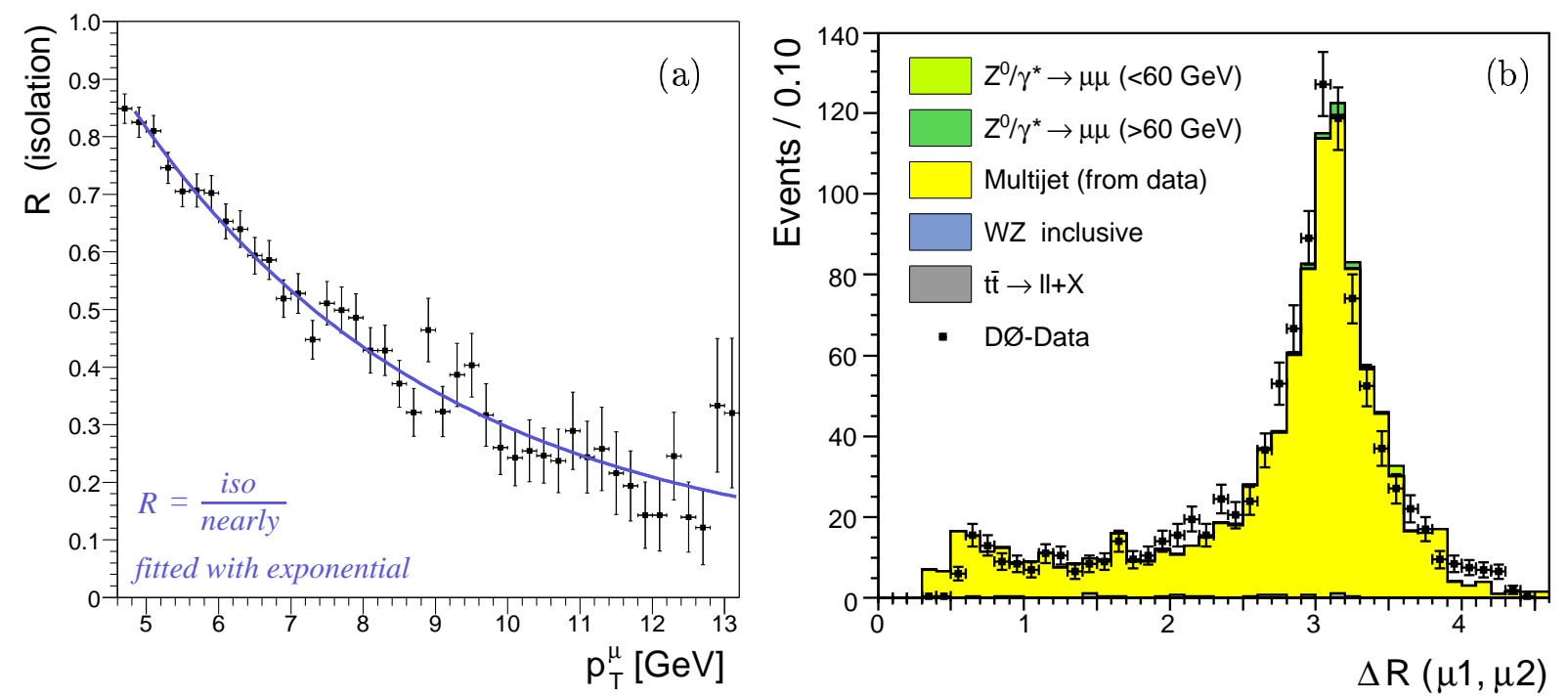

Figure 4.17: Estimation of the multijet background with the first method, using a like-sign dimuon sample. (a) The ratio of the iso and nearly-sample, where the data is fitted with an exponential function. (b) The reweighed nearly-sample scaled to the like-sign data in the distribution of the distance between the two leading muons.

However, since the first method revealed statistical insufficiencies, a second method has been developed, which makes use of an entirely independent dimuon sample, enriched with $b \bar{b}(c \bar{c})$ events due to the additional requirement of a secondary (displaced) vertex $(\rightarrow$ loose $b$-tag). The isolation criteria for both muons are loosened to $\sum p_{\mathrm{T}}^{t r}<15 \mathrm{GeV}$ and $E_{\mathrm{T}}^{\text {halo }}<10 \mathrm{GeV}$. In order to ensure, that none of the dimuon events of the analysis sample is also present in the new b-tag sample, the most energetic muon of the b-tag sample must be less isolated than required in the trilepton selection, which translates to $\sum p_{\mathrm{T}}^{t r}>2.5 \mathrm{GeV}$ compared to the orthogonal requirement for the trilepton selection. The dimuon events selected in this way are scaled by a factor of 0.19 , such that the sum of SM process MC events plus the estimated multijet background describe the data. A variation of the upper bound of the isolation criteria leads to a systematic uncertainty of roughly $\approx 20 \%$.

\begin{tabular}{lcc}
\hline \hline Isolation Criterion & Trilepton analysis & Second method: soft isolation \\
\hline \hline Tracks: & $\sum p_{\mathrm{T}}^{t r}<2.5 \mathrm{GeV} \mathrm{\&} \sum p_{\mathrm{T}}^{t r} / p_{\mathrm{T}}^{\mu}<6 \%$ & $\sum p_{\mathrm{T}}^{t r}<15 \mathrm{GeV}$ \\
Calorimeter: & $E_{\mathrm{T}}^{\text {halo }}<2.5 \mathrm{GeV} \mathrm{\&} E_{\mathrm{T}}^{\text {halo }} / p_{\mathrm{T}}^{\mu}<8 \%$ & $E_{\mathrm{T}}^{\text {halo }}<10 \mathrm{GeV}$ \\
& \multicolumn{2}{c}{ additionally for $1^{\text {st }}$ muon: $\sum p_{\mathrm{T}}^{\text {tr }}>2.5 \mathrm{GeV}$ for $1^{\text {st }}$ muon. } \\
\hline \hline
\end{tabular}

Table 4.12: Overview of the different isolation criteria, used with the second method to estimate the contribution of the multijet background to the expectation of the SM background. 
One problem of the b-tag sample is the potentially different event kinematics due to the indirect requirement of at least one jet per event. However, this is not studied in greater detail, since this analysis does not require the presence or absence of any jets, and for further reference about the dependence on the jet-multiplicity the reader is referred to [65].

Since very tight isolation criteria are employed in the following dimuon and later trilepton selection (at least two muons), the overall contribution of multijet background is very small. This is visible in Figures 4.14 and 4.15, and it is further verified in the cutflow Tables 4.13 and 4.14 and Figures 4.18 and 4.19 in section 4.5.1.

\subsection{The Selection of Dimuon \& Electron-Muon Events}

The selection of trilepton events is realised in two steps: in a first step events with either two »high quality « muons, or one »good « electron and one »high quality « muon are selected. A muon (electron) candidate is tagged as a high quality muon (good electron), if it satisfies the respective quality criteria discussed in section 4.3.2. Muons are considered within the full coverage of the DØ muon system, i.e. within $\left|\eta_{\text {det }}\right|<2$, while electrons are considered within the CC and EC regions of the calorimeter, corresponding to $\left|\eta_{\text {det }}\right|<1.1$ and $1.5<\left|\eta_{\text {det }}\right|<3.0$, respectively without the inter-cryostat region. In a second step, the third charged lepton is required, as will be discussed in detail in section 4.5.3. The selection is performed in two parallel streams, the dimu and the emu streams, which are described in more detail in the following subsections.

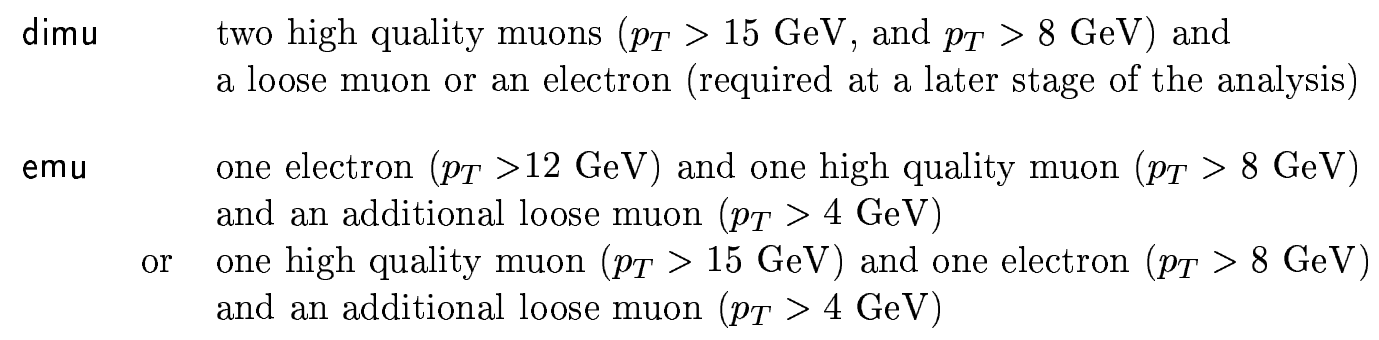

However, since always at least two muons must be present in an event, of which one has to be of medium quality and with a transverse momentum of at least $p_{\mathrm{T}}>15 \mathrm{GeV}$ (due to the dimuon trigger requirement and especially the Level 2 condition as explained in section 4.1), only very few events are selected from data and SM processes in the emu stream.

The reconstructed dimuon invariant mass of the two leading high quality muons at preselection level is shown in Figure 4.18 (a,b) on a linear and a logarithmic scale (b), where the tails of the distribution are also visible. A good agreement of data and SM background processes can be observed. In addition, the distributions of six further observables are displayed in Figure 4.19, again after the preselection and with all corrections applied. The distributions of the transverse momentum (a,b), $\eta(\mathrm{c}, \mathrm{d})$ and $\varphi(\mathrm{e}, \mathrm{f})$ of the leading (a,c,e) and the next-to-leading muon (b,d,f) are shown. In Figure 4.18, the distributions of two signal points, $\mathcal{A} 5$ pos and $\mathcal{B} 16$ pos, (red/darkred solid lines) are overlaid for reference. However, since they would hardly be visible otherwise, they are scaled by a factor of 400 . The second scale on the right-hand side indicates the number of events in the signal distributions.

The analysed data, collected with the D $\varnothing$ detector between April 2002 and August 2004 during the Run II of the Tevatron Collider, corresponds to an integrated luminosity of $\int \mathcal{L} d t=$ $361.3 \pm 23.5 \mathrm{pb}^{-1}$. With the previously defined quality criteria 19,283 data events compared to 19,588.2 expected events from SM-processes are selected and constitute what is referred to as the preselection sample; more exactly these events pass either $\mu \mu$-cut 1 or $e \mu$-cut 1 (see the following 
subsections 4.5.1 and 4.5.2). In Tables 4.13 and 4.14, Cut 1 , the combination of the first $\mu \mu$ and the first $e \mu$ cuts, represent this preselection sample. Most of the selected events are found in the so-called dimu stream, which is also the one suffering from the highest SM background, like the $Z^{0}$ and $\Upsilon$ resonances as well as Drell-Yan background. In the emu stream the second, loose quality muon is required from the beginning in order to satisfy the requirements of the dimuon trigger set. In the following two subsections a more detailed description of the two selection streams will be given.

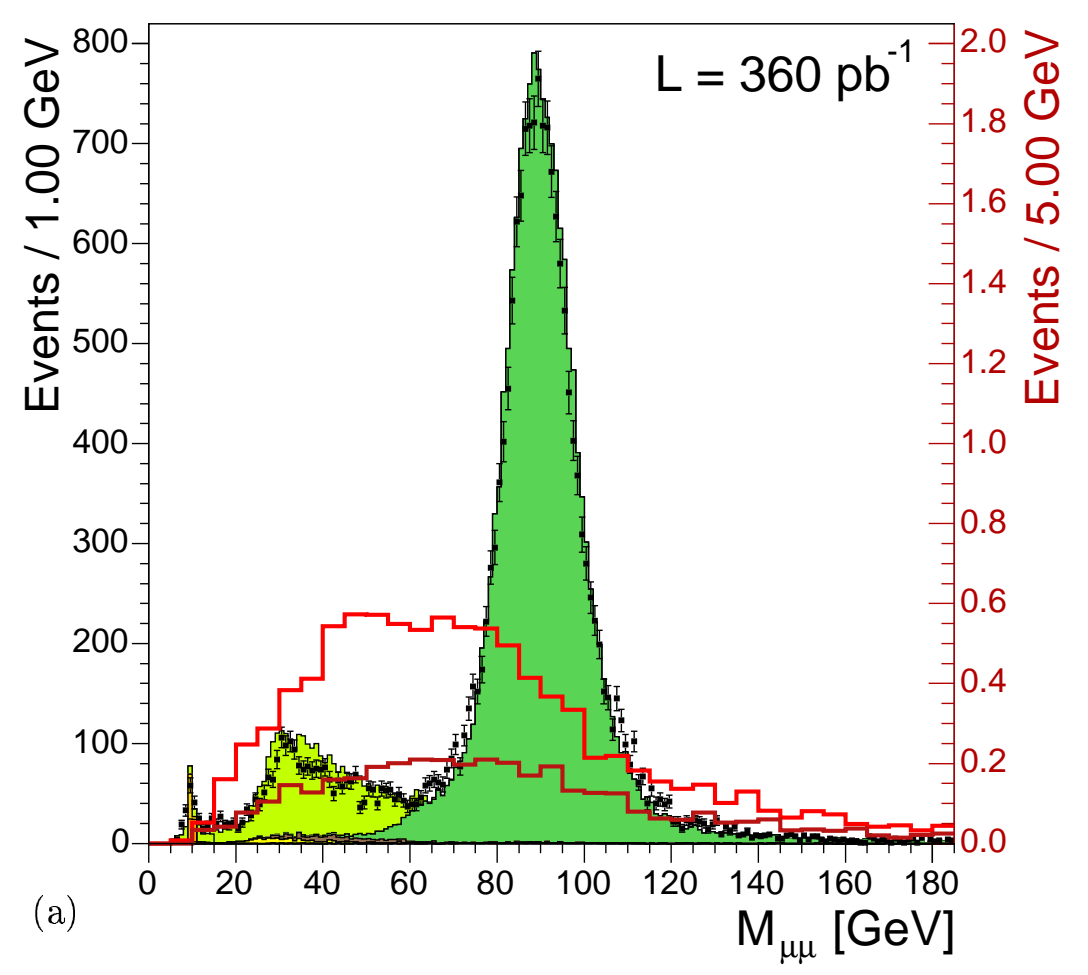

Figure 4.18: The distribution of the dimuon invariant mass $M_{\mu \mu}$ after the preselection (see Tables 4.13 and 4.14) and with all corrections applied (a) on a linear scale and (b) on a logarithmic scale, so that the tails of the distribution are also visible. Two signal distributions, again scaled by a factor of 400, are overlaid (red/darkred solid lines, right scales): $\mathcal{B} 16$ pos corresponds to an mSUGRA point with low scalar mass $m_{0}=100 \mathrm{GeV}$ $\left(\sigma_{N L O}=0.064\right)$, while $\mathcal{A} 5$ pos has $m_{0}=1000 \mathrm{GeV}\left(\sigma_{N L O}=\right.$ $0.034)$. The right-hand scale relates to the signal distributions.

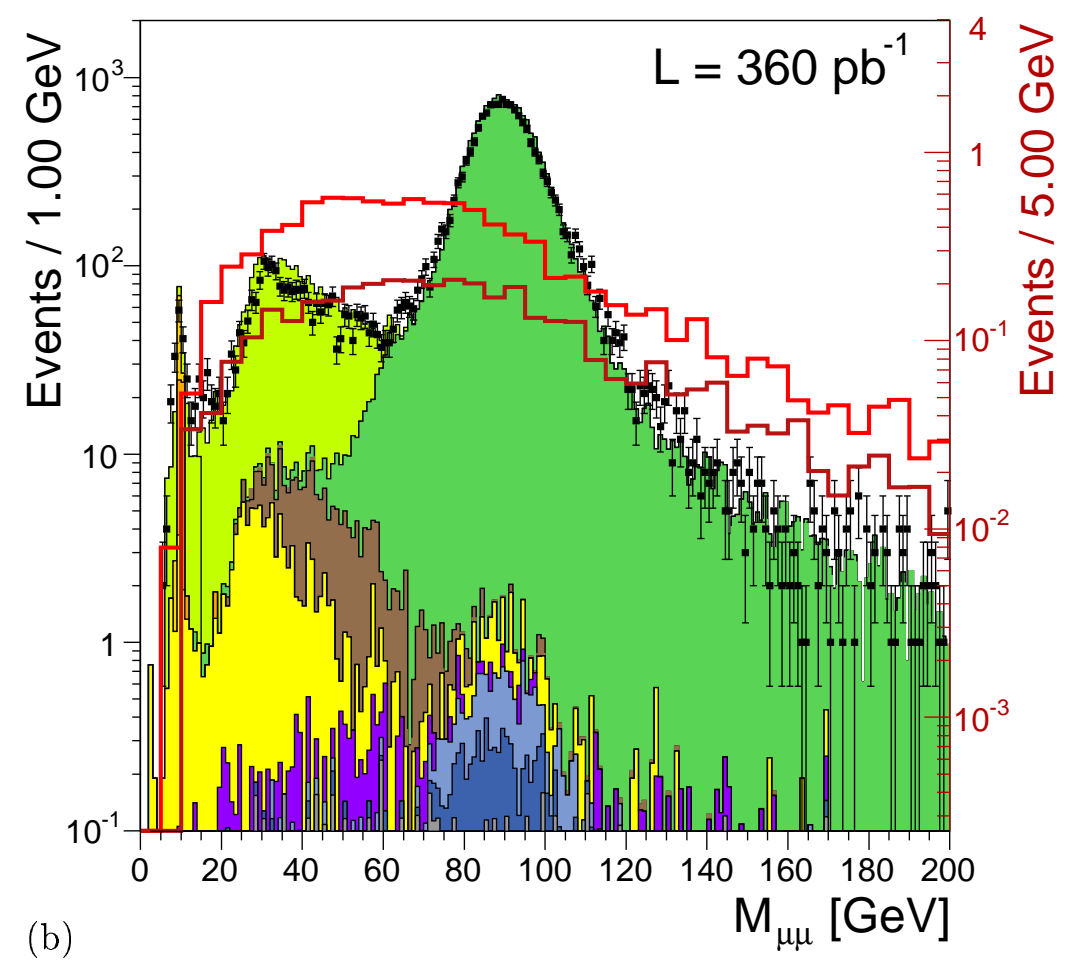

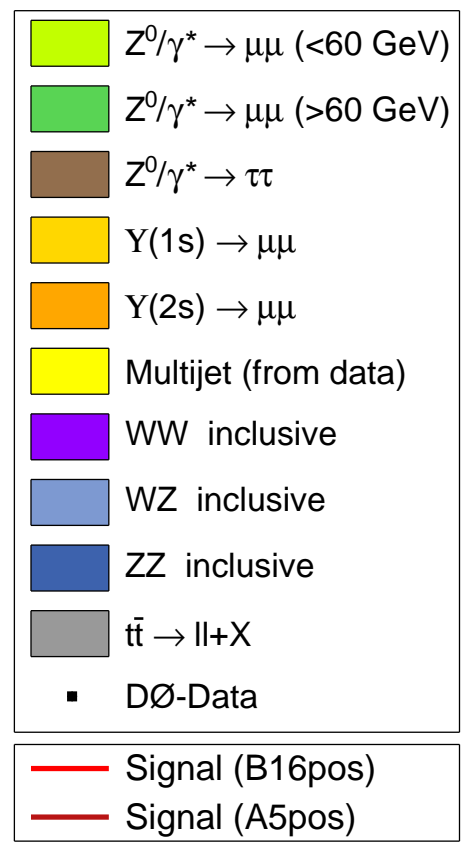



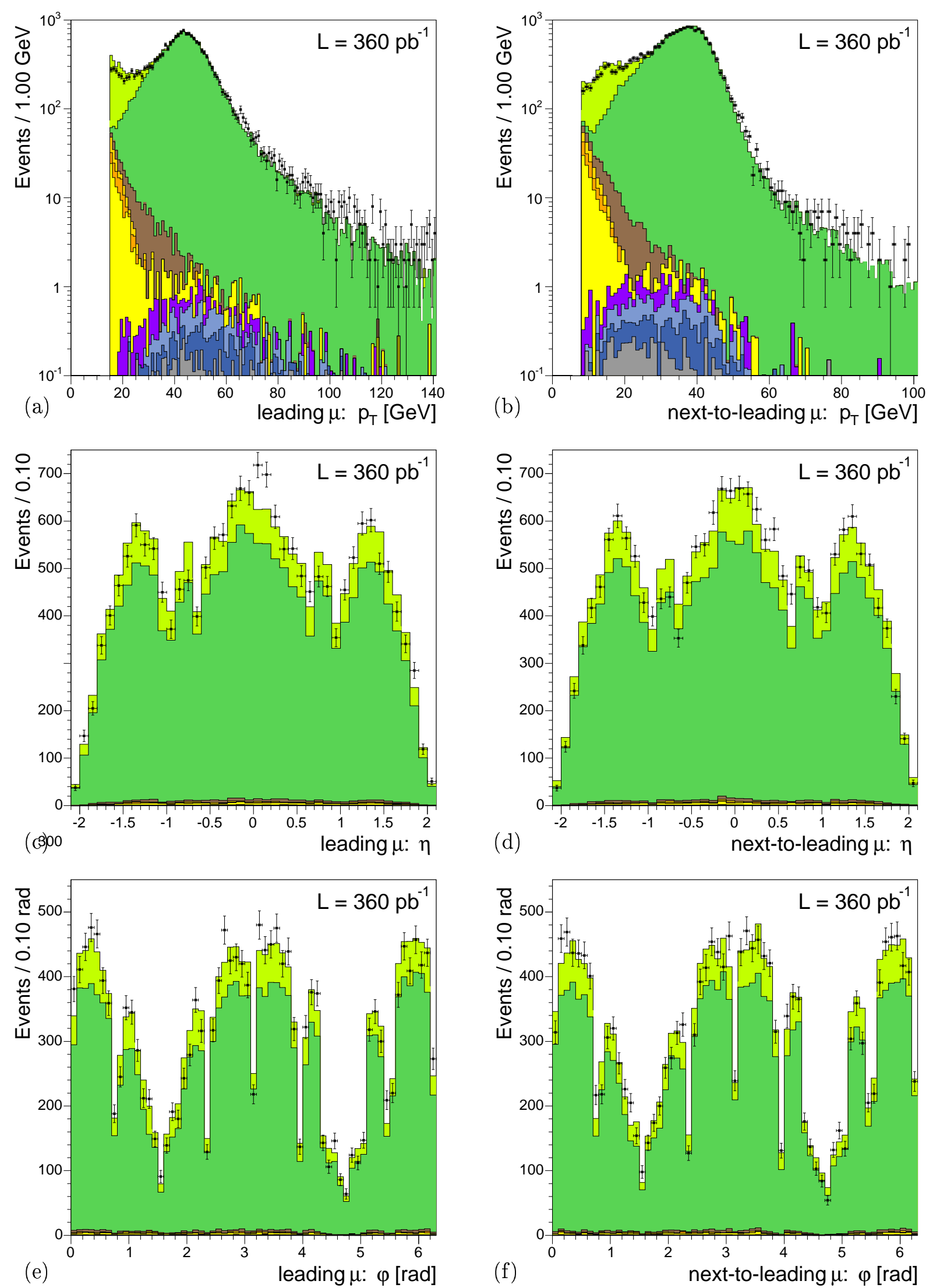

Figure 4.19: Control distributions after the preselection of two muons: (a,c,e) the transverse momentum, $\eta$ and $\varphi$ distributions of the leading muon and $(b, d, f)$ the transverse momentum, $\eta$ and $\varphi$ distributions of the next-to-leading muon. Data and coloured histograms as listed in the legend of Fig. 4.18. 


\subsubsection{The Dimuon Selection: dimu}

Since the dimu selection is the one with the highest SM background expectation, more advanced selection criteria are applied to reduce the SM background and enrich the sample in signal events. The following criteria are applied successively to events with at least two high quality muons:

$\boldsymbol{\mu} \boldsymbol{\mu}$-cut 1 (Preselection): $\quad p_{T}(\mu 1)>15 \mathrm{GeV}, p_{T}(\mu 2)>8 \mathrm{GeV}$, each matched to a central track and tightly isolated according to Table 4.11 in section 4.4 ;

$\boldsymbol{\mu} \boldsymbol{\mu}$-cut 2: $\quad$ Spatial and angular distances between the leading muons themselves and between the muons and other objects in the event (see also Figure 4.20):

$\Delta \mathcal{R}(\mu 1, \mu 2)>0.2, \Delta \mathcal{R}(\mu 1 / \mu 2, j e t)>0.5$ and $\Delta \varphi\left(\mu 1 / \mu 2, \not_{T}^{j e s}\right)>0.1$;

$\boldsymbol{\mu \mu}$-cut 3: $\quad\left|z_{\text {vertex }}\right|<60 \mathrm{~cm}$, relative to the nominal interaction point at $z=0$;

$\boldsymbol{\mu} \boldsymbol{\mu}$-cut 4 (anti- $\boldsymbol{\Upsilon}$ ): $\quad$ For oppositely charged dimuons with $\mathbb{\#}_{T}^{\text {jes }}<60 \mathrm{GeV}$, a two-dimensional cut in the $\left(M_{\mu \mu}, \not t_{T}\right)$-plane is used to reject events from the $\Upsilon$ resonances, low- $p_{\mathrm{T}}$ Drell-Yan and multijet background (see Figure 4.21):

$$
M_{\mu \mu}>0.0095 \cdot\left(\mathbb{H}_{T}^{j e s}\right)^{2} / \mathrm{GeV}-1.29 \cdot \mathbb{H}_{T}^{j e s}+48 \mathrm{GeV}
$$

$\boldsymbol{\mu} \boldsymbol{\mu}$-cut 5: Two-dimensional cut in the $\left(\Delta \varphi_{\mu \mu}, \not_{T}\right)$-plane to reject $Z / \gamma^{*}$ background (Fig. 4.23 (b)), as well as other events with only little missing transverse energy and that are mostly back-to-back in $\Delta \varphi$, e.g. events from multijet and diboson production, see Figure 4.23 (c) and (d), respectively:

$$
\Delta \varphi_{\mu \mu}<2.53 \text { for } \not_{T}^{j e s}<44 \mathrm{GeV}
$$

$\boldsymbol{\mu} \boldsymbol{\mu}$-cut 6 (anti- $\left.\boldsymbol{Z}^{\mathbf{0}}\right)$ : Another two-dimensional cut in the $\left(M_{\mu \mu}, \mathbb{F}_{T}\right)$-plane is applied for oppositely charged dimuons with an invariant mass below $M_{\mu \mu}<180 \mathrm{GeV}$ to veto events from the $Z^{0}$ resonance and from diboson production:

$$
\mathbb{E}_{T}^{\text {jes }}>48 \mathrm{GeV}-0.105 \cdot\left(M_{\mu \mu}^{2}-90 \mathrm{GeV}\right)^{2} / \mathrm{GeV} \text {; }
$$

Additionally, loose $\not_{T}$-criteria are applied: $\not_{T}^{j e s}>8 \mathrm{GeV}$, and $\not_{T}^{\mu}>9 \mathrm{GeV}$ and the dimuon invariant mass is required to be smaller than $200 \mathrm{GeV}$.

While the first three selection criteria are additional quality requirements, the last three criteria, i.e. the two-dimensional cuts, have been developed to reject as many events from SM background as possible, while keeping a high signal efficiency. The second criterion, $\mu \mu$-cut 2 , is necessary to ensure that the two muon candidates are well separated and that they are not misidentified jets or $\mathbb{F}_{T}$ objects. A high-momentum jet, that is not entirely stopped in the calorimeter, but leaks into the muon chambers may $»$ fake « a muon. The third criterion, $\mu \mu$-cut 3 , ensures that the tracks lie within the fiducial volume of the inner tracking detectors (SMT and CFT), so that a reliable tracking is guaranteed and the previously discussed corrections to the track-finding/matching efficiency are applicable.

The two-dimensional cuts are chosen to improve the signal selection efficiency and at the same time the background rejection compared to simple one-dimensional cuts. For example, cut 4, is very efficient in reducing the number of events from $\Upsilon$ production, low- $p_{\mathrm{T}} Z / \gamma^{*}$ and multijet background. The second cut in the $\left(M_{\mu \mu}, \not_{T}\right)$-plane, $\mu \mu$-cut 6 , largely reduces the background from $Z^{0}$ bosons, but also from $W Z$ and $Z Z$ processes. In addition, the requirement $M_{\mu \mu}<200 \mathrm{GeV}$ of $\mu \mu$-cut 6 leads to a significant reduction of $\approx 70 \%$ for the $Z / \gamma^{*}$ and $W Z$ background at high dimuon invarinat masses, while the signal selection efficiency remains nearly unchanged. Cut 5 in 

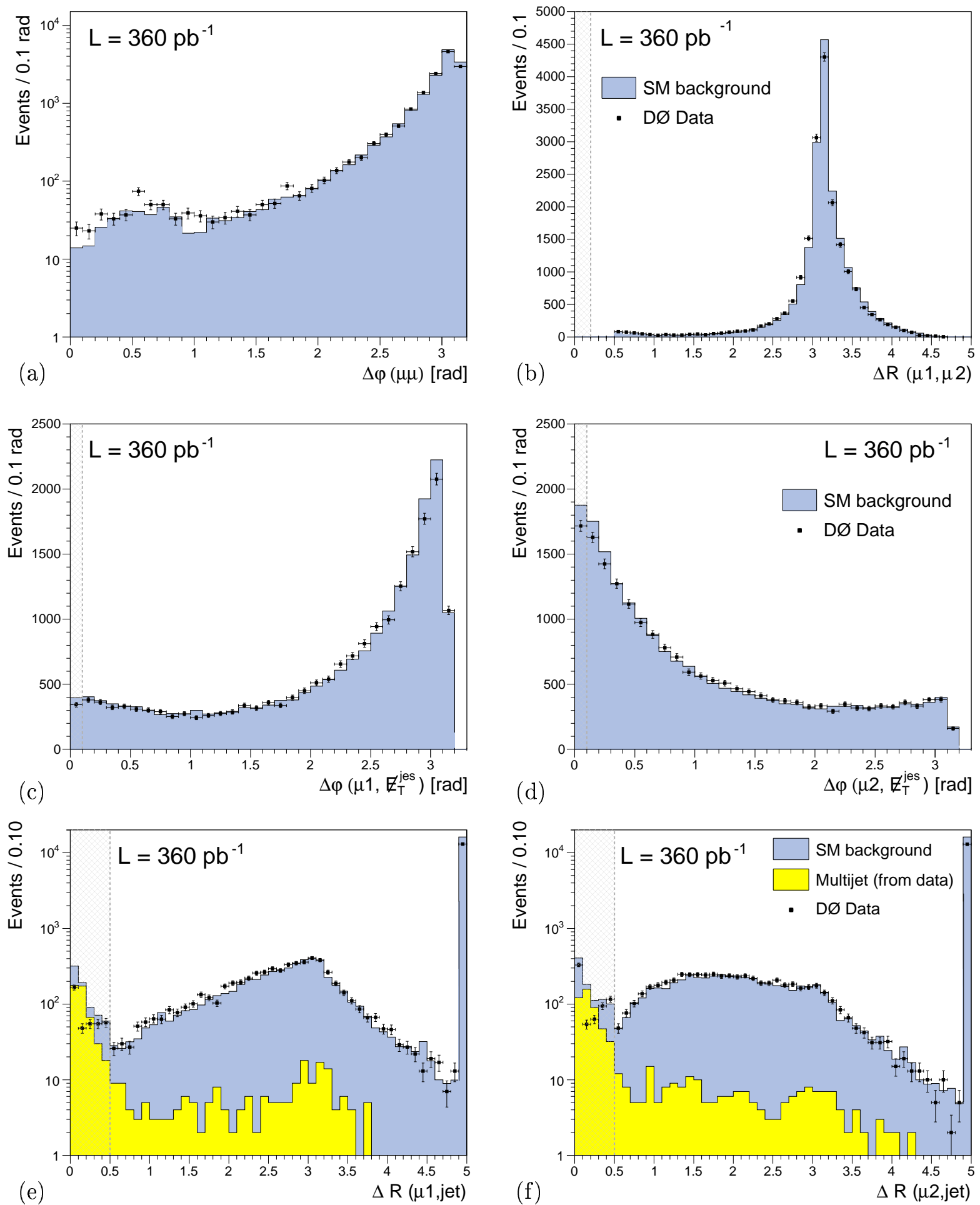

Figure 4.20: Spatial and angular distances between the two leading muons themselves and other objects in the event: (a) angular and (b) spatial distance between the leading and the next-to-leading muon, (c,d) angular distance between the leading (resp. next-to-leading) muon and the missing transverse energy vector, and $(e, f)$ spatial distance between the leading (resp. next-to-leading) muon and the closest jet in the event. In case there are no reconstructed jets in the event, the values are set to $\Delta \mathcal{R}(\mu 1 / \mu 2$, jet $)=5$. The multijet background is shown separately in $(e, f)$ since it accumulates in the rejected area $(\Delta \mathcal{R}(\mu 1 / \mu 2, j e t)<0.5$. $)$,

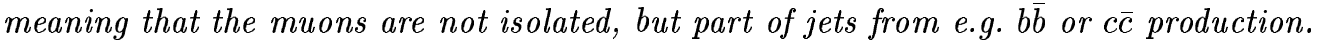


the $\left(\Delta \varphi_{\mu \mu}, \not_{T}\right)$-plane makes use of the fact that decay muons from $Z^{0}$ bosons are predominantly back-to-back in the transverse plane and usually exhibit no or only very little $\not_{T}$. Hence, this requirement additionally reduces the background from $Z / \gamma^{*}$ and $Z Z$ processes. Figures 4.21 and 4.22 illustrate the two-dimensional cuts in the $\left(M_{\mu \mu}, \not_{T}\right)$-plane, separately for data, SM background processes, and signal MC simulations. The two-dimensional cut in the $\left(\Delta \varphi_{\mu \mu}, \not_{T}\right)$ plane is displayed in Figures 4.23 and 4.24, again separately for data, SM backgrounds and six SUSY signal points, respectively.

For all of the two-dimensional figures the colours are chosen in the following way: SM backgrounds, data and signal distributions are shown in grey (black) dots or small open circles depending on the statistics, i.e. dots for SM Monte Carlo simulations with high statistics (e.g. $Z / \gamma^{*} \rightarrow \mu \mu$ ) and for data, and small open circles for low statistic processes (e.g. diboson or $t \bar{t}$ production) and for different signal points. Where two different processes are plotted together in one pad, grey and black is used to distinguish the two processes, e.g. the $Z / \gamma^{*} \rightarrow \mu \mu$ (grey dots) and $Z / \gamma^{*} \rightarrow \tau \tau$ (black circles) background in Figures 4.21 and 4.23 (b), and in Fig. 4.21 (c), the multijet background estimated from the data is shown as grey circles, while the $\Upsilon \rightarrow \mu \mu$ Monte Carlo is represented by black circles.

The coloured lines represent different cut values of the two-dimensional selection criteria. The one dark red curve indicates the chosen cut value, while green and bluish nuances indicate the tested values that are not used in the end. In Figures 4.21 and 4.22 , the area to the left of the red curve is rejected and in Figures 4.23 and 4.24 the area surrounded by the red box is cut away.

As can be seen quite clearly from the Figures 4.21-4.24 and also from the event numbers in Tables 4.13 and 4.14, the two-dimensional criteria are especially useful to reduce SM backgrounds while keeping a high selection efficiency for signal events of about $30-40 \%$.

\subsubsection{The Electron-Muon Selection: emu}

The main (irreducible) backgrounds for the emu selection are diboson production ( $W Z$ and $Z Z)$ and $t \bar{t} \rightarrow \ell \ell+X$ production. Since the cross sections of all these processes are small, no special criteria are developed for their specific reduction as compared to the dimu selection with the two-dimensional cuts. However, as pointed out previously, the third charged lepton, a loose muon, is required from the beginning in case of the emu selection. This is primarily due to the trigger requirements, but also because in this way the $e \mu$-skim need not be considered and data and Monte Carlo samples can be kept at a manageable size.

\section{e $\mu$-cut 1 (Preselection):}

$p_{T}(e)>12 \mathrm{GeV}, p_{T}(\mu)>8 \mathrm{GeV}, \quad$ or $\quad p_{T}(\mu)>15 \mathrm{GeV}, p_{T}(e)>8 \mathrm{GeV}$

and one additional loose muon with: $p_{T}^{\mu}>4 \mathrm{GeV}$, and $p_{\mathrm{T}}$-independent, loosened isolation criteria: $\sum p_{\mathrm{T}}^{t r}<9 \mathrm{GeV}$ and $E_{\mathrm{T}}^{\text {halo }}<15 \mathrm{GeV}$;

$\boldsymbol{e} \boldsymbol{\mu}$-cut 2: Distances between the leading muon or electron and other objects in the event (see Figure 4.25): $\Delta \mathcal{R}\left(\mu^{\text {good }}, e\right)>0.2, \Delta \mathcal{R}\left(\mu^{\text {good }}, j e t\right)>0.5, \Delta \varphi\left(\mu^{\text {good }} / e, \not_{T}^{j e s}\right)>0.1$;

$\boldsymbol{e} \boldsymbol{\mu}$-cut 3: $\quad\left|z_{\text {vertex }}\right|<60 \mathrm{~cm}$, relative to the nominal interaction point at $z=0$;

e $\boldsymbol{\mu}$-cut 4: $\quad$ Loose $\not_{T}$-criteria: $\not_{T}^{j e s}>8 \mathrm{GeV}$, and $\not_{T}^{\mu}>9 \mathrm{GeV}$.

Selection criteria corresponding to the two-dimensional $\mu \mu$-cuts 4,5 and 6 are not applied to the emu-selected events. Nevertheless one more selection criterion is applied to the events in 

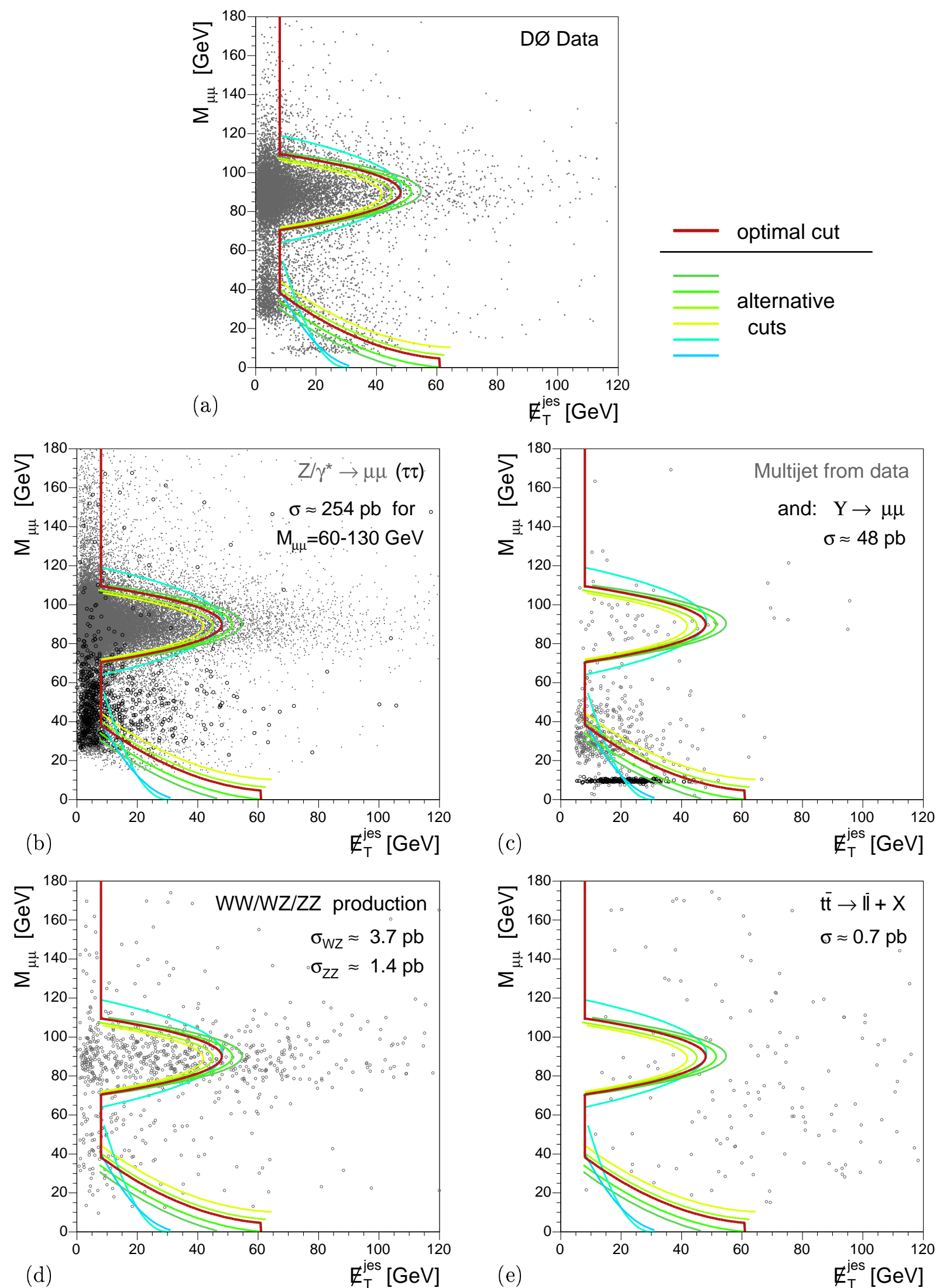

Figure 4.21: The two-dimensional criteria in the $\left(M_{\mu \mu}, \not_{T}\right)$-plane, i.e. $\mu \mu$-cut 4 and $\mu \mu$-cut 6: (a) for data and (b,c,d,e) for the respective SM backgrounds. The area to the left of the dark red curve is rejected. (See text for an explanation of the grey dots and grey/black circles.) 
4.5 The Selection of Dimuon ES Electron-Muon Events
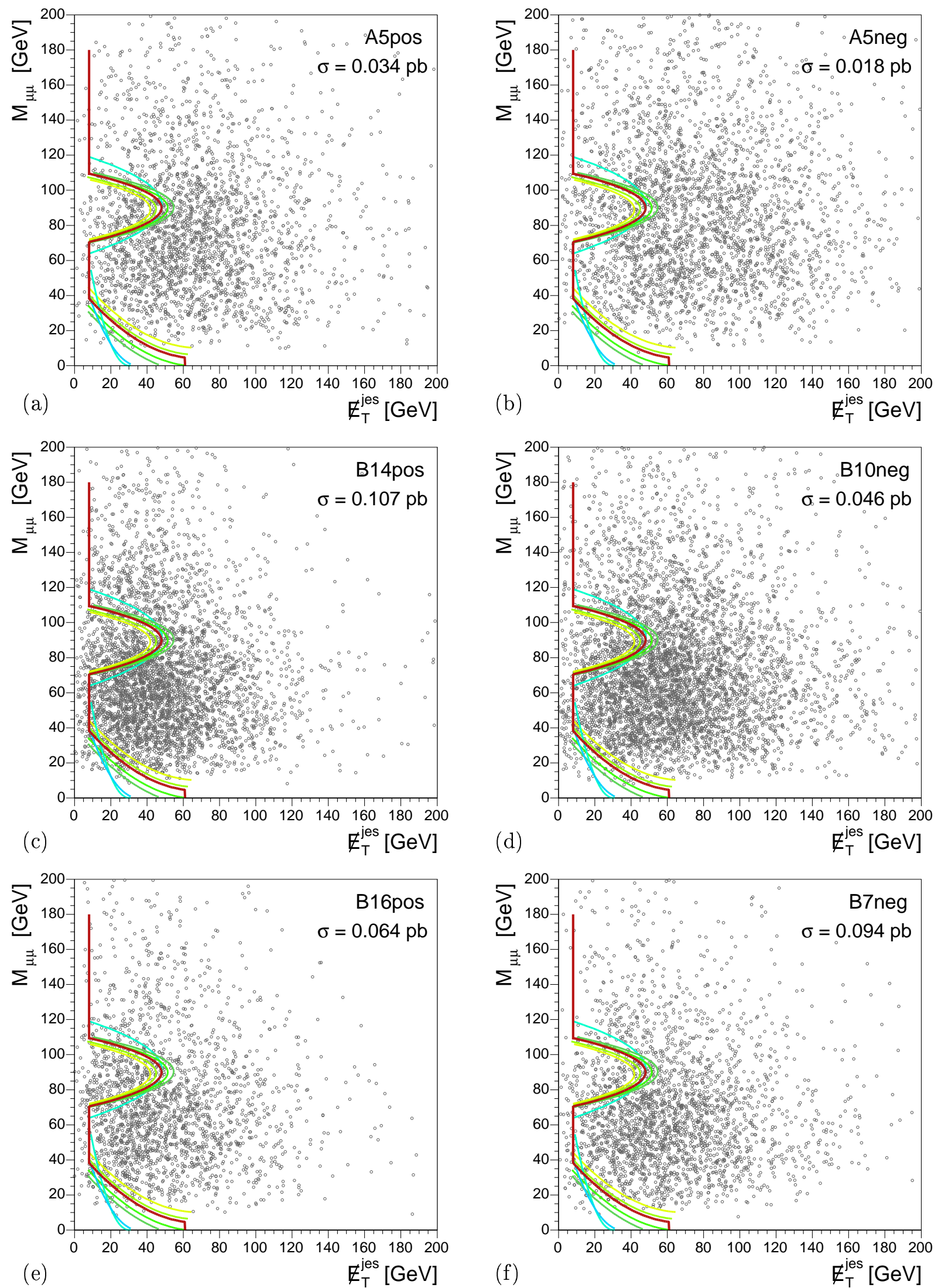

Figure 4.22: The two-dimensional criteria in the $\left(M_{\mu \mu}, \Phi_{T}\right)$-plane, i.e. $\mu \mu$-cut 4 and $\mu \mu$-cut 6 for some selected mSUGRA signal points: (a,c,e) for positive and $(b, d, f)$ for negative Higgs mixing parameter $\mu$. The area to the left of the dark red line is rejected. 

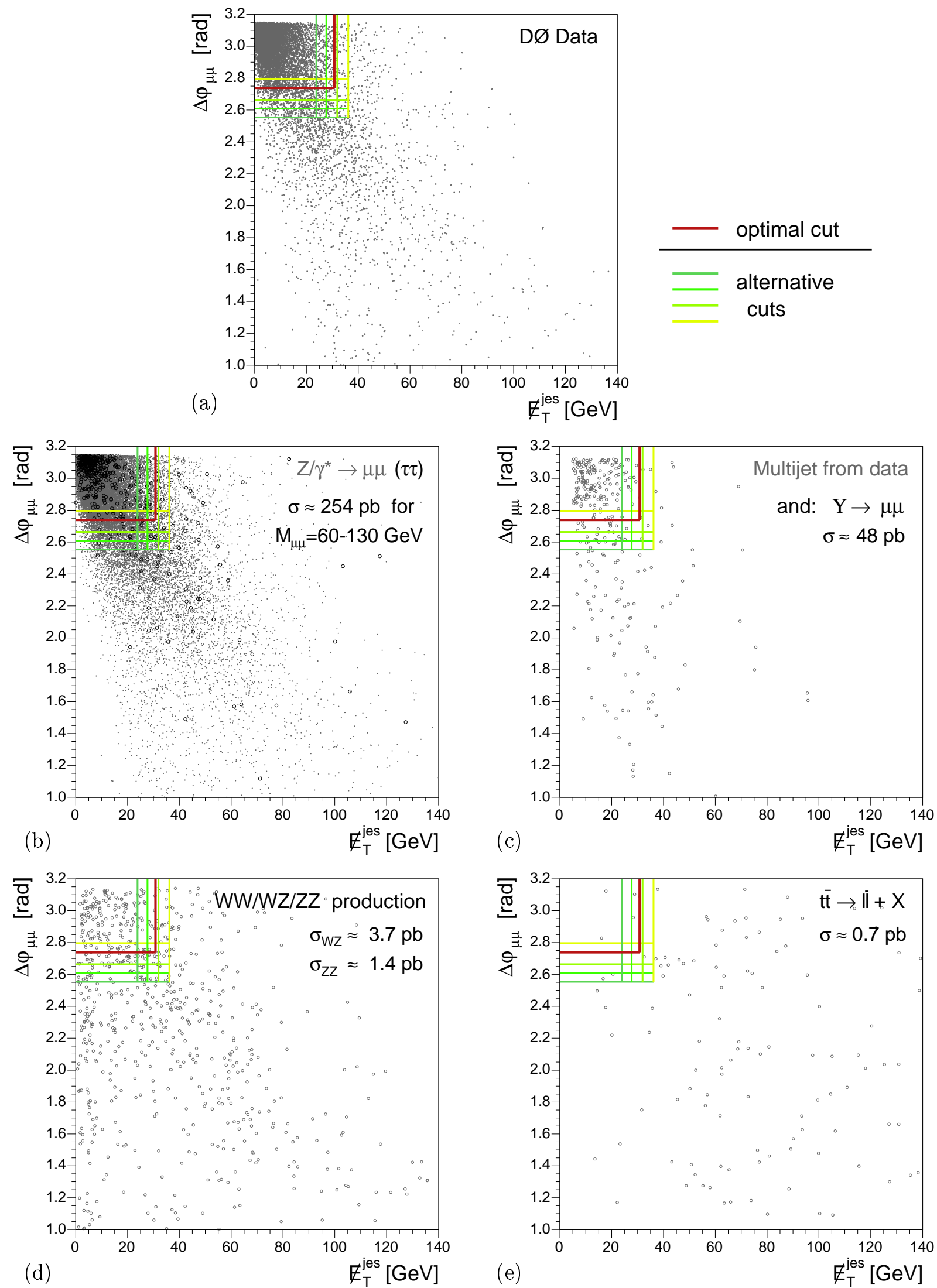

Figure 4.23: The two-dimensional criteria in the $\left(\Delta \varphi_{\mu \mu}, \not_{T}\right)$-plane, i.e. $\mu \mu$-cut 5: (a) for data and $(b, c, d, e)$ for the respective SM backgrounds. The area within the red coloured box is cut away. (See text for an explanation of the grey dots and grey/black circles.) 

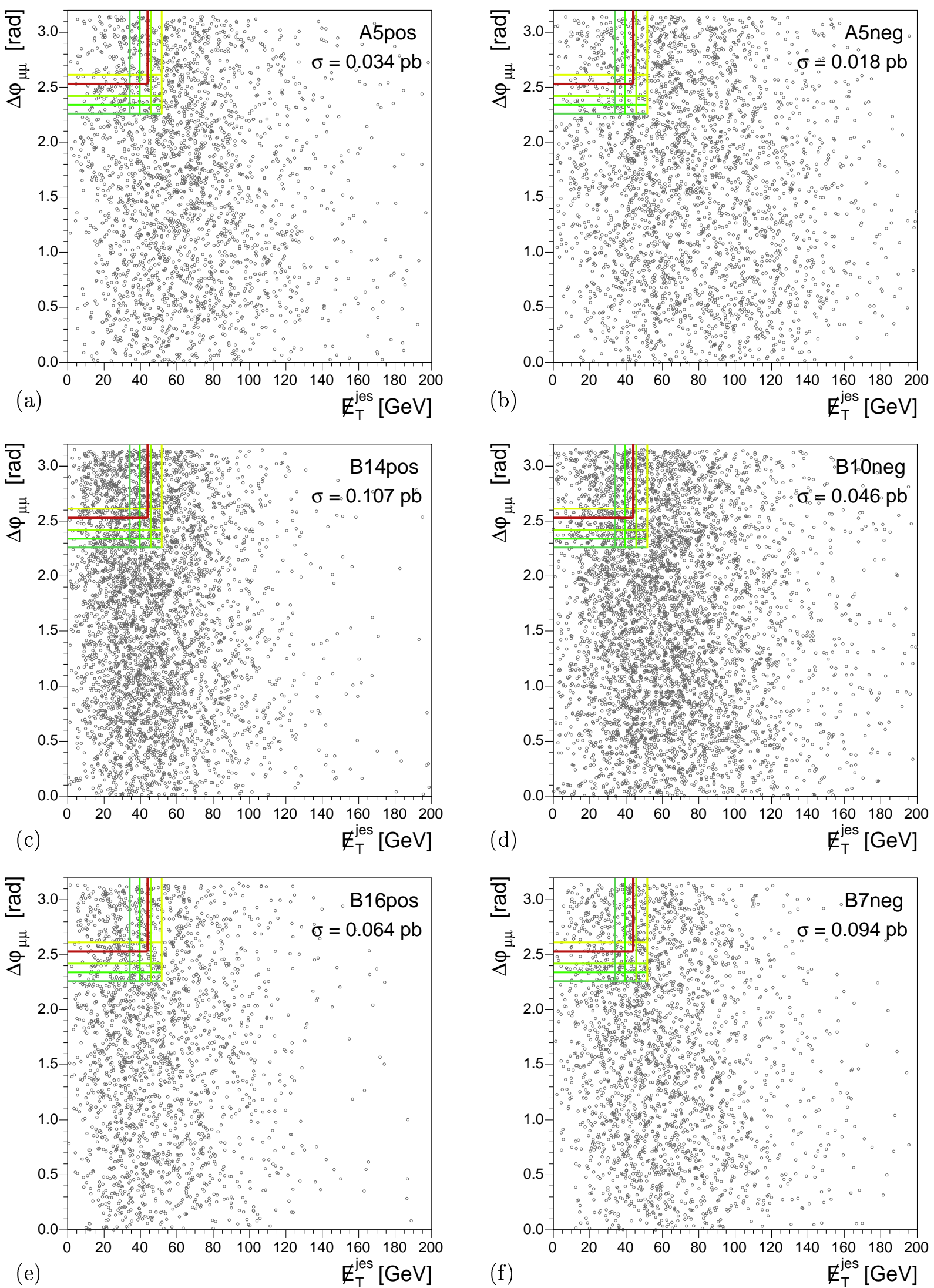

Figure 4.24: The two-dimensional criteria in the $\left(\Delta \varphi_{\mu \mu}, \Phi_{T}\right)$-plane, i.e. $\mu \mu$-cut 5 for some selected mSUGRA signal points: (a,c,e) for positive and $(b, d, f)$ for negative Higgs mixing parameter $\mu$. The area within the red coloured box is rejected. 

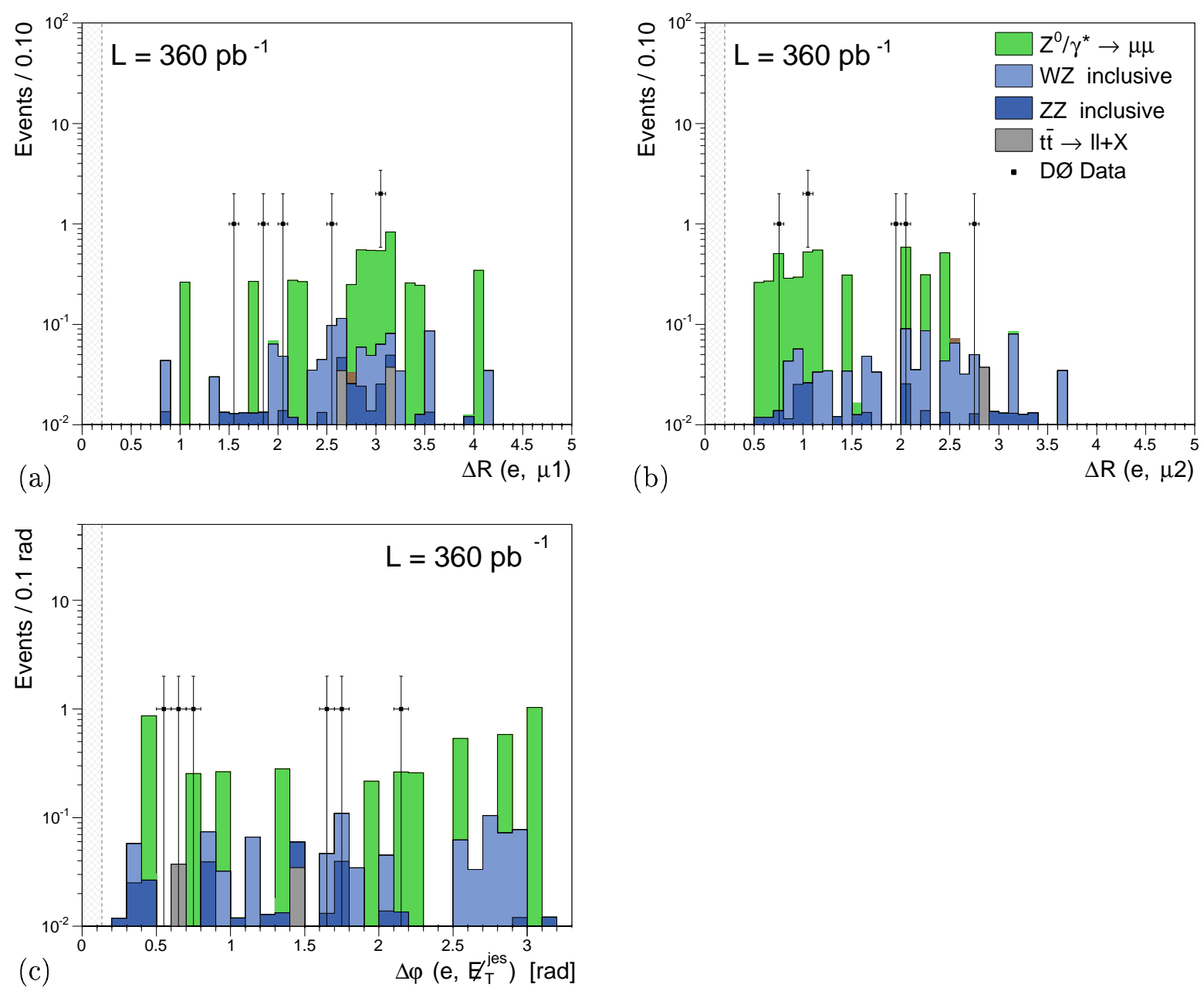

Figure 4.25: Spatial and angular distances of the electron (muon) in the emu selection: (a,b) spatial distance between the electron and the leading (resp. next-to-leading) muon, and (c) angular distance between the electron and the missing transverse energy vector.

both selection streams: the invariant mass of any $\mu \mu$ or $e \mu$ pair is required to be smaller than $200 \mathrm{GeV}$ for the dimu, as well as for the emu selection. A significant reduction of the high- $p_{\mathrm{T}}$ $Z / \gamma^{*}$ background $(\approx 70 \%)$ and events from $W Z$ and $Z Z$ production $(\approx 50 \%)$ is achieved, while the signal selection efficiency remains nearly unchanged.

After all dimu+emu selection cuts $506.4 \pm 12.5$ (stat) \pm 86.1 (sys) events are expected from SM backgrounds, while 564 events are observed in data. An overview of event numbers is presented in Tables 4.13 and 4.14, separately for all SM background processes, the data and two selected mSUGRA signal points. The signal points are chosen to show extreme examples: $\mathcal{B} 16$ pos, with a low value of $m_{0}=100 \mathrm{GeV}$ and a cross section of $\sigma_{N L O}=0.064$ and $\mathcal{A} 5$ pos for a high value of $m_{0}=1000 \mathrm{GeV}$ and with an even smaller cross section of $\sigma_{N L O}=0.034$.

Figure 4.26 illustrates the good agreement between data and the SM background expectation after the dimu+emu selection cuts: (a) the dimuon invariant mass, (b) the missing transverse energy corrected for the EM and jet energy scales $\not_{T}^{\text {jes }},(\mathrm{c}, \mathrm{d})$ the transverse momentum of the leading and next-to-leading muon, and (e,f) the angular distance of the leading (resp. next-toleading) muon to the reconstructed missing energy $\mathbb{H}_{T}^{\text {jes }}$. 


\begin{tabular}{|c|c|c|c|c|c|c|}
\hline dimu+emu & $Z / \gamma^{*} \rightarrow \mu \mu$ & $Z / \gamma^{*} \rightarrow \tau \tau$ & $\bar{W} W W, W Z, Z Z$ & $\overline{t \bar{t}} \rightarrow \ell \ell+X$ & $\bar{\Upsilon} \Upsilon(1 \mathrm{~s}), \Upsilon(2 \mathrm{~s})$ & QCD \\
\hline combined & $N \pm$ stat \pm sys & $N \pm$ stat \pm sys & $N \pm$ stat \pm sys & $N \pm$ stat \pm sys & $N \pm$ stat \pm sys & $N \pm$ stat \pm sys \\
\hline Cut 1 & $19182.2 \pm 80.5 \pm 2337.9$ & $147.9 \pm 5.2 \pm 18.3$ & $29.9 \pm 1.4 \pm 3.2$ & $8.9 \pm 0.76 \pm 1.71$ & $93.3 \pm 6.3 \pm 29.6$ & $126.0 \pm 4.9 \pm 25.2$ \\
\hline Cut 2 & $16406.3 \pm 75.7 \pm 1999.6$ & $112.2 \pm 4.6 \pm 13.9$ & $26.8 \pm 1.3 \pm 2.9$ & $8.0 \pm 0.74 \pm 1.53$ & $88.3 \pm 6.1 \pm 28.0$ & $111.5 \pm 4.6 \pm 22.3$ \\
\hline Cut 3 & $14959.0 \pm 71.4 \pm 1823.2$ & $97.2 \pm 4.2 \pm 12.0$ & $25.8 \pm 1.3 \pm 2.8$ & $7.6 \pm 0.73 \pm 1.45$ & $85.7 \pm 6.0 \pm 27.2$ & $99.8 \pm 4.4 \pm 20.0$ \\
\hline Cut 4 & $13697.3 \pm 61.9 \pm 1669.4$ & $64.4 \pm 3.4 \pm 8.0$ & $23.8 \pm 1.2 \pm 2.6$ & $7.5 \pm 0.73 \pm 1.44$ & $0.5 \pm 0.5 \pm 0.2$ & $65.2 \pm 3.5 \pm 13.0$ \\
\hline Cut 5 & $1434.7 \pm 21.8 \pm 174.9$ & $7.9 \pm 1.2 \pm 1.0$ & $14.8 \pm 0.9 \pm 1.6$ & $7.0 \pm 0.72 \pm 1.44$ & $0.5 \pm 0.5 \pm 0.2$ & $27.2 \pm 2.3 \pm 5.4$ \\
\hline Cut 6 & $470.6 \pm 12.3 \pm$ & $7.2 \pm 1.1 \pm 0.9$ & $6.8 \pm 0.6 \pm 0.7$ & $6.6 \pm 0.71 \pm 1.27$ & $<0.001$ & $15.2 \pm 1.7 \pm 3.0$ \\
\hline
\end{tabular}

Table 4.13: Overview of the dimu+emu selection separately for all SM background processes. All systematic effects discussed in section 4.6 are taken into account. The preselection sample is defined by Cut 1 and contains all events passing either $\mu \mu$-cut1 or e $\mu$-cut1.

\begin{tabular}{|c|c|c|c|c|c|c|}
\hline \multirow{2}{*}{$\begin{array}{l}\text { dimu+emu } \\
\text { combined }\end{array}$} & \multirow{2}{*}{$\begin{array}{c}\sum \text { SM backgrounds } \\
N \pm \text { stat } \pm \text { sys }\end{array}$} & \multirow{2}{*}{$\begin{array}{c}\text { Data } \\
N \\
\end{array}$} & \multicolumn{2}{|c|}{ "SUSY signal point: $\mathcal{B} 16 p o s$} & \multicolumn{2}{|c|}{ SUSY signal point: $\mathcal{A} 5 p o s$} \\
\hline & & & $\varepsilon \pm s t a t \pm s y s$ & $\left(N_{\text {sel }}\right)$ & $\varepsilon \pm s t a t \pm s y s$ & $\left(N_{\text {sel }}\right)$ \\
\hline Cut 1 & $19588.2 \pm 81.1 \pm 3331.9$ & 19283 & $49.0 \pm 0.6 \pm 4.6 \%$ & $(11.8)$ & $36.3 \pm 0.5 \pm 2.8 \%$ & $(4.6)$ \\
\hline Cut 2 & $16753.1 \pm 76.2 \pm 2849.7$ & 16284 & $45.3 \pm 0.6 \pm 4.3 \%$ & $(10.9)$ & $33.3 \pm 0.5 \pm 2.6 \%$ & $(4.3)$ \\
\hline Cut 3 & $15275.0 \pm 71.9 \pm 2598.2$ & 14918 & $44.4 \pm 0.6 \pm 4.2 \%$ & $(10.7)$ & $32.8 \pm 0.5 \pm 2.5 \%$ & $(4.2)$ \\
\hline Cut 4 & $13858.8 \pm 62.1 \pm 2357.3$ & 13749 & $44.0 \pm 0.6 \pm 4.1 \%$ & $(10.6)$ & $32.6 \pm 0.5 \pm 2.5 \%$ & $(4.2)$ \\
\hline Cut 5 & $1492.1 \pm 22.0 \pm 253.8$ & 1601 & $39.9 \pm 0.5 \pm 3.7 \%$ & $(9.6)$ & $30.9 \pm 0.5 \pm 2.4 \%$ & $(3.9)$ \\
\hline Cut 6 & $506.4 \pm 12.5 \pm 86.1$ & 564 & $35.0 \pm 0.5 \pm 3.3 \%$ & $(8.4)$ & $27.7 \pm 0.5 \pm 2.2 \%$ & $(3.5)$ \\
\hline
\end{tabular}

Table 4.14: Overview of the dimu+emu selection: the sum of the expected backgrounds in comparison with the observed number of events in data. Also shown are the selection efficiencies for two different signal points $\mathcal{B} 16$ pos and $\mathcal{A} 5$ pos, corresponding to rather small cross sections of $\sigma_{N} L O=0.064$ and $\sigma_{N L O}=0.034$, respectively. The values in parenthesis $N_{\text {sel }}$ indicate the number of events expected for the respective SUSY signal. All systematic effects (see section 4.6) are considered and for the sum of the background expectation the uncertainty due to the luminosity measurement is added in quadrature. The preselection sample is defined by Cut 1 and contains all events passing either $\mu \mu$-cut1 or e $\mu$-cut1. 

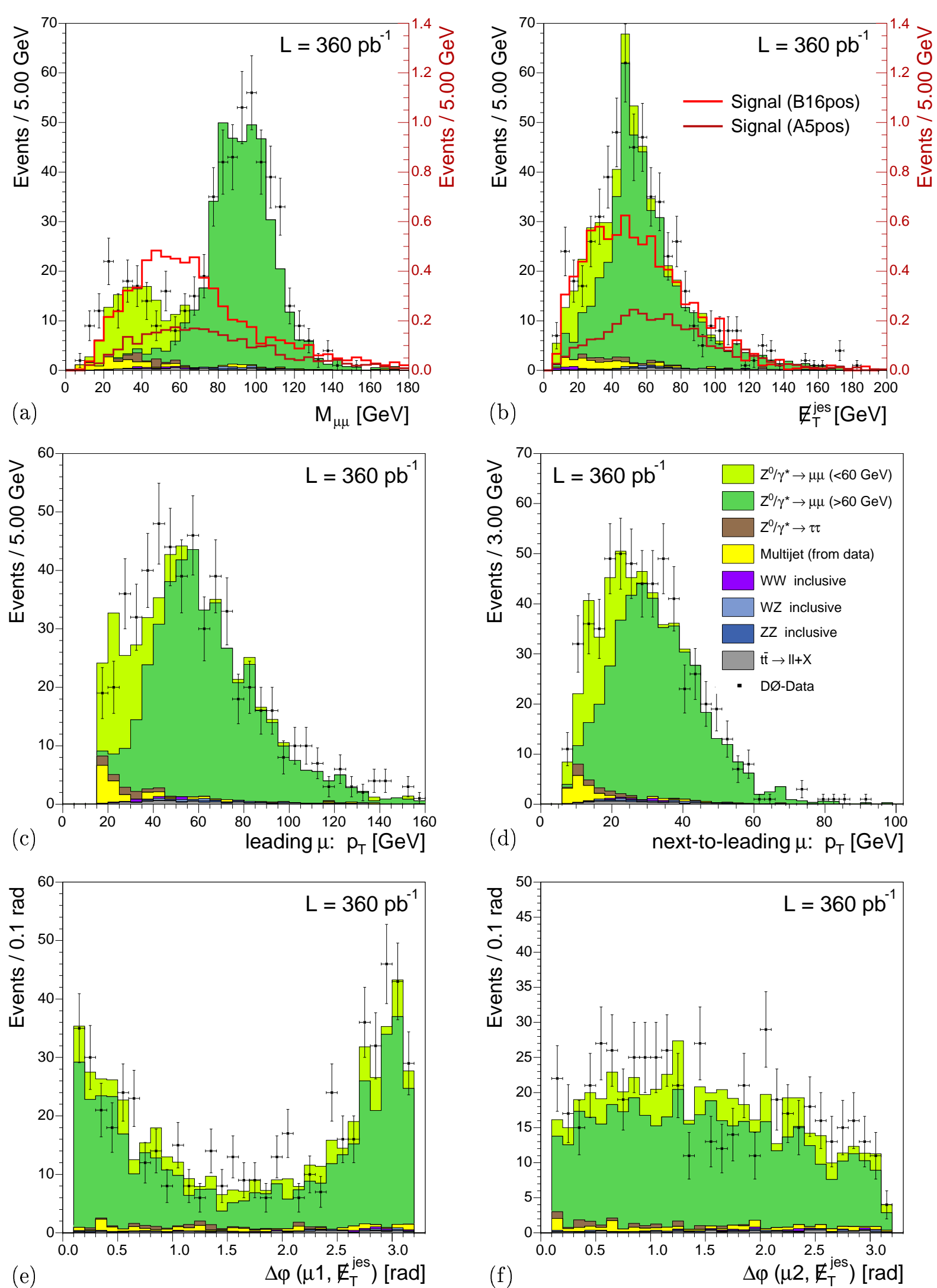

Figure 4.26: Control distributions after the application of all dimu+emu selection criteria: (a) the dimuon invariant mass and (b) the missing transverse energy $\mathbb{Z}_{T}^{\text {jes }}$ The two signal distributions are scaled by a factor of 50. $(c, d)$ the $p_{\mathrm{T}}$-distributions of the leading and next-to-leading muon, and $(e, f)$ the angular distance $\Delta \varphi$ between the leading (resp. next-to-leading) muon and $\not_{T}^{\text {jes }}$. 


\subsubsection{The Trilepton Selection}

Only very few SM background processes still contribute at this stage of the analysis, where three charged leptons are required to be present in the final state. All of them, like $t \bar{t}$ production, followed by a leptonic decay of both $W$ bosons, i.e. $t \bar{t} \rightarrow W b+W b \rightarrow \ell \nu b+\ell \nu b$, or the pair production of gauge bosons, especially $W Z \rightarrow \ell \nu+\ell \ell$ and $Z Z \rightarrow \ell \ell \ell \ell$ have very small cross sections. Other contributions may come from instrumental backgrounds, like electronic noise in the different subdetectors, or from mismeasured / misidentified physics objects. However, the misidentification rate for a muon with a matched central track, or an electron with a likelihood value of more than 0.2 , and a matched central track, is negligibly small $(\mu: 0.2 \%, e: 0.4 \%)$ and thus not taken into account here.

The separate searches for either an electron or an additional muon are reflected in the selection criteria, which are listed in Table 4.15. In case the leading leptons are an electron and a muon, the third charged lepton must be a muon - and it is required from the beginning as already mentioned. However, if both leading leptons are muons, the third may either be an electron or a muon, which is treated separately to ensure the quality of the additional charged lepton.

\begin{tabular}{ll}
\hline \hline muon criteria: $\mu \mu \mu, e \mu \mu$, or $\mu e \mu$ & electron criteria: $\mu \mu e$ \\
\hline \hline additional muon, with loose quality & additional electron (see p. 4.3.2) \\
$p_{T}(\mu)>4 \mathrm{GeV}$ & $p_{T}(e)>5 \mathrm{GeV}$ \\
angular distance: $\Delta \varphi\left(\mu, \not_{T}^{j e s}\right)>0.1$ & angular distance: $\Delta \varphi\left(e, \not_{T}^{j e s}\right)>0.2$ \\
spatial distance: $\Delta \mathcal{R}(\ell 1 / \ell 2, \mu 3)>0.1$ & spatial distance: $\Delta \mathcal{R}(\ell 1 / \ell 2, e)>0.2$ \\
spatial distance to any jet in the event: & $\Delta \mathcal{R}(\ell 3$, jet) $>0.5$ \\
$\quad$ matched to a central track according to section 4.3 .2 \\
loosely isolated: $\quad$ isolated as defined: iso $<0.2$ \\
$\sum p_{\mathrm{T}}^{\text {tr }}<9 \mathrm{GeV}$ and $E_{\mathrm{T}}^{\text {halo }}<15 \mathrm{GeV}$ \\
\hline \multicolumn{2}{c}{ scalar sum of the transverse momenta of all leptons: $\sum p_{\mathrm{T}}^{\text {leptons }}>50 \mathrm{GeV}$} \\
\hline \hline
\end{tabular}

Table 4.15: Overview of the the final trilepton selection criteria: for an additional muon on the left-hand side and an electron on the right-hand side. The $p_{\mathrm{T}}$-threshold of the third lepton, as well as the scalar sum of the transverse momenta of all charged leptons identified in an event are also varied during the previously discussed optimisation.

The $p_{T}$ threshold for the loose muon is reduced as much as possible to maximize the acceptance while still requiring that the muon hits at least the first layer of the muon chambers (A-layer). Muons with transverse momenta below $\approx 2 \mathrm{GeV}$ do not reach the muon system, but are absorbed in the calorimeter. For the $\mu \mu e$ final state it is indispensable to require not only a slightly higher $p_{\mathrm{T}}$-threshold to obtain reasonably pure electrons, but also a relatively large spatial distance between the electron candidate and the two high quality muons. This ensures that the same object is not selected twice, once as a muon and once as an electron candidate.

Since the quality requirements for the additional third charged lepton are less stringent, a comparison of data and SM model backgrounds is shown in Figure 4.27 at preselection level $(\mu \mu / e \mu-$ cut 1), but with the inclusion of a third charged lepton as defined in Table 4.15 above for either a muon or an electron. 

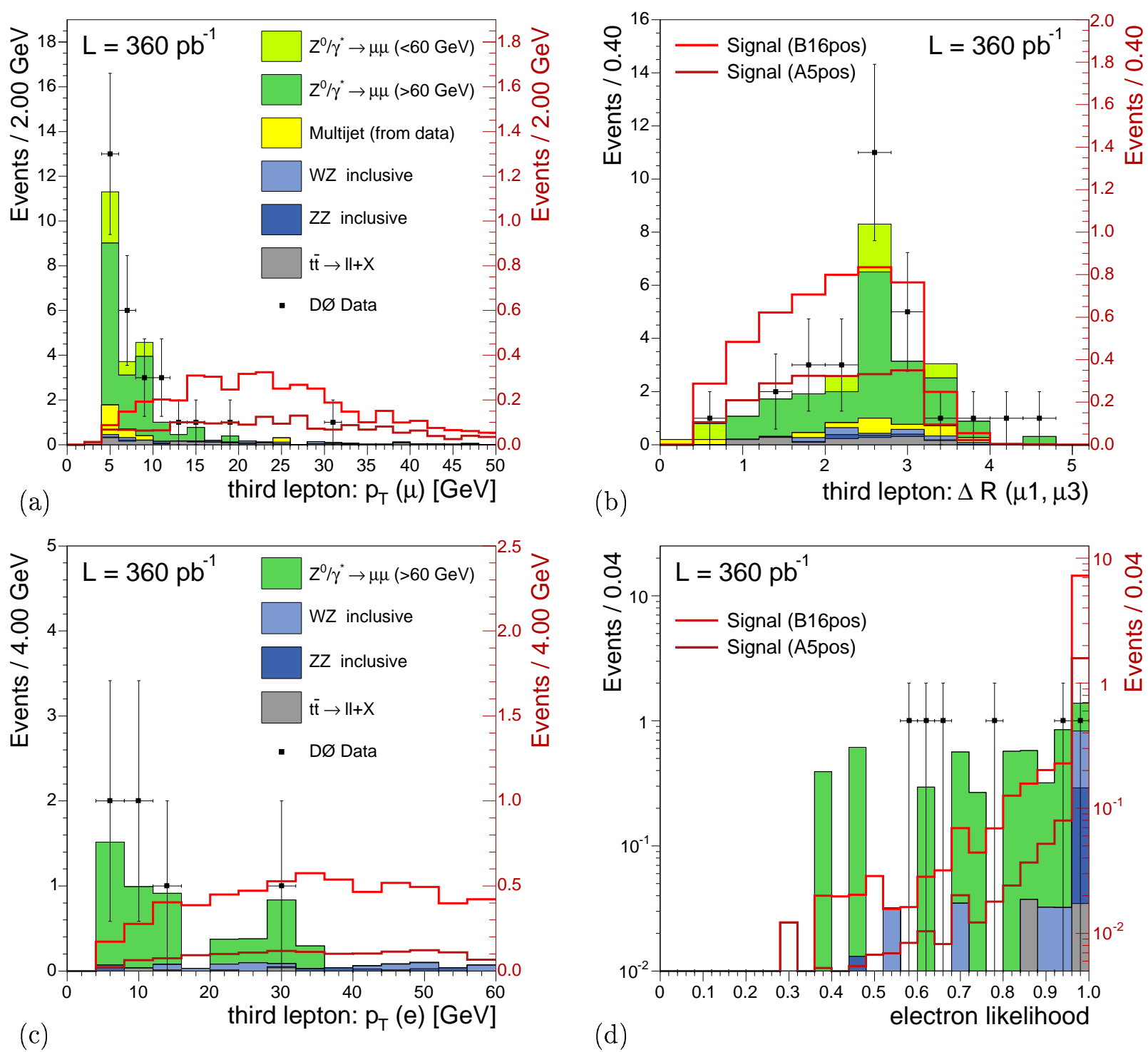

Figure 4.27: Third lepton properties at preselection level, but including an additional muon or electron: (a) the transverse momentum of the additional loose muon, (b) the spatial distance between the most energetic high quality muon and the third muon $\Delta \mathcal{R}(\mu 1, \mu 3)$ (c) the transverse momentum of the additional electron candidate $\left(p_{T}(e)>5 \mathrm{GeV}\right)$, and $(d)$ the electron likelihood as defined in section 4.3.2. The overlaid signal distributions are scaled by a factor of 10, 8, and 2 in (a), (b) and (c,d), respectively.

The last selection criterion of Table 4.15, the scalar sum of all lepton transverse momenta, is especially useful, since the probability for three charged leptons in the final state is much higher for signal events than for SM backgrounds. Figure 4.28 illustrates this last trilepton selection criterion $\sum p_{\mathrm{T}}^{\text {leptons }}>50 \mathrm{GeV}$ : (a,c,e) at preselection level and in (b,d,f) right before the application of this cut, when already three charged leptons are required. From the distributions for different mSUGRA points, it is clearly visible that the cut value of $50 \mathrm{GeV}$ has been optimised for signal points with large values of $m_{1 / 2}$, since the cross sections of points with considerably lower values of $m_{1 / 2}$ are already a factor of 4-10 larger (Tables A.1 and A.2 in the appendix). 
4.5 The Selection of Dimuon ES Electron-Muon Events
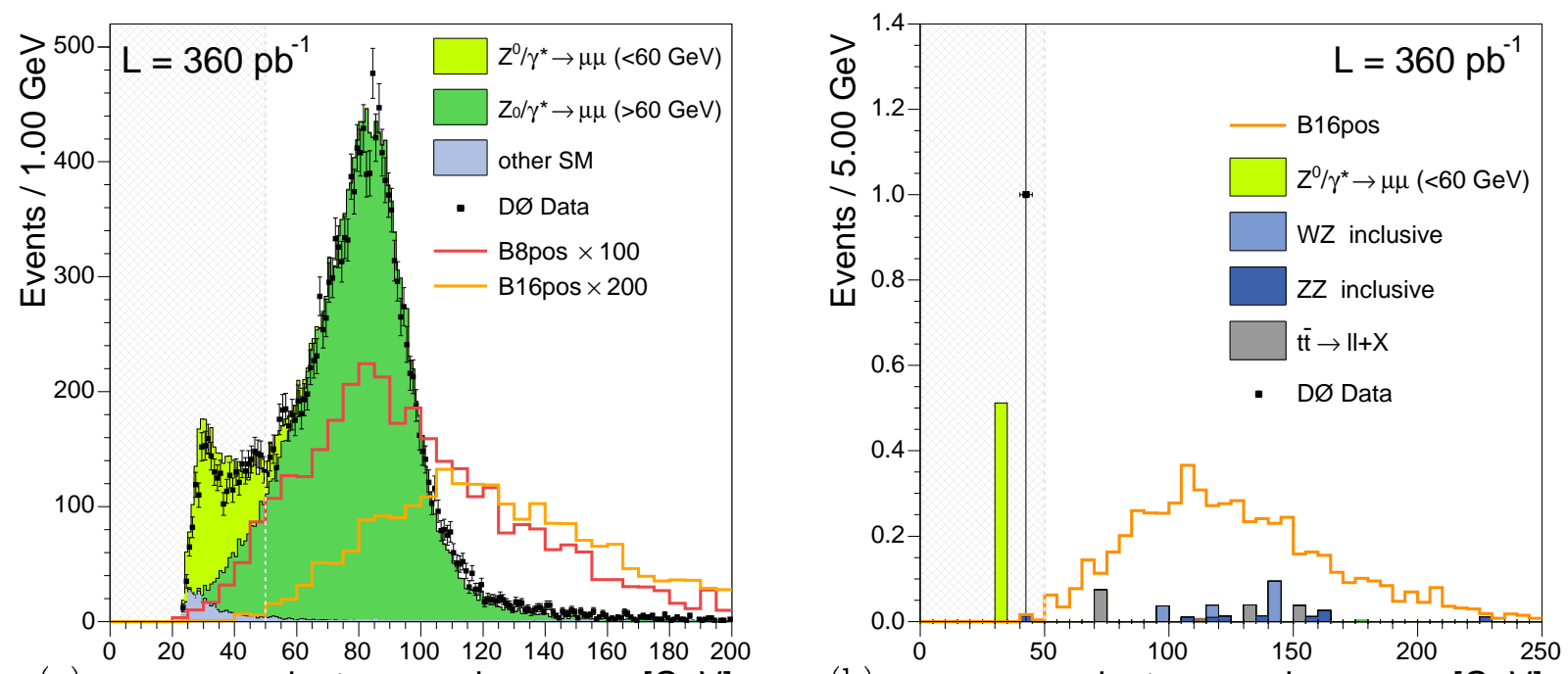

(a)

(b)

leptons: scalar $\mathrm{p}_{\mathrm{T}}$ sum $[\mathrm{GeV}]$
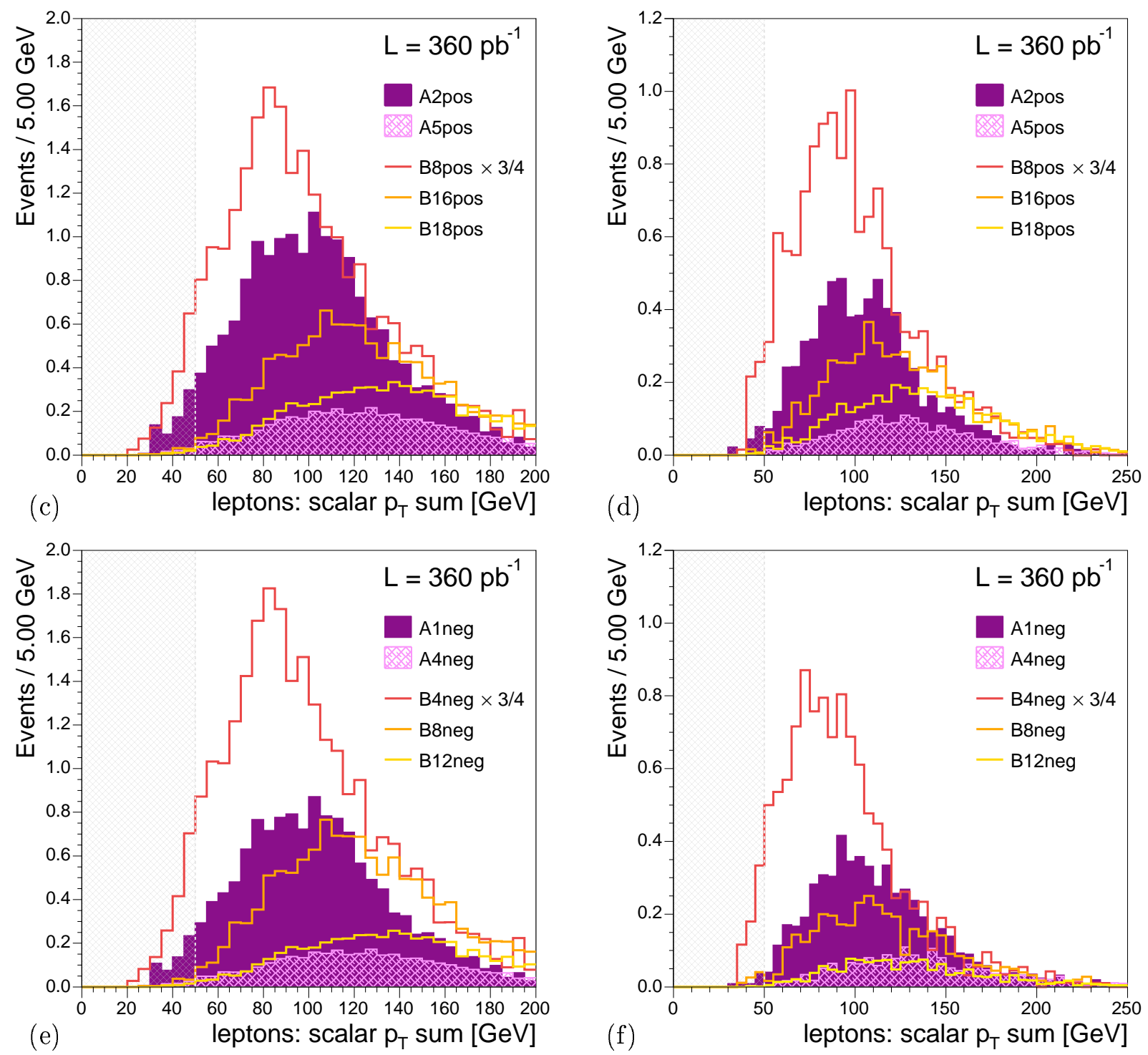

Figure 4.28: The last trilepton selection criterion $\sum p_{\mathrm{T}}^{\text {leptons }}>50 \mathrm{GeV}$ at preselection level $(a, c, e)$ and right before the cut is applied $(b, d, f)$. In $(a, b)$ a comparison of data and SM backgrounds is shown together with one (two) signal points for reference. Different mSUGRA signal distributions are presented in $(c, d)$ for positive and in $(e, f)$ for negative Higgs mixing parameter $\mu$. 


\subsubsection{The Optimisation of the dimu+emu Selection Cuts}

The optimisation is performed, using $1 / 3$ of the signal and background Monte Carlo samples, while the remaining $2 / 3$ are utilised as analysis samples. All two-dimensional selection criteria, i.e. $\mu \mu$-cuts 4,5 , and 6 , the two $\not_{T}^{t}$ requirements $\left(\not_{T}^{j e s}\right.$ and $\not_{T}^{\mu}$ of $\mu \mu$-cut 6 and e $\mu$-cut 4 ), the transverse momentum of the third charged lepton $\left(p_{\mathrm{T}}^{\mu}\right.$ or $\left.p_{\mathrm{T}}^{e}\right)$ and the scalar sum of the transverse momenta of all electrons and muons above the respective $p_{\mathrm{T}}$-threshold (see also section 4.5.3) are optimised w.r.t. the signal selection efficiency times the signal purity after all cuts:

$$
\varepsilon_{S} \cdot p_{S}=\varepsilon_{S} \cdot \frac{S}{S+B},
$$

where $S$ and $B$ denote the selected signal and background, respectively. This optimisation criterion is chosen for its robustness, since the selection requirements are not optimised separately for each signal point, but rather the cut combination is chosen which gives a high $\varepsilon_{S} \cdot p_{S}$ for the majority of the studied signal points (see the small crosses in Tables A.1 and A.2 in the appendix). Priority is given to those signal points with high values of $m_{0}$ and $m_{1 / 2}$, so that the efficiency for points with small values (especially with small $m_{1 / 2}$ ) could doubtless be higher if the criteria were adjusted. However, since the expected upper limits on the cross sections for these points are already much smaller than the respective cross sections themselves, variable criteria, such as sliding cuts in dependence of the mSUGRA parameters, have not been considered. Additionally, the maximum of $\varepsilon_{S} \cdot p_{S}$ minimises the expected error on the signal cross section, or for this reason, on the expected cross section limit.

Various cut combinations are tested in an iterative way, so that the computing time does not become too large. Each cut parameter is varied in a reasonable range. For the two-dimensional criteria this is probably best illustrated by the previous Figures $4.21,4.22,4.23$, and 4.24 , since the mere parameters are not very helpful in visualising the two-dimensional cuts.

\begin{tabular}{llr}
\hline \hline Selection criterion & Parameters and/or combinations & Perm. \\
\hline \hline$\mu \mu$-cuts 4 and $6\left(M_{\mu \mu}, \not_{T}\right)$-plane & 7 low- $p_{\mathrm{T}} Z / \gamma^{*}, \Upsilon \times 5$ anti $Z$ resonance & 35 \\
$\mu \mu$-cut $5\left(\Delta \varphi_{\mu \mu}, \not_{T}\right)$-plane & $4 \Delta \varphi \times 4 \not_{T}$ variations & 16 \\
$\not_{T}^{\text {jes }}>$ & $7,7.5,8,8.5,9,10,12,14 \mathrm{GeV}$ & 8 \\
$\not_{T}^{\mu}>$ & $8,8.5,9,9.5,10,11,12 \mathrm{GeV}$ & 7 \\
\hline third lepton $p_{\mathrm{T}}>$ & $p_{\mathrm{T}}^{\mu}>3,4,5,6 \mathrm{GeV}$ or & 4 \\
& $p_{\mathrm{T}}^{e}>5,6,7 \mathrm{GeV}$ & 3 \\
$\sum p_{\mathrm{T}}^{\text {leptons }}>$ & $35,40,45,50,55,60,70 \mathrm{GeV}$ & 7 \\
\hline \hline
\end{tabular}

Table 4.16: Overview of the different cut combinations for the two-dimensional cuts, and the actual parameter values for the one-dimensional cuts that have been tested during the optimisation.

In the beginning, values at the extremes of the cut parameter ranges are tested in order to possibly narrow the parameter space for a complete scan. For example, the range of the missing transverse energy criteria $\left(E_{\mathrm{T}}^{\text {jes }}\right.$ and $E_{\mathrm{T}}^{\mu}$, see Table 4.16 ) has been constrained to values below $11 \mathrm{GeV}$ before the fine scan was started. Similarly, the two extreme values of the last selection criterion, the cut on the scalar sum of the lepton transverse momentum $\left(\sum p_{\mathrm{T}}^{\text {leptons }}>35\right.$ and $>70)$ have also been ruled out beforehand. With a cut at $\sum p_{\mathrm{T}}^{\text {leptons }}>35$, there is too much low- $p_{\mathrm{T}} Z / \gamma^{*}$ background left and for $\sum p_{\mathrm{T}}^{\text {leptons }}>70$ the signal selection efficiency for points with intermediate values of $m_{1 / 2}$ becomes too low, so that the expected cross section limits are of the same order as the signal cross sections. 
Figure 4.29 illustrates the optimisation for different signal points. The open triangles indicate the highest value of $\varepsilon_{S} \cdot p_{S}$ per signal, whereas the actually chosen cut combination (indicated by the red stars) differs slightly from the best possible combination. This is due to the fact, that the efficiency times purity is optimised especially for signal points with large values of $m_{0}$ and intermediate to large values of $m_{1 / 2}$ (small cross sections), i.e. in the region of the expected cross section limits.
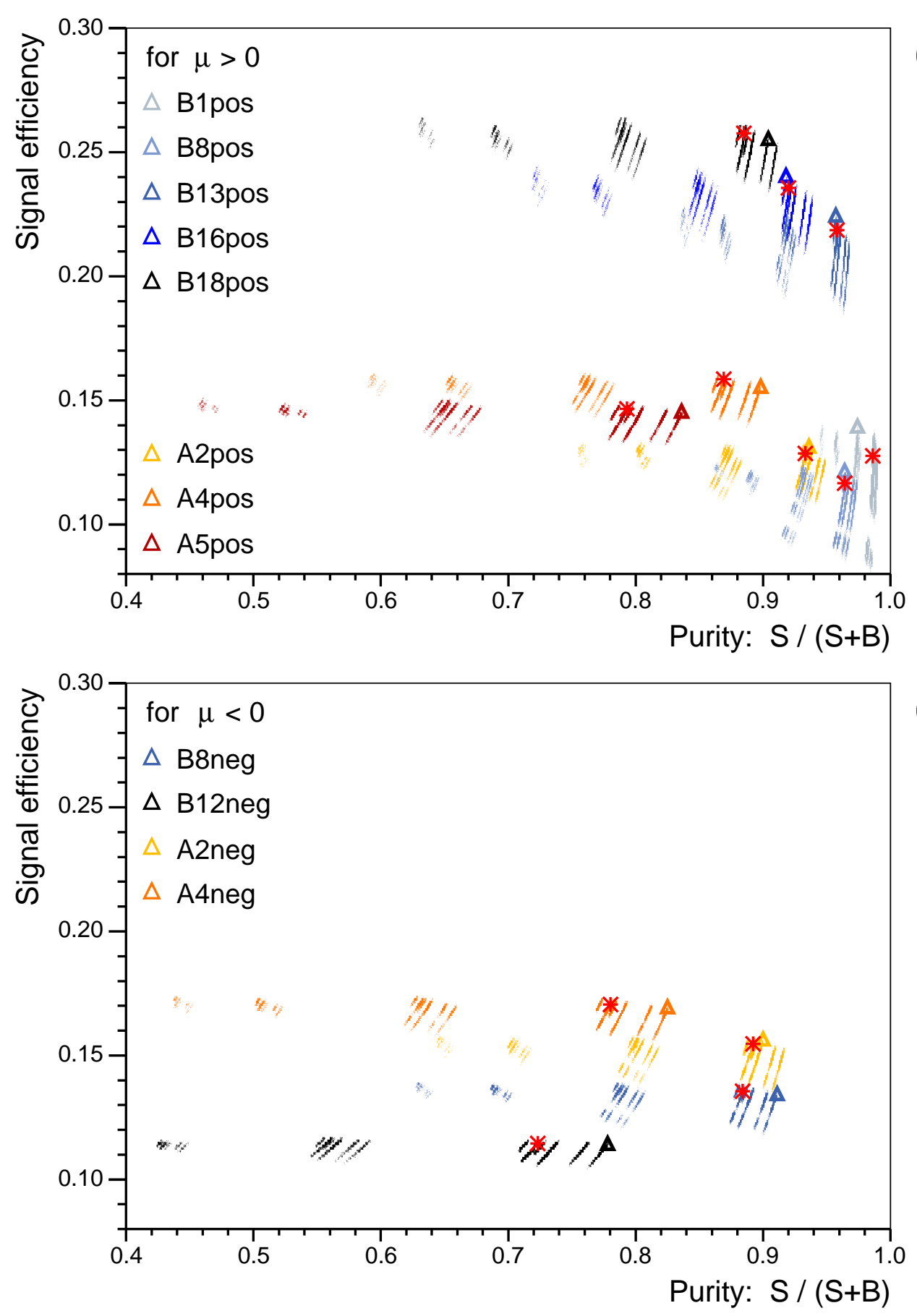

(b)

Figure 4.29: Illustration of the optimisation for different signal points. Each dot stands for a cut combination, the open triangles indicate the highest value of $\varepsilon_{S} \cdot p_{S}$ per signal, and the red stars denote the actually chosen cut combination. As can be seen, the stars and open triangles deviate slightly, see text for further details. 


\subsection{Systematic Uncertainties}

The numbers of events expected from SM background processes and from signal depend on several quantities, of which each one introduces a systematic uncertainty. This section serves to discuss the systematic uncertainties studied for the present analysis and to describe which effects are considered and how they are taken into account for the calculation of exclusion limits [169]. The following effects have been studied / are considered:

- the relative uncertainty due to the dimuon trigger efficiency: $\rightarrow \approx 11 \%$ for $Z / \gamma^{*}$ background with low invariant masses and about $2 \%$ for signal MC;

- the uncertainties due to imperfections in the detector simulation, as summarised in Table 4.7 for the muon efficiencies; for electrons the $1 \sigma$ uncertainty on the fit parameters is utilised (see Table 4.9 and also [73]):

$\rightarrow \approx 8-11 \%$, depending on the event topology, i.e. $\mu \mu \mu$ or $\mu \mu e$;

- the uncertainty due to the $p_{\mathrm{T}}, E_{\mathrm{T}}$ resolution of muons and electrons, respectively:

$\rightarrow$ amounting to $\approx 1.5 \%$ for muons and $1 \%$ for electrons;

- the uncertainty due to the modeling of the transverse momentum of the $Z / \gamma^{*}$-system: $\rightarrow$ approximately $12 \%$ for low- $p_{\mathrm{T}} Z / \gamma^{*}$ Monte Carlo samples;

- all uncertainties (e.g. scale, PDF) of the MC cross sections listed in Table 4.2;

- the uncertainty due to the measurement of the luminosity: $6.5 \%$ [114].

These effects will be discussed in more detail in the following sections.

\subsubsection{Uncertainties on the Dimuon Trigger Efficiencies}

The relative uncertainty on the dimuon trigger efficiency ranges from about $11 \%$ for $Z / \gamma^{*}$ background with low dimuon invariant masses $\left(15 \mathrm{GeV}<M_{\ell \ell}<60 \mathrm{GeV}\right)$ to about $2 \%$ for the different SUSY signals. It is estimated from variations of the dimuon trigger parametrisations described in Equations 4.6 and 4.7, as well as in Table 4.6. All parameters are varied by $\pm 1 \sigma$ and the resulting deviations are added in quadrature to account for the uncertainty of the dimuon trigger efficiency. This procedure slightly overestimates the real uncertainty on the trigger efficiency, which was found to be about 2-3\% in more recent studies [170]. In principle, larger uncertainties only lead to more conservative limits, and are thus not problematic as long as they are in a reasonable range.

\subsubsection{Uncertainties on Object ID Efficiencies}

The uncertainties on all detector related efficiencies discussed in section 4.3 .2 are added in quadrature following Equation 4.22 for the dimu, Equation 4.23 for the emu selection, and Equations 4.24 and 4.25 for the trilepton analyses, i.e. for the final states $\mu \mu \mu$ and $\mu \mu e$, respectively. Since all correction factors are calculated per muon, each muon uncertainty is summed linearly (twice, resp. thrice) and then added in quadrature to the remaining electron or muon object ID uncertainties, thus assuming full correlation between different muon identification and reconstruction corrections. 


$$
\begin{aligned}
& \frac{d \varepsilon_{\mu \mu}}{\varepsilon_{\mu \mu}}=\sqrt{\left(\sum_{i=1}^{2} \frac{d \varepsilon_{r e c o}^{\mu}}{\varepsilon_{r e c o}^{\mu}}\right)^{2}+\left(\sum_{i=1}^{2} \frac{d \varepsilon_{m e d}^{\mu}}{\varepsilon_{m e d}^{\mu}}\right)^{2}+\left(\sum_{i=1}^{2} \frac{d \varepsilon_{i s o}^{\mu}}{\varepsilon_{i s o}^{\mu}}\right)^{2}+\left(\sum_{i=1}^{2} \frac{d \varepsilon_{t r k m}^{\mu}}{\varepsilon_{t r k m}^{\mu}}\right)^{2}} \\
& \frac{d \varepsilon_{e \mu(\mu)}}{\varepsilon_{e \mu(\mu)}}=\sqrt{\left(\sum_{i=1}^{2} \frac{d \varepsilon_{r e c o}^{\mu}}{\varepsilon_{\text {reco }}^{\mu}}\right)^{2}+\left(\frac{d \varepsilon_{m e d}^{\mu}}{\varepsilon_{\text {med }}^{\mu}}\right)^{2}+\left(\sum_{i=1}^{2} \frac{d \varepsilon_{i s o}^{\mu}}{\varepsilon_{i s o}^{\mu}}\right)^{2}+\left(\sum_{i=1}^{2} \frac{d \varepsilon_{t r k m}^{\mu}}{\varepsilon_{t r k m}^{\mu}}\right)^{2}} \\
& \overline{+\left(\frac{d \varepsilon_{I D}^{e}}{\varepsilon_{I D}^{e}}\right)^{2}+\left(\frac{d \varepsilon_{t r k m}^{e}}{\varepsilon_{t r k m}^{e}}\right)^{2}} \\
& \frac{d \varepsilon_{\mu \mu \mu}}{\varepsilon_{\mu \mu \mu}}=\sqrt{\left(\sum_{i=1}^{3} \frac{d \varepsilon_{r e c o}^{\mu}}{\varepsilon_{r e c o}^{\mu}}\right)^{2}+\left(\sum_{i=1}^{2} \frac{d \varepsilon_{m e d}^{\mu}}{\varepsilon_{m e d}^{\mu}}\right)^{2}+\left(\sum_{i=1}^{3} \frac{d \varepsilon_{i s o}^{\mu}}{\varepsilon_{i s o}^{\mu}}\right)^{2}+\left(\sum_{i=1}^{3} \frac{d \varepsilon_{t k m}^{\mu}}{\varepsilon_{t r k m}^{\mu}}\right)^{2}} \\
& \frac{d \varepsilon_{\mu \mu e}}{\varepsilon_{\mu \mu e}}=\sqrt{\left(\sum_{i=1}^{2} \frac{d \varepsilon_{r e c o}^{\mu}}{\varepsilon_{\text {reco }}^{\mu}}\right)^{2}+\left(\frac{d \varepsilon_{\text {med }}^{\mu}}{\varepsilon_{\text {med }}^{\mu}}\right)^{2}+\left(\sum_{i=1}^{2} \frac{d \varepsilon_{i s o}^{\mu}}{\varepsilon_{i s o}^{\mu}}\right)^{2}+\left(\sum_{i=1}^{2} \frac{d \varepsilon_{\text {trkm }}^{\mu}}{\varepsilon_{t r k m}^{\mu}}\right)^{2}} \\
& \overline{+\left(\frac{d \varepsilon_{I D}^{e}}{\varepsilon_{I D}^{e}}\right)^{2}+\left(\frac{d \varepsilon_{t k m}^{e}}{\varepsilon_{t r k m}^{e}}\right)^{2}}
\end{aligned}
$$

The uncertainties on the lepton identification/reconstruction efficiencies account for $2.6 \%(e)$ and $3.2-3.8 \%(\mu)$ per lepton candidate. This also explains the rather wide range of uncertainty of approximately $8-12 \%$ for the different event topologies with two muons and an electron in the final state ( $\mu \mu e, \mu e \mu$, or $e \mu \mu$ events) and only muons ( $\mu \mu \mu$ events).

\subsubsection{Uncertainties due to the $p_{\mathrm{T}} / \boldsymbol{E}_{\mathrm{T}}$ Resolution for Muons/Electrons}

The relative systematic uncertainties due to the resolution of the muon transverse momentum measurement and the electron $E_{\mathrm{T}}$ are estimated by varying the resolutions in the MC simulation and are found to be less than $1.5 \%$ for the muon $p_{\mathrm{T}}$ and about $1 \%$ for the electron $E_{\mathrm{T}}$.

For the complementary analyses of the eel and ee $\tau$ final states, it was found to be not sufficient to propagate the smearing of the electron (tau) energy resolution to the missing transverse energy, but $\not_{T}$ had to be »oversmeared «, see $[1,73]$ and $[2,74]$, leading to an additional systematic uncertainty of $2 \%$ for the eel and eer analyses.

\subsubsection{Uncertainties due to the Modeling of the $Z$ boson $p_{\mathrm{T}}$}

Since it is known that PythiA does not model the $p_{\mathrm{T}}$ distribution of the $Z$ boson and the photon correctly, this systematic effect was studied extensively in DØ Note 4660 [171], and a reweighting method developed. While the application of this reweighting method shows an improvement in the high- $p_{\mathrm{T}}$ region of the transverse momentum distribution of $Z / \gamma^{*}$ background, it did not satisfactorily describe the low- $p_{\mathrm{T}}$ region (over-corrected).

Figure 4.30 illustrates two different distributions at preselection level: (a,c,e) without and (b,d,f) with the correction applied; (a,b) show the $Z$ boson transverse momentum, i.e. the transverse momentum of the dimuon system on linear and $(c, d)$ on logarithmic scale, whereas $(e, f)$ show the azimuthal distance between the two leading muons. As can be seen the $\gg$ correction « underestimates the contribution of $Z / \gamma^{*}$ events with small transverse momenta (Fig. $4.30(\mathrm{a}, \mathrm{b})$ ), which seemingly corresponds to the region with intermediate and large azimuthal distances between the two muons. On the other hand, data at large transverse momenta and, correspondingly, small 
dimuon azimuthal distances are described slightly better by the reweighed MC distributions, compared to the unweighted PYTHIA distributions.
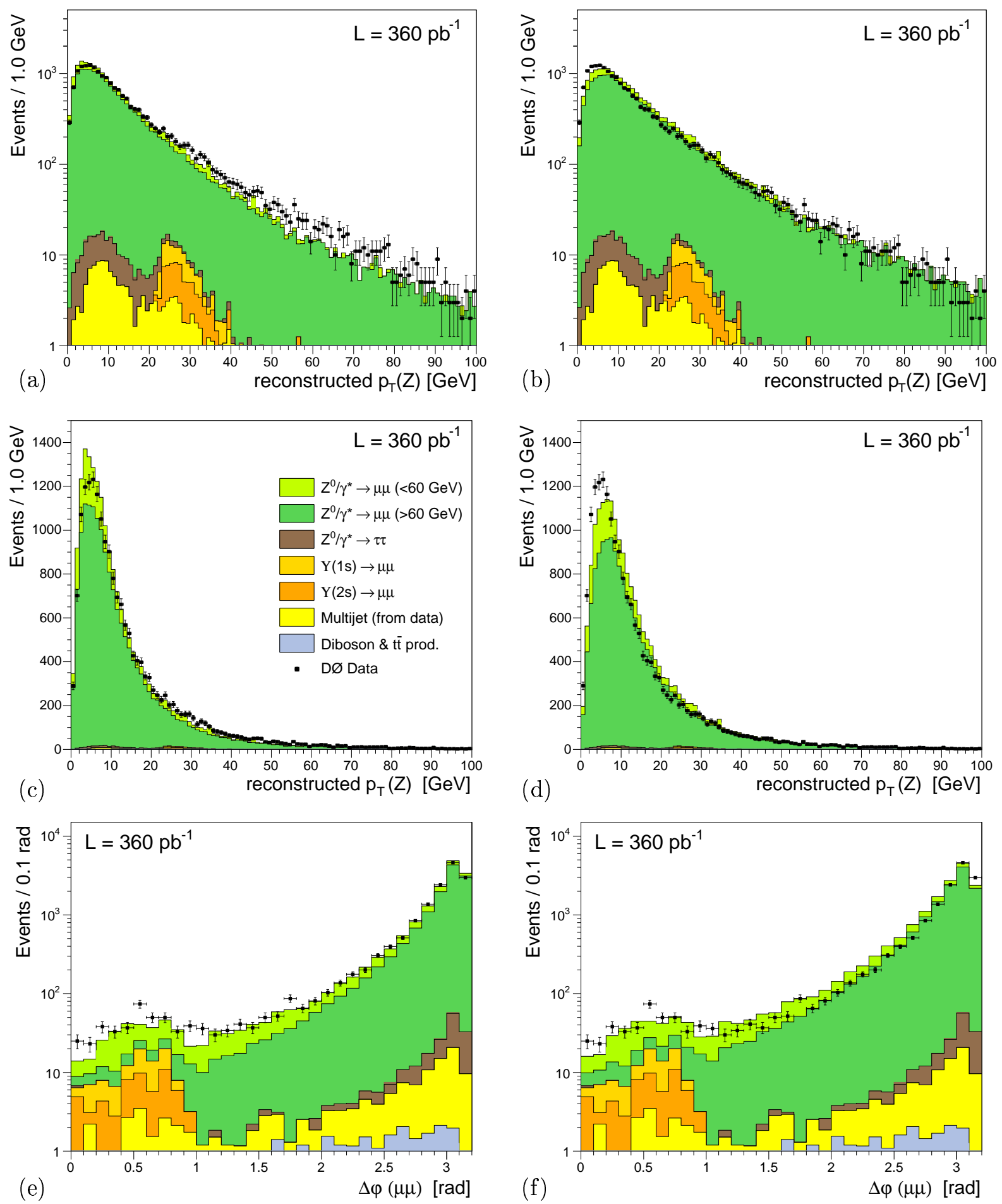

Figure 4.30: The distribution (a,c,e) on the left-hand side are for a $Z$ boson transverse momentum as generated with PYTHIA and reconstructed in DØ (i.e. uncorrected spectrum), while the right-hand side distributions $(b, d, f)$ are those for which the $p_{\mathrm{T}}$ of the $Z$ boson has been reweighed for all $Z / \gamma^{*}$ events with $M_{\mu \mu}>15 \mathrm{GeV}$ according to [171]. The $p_{\mathrm{T}}$-distribution of the $Z$ boson on logarithmic $(a, b)$ and linear $(c, d)$ scale and $(e, f)$ the angular distance between the two leading muons, which are most likely those from the $Z$ boson decay; all quantities shown at preselection level. 
The same behaviour is observed for the $\not_{T}$ distribution, which led to the decision to only include an overall systematic uncertainty instead of applying the reweighting method to »correct « for the imperfect Pythia description of the $Z$ boson transverse momentum. From a comparison of the expected $Z / \gamma^{*}$ background with and without applying the reweighting, an uncertainty of $12 \%$ is found. This uncertainty is also taken into account for the limit calculation where it it is almost negligible due to the small relative fraction of the $Z / \gamma^{*}$ background at the final stage of the selection (see Table 4.17).

\subsubsection{Systematic Uncertainties Specific to the Signal}

These systematic uncertainties have to be split in two: on the one hand, the uncertainties on the total signal cross section and on the other hand the uncertainty on the signal acceptance, which is entirely dominated by the influence of the PDF uncertainties.

The uncertainties on the signal cross sections are mostly due to theoretical uncertainties, like the variations of the renormalization and factorization scales (5\%), the LO cross section (2\%), and the determination of the $K$-factor (3\%) [172].

The choice of PDF used for the generation of the signal Monte Carlo samples adds another $\approx 9 \%$. This latter uncertainty is deduced from studies of the $Z / \gamma^{*}$ cross section at similar masses, since gaugino pair production mostly proceeds via $s$-channel exchange of virtual $\gamma, W$, or $Z$ bosons. The associated uncertainty on the $Z / \gamma^{*}$ cross section due to the PDFs is estimated to be $6 \%$, using the CTEQ6.1M uncertainty function set [87] as recommended by the CTEQ Collaboration and in [173]. This new set of PDFs includes 20 pairs of eigenvectors, where each of these pairs tests one free parameter by varying it by $1 \sigma$ of its uncertainty: $1 \sigma$-up corresponds to $S^{+}$and $1 \sigma$-down to $S^{-}$. The systematic effect on an observable $X$ is then described by:

$$
\Delta X_{ \pm}=\sqrt{\sum_{i=1}^{N<20}\left[X\left(S_{i}^{ \pm}\right)-X\left(S_{0}\right)\right]^{2}},
$$

where the sum runs over the pairs of PDF error functions and $X\left(S_{i}^{ \pm}\right)$are the values of $X$ obtained using a certain set $S_{i}^{ \pm}$of these error functions. Another $3 \%$ are added linearly to account for the lower DY cross section if calculated with CTEQ6 PDFs, compared to its estimation with CTEQ5 PDFs, which are used for the signal MC generation.

An additional, conservative systematic uncertainty of $+10 \%$ is added to account for the lower LO cross section from the SUSYGEN event generator compared to those obtained with either Pythia, or Gauginos.

All of these uncertainties are assumed to be independent, and are added in quadrature, thus a total systematic uncertainty of $-11 \%$ and $+15 \%$ is determined for the signal cross sections. (In Chapter 5, these uncertainties are represented by the grey-shaded bands of the signal cross section curves in the corresponding figures.)

The influence of PDF uncertainties on the signal acceptance has not been studied. However, the effect is expected to be small and is estimated from measurements of the processes $Z \rightarrow \mu \mu$, $Z / \gamma^{*} \rightarrow \mu \mu$, and $W \rightarrow \mu \nu$ [174], where the influence of PDF uncertainties on the acceptance was estimated by using the CTEQ6.1M set of PDF error functions. For the on-shell production of $W$ and $Z$ bosons a systematic uncertainty of $1.4 \%(W \rightarrow \mu \nu)$ and $1.7 \%(Z \rightarrow \mu \mu)$ was found.

Since the exchanged gauge bosons in gaugino pair production are usually produced off-shell, mostly shifted to higher values of the Bjørken $x$, the PDF induced uncertainty on the signal 
acceptance for gaugino pair production is conservatively estimated to be about $4 \%$. This uncertainty is also taken into account in the calculation of the expected and observed cross section limits, but it is not displayed as part of the uncertainty on the signal cross section.

\subsubsection{Other Uncertainties}

Further systematic uncertainties on the experimental cross section limits concern the theoretical uncertainties on SM background Monte Carlo cross sections, ranging from $3 \%$ to $17 \%$, depending on the process, and including PDF uncertainties. For the determination of the uncertainties on the NNLO $Z / \gamma^{*}$ cross sections, the CTEQ6.1M error functions are used, as described in DØ Note 4476 [148].

The uncertainty on the multijet background is estimated to be about $20 \%$, however, since the multijet contribution to the final background expectation is negligible, this uncertainty is of no further importance.

The absolute uncertainty of the luminosity measurement is estimated to be $\Delta \mathcal{L}=6.5 \%$ [114].

All systematic uncertainties are added in quadrature. When calculating the upper cross section limits, the total systematic uncertainties on the expected numbers of background and signal events are assumed to be fully correlated with the exception of the theoretical uncertainties of the LO cross section calculations.

\section{7 $\quad$ Facts and Figures}

After the trilepton selection, no event is observed in data, while $0.41 \pm 0.11 \pm 0.07$ events are expected from SM processes. The corresponding numbers of selected events are given in Table 4.17 separately for those SM backgrounds that still contribute to the trilepton final state, while Table 4.18 provides a comparison of data, the sum of all expected backgrounds and the selection efficiencies for two mSUGRA signal points (B16pos and $\mathcal{A} 5$ pos). Again, the values in parenthesis show how many events are expected for the respective SUSY signals.

As can be seen from the separate listing of $\mu \mu \mu$ and $\mu \mu e$ events, the largest SM background contributions are either due to the irreducible »real « trilepton background from $W Z, Z Z$ diboson production for $\mu \mu e$ final states or from $t \bar{t} \rightarrow \ell \ell+X$ production, with two real leptons from the $W$ decays and an additional muon from a b-jet for the $\mu \mu \mu$ signature. In case of the $t \bar{t}$ production, the contribution to $\mu \mu \mu$ final states is larger, since it is more likely to identify a muon from a semileptonic b-decay (b-jet muon) as an isolated muon than it is to identify a b-jet electron as isolated electron.

In addition, the numbers of events selected from all three Monte Carlo samples, i.e. $W Z, Z Z$, and $t \bar{t} \rightarrow \ell \ell+X$ are very small, of the order of less than 10 unweighted events, so that the different fractions of events expected from each sample may also be partially explained by statistical fluctuations.

The predicted number of events from the multijet sample is negligible after requiring three charged leptons $(\mu \mu \mu, \mu \mu e, \mu e \mu, e \mu \mu)$. The amount of multijet background at all stages of the selection is less than about $3 \%$ of all other known backgrounds. This is further confirmed by testing the influence of the two-dimensional criteria of which one ( $\mu \mu$-cut 4$)$ is especially designed to reduce the contribution of low- $p_{\mathrm{T}}$ multijet events. Early on in the selection, i.e. right after $\mu \mu / e \mu$-cuts 3 , a third charged lepton is required additionally. In this case only one event of this 
background is selected and would have to be weighted with the scale factor of 0.19 . This number has to be compared to about 20.2 events from all other SM background processes. Since the contribution of the multijet background accounts for only about $1 \%$ of the other backgrounds, it is considered negligible for this trilepton analysis.

\begin{tabular}{lccc}
\hline \hline & $Z / \gamma^{*} \rightarrow \mu \mu$ & $W Z, Z Z$ & $t \bar{t} \rightarrow \ell \ell+X$ \\
Type & $N \pm$ stat \pm sys & $N \pm$ stat \pm sys & $N \pm$ stat \pm sys \\
\hline \hline$\mu \mu \mu$ & $<0.005$ & $0.064 \pm 0.042 \pm 0.008$ & $0.116 \pm 0.067 \pm 0.023$ \\
$\mu \mu e$ & $0.011 \pm 0.010 \pm 0.004$ & $0.189 \pm 0.067 \pm 0.024$ & $0.033 \pm 0.033 \pm 0.007$ \\
Total & $0.011 \pm 0.010 \pm 0.004$ & $0.251 \pm 0.078 \pm 0.031$ & $0.149 \pm 0.075 \pm 0.030$ \\
\hline \hline
\end{tabular}

Table 4.17: Overview of the expected $S M$ background in the trilepton selection: for $\mu \mu \mu$ events the dominant $S M$ background is from $t \bar{t} \rightarrow \ell \ell+X$ production and for $\mu \mu e$ events diboson production constitutes the largest fraction of the background. All systematic effects that have been studied are taken into account; see section 4.6 for further details.

\begin{tabular}{|c|c|c|c|c|}
\hline & $\sum \mathrm{SM}$ backgrounds & Data & Signal point: $\mathcal{B} 16 p o s$ & Signal point: $\mathcal{A} 5$ pos \\
\hline Type & $N \pm$ stat \pm sys & $N$ & $\varepsilon \pm$ stat \pm sys $\quad\left(N_{\text {sel }}\right)$ & $\varepsilon \pm$ stat \pm sys $\quad\left(N_{s e l}\right)$ \\
\hline$\mu \mu \mu$ & $0.180 \pm 0.079 \pm 0.033$ & $\mathbf{0}$ & $10.8 \pm 0.3 \pm 1.27 \%$ & $8.2 \pm 0.3 \pm 0.96 \%$ \\
\hline$\mu \mu e$ & $0.233 \pm 0.075 \pm 0.042$ & $\mathbf{0}$ & $1.20 \%(3.1)$ & $0.60 \% \quad(0.8)$ \\
\hline Total & $0.411 \pm 0.108 \pm 0.074$ & $\mathbf{0}$ & $23.7 \pm 0.5 \pm 2.65 \%$ & $14.7 \pm 0.4 \pm 1.65 \%$ \\
\hline
\end{tabular}

Table 4.18: Comparison of the observed data with the sum of all expected backgrounds from SM processes, including the statistical and systematic uncertainties. The selection efficiencies and expected numbers of events for two different $m S U G R A$ signal points are also listed, corresponding to rather small cross sections of $\sigma_{N L O}=0.064 \mathrm{pb}$ (for $\mathcal{B} 16$ pos) and $\sigma_{N L O}=0.034 \mathrm{pb}$ (for $\left.\mathcal{A} 5 p o s\right)$. The first point, $\mathcal{B} 16$ pos, can be excluded, while $\mathcal{A} 5$ pos lies just above the limit curve, see Figure 5.3 in chapter 5.

The one »candidate« event, displayed in Figure 4.31, fails the cut on the sum of the transverse momenta of all leptons $\left(\sum p_{\mathrm{T}}^{\text {leptons }}>50 \mathrm{GeV}\right)$. This event may stem from $Z / \gamma^{*} \rightarrow \mu \mu$ production with an additional photon radiated off one of the decay muons and converted into an electron-positron pair in the inner detector region. Of this $e^{+} e^{-}$pair only the low- $p_{\mathrm{T}}$ electron is identified as such. The invariant mass of the three charged leptons is $M_{i n v}(\mu \mu e)=80.6 \mathrm{GeV}$, which would further support this hypothesis, since some of the energy is missing due to the positron. As alternative interpretations and although the momenta and the dimuon invariant mass are rather low, the event could also be due to $W Z$ production with both gauge bosons produced off-shell, or due to $Z / \gamma^{*}+$ jet production with the jet misidentified as an electron.

Since no events and in particular no excess of events is observed in data, any new physics with a trilepton signature and a selection efficiency times acceptance between $5 \%$ and $26 \%$ for all three analyses combined, corresponding to cross section limits of $0.136 \mathrm{pb}$ and $0.020 \mathrm{pb}$, respectively, can be excluded. Similarly, cross sections between $0.051 \mathrm{pb}$ and $0.029 \mathrm{pb}$ (for selection efficiencies of $16 \%$ and $29 \%$, respectively) can be excluded due to the results of the $\mu \mu \ell$ analysis alone.

In the following chapter 5 , the above presented results are interpreted in two different $\not_{p}$-SUSY models. Upper limits on the signal cross sections are calculated and additionally lower bounds on the masses of the lightest chargino and/or neutralino are extracted within these two SUSY scenarios. 


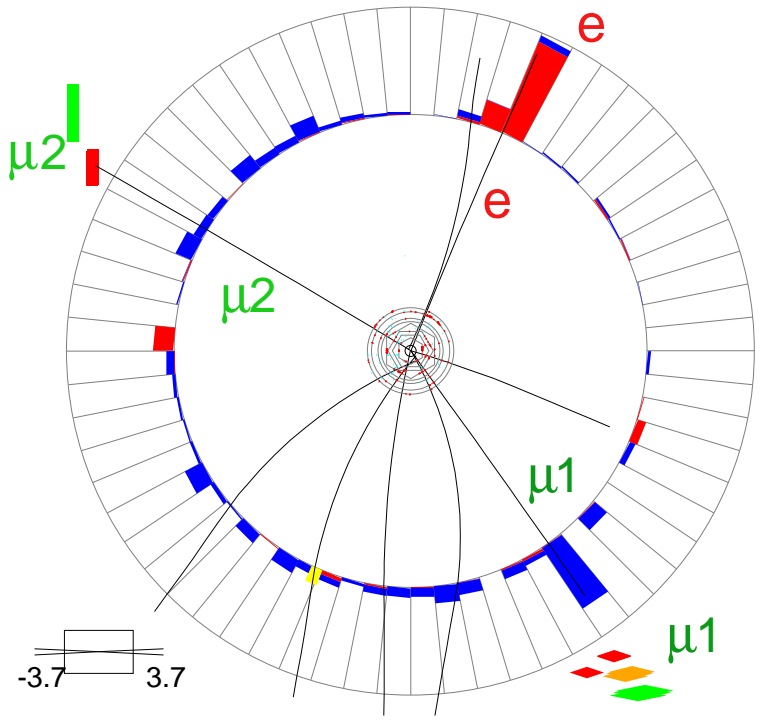

Run: $189393 \quad$ Event: 10098024
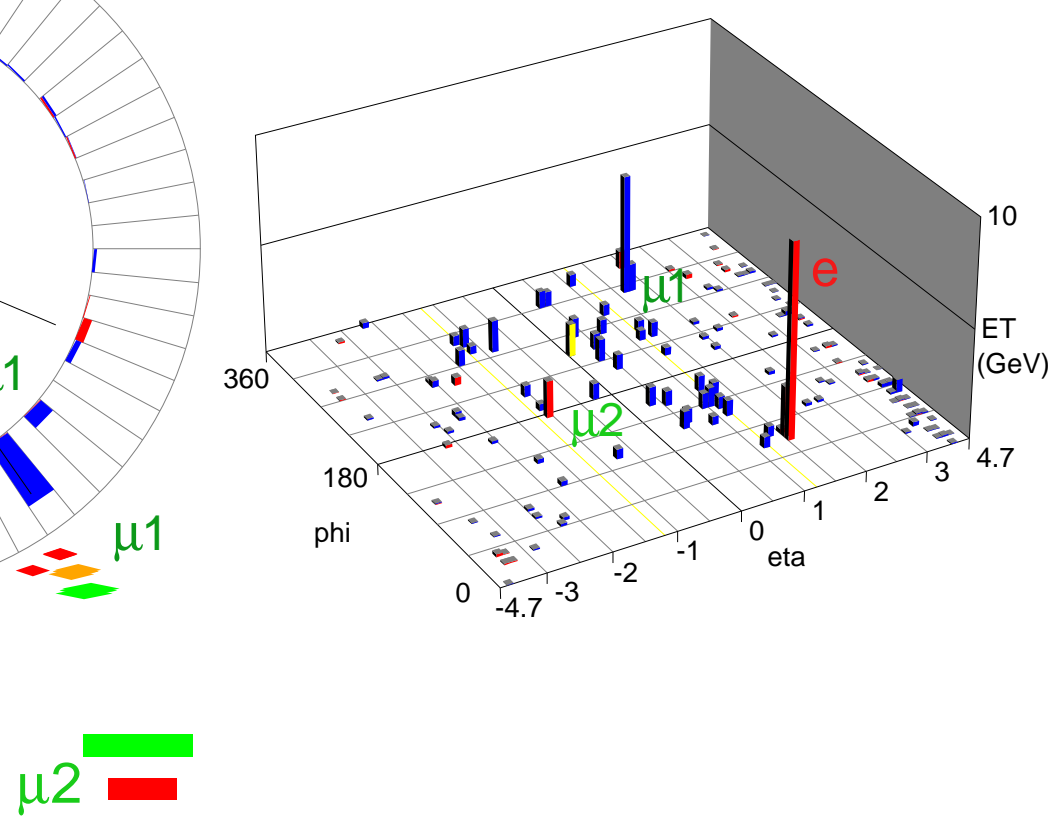

$\underline{\mu \mu e \text { - event: }}$

$$
\begin{aligned}
& p_{T}(\mu 1)=17.3 \mathrm{GeV}(-) \\
& p_{T}(\mu 2)=15.7 \mathrm{GeV}(+) \\
& p_{T}(e)=11.6 \mathrm{GeV}(-) \\
& M_{\text {inv }}(\mu 1, \mu 2)=61.5 \mathrm{GeV} \\
& M_{\text {inv }}(\mu 1, e)=24.9 \mathrm{GeV} \\
& M_{\text {inv }}(\mu 2, e)=45.7 \mathrm{GeV} \\
& \sum_{\left.p_{T}^{(l e p t o n s}\right)}=44.6 \mathrm{GeV} \\
& \not_{T}^{\text {jes }}=13.8 \mathrm{GeV}
\end{aligned}
$$

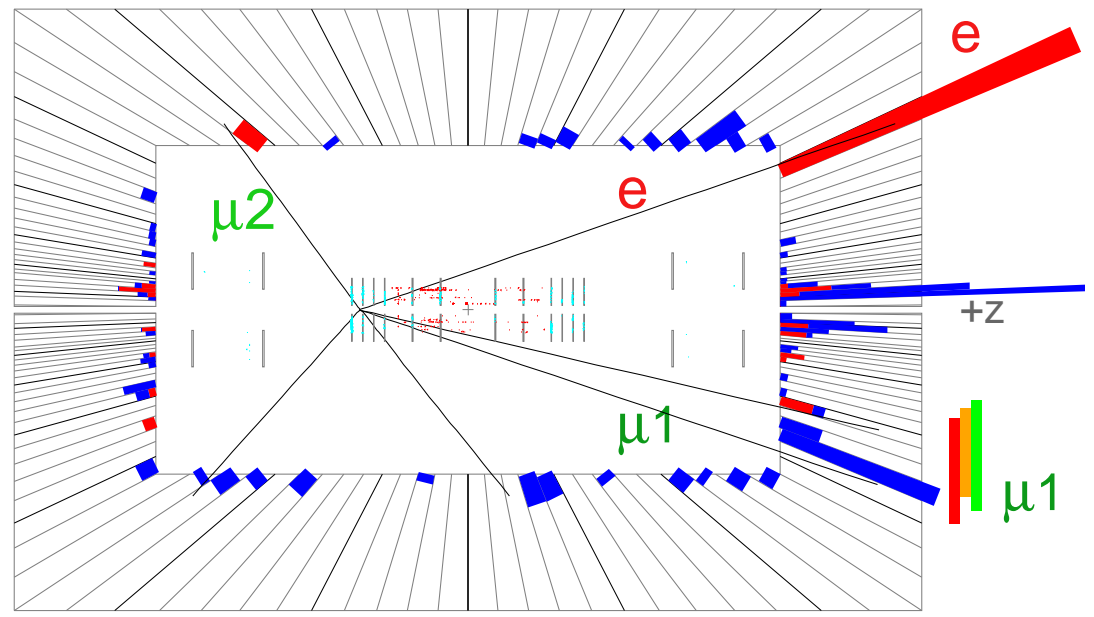

Figure 4.31: Event display of the candidate event of type $\mu \mu$. This last event in the trilepton sample fails the $\sum p_{\mathrm{T}}^{\text {leptons }}$-cut. Other relevant variables are given in the event display. The event may be due to $Z / \gamma^{*} \rightarrow \mu \mu$ production with a final state photon converted into an $e^{+} e^{-}$pair, of which only the low- $p_{\mathrm{T}}$ electron is identified. The invariant mass of the three charged leptons, $M_{i n v}(\mu \mu e)=80.6 \mathrm{GeV}$, supports this hypothesis, since the energy of the positron is missing. 


\title{
Chapter 5
}

\section{The Results and Their Interpretation}

\author{
It is remarkable that a science which began with the consideration of games of chance \\ should have become the most important object of human knowledge. \\ Pierre-Simon, Marquis de Laplace in \\ »Théorie Analytique des Probabilités « (1812)
}

In this chapter, the results of the previous chapter are translated into upper cross section limits at $95 \%$ confidence level and additionally lower bounds on the masses of the lightest neutralino and/or chargino are derived.

In the first section, the statistical method for the calculation of the expected and observed cross section limits is described, as well as the utilised procedure of combining the results of this analysis with two complementary searches for the signatures eel (via $\lambda_{121}$ ) by Anne-Marie Magnan [1,73] and eet (via $\lambda_{133}$ ) by Anne-Catherine Le-Bihan [2, 74]. Essential resources for this section are $[175,176]$ and [23]. The second section serves to interprete the results in the framework of mSUGRA, where lower bounds on the masses of the lightest neutralino and chargino are extracted, while section three presents the results in the previously defined $\gg$ noGUT « MSSM scenario (see also section 1.3.5). Improvements achieved by the combination of the three different analyses are discussed in each section where appropriate.

\subsection{The Statistical Method}

Usually the degree of agreement between data and expected SM background, or, for this reason, with the expected background plus signal, is expressed in terms of probability. According to the frequentist approach, the probability for a certain observation, i.e. the outcome of an experiment, is defined as the limit of the frequency with which this observation occurs if the experiment was repeated an infinite number of times. For a random variable $x$, the probability density function $\left(\mathrm{pdf}^{1}\right)$ then equals the limit of the frequency distribution for this infinite series of experiments $(\mathrm{n} \rightarrow \infty)$. The expectation value for any function $a(x)$ of the random variable $x$, described by the pdf $f(x)$ is thus defined as

$$
E[a(x)]=\int_{-\infty}^{+\infty} a(x) f(x) d x .
$$

\footnotetext{
${ }^{1}$ Not to be confused with the parton density function (PDF), which has been defined in section 2.1.1.
} 
The mean of this distribution is defined as the expectation value $E[x]$ of the random variable $x$ : $\mu=E[x]$, while the statistical dispersion, indicating how far away from the expected value the measurements typically are, is given by the variance

$$
V[x]=E\left[(x-E[x])^{2}\right]=E\left[x^{2}\right]-\mu^{2}=\sigma^{2}(x) .
$$

The variance of a random variable is identical to the square of its standard deviation $\sigma_{x}^{2}$. If the frequency distribution depends on more than one random variable, the variance is given by the covariance matrix $V_{i j}$

$$
V_{i j}=E\left[\left(x_{i}-\mu_{i}\right)\left(x_{j}-\mu_{j}\right)\right]=E\left[x_{i} x_{j}\right]-\mu_{i} \mu_{j} .
$$

In this case the variables may also be correlated. A measure for the correlation between any two variables is the so-called correlation coefficient, defined as $\rho_{i j}=V_{i j} /\left(\sigma_{i} \sigma_{j}\right)$.

Probability density functions can take various forms: binomial, Poisson, Gaussian, uniform distribution, and more. Depending on the situation of the experimental measurement and the nature of the random variable, which may be continuous or discrete, a different form is used. The Poisson distribution, for example, models (some) discrete random variables. Typically, a Poisson random variable is a count of the number of events that occur in a certain time interval or spatial area. A discrete random variable $x$ is said to follow a Poisson distribution with the parameter $\mu$ (mean), if it has the probability distribution:

$$
P(n ; \mu)=\frac{e^{-\mu} \mu^{n}}{n !}
$$

with $n=0,1, . . n$ and a positive mean $\mu>0$. The Poisson distribution can sometimes be used to approximate the binomial distribution with parameters $n$ and $p$. When the number of observations $n$ is large, and the success probability $p$ is small, the binomial distribution approaches the Poisson distribution with the parameter given by $\mu=n p$. On the other hand, for large mean values the Poisson distribution evolves into a Gaussian distribution.

For small mean values, however, as is the case in the present analysis (and also for the combination with two further searches), the Poisson distribution is more appropriate. Only very small numbers of events are expected from SM backgrounds, corresponding to the mean value $\mu$.

\subsubsection{The Concept of Hypothesis Tests}

To decide whether or not the observed data agree with the standard theory or rather with a new theory a test is needed that gives different probabilities in both cases and is reproducible. Each theory is usually expressed in terms of certain parameters, corresponding to a certain statistical hypothesis, which predicts the pdf of the variable $x$ that is measured. The established theory is usually referred to as the null hypothesis $H_{0}$, whereas a new theory is called alternative hypothesis, denoted $H_{A}$. On the other side, there is the experiment which produces a finite amount of data, corresponding to a frequency distribution $\hat{f}(x)$. The problem is to derive any conclusions about the theory or its validity from this distribution. Additionally, the distribution that is analysed, is mostly not the distribution of the random variable $x$ itself, but rather a function thereof, e.g. $\mathrm{t}(\mathrm{x})$, the so-called test statistic. This quantity is used to decide whether or not the null hypothesis (the established theory) should be rejected. Usually, the choice of the test statistic not only depends on the assumed probability model, but also on the hypotheses under question; it can be an estimator for a parameter of the theory, or a variable with maximal different pdfs for the two competing hypotheses $H_{0}$ and $H_{A}$. 
The two hypothesis are not, however, treated on an equal basis, special consideration is always given to the null hypothesis. This is due to the fact that the null hypothesis relates to the statement being tested, whereas the alternative hypothesis relates to the statement to be accepted if $H_{0}$ is rejected. The final conclusion is always given in terms of the null hypothesis: it either is »reject $H_{0}$ in favour of $H_{A}$ « or »do not reject $H_{0}$ «, but never » reject $H_{A} \ll$, or even »accept $H_{A}$ «. If it is concluded $\gg$ do not reject $H_{0}$ «, this does not necessarily mean that the null hypothesis is true, it only suggests that there is not sufficient evidence against it in favour of $H_{A}$. Rejecting the null hypothesis, only suggests that the alternative hypothesis may be true.

Before actually performing the experiment, a critical region, $C R$, is defined such that the sample space for the test statistic is partitioned into two regions: one region (the critical region) will lead to the rejection of the null hypothesis $H_{0}$, the other not. Now it is, of course, possible to wrongly either reject or not reject $H_{0}$. The first possibility of $\gg$ rejecting $H_{0}$, although no signal is present « is referred to as type I error, while the second decision »do not reject $H_{0}$, although there is a signal « is called type II error, see also Table 5.1. A type I error, which is also known as false discovery is often considered to be more serious, and therefore more important to avoid, than a type II error.

\begin{tabular}{lll}
\hline \hline Reality (unknown) & Reject $H_{0}$ & Do not reject $H_{0}$ \\
\hline$H_{0}$ is true & type I error & correct decision \\
$H_{A}$ is true & correct decision & type II error \\
\hline \hline
\end{tabular}

Table 5.1: Summary of the possible conclusions of a hypothesis test. Wrong decisions are connected with the two error types in statistics: the type I error for wrongly rejecting the null hypothesis (i.e. false discovery), and the type II error for not rejecting the null hypothesis, although a signal is present.

The hypothesis test procedure is therefore adjusted so that there is a guaranteed low probability of wrongly rejecting the null hypothesis. However, this probability is never zero, but is fixed by the experimenter. Usually, it is chosen to be smaller than $\alpha<5.7 \cdot 10^{-5} \%(5 \sigma)$ for the rejection of an established theory, whereas $\alpha<0.05=5 \%$ is sufficient to $»$ reject « an alternative theory (see section 5.1.2). The very small fixed probability $(\alpha)$ of committing a type I error is sometimes also called significance level. The two probabilities $\alpha$ and $\beta$ can be expressed as:

$$
\begin{array}{ll}
\alpha=P\left(x \in C R \mid H_{0}\right)<5.7 \cdot 10^{-5} \% & \text { type I error probability } \\
\beta=P\left(x \notin C R \mid H_{A}\right)=? & \text { type II error probability }
\end{array}
$$

On the other hand, the probability of a type II error, denoted as $\beta$, is generally unknown. The probability to not commit a type II error is also called the power of a statistical test; it is written as $1-\beta$. In any case, the two types of error are inversely related for any given set of data, i.e. the smaller the risk of one, the higher the risk of the other. As can be seen from Eqs. (5.5) and (5.6), the significance level only depends on the null hypothesis, whereas the statistical power depends on the alternative hypothesis.

Having said this, it is clear that the search for new physics, e.g. new particles such as neutralinos or charginos (or their respective signatures in the detector) also corresponds to a hypothesis test. On the one hand there is the established theory, the SM, which predicts a certain amount of background, which is - in this case - the null hypothesis or the so-called background only hypothesis; on the other hand, there is the new theory, e.g. $\not R_{p}$-SUSY, predicting a larger amount of events, corresponding to the alternative or the signal+background hypothesis. 
In an ideal world, one would now choose a test statistic $t(x)$, whose pdfs are maximally separated for $H_{0}$ (the SM) and $H_{A}$ ( $\not R_{p}$-SUSY). According to the Neyman-Pearson lemma [177] the optimal test statistic would be the ratio of the probability densities for $H_{0}$ and $H_{A}$ :

$$
\Lambda(x)=\frac{f\left(x \mid H_{0}\right)}{f\left(x \mid H_{A}\right)}
$$

This test statistic maximises the power $(1-\beta)$ of the hypothesis test, and thus minimises the probability of committing a type II error $(\beta)$ for a given $\alpha$, i.e. the chosen small probability for a false discovery. In other words, the optimal test statistic is the most powerful test for distinguishing the signal+background hypothesis from the background only hypothesis at the given level of significance $\alpha$. In addition, it does not depend on the range of possible models of new physics considered when testing a particular signal hypothesis.

In practice, however, it can be difficult to determine $\Lambda(x)$, since this requires knowledge of both probability density functions $f\left(x \mid H_{0}\right)$ and $f\left(x \mid H_{A}\right)$. Thus a different test statistic is constructed instead. One of the most-widely used test statistics in searches for small signals is simply the number of events $N$ for which the variable $x$ lies in a certain region, the signal region. The SM $\left(H_{0}\right)$ predicts a Poisson distribution of $N$ events with a mean value $b$ for the background only hypothesis, while $\not_{p}$-SUSY $\left(H_{A}\right)$ predicts a Poisson distribution with a mean value $b+s$ for the signal+background hypothesis.

\subsubsection{Confidence Levels and Exclusion Limits}

Unfortunately, in real life, it is even more complicated and the alternative hypothesis usually depends on a number of parameters; all resulting in different predictions for the mean value $s$ and thus in various alternative hypothesis. In addition, it is usually also impossible to meet the levels of significance for the established theory $\left(\alpha<5.7 \cdot 10^{-5}\right)$ and the alternative theory/theories $(\beta<0.05)$, in a single statistical test. Hence, a series of tests is performed for each alternative hypothesis. For each one of these »pseudo-experiments «, the critical region is adjusted such, that the power of the test is large enough, i.e. $\beta<0.05$.

The series starts with those alternative hypotheses predicting small values of mean $s$ for the signal distribution and continues to hypotheses predicting ever larger mean values $s$ until a certain $s=s_{\max }$ is reached for which the observed number of data events is lower than the boundary of the critical region. At this point, all hypothesis predicting larger mean values than $s_{\max }$ for the signal distribution are excluded at the previously chosen level of significance $\beta<0.05$, while still nothing can be inferred for hypotheses predicting smaller $s$. The procedure is equivalent to constructing the minimal acceptance ${ }^{2}$ region for the null hypothesis that still contains the observed number of events $N_{o b s}$. This is achieved by the confidence level of the signal+background hypothesis $C L_{s+b}$, written as:

$$
C L_{s+b}=\sum_{n=0}^{N_{\text {obs }}} P\left(n \mid s_{\text {max }}+b\right) \leq 0.05 .
$$

The confidence level $C L_{s+b}$ is also referred to as the upper limit on the expected signal $s$, which, in turn, corresponds to interpreting the region with $s<s_{\max }$ as the confidence region for the true value of the mean of the signal distribution $s$. The confidence level for the background only hypothesis $C L_{b}$ describes the agreement of the data with the predicted SM background; it is defined as $1-\alpha$.

\footnotetext{
${ }^{2}$ The acceptance region is the complement of the critical region $C R$
} 
The so-called $C L_{s}$ method [178], which has been used for measurements at LEP to set limits on the mass of the Higgs boson, was developed in order not to overestimate statistical downward fluctuations of the background expectation. This is sometimes also called the modified frequentist approach, since the simple frequentist approach, i.e. Equation (5.8) is modified as:

$$
C L_{s}=\frac{\beta}{1-\alpha}=\frac{C L_{s+b}}{C L_{b}}=\frac{\sum_{n=0}^{N_{o b s} P\left(n \mid s_{\max }+b\right)}}{\sum_{n=0}^{N_{o b s}} P(n \mid b)} \leq 0.05
$$

The $C L_{s+b}$ is normalised to the confidence level of the background only hypothesis $C L_{b}$, essentially increasing the required power of the test to obtain more conservative results.

Figure 5.1 illustrates the various quantities encountered so far: the two probabilities for errors of type I $(\alpha)$ and type II $(\beta)$, corresponding to the definition of the two confidence levels $C L_{b}=1-\alpha$ and $C L_{s+b}=\beta$, the critical region, and the procedure used for the many pseudo-experiments. All this results from two overlapping distributions of Poisson probabilities: one of them, $P_{s+b}$, is generated assuming signal+background to be present and the other, $P_{b}$, is produced under the assumption that only background is present; both are centered about the respective means $s+b$ and $b$. An observed number of events in data $N_{o b s}$ larger than the mean $b$ indicates that there may be more than only background and results in a large value of $C L_{b}$, since there is enough »room « to accommodate signal events in addition to the background events. In the opposite case, i.e. observing a number of data events smaller than the mean $b$ indicates that the data do not even agree with the expected background. If this difference is large, one should check the assumptions and the analysis for possible errors...
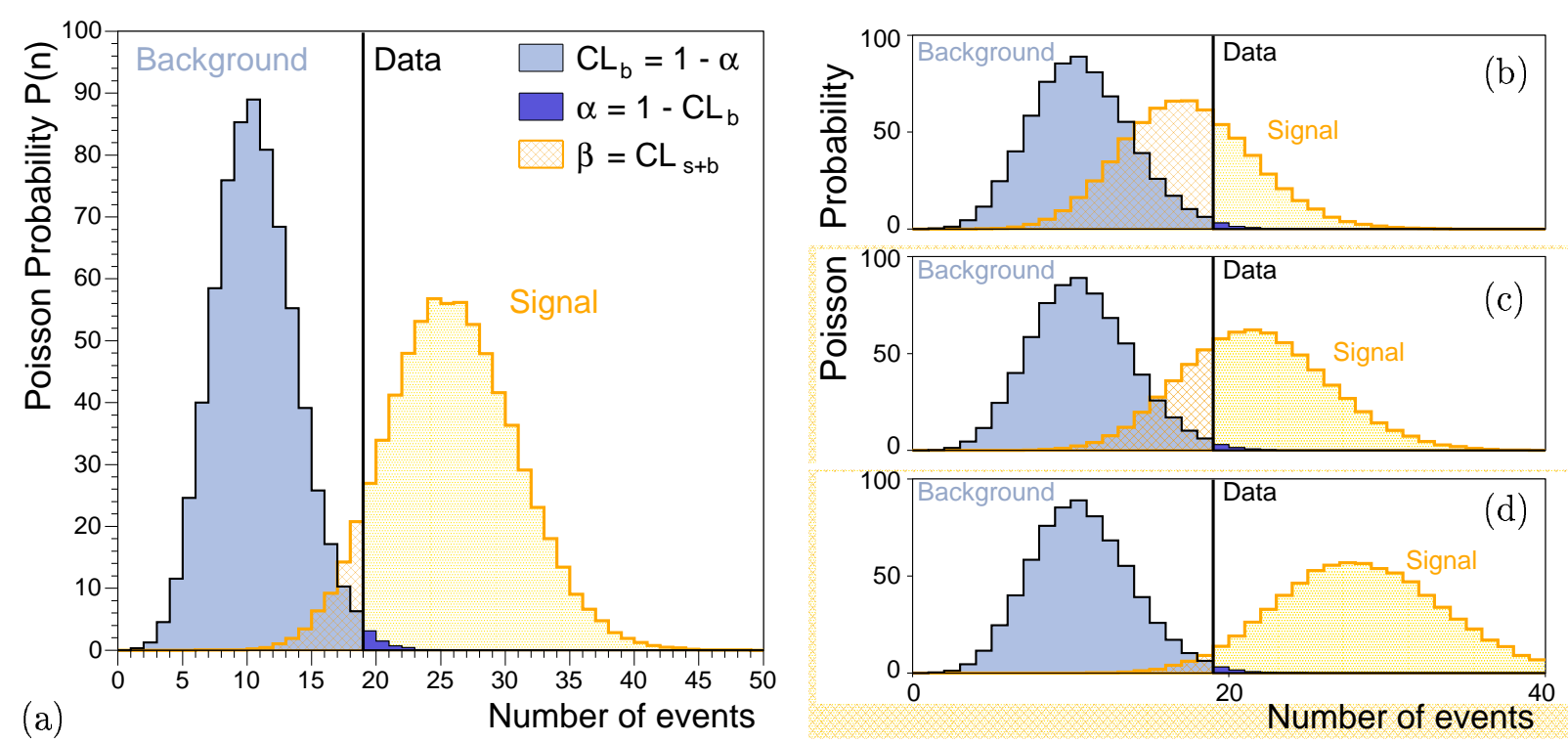

Figure 5.1: Illustration of two overlapping distributions of Poisson probabilities and various of the resulting statistical quantities: (a) the probabilities for errors of type I $(\alpha)$ and type II $(\beta)$, the corresponding definitions of the confidence intervals $C L_{b}=1-\alpha$ and $C L_{s+b}=\beta$, and the critical region $C R$. The procedure of a series of pseudo-experiments is indicated as follows: (b) for initial small $s$, (c) for medium $s$, and (d) for $s=s_{\max }$. 


\subsubsection{Combining Different Analysis Channels}

For further applications it is easier to use the likelihood ratio $Q(n)$ of the Poisson probabilities for the two competing hypotheses, $s+b$ and $b$, as a test statistic as it is advocated by the Neyman-Pearson lemma. This means, one simply has to exchange the above mentioned counter $N$ for the number of events by the likelihood ratio $Q(n)$

$$
Q(n)=\frac{P(n \mid s+b)}{P(n \mid b)}=\frac{(s+b)^{n} e^{-(s+b)}}{n !} \cdot \frac{b^{n} e^{-b}}{n !}=\left(1+\frac{s}{b}\right)^{n} e^{-s} .
$$

One important advantage of this test statistic is the fact that it can easily be extended in order to combine, e.g. a number of different analysis channels, different bins of one distribution and/or entirely different experiments. This is because the likelihood ratio has the property that the joint test statistic $Q$ for the outcome of, say $i=0,1, \ldots, N$ channels is the same as the product of the test statistics for all channels separately

$$
Q^{c o m b}=\prod_{i=1}^{N} Q\left(n_{i}\right)=\prod_{i=1}^{N}\left(1+\frac{s_{i}}{b_{i}}\right)^{n_{i}} e^{-s_{i}}
$$

where the index $i$ runs over the different analysis channels (bins, experiments). To further simplify the calculation, the natural logarithm of the likelihood ratio is used, $\ln Q(n)$, which reduces Equation (5.12) to a simple difference of the sum of candidate events, where each event is weighted with $\ln \left(1+s_{i} / b_{i}\right)$, and the number of candidate events expected solely from the signal:

$$
\ln Q^{c o m b}=\sum_{i=1}^{N} n_{i} \cdot \ln \left(1+\frac{s_{i}}{b_{i}}\right)-s_{i} .
$$

In case of the combination of the three different $\not R_{p}$-trilepton searches, the dependence of the event weights on the local (i.e. the per analysis) signal-to-background ratio $s_{i} / b_{i}$ is used to find the order in which the three analyses ought to be combined to give the best result for each coupling study. The technical realisation is described in the following section 5.1.4.

The confidence level $C L_{s}$ of Equation (5.9) for excluding the possibility of the simultaneous presence of the production of new particles and SM background ( $s+b$ hypothesis), while taking into account possible fluctuations in the background expectation, is then given by:

$$
C L_{s}=\frac{\beta}{1-\alpha}=\frac{C L_{s+b}}{C L_{b}}=\frac{P\left(Q \leq Q_{o b s} \mid s+b\right)}{P\left(Q \leq Q_{o b s} \mid b\right)} \leq 0.05,
$$

where $Q_{o b s}$ is the observed likelihood ratio.

The pdfs of the likelihood ratio in the signal+background and background only hypotheses also allow calculating the expected levels of confidence $\left\langle C L_{s+b}\right\rangle,\left\langle C L_{b}\right\rangle$, and $\left\langle C L_{s}\right\rangle$ under the assumption that only background is present. These confidence levels are a measure of how well (or poorly) an experiment or analysis would perform on average if there really was no signal at all.

Statistical and systematic uncertainties on the background, as well as on the signal expectation are also taken into account (see again section 5.1.4). Since both kinds of uncertainties lead to systematic uncertainties of the predicted mean values $b_{i}$ and $s_{i}$, an integration over all possible values of the signal and background given by their respective systematic uncertainty probability distributions is performed. The utilised probability distributions are assumed to be Gaussian, with the lower tail cut off at zero to avoid negative values for $b_{i}$ or $s_{i}$. 


\subsubsection{Technical Realisation of the $\mathrm{CL}_{S}$ Method}

Technically the above described $C L_{s}$ method is realised using an iterative procedure to find the upper cross section limit at the $95 \%$ confidence level. The utilised program is based on a modified version of the $\mathrm{C}++$ class TLimit [179] from ROOT. In the present case, the tested hypothesis $H_{A}$ for the computation of exclusion limits is »there is a signal «. As mentioned, the rejection of this hypothesis, i.e. the exclusion of a certain parameter region is performed at a confidence level of $95 \%$, which means, the probability to wrongly exclude a signal that is in fact there is, according to Equation (5.13), $C L_{s}<5 \%$. If the number of observed events in data would indicate the presence of a signal, the test hypothesis to decide whether or not the deviation is sufficient to claim a discovery, has to be the opposite statement, i.e. »there is no signal «. To reject this test hypothesis (where the underlying theory is the established one) a confidence level of $5 \sigma$ is needed, corresponding to the probability of observing this many events in data, when only background is expected $P\left(n \geq N_{o b s} \mid b\right)<5.7 \cdot 10^{-5} \%=5 \sigma$.

Since no events are observed in data $\left(N_{o b s}=0\right)$ and only a small number of events is expected from SM background, the obvious test hypothesis for the extraction of exclusion limits is the first one: »there is a signal «.

The following input variables are needed by the program:

- the expected selection efficiency for signal events, including the respective statistical and systematic uncertainties: $\varepsilon_{S}, \delta \varepsilon_{S}^{s t a t}, \delta \varepsilon_{S}^{s y s}$;

- the expected selection efficiency for background events, separately for each background and again including statistical and systematic uncertainties: $\varepsilon_{B}, \delta \varepsilon_{B}^{s t a t}, \delta \varepsilon_{B}^{\text {sys }}$;

- the theoretical cross section for each background process;

- the luminosity with its respective uncertainty: $\mathcal{L}, \delta_{\text {sys }}^{\mathcal{L}}$.

- and the observed number of events in data: $N_{\text {obs }}$;

For a given signal point, the program now starts testing a certain signal cross section $\sigma_{S}^{\text {test }}$ by iterating the following list of points:

Step 1: The effective luminosity $\mathcal{L}_{\text {eff }}$, the effective number of total expected events $N_{\text {tot }}^{\text {eff }}$ and the resulting probabilities $P_{s+b}$ and $P_{b}$ are calculated and the values of the latter two are filled into separate histograms.

- The effective luminosity $\mathcal{L}_{\text {eff }}$ is determined by generating a Gaussian distributed random number according to: $\mathcal{L}_{\text {eff }}=\operatorname{Gauss}\left(\mathcal{L}, \delta_{\text {sys }}^{\mathcal{L}}\right)$ with mean $\mathcal{L}$ and width $\delta_{\text {sys }}^{\mathcal{L}}$. This luminosity value then allows calculating the numbers of events expected from $\mathrm{SM}$ background $N_{B}^{e f f}$, as well as from the signal process $N_{S}^{e f f}$.

- The effective number of expected signal events $N_{S}^{e f f}$ is determined by generating Gaussian distributed random numbers for the statistical and the systematic uncertainty of the observed signal efficiency $\varepsilon_{S}$, each one with mean zero and the width equal to the respective uncertainty, and then calculating:

$N_{S}^{e f f}=\left(\varepsilon_{S}+\delta \varepsilon_{S, s t a t}^{e f f}+\delta \varepsilon_{S, s y s}^{e f f}\right) \cdot \sigma_{S}^{t e s t} \cdot \mathcal{L}_{e f f}$.

- The effective number of expected background events $N_{B}^{e f f}$ is determined in an analogous manner, only that this is done separately for the selection efficiency of each contributing background process $\varepsilon_{B, i}$, resulting in:

$N_{B}^{e f f}=\left[\sum_{i}\left(\varepsilon_{B, i}+\delta \varepsilon_{B, i, s t a t}^{e f f}+\delta \varepsilon_{B, i, s y s}^{e f f}\right)\right] \cdot \sigma_{S}^{\text {test }} \cdot \mathcal{L}_{e f f}$ 
- The effective total number of expected events is calculated as: $N_{\text {tot }}^{e f f}=N_{S}^{e f f}+N_{B}^{e f f}$.

- The two probabilities $P\left(n \leq N_{o b s} \mid s+b\right)$ and $P\left(n \leq N_{o b s} \mid b\right)$ of seeing at most the observed number of data events $N_{o b s}$ are calculated under the assumption that signal+background is present or only background, respectively. Each one of the two values is filled into a separate histogram.

Step 2: After generating 10,000 of these pseudo-experiments the $C L_{s}^{\text {test }}$ is calculated from the resulting probability distributions and compared to the pre-specified value of $\alpha=0.05$. This is equivalent to a confidence level of $95 \%$, which means nothing else than - in case the signal indeed existed - one would observe, in $95 \%$ of all cases, a number of events equal or less than the actually observed number of events $N_{o b s}$.

Step 3: In case $C L_{s}^{\text {test }}<0.05-\varepsilon$, the tested signal cross section $\sigma_{S}^{\text {test }}$ is increased, otherwise, for $C L_{s}^{\text {test }}>0.05+\varepsilon$, it is decreased. Thus, the above steps 1 and 2 are reiterated until $C L_{s}^{\text {test }}$ equals $5 \%$ with the desired precision of $\varepsilon=10^{-8}$ as chosen here. In this case, the 95\% CL upper limit on the signal cross section $\sigma_{o b s}^{95 \%}$ corresponds to the statement $\gg$ In $95 \%$ of all cases, the considered signal cannot have a cross section larger than $\sigma_{o b s}^{95 \%} \ll$.

An identical procedure is utilised for the computation of the expected upper cross section limit $\sigma_{e x p}^{95 \%}$, which is the limit one would obtain if data and expected background agreed well. The only difference in the above described procedure is the replacement of $N_{o b s}$ (the observed number of data events) with another random number $N_{o b s}^{B}$. Contrary to the other random numbers $N_{o b s}^{B}$ can only be an integer and is Poisson distributed with mean $N_{B}$ and a width corresponding to the statistical and systematic uncertainties on the background expectation added in quadrature.

\section{The Combination of Different Analysis Channels}

Two complementary $\not R_{p}$-SUSY trilepton searches have been developed within the D $\varnothing$ collaboration. One of them, the search for eel final states is designed and optimised to study the dominant signature of neutralino $\not R_{p}$-decays via the coupling $\lambda_{121}$ and the other one, a search for eet final states is devised to study the coupling $\lambda_{133}$. A combination of all three analyses is performed to achieve the best possible sensitivity for each $\not R_{p}$-coupling [180]. Since, due to the cascade decays of heavier gauginos, all three lepton flavours may be present in the final state produced via any one of the three considered couplings $\left(\lambda_{121}, \lambda_{122}\right.$, and $\left.\lambda_{133}\right)$ it is only natural that each additional analysis channel enhances the signal sensitivity by covering small inefficiencies of the other analyses. This argument is particularly important in understanding that even the eer analysis improves the sensitivity of the present $\mu \mu \ell$ analysis, even though the final states produced via the $\lambda_{122}$ coupling consist predominantly of electrons and muons.

However, before the results of the three analyses can be combined for one $\lambda$ coupling, all three analyses need to process the same background and signal samples, as well as data. No common background or data event is found. Simulated signal events selected in multiple channels, e.g. in eel and $\mu \mu \ell$, are assigned to the analysis with the largest signal-to-background ratio, and are removed from the other two analyses, which are then rerun, so that the efficiencies are lowered automatically to take the smaller number of events correctly into account. The percentage of common signal events for the eel and $\mu \mu \ell$ analyses ranges from about $5-8 \%$ (in the »corridor region $«, m_{0}=100 \mathrm{GeV}, \tan \beta=5, \mu>0$ and also for $m_{0}=100 \mathrm{GeV}$, $\tan \beta=5, \mu<0$ ) to about $15-24 \%$ (for $m_{0}=1000 \mathrm{GeV}, \tan \beta=5, \mu>0$ ), see also the tables in section 5.2 and in the appendix $\mathrm{C}$, whereas only in between $1-2 \%$ of events are selected by $\mu \mu \ell$ and $e e \tau$, respectively. After the removal of these multiply selected signal events from the analyses with the lower $S / B$-ratio, the same iterative method for the calculation of the 
95\% CL upper cross section limits is applied as described above for the single analysis. For the $\lambda_{122}$ study, the present analysis is always the one with the largest $S / B$-ratio. This is expected, since $\lambda_{122}$ leads predominantly to final states with muons. However, as can be seen from the percentage of common events in the eel and $\mu \mu \ell$ analyses, the eel analysis is also sensitive to $\lambda_{122}$, due to the signatures with electrons and muons (see section 3.3 for further details).

\section{Treatment of the Systematic Uncertainties}

The correlation between the signal efficiencies in the three analyses is taken into account for the calculation of the systematic uncertainties and thus for the extraction of exclusion limits.

All systematic uncertainties are assumed to be fully correlated among all three analyses. This overestimates the correlation of systematic uncertainties of the eel/eet analyses and the $\mu \mu \ell$ analysis slightly, but since nearly all systematic uncertainties of the eel and ee $\tau$ analyses are fully correlated, this procedure was adopted to simplify the calculation. The following list provides an overview of the correlation of systematic uncertainties between the three different analyses.

\section{Quantities that are fully correlated among eel, $\mu \mu \ell$, and $e e \tau$ :}

- The luminosity

- Electron identification \& reconstruction efficiencies: emf, iso, $H M x 7$, and lhood

- Electron resolution effects ( $E_{\mathrm{T}}$ resol. \& smearing)

- The modelling of the $Z$ boson $p_{\mathrm{T}}$ distribution

- The signal acceptance due to PDF uncertainties

\section{Quantities that are fully correlated among eel and $\mu \mu \ell$ :}

- Muon identification \& quality efficiencies: reconstruction, »medium« hit pattern, isolation and trackfinding/-matching

- Muon $p_{\mathrm{T}}$ resolution and smearing

\section{Quantities that are fully correlated among eel and $e e \tau$ :}

- Trigger parametrisation for single and dielectron triggers

- The »oversmearing«/reweighting of the $\not_{T}$ distribution in eel and ee $\tau$ events

\subsection{Interpretation within the mSUGRA Scenario}

Expected and observed upper cross section limits (exclusion limits) are calculated at 95\% CL for all mSUGRA signal points listed in Tables A.1 and A.2 in the appendix. To obtain conservative results, all limits are calculated with respect to the next-to-leading order (NLO) cross section reduced by its total systematic uncertainty. The NLO cross section is the SUSYGEN LO cross section multiplied by the corresponding $K$-factor, which lies between 1.13 and 1.28 for all mSUGRA points. It has been obtained using GaUGinos, see also section 4.2.2). The considered systematic uncertainties are due to the leading order cross section, the determination of the $K$-factor, the variations of the factorization and renormalization scales and the choice of PDF (CTEQ5 versus CTEQ6), compare section 4.6.5. This leads to signal cross sections, which are $11 \%$ lower than the respective NLO cross sections. In the following one-dimensional figures the cross section curves refer to the unreduced NLO cross sections, while the lower mass bounds are given by the intersection of the observed $\sigma_{S}^{95 \%}$ curve with the lower edge of the cross section error band.

Table 5.2 provides an overview of the results for some selected mSUGRA points, with the parameters $m_{0}=100 \mathrm{GeV}$ and $1000 \mathrm{GeV}$ for $\tan \beta=5$ and both signs of the Higgs mixing parameter $\mu$. Listed are for each point the signal selection efficiency, including the statistical and systematic 
uncertainties, the NLO cross section and the expected and observed upper cross section limits obtained from this analysis alone, as well as the corresponding expected and observed limits obtained from a combination of this analysis with the two complementary analyses of eel and $e e \tau$ final states, by Anne-Marie Magnan and Anne-Catherine Le-Bihan, respectively. For those points, where combined limits are available, the percentage of overlap events between the eel and the $\mu \mu \ell$ analysis (this work) is also provided. The corresponding complete tables for all points can be found in appendix $\mathrm{C}$.

\begin{tabular}{|c|c|c|c|c|c|c|c|}
\hline \multirow[b]{2}{*}{ Name } & \multirow{2}{*}{$\begin{array}{r}\sigma_{N L O} \\
{[\mathrm{pb}]} \\
\end{array}$} & \multirow{2}{*}{$\begin{array}{c}\varepsilon_{\text {Signal }}[\%] \\
\varepsilon_{S} \pm \text { stat } \pm \text { sys } \\
\end{array}$} & \multicolumn{2}{|c|}{$\mu \mu \ell$ limits [pb] } & \multicolumn{2}{|c|}{ comb. limits [pb] } & \multirow{2}{*}{$\begin{array}{l}\text { Ov. } \\
{[\%]} \\
\end{array}$} \\
\hline & & & $\sigma_{e x p}^{95 \%}$ & $\sigma_{S}^{95 \%}$ & $\sigma_{\exp }^{95 \%}$ & $\sigma_{S}^{95 \%}$ & \\
\hline $\mathcal{A} 2 p o s$ & 0.143 & $12.7 \pm 0.3 \pm 1.4$ & 0.081 & 0.065 & 0.069 & 0.050 & 7.2 \\
\hline $\mathcal{A} 3 p o s$ & 0.088 & $14.9 \pm 0.4 \pm 1.7$ & 0.069 & 0.055 & 0.060 & 0.044 & 8.3 \\
\hline $\mathcal{A} 4 p o s$ & 0.055 & $16.1 \pm 0.4 \pm 1.8$ & 0.064 & 0.051 & 0.055 & 0.040 & 7.8 \\
\hline $\mathcal{A} 5 p o s$ & 0.034 & $14.7 \pm 0.4 \pm 1.6$ & 0.070 & 0.056 & 0.060 & 0.044 & 8.7 \\
\hline $\mathcal{A} 6 p o s$ & 0.022 & $14.6 \pm 0.4 \pm 1.6$ & 0.071 & 0.056 & 0.060 & 0.043 & 9.8 \\
\hline $\mathcal{B} 1$ pos & 0.766 & $12.8 \pm 0.3 \pm 1.4$ & 0.081 & 0.064 & 0.068 & 0.049 & 5.8 \\
\hline $\mathcal{B} 3 p$ os & 0.653 & $13.2 \pm 0.3 \pm 1.5$ & 0.078 & 0.062 & 0.068 & 0.050 & 8.0 \\
\hline $\mathcal{B} 6$ pos & 0.557 & $9.9 \pm 0.3 \pm 1.1$ & 0.105 & 0.083 & 0.092 & 0.068 & 4.9 \\
\hline $\mathcal{B} 8$ pos & 0.309 & $11.2 \pm 0.3 \pm 1.3$ & 0.093 & 0.074 & 0.077 & 0.056 & 6.5 \\
\hline $\mathcal{B} 10$ pos & 0.178 & $11.2 \pm 0.3 \pm 1.2$ & 0.092 & 0.074 & 0.075 & 0.053 & 7.3 \\
\hline $\mathcal{B} 12$ pos & 0.151 & $13.0 \pm 0.4 \pm 1.5$ & 0.079 & 0.063 & 0.066 & 0.048 & 7.3 \\
\hline $\mathcal{B} 13$ pos & 0.137 & $21.8 \pm 0.4 \pm 2.4$ & 0.047 & 0.038 & 0.032 & 0.022 & 14.9 \\
\hline B14pos & 0.107 & $24.6 \pm 0.4 \pm 2.8$ & 0.042 & 0.034 & 0.031 & 0.021 & 23.0 \\
\hline $\mathcal{B} 16$ pos & 0.064 & $23.7 \pm 0.5 \pm 2.6$ & 0.044 & 0.035 & 0.029 & 0.020 & 16.3 \\
\hline $\mathcal{B} 17$ pos & 0.050 & $21.4 \pm 0.4 \pm 2.4$ & 0.048 & 0.038 & 0.034 & 0.023 & 21.8 \\
\hline $\mathcal{B} 18$ pos & 0.039 & $25.8 \pm 0.5 \pm 2.9$ & 0.040 & 0.032 & 0.030 & 0.021 & 21.4 \\
\hline $\mathcal{B} 19$ pos & 0.031 & $25.1 \pm 0.4 \pm 2.8$ & 0.041 & 0.033 & 0.028 & 0.019 & 23.8 \\
\hline $\mathcal{B} 21$ pos & 0.025 & $24.6 \pm 0.5 \pm 2.8$ & 0.042 & 0.033 & 0.029 & 0.020 & 23.8 \\
\hline $\mathcal{B} 22$ pos & 0.019 & $24.7 \pm 0.5 \pm 2.8$ & 0.042 & 0.033 & 0.029 & 0.020 & 23.5 \\
\hline $\mathcal{B} 23$ pos & 0.016 & $24.3 \pm 0.4 \pm 2.7$ & 0.042 & 0.034 & 0.029 & 0.020 & 23.1 \\
\hline $\mathcal{A} 1$ neg & 0.112 & $13.5 \pm 0.4 \pm 1.5$ & 0.077 & 0.061 & 0.066 & 0.048 & 9.2 \\
\hline $\mathcal{A} 2 n e g$ & 0.070 & $15.5 \pm 0.4 \pm 1.7$ & 0.066 & 0.053 & 0.056 & 0.041 & 7.9 \\
\hline A3neg & 0.044 & $15.9 \pm 0.4 \pm 1.8$ & 0.065 & 0.052 & 0.056 & 0.041 & 9.8 \\
\hline $\mathcal{A} 4 n e g$ & 0.027 & $17.2 \pm 0.4 \pm 1.9$ & 0.060 & 0.048 & 0.051 & 0.037 & 7.6 \\
\hline $\mathcal{A} 5 n e g$ & 0.018 & $16.8 \pm 0.4 \pm 1.9$ & 0.061 & 0.049 & 0.053 & 0.039 & 10.9 \\
\hline B3neg & 0.587 & $8.6 \pm 0.3 \pm 1.0$ & 0.120 & 0.095 & 0.106 & 0.078 & 5.4 \\
\hline $\mathcal{B} 4$ neg & 0.335 & $8.6 \pm 0.3 \pm 1.0$ & 0.120 & 0.095 & 0.103 & 0.075 & 7.2 \\
\hline $\mathcal{B} 5$ neg & 0.199 & $9.2 \pm 0.3 \pm 1.0$ & 0.112 & 0.090 & 0.092 & 0.066 & 6.2 \\
\hline $\mathcal{B} 6$ neg & 0.121 & $12.0 \pm 0.4 \pm 1.4$ & 0.086 & 0.068 & 0.074 & 0.054 & 6.6 \\
\hline $\mathcal{B} 8$ neg & 0.074 & $13.8 \pm 0.3 \pm 1.5$ & 0.075 & 0.060 & 0.066 & 0.049 & 5.1 \\
\hline $\mathcal{B} 9$ neg & 0.060 & $12.7 \pm 0.3 \pm 1.4$ & 0.082 & 0.065 & 0.072 & 0.054 & 5.1 \\
\hline B10neg & 0.046 & $14.6 \pm 0.3 \pm 1.6$ & 0.070 & 0.056 & 0.063 & 0.047 & 5.5 \\
\hline B12neg & 0.030 & $12.2 \pm 0.3 \pm 1.4$ & 0.084 & 0.067 & 0.075 & 0.056 & 3.4 \\
\hline
\end{tabular}

Table 5.2: Example overview of the upper cross section limits for some selected mSUGRA signal points: expected $\left(\sigma_{e x p}^{95 \%}\right)$ and observed $\left(\sigma_{S}^{95 \%}\right)$ limits from this analysis alone and from the combintion with the searches for eel and eet final states. Also listed are the NLO cross section, the signal selection efficiency of the $\mu \mu \ell$ analysis, and the fractional overlap between the eel and the $\mu \mu \ell$ analyses (last column). 
For most mSUGRA points, the selection efficiency lies in between $\varepsilon_{S} \approx 10-15 \%$ (see Tables C.1 and C.2 in the appendix). However, for points with $m_{0}=250 \mathrm{GeV}$, positive $\mu$, and $\tan \beta=5$ the selection efficiency increases up to $\approx 26 \%$ for $m_{1 / 2}$ values above $m_{1 / 2}=250 \mathrm{GeV}$, while the contrary is true for mSUGRA points with negative $\mu$ (again $m_{0}=250 \mathrm{GeV}$ and $\tan \beta=5$ ) and low values of $m_{1 / 2}$, where the selection efficiency drops to $\approx 8.5 \%$. In either case, the statistical uncertainty on the selection efficiency is small due to the, in general, large number of generated events per signal point. The systematic uncertainty, on the other hand, is rather large with only small variations. For a majority of the mSUGRA signal points, it lies at about $\delta \varepsilon_{S, \text { sefs }}^{\text {eff }} \approx 11.2 \%$. This is due to the fact that the dominant uncertainty is the one of the muon identification \& quality criteria, making up nearly $9-10 \%$ of the total $\approx 11 \%$.

For $\tan \beta=5$, the signal points with $m_{0}$ values of $m_{0}=100$ and $1000 \mathrm{GeV}$ have been processed through the eel and ee $\tau$ analyses, and in addition, all $\tan \beta=5$ signal points have been processed through the eel analyses, so that the combined limits refer to a combination of all three analyses $(\mu \mu \ell, e e \ell$, and $e e \tau)$ or to a combination of the two most sensitive analyses in case of $\lambda_{122}$, which are the $\mu \mu \ell$ and eel analyses.

The upper cross section limits (expected and observed) are also displayed in the following Figures 5.2 and 5.3 for mSUGRA signals with positive Higgs mixing parameter $\mu$ and Figure 5.4 with negative $\mu$, respectively. The NLO signal cross section is reprented by a solid dark grey curve with dashed grey lines indicating the positive and negative systematic uncertainties. All limit curves are shown in different shades of magenta/violet: the limit curves for this analysis alone are in a lighter tone and the limit curves for all three analyses combined in a darker violet. Expected limits are represented by wide, dashed curves, and observed limits by narrower, solid curves, with open circles ( $\mu \mu \ell$ analysis alone) or triangles (all analysis combined) to indicate where exactly a signal point was generated.

The resulting lower bounds on the masses of the lightest chargino and neutralino are indicated by the shaded regions, terminated by coloured arrows at the right end. The arrows at lower mass values correspond to the lower mass bound set by this analysis alone, and the darker arrows at higher mass values indicate the bound obtained by combining all three $\mathbb{R}_{p}$-trilepton analyses, respectively. The allowed mass ranges are to the right of the shaded areas.

However, for points with $m_{0} \leq 100 \mathrm{GeV}, \tan \beta=5$ and for both signs of $\mu$, it should be noted, that the selection efficiency drops abruptly in the »corridor region « (with $m_{1 / 2} \approx 200-250 \mathrm{GeV}$, $m\left(\widetilde{\chi}_{1}^{ \pm}\right) \approx 130-180 \mathrm{GeV}$, and $\left.m\left(\widetilde{\chi}_{1}^{0}\right) \approx 74-96 \mathrm{GeV}\right)$, while increasing again to nearly $25 \%(\mu>0)$ and to about $14 \%(\mu<0)$ for values above $m_{1 / 2} \geq 250 \mathrm{GeV}$. This effect can be observed very clearly in Figure 5.2 (a) corresponding to $\mu>0$ and $m_{0}=100 \mathrm{GeV}$, but is also still visible in Figure 5.2 (b) for $\mu>0$ and $m_{0}=50 \mathrm{GeV}$, and in Figure 5.4 (a) for $\mu<0$ and $m_{0}=100 \mathrm{GeV}$.

This behaviour is explained by the distributions of branching ratios of the second lightest neutralino and the lightest chargino and the multiplicity of the so-called »additional « leptons as detailed in section 3.3. In this corridor region, the dominant decays are $\widetilde{\chi}_{1}^{ \pm} \rightarrow \widetilde{\tau}_{1} \nu_{\tau}$ and $\widetilde{\chi}_{2}^{0} \rightarrow \widetilde{\tau}_{1} \tau$, both of which do not produce any »additional « leptons. For $\mu>0$, the channel $\widetilde{\chi}_{2}^{0} \rightarrow(\widetilde{e}, \widetilde{\mu})_{R}+(e, \mu)$ opens at about $m_{1 / 2}=200-210 \mathrm{GeV}$, but although it produces up to two additional charged leptons in the final state the increase in efficiency is hardly visible. This is because the effect is weakened as long as the channel $\widetilde{\chi}_{1}^{ \pm} \rightarrow \widetilde{\tau}_{1} \nu_{\tau}$ remains open, i.e. again in the corridor region. The effect is not as pronounced for $\mu<0$ and the entire region is slightly shifted towards lower values of $m_{1 / 2},\left(m\left(\widetilde{\chi}_{1}^{ \pm}\right), m\left(\widetilde{\chi}_{1}^{0}\right)\right)$, but the effect is still visible in Figure 5.4 (a) corresponding to $m_{0}=100 \mathrm{GeV}$ and $\mu<0$. 

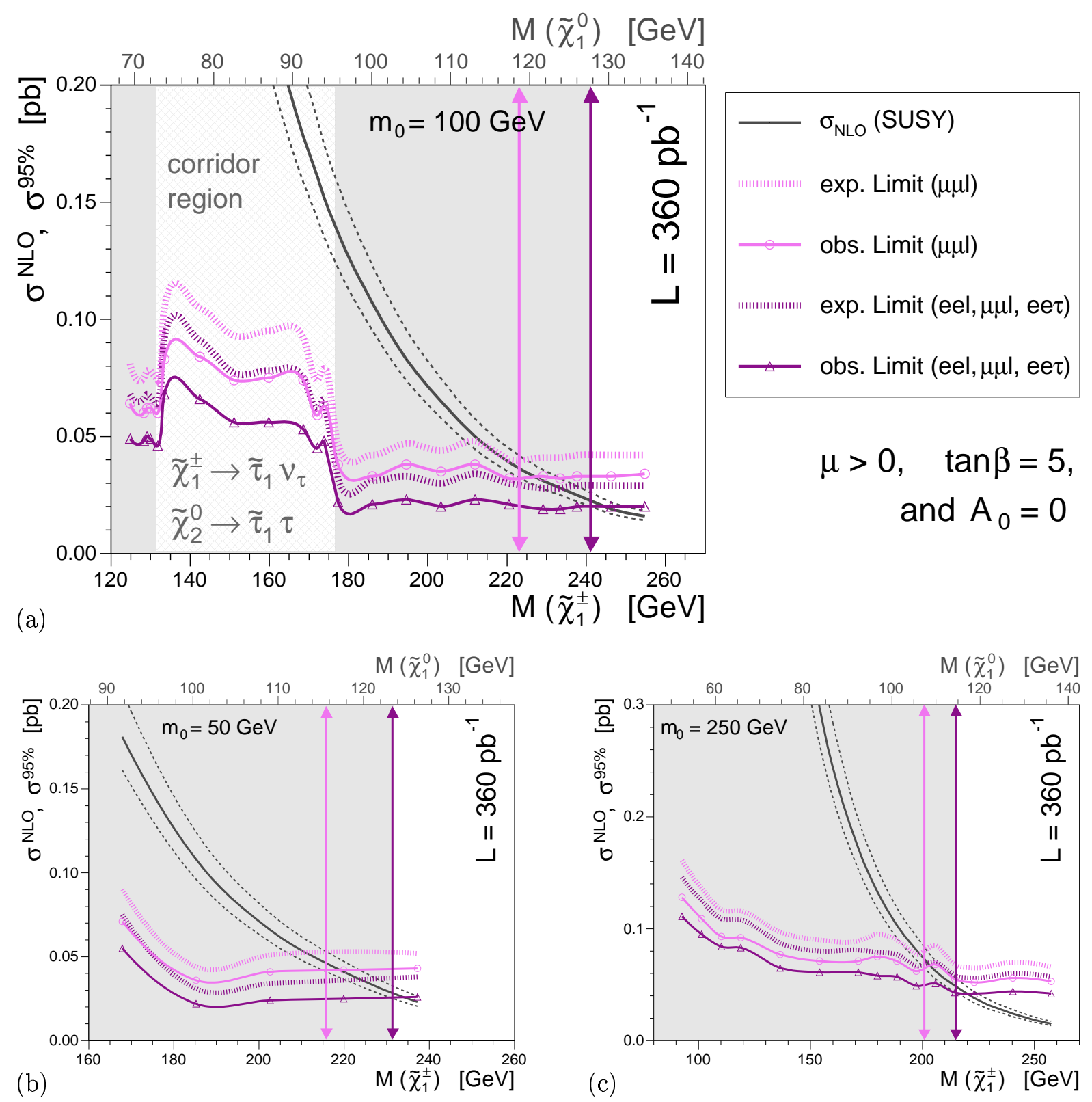

Figure 5.2: Upper limits at 95\% CL on the mSUGRA cross section for various values of $m_{0}$ and fixed parameters: $\tan \beta=5, A_{0}=0$, and $\mu>0$. The resulting lower bounds on the masses of the lightest chargino and neutralino are indicated by the shaded regions. The allowed mass ranges are to the right of these regions. (a) for $m_{0}=100 \mathrm{GeV}$, since the $»$ corridor region « is visible here, (b) for $m_{0}=50 \mathrm{GeV}$, and (c) for $m_{0}=250 \mathrm{GeV}$. See the legend for details on the different curves and the colour code.

In the adjacent region $m_{1 / 2} \approx 250-350 \mathrm{GeV}$, corresponding to $m\left(\widetilde{\chi}_{1}^{ \pm}\right) \approx 180-260 \mathrm{GeV}$, and $m\left(\widetilde{\chi}_{1}^{0}\right) \approx 96-140 \mathrm{GeV}$, the dominant decay of the lightest chargino is $\widetilde{\chi}_{1}^{ \pm} \rightarrow \widetilde{\chi}_{1}^{0} W$, since it is possible to produce the $W$ boson on-shell there. The observed large increase in efficiency is due to the fact that about $20 \%$ of the time, the $W$ boson contributes another charged lepton to the final state, enlarging the probability to detect/reconstruct three charged leptons. In addition, the channels $\widetilde{\chi}_{1}^{ \pm} \rightarrow \widetilde{\nu}_{i} \ell_{i}$ and $\widetilde{\chi}_{1}^{ \pm} \rightarrow(\widetilde{e}, \widetilde{\mu})+\left(\nu_{e}, \nu_{\mu}\right)$ open at about $m_{1 / 2} \approx 300 \mathrm{GeV}\left(m\left(\widetilde{\chi}_{1}^{ \pm}\right) \approx\right.$ $220 \mathrm{GeV})$ and $350 \mathrm{GeV}(260 \mathrm{GeV})$, respectively, so that it is possible to detect additional leptons in the final state for very large values of $m_{1 / 2}$, explaining the relatively low value of the upper cross section limit $\sigma_{S}^{95 \%}$. 

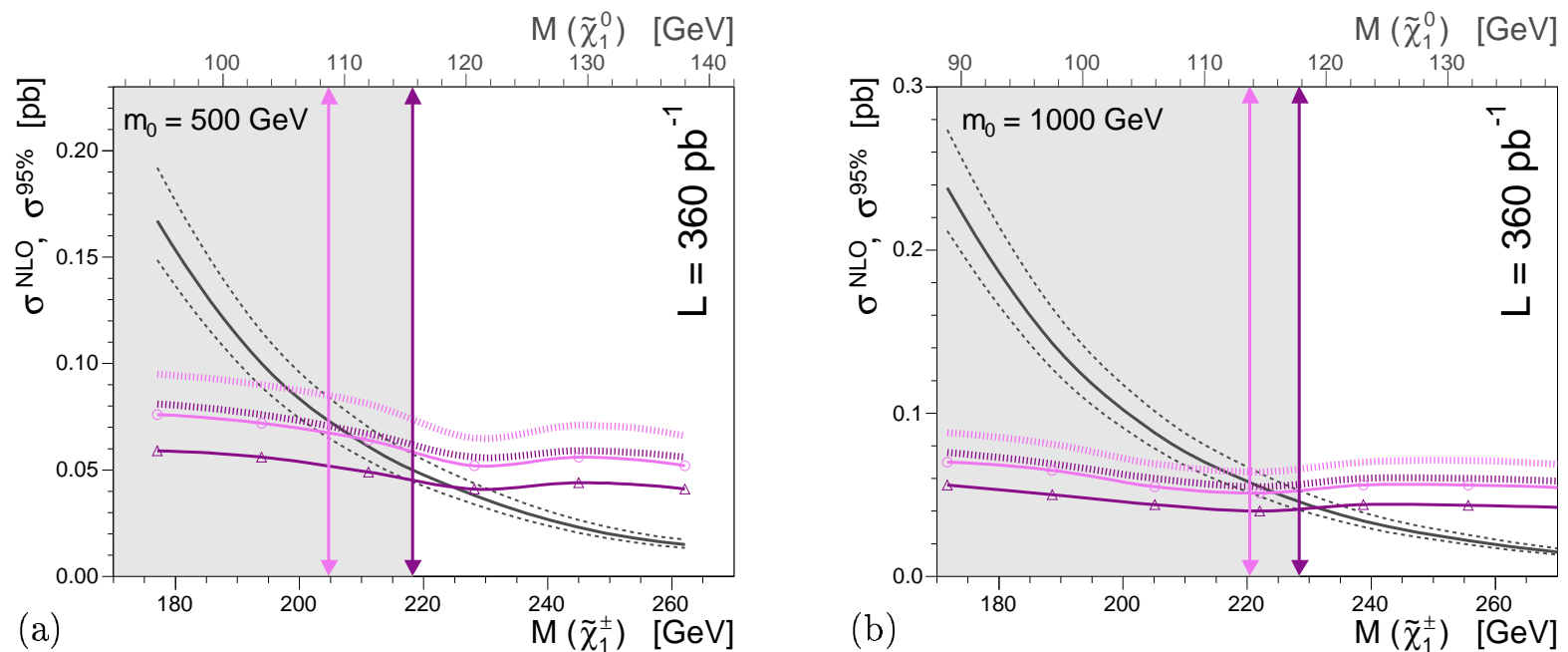

Figure 5.3: Upper limits at 95\% CL on the mSUGRA cross section for various values of $m_{0}$ and fixed parameters: $\tan \beta=5, A_{0}=0$, and $\mu>0$ for (a) $m_{0}=500 \mathrm{GeV}$ and (b) for $m_{0}=1000 \mathrm{GeV}$. The lower bounds on $m\left(\widetilde{\chi}_{1}^{ \pm}\right)$and $m\left(\widetilde{\chi}_{1}^{0}\right)$ are again indicated by the shaded regions.
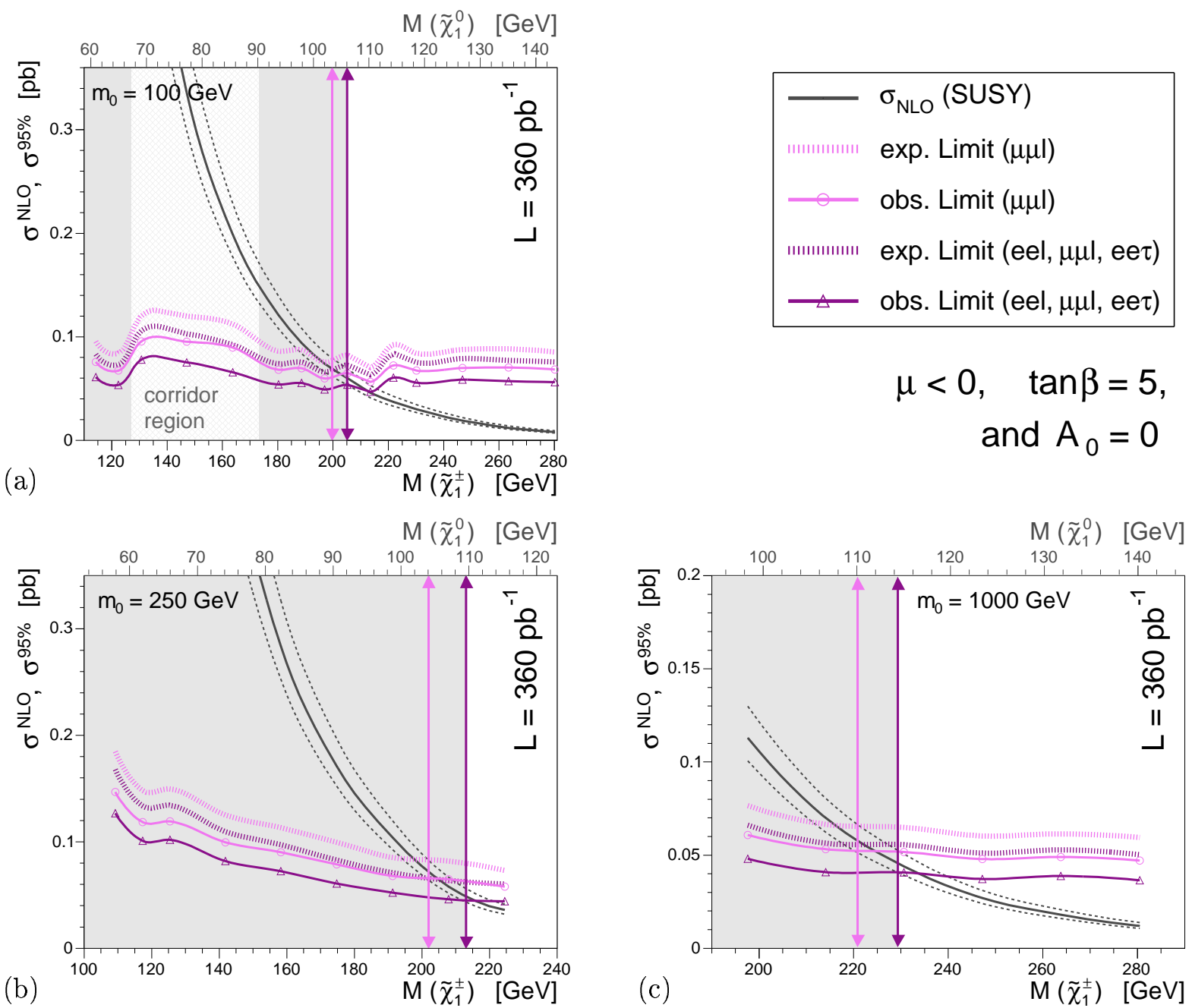

Figure 5.4: Upper limits at 95\% CL on the mSUGRA cross section for various values of $m_{0}$ and fixed parameters: $\tan \beta=5, A_{0}=0$, and $\mu<0$ for (a) $m_{0}=100 \mathrm{GeV}$, (b) $m_{0}=250 \mathrm{GeV}$, and (c) for $m_{0}=1000 \mathrm{GeV}$. The lower bounds on on $m\left(\widetilde{\chi}_{1}^{ \pm}\right)$and $m\left(\widetilde{\chi}_{1}^{0}\right)$ are indicated by the shaded regions. 
The resulting lower bounds on the masses of the lightest chargino and neutralino are summarised in Table 5.3 for the mSUGRA parameters $\tan \beta=5, A_{0}=0$, and for both signs of the Higgs mixing parameter.

\begin{tabular}{rcccc}
\hline \hline$\mu>0$ & \multicolumn{2}{c}{$\mu \mu \ell$ analysis alone } & \multicolumn{2}{c}{ Comb. analyses: $\mu \mu \ell+e e \ell(+e e \tau)$} \\
$m_{0}[\mathrm{GeV}]$ & $m\left(\widetilde{\chi}_{1}^{0}\right)[\mathrm{GeV}]$ & $m\left(\widetilde{\chi}_{1}^{ \pm}\right)[\mathrm{GeV}]$ & $m\left(\widetilde{\chi}_{1}^{0}\right)[\mathrm{GeV}]$ & $m\left(\widetilde{\chi}_{1}^{ \pm}\right)[\mathrm{GeV}]$ \\
\hline 50 & 115.6 & 215.8 & 123.5 & 231.5 \\
100 & 119.3 & 223.3 & 128.3 & 241.3 \\
250 & 107.8 & 200.9 & 114.8 & 215.0 \\
500 & 108.8 & 204.8 & 115.6 & 218.3 \\
1000 & 113.8 & 220.6 & 117.9 & 228.5 \\
\hline$\mu<0$ & $\mu \mu \ell$ analysis alone & Comb. analyses: $\mu \mu \ell+e e \ell(+e e \tau)$ \\
\hline 50 & 96.8 & 186.4 & 100.6 & 193.8 \\
100 & 103.4 & 199.8 & 106.0 & 205.1 \\
250 & 104.2 & 202.1 & 109.7 & 213.1 \\
500 & 107.1 & 209.9 & 109.6 & 229.4 \\
1000 & 110.0 & 221.0 & 114.3 & \\
\hline \hline
\end{tabular}

Table 5.3: Summary of the lower bounds on $m\left(\widetilde{\chi}_{1}^{ \pm}\right)$and $m\left(\widetilde{\chi}_{1}^{0}\right)$ in the $m S U G R A$ scenario for all signal points with parameters: $\tan \beta=5, A_{0}=0$, and for both signs of $\mu$.

The expected and observed cross section limits for mSUGRA points with $\tan \beta=20$ are about $25 \%-30 \%$ lower. Since the masses of the gauginos are roughly about $10 \%$ larger for $\tan \beta=20$, the resulting lower bounds on the masses of the lightest neutralino and chargino are slightly higher than for $\tan \beta=5$.

In order to provide a coherent picture and also to allow for an easier comparison with other analyses, e.g. the D $\varnothing$ Run I analysis [152], or the exclusion region of the $\not R_{p}$-SUSY searches at LEP [138] and [153], the obtained exclusion contours are presented in two-dimensional figures. For mSUGRA signal points with $\tan \beta=5$ and both signs of the Higgs mixing parameter $\mu$, the exclusion areas are presented in the $\left(m_{0}, m_{1 / 2}\right)$ plane (see Fig. 5.5), the $\left(m_{0}, m\left(\widetilde{\chi}_{1}^{ \pm}\right)\right)$plane (see Fig. 5.6), and in the $\left(m_{0}, m\left(\widetilde{\chi}_{1}^{0}\right)\right)$ plane (see Fig. 5.7), respectively. A linear interpolation between the generated points (black crosses) is used to obtain the final contours. The observed exclusion from the combined analyses is represented in light blue and the expected lower mass bounds are shown as dark blue, dashed line. Signal points excluded by the combined analyses are shown as dark red stars and signal points excluded by the $\mu \mu \ell$ analysis alone are represented as light red open circles. All other contours, slepton mass limits, the chargino mass limit from measurements at LEP [138] and the results from the D $\varnothing$ Run I analysis are shown as grey shaded (hatched) areas or lines. The area labeled $\tilde{\ell}_{R}$ (at low $m_{0}$ and $m_{1 / 2}$ values) is the combined exclusion region of searches for $\widetilde{e}_{R}, \widetilde{\mu}_{R}$, and $\widetilde{\tau}_{R}[181]$.

The obtained exclusion limits from this analysis alone - and even more so in combination with the two other $\not R_{p}$-trilepton searches - are the most stringent limits on gaugino pair production with a subsequent decay via the $L L \bar{E}$ coupling $\lambda_{122}$. Since the strength of the coupling only enters in the decay, the analysis is sensitive down to neutralino masses of $m\left(\widetilde{\chi}_{1}^{0}\right) \approx 50 \mathrm{GeV}$. The sensitivity probably even extends to lower masses, since the ratio between cross section and exclusion limit for points with $m_{0}=50 \mathrm{GeV}$ points at the lower edge (see Figure5.5) permits this conclusion. However, signal points with lower masses have not been studied explicitely. 


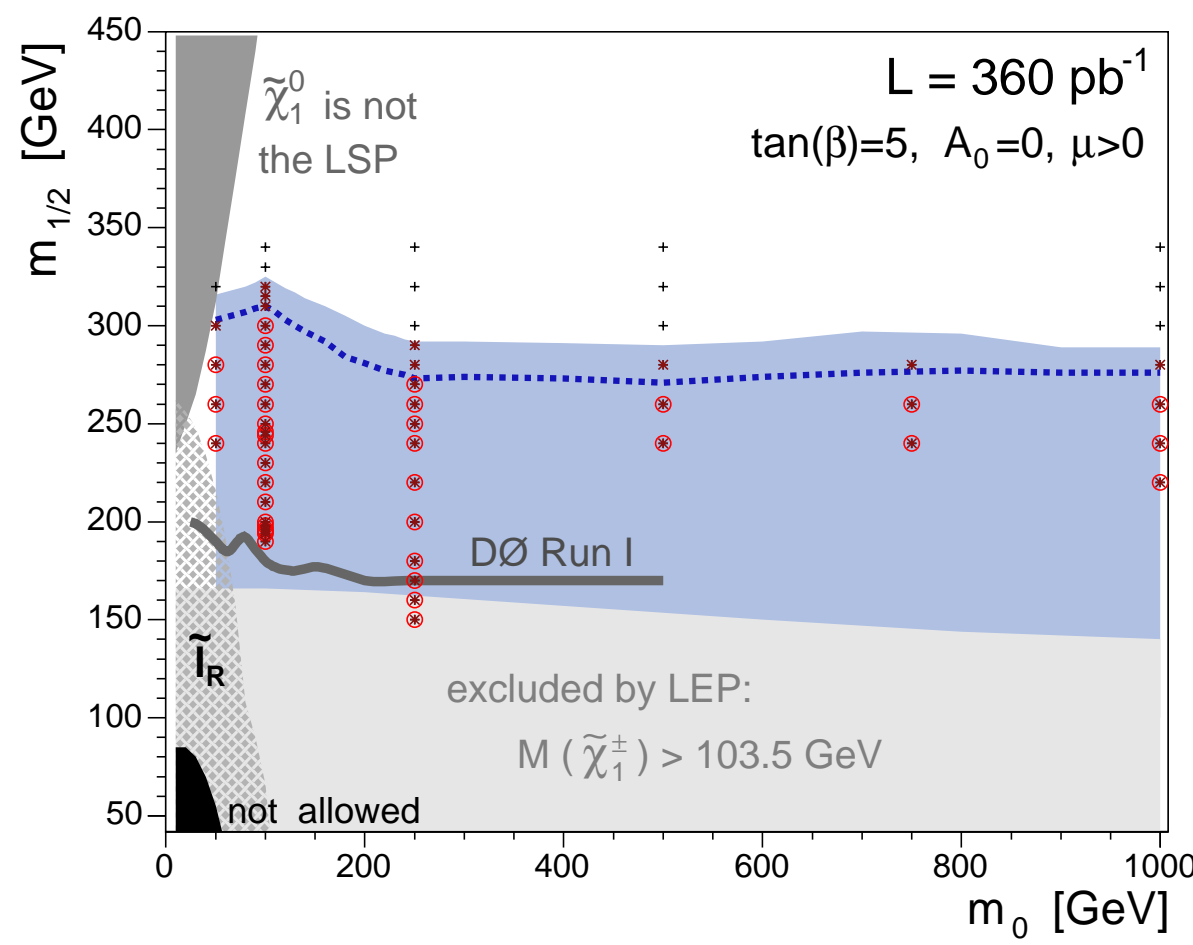

(a)

+ generated points

$\mu \mu \mathrm{l}$ alone:

- excluded points

$\mu \mu \mathrm{l}$, eel, ee $\tau$ combined:

* excluded points

obs. exclusion

exp. exclusion

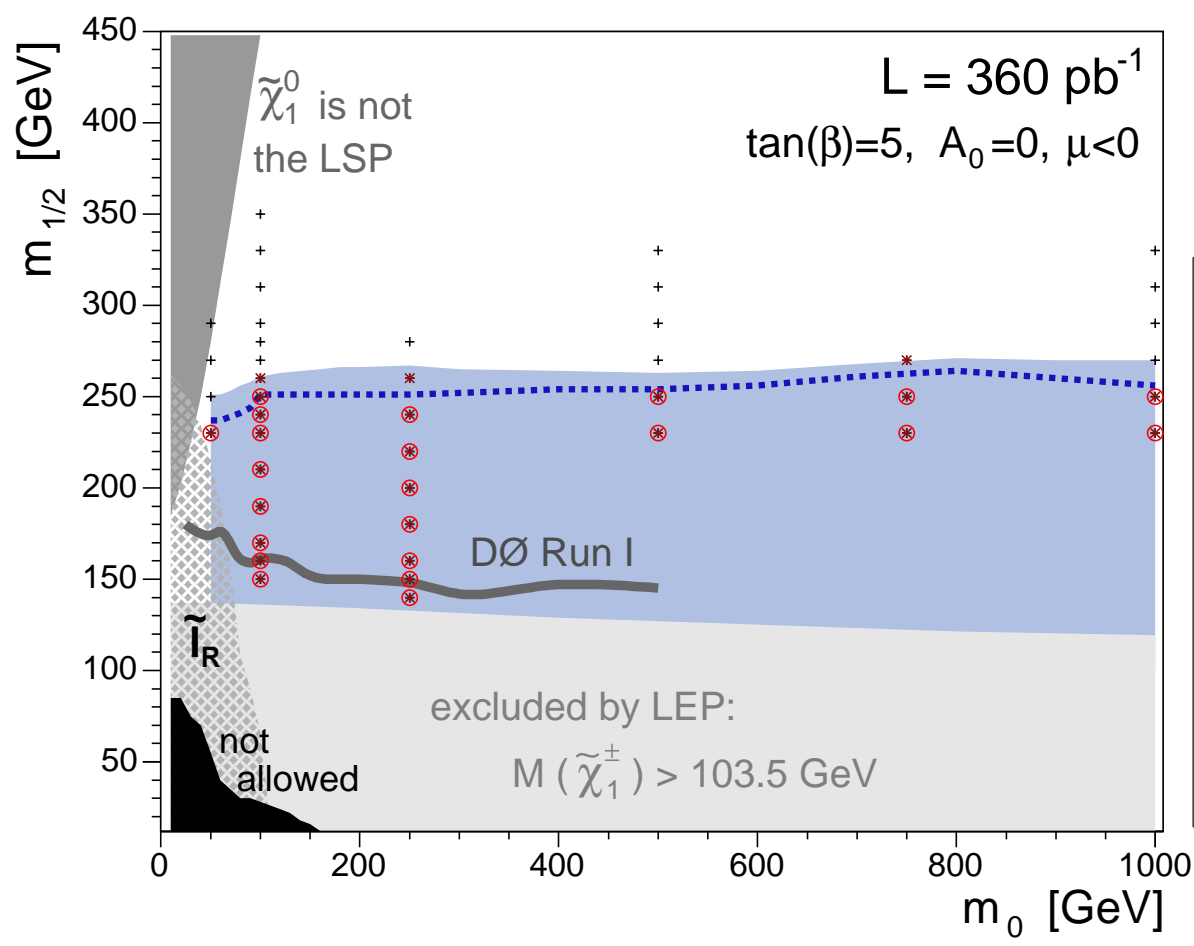

(b)

+ generated points

$\mu \mu$ l alone:

○ excluded points

$\mu \mu \mathrm{l}$, eel, ee $\tau$ combined:

* excluded points

obs. exclusion

exp. exclusion

Figure 5.5: Exclusion contour in the $\left(m_{0}, m_{1 / 2}\right)$ plane of the mSUGRA parameter space with the fixed parameters $\tan \beta=5$, and $A_{0}=0$ : (a) for positive Higgs mixing parameter $\mu$ and (b) for negative $\mu$. The different exclusion areas and lines are either labeled in the plot itself or detailed in the legend. 


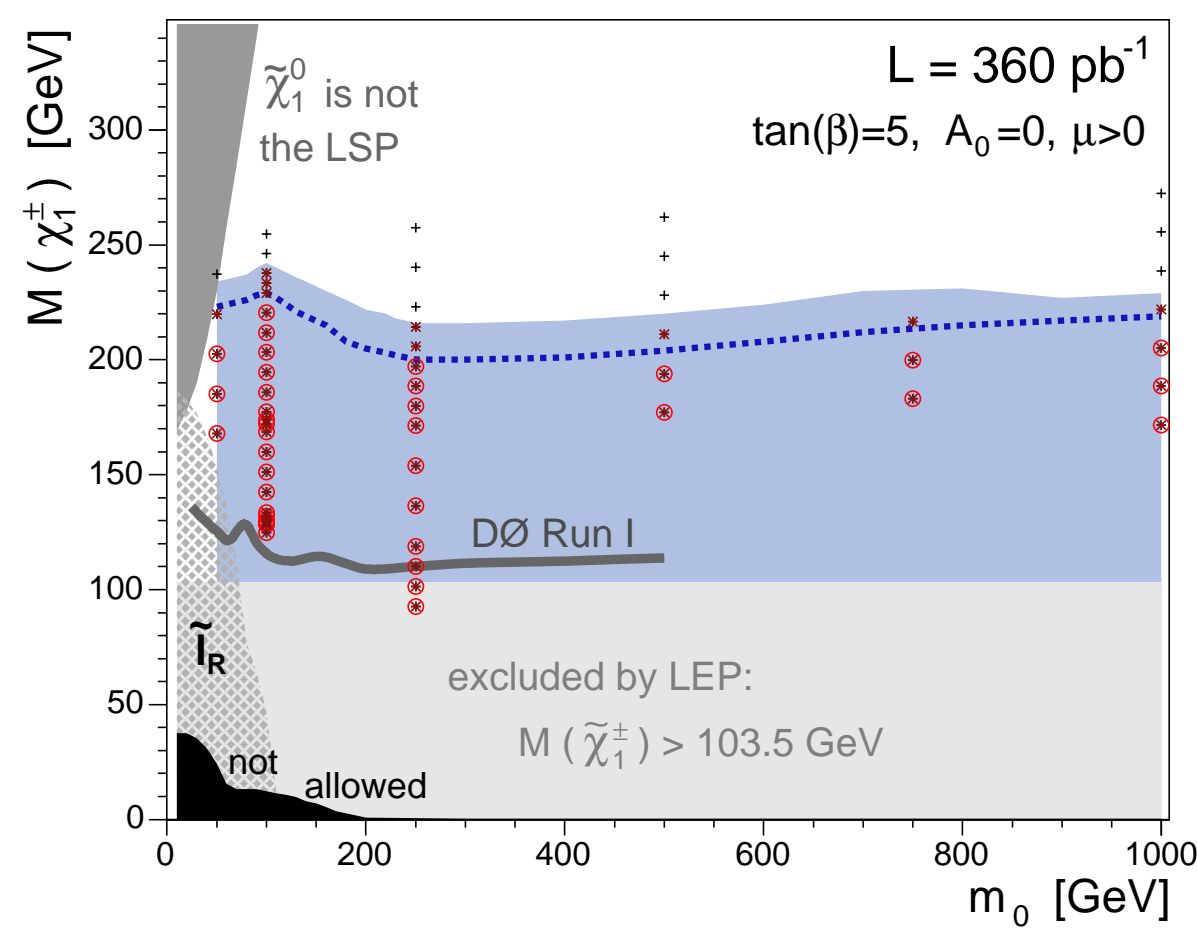

(a)

+ generated points

$\mu \mu$ I alone:

- excluded points

$\mu \mu$ I, eel, ee $\tau$ combined:

* excluded points

obs. exclusion

exp. exclusion

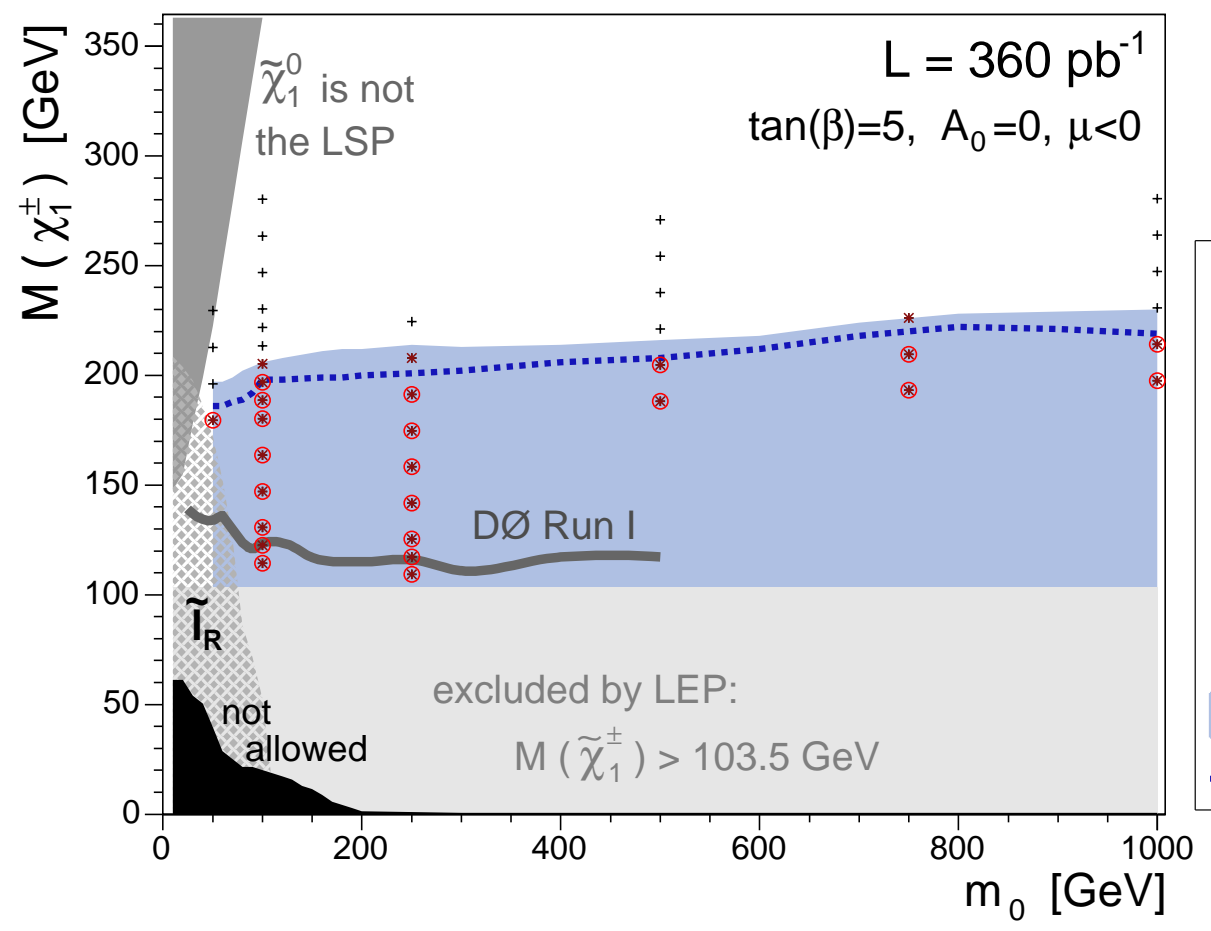

(b)

$+\quad$ generated points

$\mu \mu$ I alone:

$\circ$ excluded points

$\mu \mu$ I, eel, ee $\tau$ combined:

* excluded points

obs. exclusion

exp. exclusion

Figure 5.6: Exclusion contour in the $\left(m_{0}, m\left(\widetilde{\chi}_{1}^{ \pm}\right)\right)$plane of the mSUGRA parameter space with the fixed parameters $\tan \beta=5$, and $A_{0}=0$ : (a) for positive Higgs mixing parameter $\mu$ and (b) for negative $\mu$. The different exclusion areas and lines are either labeled in the plot itself or detailed in the legend. 


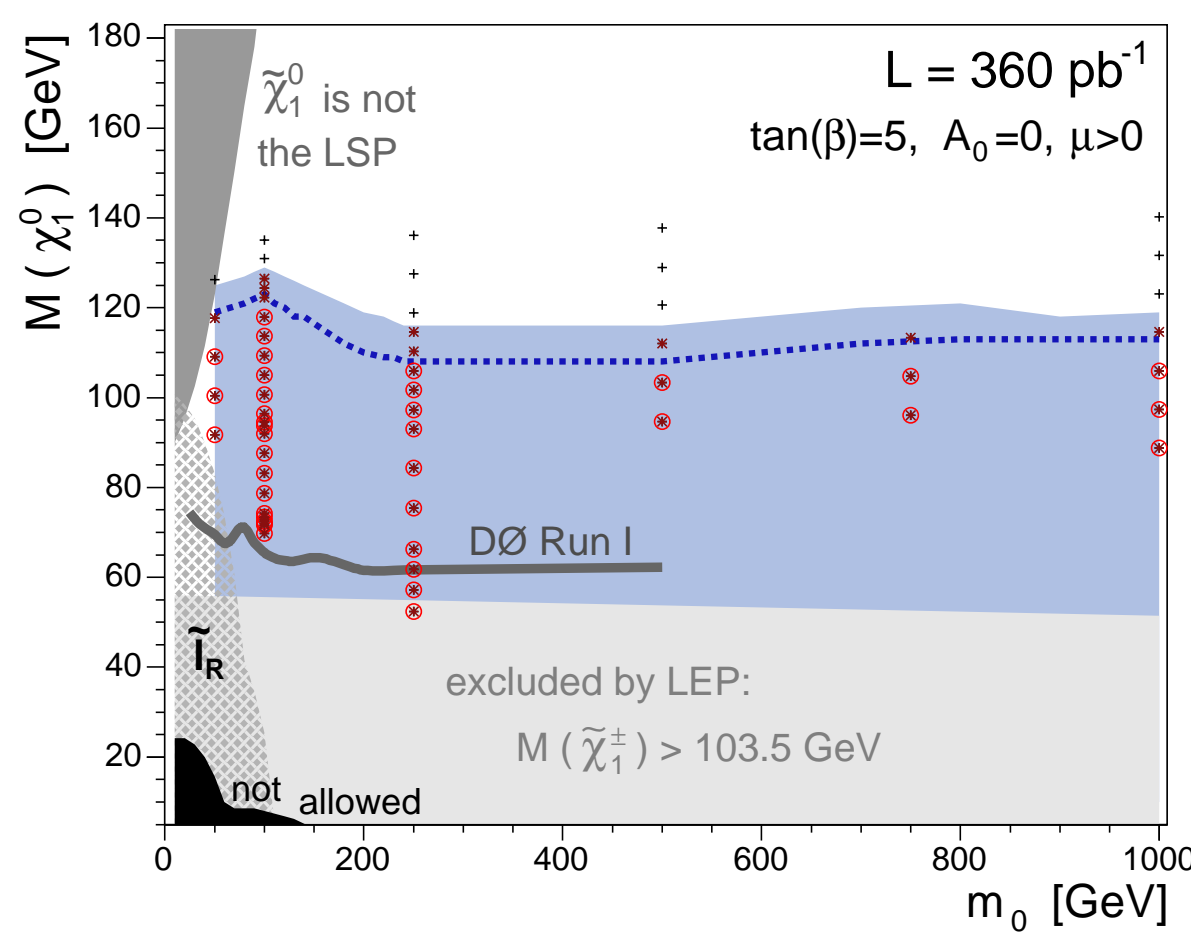

(a)

$+\quad$ generated points

$\mu \mu \mathrm{I}$ alone:

- excluded points

$\mu \mu$ l, eel, ee $\tau$ combined:

* excluded points

obs. exclusion

exp. exclusion

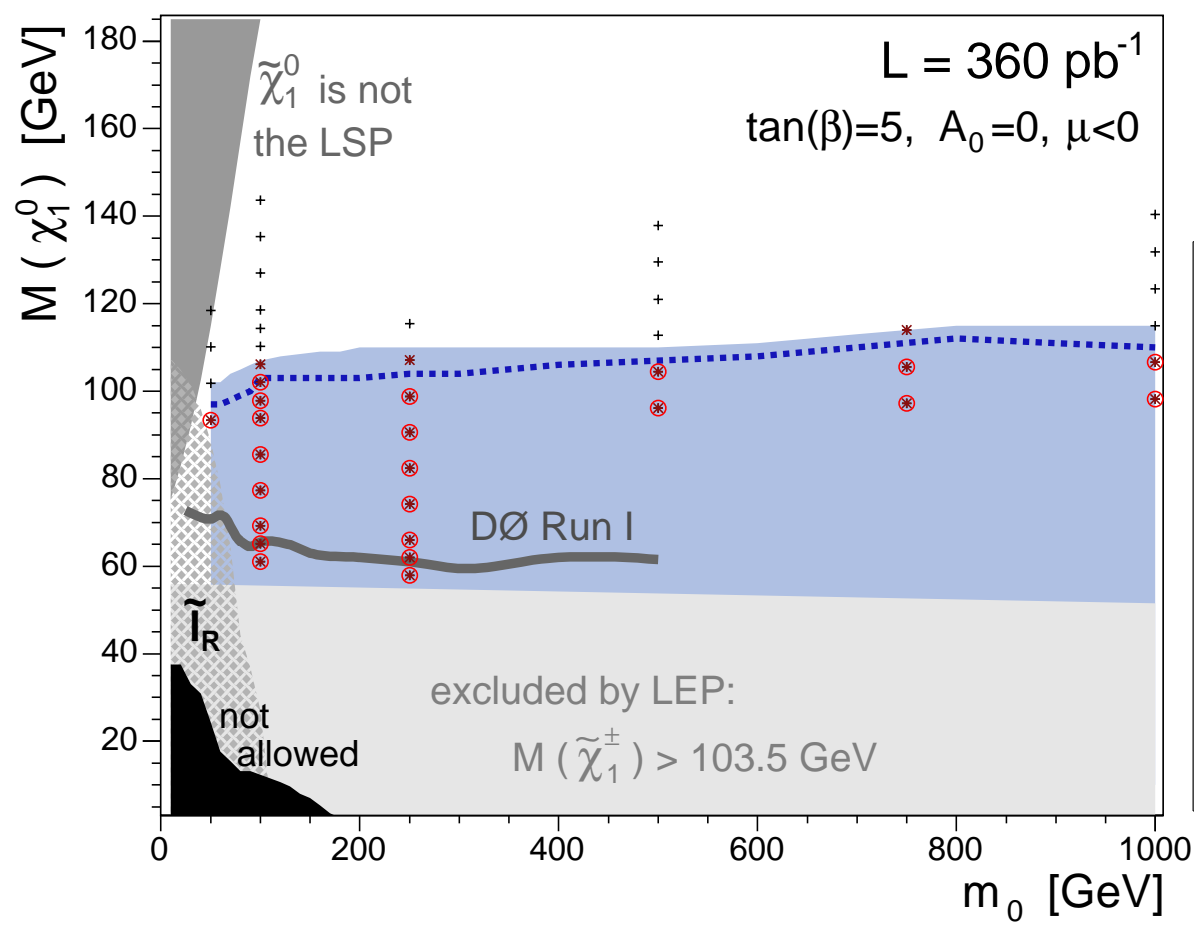

(b)

+ generated points

$\mu \mu$ I alone:

- excluded points

$\mu \mu$ l, eel, ee $\tau$ combined:

* excluded points

obs. exclusion

exp. exclusion

Figure 5.7: Exclusion contour in the $\left(m_{0}, m\left(\widetilde{\chi}_{1}^{0}\right)\right)$ plane of the mSUGRA parameter space with the fixed parameters $\tan \beta=5$, and $A_{0}=0$ : (a) for positive Higgs mixing parameter $\mu$ and (b) for negative $\mu$. The different exclusion areas and lines are either labeled in the plot itself or detailed in the legend. 


\subsection{Interpretation within the »no-GUT « MSSM Scenario}

The cross section limits at $95 \% \mathrm{CL}$ are also interpreted in the $\gg$ no-GUT $«$ MSSM model, introduced in section 1.3.5. The model parameters for the study of the MSSM scenario are chosen in the following way: the ratio of the Higgs VEV's is set to $\tan \beta=5$, the Higgs mixing parameter is $\mu=1 \mathrm{TeV}$, the trilinear coupling at the GUT scale is given by $A_{0}=0$, and the pseudoscalar Higgs mass is fixed at $m_{A}=400 \mathrm{GeV}$.

As for the mSUGRA model, all limits are calculated with respect to the next-to-leading cross section reduced by its total systematic uncertainty (see section 4.6.5). The NLO cross section is given by the SusYGEN LO cross section, which is then multiplied by the corresponding $K$-factor of 1.16 (large values of $M_{1}$ and $M_{2}$ ) up to to 1.28 (small $M_{1}$ and $M_{2}$ ). The factor is again obtained using the package GaUGinos (see section 4.2.2). For the actual cross section limits calculated for all MSSM signal points, the reader is referred to the Tables D.1, D.2 and D.3 in appendix D. All tables contain the NLO cross section of the studied point, the expected and observed upper cross section limits at $95 \%$ CL for the $\mu \mu \ell$ analysis alone and for the combination of this analysis with the two complementary analyses of eel and $e e \tau$ final states. In addition, the $\mu \mu \ell$ signal selection efficiency is provided and the fractional overlap between this analysis $(\mu \mu \ell)$ and the eel analysis. (The overlap with the eer analysis is at most 1-2\% and not detailed in the tables.)

All systematic uncertainties discussed in section 4.6 are considered in the calculation of the limits. For the extraction of the mass bounds, the signal cross sections are again reduced by their respective systematic uncertainty (see also section 5.2), so that the lower mass bounds in the following one-dimensional figures are given by the intersection of the observed $\sigma_{S}^{95 \%}$ curve with the lower edge of the cross section error band.

A variation of the chargino mass, with the neutralino mass fixed to $m\left(\widetilde{\chi}_{1}^{0}\right)=80 \mathrm{GeV}$ and $100 \mathrm{GeV}$, leads to Figure 5.8. The limit curves (expected and observed limits) are nearly constant as a function of the chargino mass, while the cross section shows an exponential decrease with increasing chargino mass.
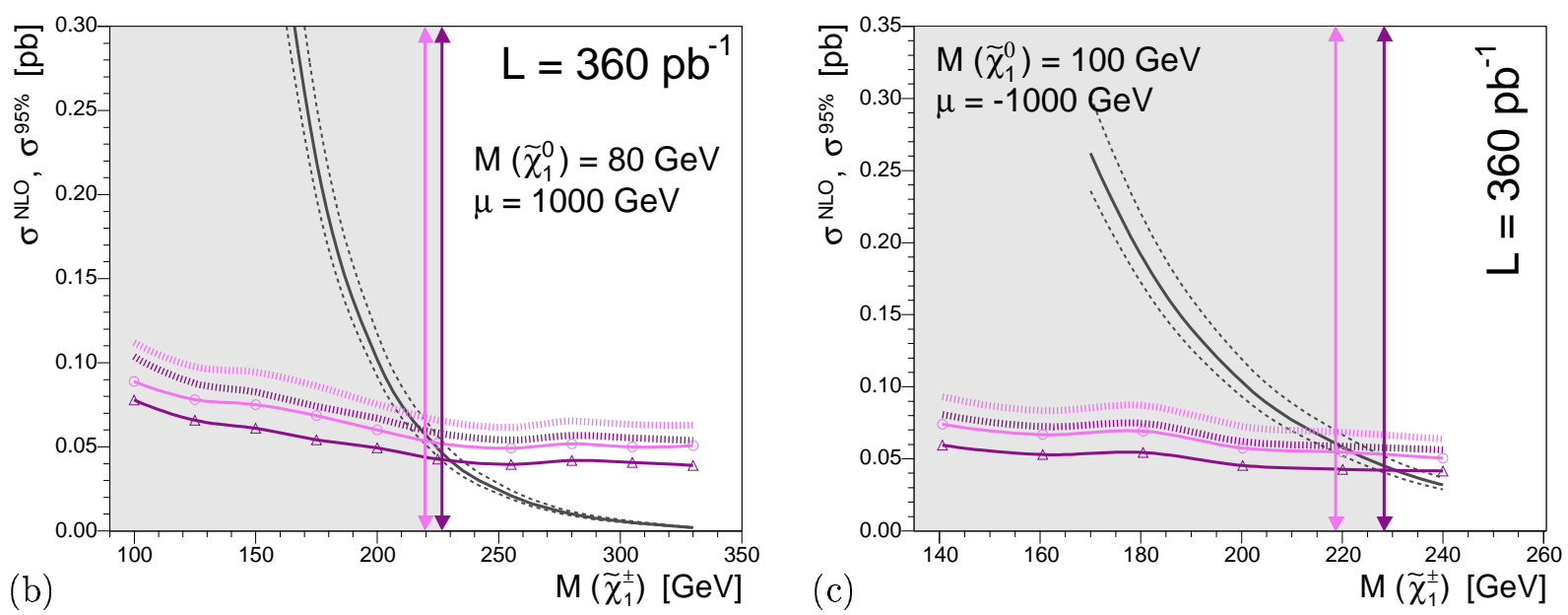

Figure 5.8: Upper cross section limits at 95\% CL for MSSM signal points with the neutralino mass fixed to $(\mathrm{a}) m\left(\widetilde{\chi}_{1}^{0}\right)=80 \mathrm{GeV}\left(M_{1} \approx 81 \mathrm{GeV}\right)$ and $(\mathrm{b}) m\left(\widetilde{\chi}_{1}^{0}\right)=100 \mathrm{GeV}\left(M_{1} \approx 100 \mathrm{GeV}\right)$. The remaining MSSM model parameters are set to: $\tan \beta=5, A_{0}=0$, and $M_{A}=400 \mathrm{GeV}$. (See the legend in Fig. 5.9 for the different curves and the colour code.) 
Figure 5.9 illustrates the lower mass bounds on the mass of the lightest neutralino derived for chargino masses fixed to $m\left(\widetilde{\chi}_{1}^{ \pm}\right)=103.5 \mathrm{GeV}$ (LEP-limit [138]), $200 \mathrm{GeV}$, and $225 \mathrm{GeV}$. The cross section curves for fixed chargino masses are flat as a function of the neutralino mass. As can be seen, the cross section lies far above the expected and observed limit curves for $m\left(\widetilde{\chi}_{1}^{ \pm}\right)=103.5 \mathrm{GeV}$, so that the neutralino masses for all studied points are clearly excluded. In case of $m\left(\widetilde{\chi}_{1}^{ \pm}\right)=200 \mathrm{GeV}$, the cross sections are only slightly larger than the observed limits and neutralino masses above $m\left(\widetilde{\chi}_{1}^{0}\right)=30 \mathrm{GeV}$ are excluded. Finally, for $m\left(\widetilde{\chi}_{1}^{ \pm}\right)=225 \mathrm{GeV}$, the cross sections and limits are of the same size, so that only very few signal points can be excluded.

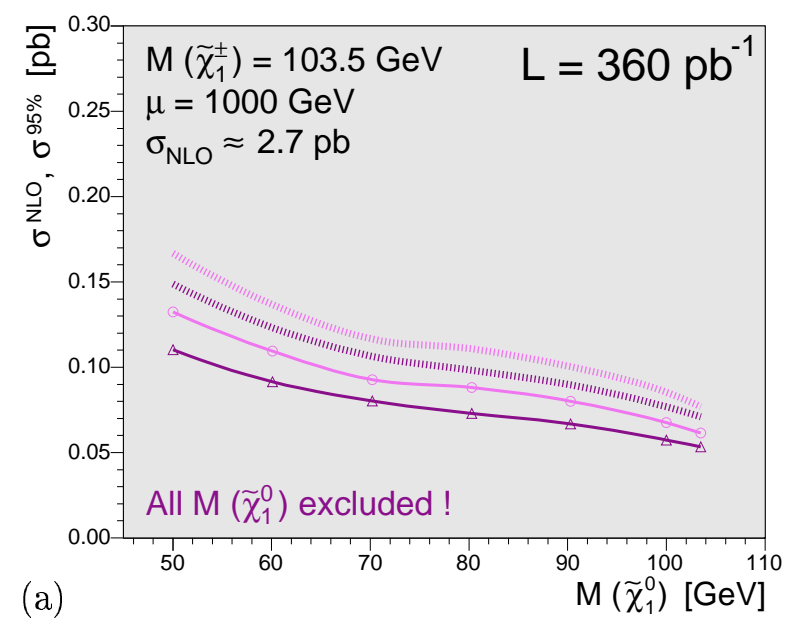

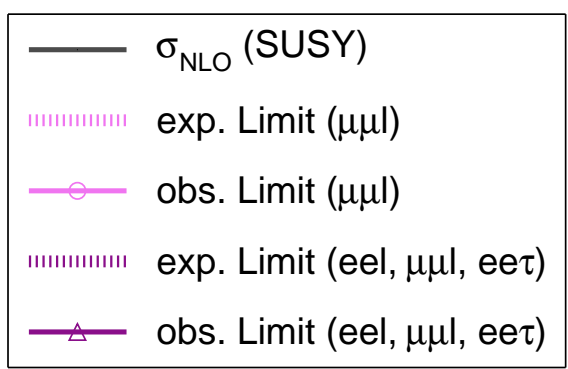

$\tan \beta=5, \quad \mu=1 \mathrm{TeV}$,
$\mathrm{A}_{0}=0, \quad \mathrm{M}_{\mathrm{A}}=400 \mathrm{GeV}$
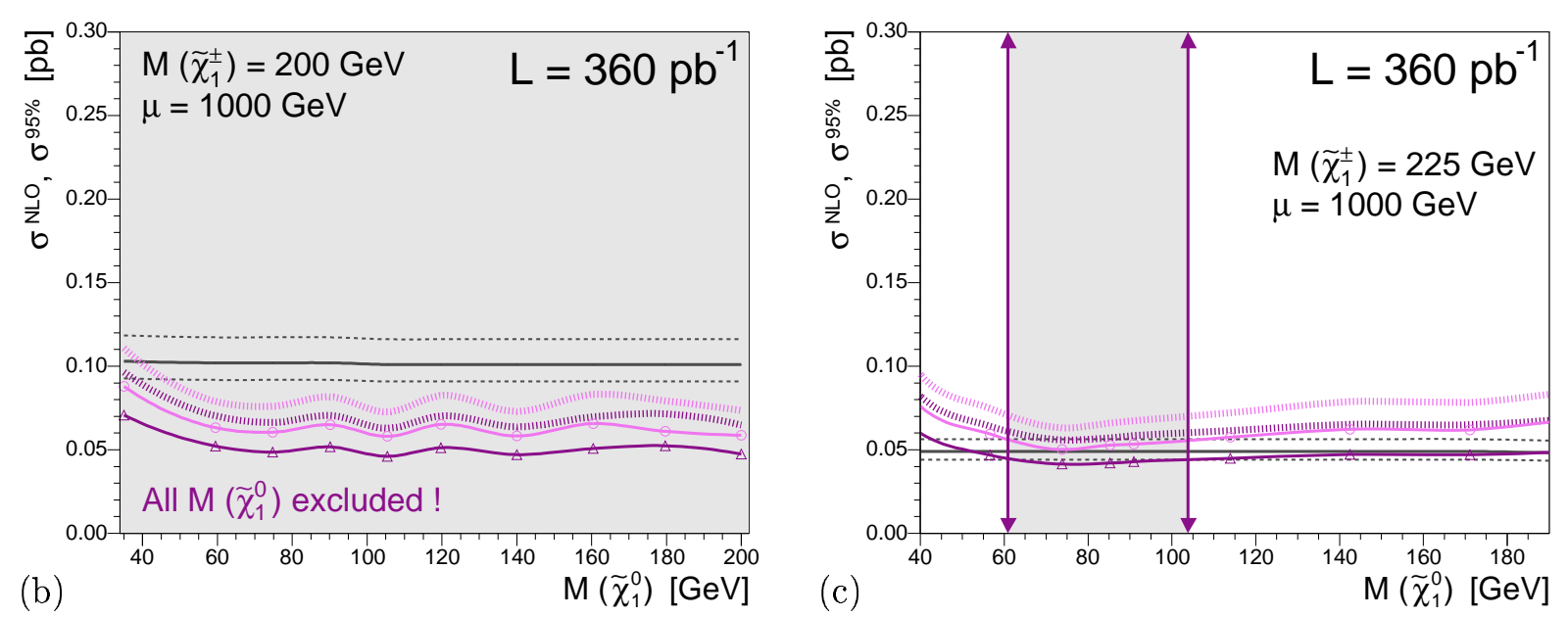

Figure 5.9: Upper cross section limits at 95\% CL for MSSM signal points with the chargino mass fixed to (a) $m\left(\widetilde{\chi}_{1}^{ \pm}\right)=103.5 \mathrm{GeV}\left(M_{2}=106.7 \mathrm{GeV}\right)$, (b) $m\left(\widetilde{\chi}_{1}^{ \pm}\right)=200 \mathrm{GeV}\left(M_{2}=204 \mathrm{GeV}\right)$, and (c) $m\left(\widetilde{\chi}_{1}^{ \pm}\right)=225 \mathrm{GeV}\left(M_{2}=229 \mathrm{GeV}\right)$. The remaining MSSM model parameters are set to: $\tan \beta=5$, $\mu=1 \mathrm{TeV}, A_{0}=0$, and $M_{A}=400 \mathrm{GeV}$. The cross section curves are flat as a function of the neutralino mass, leading to the observed regional exclusion for $m\left(\widetilde{\chi}_{1}^{ \pm}\right)=225 \mathrm{GeV}$.

The two-dimensional Figure 5.10 illustrates the interpretation of the cross section limits in the $\left(\widetilde{\chi}_{1}^{0}, \widetilde{\chi}_{1}^{ \pm}\right)$-mass plane of the MSSM scenario. Analogously to the two-dimensional mSUGRA figures, a linear interpolation between the generated points (black crosses) is used to obtain the final contours. The observed exclusion from the combined analyses is represented in light blue and the expected lower bound on the mass of the lightest chargino is shown as dark blue, dashed line. Signal points excluded by the combined analyses are shown as dark red stars and signal points excluded by the $\mu \mu \ell$ analysis alone are represented as light red open circles. 
Contrary to mSUGRA, no absolute lower mass bounds can be set in the MSSM scenario. This is due to the fact, that the analysis is only sensitive down to neutralino masses of $m\left(\widetilde{\chi}_{1}^{0}\right) \approx 30 \mathrm{GeV}$. Lower neutralino masses cannot be investigated, because of the combined effect of the lifetime of the lightest neutralino and the choice of the coupling strength for the $\not_{p}$-coupling $\lambda_{122}$. For a coupling strength of $\lambda_{122}=0.01$, the decay length of the neutralino becomes larger than $1 \mathrm{~cm}$, which was chosen as cutoff, to be able to rely on the Tracker and the tracking algorithms of the D $\varnothing$ experiment. Neutralinos with decay lengths above $1 \mathrm{~cm}$ are then simply passed on to the detector simulation software, which does not recognise these particles, so that they remain stable. As a result, there are no charged leptons from the neutralino $\mathbb{R}_{p}$-decays and thus the signal selection efficiency drops to zero (or close to zero) right below $m\left(\widetilde{\chi}_{1}^{0}\right)=30 \mathrm{GeV}$, see also section 3.1. From measurements by the four LEP experiments the region below a chargino mass of $m\left(\widetilde{\chi}_{1}^{ \pm}\right)=103.5$ is excluded [138], corresponding to the grey hatched area in Figure 5.10.

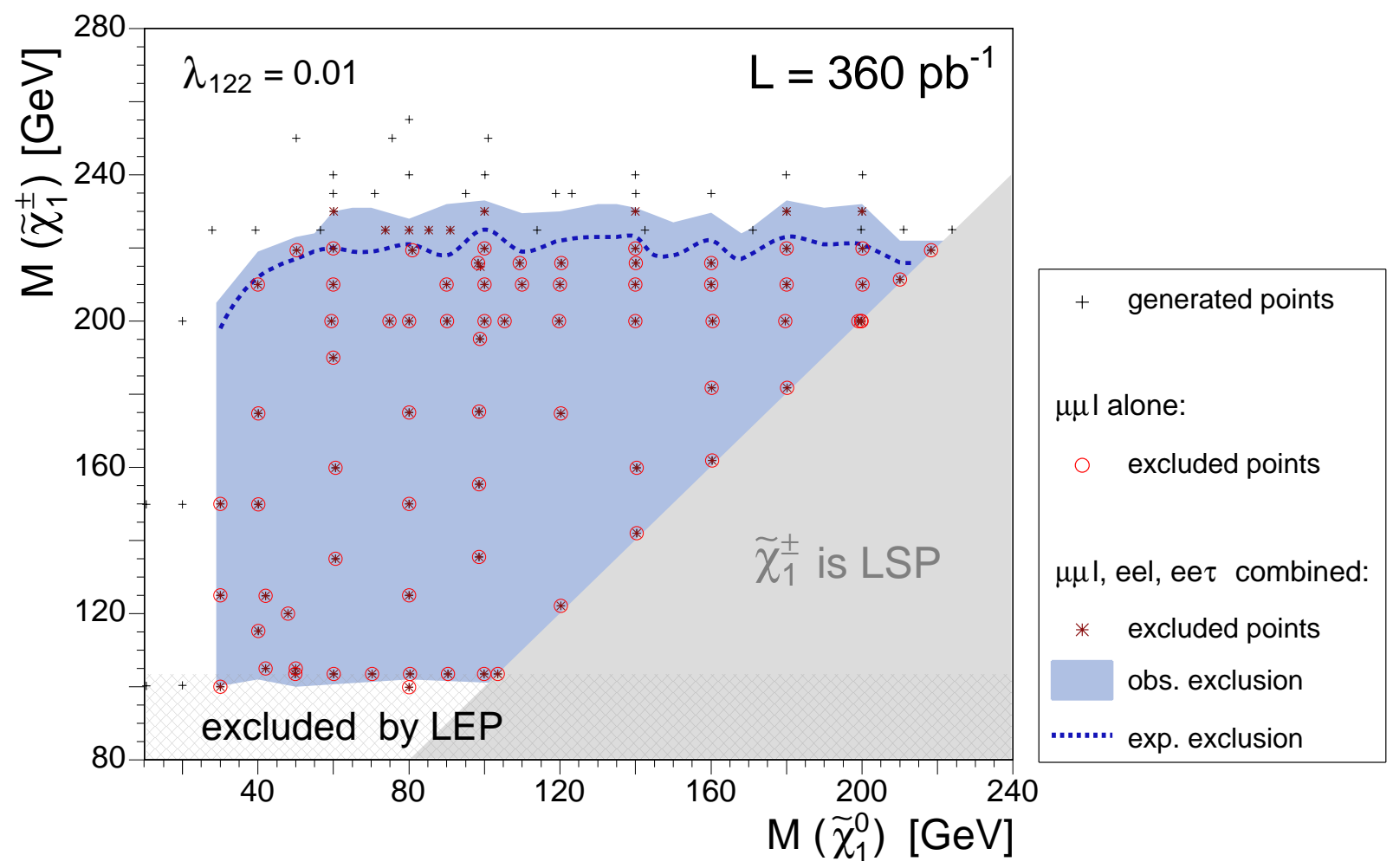

Figure 5.10: Exclusion contour in the $\left(\widetilde{\chi}_{1}^{0}, \widetilde{\chi}_{1}^{ \pm}\right)$-mass plane of the MSSM model. The parameters are chosen as follows: $\tan \beta=5, \mu=1 \mathrm{TeV}, A_{0}=0$, and $M_{A}=400 \mathrm{GeV}$.

The sensitivity and exclusion area for the $\not R p_{p}$-coupling $\lambda_{121}$ is very similar. In this case, the eel analysis has the highest signal-to-background ratio, compare also [180]. Contrary to this the signal selection efficiency of the eet analysis is lower, hence the exclusion area for the coupling $\lambda_{133}$ considerably smaller. 


\section{Conclusion and Outlook}

A search for the trilepton signature $\mu \mu \ell$ (with $\ell=e$, or $\mu$ ) from chargino-neutralino production with a subsequent lepton number violating decay via the operator $L L \bar{E}$ has been presented. The coupling strength was chosen to be $\lambda_{122}=0.001$ in case of the mSUGRA scenario and $\lambda_{122}=0.01$ in case of the MSSM model. In both these models, the lightest neutralino is assumed to be the lightest supersymmetric particle that decays via the $L L \bar{E}$ operator into SM leptons. Since the operator couples two weak isospin doublet and one singlet (s)lepton fields, two charged leptons and one neutrino are produced from a neutralino decay, while three charged leptons or one charged and two neutral leptons are produced from a direct $\not \mathbb{p}_{p}$-decay of a chargino. The flavour of the leptons depends on the generation indices of the operator and the $\lambda_{i j k}$ coupling, so that that an $L L \bar{E}$ operator with a $\lambda_{122}$ coupling produces leptons of the first two generations, i.e. electrons and muons together with their respective neutrinos.

The data, corresponding to an integrated luminosity of $\int \mathcal{L} d t=360 \pm 23 \mathrm{pb}^{-1}$, was collected with the DØ detector at the Fermilab $p \bar{p}$ collider Tevatron form April 2002 until August 2004. While no event is observed in the data, $0.41 \pm 0.11$ (stat) \pm 0.07 (sys) events are expected from SM background processes. Due to the non-observation of an excess of events in the data, any new physics producing a $\mu \mu \ell$ trilepton signature in the detector that is detected with an efficiency times acceptance ranging from $5 \%$ and $26 \%$, is excluded as long as the cross section is larger than the corresponding cross section upper limits of $0.151 \mathrm{pb}$ and $0.029 \mathrm{pb}$, respectively.

These cross section upper limits are interpreted in two $\not R_{p}$-SUSY scenarios: the mSUGRA and the MSSM model. Lower bounds on the masses of the lightest neutralino and chargino are extracted. For the mSUGRA parameters $\tan \beta=5$, and $A_{0}=0$, the lower mass bounds are found to lie within the following ranges, depending on the values of $m_{0}, m_{1 / 2}$, and $\tan \beta$ :

$$
\begin{array}{llll}
\text { mSUGRA, } \mu>0: & \mathrm{M}\left(\widetilde{\chi}_{1}^{0}\right) \gtrsim 115-128 \mathrm{GeV} & \text { and } & \mathrm{M}\left(\widetilde{\chi}_{1}^{ \pm}\right) \gtrsim 215-241 \mathrm{GeV} \\
\text { mSUGRA }, \mu<0: & \mathrm{M}\left(\widetilde{\chi}_{1}^{0}\right) \gtrsim 101-114 \mathrm{GeV} & \text { and } & \mathrm{M}\left(\widetilde{\chi}_{1}^{ \pm}\right) \gtrsim 194-230 \mathrm{GeV}
\end{array}
$$

The expected and observed cross section limits for mSUGRA points with $\tan \beta=20$ are about $25 \%-30 \%$ lower. This is explained by the fact, that the gaugino masses are roughly about $10 \%$ larger for $\tan \beta=20$, compared to $\tan \beta=5$. Hence, the resulting lower bounds on the masses of the lightest neutralino and chargino are slightly higher than for $\tan \beta=5$, so that the results quoted above represent the most conservative scenario.

Contrary to the case of the mSUGRA model, no absolute lower bounds on the masses of the lightest neutralino or chargino can be derived within the MSSM scenario. This is due to the fact, that the analysis is not sensitive for neutralino masses below $m\left(\widetilde{\chi}_{1}^{0}\right) \approx 30 \mathrm{GeV}$. In case of the mSUGRA model, this was not problematic, since from measurements by the four LEP experiments the region below a chargino mass of $m\left(\widetilde{\chi}_{1}^{ \pm}\right)=103.5$ is excluded [138]. Due to the GUT-relation, this chargino lower mass bound can be translated into a neutralino mass bound of $m\left(\widetilde{\chi}_{1}^{0}\right)>52 \mathrm{GeV}$ for $\tan \beta \geq 5$. However, this is impossible in case of the MSSM model, 
since the GUT-relation between $M_{1}$ and $M_{2}$, the two parameters, governing the masses of the superpartners of the $\mathrm{U}(1)_{Y}$ and $\mathrm{SU}(2)_{L}$ gauge bosons, is assumed to be non-existent. Therefore no lower bound on the mass of the lightest neutralino exists and a lower bound on the mass of the lightest chargino can only be formulated for neutralino masses above $30 \mathrm{GeV}$.

The lower bound on the mass of the lightest chargino in the MSSM model is found to be:

and increases to:

$$
\begin{array}{lll}
m\left(\widetilde{\chi}_{1}^{ \pm}\right)>205 \mathrm{GeV} & \text { for } & m\left(\widetilde{\chi}_{1}^{0}\right)=30 \mathrm{GeV} \\
m\left(\widetilde{\chi}_{1}^{ \pm}\right)>232 \mathrm{GeV} & \text { for } & m\left(\widetilde{\chi}_{1}^{0}\right)=200 \mathrm{GeV} .
\end{array}
$$

The achieved cross section upper limits are the most stringent limits on gaugino pair production with a subsequent lepton number violating decay via an $L L \bar{E}$ operator with a $\lambda_{122}$ coupling. To date, the extracted lower bounds on the masses of neutralino and chargino are the highest lower mass bounds on this type of SUSY particles.

However, since the data collected with the $\mathrm{D} \emptyset$ detector has more than tripled since the shutdown in August 2004 - by now more than $1200 \mathrm{pb}^{-1}$ of data are available for analysis - it is clear, that the above limits will either be improved, or supersymmetry be discovered at the Tevatron within the next two to three years.

In case, no supersymmetric particle is found at the Tevatron, neither by the D $\varnothing$, nor the CDF collaborations, it will be found at the LHC, whose start-up is planned for the year 2007. Even if the lightest SUSY particle would be extremely heavy, but still compatible with a »light SUSY scenario «, where at least some SUSY particles should have masses below $1 \mathrm{TeV}$, it might take a little longer - in between two to three years of LHC data taking - before SUSY is discovered. In any case, it will be essential to further reduce the so-called irreducible SM background for a trilepton signature, from, e.g. diboson and $t \bar{t}$ production since the production cross sections of these processes increase dramatically with the CM-energy.

I think physicists are the Peter Pans of the human race.

They never grow up and they keep their curiosity.

Isidor Isaac Rabi (American Physicist, 1898-1988) 


\section{Appendix A}

\section{Simulated mSUGRA Signal Points}

All generated mSUGRA signal points are summarised in Table A.1 for $\mu>0$ and in Table A.2 for $\mu<0$. The relevant parameters, masses and cross sections, as well as the equivalent luminosity $\left(\mathcal{L}_{M C}\right)$ and the number of generated events are listed for $\tan \beta=5$ and 20 . The LO cross sections for the two dominant production processes, i.e. the pair production of the lightest chargino, $\widetilde{\chi}_{1}^{ \pm} \widetilde{\chi}_{1}^{\mp}$ $(C C)$, and the second lightest neutralino and lightest chargino, $\widetilde{\chi}_{2}^{0} \widetilde{\chi}_{1}^{ \pm}(N C)$, as well as the gaugino masses are taken from the Monte Carlo event generator Susygen. Scale factors between LO and NLO cross sections, $K$-factors, are computed per point with the program Gauginos [140] and are then multiplied with the total SUSYGEN LO cross section to give the NLO cross section listed in the following tables.

The small crosses $(x)$ in the following tables indicate those mSUGRA points for which detailed studies were performed, optimising the product of the signal selection efficiency and the achieved purity, i.e. $\varepsilon_{S} \cdot \frac{S}{S+B}$ for the combined dimuon and trilepton selections.

\begin{tabular}{l||rccc|cccc|cr}
\hline \hline$\mu>0$ & \multicolumn{5}{|c|}{ Masses are in GeV } & \multicolumn{3}{c|}{ Cross Sections are in pb } & \multicolumn{2}{c}{$\mathcal{L}_{M C}$} \\
Name & $m_{0}$ & $m_{1 / 2}$ & $m\left(\widetilde{\chi}_{1}^{0}\right)$ & $m\left(\widetilde{\chi}_{1}^{ \pm}\right)$ & $\sigma_{C C}$ & $\sigma_{N C}$ & $K$ & $\sigma_{N L O}$ & {$\left[\mathrm{fb}^{-1}\right]$} & $N_{g e n}$ \\
\hline \hline $\mathcal{A} 1$ pos & 1000 & 220 & 88.8 & 171.7 & 0.078 & 0.115 & 1.24 & 0.239 & $\approx 39.7$ & 9,500 \\
$\mathcal{A} 2$ pos $\times$ & 1000 & 240 & 97.4 & 188.6 & 0.048 & 0.069 & 1.22 & 0.143 & $\approx 70.0$ & 10,000 \\
$\mathcal{A} 3$ pos & 1000 & 260 & 105.9 & 205.1 & 0.031 & 0.042 & 1.21 & 0.088 & $\approx 113.6$ & 10,000 \\
$\mathcal{A} 4$ pos $\times$ & 1000 & 280 & 114.6 & 222.0 & 0.019 & 0.026 & 1.20 & 0.055 & $\approx 172.7$ & 9,500 \\
$\mathcal{A} 5$ pos $\times$ & 1000 & 300 & 123.1 & 238.7 & 0.013 & 0.016 & 1.18 & 0.034 & $\approx 250.0$ & 8,500 \\
$\mathcal{A} 6$ pos & 1000 & 320 & 131.6 & 255.6 & 0.008 & 0.010 & 1.17 & 0.022 & $\approx 454.5$ & 10,000 \\
$\mathcal{A} 7$ pos & 1000 & 340 & 140.2 & 272.3 & 0.005 & 0.007 & 1.16 & 0.014 & $\approx 714.3$ & 10,000 \\
$\mathcal{B} 1$ pos $\times$ & 100 & 190 & 69.8 & 124.8 & 0.235 & 0.372 & 1.24 & 0.766 & $\approx 12.4$ & 9,500 \\
$\mathcal{B} 2$ pos & 100 & 194 & 71.6 & 128.3 & 0.209 & 0.326 & 1.24 & 0.675 & $\approx 14.1$ & 9,500 \\
$\mathcal{B} 3$ pos & 100 & 195 & 72.0 & 129.1 & 0.202 & 0.316 & 1.24 & 0.653 & $\approx 15.3$ & 10,000 \\
$\mathcal{B} 4$ pos & 100 & 196 & 72.5 & 130.0 & 0.197 & 0.308 & 1.24 & 0.632 & $\approx 12.7$ & 8,000 \\
$\mathcal{B} 5$ pos & 100 & 198 & 73.4 & 131.8 & 0.186 & 0.289 & 1.23 & 0.594 & $\approx 10.1$ & 6,000 \\
$\mathcal{B} 6$ pos & 100 & 200 & 74.2 & 133.5 & 0.174 & 0.271 & 1.23 & 0.557 & $\approx 16.1$ & 9,000 \\
$\mathcal{B} 7$ pos & 100 & 210 & 78.7 & 142.4 & 0.132 & 0.199 & 1.22 & 0.415 & $\approx 21.7$ & 9,000 \\
$\mathcal{B} 8$ pos $\times$ & 100 & 220 & 83.2 & 151.1 & 0.100 & 0.148 & 1.22 & 0.309 & $\approx 30.7$ & 9,500 \\
$\mathcal{B} 9$ pos & 100 & 230 & 87.6 & 159.8 & 0.077 & 0.112 & 1.21 & 0.234 & $\approx 38.5$ & 9,000 \\
$\mathcal{B} 10$ pos & 100 & 240 & 91.9 & 168.6 & 0.060 & 0.086 & 1.20 & 0.178 & $\approx 56.2$ & 10,000 \\
$\mathcal{B} 11$ pos & 100 & 244 & 93.7 & 172.1 & 0.054 & 0.077 & 1.20 & 0.161 & $\approx 37.3$ & 6,000 \\
$\mathcal{B} 12$ pos & 100 & 246 & 94.6 & 173.8 & 0.051 & 0.073 & 1.20 & 0.151 & $\approx 39.7$ & 6,000
\end{tabular}




\begin{tabular}{|c|c|c|c|c|c|c|c|c|c|c|}
\hline \multirow{2}{*}{$\begin{array}{l}\mu>0 \\
\text { Name }\end{array}$} & \multicolumn{4}{|c|}{ Masses are in $\mathrm{GeV}$} & \multicolumn{4}{|c|}{ Cross Sections in pb } & \multirow{2}{*}{$\begin{array}{c}\mathcal{L}_{M C} \\
{\left[\mathrm{fb}^{-1}\right]} \\
\end{array}$} & \multirow[b]{2}{*}{$N_{g e n}$} \\
\hline & $m_{0}$ & $m_{1 / 2}$ & $m\left(\widetilde{\chi}_{1}^{0}\right)$ & $m\left(\widetilde{\chi}_{1}^{ \pm}\right)$ & $\sigma_{C C}$ & $\sigma_{N C}$ & $K$ & $\sigma_{N L O}$ & & \\
\hline $\mathcal{B} 13$ pos $\mathrm{x}$ & 100 & 250 & 96.4 & 177.3 & 0.047 & .066 & 1.19 & 0.137 & $\approx 65.7$ & 9,000 \\
\hline $\mathcal{B} 14$ pos & 100 & 260 & 100.7 & 186.0 & 037 & 0.051 & 1.19 & 107 & $\approx 93.5$ & 10,000 \\
\hline $\mathcal{B} 15$ pos & 100 & 270 & & 194.6 & .029 & 0.040 & 1.18 & & $\approx 114.5$ & 9,500 \\
\hline $\mathcal{B} 16$ pos $\mathrm{x}$ & 100 & 280 & 109.3 & 203.3 & 0.023 & 0.031 & 1.17 & 0.064 & 125.0 & 8,000 \\
\hline $\mathcal{B} 17$ pos & 100 & 290 & 113.7 & 211.9 & 0.018 & 0.024 & 1.17 & & 190.0 & 9,500 \\
\hline $\mathcal{B} 18$ pos $\mathrm{x}$ & 100 & 300 & 117.9 & 220.5 & 0.014 & 0.019 & 1.16 & 039 & $\approx 217.9$ & 8,500 \\
\hline $\mathcal{B} 19$ pos & 100 & 310 & 122.3 & 229.1 & 0.011 & 0.015 & 1.15 & 031 & $\approx 483.9$ & 15,000 \\
\hline $\mathcal{B} 20$ pos & 100 & 315 & 124.4 & 233.4 & 0.010 & .013 & 1.15 & 28 & 339.3 & 9,500 \\
\hline B21pos & 100 & 320 & & 237.7 & .009 & 0.012 & 1.14 & & 360.0 & 000 \\
\hline $\mathcal{B} 22$ pos & 100 & 33 & 13 & 246.3 & 0.008 & 0.009 & 1.14 & 19 & $\approx 473.7$ & 9,000 \\
\hline B23pos & 100 & 34 & 135.1 & 254.8 & 0.006 & 0.007 & 1.13 & 016 & $\approx 593.8$ &, 500 \\
\hline & 250 & 15 & & 92.6 & 0.871 & 537 & 8 & 16 & $\approx 2.9$ & 00 \\
\hline$o s$ & 250 & 16 & & 101.4 & 599 & 017 & 1.27 & & $\approx 2.6$ & 500 \\
\hline & 250 & 17 & & 110.2 & 422 & .698 & 1.26 & & $\approx 3.5$ & 500 \\
\hline & 250 & 18 & & 118.9 & 0.307 & 0.493 & 1.25 & & $\approx 6.7$ & 500 \\
\hline & 250 & 20 & & 136.5 & 167 & 260 & 1.24 & & $\approx 15.9$ & 600 \\
\hline & 250 & 22 & & 154.0 & 096 & 144 & 1.22 & & $\approx 18.0$ & 000 \\
\hline$o s \mathrm{x}$ & 250 & 24 & & 171.3 & 0.058 & 83 & 1.21 & & $\approx 46.6$ & 000 \\
\hline & 250 & 25 & & 179.9 & 0.045 & 0.064 & 1.20 & & $\approx 67.7$ &, 000 \\
\hline & 250 & 26 & & & 35 & & 1.19 & & $\approx 83.3$ & 500 \\
\hline & 250 & 27 & & 197.2 & 028 & & & & 118.7 & 00 \\
\hline os $\mathrm{x}$ & 250 & 28 & & 205.8 & 022 & 0.030 & 1.18 & 63 & 142.8 & 000 \\
\hline & 250 & 29 & & 214.4 & 0.018 & 0.023 & 1.17 & & $\approx 204.1$ & 10,000 \\
\hline & 250 & 30 & & 2 & 014 & 19 & 1.16 & & 263.1 & 10,000 \\
\hline & 250 & 32 & & 240.3 & & & & & 291.7 & 7,000 \\
\hline & 2 & 34 & & .4 & 16 & 7 & 1.14 & & $\approx 600.0$ & 9,000 \\
\hline & & & & & & & & & $\approx 5$ & \\
\hline & 5( & 26 & & .9 & 34 & 48 & 0 & & $\approx 90.0$ & 000 \\
\hline & 50 & 28 & & .1 & 22 & 29 & 9 & & $\approx 147.5$ & 9,000 \\
\hline & 50 & 30 & & & 0.014 & & & & $\approx 216.2$ & 8,000 \\
\hline & 50 & 32 & & & & & & & $\approx 413.0$ & 9,500 \\
\hline D6pos & 500 & 34 & & .1 & 0.006 & 0.007 & 1.10 & 15 & $\approx 600.0$ & 9,000 \\
\hline & & & & & & & & & $\approx 5$ & \\
\hline & 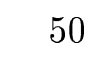 & 26 & & & & & & & $\approx 92.3$ & \\
\hline & 5 & 28 & & 6 & 23 & & 7 & & $\approx 143.4$ & 9,500 \\
\hline & 50 & 300 & & & & & & & $\approx 231.7$ & 9,500 \\
\hline $\mathcal{E} 5$ pos & 50 & 320 & 126.3 & 237.2 & 0.008 & 0.010 & 1.16 & 0.023 & $\approx 208.3$ & 5,000 \\
\hline & & & & & & & & & $\approx 61.3$ & 9,500 \\
\hline & & 26 & & 199.9 & & & & & $\approx 101.1$ & 9,500 \\
\hline & 750 & 280 & & 216.6 & 0.021 & 0.028 & 1.19 & 058 & $\approx 250.0$ & 14,500 \\
\hline \multicolumn{11}{|c|}{$\tan \beta=20$} \\
\hline & & & & & & & & & & \\
\hline & & 260 & & & & & & & $\approx 68.4$ & 6,500 \\
\hline $\mathcal{A} 3$ pos 20 & 1000 & 280 & & 217.8 & 0.021 & 0.028 & 1.20 & & $\approx 169.5$ & 10,000 \\
\hline os 20 & 1000 & 300 & 123 & 234.9 & 0.013 & 0.017 & 1.17 & 0.036 & $\approx 263.9$ & 9,500 \\
\hline $\mathcal{A} 5$ pos 20 & 1000 & 320 & 132.4 & 252.6 & 0.008 & 0.011 & 1.16 & 0.022 & $\approx 363.6$ & 8,000 \\
\hline
\end{tabular}


Appendix A: Simulated mSUGRA Signal Points

\begin{tabular}{l||cccc|cccc|cr}
\hline \hline \multicolumn{1}{l||}{$\mu$} & \multicolumn{4}{|c|}{ Masses are in GeV } & \multicolumn{4}{c|}{ Cross Sections in pb } & \multicolumn{2}{c}{$\mathcal{L}_{M C}$} \\
Name & $m_{0}$ & $m_{1 / 2}$ & $m\left(\widetilde{\chi}_{1}^{0}\right)$ & $m\left(\widetilde{\chi}_{1}^{ \pm}\right)$ & $\sigma_{C C}$ & $\sigma_{N C}$ & $K$ & $\sigma_{N L O}$ & {$\left[\mathrm{fb}^{-1}\right]$} & $N_{\text {gen }}$ \\
\hline \hline $\mathcal{B} 1$ pos 20 & 100 & 240 & 94.0 & 173.7 & 0.051 & 0.072 & 1.19 & 0.151 & $\approx 66.2$ & 10,000 \\
$\mathcal{B} 2$ pos 20 & 100 & 260 & 102.6 & 190.9 & 0.032 & 0.043 & 1.19 & 0.092 & $\approx 108.7$ & 10,000 \\
$\mathcal{B} 3$ pos 20 & 100 & 280 & 111.1 & 208.0 & 0.020 & 0.027 & 1.18 & 0.057 & $\approx 175.4$ & 10,000 \\
$\mathcal{B} 4$ pos 20 & 100 & 300 & 119.6 & 225.0 & 0.013 & 0.017 & 1.16 & 0.035 & $\approx 271.4$ & 9,500 \\
$\mathcal{B} 5$ pos 20 & 100 & 320 & 128.1 & 242.0 & 0.008 & 0.010 & 1.14 & 0.022 & $\approx 454.5$ & 10,000 \\
$\mathcal{D} 1$ pos 20 & 500 & 240 & 96.2 & 178.8 & 0.052 & 0.073 & 1.20 & 0.152 & $\approx 65.8$ & 10,000 \\
$\mathcal{D} 2$ pos 20 & 500 & 260 & 104.7 & 195.8 & 0.032 & 0.044 & 1.18 & 0.092 & $\approx 97.8$ & 9,000 \\
$\mathcal{D} 3$ pos 20 & 500 & 280 & 113.4 & 213.1 & 0.020 & 0.027 & 1.18 & 0.057 & $\approx 166.7$ & 9,500 \\
$\mathcal{D} 4$ pos 20 & 500 & 300 & 122.0 & 230.3 & 0.013 & 0.017 & 1.17 & 0.035 & $\approx 271.4$ & 9,500 \\
$\mathcal{D} 5$ pos 20 & 500 & 320 & 130.5 & 247.4 & 0.008 & 0.010 & 1.15 & 0.022 & $\approx 409.1$ & 9,000 \\
\hline \hline
\end{tabular}

Table A.1: Signal Monte Carlo simulations for the following mSUGRA parameters: $\mu<0, A_{0}=0$ and two values of $\tan \beta=5$ and 20. The production of pairs of the lightest chargino $\left(\widetilde{\chi}_{1}^{ \pm} \widetilde{\chi}_{1}^{\mp}\right)$ and pairs of the second lightest neutralino and lightest chargino $\left(\widetilde{\chi}_{2}^{0} \widetilde{\chi}_{1}^{ \pm}\right)$is denoted by $C C(N C)$, with $C$ being either $\widetilde{\chi}_{1}^{ \pm}$or $\widetilde{\chi}_{2}^{ \pm}$, and $N$ being one of the four neutralinos: $\widetilde{\chi}_{1}^{0}, \widetilde{\chi}_{2}^{0}, \widetilde{\chi}_{3}^{0}$, or $\widetilde{\chi}_{4}^{0}$. The masses, as well as the LO cross sections are taken from SUSYGEN and the total SUSYGEN LO cross section is then multiplied by the appropriate $N L O / L O K$-factor, obtained with the program GAUGINOs.

\begin{tabular}{|c|c|c|c|c|c|c|c|c|c|c|}
\hline \multirow{2}{*}{$\begin{array}{l}\mu<0 \\
\text { Name }\end{array}$} & \multicolumn{4}{|c|}{ Masses are in $\mathrm{GeV}$} & \multicolumn{4}{|c|}{ Cross Sections are in pb } & \multirow{2}{*}{$\begin{array}{r}\mathcal{L}_{M C} \\
{\left[\mathrm{fb}^{-1}\right]} \\
\end{array}$} & \multirow[b]{2}{*}{$N_{g e n}$} \\
\hline & $m_{0}$ & $m_{1 / 2}$ & $m\left(\widetilde{\chi}_{1}^{0}\right)$ & $m\left(\widetilde{\chi}_{1}^{ \pm}\right)$ & $\sigma_{C C}$ & $\sigma_{N C}$ & $K$ & $\sigma_{N L O}$ & & \\
\hline $\mathcal{A} 1$ neg & 1000 & 230 & 98.2 & 197.6 & 0.038 & 0.054 & 1.22 & 0.112 & $\approx 80.4$ & 9,000 \\
\hline $\mathcal{A} 2 n e g \times$ & 1000 & 250 & 106.6 & 214.1 & 0.025 & 0.033 & 1.21 & 0.070 & $\approx 128.6$ & 9,000 \\
\hline $\mathcal{A} 3$ neg & 1000 & 270 & 114.9 & 230.7 & 0.016 & 0.021 & 1.20 & 0.044 & $\approx 215.9$ & 9,500 \\
\hline $\mathcal{A} 4 n e g \mathrm{x}$ & 1000 & 290 & 123.4 & 247.2 & 0.010 & 0.013 & 1.18 & 0.027 & $\approx 277.8$ & 7,500 \\
\hline $\mathcal{A} 5 n e g$ & 1000 & 310 & 131.8 & 263.9 & 0.007 & 0.008 & 1.17 & 0.018 & $\approx 500.0$ & 9,000 \\
\hline A6neg & 1000 & 330 & 140.4 & 280.5 & 0.004 & 0.005 & 1.16 & 0.012 & $\approx 791.7$ & 9,500 \\
\hline $\mathcal{B} 1$ eg & 100 & 150 & 61.0 & 114.2 & 0.331 & 0.506 & 1.24 & 1.068 & $\approx 8.9$ &, 500 \\
\hline $\mathcal{B} 2 n e g$ & 100 & 160 & 65.1 & 122.4 & 0.248 & 0.372 & 1.24 & 0.790 & $\approx 10.7$ & 8,500 \\
\hline B3neg & 100 & 170 & 69.2 & 130.7 & 0.187 & 0.276 & 1.23 & 0.587 & $\approx 15.3$ & 9,000 \\
\hline $\mathcal{B} 4 n e g$ & 100 & 190 & 77.3 & 147.1 & 0.110 & 0.159 & 1.21 & 0.335 & $\approx 28.4$ & 9,500 \\
\hline B5neg & 100 & 210 & 85.5 & 163.7 & 0.067 & 0.094 & 1.20 & 0.199 & $\approx 45.2$ & 9,000 \\
\hline B6neg & 100 & 230 & 93.8 & 180.3 & 0.042 & 0.057 & 1.19 & 0.121 & $\approx 66.1$ & 8,000 \\
\hline $\mathcal{B} 7$ neg & 100 & 240 & 97.8 & 188.6 & 0.033 & 0.045 & 1.18 & 0.094 & $\approx 106.4$ & 10,000 \\
\hline $\mathcal{B} 8$ neg $\mathrm{x}$ & 100 & 250 & 102.0 & 196.9 & 0.026 & 0.035 & 1.17 & 0.074 & $\approx 135.1$ & 10,000 \\
\hline $\mathcal{B} 9$ neg & 100 & 260 & 106.1 & 205.2 & 0.021 & 0.028 & 1.17 & 0.060 & $\approx 158.3$ & 9,500 \\
\hline B10neg & 100 & 270 & 110.3 & 213.5 & 0.017 & 0.022 & 1.16 & 0.046 & $\approx 358.7$ & 16,500 \\
\hline $\mathcal{B} 11$ neg & 100 & 280 & 114.4 & 221.8 & 0.014 & 0.018 & 1.16 & 0.037 & $\approx 256.7$ & 9,500 \\
\hline $\mathcal{B} 12$ neg $\mathrm{x}$ & 100 & 290 & 118.6 & 230.2 & 0.011 & 0.014 & 1.15 & 0.030 & $\approx 300.0$ & 9,000 \\
\hline $\mathcal{B} 13$ neg & 100 & 310 & 127.0 & 246.8 & 0.007 & 0.009 & 1.14 & 0.019 & $\approx 421.0$ & 8,000 \\
\hline $\mathcal{B} 14$ neg & 100 & 330 & 135.3 & 263.5 & 0.005 & 0.006 & 1.13 & 0.012 & $\approx 791.7$ & 9,500 \\
\hline $\mathcal{C} 1$ neg & 250 & 140 & 57.9 & 109.2 & 0.435 & 0.688 & 1.26 & 1.443 & $\approx 3.8$ & 5,500 \\
\hline $\mathcal{C} 2 n e g$ & 250 & 150 & 62.0 & 117.3 & 0.321 & 0.498 & 1.26 & 1.045 & $\approx 5.3$ & 5,500 \\
\hline C3neg & 250 & 160 & 66.0 & 125.4 & 0.242 & 0.368 & 1.25 & 0.779 & $\approx 6.4$ & 5,000 \\
\hline $\mathcal{C} 4 n e g$ & 250 & 180 & 74.2 & 141.8 & 0.140 & 0.207 & 1.23 & 0.486 & $\approx 12.3$ & 6,000 \\
\hline $\mathcal{C} 5 n e g$ & 250 & 200 & 82.4 & 158.2 & 0.084 & 0.121 & 1.22 & 0.284 & $\approx 35.2$ & 10,000 \\
\hline $\mathcal{C} 6 n e g$ & 250 & 220 & 90.6 & 174.8 & 0.052 & 0.072 & 1.20 & 0.171 & $\approx 52.6$ & 9,000 \\
\hline
\end{tabular}




\begin{tabular}{|c|c|c|c|c|c|c|c|c|c|c|}
\hline \multirow{2}{*}{$\begin{array}{l}\mu<0 \\
\text { Name }\end{array}$} & \multicolumn{4}{|c|}{ Masses are in $\mathrm{GeV}$} & \multicolumn{4}{|c|}{ Cross Sections in pb } & \multirow{2}{*}{$\begin{array}{c}\mathcal{L}_{M C} \\
{\left[\mathrm{fb}^{-1}\right]} \\
\end{array}$} & \multirow[b]{2}{*}{$N_{g e n}$} \\
\hline & $m_{0}$ & $m_{1 / 2}$ & $m\left(\widetilde{\chi}_{1}^{0}\right)$ & $m\left(\widetilde{\chi}_{1}^{ \pm}\right)$ & $\sigma_{C C}$ & $\sigma_{N C}$ & $K$ & $\sigma_{N L O}$ & & \\
\hline $\mathcal{C} 7 n e g$ & 250 & 240 & 98.8 & 191.3 & 0.033 & 0.044 & 1.19 & 0.105 & $\approx 71.4$ & 7,500 \\
\hline $\mathcal{C} 8 n e g$ & 250 & 260 & 107.1 & 207.9 & 0.021 & 0.028 & 1.17 & 0.057 & $\approx 140.3$ & 8,000 \\
\hline $\mathcal{C} 9 n e g$ & 250 & 280 & 115.4 & 224.5 & 0.013 & 0.017 & 1.16 & 0.036 & $\approx 263.9$ & 9,500 \\
\hline $\mathcal{D} 1$ neg & 500 & 230 & 96.2 & 188.2 & 0.041 & 0.057 & 1.21 & 0.119 & $\approx 79.8$ & 9,500 \\
\hline $\mathcal{D} 2 n e g \mathrm{x}$ & 500 & 250 & 104.5 & 204.7 & 0.026 & 0.035 & 1.20 & 0.073 & $\approx 123.3$ & 9,000 \\
\hline D3neg & 500 & 270 & 112.8 & 221.2 & 0.016 & 0.022 & 1.18 & 0.046 & $\approx 152.2$ & 7,000 \\
\hline $\mathcal{D} 4 n e g \times$ & 500 & 290 & 121.0 & 237.7 & 0.011 & 0.014 & 1.17 & 0.029 & $\approx 327.6$ & 9,500 \\
\hline $\mathcal{D} 5 n e g$ & 500 & 310 & 129.5 & 254.3 & 0.007 & 0.009 & 1.15 & 0.018 & $\approx 527.8$ & 9,500 \\
\hline D6neg & 500 & 330 & 137.9 & 271.0 & 0.005 & 0.006 & 1.14 & 0.011 & $\approx 818.2$ & 9,000 \\
\hline $\mathcal{E} 1$ neg & 50 & 230 & 93.4 & 179.5 & 0.042 & 0.058 & 1.19 & 0.123 & $\approx 36.6$ & 4,500 \\
\hline $\mathcal{E} 2 n e g$ & 50 & 250 & 101.8 & 196.2 & 0.027 & 0.036 & 1.17 & 0.075 & $\approx 66.7$ & 5,000 \\
\hline $\mathcal{E} 3 n e g$ & 50 & 270 & 110.1 & 212.8 & 0.017 & 0.023 & 1.16 & 0.048 & $\approx 104.2$ & 5,000 \\
\hline $\mathcal{E} 4 n e g$ & 50 & 290 & 118.4 & 229.5 & 0.011 & 0.014 & 1.15 & 0.030 & $\approx 166.7$ & 5,000 \\
\hline $\mathcal{F} 1$ neg & 750 & 230 & 97.2 & 193.2 & 0.040 & 0.056 & 1.22 & 0.117 & $\approx 72.6$ & 8,500 \\
\hline $\mathcal{F} 2$ neg & 750 & 250 & 105.6 & 209.7 & 0.025 & 0.035 & 1.21 & 0.073 & $\approx 130.1$ & 9,500 \\
\hline $\mathcal{F} 3$ neg & 750 & 270 & 114.0 & 226.2 & 0.016 & 0.021 & 1.19 & 0.045 & $\approx 200.0$ & 9,000 \\
\hline \multicolumn{11}{|c|}{$\tan \beta=20$} \\
\hline $\mathcal{A} 1$ neg 20 & 1000 & 230 & 95.2 & 180.4 & 0.057 & 0.082 & 1.20 & 0.170 & $\approx 55.9$ & 9,500 \\
\hline $\mathcal{A} 2$ neg 20 & 1000 & 250 & 103.7 & 197.4 & 0.036 & 0.051 & 1.20 & 0.104 & $\approx 96.2$ & 10,000 \\
\hline $\mathcal{A} 3$ neg 20 & 1000 & 270 & 112.4 & 215.0 & 0.023 & 0.030 & 1.19 & 0.064 & $\approx 156.3$ & 10,000 \\
\hline $\mathcal{A} 4$ neg 20 & 1000 & 290 & 120.9 & 232.0 & 0.014 & 0.019 & 1.17 & 0.040 & $\approx 250.0$ & 10,000 \\
\hline $\mathcal{A} 5$ neg 20 & 1000 & 310 & 129.4 & 249.2 & 0.009 & 0.012 & 1.16 & 0.024 & $\approx 375.0$ & 9,000 \\
\hline $\mathcal{B} 1$ neg 20 & 100 & 230 & 91.4 & 170.6 & 0.056 & 0.078 & 1.18 & 0.164 & $\approx 61.0$ & 10,000 \\
\hline $\mathcal{B} 2$ neg 20 & 100 & 250 & 100.0 & 187.6 & 0.035 & 0.047 & 1.17 & 0.098 & $\approx 102.0$ & 10,000 \\
\hline $\mathcal{B} 3$ neg 20 & 100 & 270 & 108.3 & 204.5 & 0.022 & 0.029 & 1.15 & 0.060 & $\approx 158.3$ & 9,500 \\
\hline $\mathcal{B} 4$ neg 20 & 100 & 290 & 116.7 & 221.5 & 0.014 & 0.018 & 1.14 & 0.038 & $\approx 263.1$ & 10,000 \\
\hline $\mathcal{B} 5 n e g 20$ & 100 & 310 & 125.1 & 238.4 & 0.009 & 0.011 & 1.14 & 0.034 & $\approx 294.1$ & 10,000 \\
\hline $\mathcal{D} 1$ neg 20 & 500 & 230 & 93.6 & 175.8 & 0.057 & 0.081 & 1.19 & 0.167 & $\approx 56.9$ & 9,500 \\
\hline $\mathcal{D} 2$ neg 20 & 500 & 250 & 102.1 & 192.9 & 0.035 & 0.048 & 1.18 & 0.100 & $\approx 90.0$ & 9,000 \\
\hline $\mathcal{D} 3$ neg 20 & 500 & 270 & 110.5 & 209.8 & 0.022 & 0.030 & 1.17 & 0.062 & $\approx 153.2$ & 9,500 \\
\hline $\mathcal{D} 4 n e g 20$ & 500 & 290 & 118.9 & 226.8 & 0.014 & 0.018 & 1.16 & 0.038 & $\approx 263.2$ & 10,000 \\
\hline D5neg 20 & 500 & 310 & 127.5 & 244.0 & 0.009 & 0.011 & 1.15 & 0.024 & $\approx 395.8$ & 9,500 \\
\hline
\end{tabular}

Table A.2: Signal Monte Carlo simulations for the following mSUGRA parameters: $\mu<0, A_{0}=0$ and two values of $\tan \beta=5$ and 20. The production of pairs of the lightest chargino ( $\widetilde{\chi}_{1}^{ \pm} \widetilde{\chi}_{1}^{\mp}$ ) and pairs of the second lightest neutralino and lightest chargino $\left(\widetilde{\chi}_{2}^{0} \widetilde{\chi}_{1}^{ \pm}\right)$is denoted by $C C(N C)$, with $C$ being either $\widetilde{\chi}_{1}^{ \pm}$or $\widetilde{\chi}_{2}^{ \pm}$, and $N$ being one of the four neutralinos: $\widetilde{\chi}_{1}^{0}, \widetilde{\chi}_{2}^{0}, \widetilde{\chi}_{3}^{0}$, or $\widetilde{\chi}_{4}^{0}$. The masses, as well as the LO cross sections are taken from SUSYGEN and the total SUSYGEN LO cross section is then multiplied by the appropriate $N L O / L O K$-factor, obtained with the program GAUGINOS. 


\section{Appendix B}

\section{Simulated MSSM Signal Points (no-GUT Scenario)}

The MSSM signal points generated for a fixed chargino or a fixed neutralino mass are summarised in Tables B.1 and B.2, respectively. Signal points corresponding to a scan of the complete $\left(\widetilde{\chi}_{1}^{0}, \widetilde{\chi}_{1}^{ \pm}\right)$-mass plane are listed in Table B.3.

The following parameters are provided in the tables: the mass parameters and masses of the $\mathrm{U}(1)_{Y}$ and $\mathrm{SU}(2)_{L}$ gauge bosons: $M_{1} \rightarrow m\left(\widetilde{\chi}_{1}^{0}\right)$, and $M_{2} \rightarrow m\left(\widetilde{\chi}_{1}^{ \pm}\right)$, the LO cross sections of the dominant $C C$ and $N C$ pair production processes $\left(\widetilde{\chi}_{1}^{ \pm} \widetilde{\chi}_{1}^{\mp}\right.$ and $\left.\widetilde{\chi}_{2}^{0} \widetilde{\chi}_{1}^{ \pm}\right)$, the NLO/LO $K$-factor, again computed with GaUGinos [140], the resulting NLO total cross section, the equivalent luminosity $\left(\mathcal{L}_{M C}\right)$ and the number of generated events are also given. For all MSSM signal simulations, the ratio of the Higgs field VEVs is set to $\tan \beta=5$ and the universal, trilinear coupling is set to $A_{0}=0$.

\begin{tabular}{l||rrrr|rrrr|cc}
\hline \hline \multicolumn{1}{l||}{$\mu=1 \mathrm{TeV}$} & \multicolumn{9}{|c|}{ Masses are in GeV } & \multicolumn{3}{c|}{ Cross Sections are in pb } & \multicolumn{2}{|c}{$\mathcal{L}_{M C}$} \\
Name & $M_{2}$ & $m\left(\widetilde{\chi}_{1}^{ \pm}\right)$ & $M_{1}$ & $m\left(\widetilde{\chi}_{1}^{0}\right)$ & $\sigma_{C C}$ & $\sigma_{N C}$ & $K$ & $\sigma_{N L O}$ & {$\left[\mathrm{fb}^{-1}\right]$} & $N_{\text {gen }}$ \\
\hline \hline $\mathrm{C} 103-M S 1$ & 106.7 & 103.5 & 50.8 & 50 & 0.796 & 1.323 & 1.28 & 2.716 & $\approx 1.5$ & 4,000 \\
$\mathrm{C} 103-M S 2$ & 106.7 & 103.5 & 61.0 & 60 & 0.796 & 1.323 & 1.28 & 2.712 & $\approx 1.5$ & 4,000 \\
$\mathrm{C} 103-M S 3$ & 106.7 & 103.5 & 71.1 & 70 & 0.796 & 1.325 & 1.28 & 2.714 & $\approx 1.5$ & 4,000 \\
$\mathrm{C} 103-M S 4$ & 106.7 & 103.5 & 81.3 & 80 & 0.796 & 1.323 & 1.28 & 2.711 & $\approx 1.5$ & 4,000 \\
$\mathrm{C} 103-M S 5$ & 106.7 & 103.5 & 91.5 & 90 & 0.796 & 1.330 & 1.28 & 2.719 & $\approx 1.5$ & 4,000 \\
$\mathrm{C} 103-M S 6$ & 106.7 & 103.5 & 101.6 & 100 & 0.796 & 1.325 & 1.28 & 2.714 & $\approx 1.1$ & 3,000 \\
$\mathrm{C} 103-M S 7$ & 106.7 & 103.5 & 267.5 & 104 & 0.796 & 1.320 & 1.28 & 2.711 & $\approx 0.9$ & 2,500 \\
$\mathrm{C} 200-M S 1$ & 204.0 & 200 & 35.8 & 35 & 0.035 & 0.049 & 1.23 & 0.103 & $\approx 38.8$ & 4,000 \\
$\mathrm{C} 200-M S 2$ & 204.0 & 200 & 60.3 & 60 & 0.035 & 0.049 & 1.22 & 0.102 & $\approx 39.2$ & 4,000 \\
$\mathrm{C} 200-M S 3$ & 204.0 & 200 & 75.7 & 75 & 0.035 & 0.049 & 1.21 & 0.102 & $\approx 34.3$ & 3,500 \\
$\mathrm{C} 200-M S 4$ & 204.0 & 200 & 91.0 & 90 & 0.035 & 0.049 & 1.21 & 0.102 & $\approx 34.3$ & 3,500 \\
$\mathrm{C} 200-M S 5$ & 204.0 & 200 & 106.4 & 105 & 0.035 & 0.049 & 1.21 & 0.101 & $\approx 34.6$ & 3,500 \\
$\mathrm{C} 200-M S 6$ & 204.0 & 200 & 120.7 & 120 & 0.035 & 0.049 & 1.21 & 0.101 & $\approx 49.5$ & 5,000 \\
$\mathrm{C} 200-M S 7$ & 204.0 & 200 & 141.1 & 140 & 0.035 & 0.049 & 1.20 & 0.101 & $\approx 29.7$ & 3,000 \\
$\mathrm{C} 200-M S 8$ & 204.0 & 200 & 161.6 & 160 & 0.035 & 0.049 & 1.20 & 0.101 & $\approx 24.7$ & 2,500 \\
$\mathrm{C} 200-M S 9$ & 204.0 & 200 & 181.0 & 180 & 0.035 & 0.049 & 1.20 & 0.101 & $\approx 29.7$ & 3,000 \\
$\mathrm{C} 200-M S 10$ & 204.0 & 200 & 204.5 & 199 & 0.035 & 0.049 & 1.20 & 0.101 & $\approx 39.6$ & 4,000 \\
$\mathrm{C} 200-M S 11$ & 204.0 & 200 & 214.8 & 200 & 0.035 & 0.049 & 1.20 & 0.101 & $\approx 39.6$ & 4,000 \\
$\mathrm{C} 200-M S 12$ & 204.0 & 200 & 225.0 & 200 & 0.035 & 0.049 & 1.21 & 0.102 & $\approx 34.3$ & 3,500
\end{tabular}


Appendix B: Simulated MSSM Signal Points (no-GUT Scenario)

\begin{tabular}{l||rrrr|rrrr|cc}
\hline \hline \multicolumn{1}{l||}{$\mu=1 \mathrm{TeV}$} & \multicolumn{9}{c|}{ Masses are in GeV } & \multicolumn{3}{c|}{ Cross Sections are in pb } & \multicolumn{2}{c}{$\mathcal{L}_{M C}$} \\
Name & $M_{2}$ & $m\left(\widetilde{\chi}_{1}^{ \pm}\right)$ & $M_{1}$ & $m\left(\tilde{\chi}_{1}^{0}\right)$ & $\sigma_{C C}$ & $\sigma_{N C}$ & $K$ & $\sigma_{N L O}$ & {$\left[\mathrm{fb}^{-1}\right]$} & $N_{\text {gen }}$ \\
\hline \hline $\mathrm{C} 225-M S 1$ & 229.1 & 225 & 28.7 & 28 & 0.018 & 0.024 & 1.21 & 0.050 & $\approx 80.0$ & 4,000 \\
$\mathrm{C} 225-M S 2$ & 229.1 & 225 & 40.2 & 39 & 0.018 & 0.024 & 1.21 & 0.050 & $\approx 50.0$ & 2,500 \\
$\mathrm{C} 225-M S 3$ & 229.1 & 225 & 57.4 & 57 & 0.018 & 0.024 & 1.20 & 0.049 & $\approx 81.6$ & 4,000 \\
$\mathrm{C} 225-M S 4$ & 229.1 & 225 & 74.7 & 74 & 0.018 & 0.024 & 1.20 & 0.049 & $\approx 81.6$ & 4,000 \\
$\mathrm{C} 225-M S 5$ & 229.1 & 225 & 86.2 & 85 & 0.018 & 0.024 & 1.19 & 0.049 & $\approx 71.4$ & 3,500 \\
$\mathrm{C} 225-M S 6$ & 229.1 & 225 & 91.9 & 91 & 0.018 & 0.024 & 1.19 & 0.049 & $\approx 71.4$ & 3,500 \\
$\mathrm{C} 225-M S 7$ & 229.1 & 225 & 114.9 & 114 & 0.018 & 0.024 & 1.19 & 0.049 & $\approx 71.4$ & 3,500 \\
$\mathrm{C} 225-M S 8$ & 229.1 & 225 & 143.6 & 143 & 0.018 & 0.024 & 1.18 & 0.049 & $\approx 81.6$ & 4,000 \\
$\mathrm{C} 225-M S 9$ & 229.1 & 225 & 172.3 & 171 & 0.018 & 0.024 & 1.18 & 0.049 & $\approx 71.4$ & 3,500 \\
$\mathrm{C} 225-M S 10$ & 229.1 & 225 & 201.0 & 200 & 0.018 & 0.024 & 1.18 & 0.048 & $\approx 83.3$ & 4,000 \\
$\mathrm{C} 225-M S 11$ & 229.1 & 225 & 212.5 & 211 & 0.018 & 0.024 & 1.18 & 0.048 & $\approx 83.3$ & 4,000 \\
$\mathrm{C} 225-M S 12$ & 229.1 & 225 & 229.7 & 224 & 0.018 & 0.024 & 1.18 & 0.048 & $\approx 52.1$ & 2,500 \\
\hline \hline
\end{tabular}

Table B.1: Signal Monte Carlo simulations for fixed values of the chargino mass in the context of the no-GUT MSSM. The neutralino mass is varied for three different values of the chargino mass: $m\left(\widetilde{\chi}_{1}^{ \pm}\right)=$ $103.5 \mathrm{GeV}$ (LEP limit [138]), and $m\left(\widetilde{\chi}_{1}^{ \pm}\right)=200 \mathrm{GeV}$ and $225 \mathrm{GeV}$. For all points, the trilinear coupling is set to $A_{0}=0$, the ratio of the Higgs field VEVs to $\tan \beta=5$, and the mass of the pseudoscalar Higgs boson is chosen to be $m_{A}=400 \mathrm{GeV}$, while the remaining three MSSM parameters $M_{1}, M_{2}$ and $\mu$ are given in the table.

\begin{tabular}{|c|c|c|c|c|c|c|c|c|c|c|}
\hline \multirow{2}{*}{$\begin{array}{l}\mu=1 \mathrm{TeV} \\
\text { Name }\end{array}$} & \multicolumn{4}{|c|}{ Masses are in $\mathrm{GeV}$} & \multicolumn{4}{|c|}{ Cross Sections are in pb } & \multirow{2}{*}{$\begin{array}{c}\mathcal{L}_{M C} \\
{\left[\mathrm{fb}^{-1}\right]} \\
\end{array}$} & \multirow[b]{2}{*}{$N_{g e n}$} \\
\hline & $M_{1}$ & $m\left(\widetilde{\chi}_{1}^{0}\right)$ & $M_{2}$ & $m\left(\widetilde{\chi}_{1}^{ \pm}\right)$ & $\sigma_{C C}$ & $\sigma_{N C}$ & $K$ & $\sigma_{N L O}$ & & \\
\hline N80-MS1 & 81.0 & 80 & 103.1 & 100 & 0.927 & 1.546 & 1.28 & 3.164 & $\approx 1.3$ & 4,000 \\
\hline$M S 2$ & 80.9 & 80 & 128.3 & 125 & 0.353 & 0.567 & 1.26 & 1.161 & $\approx 3.4$ & 4,000 \\
\hline N80-MS3 & 80.9 & 80 & 153.6 & 150 & 0.155 & 0.237 & 1.24 & 0.488 & $\approx 8.2$ & 4,000 \\
\hline N80-MS4 & 80.9 & 80 & 178.7 & 175 & 0.073 & 0.106 & 1.23 & 0.220 & $\approx 18.2$ & 4,000 \\
\hline$M S 5$ & 81.0 & 80 & 204.0 & 200 & 0.035 & 0.049 & 1.21 & 0.102 & $\approx 39.2$ & 4,000 \\
\hline $4 S 6$ & 81.0 & 80 & 229.1 & 226 & 0.018 & 0.024 & 1.20 & 0.049 & $\approx 81.6$ & 4,000 \\
\hline $1 S 7$ & 81.1 & 80 & 259.5 & 255 & 0.008 & 0.010 & 1.18 & 0.021 & $\approx 166.7$ & 3,500 \\
\hline N80-MS8 & 80.8 & 80 & 284.7 & 280 & 0.004 & 0.005 & 1.16 & 0.010 & $\approx 350.0$ & 3,500 \\
\hline N80-MS9 & 80.8 & 80 & 309.9 & 305 & 0.002 & 0.002 & 1.15 & 0.005 & $\approx 700.0$ & 3,500 \\
\hline N80-MS10 & 81.0 & 80 & 335.2 & 330 & 0.001 & 0.001 & 1.13 & 0.002 & $\approx 1750$ & 3,500 \\
\hline \multicolumn{11}{|c|}{$\mu=-1 \mathrm{TeV}$} \\
\hline $\mathrm{N} 100-M S 1$ & 99.7 & 100 & 139.0 & 141 & 0.247 & 0.389 & 1.27 & 0.683 & $\approx 4.4$ & 3,000 \\
\hline N100-MS2 & 99.6 & 100 & 159.0 & 160 & 0.131 & 0.199 & 1.25 & 0.351 & $\approx 8.5$ & 3,000 \\
\hline $\mathrm{N} 100-M S 3$ & 99.6 & 100 & 179.0 & 180 & 0.072 & 0.106 & 1.24 & 0.189 & $\approx 15.9$ & 3,000 \\
\hline $\mathrm{N} 100-M S 4$ & 99.8 & 100 & 199.0 & 200 & 0.040 & 0.057 & 1.23 & 0.103 & $\approx 29.1$ & 3,000 \\
\hline N100-MS5 & 99.9 & 100 & 219.0 & 220 & 0.023 & 0.032 & 1.23 & 0.058 & $\approx 51.7$ & 3,000 \\
\hline N100-MS6 & 99.5 & 100 & 239.0 & 240 & 0.013 & 0.018 & 1.20 & 0.033 & $\approx 90.9$ & 3,000 \\
\hline
\end{tabular}

Table B.2: Signal Monte Carlo simulations for fixed values of the neutralino mass in the context of the no-GUT MSSM model. The chargino mass is varied for two different neutralino masses of $m\left(\widetilde{\chi}_{1}^{0}\right)=$ $80 \mathrm{GeV}$ and $100 \mathrm{GeV}$. For all points, the trilinear coupling is set to $A_{0}=0$, the ratio of the Higgs field $V E V s$ to $\tan \beta=5$, and the mass of the pseudoscalar Higgs boson is chosen to be $m_{A}=400 \mathrm{GeV}$. The remaining three MSSM parameters $M_{1}, M_{2}$ and $\mu$ are given in the table. 
Appendix B: Simulated MSSM Signal Points (no-GUT Scenario)

\begin{tabular}{|c|c|c|c|c|c|c|c|c|c|c|}
\hline \multirow{2}{*}{$\begin{array}{l}\mu=1 \mathrm{TeV} \\
\text { Name }\end{array}$} & \multicolumn{4}{|c|}{ Masses are in $\mathrm{GeV}$} & \multicolumn{4}{|c|}{ Cross Sections are in $\mathrm{pb}$} & \multirow{2}{*}{$\begin{array}{r}\mathcal{L}_{M C} \\
{\left[\mathrm{fb}^{-1}\right]} \\
\end{array}$} & \multirow[b]{2}{*}{$N_{g e n}$} \\
\hline & $M_{2}$ & $m\left(\widetilde{\chi}_{1}^{ \pm}\right)$ & $M_{1}$ & $m\left(\widetilde{\chi}_{1}^{0}\right)$ & $\sigma_{C C}$ & $\sigma_{N C}$ & $K$ & $\sigma_{N L O}$ & & \\
\hline$M S$ map1 & 103.3 & 100 & 10.8 & 10 & 0.908 & 1.532 & 1.28 & 3.126 & $\approx 1.0$ & 3.000 \\
\hline$M S$ map2 & 153.5 & 150 & 10.8 & 10 & 0.155 & 0.239 & 1.25 & 0.494 & $\approx 6.1$ & 3.000 \\
\hline$M S$ map3 & 103.5 & 100 & 20.8 & 20 & 0.906 & 1.509 & 1.28 & 3.091 & $\approx 0.8$ & 2.500 \\
\hline$M S m a p 4$ & 153.5 & 150 & 20.8 & 20 & 0.155 & 0.239 & 1.25 & 0.492 & $\approx 5.1$ & 2.500 \\
\hline$M S m a p 5$ & 204.0 & 200 & 20.8 & 20 & 0.035 & 0.049 & 1.21 & 0.102 & $\approx 19.6$ & 2.000 \\
\hline$M S m a p 6$ & 103.5 & 100 & 30.8 & 30 & 0.906 & 1.505 & 1.28 & 3.086 & $\approx 0.6$ & 2.000 \\
\hline$M S$ map7 & 128.3 & 125 & 30.8 & 30 & 0.356 & 0.567 & 1.26 & 1.163 & $\approx 1.7$ & 2.000 \\
\hline$M S$ map8 & 153.5 & 150 & 30.8 & 30 & 0.146 & 0.243 & 1.25 & 0.492 & $\approx 5.1$ & 2.500 \\
\hline$M S m a p 10$ & 108.2 & 105 & 42.8 & 42 & 0.751 & 1.244 & 1.28 & 2.537 & $\approx 1.4$ & 3.500 \\
\hline$M S m a p 11$ & 108.2 & 105 & 50.9 & 50 & 0.751 & 1.243 & 1.28 & 2.536 & $\approx 2.0$ & 5.000 \\
\hline$M S m a p 12$ & 118.5 & 115 & 40.9 & 40 & 0.504 & 0.826 & 1.27 & 1.678 & $\approx 4.0$ & 6.750 \\
\hline$M S$ map13 & 123.3 & 120 & 48.8 & 48 & 0.423 & 0.686 & 1.26 & 1.400 & $\approx 2.8$ & 4.000 \\
\hline$M S$ map14 & 125.5 & 122 & 122.8 & 120 & 0.393 & 0.634 & 1.26 & 1.295 & $\approx 5.4$ & 7.000 \\
\hline$M S$ map15 & 128.3 & 125 & 42.8 & 42 & 0.356 & 0.567 & 1.27 & 1.156 & $\approx 3.0$ & 3.500 \\
\hline$M S$ map16 & 138.5 & 135 & 61.4 & 61 & 0.252 & 0.396 & 1.26 & 0.810 & $\approx 8.0$ & 6.500 \\
\hline$M S$ map17 & 145.5 & 142 & 143.3 & 140 & 0.200 & 0.311 & 1.25 & 0.639 & $\approx 10.6$ & 6.750 \\
\hline$M S$ map18 & 153.5 & 150 & 40.9 & 40 & 0.155 & 0.238 & 1.25 & 0.491 & $\approx 12.7$ & 6.250 \\
\hline$M S$ map19 & 163.5 & 160 & 61.4 & 60 & 0.114 & 0.172 & 1.24 & 0.355 & $\approx 19.7$ & 7.000 \\
\hline$M S$ map20 & 163.5 & 160 & 141.6 & 140 & 0.114 & 0.172 & 1.24 & 0.355 & $\approx 19.0$ & 6.750 \\
\hline$M S m a p 21$ & 165.5 & 162 & 163.7 & 160 & 0.107 & 0.160 & 1.23 & 0.331 & $\approx 22.6$ & 7.500 \\
\hline$M S$ map22 & 178.5 & 175 & 40.9 & 40 & 0.073 & 0.107 & 1.23 & 0.223 & $\approx 31.4$ & 7.000 \\
\hline$M S$ map23 & 178.5 & 175 & 121.2 & 120 & 0.073 & 0.107 & 1.22 & 0.223 & $\approx 33.6$ & 7.500 \\
\hline$M S$ map24 & 185.5 & 182 & 161.4 & 160 & 0.059 & 0.086 & 1.21 & 0.180 & $\approx 38.9$ & 7.000 \\
\hline$M S$ map25 & 185.5 & 182 & 184.1 & 180 & 0.059 & 0.086 & 1.21 & 0.180 & $\approx 41.7$ & 7.500 \\
\hline$M S$ map26 & 193.9 & 190 & 60.8 & 60 & 0.047 & 0.067 & 1.21 & 0.140 & $\approx 35.7$ & 5.000 \\
\hline$M S$ map 27 & 204.0 & 200 & 60.3 & 60 & 0.035 & 0.049 & 1.22 & 0.102 & $\approx 44.1$ & 4.500 \\
\hline$M S$ map28 & 204.0 & 200 & 100.9 & 100 & 0.035 & 0.049 & 1.23 & 0.102 & $\approx 44.1$ & 4.500 \\
\hline$M S$ map29 & 204.0 & 200 & 141.1 & 140 & 0.035 & 0.049 & 1.20 & 0.102 & $\approx 44.1$ & 4.500 \\
\hline$M S$ map30 & 204.0 & 200 & 181.0 & 180 & 0.035 & 0.049 & 1.20 & 0.102 & $\approx 49$ & 5.000 \\
\hline$M S$ map31 & 204.0 & 200 & 204.5 & 199 & 0.035 & 0.049 & 1.20 & 0.102 & $\approx 49$ & 5.000 \\
\hline$M S m a p 32$ & 214.0 & 210 & 40.9 & 40 & 0.027 & 0.037 & 1.20 & 0.077 & $\approx 58$ & 4.500 \\
\hline$M S m a p 33$ & 214.0 & 210 & 60.8 & 60 & 0.027 & 0.037 & 1.20 & 0.077 & $\approx 65$ & 5.000 \\
\hline$M S$ map34 & 214.0 & 210 & 91.0 & 90 & 0.027 & 0.037 & 1.20 & 0.077 & $\approx 46$ & 3.500 \\
\hline$M S$ map35 & 214.0 & 210 & 100.9 & 100 & 0.027 & 0.037 & 1.20 & 0.077 & $\approx 58$ & 4.500 \\
\hline$M S$ map36 & 214.0 & 210 & 111.0 & 110 & 0.027 & 0.037 & 1.20 & 0.077 & $\approx 52$ & 4.000 \\
\hline$M S$ map37 & 214.0 & 210 & 121.0 & 120 & 0.027 & 0.037 & 1.20 & 0.077 & $\approx 52$ & 4.000 \\
\hline$M S m a p 38$ & 214.0 & 210 & 141.1 & 140 & 0.027 & 0.037 & 1.20 & 0.077 & $\approx 104$ & 8.000 \\
\hline$M S m a p 39$ & 214.0 & 210 & 161.1 & 160 & 0.027 & 0.037 & 1.20 & 0.077 & $\approx 58$ & 4.500 \\
\hline$M S m a p 40$ & 214.0 & 210 & 181.3 & 180 & 0.027 & 0.037 & 1.20 & 0.077 & $\approx 65$ & 5.000 \\
\hline$M S m a p 41$ & 214.0 & 210 & 201.7 & 200 & 0.027 & 0.037 & 1.19 & 0.077 & $\approx 65$ & 5.000 \\
\hline$M S$ map42 & 215.5 & 211 & 214.9 & 210 & 0.025 & 0.035 & 1.19 & 0.073 & $\approx 103$ & 7.500 \\
\hline$M S$ map43 & 220.0 & 216 & 99.3 & 98 & 0.022 & 0.031 & 1.20 & 0.064 & $\approx 62$ & 4.000 \\
\hline$M S m a p 44$ & 220.0 & 216 & 110.3 & 109 & 0.022 & 0.031 & 1.20 & 0.064 & $\approx 86$ & 5.500 \\
\hline$M S \operatorname{map} 45$ & 220.0 & 216 & 121.3 & 120 & 0.022 & 0.031 & 1.20 & 0.064 & $\approx 117$ & 7.500 \\
\hline$M S m a p 46$ & 220.0 & 216 & 141.2 & 140 & 0.022 & 0.031 & 1.20 & 0.064 & $\approx 94$ & 6.000 \\
\hline$M S$ map47 & 220.0 & 216 & 161.1 & 160 & 0.022 & 0.031 & 1.20 & 0.064 & $\approx 86$ & 5.500 \\
\hline$M S$ map48 & 223.5 & 219 & 51.2 & 50 & 0.020 & 0.028 & 1.21 & 0.058 & $\approx 86$ & 5.000 \\
\hline
\end{tabular}




\begin{tabular}{|c|c|c|c|c|c|c|c|c|c|c|}
\hline \multirow{2}{*}{$\begin{array}{l}\mu=1 \mathrm{TeV} \\
\text { Name }\end{array}$} & \multicolumn{4}{|c|}{ Masses are in $\mathrm{GeV}$} & \multicolumn{4}{|c|}{ Cross Sections are in pb } & \multirow{2}{*}{$\begin{array}{r}\mathcal{L}_{M C} \\
{\left[\mathrm{fb}^{-1}\right]} \\
\end{array}$} & \multirow[b]{2}{*}{$N_{g e n}$} \\
\hline & $M_{2}$ & $m\left(\widetilde{\chi}_{1}^{ \pm}\right)$ & $M_{1}$ & $m\left(\widetilde{\chi}_{1}^{0}\right)$ & $\sigma_{C C}$ & $\sigma_{N C}$ & $K$ & $\sigma_{N L O}$ & & \\
\hline$M S \operatorname{map} 49$ & 223.5 & 219 & 81.8 & 81 & 0.020 & 0.028 & 1.20 & 0.058 & $\approx 125$ & 7.250 \\
\hline$M S \operatorname{map} 50$ & 223.5 & 219 & 224.1 & 218 & 0.020 & 0.028 & 1.19 & 0.058 & $\approx 130$ & 7.500 \\
\hline$M S$ map51 & 224.0 & 220 & 60.9 & 60 & 0.020 & 0.027 & 1.19 & 0.056 & $\approx 80$ & 4.500 \\
\hline$M S$ map52 & 224.0 & 220 & 100.9 & 100 & 0.020 & 0.027 & 1.23 & 0.056 & $\approx 80$ & 4.500 \\
\hline$M S m a p 53$ & 224.0 & 220 & 141.0 & 140 & 0.020 & 0.027 & 1.21 & 0.056 & $\approx 89$ & 5.000 \\
\hline$M S \operatorname{map} 54$ & 224.0 & 220 & 181.2 & 180 & 0.020 & 0.027 & 1.20 & 0.056 & $\approx 89$ & 5.000 \\
\hline$M S \operatorname{map} 55$ & 224.0 & 220 & 201.5 & 200 & 0.020 & 0.027 & 1.19 & 0.056 & $\approx 71$ & 4.000 \\
\hline$M S \operatorname{map} 56$ & 234.2 & 230 & 60.9 & 60 & 0.015 & 0.020 & 1.18 & 0.042 & $\approx 107$ & 500 \\
\hline$M S \operatorname{map} 57$ & 234.2 & 230 & 101.0 & 100 & 0.015 & 0.020 & 1.18 & 0.042 & $\approx 119$ & 5.000 \\
\hline$M S$ map58 & 234.2 & 230 & 141.0 & 140 & 0.015 & 0.020 & 1.18 & 0.042 & $\approx 119$ & 5.000 \\
\hline$M S$ map59 & 234.2 & 230 & 181.2 & 180 & 0.015 & 0.020 & 1.18 & 0.042 & $\approx 107$ & 4.500 \\
\hline$M S \operatorname{map} 60$ & 234.2 & 230 & 201.4 & 200 & 0.015 & 0.020 & 1.18 & 0.042 & $\approx 107$ & 4.500 \\
\hline$M S \operatorname{map} 61$ & 239.2 & 235 & 60.9 & 60 & 0.013 & 0.018 & 1.18 & 0.037 & $\approx 122$ & 4.500 \\
\hline$M S \operatorname{map} 62$ & 239.2 & 235 & 71.9 & 71 & 0.013 & 0.018 & 1.18 & 0.037 & $\approx 135$ & 5.000 \\
\hline$M S$ map63 & 239.2 & 235 & 95.9 & 95 & 0.013 & 0.018 & 1.18 & 0.037 & $\approx 108$ & 4.000 \\
\hline$M S m a p 64$ & 239.2 & 235 & 119.9 & 119 & 0.013 & 0.018 & 1.17 & 0.037 & $\approx 122$ & 4.500 \\
\hline$M S$ map65 & 239.2 & 235 & 124.1 & 123 & 0.013 & 0.018 & 1.17 & 0.037 & $\approx 135$ & 5.000 \\
\hline$M S \operatorname{map} 66$ & 239.2 & 235 & 141.1 & 140 & 0.013 & 0.018 & 1.16 & 0.037 & $\approx 122$ & 4.500 \\
\hline$M S \operatorname{map} 67$ & 239.2 & 235 & 161.2 & 160 & 0.013 & 0.018 & 1.16 & 0.037 & $\approx 135$ & 5.000 \\
\hline$M S$ map68 & 244.3 & 240 & 60.9 & 60 & 0.012 & 0.015 & 1.17 & 0.032 & $\approx 156$ & 5.000 \\
\hline$M S \operatorname{map} 69$ & 244.3 & 240 & 80.9 & 80 & 0.012 & 0.015 & 1.20 & 0.032 & $\approx 156$ & 5.000 \\
\hline$M S$ map70 & 244.3 & 240 & 101.0 & 100 & 0.012 & 0.015 & 1.20 & 0.032 & $\approx 140$ & 4.500 \\
\hline$M S$ map71 & 244.3 & 240 & 141.1 & 140 & 0.012 & 0.015 & 1.19 & 0.032 & $\approx 156$ & 5.000 \\
\hline$M S$ map72 & 244.3 & 240 & 181.1 & 180 & 0.012 & 0.015 & 1.18 & 0.032 & $\approx 156$ & 5.000 \\
\hline$M S$ map73 & 244.3 & 240 & 201.4 & 200 & 0.012 & 0.015 & 1.17 & 0.032 & $\approx 125$ & 4.000 \\
\hline$M S$ map74 & 254.4 & 250 & 51.0 & 50 & 0.009 & 0.011 & 1.20 & 0.024 & $\approx 208$ & 5.000 \\
\hline$M S$ map75 & 254.4 & 250 & 76.5 & 76 & 0.009 & 0.011 & 1.19 & 0.024 & $\approx 187$ & 4.500 \\
\hline$M S \operatorname{map} 76$ & 254.4 & 250 & 102.0 & 101 & 0.009 & 0.011 & 1.19 & 0.024 & $\approx 208$ & 5.000 \\
\hline
\end{tabular}

Table B.3: Signal Monte Carlo simulations for the mapping of the $\left(\widetilde{\chi}_{1}^{0}, \widetilde{\chi}_{1}^{ \pm}\right)$-mass plane in the framework of the no-GUT MSSM scenario. Both mass parameters $M_{1}$ and $M_{2}$ are varied, resulting in an independent variation of the masses of the lightest neutralino and chargino. The remaining MSSM parameters are chosen as follows: the ratio of the Higgs field VEVs is set to $\tan \beta=5$, the Higgs mixing parameter to $\mu=1 \mathrm{TeV}$, the trilinear coupling to $A_{0}=0$, and the mass of the pseudoscalar Higgs boson is chosen as $m_{A}=400 \mathrm{GeV}$. 


\section{Appendix C}

\section{Results within the mSUGRA Scenario}

Expected and observed cross section upper limits are calculated at 95\% CL for all generated mSUGRA signal points. The results are summarised in the following Table C.1 for positive Higgs mixing parameter $\mu>0$ and Table C. 2 for $\mu<0$.

The signal selection efficiency for the $\mu \mu \ell$ analysis and the resulting expected and observed limits are listed in columns three, four and five, respectively. For those signal points, where results are also available from the eel and $e e \tau$ analyses (or the eel analysis alone), the combined limits are given in columns six and seven. Besides, the fractional overlap with the eel analysis is provided in the last column, in case combined results exist.

\begin{tabular}{lc||rcccc|r}
\hline \hline \multicolumn{1}{|c||}{$\sigma_{N L O}$} & \multicolumn{2}{c}{$\varepsilon_{\text {Signal }}[\%]$} & \multicolumn{2}{c}{$\mu \mu \ell$ limits $[\mathrm{pb}]$} & \multicolumn{2}{c}{ comb. limits [pb] } & Ov. \\
Name & {$[\mathrm{pb}]$} & $\varepsilon_{S} \pm$ stat \pm sys & $\sigma_{\text {exp }}^{95 \%}$ & $\sigma_{S}^{95 \%}$ & $\sigma_{\text {exp }}^{95 \%}$ & $\sigma_{S}^{95 \%}$ & {$[\%]$} \\
\hline \hline $\mathcal{A} 1$ pos & 0.239 & $11.7 \pm 0.3 \pm 1.3$ & 0.088 & 0.070 & 0.076 & 0.056 & 8.7 \\
$\mathcal{A} 2$ pos & 0.143 & $12.7 \pm 0.3 \pm 1.4$ & 0.081 & 0.065 & 0.069 & 0.050 & 7.2 \\
$\mathcal{A} 3$ pos & 0.088 & $14.9 \pm 0.4 \pm 1.7$ & 0.069 & 0.055 & 0.060 & 0.044 & 8.3 \\
$\mathcal{A} 4$ pos & 0.055 & $16.1 \pm 0.4 \pm 1.8$ & 0.064 & 0.051 & 0.055 & 0.040 & 7.8 \\
$\mathcal{A} 5$ pos & 0.034 & $14.7 \pm 0.4 \pm 1.6$ & 0.070 & 0.056 & 0.060 & 0.044 & 8.7 \\
$\mathcal{A} 6$ pos & 0.022 & $14.6 \pm 0.4 \pm 1.6$ & 0.071 & 0.056 & 0.060 & 0.043 & 9.8 \\
$\mathcal{A} 7$ pos & 0.014 & $15.2 \pm 0.4 \pm 1.7$ & 0.068 & 0.054 & 0.058 & 0.042 & 10.2 \\
$\mathcal{B} 1$ pos & 0.766 & $12.8 \pm 0.3 \pm 1.4$ & 0.081 & 0.064 & 0.068 & 0.049 & 5.8 \\
$\mathcal{B} 2$ pos & 0.675 & $13.7 \pm 0.4 \pm 1.5$ & 0.075 & 0.060 & 0.066 & 0.048 & 9.1 \\
$\mathcal{B} 3$ pos & 0.653 & $13.2 \pm 0.3 \pm 1.5$ & 0.078 & 0.062 & 0.068 & 0.050 & 8.0 \\
$\mathcal{B} 4$ pos & 0.632 & $13.3 \pm 0.4 \pm 1.5$ & 0.078 & 0.062 & 0.067 & 0.049 & 8.7 \\
$\mathcal{B} 5$ pos & 0.594 & $14.8 \pm 0.5 \pm 1.7$ & 0.074 & 0.060 & 0.064 & 0.046 & 9.0 \\
$\mathcal{B} 6$ pos & 0.557 & $9.9 \pm 0.3 \pm 1.1$ & 0.105 & 0.083 & 0.092 & 0.068 & 4.9 \\
$\mathcal{B} 7$ pos & 0.415 & $9.8 \pm 0.3 \pm 1.1$ & 0.105 & 0.084 & 0.091 & 0.066 & 5.2 \\
$\mathcal{B} 8$ pos & 0.309 & $11.2 \pm 0.3 \pm 1.3$ & 0.093 & 0.074 & 0.077 & 0.056 & 6.5 \\
$\mathcal{B} 9$ pos & 0.234 & $11.0 \pm 0.3 \pm 1.2$ & 0.095 & 0.075 & 0.078 & 0.056 & 7.0 \\
$\mathcal{B} 10$ pos & 0.178 & $11.2 \pm 0.3 \pm 1.2$ & 0.092 & 0.074 & 0.075 & 0.053 & 7.3 \\
$\mathcal{B} 11$ pos & 0.161 & $13.9 \pm 0.4 \pm 1.6$ & 0.074 & 0.059 & 0.062 & 0.045 & 8.6 \\
$\mathcal{B} 12$ pos & 0.151 & $13.0 \pm 0.4 \pm 1.5$ & 0.079 & 0.063 & 0.066 & 0.048 & 7.3 \\
$\mathcal{B} 13$ pos & 0.137 & $21.8 \pm 0.4 \pm 2.4$ & 0.047 & 0.038 & 0.032 & 0.022 & 14.9 \\
$\mathcal{B} 14$ pos & 0.107 & $24.6 \pm 0.4 \pm 2.8$ & 0.042 & 0.034 & 0.031 & 0.021 & 23.0 \\
$\mathcal{B} 15$ pos & 0.083 & $21.7 \pm 0.4 \pm 2.4$ & 0.047 & 0.038 & 0.033 & 0.023 & 20.6 \\
$\mathcal{B} 16$ pos & 0.064 & $23.7 \pm 0.5 \pm 2.6$ & 0.044 & 0.035 & 0.029 & 0.020 & 16.3
\end{tabular}




\begin{tabular}{|c|c|c|c|c|c|c|c|}
\hline \multirow{2}{*}{ Name } & \multirow{2}{*}{$\begin{array}{r}\sigma_{N L O} \\
{[\mathrm{pb}]} \\
\end{array}$} & \multirow{2}{*}{$\begin{array}{c}\varepsilon_{\text {Signal }}[\%] \\
\varepsilon_{S} \pm \text { stat } \pm \text { sys }\end{array}$} & \multicolumn{2}{|c|}{ 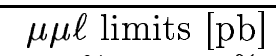 } & \multicolumn{2}{|c|}{ comb. limits $[\mathrm{pb}]$} & \multirow{2}{*}{$\begin{array}{l}\text { Ov. } \\
{[\%]}\end{array}$} \\
\hline & & & $\sigma_{e x p}^{95 \%}$ & $\sigma_{S}^{95 \%}$ & $\sigma_{e x p}^{95 \%}$ & $\sigma_{S}^{95 \%}$ & \\
\hline $\mathcal{B} 17$ pos & 0.050 & $21.4 \pm 0.4 \pm 2.4$ & 0.048 & 0.038 & 0.034 & 0.023 & 21.8 \\
\hline B18pos & 0.039 & $25.8 \pm 0.5 \pm 2.9$ & 0.040 & 0.032 & 0.030 & 0.021 & 21.4 \\
\hline $\mathcal{B} 19$ pos & 0.031 & $25.1 \pm 0.4 \pm 2.8$ & 0.041 & 0.033 & 0.028 & 0.020 & 23.8 \\
\hline $\mathcal{B} 20$ pos & 0.028 & $25.4 \pm 0.4 \pm 2.8$ & 0.041 & 0.032 & 0.028 & 0.019 & 23.9 \\
\hline $\mathcal{B} 21$ pos & 0.025 & $24.6 \pm 0.5 \pm 2.8$ & 0.042 & 0.033 & 0.029 & 0.020 & 23.8 \\
\hline B22pos & 0.019 & $24.7 \pm 0.5 \pm 2.8$ & 0.042 & 0.033 & 0.029 & 0.020 & 23.5 \\
\hline $\mathcal{B} 23$ pos & 0.016 & $24.3 \pm 0.4 \pm 2.7$ & 0.042 & 0.034 & 0.029 & 0.020 & 23.1 \\
\hline $\mathcal{C} 1$ pos & 3.116 & $6.4 \pm 0.3 \pm 0.7$ & 0.161 & 0.128 & 0.146 & 0.111 & 3.0 \\
\hline $\mathcal{C} 2 p$ os & 2.076 & $7.6 \pm 0.4 \pm 0.8$ & 0.136 & 0.109 & 0.125 & 0.095 & 2.9 \\
\hline $\mathcal{C} 3$ pos & 1.589 & $8.8 \pm 0.4 \pm 1.0$ & 0.117 & 0.093 & 0.109 & 0.083 & 2.7 \\
\hline $\mathcal{C} 4$ pos & 1.127 & $8.9 \pm 0.3 \pm 1.0$ & 0.116 & 0.092 & 0.108 & 0.083 & 3.1 \\
\hline $\mathcal{C} 5$ pos & 0.598 & $10.7 \pm 0.3 \pm 1.2$ & 0.097 & 0.077 & 0.086 & 0.065 & 2.5 \\
\hline $\mathcal{C} 6$ pos & 0.334 & $11.6 \pm 0.4 \pm 1.3$ & 0.089 & 0.071 & 0.080 & 0.061 & 3.3 \\
\hline $\mathcal{C} 7$ pos & 0.193 & $11.5 \pm 0.3 \pm 1.3$ & 0.089 & 0.071 & 0.081 & 0.061 & 2.9 \\
\hline $\mathcal{C} 8$ pos & 0.133 & $11.0 \pm 0.3 \pm 1.2$ & 0.094 & 0.075 & 0.079 & 0.058 & 8.9 \\
\hline $\mathcal{C} 9$ pos & 0.102 & $11.5 \pm 0.3 \pm 1.3$ & 0.090 & 0.071 & 0.077 & 0.057 & 9.1 \\
\hline C10pos & 0.080 & $13.3 \pm 0.3 \pm 1.5$ & 0.077 & 0.062 & 0.067 & 0.049 & 9.4 \\
\hline $\mathcal{C} 11$ pos & 0.063 & $12.2 \pm 0.3 \pm 1.4$ & 0.085 & 0.068 & 0.070 & 0.051 & 8.8 \\
\hline $\mathcal{C} 12$ pos & 0.049 & $15.0 \pm 0.4 \pm 1.7$ & 0.068 & 0.055 & 0.058 & 0.042 & 9.1 \\
\hline $\mathcal{C} 13$ pos & 0.038 & $15.9 \pm 0.4 \pm 1.8$ & 0.065 & 0.052 & 0.056 & 0.041 & 9.2 \\
\hline C14pos & 0.024 & $14.7 \pm 0.4 \pm 1.6$ & 0.070 & 0.056 & 0.060 & 0.044 & 8.0 \\
\hline $\mathcal{C} 15$ pos & 0.015 & $15.5 \pm 0.4 \pm 1.7$ & 0.066 & 0.053 & 0.057 & 0.042 & 9.4 \\
\hline $\mathcal{D} 1$ pos & 0.167 & $10.9 \pm 0.3 \pm 1.2$ & 0.095 & 0.076 & 0.081 & 0.059 & 9.3 \\
\hline $\mathcal{D} 2 p o s$ & 0.100 & $11.4 \pm 0.3 \pm 1.3$ & 0.091 & 0.072 & 0.076 & 0.056 & 9.1 \\
\hline $\mathcal{D} 3 p o s$ & 0.061 & $12.8 \pm 0.4 \pm 1.4$ & 0.081 & 0.064 & 0.067 & 0.049 & 9.5 \\
\hline $\mathcal{D} 4$ pos & 0.037 & $15.9 \pm 0.4 \pm 1.8$ & 0.065 & 0.052 & 0.056 & 0.041 & 10.1 \\
\hline $\mathcal{D} 5 p o s$ & 0.023 & $14.6 \pm 0.4 \pm 1.6$ & 0.071 & 0.056 & 0.059 & 0.044 & 8.6 \\
\hline $\mathcal{D} 6$ pos & 0.015 & $15.7 \pm 0.4 \pm 1.8$ & 0.066 & 0.052 & 0.056 & 0.041 & 9.5 \\
\hline $\mathcal{E} 1 p o s$ & 0.181 & $11.5 \pm 0.3 \pm 1.3$ & 0.090 & 0.071 & 0.075 & 0.055 & 7.5 \\
\hline $\mathcal{E} 2 p o s$ & 0.108 & $23.2 \pm 0.4 \pm 2.6$ & 0.045 & 0.036 & 0.031 & 0.022 & 23.0 \\
\hline $\mathcal{E} 3 p o s$ & 0.066 & $20.2 \pm 0.4 \pm 2.3$ & 0.051 & 0.041 & 0.034 & 0.024 & 20.8 \\
\hline $\mathcal{E} 4$ pos & 0.041 & $19.5 \pm 0.4 \pm 2.2$ & 0.053 & 0.042 & 0.036 & 0.025 & 21.0 \\
\hline $\mathcal{E} 5 p o s$ & 0.023 & $19.5 \pm 0.4 \pm 2.2$ & 0.054 & 0.043 & 0.037 & 0.026 & 19.8 \\
\hline $\mathcal{F} 1$ pos & 0.155 & $12.0 \pm 0.3 \pm 1.3$ & 0.086 & 0.069 & 0.074 & 0.054 & 8.8 \\
\hline $\mathcal{F} 2$ pos & 0.094 & $13.7 \pm 0.4 \pm 1.5$ & 0.075 & 0.060 & 0.065 & 0.048 & 8.3 \\
\hline \multirow[t]{2}{*}{$\mathcal{F} 3$ pos } & 0.058 & $15.3 \pm 0.3 \pm 1.7$ & 0.067 & 0.054 & 0.057 & 0.043 & 9.6 \\
\hline & \multicolumn{7}{|c|}{$\tan \beta=20$} \\
\hline $\mathcal{A} 1$ pos 20 & 0.156 & $17.1 \pm 0.4 \pm 1.9$ & 0.060 & 0.048 & - & - & - \\
\hline $\mathcal{A} 2 p o s 20$ & 0.095 & $18.2 \pm 0.5 \pm 2.0$ & 0.057 & 0.045 & - & - & - \\
\hline $\mathcal{A} 3 p o s 20$ & 0.059 & $20.6 \pm 0.4 \pm 2.3$ & 0.051 & 0.040 & - & - & - \\
\hline $\mathcal{A} 4$ pos 20 & 0.036 & $20.8 \pm 0.4 \pm 2.3$ & 0.050 & 0.039 & - & - & - \\
\hline $\mathcal{A} 5$ pos 20 & 0.022 & $19.9 \pm 0.4 \pm 2.2$ & 0.052 & 0.041 & - & - & - \\
\hline $\mathcal{B} 1$ pos 20 & 0.151 & $16.1 \pm 0.4 \pm 1.8$ & 0.064 & 0.051 & - & - & - \\
\hline $\mathcal{B} 2$ pos 20 & 0.092 & $25.7 \pm 0.4 \pm 2.9$ & 0.040 & 0.032 & - & - & - \\
\hline $\mathcal{B} 3$ pos 20 & 0.057 & $28.2 \pm 0.5 \pm 3.2$ & 0.037 & 0.029 & - & - & - \\
\hline
\end{tabular}


Appendix C: Results Within the mSUGRA Scenario

\begin{tabular}{|c|c|c|c|c|c|c|c|}
\hline \multirow[b]{2}{*}{ Name } & \multirow{2}{*}{$\begin{array}{r}\sigma_{N L O} \\
{[\mathrm{pb}]}\end{array}$} & \multirow{2}{*}{$\begin{array}{c}\varepsilon_{\text {Signal }}[\%] \\
\varepsilon_{S} \pm \text { stat } \pm \text { sys }\end{array}$} & \multicolumn{2}{|c|}{$\mu \mu \ell$ limits $[\mathrm{pb}]$} & \multicolumn{2}{|c|}{ comb. limits [pb } & \multirow{2}{*}{$\begin{array}{l}\text { Ov. } \\
{[\%]}\end{array}$} \\
\hline & & & $\sigma_{e x p}^{95 \%}$ & $\sigma_{S}^{95 \%}$ & $\sigma_{e x p}^{95 \%}$ & $\sigma_{S}^{95 \%}$ & \\
\hline $\mathcal{B} 4$ pos 20 & 0.035 & $28.2 \pm 0.5 \pm 3.2$ & 0.037 & 0.029 & - & - & - \\
\hline $\mathcal{B} 5$ pos 20 & 0.022 & $28.8 \pm 0.5 \pm 3.2$ & 0.036 & 0.029 & - & - & - \\
\hline $\mathcal{D} 1$ pos 20 & 0.152 & $16.2 \pm 0.4 \pm 1.8$ & 0.064 & 0.051 & - & - & - \\
\hline $\mathcal{D} 2 p o s 20$ & 0.092 & $18.6 \pm 0.4 \pm 2.1$ & 0.055 & 0.044 & - & - & - \\
\hline $\mathcal{D} 3$ pos 20 & 0.057 & $19.7 \pm 0.4 \pm 2.2$ & 0.052 & 0.042 & - & - & - \\
\hline $\mathcal{D} 4$ pos 20 & 0.035 & $21.1 \pm 0.4 \pm 2.4$ & 0.049 & 0.039 & - & - & - \\
\hline $\mathcal{D} 5$ роs 20 & 0.022 & $20.0 \pm 0.4 \pm 2.2$ & 0.051 & 0.041 & - & - & - \\
\hline
\end{tabular}

Table C.1: The cross section upper limits for all mSUGRA signal points with positive Higgs mixing parameter $\mu$. Listed are the expected $\left(\sigma_{e x p}^{95 \%}\right)$ and observed $\left(\sigma_{S}^{95 \%}\right)$ limits from this analysis alone $(\mu \mu \ell)$ and in combination with the results from the searches for eel and eec final states. In column three, the selection efficiency in the $\mu \mu \mathrm{\ell}$ analysis is provided additionally, as is the factional overlap with the eel analysis in the last column.

\begin{tabular}{|c|c|c|c|c|c|c|c|}
\hline \multirow{2}{*}{$\begin{array}{l}\mu>0 \\
\text { Name }\end{array}$} & \multirow{2}{*}{$\begin{array}{r}\sigma_{N L O} \\
{[\mathrm{pb}]}\end{array}$} & \multirow{2}{*}{$\begin{array}{c}\varepsilon_{\text {Signal }}[\%] \\
\varepsilon_{S} \pm \text { stat } \pm \text { sys }\end{array}$} & \multicolumn{2}{|c|}{ 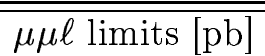 } & \multicolumn{2}{|c|}{ comb. limits [pb] } & \multirow{2}{*}{$\begin{array}{l}\text { Ov. } \\
{[\%]}\end{array}$} \\
\hline & & & $\sigma_{e x p}^{95 \%}$ & $\sigma_{S}^{95 \%}$ & $\sigma_{e x p}^{95 \%}$ & $\sigma_{S}^{95 \%}$ & \\
\hline $\mathcal{A} 1$ neg & 0.112 & $13.5 \pm 0.4 \pm 1.5$ & 0.077 & 0.061 & 0.066 & 0.048 & 9.2 \\
\hline $\mathcal{A} 2$ neg & 0.070 & $15.5 \pm 0.4 \pm 1.7$ & 0.066 & 0.053 & 0.056 & 0.041 & 7.9 \\
\hline $\mathcal{A} 3$ neg & 0.044 & $15.9 \pm 0.4 \pm 1.8$ & 0.065 & 0.052 & 0.056 & 0.041 & 9.8 \\
\hline $\mathcal{A} 4 n e g$ & 0.027 & $17.2 \pm 0.4 \pm 1.9$ & 0.060 & 0.048 & 0.051 & 0.037 & 7.6 \\
\hline $\mathcal{A} 5$ neg & 0.018 & $16.8 \pm 0.4 \pm 1.9$ & 0.061 & 0.049 & 0.053 & 0.039 & 10.9 \\
\hline $\mathcal{A} 6 n e g$ & 0.012 & $17.4 \pm 0.4 \pm 2.0$ & 0.060 & 0.047 & 0.050 & 0.036 & 9.3 \\
\hline $\mathcal{B} 1$ neg & 1.068 & $10.8 \pm 0.3 \pm 1.2$ & 0.095 & 0.076 & 0.083 & 0.061 & 7.6 \\
\hline $\mathcal{B} 2 n e g$ & 0.790 & $12.2 \pm 0.4 \pm 1.4$ & 0.084 & 0.067 & 0.073 & 0.053 & 8.5 \\
\hline B3neg & 0.587 & $8.6 \pm 0.3 \pm 1.0$ & 0.120 & 0.095 & 0.106 & 0.078 & 5.4 \\
\hline B4neg & 0.335 & $8.6 \pm 0.3 \pm 1.0$ & 0.120 & 0.095 & 0.103 & 0.075 & 7.2 \\
\hline B5neg & 0.199 & $9.2 \pm 0.3 \pm 1.0$ & 0.112 & 0.090 & 0.092 & 0.066 & 6.2 \\
\hline B6neg & 0.121 & $12.0 \pm 0.4 \pm 1.4$ & 0.086 & 0.068 & 0.074 & 0.054 & 6.6 \\
\hline $\mathcal{B} 7$ neg & 0.094 & $11.8 \pm 0.3 \pm 1.3$ & 0.087 & 0.070 & 0.075 & 0.055 & 7.4 \\
\hline B8neg & 0.074 & $13.8 \pm 0.3 \pm 1.5$ & 0.075 & 0.060 & 0.066 & 0.049 & 5.1 \\
\hline $\mathcal{B} 9$ neg & 0.060 & $12.7 \pm 0.3 \pm 1.4$ & 0.082 & 0.065 & 0.072 & 0.054 & 5.1 \\
\hline B10neg & 0.046 & $14.6 \pm 0.3 \pm 1.6$ & 0.070 & 0.056 & 0.063 & 0.047 & 5.5 \\
\hline B11neg & 0.037 & $10.4 \pm 0.3 \pm 1.2$ & 0.099 & 0.079 & 0.089 & 0.066 & 5.2 \\
\hline $\mathcal{B} 12 n e g$ & 0.030 & $12.2 \pm 0.3 \pm 1.4$ & 0.084 & 0.067 & 0.075 & 0.056 & 3.4 \\
\hline $\mathcal{B} 13$ neg & 0.019 & $11.8 \pm 0.4 \pm 1.3$ & 0.088 & 0.070 & 0.079 & 0.059 & 5.2 \\
\hline $\mathcal{B} 14 n e g$ & 0.012 & $11.7 \pm 0.3 \pm 1.3$ & 0.088 & 0.070 & 0.077 & 0.057 & 4.7 \\
\hline $\mathcal{C} 1$ neg & 1.443 & $5.6 \pm 0.3 \pm 0.6$ & 0.185 & 0.147 & 0.168 & 0.127 & 3.2 \\
\hline $\mathcal{C} 2 n e g$ & 1.045 & $7.0 \pm 0.3 \pm 0.8$ & 0.148 & 0.119 & 0.134 & 0.101 & 1.8 \\
\hline C3neg & 0.779 & $6.9 \pm 0.4 \pm 0.8$ & 0.150 & 0.119 & 0.134 & 0.102 & 3.2 \\
\hline $\mathcal{C} 4$ neg & 0.486 & $8.3 \pm 0.4 \pm 0.9$ & 0.125 & 0.100 & 0.110 & 0.082 & 5.4 \\
\hline $\mathcal{C} 5$ neg & 0.284 & $9.1 \pm 0.3 \pm 1.0$ & 0.113 & 0.090 & 0.097 & 0.072 & 6.1 \\
\hline C6neg & 0.171 & $10.4 \pm 0.3 \pm 1.2$ & 0.100 & 0.079 & 0.083 & 0.061 & 8.1 \\
\hline $\mathcal{C} 7$ neg & 0.105 & $12.1 \pm 0.4 \pm 1.4$ & 0.085 & 0.068 & 0.071 & 0.052 & 9.5 \\
\hline C8neg & 0.057 & $12.7 \pm 0.4 \pm 1.4$ & 0.082 & 0.065 & 0.064 & 0.046 & 8.6 \\
\hline $\mathcal{C} 9$ neg & 0.036 & $14.2 \pm 0.4 \pm 1.6$ & 0.073 & 0.058 & 0.060 & 0.044 & 10.4 \\
\hline
\end{tabular}




\begin{tabular}{|c|c|c|c|c|c|c|c|}
\hline \multirow{2}{*}{$\begin{array}{l}\mu>0 \\
\text { Name }\end{array}$} & \multirow{2}{*}{$\begin{array}{r}\sigma_{N L O} \\
{[\mathrm{pb}]}\end{array}$} & \multirow{2}{*}{$\begin{array}{c}\varepsilon_{\text {Signal }}[\%] \\
\varepsilon_{S} \pm \text { stat } \pm \text { sys }\end{array}$} & \multicolumn{2}{|c|}{$\bar{\mu} \mu \mu \ell$ limits [pb] } & \multicolumn{2}{|c|}{ comb. limits [pb] } & \multirow{2}{*}{$\begin{array}{l}\text { Ov. } \\
{[\%]}\end{array}$} \\
\hline & & & $\sigma_{e x p}^{95 \%}$ & $\sigma_{S}^{95 \%}$ & $\sigma_{e x p}^{95 \%}$ & $\sigma_{S}^{95 \%}$ & \\
\hline $\bar{D} 1$ 1neg & 0.119 & $11.4 \pm 0.3 \pm 1.3$ & 0.091 & 0.072 & 0.077 & 0.056 & 8.5 \\
\hline $\mathcal{D} 2 n e g$ & 0.073 & $14.8 \pm 0.4 \pm 1.7$ & 0.069 & 0.056 & 0.060 & 0.044 & 9.2 \\
\hline D3neg & 0.046 & $13.2 \pm 0.4 \pm 1.5$ & 0.078 & 0.062 & 0.071 & 0.054 & 9.0 \\
\hline $\mathcal{D} 4 n e g$ & 0.029 & $15.9 \pm 0.4 \pm 1.8$ & 0.065 & 0.052 & 0.055 & 0.040 & 9.8 \\
\hline $\mathcal{D} 5 n e g$ & 0.018 & $16.5 \pm 0.4 \pm 1.8$ & 0.062 & 0.050 & 0.053 & 0.039 & 9.8 \\
\hline D6neg & 0.011 & $16.5 \pm 0.4 \pm 1.8$ & 0.063 & 0.050 & 0.053 & 0.039 & 9.6 \\
\hline $\mathcal{E} 1$ neg & 0.123 & $9.0 \pm 0.4 \pm 1.0$ & 0.116 & 0.092 & 0.102 & 0.076 & 4.7 \\
\hline $\mathcal{E} 2 n e g$ & 0.075 & $9.2 \pm 0.4 \pm 1.0$ & 0.112 & 0.090 & 0.097 & 0.072 & 5.1 \\
\hline $\mathcal{E} 3$ neg & 0.048 & $9.6 \pm 0.4 \pm 1.1$ & 0.108 & 0.086 & 0.094 & 0.070 & 4.9 \\
\hline $\mathcal{E} 4 n e g$ & 0.030 & $10.0 \pm 0.4 \pm 1.1$ & 0.103 & 0.082 & 0.090 & 0.066 & 4.3 \\
\hline $\mathcal{F} 1$ neg & 0.117 & $13.9 \pm 0.4 \pm 1.6$ & 0.074 & 0.059 & 0.064 & 0.048 & 9.2 \\
\hline $\mathcal{F} 2$ neg & 0.073 & $14.8 \pm 0.4 \pm 1.7$ & 0.069 & 0.056 & 0.060 & 0.045 & 8.7 \\
\hline \multirow[t]{2}{*}{$\mathcal{F} 3$ neg } & 0.045 & $16.2 \pm 0.4 \pm 1.8$ & 0.064 & 0.051 & 0.055 & 0.041 & 10.0 \\
\hline & \multicolumn{7}{|c|}{$\tan \beta=20$} \\
\hline $\mathcal{A} 1$ neg 20 & 0.170 & $17.4 \pm 0.4 \pm 1.9$ & 0.059 & 0.047 & - & - & - \\
\hline $\mathcal{A} 2$ neg 20 & 0.104 & $18.6 \pm 0.4 \pm 2.1$ & 0.055 & 0.044 & - & - & - \\
\hline $\mathcal{A} 3$ neg 20 & 0.064 & $19.8 \pm 0.4 \pm 2.2$ & 0.052 & 0.041 & - & - & - \\
\hline $\mathcal{A} 4 n e g 20$ & 0.040 & $20.6 \pm 0.4 \pm 2.3$ & 0.050 & 0.040 & - & - & - \\
\hline $\mathcal{A} 5$ neg 20 & 0.024 & $20.3 \pm 0.4 \pm 2.3$ & 0.051 & 0.041 & - & - & - \\
\hline $\mathcal{B} 1$ neg 20 & 0.164 & $16.3 \pm 0.4 \pm 1.8$ & 0.064 & 0.050 & - & - & - \\
\hline $\mathcal{B} 2$ neg 20 & 0.098 & $27.8 \pm 0.4 \pm 3.1$ & 0.037 & 0.030 & - & - & - \\
\hline B3neg 20 & 0.060 & $27.8 \pm 0.5 \pm 3.1$ & 0.038 & 0.030 & - & - & - \\
\hline $\mathcal{B} 4 n e g 20$ & 0.038 & $28.2 \pm 0.4 \pm 3.2$ & 0.037 & 0.029 & - & - & - \\
\hline $\mathcal{B} 5$ neg 20 & 0.034 & $24.3 \pm 0.4 \pm 2.7$ & 0.042 & 0.034 & - & - & - \\
\hline $\mathcal{D} 1$ neg 20 & 0.167 & $17.1 \pm 0.4 \pm 1.9$ & 0.060 & 0.048 & - & - & - \\
\hline $\mathcal{D} 2 n e g 20$ & 0.100 & $17.9 \pm 0.4 \pm 2.0$ & 0.057 & 0.046 & - & - & - \\
\hline $\mathcal{D} 3$ neg 20 & 0.062 & $20.0 \pm 0.4 \pm 2.2$ & 0.052 & 0.041 & - & - & - \\
\hline $\mathcal{D} 4 n e g 20$ & 0.038 & $21.1 \pm 0.4 \pm 2.4$ & 0.049 & 0.039 & - & - & - \\
\hline D5neg 20 & 0.024 & $20.0 \pm 0.4 \pm 2.2$ & 0.051 & 0.041 & - & - & - \\
\hline
\end{tabular}

Table C.2: The cross section upper limits for all mSUGRA signal points with negative Higgs mixing parameter $\mu$. The expected $\left(\sigma_{e x p}^{95 \%}\right)$ and observed $\left(\sigma_{S}^{95 \%}\right)$ limits from this analysis alone $(\mu \mu \ell)$ and in combination with the results from the searches for eel and eet final states are listed. Column three additionally provides the selection efficiency of the $\mu \mu \ell$ analysis, and the last column shows the factional overlap between the $\mu \mu \ell$ and the eel analyses. 


\section{Appendix D}

\section{Results within the no-GUT MSSM Scenario}

The expected and observed 95\% CL cross section upper limits for all MSSM signal points are presented in the following tables. The first two columns hold the signal point name and NLO cross section, while the $\mu \mu \ell$ signal selection efficiency can be found in column three. The resulting expected and observed limits for this analysis $(\mu \mu \ell)$ and for the combination of the three

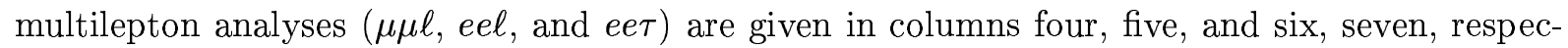
tively. In case the combined results are available, the fractional overlap between the $\mu \mu \ell$ and the eel analysis is also given in the last column.

Tables D.1 and D.2 summarise the results for fixed chargino and neutralino masses, while Table D.3 holds the results for the complete scan of the $\left(\widetilde{\chi}_{1}^{0}, \widetilde{\chi}_{1}^{ \pm}\right)$-mass plane, where both mass parameters, $M_{1}$ and $M_{2}$ are varied independently. The remaining MSSM parameters are chosen as $\tan \beta=5, \mu=1 \mathrm{TeV}, A_{0}=0$, and $m_{A}=400 \mathrm{GeV}$.

\begin{tabular}{lc||rcccc|r}
\hline \hline \multicolumn{1}{l||}{} & \multicolumn{1}{c||}{$\sigma_{N L O}$} & \multicolumn{2}{c}{$\varepsilon_{\text {Signal }}[\%]$} & \multicolumn{3}{c|}{$\mu \mu \ell$ limits [pb] } & \multicolumn{3}{c}{ comb. limits [pb] } & Ov. \\
Name & {$[\mathrm{pb}]$} & $\varepsilon_{S} \pm$ stat \pm sys & $\sigma_{\text {exp }}^{95 \%}$ & $\sigma_{S}^{95 \%}$ & $\sigma_{e x p}^{95 \%}$ & $\sigma_{S}^{95 \%}$ & {$[\%]$} \\
\hline \hline $\mathrm{C} 103-M S 1$ & 2.716 & $6.2 \pm 0.4 \pm 0.7$ & 0.1668 & 0.1324 & 0.1488 & 0.1102 & 6.2 \\
$\mathrm{C} 103-M S 2$ & 2.712 & $7.6 \pm 0.4 \pm 0.8$ & 0.1367 & 0.1094 & 0.1232 & 0.0915 & 3.9 \\
$\mathrm{C} 103-M S 3$ & 2.714 & $8.9 \pm 0.4 \pm 1.0$ & 0.1165 & 0.0927 & 0.1064 & 0.0802 & 3.0 \\
$\mathrm{C} 103-M S 4$ & 2.711 & $9.3 \pm 0.5 \pm 1.0$ & 0.1108 & 0.0881 & 0.0982 & 0.0730 & 3.6 \\
$\mathrm{C} 103-M S 5$ & 2.719 & $10.3 \pm 0.5 \pm 1.2$ & 0.1003 & 0.0801 & 0.0897 & 0.0668 & 3.9 \\
$\mathrm{C} 103-M S 6$ & 2.714 & $12.2 \pm 0.6 \pm 1.4$ & 0.0853 & 0.0675 & 0.0768 & 0.0574 & 5.2 \\
$\mathrm{C} 103-M S 7$ & 2.711 & $13.4 \pm 0.7 \pm 1.5$ & 0.0769 & 0.0614 & 0.0709 & 0.0533 & 8.3 \\
$\mathrm{C} 200-M S 1$ & 0.103 & $9.4 \pm 0.5 \pm 1.0$ & 0.1108 & 0.0880 & 0.0965 & 0.0708 & 7.2 \\
$\mathrm{C} 200-M S 2$ & 0.102 & $13.0 \pm 0.5 \pm 1.5$ & 0.0790 & 0.0632 & 0.0703 & 0.0522 & 7.2 \\
$\mathrm{C} 200-M S 3$ & 0.102 & $13.6 \pm 0.6 \pm 1.5$ & 0.0760 & 0.0605 & 0.0663 & 0.0486 & 6.3 \\
$\mathrm{C} 200-M S 4$ & 0.102 & $12.7 \pm 0.6 \pm 1.4$ & 0.0817 & 0.0650 & 0.0705 & 0.0517 & 8.9 \\
$\mathrm{C} 200-M S 5$ & 0.101 & $14.2 \pm 0.6 \pm 1.6$ & 0.0727 & 0.0580 & 0.0629 & 0.0461 & 10.8 \\
$\mathrm{C} 200-M S 6$ & 0.101 & $12.6 \pm 0.5 \pm 1.4$ & 0.0824 & 0.0652 & 0.0702 & 0.0512 & 9.6 \\
$\mathrm{C} 200-M S 7$ & 0.101 & $14.2 \pm 0.6 \pm 1.6$ & 0.0730 & 0.0582 & 0.0637 & 0.0471 & 9.1 \\
$\mathrm{C} 200-M S 8$ & 0.101 & $12.6 \pm 0.7 \pm 1.4$ & 0.0831 & 0.0657 & 0.0697 & 0.0507 & 10.5 \\
$\mathrm{C} 200-M S 9$ & 0.101 & $12.2 \pm 0.6 \pm 1.4$ & 0.0855 & 0.0676 & 0.0715 & 0.0524 & 6.5 \\
$\mathrm{C} 200-M S 10$ & 0.101 & $12.3 \pm 0.5 \pm 1.4$ & 0.0833 & 0.0668 & 0.0727 & 0.0531 & 7.5 \\
$\mathrm{C} 200-M S 11$ & 0.101 & $14.0 \pm 0.5 \pm 1.6$ & 0.0735 & 0.0587 & 0.0648 & 0.0474 & 8.4
\end{tabular}


Appendix D: Results Within the No-GUT MSSM Scenario

\begin{tabular}{lc||rrrrr|r}
\hline \hline \multicolumn{1}{l||}{} & \multicolumn{1}{c||}{$\sigma_{N L O}$} & \multicolumn{1}{c}{$\varepsilon_{\text {Signal }}[\%]$} & \multicolumn{2}{c}{$\mu \mu \ell$ limits [pb] } & \multicolumn{2}{c}{ comb. limits [pb] } & Ov. \\
Name & {$[\mathrm{pb}]$} & $\varepsilon_{S} \pm$ stat \pm sys & $\sigma_{e x p}^{95 \%}$ & $\sigma_{S}^{95 \%}$ & $\sigma_{e x p}^{95 \%}$ & $\sigma_{S}^{95 \%}$ & {$[\%]$} \\
\hline \hline $\mathrm{C} 200-M S 12$ & 0.102 & $14.5 \pm 0.6 \pm 1.6$ & 0.0709 & 0.0567 & 0.0636 & 0.0468 & 10.1 \\
$\mathrm{C} 225-M S 1$ & 0.050 & $6.8 \pm 0.4 \pm 0.8$ & 0.1528 & 0.1213 & 0.1262 & 0.0904 & 4.7 \\
$\mathrm{C} 225-M S 2$ & 0.050 & $10.6 \pm 0.6 \pm 1.2$ & 0.0970 & 0.0776 & 0.0835 & 0.0611 & 7.0 \\
$\mathrm{C} 225-M S 3$ & 0.049 & $13.8 \pm 0.5 \pm 1.5$ & 0.0747 & 0.0594 & 0.0641 & 0.0469 & 7.3 \\
$\mathrm{C} 225-M S 4$ & 0.049 & $16.4 \pm 0.6 \pm 1.8$ & 0.0631 & 0.0501 & 0.0559 & 0.0414 & 9.0 \\
$\mathrm{C} 225-M S 5$ & 0.049 & $15.7 \pm 0.6 \pm 1.7$ & 0.0660 & 0.0527 & 0.0572 & 0.0420 & 7.3 \\
$\mathrm{C} 225-M S 6$ & 0.049 & $15.4 \pm 0.6 \pm 1.7$ & 0.0673 & 0.0533 & 0.0584 & 0.0429 & 6.8 \\
$\mathrm{C} 225-M S 7$ & 0.049 & $14.3 \pm 0.6 \pm 1.6$ & 0.0721 & 0.0575 & 0.0615 & 0.0449 & 9.0 \\
$\mathrm{C} 225-M S 8$ & 0.049 & $13.2 \pm 0.5 \pm 1.5$ & 0.0788 & 0.0623 & 0.0652 & 0.0472 & 10.5 \\
$\mathrm{C} 225-M S 9$ & 0.049 & $13.3 \pm 0.6 \pm 1.5$ & 0.0783 & 0.0619 & 0.0650 & 0.0471 & 7.3 \\
$\mathrm{C} 225-M S 10$ & 0.048 & $11.9 \pm 0.5 \pm 1.3$ & 0.0861 & 0.0691 & 0.0698 & 0.0500 & 6.3 \\
$\mathrm{C} 225-M S 11$ & 0.048 & $11.6 \pm 0.5 \pm 1.3$ & 0.0885 & 0.0707 & 0.0734 & 0.0531 & 6.9 \\
$\mathrm{C} 225-M S 12$ & 0.048 & $10.2 \pm 0.6 \pm 1.1$ & 0.1016 & 0.0812 & 0.0853 & 0.0615 & 7.0 \\
\hline \hline
\end{tabular}

Table D.1: The cross section upper limits for MSSM signal points with the lightest chargino mass fixed to $m\left(\widetilde{\chi}_{1}^{ \pm}\right)=103.5 \mathrm{GeV}$ (LEP limit [138]), $200 \mathrm{GeV}$, and $225 \mathrm{GeV}$. The expected $\left(\sigma_{\text {exp }}^{95 \%}\right)$ and observed $\left(\sigma_{S}^{95 \%}\right)$ limits are listed for this analysis alone $(\mu \mu \ell)$ and for the combination of the three trilepton analyses: $\mu \mu \ell$,

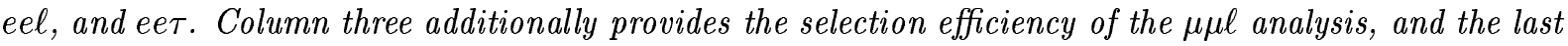
column shows the factional overlap between the $\mu \mu \ell$ and the eel analyses.

\begin{tabular}{|c|c|c|c|c|c|c|c|}
\hline \multirow{2}{*}{ Name } & \multirow{2}{*}{$\begin{array}{r}\sigma_{N L O} \\
{[\mathrm{pb}]} \\
\end{array}$} & \multirow{2}{*}{$\begin{array}{c}\varepsilon_{\text {Signal }}[\%] \\
\varepsilon_{S} \pm \text { stat } \pm \text { sys }\end{array}$} & \multicolumn{2}{|c|}{ 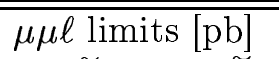 } & \multicolumn{2}{|c|}{ comb. limits [pb] } & \multirow{2}{*}{$\begin{array}{l}\mathrm{Ov} . \\
{[\%]}\end{array}$} \\
\hline & & & $\sigma_{e x p}^{95 \%}$ & $\sigma_{S}^{95 \%}$ & $\sigma_{e x p}^{95 \%}$ & $\sigma_{S}^{95 \%}$ & \\
\hline N80-MS1 & 3.164 & $9.2 \pm 0.5 \pm 1.0$ & 0.1121 & 0.0890 & 0.1037 & 0.0779 & 5.2 \\
\hline N80-MS2 & 1.161 & $10.6 \pm 0.5 \pm 1.2$ & 0.0976 & 0.0781 & 0.0878 & 0.0658 & 5.4 \\
\hline $\mathrm{N} 80-M S 3$ & 0.488 & $11.0 \pm 0.5 \pm 1.2$ & 0.0944 & 0.0751 & 0.0826 & 0.0610 & 6.7 \\
\hline $\mathrm{N} 80-M S 4$ & 0.220 & $12.0 \pm 0.5 \pm 1.3$ & 0.0862 & 0.0686 & 0.0741 & 0.0542 & 7.3 \\
\hline N80- $M S 5$ & 0.102 & $13.7 \pm 0.5 \pm 1.5$ & 0.0753 & 0.0601 & 0.0669 & 0.0494 & 7.5 \\
\hline N80- $M S 6$ & 0.049 & $15.8 \pm 0.6 \pm 1.8$ & 0.0659 & 0.0522 & 0.0583 & 0.0427 & 7.8 \\
\hline $\mathrm{N} 80-M S 7$ & 0.021 & $16.8 \pm 0.6 \pm 1.9$ & 0.0616 & 0.0492 & 0.0541 & 0.0396 & 9.2 \\
\hline N80- $M S 8$ & 0.010 & $15.9 \pm 0.6 \pm 1.8$ & 0.0655 & 0.0518 & 0.0568 & 0.0419 & 8.2 \\
\hline N80- $M S 9$ & 0.005 & $16.5 \pm 0.6 \pm 1.8$ & 0.0632 & 0.0499 & 0.0553 & 0.0408 & 8.3 \\
\hline \multirow[t]{2}{*}{ N80-MS10 } & 0.002 & $16.2 \pm 0.6 \pm 1.8$ & 0.0631 & 0.0507 & 0.0538 & 0.0389 & 8.3 \\
\hline & \multicolumn{6}{|c|}{$\mu=-1 \mathrm{TeV}$} & \\
\hline $\mathrm{N} 100-M S 1$ & 0.683 & $11.1 \pm 0.6 \pm 1.2$ & 0.0932 & 0.0740 & 0.0808 & 0.0595 & 7.2 \\
\hline N100-MS2 & 0.351 & $12.4 \pm 0.6 \pm 1.4$ & 0.0835 & 0.0668 & 0.0724 & 0.0530 & 7.2 \\
\hline N100-MS3 & 0.189 & $11.9 \pm 0.6 \pm 1.3$ & 0.0870 & 0.0692 & 0.0746 & 0.0544 & 9.3 \\
\hline N100-MS4 & 0.103 & $14.3 \pm 0.6 \pm 1.6$ & 0.0723 & 0.0575 & 0.0620 & 0.0454 & 10.3 \\
\hline N100-MS5 & 0.058 & $15.1 \pm 0.7 \pm 1.7$ & 0.0684 & 0.0546 & 0.0588 & 0.0427 & 7.4 \\
\hline N100-MS6 & 0.033 & $16.3 \pm 0.7 \pm 1.8$ & 0.0638 & 0.0505 & 0.0563 & 0.0416 & 9.6 \\
\hline
\end{tabular}

Table D.2: The cross section upper limits for MSSM signal points with the lightest neutralino mass fixed to $m\left(\widetilde{\chi}_{1}^{0}\right)=80 \mathrm{GeV}$, and $100 \mathrm{GeV}$. The expected $\left(\sigma_{e x p}^{95 \%}\right)$ and observed $\left(\sigma_{S}^{95 \%}\right)$ limits are listed for this analysis alone ( $\mu \mu \ell)$ and for the combination of the three trilepton analyses: $\mu \mu \ell$, eel, and eer. Column three additionally provides the selection efficiency of the $\mu \mu \ell$ analysis, and the last column shows the factional overlap between the $\mu \mu \ell$ and the eel analyses. 
Appendix D: Results Within the No-GUT MSSM Scenario

\begin{tabular}{|c|c|c|c|c|c|c|c|}
\hline \multirow[b]{2}{*}{ Name } & \multirow{2}{*}{$\begin{array}{r}\sigma_{N L O} \\
{[\mathrm{pb}]}\end{array}$} & \multirow{2}{*}{$\begin{array}{c}\varepsilon_{\text {Signal }}[\%] \\
\varepsilon_{S} \pm \text { stat } \pm \text { sys }\end{array}$} & \multicolumn{2}{|c|}{ 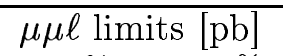 } & \multicolumn{2}{|c|}{ comb. limits $[\mathrm{pb}]$} & \multirow{2}{*}{$\begin{array}{l}\text { Ov. } \\
{[\%]}\end{array}$} \\
\hline & & & $\sigma_{e x p}^{95 \%}$ & $\sigma_{S}^{95 \%}$ & $\sigma_{e x p}^{95 \%}$ & $\sigma_{S}^{95 \%}$ & \\
\hline$M S$ map1 & 3.126 & - & - & - & - & - & - \\
\hline$M S$ map2 & 0.494 & - & - & - & - & - & - \\
\hline$M S$ map3 & 3.091 & - & - & - & - & - & - \\
\hline$M S$ map4 & 0.492 & - & - & - & - & - & - \\
\hline$M S$ map5 & 0.102 & - & - & - & - & - & - \\
\hline$M S m a p 6$ & 3.086 & $5.3 \pm 0.5 \pm 0.6$ & 0.1984 & 0.1578 & 0.1820 & 0.1358 & 3.7 \\
\hline$M S \operatorname{map} 7$ & 1.163 & $4.7 \pm 0.5 \pm 0.5$ & 0.2211 & 0.1768 & 0.2083 & 0.1589 & 5.4 \\
\hline$M S$ map8 & 0.492 & $6.2 \pm 0.5 \pm 0.7$ & 0.1675 & 0.1331 & 0.1537 & 0.1150 & 4.1 \\
\hline$M S \operatorname{map} 10$ & 2.537 & $8.6 \pm 0.5 \pm 1.0$ & 0.1206 & 0.0957 & 0.1133 & 0.0859 & 2.4 \\
\hline$M S$ map11 & 2.536 & $10.8 \pm 0.4 \pm 1.2$ & 0.0958 & 0.0767 & 0.0898 & 0.0679 & 4.9 \\
\hline$M S \operatorname{map} 12$ & 1.678 & $9.4 \pm 0.4 \pm 1.1$ & 0.1094 & 0.0871 & 0.1081 & 0.0844 & 2.3 \\
\hline$M S \operatorname{map} 13$ & 1.400 & $10.5 \pm 0.5 \pm 1.2$ & 0.0987 & 0.0785 & 0.0929 & 0.0700 & 4.5 \\
\hline$M S \operatorname{map} 14$ & 1.295 & $19.1 \pm 0.5 \pm 2.1$ & 0.0540 & 0.0431 & 0.0493 & 0.0372 & 7.2 \\
\hline$M S \operatorname{map} 15$ & 1.156 & $7.1 \pm 0.4 \pm 0.8$ & 0.1469 & 0.1163 & 0.1366 & 0.1032 & 6.5 \\
\hline$M S$ map16 & 0.810 & $16.4 \pm 0.5 \pm 1.8$ & 0.0628 & 0.0501 & 0.0585 & 0.0440 & 4.7 \\
\hline$M S \operatorname{map} 17$ & 0.639 & $20.1 \pm 0.5 \pm 2.3$ & 0.0517 & 0.0409 & 0.0464 & 0.0348 & 8.2 \\
\hline$M S \operatorname{map} 18$ & 0.491 & $9.6 \pm 0.4 \pm 1.1$ & 0.1083 & 0.0856 & 0.0986 & 0.0741 & 4.3 \\
\hline$M S$ map19 & 0.355 & $15.4 \pm 0.4 \pm 1.7$ & 0.0666 & 0.0534 & 0.0618 & 0.0465 & 4.9 \\
\hline$M S \operatorname{map} 20$ & 0.355 & $20.3 \pm 0.5 \pm 2.3$ & 0.0507 & 0.0405 & 0.0460 & 0.0343 & 8.6 \\
\hline$M S \operatorname{map} 21$ & 0.331 & $19.9 \pm 0.5 \pm 2.2$ & 0.0518 & 0.0414 & 0.0470 & 0.0348 & 7.0 \\
\hline$M S$ map22 & 0.223 & $12.6 \pm 0.4 \pm 1.4$ & 0.0817 & 0.0651 & 0.0757 & 0.0566 & 4.7 \\
\hline$M S$ map23 & 0.223 & $20.5 \pm 0.5 \pm 2.3$ & 0.0505 & 0.0402 & 0.0455 & 0.0339 & 8.5 \\
\hline$M S$ map24 & 0.180 & $20.4 \pm 0.5 \pm 2.3$ & 0.0504 & 0.0405 & 0.0453 & 0.0337 & 7.5 \\
\hline$M S$ map25 & 0.180 & $19.1 \pm 0.5 \pm 2.1$ & 0.0541 & 0.0431 & 0.0482 & 0.0357 & 7.0 \\
\hline$M S$ map26 & 0.140 & $16.9 \pm 0.5 \pm 1.9$ & 0.0612 & 0.0488 & 0.0573 & 0.0431 & 6.3 \\
\hline$M S \operatorname{map} 27$ & 0.102 & $17.5 \pm 0.6 \pm 2.0$ & 0.0592 & 0.0472 & 0.0553 & 0.0416 & 7.3 \\
\hline$M S$ map28 & 0.102 & $19.2 \pm 0.6 \pm 2.1$ & 0.0537 & 0.0428 & 0.0491 & 0.0367 & 8.8 \\
\hline$M S$ map29 & 0.102 & $20.1 \pm 0.6 \pm 2.2$ & 0.0514 & 0.0410 & 0.0468 & 0.0351 & 8.8 \\
\hline$M S \operatorname{map} 30$ & 0.102 & $19.1 \pm 0.6 \pm 2.1$ & 0.0540 & 0.0430 & 0.0488 & 0.0365 & 9.8 \\
\hline$M S \operatorname{map} 31$ & 0.102 & $17.3 \pm 0.5 \pm 1.9$ & 0.0597 & 0.0477 & 0.0546 & 0.0408 & 9.3 \\
\hline$M S$ map32 & 0.077 & $14.6 \pm 0.5 \pm 1.6$ & 0.0707 & 0.0564 & 0.0652 & 0.0491 & 7.0 \\
\hline$M S$ map33 & 0.077 & $18.2 \pm 0.5 \pm 2.0$ & 0.0563 & 0.0450 & 0.0532 & 0.0398 & 8.6 \\
\hline$M S$ map34 & 0.077 & $18.0 \pm 0.6 \pm 2.0$ & 0.0573 & 0.0457 & 0.0523 & 0.0393 & 8.0 \\
\hline$M S$ map35 & 0.077 & $19.2 \pm 0.6 \pm 2.1$ & 0.0539 & 0.0428 & 0.0488 & 0.0363 & 9.1 \\
\hline$M S \operatorname{map} 36$ & 0.077 & $20.7 \pm 0.6 \pm 2.3$ & 0.0500 & 0.0398 & 0.0458 & 0.0345 & 10.2 \\
\hline$M S$ map37 & 0.077 & $17.3 \pm 0.6 \pm 1.9$ & 0.0596 & 0.0477 & 0.0526 & 0.0388 & 10.6 \\
\hline$M S$ map38 & 0.077 & $18.8 \pm 0.4 \pm 2.1$ & 0.0547 & 0.0438 & 0.0495 & 0.0369 & 9.4 \\
\hline$M S$ map39 & 0.077 & $19.0 \pm 0.6 \pm 2.1$ & 0.0544 & 0.0434 & 0.0487 & 0.0358 & 10.1 \\
\hline$M S \operatorname{map} 40$ & 0.077 & $20.1 \pm 0.6 \pm 2.2$ & 0.0512 & 0.0411 & 0.0468 & 0.0352 & 8.9 \\
\hline$M S$ map41 & 0.077 & $17.5 \pm 0.5 \pm 2.0$ & 0.0587 & 0.0470 & 0.0527 & 0.0389 & 8.5 \\
\hline$M S$ map42 & 0.073 & $16.9 \pm 0.4 \pm 1.9$ & 0.0607 & 0.0486 & 0.0532 & 0.0394 & 7.4 \\
\hline$M S \operatorname{map} 43$ & 0.064 & $19.0 \pm 0.6 \pm 2.1$ & 0.0546 & 0.0433 & 0.0494 & 0.0370 & 9.5 \\
\hline$M S$ map44 & 0.064 & $18.6 \pm 0.5 \pm 2.1$ & 0.0553 & 0.0443 & 0.0504 & 0.0376 & 9.5 \\
\hline$M S \operatorname{map} 45$ & 0.064 & $19.9 \pm 0.5 \pm 2.2$ & 0.0517 & 0.0414 & 0.0468 & 0.0350 & 10.4 \\
\hline$M S$ map46 & 0.064 & $18.6 \pm 0.5 \pm 2.1$ & 0.0552 & 0.0442 & 0.0504 & 0.0376 & 10.3 \\
\hline$M S \operatorname{map} 47$ & 0.064 & $18.4 \pm 0.5 \pm 2.1$ & 0.0561 & 0.0446 & 0.0504 & 0.0375 & 10.4 \\
\hline
\end{tabular}




\begin{tabular}{|c|c|c|c|c|c|c|c|}
\hline \multirow[b]{2}{*}{ Name } & \multirow{2}{*}{$\begin{array}{r}\sigma_{N L O} \\
{[\mathrm{pb}]}\end{array}$} & \multirow{2}{*}{$\begin{array}{c}\varepsilon_{\text {Signal }}[\%] \\
\varepsilon_{S} \pm \text { stat } \pm \text { sys }\end{array}$} & \multicolumn{2}{|c|}{ 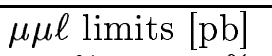 } & \multicolumn{2}{|c|}{ comb. limits $[\mathrm{pb}]$} & \multirow{2}{*}{$\begin{array}{l}\mathrm{Ov} \\
{[\%}\end{array}$} \\
\hline & & & $\sigma_{e x p}^{95 \%}$ & $\sigma_{S}^{95 \%}$ & $\sigma_{e x p}^{95 \%}$ & $\sigma_{S}^{95 \%}$ & \\
\hline$M S \operatorname{map} 48$ & 0.058 & $16.9 \pm 0.5 \pm 1.9$ & 0.0612 & 0.0487 & 0.0554 & 0.0414 & 7.9 \\
\hline$M S \operatorname{map} 49$ & 0.058 & $20.6 \pm 0.5 \pm 2.3$ & 0.0499 & 0.0399 & 0.0457 & 0.0338 & 7.0 \\
\hline$M S \operatorname{map} 50$ & 0.058 & $16.5 \pm 0.4 \pm 1.8$ & 0.0625 & 0.0500 & 0.0543 & 0.0397 & 6.9 \\
\hline$M S$ map51 & 0.056 & $19.1 \pm 0.6 \pm 2.1$ & 0.0542 & 0.0431 & 0.0507 & 0.0381 & 7.3 \\
\hline$M S$ map52 & 0.056 & $20.9 \pm 0.6 \pm 2.3$ & 0.0493 & 0.0393 & 0.0452 & 0.0336 & 9.2 \\
\hline$M S$ map53 & 0.056 & $19.5 \pm 0.6 \pm 2.2$ & 0.0528 & 0.0421 & 0.0477 & 0.0353 & 10.7 \\
\hline$M S$ map54 & 0.056 & $19.1 \pm 0.6 \pm 2.1$ & 0.0540 & 0.0431 & 0.0481 & 0.0356 & 8.8 \\
\hline$M S$ map55 & 0.056 & $18.7 \pm 0.6 \pm 2.1$ & 0.0550 & 0.0439 & 0.0489 & 0.0362 & 7.9 \\
\hline$M S \operatorname{map} 56$ & 0.042 & $19.8 \pm 0.6 \pm 2.2$ & 0.0521 & 0.0416 & 0.0485 & 0.0367 & 7.9 \\
\hline$M S$ map57 & 0.042 & $21.1 \pm 0.6 \pm 2.4$ & 0.0485 & 0.0389 & 0.0450 & 0.0340 & 9.5 \\
\hline$M S$ map58 & 0.042 & $19.5 \pm 0.6 \pm 2.2$ & 0.0528 & 0.0422 & 0.0470 & 0.0347 & 9.9 \\
\hline$M S$ map59 & 0.042 & $20.8 \pm 0.6 \pm 2.3$ & 0.0498 & 0.0396 & 0.0442 & 0.0328 & 9.9 \\
\hline$M S \operatorname{map} 60$ & 0.042 & $18.9 \pm 0.6 \pm 2.1$ & 0.0547 & 0.0435 & 0.0479 & 0.0354 & 9.8 \\
\hline$M S \operatorname{map} 61$ & 0.037 & $19.4 \pm 0.6 \pm 2.2$ & 0.0533 & 0.0424 & 0.0494 & 0.0374 & 10.2 \\
\hline$M S$ map62 & 0.037 & $20.4 \pm 0.6 \pm 2.3$ & 0.0507 & 0.0405 & 0.0464 & 0.0346 & 10.5 \\
\hline$M S$ map63 & 0.037 & $21.4 \pm 0.6 \pm 2.4$ & 0.0480 & 0.0383 & 0.0443 & 0.0332 & 8.8 \\
\hline$M S$ map64 & 0.037 & $19.9 \pm 0.6 \pm 2.2$ & 0.0519 & 0.0415 & 0.0471 & 0.0351 & 8.9 \\
\hline$M S$ map65 & 0.037 & $19.4 \pm 0.6 \pm 2.2$ & 0.0532 & 0.0424 & 0.0480 & 0.0358 & 8.3 \\
\hline$M S$ map66 & 0.037 & $20.1 \pm 0.6 \pm 2.2$ & 0.0515 & 0.0410 & 0.0463 & 0.0343 & 9.4 \\
\hline$M S \operatorname{map} 67$ & 0.037 & $18.8 \pm 0.6 \pm 2.1$ & 0.0546 & 0.0436 & 0.0489 & 0.0364 & 9.5 \\
\hline$M S$ map68 & 0.032 & $18.7 \pm 0.6 \pm 2.1$ & 0.0554 & 0.0443 & 0.0509 & 0.0384 & 9.2 \\
\hline$M S$ map69 & 0.032 & $21.2 \pm 0.6 \pm 2.4$ & 0.0488 & 0.0389 & 0.0451 & 0.0338 & 9.9 \\
\hline$M S$ map70 & 0.032 & $20.9 \pm 0.6 \pm 2.3$ & 0.0495 & 0.0393 & 0.0451 & 0.0339 & 8.6 \\
\hline$M S \operatorname{map} 71$ & 0.032 & $20.3 \pm 0.6 \pm 2.3$ & 0.0511 & 0.0404 & 0.0454 & 0.0338 & 10.4 \\
\hline$M S$ map72 & 0.032 & $19.9 \pm 0.6 \pm 2.2$ & 0.0516 & 0.0414 & 0.0463 & 0.0341 & 9.6 \\
\hline$M S$ map73 & 0.032 & $18.9 \pm 0.6 \pm 2.1$ & 0.0547 & 0.0436 & 0.0478 & 0.0352 & 9.6 \\
\hline$M S$ map74 & 0.024 & $16.1 \pm 0.5 \pm 1.8$ & 0.0638 & 0.0511 & 0.0580 & 0.0433 & 7.7 \\
\hline$M S \operatorname{map} 75$ & 0.024 & $20.8 \pm 0.6 \pm 2.3$ & 0.0495 & 0.0396 & 0.0452 & 0.0339 & 10.3 \\
\hline$M S$ map76 & 0.024 & $21.0 \pm 0.6 \pm 2.3$ & 0.0492 & 0.0391 & 0.0452 & 0.0337 & 9.8 \\
\hline
\end{tabular}

Table D.3: The cross section upper limits for MSSM signal points with independently varied mass parameters $M_{1}$ and $M_{2}$, governing the masses of the lightest neutralino $\left(m\left(\widetilde{\chi}_{1}^{0}\right)\right)$ and the lightest chargino $\left(m\left(\widetilde{\chi}_{1}^{ \pm}\right)\right)$, respectively. The remaining MSSM parameters are chosen as: $\tan \beta=5, \mu=1 \mathrm{TeV}, A_{0}=$ 0 , and $m_{A}=400 \mathrm{GeV}$. The expected $\left(\sigma_{\text {exp }}^{95 \%}\right)$ and observed $\left(\sigma_{S}^{95 \%}\right)$ limits are listed for this analysis alone $(\mu \mu \ell)$ and for the combination of the three trilepton analyses: $\mu \mu \ell$, eel, and eet. Column three additionally provides the selection efficiency of the $\mu \mu \ell$ analysis, and the last column shows the factional overlap between the $\mu \mu \ell$ and the eel analyses. 


\section{List of Figures}

1.1 The Higgs potential $\ldots \ldots \ldots \ldots \ldots \ldots$. . . . . . . . . . . . . . . . 12

1.2 Corrections to the Higgs boson mass . . . . . . . . . . . . . 15

1.3 Comparison of the scale dependence of the inverse gauge couplings in the SM and the MSSM model . . . . . . . . . . . . . . . . . . . 18

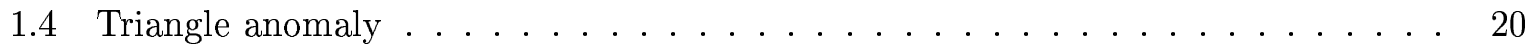

1.5 Interaction between gauge bosons and fermionic or scalar fields . . . . . . . . 26

1.6 Trilinear interactions between fermions and scalars, or of scalar fields alone . . . 26

1.7 Quadri- and trilinear interactions among gauge bosons and trilinear interactions of gauge bosons and gauginos . . . . . . . . . . . . . . 26

1.8 Interaction between a chiral scalar and fermion field . . . . . . . . . . . 26

1.9 Typical evolution of sparticle masses in a SUGRA scenario . . . . . . . . . . . . 32

1.10 Schematic mass spectrum of all supersymmetric particles and Higgs bosons with unified gaugino masses, scalar masses and trilinear couplings . . . . . . . . . . . 33

1.11 Leading order interactions associated with trilinear $\not p_{p}$-terms $\ldots \ldots \ldots$

1.12 Examples of R-parity violating processes . . . . . . . . . . . . 36

1.13 R-parity conserving gaugino pair production . . . . . . . . . . . 37

$1.14 \mathrm{R}$-parity violating decays of the lightest neutralino via the $L L \bar{E}$-coupling $\lambda_{122} \ldots 38$

$1.15 \mathrm{R}$-parity conserving cascade decays of heavier chargino/neutralino mass eigenstates 39

1.16 Direct $\not R_{p}$-decays of the lightest chargino via the $L L \bar{E}$-coupling $\lambda_{122} \ldots \ldots \ldots$

1.17 Muon decay and contributions to the Fermi constant . . . . . . . . . . . . . 40

1.18 Contributions to the neutrino mass through $\not R_{p}$-interactions via $\lambda_{122} \ldots \ldots \ldots 4$

2.1 Feynman diagram of the reaction: $p \bar{p} \rightarrow 2$ jets $\ldots \ldots \ldots \ldots \ldots$

2.2 Measurements of the proton structure functions: $F_{2}\left(x, Q^{2}\right)$ for fixed $x$, or fixed $Q^{2} \quad 46$

2.3 Distribution of parton density functions as measured by H1 and ZEUS . . . . . . 47

2.4 Overview of cross sections as a function of the CM-energy $\ldots \ldots \ldots$. . . . . . 49

2.5 Aerial view of the Fermilab site . . . . . . . . . . . . . . . 51 
2.6 Development of the instantaneous and integrated luminosity with time . . . . . 53

2.7 Schematic overview of the Fermilab accelerator chain . . . . . . . . . . . . 54

2.8 Aerial overview of the smaller accelerators at Fermilab . . . . . . . . . . . 54

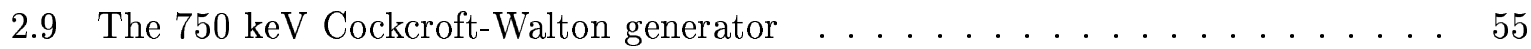

2.10 Drift-tube cavities in the first stage of the LINAC . . . . . . . . . . . . 56

2.11 Side-coupled cavities in the second LINAC stage . . . . . . . . . . . . 57

2.12 Strong focusing using alternating quadrupoles . . . . . . . . . . . . . 57

2.13 The Main Injector and Recycler rings . . . . . . . . . . . . . . . . . . 58

2.14 Schematic illustration of bunch rotation . . . . . . . . . . . . . . 60

2.15 Schematic illustration of stochastic cooling . . . . . . . . . . . . . . 60

2.16 The Debuncher and Accumulator rings and an illustration of the Accumulator stack profile ............................. 61

2.17 Schematic illustration of the bunch trains in the Tevatron . . . . . . . . 63

2.18 Beam positions in the transverse plane . . . . . . . . . . . . . . 64

2.19 Simplified $\gg$ onion skin layout of a multipurpose detector $\ldots \ldots \ldots$. . . . . . 65

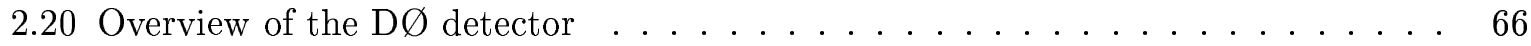

2.21 The inner tracking system of SMT and CFT . . . . . . . . . . . 67

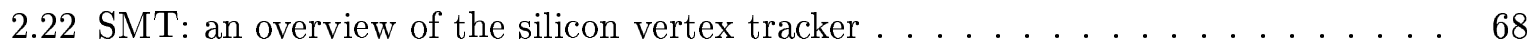

2.23 CFT: a doublet layer of the scintillating fiber tracker . . . . . . . . . . . . 69

2.24 VLPC: photograph and calibration spectrum $\ldots \ldots \ldots \ldots$

$2.25 \mathrm{SMT}$ and CFT combined resolution, schematic view of the CFT/CPS . . . . 70

2.26 CPS/FPS: the layout of the preshower detectors $\ldots \ldots \ldots$. . . . . . . 71

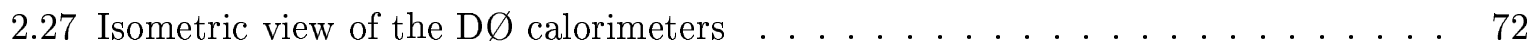

2.28 Calorimeter unit cell . . . . . . . . . . . . . . . . . . . 73

2.29 CAL quadrant: the semi-projective tower geometry . . . . . . . . . . . 74

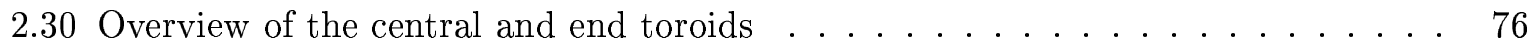

2.31 PDT cell configuration . . . . . . . . . . . . . . . . . . . 77

2.32 Overview of all muon wire chambers: PDTs and MDTs . . . . . . . . . 78

2.33 Overview of all muon scintillation counters . . . . . . . . . . . . . 80

2.34 Layout of the muon system A- $\varphi$ counters . . . . . . . . . . . . . . . . . . 82

2.35 Luminosity monitors . . . . . . . . . . . . . . . . . . . . . 84

2.36 The forward proton detector $(\mathrm{FPD}) \ldots \ldots \ldots \ldots \ldots$

2.37 The $\mathrm{D} \varnothing$ detector in open, rolled-out configuration . . . . . . . . . . . . 86

$2.38 \mathrm{D} \emptyset$ trigger \& data acquisition systems $\ldots \ldots \ldots \ldots \ldots \ldots$ 
2.39 Block diagram of the Level 1 and Level 2 trigger systems . . . . . . . . . . . . 88

2.40 Information and data flow through the L3DAQ system . . . . . . . . . . . 92

2.41 Illustrations of the physical architecture and the data flow of the online host system. 93

2.42 Physics store number 4400 , high data-taking efficiency . . . . . . . . . . . 95

2.43 Physics store number 3980, problems at Level 3 in the first three runs . . . . . . 96

2.44 Global Monitoring: low-level muon variables . . . . . . . . . . . . . . . . . 97

2.45 DØOM: The DØ Object Model . . . . . . . . . . . . . . . . . . 98

2.46 Data structure: the analysis chain for detector and Monte Carlo data . . . . . . . 101

3.1 Gaugino pair and gaugino-gluino production cross sections . . . . . . . . . 105

3.2 $K$-factors as calculated with GaUGinos as a function of the gaugino mass parameter $m_{1 / 2} \ldots \ldots \ldots \ldots \ldots \ldots \ldots \ldots \ldots \ldots$

3.3 SUSY particle masses as a function of the gaugino mass parameter $m_{1 / 2} \ldots \ldots 108$

3.4 Branching ratios of the second lightest neutralino and the lightest chargino for positive Higgs mixing parameter . . . . . . . . . . . . . . . . . . 110

3.5 Branching ratios of the second lightest neutralino and the lightest chargino for positive Higgs mixing parameter (cont'd) . . . . . . . . . . . . . . . . . . 111

3.6 Branching ratios of the second lightest neutralino and the lightest chargino for negative Higgs mixing parameter . . . . . . . . . . . . . . . . . 112

3.7 Branching ratios of the second lightest neutralino and the lightest chargino for negative Higgs mixing parameter (cont'd) . . . . . . . . . . . . . . 113

3.8 Multiplicity of additional charged leptons from the cascade decays of $\widetilde{\chi}_{2}^{0}$ and $\widetilde{\chi}_{1}^{ \pm}$. 116

3.9 Comparison of generator level and detector level lepton $(e, \mu)$ transverse momenta for $m_{0}=100 \mathrm{GeV} \ldots \ldots \ldots \ldots \ldots \ldots \ldots$

3.10 Comparison of generator level and detector level lepton $(e, \mu)$ transverse momenta for $m_{0}=1000 \mathrm{GeV} \ldots \ldots \ldots \ldots \ldots \ldots \ldots$

3.11 Comparison of generator level and detector level lepton $(e, \mu)$ transverse momenta for $m_{0}=250 \mathrm{GeV}, m_{1 / 2}=150 \mathrm{GeV}(140 \mathrm{GeV})$ for $\mu>0(\mu<0) \ldots \ldots \ldots$

3.12 Mean lepton $(e, \mu)$ transverse momentum at generator level and after reconstruction as a function of the gaugino mass parameter $m_{1 / 2}$ and for positive $\mu \ldots \ldots 121$

4.1 Data and luminosity consistency check . . . . . . . . . . . . . . 125

4.2 Comparison of two $Z / \gamma^{*}$ samples, produced with different PDF versions . . . . 128

4.3 Measurements of the L1 and L2 muon trigger efficiencies . . . . . . . . . . . . 134

4.4 Illustration of the muon isolation . . . . . . . . . . . . . . . 136

4.5 Reconstruction efficiency in data and Monte Carlo and the resulting correction factor . . . . . . . . . . . . . . . . . . . 140 
4.6 $\gg$ Medium « muon efficiency in data and Monte Carlo and the resulting correction factor . . . . . . . . . . . . . . . . . . . . . 141

4.7 Trackfinding/-matching efficiency in data and Monte Carlo and the resulting correction factor . . . . . . . . . . . . . . . . . . . . 142

4.8 Isolation efficiency in data and Monte Carlo and the resulting correction factor - 143

4.9 Illustration of infrared sensitivity . . . . . . . . . . . . . . . . . . . 144

4.10 Illustration of collinear sensitivity of the jet-finding algorithm . . . . . . . . . 145

4.11 Efficiencies and corresponding MC corrections for electron quality criteria . . . . 149

4.12 Jet energy scale correction and uncertainty for data . . . . . . . . . . . . . . . 152

4.13 Jet energy scale correction and uncertainty for MC simulated data . . . . . . 152

4.14 Missing transverse energy distribution $\left(\not_{T}^{j e s}\right)$ after preselection $\ldots \ldots \ldots \ldots 4$

4.15 Missing transverse energy distribution $\left(\not_{T}^{\mu}\right)$ after preselection . . . . . . . 154

4.16 Neural network output for the identification of $\tau$ leptons . . . . . . . . . . 155

4.17 Estimation of the multijet background via like-sign/unlike-sign dimuon samples . 157

4.18 Dimuon invariant mass distribution $M_{\mu \mu}$ after the preselection . . . . . . . . 159

4.19 Control distributions on preselection level . . . . . . . . . . . . . . . . 160

4.20 Spatial and angular distances between the two leading muons and other objects . 162

4.21 The two-dimensional selection criteria in the $\left(M_{\mu \mu}, \not_{T}\right)$-plane, $(\mu \mu$-cut 4 and 6): comparison of data and SM backgrounds . . . . . . . . . . . . . . . 164

4.22 The two-dimensional selection criteria in the $\left(M_{\mu \mu}, \not_{T}\right)$-plane, $(\mu \mu$-cut 4 and 6$)$ : for various mSUGRA signal points . . . . . . . . . . . . . . . 165

4.23 The two-dimensional criteria in the $\left(\Delta \varphi_{\mu \mu}, \not_{T}\right)$-plane, $(\mu \mu$-cut 5): comparison of data and SM backgrounds . . . . . . . . . . . . . . . . 166

4.24 The two-dimensional criteria in the $\left(\Delta \varphi_{\mu \mu}, \not_{T}\right)$-plane, $(\mu \mu$-cut 5$)$ for various mSUGRA signal points . . . . . . . . . . . . . . . . 167

4.25 Spatial and angular distances of the electron (muon) in the emu selection . . . . 168

4.26 Control distributions after the application of all dimu+emu selection criteria $\ldots$. 170

4.27 Third lepton properties for a muon and an electron candidate . . . . . . . . . . . 172

4.28 The last trilepton selection criterion $\sum p_{\mathrm{T}}^{\text {leptons }}>50 \mathrm{GeV} \ldots \ldots \ldots \ldots$

4.29 Illustration of the optimisation for different signal points . . . . . . . . . . . . 175

4.30 Control distributions for the study of the $Z$ boson $p_{\mathrm{T}}$-modeling $\ldots \ldots \ldots$

4.31 Event display of the one candidate event of $\mu \mu e$ type . . . . . . . . . . . . . 182

5.1 Illustration of two overlapping distributions of Poisson probabilities and the resulting statistical quantities: error types and confidence levels . . . . . . . . . . 187

5.2 Upper limits on the cross section and resulting lower bounds on the masses of the lightest chargino and neutralino for parameter sets with $\mu>0 \ldots \ldots$. . . . 194 
5.3 Continuation of Figure 5.2: upper limits on the cross section and resulting lower bounds on the masses of the lightest chargino and neutralino . . . . . . . . 195

5.4 Upper limits on the cross section and resulting lower bounds on the masses of the lightest chargino and neutralino for parameter sets with $\mu<0 \ldots \ldots$. . . 195

5.5 Exclusion contour in the $\left(m_{0}, m_{1 / 2}\right)$ plane of the mSUGRA parameter space . . 197

5.6 Exclusion contour in the $\left(m_{0}, m\left(\widetilde{\chi}_{1}^{ \pm}\right)\right)$plane of the mSUGRA parameter space . . 198

5.7 Exclusion contour in the $\left(m_{0}, m\left(\widetilde{\chi}_{1}^{0}\right)\right)$ plane of the mSUGRA parameter space . . 199

5.8 Upper cross section limits for MSSM signal points with fixed neutralino masses . 200

5.9 Upper cross section limits for MSSM signal points with fixed chargino masses . . 201

5.10 Exclusion contour in the $\left(\widetilde{\chi}_{1}^{0}, \widetilde{\chi}_{1}^{ \pm}\right)$-mass plane of the MSSM model . . . . . . 202 



\section{List of Tables}

1.1 The SM fermions and their quantum numbers . . . . . . . . . . 8

1.2 The symmetry eigenstates of the SM boson fields . . . . . . . . . . . . . 10

1.3 The mass eigenstates of the $\mathrm{SM}$ boson fields . . . . . . . . . . . . . . 13

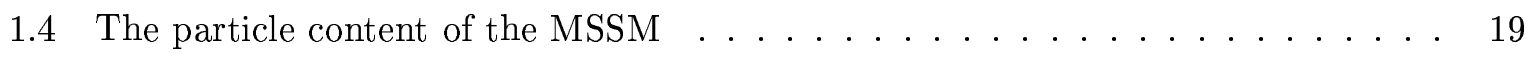

1.5 Overview of the undiscovered particles in mSUGRA and MSSM models . . . . . 34

1.6 Indirect bounds on single $\mathbb{R}_{p}$-couplings of the $L L \bar{E}$-type at the $2 \sigma$-level $\ldots \ldots$. . 41

2.1 Tevatron (and Main Injector) parameters . . . . . . . . . . . . 52

2.2 Calorimeter dimensions . . . . . . . . . . . . . . . . . 74

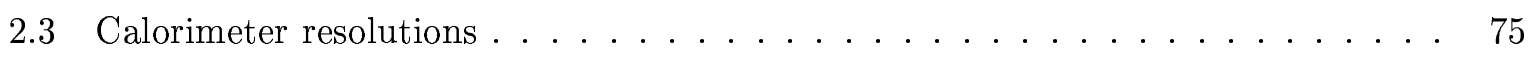

3.1 Decay length of the lightest neutralino as a function of its mass and the common sfermion mass $m_{0}$ in the mSUGRA model . . . . . . . . . . 106

4.1 Utilised dimuon triggers, run-ranges and corresponding luminosities . . . . . . . 124

4.2 The Monte Carlo simulations of Standard Model background processes . . . . . 127

4.3 Studied mSUGRA parameter sets . . . . . . . . . . . . . . . . . 129

4.4 Dependence of the LSP decay length on its mass . . . . . . . . . . . . . 130

4.5 Studied parameter sets in the no-GUT MSSM model . . . . . . . . . . . . . . 131

4.6 Parametrisation of the $\eta_{d e t}$-dependence of the L1 and L2 trigger conditions . . . 134

4.7 Average efficiencies and resulting Monte Carlo correction factors . . . . . . . . 138

4.8 Specifications of the Improved Legacy Cone Algorithm . . . . . . . . . . . . . 146

4.9 Fit parameters for the MC efficiency correction of the electron quality criteria . . 149

4.10 Parameters for the MC correction factors to the jet reconstruction efficiency . . . 151

4.11 Overview of the different isolation criteria for the estimation of the multijet background: first method . . . . . . . . . . . . . . 156

4.12 Overview of the different isolation criteria for the estimation of the multijet background: second method . . . . . . . . . . . . . . . 157 
4.13 Overview of the dimu+emu selection for all SM background processes . . . . . . 169

4.14 Overview of the dimu+emu selection: comparison of data with the sum of all SM background processes . . . . . . . . . . . . . . . . 169

4.15 Overview of the final trilepton selection criteria $\ldots \ldots \ldots \ldots \ldots$

4.16 Overview of the different cut combinations (cut values) tested during the optimisation . . . . . . . . . . . . . . . . . . . . 174

4.17 Overview of the expected SM background in the trilepton selection . . . . . . . 181

4.18 Comparison of data and SM background after the final trilepton selection . . . . 181

5.1 Summary of the possible conclusions of a hypothesis test . . . . . . . . . . . 185

5.2 Example overview of the upper cross section limits for some selected mSUGRA signal points: expected and observed limits from this analysis alone and from the combintion with the searches for eel and eer final states . . . . . . . . . . . . . 192

5.3 Summary of the lower bounds on $m\left(\widetilde{\chi}_{1}^{ \pm}\right)$and $m\left(\widetilde{\chi}_{1}^{0}\right)$ in the mSUGRA scenario . . 196

A.1 mSUGRA signal Monte Carlo simulations for $\mu>0 \ldots \ldots \ldots$. . . . . 207

A.2 mSUGRA signal Monte Carlo simulations for $\mu<0 \ldots \ldots \ldots$. . . . . 208

B.1 MSSM signal Monte Carlo samples for fixed values of $m\left(\widetilde{\chi}_{1}^{ \pm}\right) \ldots \ldots$. . . . . 210

B.2 MSSM signal Monte Carlo samples for fixed values of $m\left(\widetilde{\chi}_{1}^{0}\right) \ldots \ldots \ldots$. . . . 210

B.3 MSSM signal Monte Carlo samples for the mapping of the $\left(\widetilde{\chi}_{1}^{0}, \widetilde{\chi}_{1}^{ \pm}\right)$-mass plane . 212

C.1 Cross section upper limits for mSUGRA signal points with positive $\mu$ for the $\mu \mu \ell$

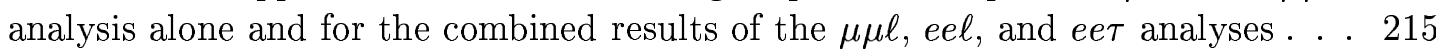

C.2 Cross section upper limits for mSUGRA signal points with negative $\mu$ for the $\mu \mu \ell$ analysis alone and for the combined results of the $\mu \mu \ell$, eel, and eet analyses . . . 216

D.1 Cross section upper limits for MSSM signal points with fixed chargino masses for

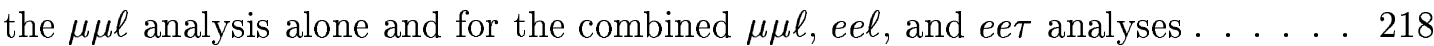

D.2 Cross section upper limits for MSSM signal points with fixed neutralino masses for the $\mu \mu \ell$ analysis alone and for the combined $\mu \mu \ell, e e \ell$, and $e e \tau$ analyses . . . . 218

D.3 Cross section upper limits for MSSM signal points with independently varied mass parameters $M_{1}$ (governing $\left.m\left(\widetilde{\chi}_{1}^{0}\right)\right)$ and $M_{2}$ (governing $m\left(\widetilde{\chi}_{1}^{ \pm}\right)$) . . . . . . . . 220 


\section{Bibliography}

[1] A.-M. Magnan and G. Sajot, »Search for RpV SUSY in the eel $(\ell=e$, or $\mu)$ Channel $\left(\lambda_{121}\right.$ Coupling) «, DØ Note 4982 (2005).

[2] A.-C. Le-Bihan and F. Charles, »Search for R-parity Violated Supersymmetry: $\lambda_{133}$ Coupling«, DØ Note 4979 (2005).

[3] I.J.R. Aitchison and A.J.G. Hey, »Gauge Theories in Particle Physics «, Vol. 1: »From Relativistic Quantum Mechanics to QED « and Vol. 2: »QCD and the Electroweak Theory «, Institute of Physics Publishing, ISBN: 075030950 4, $3^{\text {rd }}$ edition (2004); F. Halzen and A.D. Martin, »Quarks and Leptons «, John Wiley \& Sons, ISBN 0-471-88741-2, USA (1984).

[4] C.N. Yang and R.L. Mills, »Isotopic Spin Conservation and a Generalized Gauge Invariance «, Phys. Rev. 95 (1954), 631; C.N. Yang and R.L. Mills, »Conservation of Isotopic Spin and Isotopic Gauge Invariance «, Phys. Rev. 96 (1954), 191.

[5] S.L. Glashow, »Partial Symmetries of Weak Interactions «, Nucl. Phys. 22 (1961), 579; A. Salam and J.C. Ward, »On a Gauge Theory of Elementary Interactions «, Nuovo Cimento 19 (1961), 166; A. Salam and J.C. Ward, »Electromagnetic and weak interactions ", Phys. Lett. 13 (1964), 168; A. Salam and J.C. Ward, »Gauge theory of elementary interactions «, Phys. Rev. B 136 (1964), 763; S. Weinberg, »A Model of Leptons «, Phys. Rev. Lett. 19 (1967), 1264; S. Weinberg, »Effects of a Neutral Intermediate Boson in Semileptonic Processes «, Phys. Rev. D 5 (1972), 1412; A. Salam,»Weak and Electromagnetic Interactions «, originally printed in: Elementary Particle Theory, Proceedings of the Nobel Symposium Lerum, Sweden, Ed. N. Svartholm; Almquist and Wiksell, Stockholm (1968), 367.

Nobel Lectures (1979): S.L. Glashow, » Towards a Unified Theory - Threads in a Tapestry ", A. Salam, »Gauge Unification of Fundamental Forces «, S. Weinberg, »Conceptual Foundations of the Unified Theory of Weak and Electromagnetic Interactions «, published in: Nobel Lectures, Physics 1971-1980, Ed. S. Lundqvist, World Scientific Publishing Co., Singapore, 1992.

[6] P.W. Higgs, »Broken Symmetries, Massless Particles and Gauge Fields «, Phys. Lett. 12 (1964), 132; A. Klein and B.W. Lee, »Does Spontaneous Breakdown of Symmetry Imply Zero-Mass Particles?", Phys. Rev. Lett. 12 (1964), 266; F. Englert and R. Brout, »Broken Symmetry and the Mass of Gauge Vector Mesons «, Phys. Rev. Lett. 13 (1964), 321; P.W. Higgs, »Broken Symmetries and the Masses of Gauge Bosons «, Phys. Rev. Lett. 13 (1964), 508; G.S. Guralnik, C.R. Hagen and T.W.B. Kibble, »Global Conservation Laws and Massless Particles «, Phys. Rev. Lett. 13 (1964), 585; P.W. Higgs, »Spontaneous Symmetry Breakdown without Massless Bosons «, Phys. Rev. 145 (1966), 1156; T.W.B. Kibble, »Symmetry Breaking in Non-Abelian Gauge Theories «, Phys. Rev. 155 (1967), 1554.

[7] M. Gell-Mann and Y. Ne'eman, » The Eightfold Way«, New York, W.A. Benjamin (1964); 
[8] M. Gell-Mann, »Symmetries of Baryons and Mesons «, Phys. Rev. 125 (1961), 1067; H. Fritzsch, M. Gell-Mann and H. Leutwyler, »Advantages of the Color Octet Gluon Picture «, Phys. Lett. B 47 (1973), 365; D.J. Gross and F. Wilczek, »Asymptotically Free Gauge Theories.I«, Phys. Rev. D 8 (1973), 3633.

[9] D.J. Gross and F. Wilczek, »Ultraviolet Behavior of Nonabelian Gauge Theories «, Phys. Rev. Lett. 30 (1973), 1343; H.D. Politzer, »Reliable Perturbative Results for Strong Interactions? ", Phys. Rev. Lett. 30 (1973), 1346.

[10] S. Weinberg, »Nonabelian Gauge Theories of the Strong Interactions«, Phys. Rev. Lett. 31 (1973), 494.

[11] R.P. Feynman, »Space-Time Approach to Non-Relativistic Quantum Mechanics «, Rev. Mod. Phys. 20 (1948), 367; R.P. Feynman, »Relativistic Cut-Off for Quantum Electrodynamics «, Phys. Rev. 74 (1948), 1430; R.P. Feynman, »Space-Time Approach to Quantum Electrodynamics «, Phys. Rev. 76 (1949), 769; R.P. Feynman, » The Theory of Positrons «, Phys. Rev. 76 (1949),749; J. Schwinger, »Quantum Electrodynamics. I. A Covariant Formulation «, Phys. Rev. 74 (1948), 1439; J. Schwinger, »Quantum Electrodynamics. II. Vacuum Polarization and Self-Energy«, Phys. Rev. 75 (1949), 651; J. Schwinger, »Quantum Electrodynamics. III. The Electromagnetic Properties of the Electron - Radiative Corrections to Scattering «, Phys. Rev. 76 (1949), 790; S. Tomonaga, »On a Relativistically Invariant Formulation of the Quantum Theory of Wave Fields «, Prog. Theor. Phys. 1 (1946), 27; D. Itô, Z. Koba and S. Tomonaga, »Corrections due to the Reaction of "Cohesive Force Field" to the Elastic Scattering of an Electron.I«, Prog. Theor. Phys. 3 (1948), 276; Z. Koba and S. Tomonaga, »On Radiation Reactions in Collision Processes.I «, Prog. Theor. Phys. 3 (1948), 290; S. Tomonaga, »Remarks on Bloch's Method of Sound Waves applied to ManyFermion Problems «, Prog. Theor. Phys. 5 (1950), 544.

Nobel Lectures (1965): S. Tomonaga (May 6, 1966), »Development of Quantum Electrodynamics«, J. Schwinger, »Relativistic Quantum Field Theory«, R.P. Feynman, »The Development of the Space-Time View of Quantum Electrodynamics «, published in: Nobel Lectures, Physics 1963-1970, Elsevier Publishing Company, Amsterdam, 1972.

[12] G.'t Hooft, »Renormalization of Massless Yang-Mills Fields «, Nucl. Phys. B 33 (1971), 173; G.'t Hooft, »Renormalisable Lagrangians for Massive Yang-Mills Fields «, Nucl. Phys. B 35 (1971), 167.

[13] C.S. Wu, E. Ambler, R.W. Hayward, D.D. Hoppes and R.P. Hudson, » Experimental Test of Parity Conservation in Beta Decay«, Phys. Rev. 105 (1957),1413.

[14] N. Cabibbo, »Unitary Symmetry and Leptonic Decays «, Phys. Rev. Lett. 10 (1963), 531; M. Kobayashi and K. Maskawa, »CP Violation in the Renormalisable Theory of Weak Interaction «, Prog. Theor. Phys. 49 (1973), 652.

[15] The CDF Collaboration (F. Abe et al.), »Evidence for Top Quark Production in anti-p $p$ Collisions at $\sqrt{s}=1.8 \mathrm{TeV}$, Phys. Rev. D 50 (1994), 2966; »Observation of Top Quark Production in anti-p p Collisions «, Phys. Rev. Lett. 74 (1995), 2626;

The DØ Collaboration (S. Abachi et al.), »Observation of the Top Quark«, Phys. Rev. Lett. 74 (1995), 2632.

[16] The DONUT Collaboration (K. Kodama et al.), »Observation of Tau Neutrino Interactions «, Phys. Lett. B 504 (2001), 218; (R. Schwienhorst et al.), »A New Upper Limit for the Tau-Neutrino Magnetic Moment«, Phys. Lett. B 513 (2001), 23. 
[17] J.N. Bahcall, »Solar Neutrinos. I. Theoretical«, Phys. Rev. Lett. 12 (1964), 300; R. Davis, Jr., »Solar Neutrinos: II. Experimental«, Phys. Rev. Lett. 12 (1964), 303; J.N. Bahcall and R. Davis, Jr., »Solar Neutrinos: A Scientific Puzzle«, Science 191 (1976), 264; J.N. Bahcall and M.H. Pinsonneault, »Standard Solar Models, with and without Helium Diffusion, and the Solar Neutrino Problem «, Rev. Mod. Phys. 64 (1992), 885;

The GALLEX Collaboration, (P. Anselmann et al.), »GALLEX Solar Neutrino Observations: Complete Results for GALLEX II«, Phys. Lett. B 357 (1995), 237; (W. Hampel et al.), »GALLEX Solar Neutrino Observations: Results for GALLEX III «, Phys. Lett. B 388 (1996), 384;

The SAGE Collaboration, »Results from SAGE (The Russian-American Gallium Solar Neutrino Experiment) «, Phys. Lett. B 328 (1994), 234; »Measurement of the Solar Neutrino Capture Rate by SAGE and Implications for Neutrino Oscillations in Vacuum «, Phys. Rev. Lett. 83 (1999), 4686; »Measurement of the Solar Neutrino Capture Rate by the RussianAmerican Gallium Solar Neutrino Experiment During One Half of the 22-Year Cycle of Solar Activity«, J. Exp. Theor. Phys. 95 (2002), 181;

The GNO Collaboration (M. Altman et al.), »GNO Solar Neutrino Observations: Results for GNO I«, Phys. Lett. B 490 (2000), 16; [arXiv:hep-ex/0006034]; »Complete Results for Five Years of GNO Solar Neutrino Observations", Phys. Lett. B 616 (2005), 174; [arXiv:hep-ex/0504037].

[18] The Super-Kamiokande Collaboration (Y. Fukuda et al.), »Evidence for Oscillation of Atmospheric Neutrinos «, Phys. Rev. Lett. 81 (1998), 1562; »Tau Neutrinos Favored Over Sterile Neutrinos in Atmospheric Muon Neutrino Oscillations «, Phys. Rev. Lett. 85 (2000), 3999; »Solar ${ }^{8} B$ and hep Neutrino Measurements from 1258 Days of Super-Kamiokande Data«, Phys. Rev. Lett. 86 (2001), 5651; (S. Fukuda et al.), »Determination of Solar Neutrino Oscillation Parameters Using 1496 Days of Super-Kamiokande-I Data«, Phys. Lett. B 539 (2002), 179.

[19] The K2K Collaboration (M.H. Ahn et al.), »Indications of Neutrino Oscillation in a $250 \mathrm{~km}$ Long-Baseline Experiment«, Phys. Rev. Lett. 90 (2003), 041801; (E. Aliu et al.), »Evidence for Muon Neutrino Oscillation in an Accelerator-Based Experiment«, Phys. Rev. Lett. 94 (2005), 081802.

[20] The SNO Collaboration (Q.R. Ahmad et al.), »Measurement of Charged Current Interactions Produced by ${ }^{8}$ B Solar Neutrinos at the Sudbury Neutrino Observatory «, Phys. Rev. Lett. 87 (2001), 071301; »Direct Evidence for Neutrino Flavor Transformation from Neutral-Current Interactions in the Sudbury Neutrino Observatory «, Phys. Rev. Lett. 89 (2002), 011301; »Measurement of Day and Night Neutrino Energy Spectra at SNO and Constraints on Neutrino Mixing Parameters «, Phys. Rev. Lett. 89 (2002), 011302; Electron antineutrino search at the Sudbury Neutrino Observatory«, Phys. Rev. D 70 (2004), 093014; $»$ Electron Energy Spectra, Fluxes, and Day-Night Asymmetries of ${ }^{8} B$ Solar Neutrinos from the 391-Day Salt Phase SNO Data Set«, Phys. Rev. C 72 (2005), 055502.

[21] The Gargamelle Neutrino Collaboration (F.J. Hasert et al.), »Search for Elastic MuonNeutrino Electron Scattering «, Phys. Lett. B 46 (1973), 121; (F.J. Hasert et al.), »Observation of Neutrino-like Interactions without Muon or Electron in the Gargamelle Neutrino Experiment«, Phys. Lett. B 46 (1973), 138; and (T. Eichten et al.), »Measurement of the Neutrino-Nucleon Anti-Neutrino-Nucleon Total Cross Section«, Phys. Lett. B 46 (1973), 274; (F.J. Hasert et al.), »Observation of Neutrino-like Interactions without Muon or Electron in the Gargamelle Neutrino Experiment«, Nucl. Phys. B 73 (1974), 1.

[22] Y. Nambu, »Quasi-Particles and Gauge Invariance in the Theory of Superconductivity«, 
Phys. Rev. 117 (1960), 648; Y. Nambu, »Axial Vector Current Conservation in Weak Interactions «, Phys. Rev. Lett. 4 (1960), 380; Y. Nambu and G. Jona-Lasinio, » Dynamical Model of Elementary Particles Based on an Analogy with Superconductivity.I«, Phys. Rev. 122 (1961), 345. J. Goldstone, »Field Theories With 'Superconductor' Solutions « Nuovo Cimento 19 (1961), 154; J. Goldstone, A. Salam and S. Weinberg, »Broken Symmetries «, Phys. Rev. 127 (1962), 965; P.W. Anderson, »Plasmons, Gauge Invariance, and Mass «, Phys. Rev. 130 (1963), 439;

[23] The Particle Data Group Collaboration (S. Eidelmann et al.), »Review of Particle Physics «, Phys. Lett. B 592 (2004), 283; [Particle Data Group].

[24] E. Fermi, »Un Metodo Statistico per la Determinazione di alcune Priorieta dell'Atome«, Rend. Accad. Naz. Lincei 6 (1927), 602; L.H. Thomas, » The calculation of atomic fields «, Proc. Camb. Philos. Soc. 23 (1927), 542; E. Fermi and C.N. Yang, »Are Mesons Elementary Particles? «, Phys. Rev. 76 (1949), 1739; R.P. Feynman and M. Gell-Mann, » Theory of the Fermi Interaction «, Phys. Rev. 109 (1958) 193; E.H. Lieb, 》Thomas-Fermi and Related Theories of Atoms and Molecules «, Rev. Mod. Phys. 53 (1981), 603.

[25] W.J. Marciano and A. Sirlin, »Electroweak Radiative Corrections to $\tau$ Decay«, Phys. Rev. Lett. 61 (1988), 1815; T. van Ritbergen and R.G. Stuart, »Complete Two Loop Quantum Electrodynamic Contributions to the Muon Lifetime in the Fermi Model «, Phys. Rev. Lett. 82 (1999), 488 [arXiv:hep-ph/9808283].

[26] S. Weinberg, »Implications of Dynamical Symmetry Breaking«, Phys. Rev. D 13 (1976), 974; S. Weinberg, »Implications of Dynamical Symmetry Breaking: An Addendum «, Phys. Rev. D 19 (1978), 1277; L. Susskind, »Dynamics of spontaneous symmetry breaking in the Weinberg-Salam theory«, Phys. Rev. D 20 (1979), 2619; G.'t Hooft, »Naturalness, Chiral Symmetry, and Spontaneous Chiral Symmetry Breaking « in Recent Developments in Gauge Theories, Ed. G.'t Hooft et al. (Plenum Press, New York, 1980) p.135; Reprinted in Dynamical Gauge Symmetry Breaking, Eds. E. Farhi and R. Jackiw (World Scientific, Singapore, 1982) p.345; E. Witten, »Dynamical Breaking of Supersymmetry«, Nucl. Phys. B 188 (1981), 513;

[27] E. Lançona, »Electroweak fits at LEP and $S L D \ll$, Talk presented at the Siena Conference, The Legacy of LEP and SLC, Siena (Italy), 2001; P.B. Renton, »Precision Electroweak Tests of the Standard Model «, Rep. Prog. Phys. 65 (2002), 1271 [arXiv:hep-ph/0410177]; The ALEPH, DELPHI, L3, OPAL, SLD Collaborations, the LEP Electroweak Working Group, the SLD Electroweak and Heavy Flavour Groups, »Precision Electroweak Measurements on the $Z$ Resonance (2005); CERN-PH-EP/2005-041, SLAC-R-774 [arXiv:hepex/0509008].

[28] The LEP Working Group for Higgs Boson Searches, (ALEPH, DELPHI, L3 and OPAL Collaborations), »Search for the Standard Model Higgs Boson at LEP «, Phys. Lett. B 565 (2003), 61 [arXiv:hep-ex/0306033]; »Search for Neutral MSSM Higgs Bosons at LEP «, Paper contributed to ICHEP'04, Beijing (China), 2004; LHWG-Note 2004-01; ALEPH 2004008 PHYSICS 2004-007; DELPHI 2004-042 CONF 717; L3 Note 2820; OPAL Technical Note TN744; »Search for Neutral MSSM Higgs Bosons at LEP «, Paper contributed to the Lepton-Photon Symposium, Uppsala (Sweden), 2005; LHWG-Note 2005-01; ALEPH 2005002 PHYSICS 2005-002; DELPHI 2005-021 CONF 741; L3 Note 2830; OPAL Technical Note TN746.

[29] The Heidelberg-Moscow Collaboration: (L. Baudis et al.), »Limits on the Majorana Neutrino Mass in the 0.1-eV Range «, Phys. Rev. Lett. 83 (1999), 41 [arXiv:hep-ex/9902014]. 
[30] S.M. Bilenky, »Neutrinoless Double $\beta$-Decay: Status and Future (2005) [arXiv:hepph/0509098]; G. Altarelli and F. Feruglio, »Neutrino Masses and Mixings: A Theoretical Perspective «, Phys. Lett. B 439 (1998), 112 [arXiv:hep-ph/9905536]; O. Haug, » Majorana Neutrinos: Kern- und Teilchenphysikalische Aspekte «; http://w210.ub.uni-tuebingen.de/dbt/volltexte/2001/210/.

[31] C. Bennett, A. Kogut, G. Hinshaw, A. Banday, E. Wright, K. Gorski, D. Wilkinson, R. Weiss, G. Smoot, S. Meyer, J. Mather, P. Lubin, K. Loewenstein, C. Lineweaver, P. Keegstra, E. Kaita, P. Jackson and E. Cheng, »Cosmic Temperature Fluctuations from Two Years of COBE DMR Observations «, The Astrophysical Journal, 436 (1994), 423; [arXiv:astro-ph/9401012].

[32] The WMAP Collaboration (C.L. Bennet et al.), »First Year Wilkinson Microwave Anisotropy Probe (WMAP) Observations: Preliminary Maps and Basic Results «, The Astrophysical Journal, 148 (2003), 1 [arXiv:astro-ph/0302207].

[33] S.M. Carroll, »Why is the Universe Accelerating? «, Published in Carnegie Observatories Astrophysics Series, Vol. 2: Measuring and Modeling the Universe, Ed. W.L. Freedman, Cambridge Univ. Press; [arXiv:astro-ph/0310342] (2003);

S.M. Carroll, »The Cosmological Constant«, Max-Planck-Gesellschaft, ISSN 1433-8351 [arXiv:astro-ph/0004075] (2000); http://www.livingreviews .org/lrr-2001-1.

[34] Z. Maki, M. Nakagawa and S. Sakata, »Remarks on the Unified Model of Elementary Particles «, Prog. Theor. Phys. 28 (1962), 247; B. Pontecorvo, Zh. Eksp. Theor. Phys. 53 (1967), 1717; (Neutrino Experiments and the Conservation of Lepton Charge, Engl. transl. of Sov. Phys. JETP Lett. 26, 989.

[35] Y.A. Gol'Fand and E.P. Likhtman, »Extension of the Algebra of Poincare Group Generators and Violation of P Invariance «, Sov. Phys. JETP Lett. 13 (1971), 323; Reprinted in Supersymmetry, Ed. S. Ferrara, (North-Holland/World Scientific, Amsterdam/Singapore, 1987), Vol. 1, p.7; D.V. Volkov and V.P. Akulov, »Is the Neutrino a Goldstone Particle? ", Phys. Lett. B 46 (1973), 109; J. Wess and B. Zumino, »Supergauge Transformations In Four-Dimensions", Nucl. Phys. B 70 (1974), 39. J. Wess and B. Zumino, »Supergauge Invariant Extension of Quantum Electrodynamics«, Nucl. Phys. B 78 (1974), 1.

[36] S.J. Gates, M.T. Grisaru, M. Rocek and W. Siegel, »Superspace, Or One Thousand And One Lessons In Supersymmetry«, Front. Phys. 58 (1983), 1 [arXiv:hep-th/0108200].

[37] H.P. Nilles, »Supersymmetry, Supergravity and Particle Physics«, Phys. Rept. 110 (1984), 1; H.E. Haber and G.L. Kane, »The Search for Supersymmetry: Probing Physics Beyond the Standard Model«, Phys. Rept. 117 (1985), 75.

[38] S.P. Martin, »A Supersymmetry Primer «, Published in Perspectives on Supersymmetry, Ed. G. Kane, pp. 1-98. (World Scientific, Singapore, 1998) [arXiv:hep-ph/9709356].

[39] W. de Boer, »Grand Unified Theories and Supersymmetry in Particle Physics and Cosmology «, Progr. in Nucl. and Particle Phys. 33 (1994), 201 [arXiv:hep-ph/9402266]; M. Drees, $»$ An Introduction to Supersymmetry « (1996), APCTP-5 KEK-TH-501 [arXiv:hepph/9611409]; K.A. Olive, »Introduction to Supersymmetry: Astrophysical and Phenomenological Constraints «(1999), 201 [arXiv:hep-ph/9402266].

[40] H.K. Dreiner, »Hide and Seek with Supersymmetry«, Lecture at the Summer School on Hidden Symmetries and Higgs Phenomena at Zuoz, Switzerland (1998) [arXiv:hep$\mathrm{ph} / 9902347]$. 
[41] L. Susskind, »Dynamics of Spontaneous Symmetry Breaking in the Weinberg-Salam Theory «, Phys. Rev. D 20 (1979), 2619; M. Veltman, » The Infrared - Ultraviolet Connection«, Acta Phys. Pol. B 12 (1981), 437; M. Chaichian, R.G. Felipe and K. Huitu, » On Quadratic Divergences and the Higgs Mass«, Phys. Lett. B 363 (1995), 101.

[42] E. Witten, »Dynamical Breaking of Supersymmetry«, Nucl. Phys. B 188 (1981), 513; N. Sakai, » Naturalness in Supersymmetric 'GUTs' «, Z. Phys. C 11, (1981), 153; R.K. Kaul and P. Majumdar, »Cancellation of Quadratically Divergent Mass Corrections in Globally Supersymmetric Spontaneously Broken Gauge Theories«, Nucl. Phys. B 199 (1982), 36.

[43] C. Hoffmann, »Bestimmung einer unteren Masse des leichtesten Neutralinos mit dem ALEPH Detektor in $e^{+} e^{-}$-Kollisionen bei Schwerpunktsenergien bis $172 \mathrm{GeV}$ ", Dissertation, Johannes Gutenberg-Universität Mainz (1998), Shaker, Aachen (1998).

[44] C. Muñoz, »Dark Matter Detection in the Light of Recent Experimental Results «, Int. J. Mod. Phys. A 19 (2004), 3093-3170 [arXiv:hep-ph/0309346]; J.D. Wells, »Darkmatter in Gravity-mediated Supersymmetry Breaking«, Nucl. Phys. Proc. Suppl. 62 (1998), 235-240 [arXiv:hep-ph/9708284].

[45] H. Murayama and A. Pierce, »Perspectives on Supersymmetry«, Phys. Rev. D 65 (2002), 055009. S. Raby, »Proton Decay «, Talk presented at SUSY'02, DESY, Hamburg, Germany, (2002) [arXiv:hep-ph/0211024]; D.E. Morrissey, T.M.P. Tait and C.E.M. Wagner, »Proton Lifetime and Baryon Number Violating Signatures at the LHC in Gauge Extended Models", Phys. Rev. D 72 (2005), 095003 [arXiv:hep-ph/0508123].

[46] S.R. Coleman and J. Mandula, »All Possible Symmetries of the S Matrix«, Phys. Rev. 159 (1967), 1251; R. Haag, J.T. Lopuszanski and M. Sohnius, »All Possible Generators of Supersymmetries of the S Matrix«, Nucl. Phys. B 88 (1975), 257.

[47] J. Wess and B. Zumino, »Supergauge Transformations In Four-Dimensions«, Nucl. Phys. B 70 (1974), 39.

[48] P. Fayet and J. Iliopoulos, »Spontaneously Broken Supergauge Symmetries and Goldstone Spinors «, Phys. Lett. B 51 (1974), 461; P. Fayet, »Supergauge Invariant Extension of the Higgs Mechanism and a Model for the Electron and its Neutrino «, Nucl. Phys. B 90 (1975), 104; P. Fayet, » Mixing Between Gravitational and Weak Interactions Through the Massive Gravitino«, Phys. Lett. B 70 (1977), 461.

[49] L. O'Raifeartaigh, »Spontaneous Symmetry Breaking for Chiral Scalar Superfields «, Nucl. Phys. B 96 (1975), 331.

[50] S. Dimopoulos and H. Georgi, »Softly Broken Supersymmetry and SU(5)«, Nucl. Phys. B 193 (1981), 150. L. Giardello and M.T. Grisaru, »Soft Breaking of Supersymmetry«, Nucl. Phys. B 194 (1982), 65;

[51] D.Z. Freedman, P. van Nieuwenhuizen and S. Ferrara, »Progress Toward a Theory of Supergravity«, Phys. Rev. D 13 (1976), 3214; D.Z. Freedman and P. van Nieuwenhuizen, $»$ Properties of Supergravity Theory«, Phys. Rev. D 14 (1976), 912; S. Deser and B. Zumino, »Consistent Supergravity«, Phys. Lett. B 62 (1976), 335; S. Deser and B. Zumino, »Broken Supersymmetry and Supergravity«, Phys. Rev. Lett. 38 (1977), 1433; E. Cremmer, B. Julia, J. Scherk, P. van Nieuwenhuizen, S. Ferrara, and L. Girardello, »Superhiggs Effect in Supergravity with General Scalar Interactions «, Phys. Lett. B 79 (1978), 231; J. Wess and J. Bagger, »Supersymmetry and Supergravity«, Second edition, Princton University Press (1992). 
[52] Y. Okada, M. Yamaguchi and T. Yanagida, »Upper Bound of the Lightest Higgs Boson Mass in the Minimal Supersymmetric Standard Model«, Prog. Theo. Phys. 85 (1991), 1; J. Ellis, G. Ridolfi and F. Zwirner, »Radiative Corrections to the Masses of Supersymmetric Higgs Bosons «, Phys. Lett. B 257 (1991), 83; J. Ellis, G. Ridolfi and F. Zwirner, »On Radiative Corrections to Supersymmetric Higgs Boson Masses and Their Implications for LEP Searches «, Phys. Lett. B 262 (1991), 477; H.E. Haber and R. Hempfling, »Can the Mass of the Lightest Higgs Boson of the Minimal Supersymmetric Model Be Larger than $m Z$ ? «, Phys. Rev. Lett. 66 (1991), 1815; R. Barbieri, M. Frigeni and F. Caravaglios, »The Supersymmetric Higgs for Heavy Superpartners «, Phys. Lett. B 258 (1991), 167; Y. Okada, M. Yamaguchi and T. Yanagida, »Renormalization-group Analysis on the Higgs Mass in the Softly-broken Supersymmetric Standard Model«, Phys. Lett. B 262 (1991), 54.

[53] L.H. Hall, V.A. Kostalecky and S. Raby, »New Flavor Violations in Supergravity Models«, Nucl. Phys. B 267 (1986), 415; F. Gabbiani and A. Masiero, »Superheavy Contributions to FCNC in the Flipped $S U(5) \otimes U(1)$ «, Phys. Lett. B 209 (1988), 289; R. Barbieri and G.F. Giudice, $» b \rightarrow s \gamma$ Decay and Supersymmetry «, Phys. Lett. B 309 (1993), 86; R. Barbieri and L.J. Hall, »Signals For Supersymmetric Unification«, Phys. Lett. B 338 (1994), 212.

[54] J.F. Donahue, H.P. Nilles and D. Wyler, »Flavour Changes in Locally Supersymmetric Theories «, Phys. Lett. B 128 (1983), 55; F. Gabbiani and A. Masiero, »FCNC in Generalized Supersymmetric Theories«, Nucl. Phys. B 322 (1989), 235; J. Hagelin, S. Kelley and T. Tanaka, »Supersymmetric Flavor Changing Neutral Currents: Exact Amplitudes and Phenomenological Analysis «, Nucl. Phys. B 415 (1994), 293. F. Gabbiani, E. Gabrielli, A. Masiero and L. Silvestrini, »A Complete Analysis of FCNC and CP Constraints in General SUSY Extensions of the Standard Model «, Nucl. Phys. B 477 (1996), 321; [arXiv:hep$\mathrm{ph} / 9604387]$.

[55] L.E. Ibañez, »Grand Unification with Local Supersymmetry«, Nucl. Phys. B 218 (1983), 514; L. Alvarez-Gaumé, J. Polchinski and M.B. Wise, »Minimal Low-energy Supergravity «, Nucl. Phys. B 221 (1983), 495; L. Hall, J. Lykken and S. Weinberg, »Supergravity as the Messenger of Supersymmetry Breaking«, Phys. Rev. D 27 (1983), 2359; P. Nath and R. Arnowitt, »Grand Unification and B and L Conservation «, Proceedings of Lepton and Baryon Number Violation in Particle Physics, Astrophysics and Cosmology, (1999), Eds. H.V. Klapdor-Kleingrothaus and I.V. Krivosheina; [arXiv:hep-ph/9808465].

[56] J. Ellis and D.V. Nanopoulos, »Flavour Changing Neutral Interactions in Broken Supersymmetric Theories «, Phys. Lett. B 110 (1982), 44; R. Barbieri and R. Gatto, » Conservation Laws for Neutral Currents in Spontaneously Broken Supersymmetric Theories «, Phys. Lett. B 110 (1982), 211; B.A. Campbell, »Supersymmetry and Neutral Flavor Nonconservation «, Phys. Rev. D 28 (1983), 209.

[57] V. Barger, M.S. Berger, P. Ohmann and R.J.N. Phillips, »Multilepton SUSY Signals from R-parity Violation at the Fermilab Tevatron «, Phys. Rev. D 50 (1994), 4299;

[58] R. Barbier et al., »Report of SUGRA Working Group for Run II of the Tevatron«, (2000); [arXiv:hep-ph/00003154]; S. Kraml, »SUSY Part 2, SUSY Breaking Models and Their Phenomenology«, Lecture at the Linear Collider Physics School 2003, Ambleside, United Kingdom (2003)

[59] L.J. Hall and M. Suzuki, »Explicit R Parity Breaking In Supersymmetric Models «, Nucl. Phys. B 231 (1984), 419. H.K. Dreiner, »An Introduction to Explicit R-parity Violation« 
(1997), also published in: »Perspectives on Supersymmetry«, Ed. G.L. Kane [arXiv:hep$\mathrm{ph} / 9707435]$.

[60] G. Bhattacharyya, »R-parity-Violating Supersymmetric Yukawa Couplings: A MiniReview«, Nucl. Phys. Proc. Suppl. A (1997), 83 [arXiv:hep-ph/9608415].

[61] R. Barbier et al., »R-parity Violating Supersymmetry«, Phys. Rept. 420 (2005); [arXiv:hep-ph/0406039].

[62] G.R. Farrar and P. Fayet, »Phenomenology of the Production, Decay, and Detection of New Hadronic States Associated With Supersymmetry«, Phys. Lett. B 76 (1987), 575-579.

[63] J.C. Pati and A. Salam, »Is Baryon Number Conserved? «, Phys. Rev. Lett. 31 (1973), 661. S. Weinberg, »Baryon- and Lepton-Nonconserving Processes«, Phys. Rev. Lett. 43 (1979), 1566

[64] G. Bhattacharyya and P.B. Pal, »Upper Bounds on All R-parity Violating $\lambda \lambda^{\prime \prime}$ Combinations from Proton Stability«, Phys. Rev. D 59 (1999), 097701 [arXiv:hep-ph/9809493].

[65] C.T. Autermann, »Search for Second Generation Resonant Slepton Production with the DØ Detector «, Ph.D. Thesis, RWTH Aachen, Aachen/Germany (2006); H. Dreiner, P. Richardson and M. Seymour, »Resonant Slepton Production in Hadron-Hadron Collisions «, Phys. Rev. D 63 (2001), 055008.

[66] F. Ledroit and G. Sajot, »Indirect Limits on SUSY $R_{p}$ Violating Couplings $\lambda$ and $\lambda^{\prime} \ll$, Rapport GDR-Supersymétrie GDR-S-008, (ISN Grenoble, 1998).

[67] B.C. Allanach, A. Dedes and H.K. Dreiner, »Bounds on R-parity Violating Couplings at the Weak Scale and at the GUT Scale«, Phys. Rev. D 60 (1999), 075014 [arXiv:hepph/9906209]; B.C. Allanach, A. Dedes and H.K. Dreiner, » The R-parity Violating Minimal Supergravity Model «, Phys. Rev. D 69 (2004), 115002 [arXiv:hep-ph/0309196].

[68] C.S. Aulakh and R.N. Mohapatra, »Supersymmetry And The Calculation Of Neutrino Masses «, Phys. Lett. B 121 (1983) 147. R.N. Mohapatra and J.W.F. Valle, »Neutrino Mass And Baryon-Number Nonconservation In Superstring Models «, Phys. Rev. D 34 (1986), 1642; A. Masiero and J.W.F. Valle, »A Model for Spontaneous R-Parity Breaking», Phys. Lett. B 251 (1990), 273; J.C. Romao and J.W.F. Valle, »Neutrino Masses in Supersymmetry with Spontaneously Broken R-parity«, Nucl. Phys. B 381 (1992), 87; M.A. Diaz, J. Ferrandis, J.C. Romao and J.W.F. Valle, »Unification of Gauge Couplings and the Tau Neutrino Mass in Supergravity without R-parity«, Nucl. Phys. B 590 (2000), 3 [arXiv:hep-ph/9906343];

[69] O. Haug, J.D. Vergados, A. Faessler and S. Kovalenko, »Neutrino Masses Originating from SUSY R-parity Violating Terms with U(1) Flavor Symmetry«, Phys. Atom. Nucl. 63 (2000), 1078; [Yad. Fiz. 63 (2000), 1153].

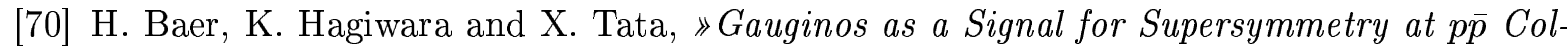
liders «, Phys. Rev. D 35 (1986), 1598; H. Baer, C. Kao and X. Tata, »Aspects of CharginoNeutralino Production at the Fermilab Tevatron Collider «, Phys. Rev. D 48 (1993), 5175; V. Barger, M.S. Berger, P. Ohmann, and R.J.N. Phillips, »Multilepton SUSY Signals from R-parity Violation at the Fermilab Tevatron«, Phys. Rev. D 50 (1994), 4299.

[71] B.C. Allanach et al., »Searching for R-parity Violation at Run II of the Tevatron « (1998) [arXiv:hep-ph/9906224]. 
[72] S. Dimopoulos and L.J. Hall, »Lepton and Baryon Number Violating Collider Signatures from Supersymmetry«, Phys. Lett. B 207 (1988), 210. H.K. Dreiner and G.G. Ross, »Rparity Violation at Hadron Colliders«, Nucl. Phys. B 365 (1991), 597-613.

[73] A.-M. Magnan, »Recherche de Particules Supersymétriques se Desintégrant en R-parité Violée (Couplage $\lambda_{121}$ ) Dans un État Final à Trois Leptons «, Ph.D. Thesis, Université Joseph Fourier, Grenoble/France (July, 2005).

[74] A.-C. Le-Bihan, »Identification des Leptons Tau dans l'Expérience DØ auprès du Tevatron et Recherche de Particules Supersymétriques se Desintégrant avec R-parité Violée (Couplage $\left.\lambda_{133}\right)$ ), Ph.D. Thesis, Université Louis Pasteur, Strasbourg/France (April, 2005).

[75] V. D. Barger, G. F. Giudice and T. Han, »Some New Aspects of Supersymmetry R-Parity Violating Interactions«, Phys. Rev. D 40 (1989), 2987.

[76] The Particle Data Group Collaboration (K. Hagiwara et al.), »Review of Particle Physics«, Phys. Rev. D 66 (2002), 010001.

[77] C.M. Ankenbrandt et al., »Clashing Gigantic Synchrotrons «, FERMILAB-PROPOSAL0491 (1976); TevatronI Group, » Design Report Tevatron 1 Project «, FERMILAB-DESIGN1984-01 (1984); L.M. Lederman, » The Fermilab Upgrade «, FERMILAB-TM-1536 (1988), Proceedings of Summer Study on High Energy Physics in the 1990's, in Snowmass, USA (1988); http://www.fnal.gov/, http://www.fnal.gov/faw/fermilab_at_work.html

[78] H.T. Edwards, » The Tevatron Energy Doubler: A Superconducting Accelerator «, Ann. Rev. Nucl. Part. Sci. 35 (1985), 605;

Fermilab Beams Division, »Run II Handbook« http://www-ad.fnal.gov/runII/index.html http://www-bdnew.fnal.gov/operations/rookie_books/rbooks.html.

[79] The D $\varnothing$ Collaboration (S. Abachi et al.), » The Do Detector «, Nucl. Instrum. Meth. A 338 (1994), 185. [FERMILAB-PUB-93-179-E].

[80] The D $\varnothing$ Collaboration (V.M. Abazov et al.), » The Upgraded D $\varnothing$ Detector «, Nucl. Instrum. Methods Phys. Res. A (to be published), [arXiv:physics/0507191].

[81] The DØ Collaboration (V.M. Abazov et al.),»The Muon System of the Run II DØ Detector«, Nucl. Instrum. Methods Phys. Res. A 552 (2005), 372 [arXiv.org:physics/0503151].

[82] Visual Media Services - Fermilab: http://www-visualmedia.fnal.gov/VMS_Site_2/index.shtml.

[83] J. D. Bjorken and E. A. Paschos, »Inelastic Electron Proton and Gamma Proton Scattering, and the Structure of the Nucleon«, Phys. Rev. 185 (1969), 1975.

[84] G. Altarelli and G. Parisi, »Asymptotic Freedom in Parton Language«, Nucl. Phys. 126 (1977), 298; V.N. Gribov and L.N. Lipatov, »Deep Inelastic ep-Scattering In Perturbation Theory«, Sov. J. Nucl. Phys. 15 (1972), 438; [Yad. Fiz. 15 (1972), 781]; Y.L. Dokshitzer, »Calculation of the Structure Functions for Deep Inelastic Scattering and $e^{+} e^{-}$Annihilation by Perturbation Theory in Quantum Chromodynamics «(Russian), Sov. Phys. JETP 46 (1977), 641; [Zh. Eksp. Teor. Fiz. 73 (1977), 1216].

[85] The H1 Collaboration (C. Adloff et al.), »Measurement and QCD Analysis of Neutral and Charged Current Cross Sections at HERA«, Eur. Phys. J. C 30 (2003), 1; [arXiv:hepex/0304003]. 
[86] The ZEUS Collaboration (S. Chekanov et al.), »A ZEUS next-to-leading-order QCD Analysis of Data on Deep Inelastic Scattering «, Phys. Rev. D 67 (2003), 012007; [arXiv:hepex/0208023]; The ZEUS Collaboration (S. Chekanov et al.), »An NLO QCD Analysis of Inclusive Cross Section and Jet Production Data from the ZEUS Experiment «, Eur. Phys. J. C 42 (2005), 1 [arXiv:hep-ph/0503274].

[87] J. Pumplin, D.R. Stump, J. Huston, H.L. Lai, P. Nadolsky and W.K. Tung, »New Generation of Parton Distributions with Uncertainties from Global QCD Analysis «, JHEP 0207 (2002), 012; [arXiv:hep-ph/0201195]; D. Stump, J. Huston, J. Pumplin, W.K. Tung, H.L. Lai, S. Kuhlmann, and J.F. Owens, »Inclusive Jet Production, Parton Distributions, and the Search for New Physics «, JHEP 0310 (2003), 046 [arXiv:hep-ph/0303013]; http://user .pa.msu.edu/wkt/cteq/cteq6/cteq6pdf.html.

[88] The Coordinated Theoretical-Experimental Project on QCD (CTEQ) Collaboration: http://www.phys.psu.edu/ ${ }^{\sim}$ cteq/.

[89] A.M. Cooper-Sarkar, A. Bodek, K. Long, E. Rizvi and H. Spiesberger, »High $Q^{2}$ Deep Inelastic Scattering at HERA«, J. Phys. G 25 (1999), 1387; [arXiv:hep-ph/9902277].

[90] E. Rizvi, »QCD Analyses of NC and CC Cross Sections, Determination of PDFs and $\alpha_{S} \lll$, Talk presented at the International Euro-Physics Conference on High Energy Physics, Aachen, Germany, (2003).

[91] V. Lendermann, »Measurement of Proton Structure at the HERA Collider «, Talk presented at the $18^{\text {th }}$ Lake Louise Winter Institute 2005 on Fundamental Interactions Alberta, Canada, (2005).

[92] S. Catani, »Aspects of QCD, from the Tevatron to the $L H C$ «, (2000) [arXiv:hep-ph/0005233].

[93] P. Skands, »Phenomenological Studies on Supersymmetry and the Strong Force«, Ph.D. Thesis, Lund University, Lund/Sweden (September, 2004).

[94] M.D. Church and J.P. Marriner, » The Antiproton Sources: Design and Operation«, Ann. Rev. Nucl. Part. Sci. 43 (1993), 253.

[95] S. van der Meer, »Stochastic Damping of Betatron Oscillations in the ISR «, CERN/ISRPO/72-31 (1972); G. Carron and L. Thorndahl, »Stochastic Cooling of Momentum Spread by Filter Techniques «, CERN/ISR-RF/78-12 (1978); S. van der Meer, »Stochastic Stacking in the Antiproton Accumulator «, CERN/PS-AA/78-22 (1978a); S. van der Meer, »Precooling in the Antiproton Accumulator «, CERN/PS-AA/78-26 (1978b); H. Herr and D. Möhl, »Bunched Beam Stochastic Cooling «, CERN/EP/Note 79-34 (1979); S. van der Meer, »A Different Formulation of the Longitudinal and Transverse Beam Response «, CERN/PSAA/80-4 (1980); D. Möhl, G. Petrucci, L. Thorndahl and S. van der Meer, »Physics and Technique of Stochastic Cooling «, Phys. Rep. 58 (1980), 73; D. Möhl, »Stochastic Cooling for Beginners «, Proceedings of the CERN Accelerator School on Antiprotons for Colliding Beam Facilities, CERN 84-15 (1984). S. van der Meer, »Stochastic Cooling and the Accumulation of Antiprotons «, Rev. Mod. Phys. 57 (1985), 689. Nobel Lecture (1984): S. van der Meer, »Stochastic Cooling and the Accumulation of Antiprotons «, Published in Nobel Lectures, Physics 1981-1990, Ed. Gösta Ekspång, World Scientific Publishing Co., Singapore, 1993.

[96] G. Budker, G. Dimov and V. Dudnikov, Proceedings of the International Symposium on Electron and Positron Storage Rings, Saclay/Paris, France, 1966, Eds. H. Zyngier and E. Cremienx-Alcan, p.II-1-1; G. Budker, G. Dimov and V. Dudnikov, Sov. At. Energy 22 
(1967), 384; G.I. Budker et al., » Experimental Studies of Electron Cooling«, PAAC 7 (1976), 197; http://www-ecool.fnal.gov/.

[97] S. Nagaitsev, »Electron Cooling Demonstration with Recycler $8.9 \mathrm{GeV} / c$ pbars «, Talk presented at Fermilab (July 2005);

http://www.fnal.gov/directorate/program_planning/all_experimenters_meetings/special_reports/Nagaitsev_Ecooling_07_18_05.pdf.

[98] D.M. Kaplan and K.S. Nelson, »Introduction to Subatomic-Particle Spectrometers « (1998), [arXiv:physics/9805026].

[99] R.C. Ruchti, »The Use of Scintillating Fibers for Charged-Particle Tracking«, Ann. Rev. Nucl. Part. Sci. 46 (1996), 281.

[100] M.D. Petroff and M.G. Stapelbroek, »Photon-Counting Solid-State Photomultiplier «, IEEE Trans. Nucl. Sci. 36 (1989), 158; M. D. Petroff and M. Atac, IEEE Trans. Nucl. Sci. 36 (1989), 163.

[101] J. Brzenziak et al., »Conceptual Design of a 2-Tesla Superconducting Solenoid for the Fermilab DØ Detector Upgrade«, Fermilab-TM-1886 (1994); B. Squires et al., »Design of the 2 Tesla Superconducting Solenoid for the Fermilab DØ Detector Upgrade«, Published in Advances in Cryogenic Engineering, Ed. P. Kittel, Plenum Press, New York, New York, 1994, p.301.

[102] P. Baringer et al., »Cosmic Ray Tests of the DØ Preshower Detector «, Nucl. Instr. Methods Phys. Res. A 469 (2001), 295.

[103] A.M. Patwa, »The Forward Preshower System and a Study of the J $\psi$ Trigger with the DØ Detector «, Ph.D. Thesis, State University of New York, Stony Brook/U.S.A. (2002); http://www-d0.fnal.gov/results/publications_talks/thesis/patwa/thesis.ps.

[104] The DØ EMID Group: http://www-d0.fnal.gov/phys_id/emid/d0_private/emid.html; http://www-d0.fnal.gov/phys_id/emid/d0_private/certification/main_v2_2.html\#resolution;

Y. Arnaud [for the D $\varnothing$ EMID Group], »Run II Calorimeter Resolution«, Talk presented in the EMID meeting at Fermilab, Oct.23, 2002;

http://www-d0.fnal.gov/phys_id/emid/d0_private/minutes/20021023yannick.pdf

[105] The DØ MuonID \& Algorithms Group: C. Clément, F. Deliot, T. Golling, K. Haganaki, B. Leonhardt, M. Mulders, E. Nurse, S. Soldner-Remboldt, and J. Stark, »MuonID Certification for p14«, DØ Note 4350 (2004);

http://www-do.fnal.gov/phys_id/muon_id/d0_private/muon_id.html; http://www-d0.fnal.gov/phys_id/muon_id/d0_private/certif/p14/index.html.

[106] http://d0.web.lal.in2p3.fr/DOLAL/Galerie/galerie.html.

[107] The DØ Collaboration (A. Brandt et al.), »A forward proton detector at D0«, FERMILABPUB-97-377.

[108] U. Amaldi et al., »Measurements of the Proton Proton Total Cross-Sections by Means of Coulomb Scattering at the CERN Intersecting Storage Rings«, Phys. Lett. B 43 (1973), 231.

[109] http://www-d0.fnal.gov/runcoor/DAQ/.

[110] http://www.pa.msu.edu/hep/d0/11/framework/tfw_tutorial_march_2003.html. 
[111] M. Fortner, A Maciel, H. Evans, B. Kothari and S. Uzunyan, » The Level-2 muon trigger at D0«, IEEE Trans. Nucl. Sci. 49 (2002), 1589.

[112] http://www-d0en.fnal.gov/enstore/.

[113] SAM: http://d0db.fnal.gov/sam; http://www-d0.fnal.gov/computing/sam/; I. Terekhov, »Distributed Processing and Analysis of Physics Data in the Dzero SAM System at Fermilab «, Fermilab-TM-2156 (2001); A. Baranovski et al., »SAM Managed Cache and Processing for Clusters in a Worldwide Grid-Enabled System «, Fermilab-TM2175 (2002); I. Terekhov et al., »SAM for Dzero - A Fully Distributed Data Access Layer for Dzero Run II» Talk presented at the VII. International Workshop on Advanced Analysis Techniques in Physics Research (ACAT) at Fermilab, Batavia (USA), 2000;

L. Loebel Carpenter et al., »SAM Overview and Operational Experience at the Dzero Experiment «, »Resource Management in SAM and the DO Particle Physics Data Grid «, and $» S A M$ and the Particle Physics Data Grid«, L. Lueking et al., »Resource Management in SAM and the DO Particle Physics Data Grid «, Talks presented at the International Symposium on Computing in High Energy Physics (CHEP) 2001, Beijing, China; Published in the Proceedings.

[114] M. Begel, D. Edmunds, P. Laurens and R. Partridge, »DØLuminosity in Run II: Delivered «, DØ Note 3970 (2003); M. Begel et al., »DØLuminosity in Run II: Recorded«, DØ Note 3972 (2003); M. Begel et al., »Luminosity and Performance of DØ During the First Year of Run II «, DØ Note 3973 (2003); T. Andeen et al., » The Updated DØ Luminosity Determination - Short summary«, DØ Note 4328 (2004); B. Casey, T. Edwards and H. Schellman, »A crosscheck of the Run II Luminosity Monitor Efficiency «, DØ Note 4329 (2004); T. Andeen et al., »Luminosity Constant for Dzero Run II«, DØ Note 4496 (2004).

[115] L. Dalesio et al., »The Experimental Physics and Industrial Control System Architecture: Past, Present, and Future «, Proceedings of the International Conference on Accelerator 8 Large Experimental Physics Control Systems (ICALEPCS'93), in Berlin, Germany, (1993), p.179.

[116] J.F. Bartlett et al., »The Control Architecture of the DØ Experiment «, Talk presented at the 8th International Conference on Accelerator \& Large Experimental Physics Control Systems (ICALEPCS'01, San Jose (CA), USA (2001); [TUCT005]; Tutorials, Talks on DØ Operations and Controls at:

tt http://www-d0.fnal.gov/runcoor/DAQ/.

[117] http://www-d0online.fnal.gov/groups/gm/GM.html, http://www-d0online.fnal.gov/groups/gm/TrigExPlotsDescription.html, http://www-d0online.fnal.gov/groups/gm/PhysExPlotsDescription.html, http://www-d0online.fnal.gov/groups/gm/muon_doc/muon.html, http://www-d0.fnal.gov/computing/data_quality/figures/figures.html.

[118] S. Snyder, »The DØ Object Model«, Talk presented at the Computing in High Energy Physics Conference (Chep'98), Chicago, USA (1998); http://www.hep.net/chep98/PDF/130.pdf.

[119] R. Hauser, »The DØ Framework Software Tutorial«, Talk / Tutorial presented during the DØ Collaboration Week, February 2003; http://www-d0.fnal.gov/computing/algorithms/howto/tutorial/rhauser1.pdf.

[120] CINT: C/C++ Interpreter; http://root.cern.ch/root/Cint.html. 
[121] S. Protopopescu, S. Baffioni, E. Nagy, E. Thomas and N. Lahrichi, »The tmb_tree Package«, DØ Note 3978 (2002); S. Protopopescu, S. Baffioni and E. Nagy, »ThumbNail: a compact data format «, DØ Note 3979 (2002);

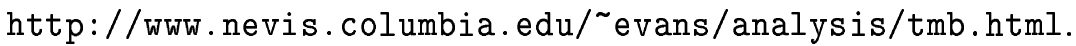

[122] R. Brun et.al., ROOT: An Object-Oriented Data Analysis Framework, Users Guide 5.08 (Dec. 2005); [Version ROOT 4.00/08 was utilised.] http://root.cern.ch/.

[123] F. Déliot, H. Greenlee, S. Kulik, A. Lyon, S. Protopopescu and G. Watts, »Report of the Data Format Working Group«, DØ Note 4473; D $\varnothing$ Data Format Working Group, »Recommendations Regarding Common Analysis Format Content «, DØ Note 4647 (2005); R. Hauser, »Common Analysis Format Tutorial«, Tutorial presented during the DØ Collaboration Week, Vancouver, 2005; http://www-d0.fnal.gov/Run2Physics/cs/caf/.

[124] T. Sjöstrand et al. Comput. Phys. Commun. 135 (2001) 238; [arXiv:hep-ph/0010017]; with L. Lönnblad, »PYTHIA 6.2 Physics and Manual«, [arXiv:hep-ph/0108264]; [Versions Pythia 6.201 and Pythia 6.202 were used.]

[125] S. Katsanevas and P. Morawitz, »Susygen 2.2, A Monte Carlo Event Generator for MSSM Sparticle Production at $e^{+} e^{-}$Colliders «, Comput. Phys. Commun. 112 (1998), 227; [arXiv:hep-ph/9711417]; N. Ghodbane, S. Katsanevas, P. Morawitz and E. Perez, »Susygen 3.0, A Monte Carlo Event Generator for Supersymmetric Particle Production at $e^{+} e^{-}$, ep and pp Colliders «; [Version Susygen 3 was used.] http://lyoinfo.in2p3.fr/susygen/susygen3.html.

[126] Y. Fisyank and J. Womersley, »DØGstar: DØGeant Simulation of the Total Apparatus Response«, DØ Note 3191 (1997); http://www-d0.fnal.gov/d0dist/dist/packages/d0gstar/devel/docs/html/d0gstar.html.

[127] R. Brun and F. Carminati, »GEANT - Detector Description and Simulation Tool «, CERN Program Library Long Writeup W5013 (1993), unpublished; [Version 3.21 was utilized.]

[128] http://www-d0.fnal.gov/computing/MonteCarlo/MonteCarlo.html; http://www.hep.ph.ic.ac.uk/ villeneu/mcprod/cook_book.html.

[129] The CDF Collaboration (T. Affolder et al.), »Charged Jet Evolution and the Underlying Event in Proton-Antiproton Collisions at 1.8 TeV«, Phys. Rev. D 65 (2002), 092002; R.D. Field [CDF Collaboration], »The Underlying Event in Hard Scattering Processes «, Contribution to the APS/DPF/DPB Summer Study on the Future of Particle Physics (Snowmass 2001), Snowmass, Colorado, U.S.A. (2001); [arXiv:hep-ph/0201192]

[130] [Version p14.06.01 was used to (re)-reconstruct real data, and all PYTHIA-generated MC events. For signal MC events, produced with the SUSYGEN generator, the production release p14.05.02 was used, since other versions did not correctly process the events.] http://www-d0.fnal.gov/computing/algorithms/status/p14.html; http://www-d0.fnal.gov/computing/algorithms/howto/howtoreco.html.

[131] http://www-d0.fnal.gov/Run2Physics/cs/index.html; http://www-d0.fnal.gov/Run2Physics/cs/skimming/pass1.html.

[132] M. Klute, M. Phaf, D. Whiteson,» TopAnalyze - A Framework Analyze Package For Top Group Analyses, and the Source Code of the TopAnalyze Package«, DØ Note 4122 (2003); Documentation and the package source code are available from: http://www-do.fnal.gov/Run2Physics/top/d0_private/wg/top_analyze. [Version Ipanema Updated was used to process data as well as MC events.] 
[133] The DØ Top Physics Working Group, »DØ Top Analysis and Data Sample for the Winter Conferences 2004«, Draft 0.3* (2004).

[134] B. Vachon et al. TriggerEfficiency Class, containing parametrisations of the Level 1, Level 2 and Level 3 trigger turn-on curves for a variety of triggers, including a set of dimuon triggers used in the present analysis:

http://www-d0.fnal.gov/Run2Physics/top/d0_private/wg/triggers/.

[135] H. Schellmann and M. Verzocchi: http://www-do.fnal.gov/phys_id/luminosity/data_access/lm_access/doc/runrange_luminosity.html.

[136] A. Djouadi, J.-L. Kneur and G. Moultaka, »SUSPECT: A Fortran Code for the Supersymmetric and Higgs Particle Spectrum in the MSSM « (2002); [arXiv:hep-ph/0211331];

[Version Suspect 2.2 was interfaced to the SusYgen event generator.]

[137] S. Dawson, »R-parity Breaking in Supersymmetric Theories«, Nucl. Phys. B 261 (1985), 297.

[138] The LEP2 SUSY Working Group (LEPSUSYWG) (M. Berggren et al.), and the ALEPH, DELPHI, L3, and OPAL experiments, »R-parity Violation with LLE Couplings «, (LEPSUSYWG/02-10.1); http://lepsusy.web.cern.ch/lepsusy/Welcome.html.

The ALEPH Collaboration (D. Buskulic et al.), »Search for R-Parity Violating Decays of Supersymmetric Particles in $e^{+} e^{-}$Collisions at Centre-of-Mass Energies from $189 \mathrm{GeV}$ to 202 GeV«, Eur. Phys. J.C 19 (2001), 415; (A. Heister et al.), »Search for Supersymmetric Particles with R-Parity Violating Decays in $e^{+} e^{-}$Collisions at $\sqrt{s}$ up to $209 \mathrm{GeV} \ll$, Eur. Phys. J.C 31 (2003), 1;

The DELPHI Collaboration (P. Abreu et al.), »Search for Supersymmetry with R-parity Violating LLE Couplings at $\sqrt{s}=183 \mathrm{GeV}$ ", Eur. Phys. J.C 13 (2000), 591; »Search for SUSY with R-parity Violating LLE Couplings at $\sqrt{s}=189 \mathrm{GeV}$ «, Phys. Lett. B 487 (2000), 36 ; »Search for Spontaneous R-parity Violation at $\sqrt{s}=183 \mathrm{GeV}$ and $189 \mathrm{GeV}$ «, Phys. Lett. B 502 (2001), 24; »Search for Supersymmetry with R-parity Violation at $\sqrt{s}=192$ to $208 \mathrm{GeV} \ll$, [DELPHI 2002-036-CONF-570];

The L3 Collaboration (M. Acciarri et al.), »Search for R-parity Violating Chargino and Neutralino Decays in $e^{+} e^{-}$Collisions up to $\sqrt{s}=183 \mathrm{GeV}$, Phys. Lett. B 459 (1999), 354 ; »Search for R-Parity Violating Decays of Supersymmetric Particles in $e^{+} e^{-}$Collisions at $\sqrt{s}=189 \mathrm{GeV}$, Eur. Phys. J.C 19 (2001), 397; (P. Achard et al.), »Search for $R$-parity Violating Decays of Supersymmetric Particles in $e^{+} e^{-}$Collision at LEP $\ll$, Phys. Lett. B 524 (2001), 65;

The OPAL Collaboration (P.D. Acton et al.), (G. Abbiendi et al.), »Tests of the Standard Model and Constraints on New Physics from Measurements of Fermion-pair Production at 189 GeV«, Eur. Phys. J.C 13 (2000), 553; »Search for R-parity Violating Decays of Scalar Fermions at LEP«, Eur. Phys. J.C 33 (2004), 149.

[139] The DELPHI Collaboration (P. Abreu et al.), »Search for SUSY with R-parity Violating $L L \bar{E}$ Couplings at $\sqrt{s}=189 \mathrm{GeV}$ «, Phys. Lett. B 487 (2000), 36; [arXiv:hep-ex/0103006].

[140] W. Beenakker, M. Klasen, M. Krämer, T. Plehn, M. Spira, and P.M. Zerwas, »The Production of Charginos/Neutralinos and Sleptons at Hadron Colliders «, Phys. Rev. Lett. 83 (1999) 3780; [arXiv:hep-ph/9906298].

[141] The DØ Common Sample Group (CSG); http://www-d0.fnal.gov/Run2Physics/cs/index.html;

http://www-d0.fnal.gov/Run2Physics/cs/skimming/skimming.html 
[142] http://d0db.fnal.gov/qualitygrabber/qualQueries.html;

Run II data-taking epochs:

http://www-d0.fnal.gov/computing/data_quality/punch/DataSets.txt.

[143] http://www-clued0.fnal.gov/ pverdier/d0_private/MetRunSel/;

http://www-clued0.fnal.gov/ pverdier/d0_private/MetRunSel/pass1_cert6.0.php

[144] DØ Monte Carlo request/production page, and MC Catalog:

http://www-d0.fnal.gov/computing/mcprod/mcc.html;

http://d0-france.in2p3.fr/WORKING_GROUPS/MONTECARLO/Catalog/current/.

[145] M.L. Mangano, M. Moretti, F. Piccinini, R. Pittau and A.D. Polosa, »ALPGEN, a Generator for Hard Multiparton Processes in Hadronic Collisions «, JHEP 0307 (2003), 001; CERN-TH-2002-129; [arXiv:hep-ph/0206293].

[146] The CTEQ Collaboration: (H. L. Lai et al.), »Global QCD Analysis of Parton Structure of the Nucleon: CTEQ5 Parton Distributions «, Eur. Phys. J.C12 (2000), 375;

[arXiv:hep-ph/9903282]; http://zebu.uoregon.edu/ parton/partonCTEQ.html.

[147] H.L. Lai, J. Huston, S. Kuhlmann, F. Olness, J. Owens, D. Soper, W.K. Tung, and H. Weerts, »Improved Parton Distributions from Global Analysis of Recent Deep Inelastic Scattering and Inclusive Jet Data «, Phys. Rev. D 55 (1997), 1280; [arXiv:hep-ph/9606399]; H.L. Lai and W.K. Tung, »Charm Production and Parton Distributions «, Z. Phys. C 74 (1997), 463. [arXiv:hep-ph/9701256];

[148] T. Nunnemann, »NNLO Cross-Sections for Drell-Yann, $Z$ and $W$ Production using Modern Parton Distribution Functions «, D $\varnothing$ Note 4476 (2004);

http://www-clued0.fnal.gov/〜nunne/cross-sections/dy_cross-sections.html.

[149] R. Hamberg, W.L. van Neerven and T. Matsuura, »A Complete Calculation of the Order $\alpha_{s}^{2}$ Correction to the Drell-Yan K-factor «, Nucl. Phys. B 359 (1991), 343; [Erratum-ibid. B 644 (2002), 403]. Program code: http://www.lorentz.leidenuniv.nl/neerven.

[150] NLO cross sections of diboson production (T. Nunnemann) http://www-clued0.fnal.gov/\%7Enunne/cross-sections/mcfm_cross-sections.html.

[151] N. Kidonakis and R. Vogt, »Next-to-Next-to-Leading Order Soft-Gluon Corrections in Top Quark Hadroproduction «, Phys. Rev. D 68 (2003), 114014;

http://www-d0.fnal.gov/Run2Physics/top/d0_private/wg/singletop/singletop_corrmc_strad.html.

[152] The DØ Collaboration (B. Abbott et al.), »Search for R-parity Violation in Multilepton Final States in pp Collisions at $\sqrt{s}=1.8$ TeV«, Phys. Rev. D Rapid. Comm. 62 (2000), 071701; E. Nagy and E. H. Smith, »Study of R-parity Violation Through Yukawa $\lambda$ Couplings «, DØ Note 3656 (1999).

[153] The ALEPH, DELPHI, L3 and OPAL Collaborations and the LEP Working Group for Higgs Boson Searches, »Search for Neutral MSSM Higgs Bosons at LEP «, Submitted to Eur. Phys. J. C; [arXiv:hep-ex/0602042]. LEP SUSY Working Group on the WEB: http://lepsusy.web.cern.ch/lepsusy/www/lsp_cmssm_summer04/cMSSM_final.html http://lepsusy.web.cern.ch/lepsusy/www/sleptons_budapest01/sleptons_pub.html.

[154] DØ Jet Energy Scale Group: http://www-d0.fnal.gov/phys_id/jes/d0_private/jes.html; http://www-d0.fnal.gov/phys_id/jes/d0_private/certified/certified.html; 
[Version JetCorr v5.3 is used in the framework package top_analyze.] http://www-d0.fnal.gov/phys_id/jes/d0_private/certified/v5.3/links.html.

[155] D. Chapin, H. Fox, J. Gardner, R. Illingworth, A. Lyon, and J. Zhu, » Measurement of

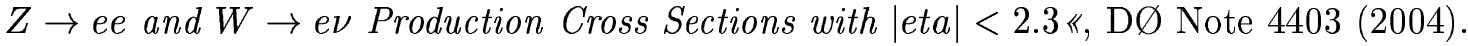

[156] R. Schwienhorst, »Top Trigger Selection and Application of Turn On Curves to the Monte Carlo«, DØ Note 4508 (2004); M. Agelou et al., » Top Trigger Efficiency Measurements and the top_trigger package«, DØ Note 4512 (2004);

Trigger list versions as a function of the run number: http://www-d0.fnal.gov/Run2Physics/top/d0_private/wg/triggers/other/trigger_list_summary.html.

[157] G.C. Blazey et al., »Run II Jet Physics«, Proceedings of the workshop QCD and Weak Boson Physics in Run II, Eds. U. Baur, R.K. Ellis and D. Zeppenfeld, Batavia (2000), p.47; [arXiv:hep-ex/0005012] and references [16] and [19] therein.

[158] G.C. Blazey and B.L. Flaugher, »Inclusive Jet and Dijet Production at the Tevatron «, Ann. Rev. Nucl. Part. Sci. 49 (1999), 633; [FERMILAB-PUB-99/038-E], [arXiv:hep-ex/9903058]; based on the Snowmass Algorithm: J.E. Huth et al. in the Proceedings of Research Directions for the Decade: Snowmass 1990, July 1990, Ed. E.L. Berger (World Scientific, Singapore, 1992), p. 134; [FERMILAB-CONF-90-249-E]; B. Andrieu, » Jet Finding Algorithms at Tevatron «, Talk presented at the XXXIV. International Symposium on Multiparticle Dynamics 2004 (ISMD04), Sonoma, California, U.S.A. (2004).

[159] G. Steinbrück, »Measurement of the Angular Distribution of Electrons from $W$ Boson Decays at $D \varnothing \lll$, Ph.D.-Thesis, University of Oklahoma, Oklahoma/U.S.A. (Sep. 1999). http://www-d0.fnal.gov/results/publications_talks/thesis/steinbrueck/thesis.ps

[160] D. Whitson and L. Phaf, »Electron Likelihood«, DØ Note 4184 (2003); J. Kozminski, R. Kehoe, H. Weerts, S.-J. Park, A. Quadt, J. Gardner and S. Jabeen, »Electron Likelihood in $p 14 \ll, \mathrm{D} \emptyset$ Note 4449 (2004); http://www-d0.fnal.gov/phys_id/emid/d0_private/EM_Particle_Documentation_EMID.html

[161] DØ JetID group: http://www-d0.fnal.gov/phys_id/jets/jetid-projects.html; http://www-d0.fnal.gov/computing/algorithms/calgo/jet/jetID_p14.html.

[162] N. Buchanan, »DØ Jet Energy Scale and Inclusive Jet Cross Section «, Talk presented at the Hadron Collider Physics Symposium 2005 (HCPS'05), Les Diablerets, Switzerland (2005);

[163] M. Agelou, J.-L. Agram, and C. Royon [for the DØ Jet Energy Scale Group], 0.5 and 0.7 jet $p_{T}$ resolution using JES v05-03-00«, DØ Note 4775 (2005).

[164] The DØ Collaboration (V. Abazov et al.), »Measurement of $\sigma(p \bar{p} \rightarrow Z) \times B r(Z \rightarrow \tau \tau)$ at $\sqrt{(s)}=1.96 \mathrm{Te} V \ll$, Phys. Rev. D 71 (2005) 072004.

[165] Private communication by Dr. Anne-Catherine Le-Bihan.

[166] D. Käfer and A. Meyer, $\gg A$ Search for RPV-SUSY in the $\mu \mu \ell(\ell=e, \mu)$ Channel from Decays via the $L L \bar{E}$-coupling $\lambda_{122} \ll$, DØ Note 4490 (2004); D. Käfer and A. Meyer, »A Search for R-parity Violating Supersymmetry in the $\mu \mu \ell(\ell=e, \mu)$ Channel«, DØ Note 4490 CONF (2004).

[167] [D $\varnothing$ Collaboration] A. Yurkewicz, R. Hauser, J. Linnemann and R. Moore, »Search for mSUGRA SUSY in the Like-Sign Dimuon Channel «, DØ Note 4408. 
[168] K. Anikeev et al., »B Physics at the Tevatron: Run II and Beyond«, p.441 ff. (2002) [FERMILAB-Pub-01/197], [arXiv:hep-ph/0201071]; see also the talk: »Beauty and Charm Production at the Fermilab Tevatron «; http://www-cdf.fnal.gov/physics/talks_transp/2004/rhic_bcprod_bishai.pdf.

[169] R.J. Barlow, »Systematic Errors: Facts and Fiction «, Talk presented at the Advanced Statistical Techniques in HEP Conference, Durham, Ireland (2002); [arXiv:hep-ex/0207026]. R.J. Barlow, »Lecture 5: Systematic Errors «, Lecture held at the SLAC Users Organisation: Lectures on Statistics and Numerical Methods in HEP, SLAC, Stanford, California, U.S.A. (2000);

[170] L. Christofek, » Text and Trigger Tables for Publications for the Top Quark Physics Group «, DØ Note 4978 (2006), and references to DØ Notes therein.

[171] B. Tiller and T. Nunnemann, »Measurement of the Differential $Z^{0}$-boson Production Cross Section as a Function of Transverse Momentum «, DØ Note 4660 (2004).

[172] Private communication by Prof. Dr. Gérard Sajot.

[The $5 \%$ uncertainty on the signal cross section due to a variation of the renormalization and factorization scales was obtained by varying both scales independently from $m / 2$ to $2 \mathrm{~m}$. The $3 \%$ uncertainty due to the $K$-factor was estimated by summing the respective uncertainties for the GaUginos NLO and LO cross sections in quadrature; and the remaining $2 \%$ uncertainty due to the SUSYGEN LO cross section covers merely the statistical uncertainty of the Monte Carlo Generator.]

[173] V. Büscher, J.-F. Grivaz, T. Nunnemann, and M. Wobisch, »Conclusions of the MiniWorkshop on PDF Uncertainties and Related Topics «, DØ Note 4618 (2004).

[174] E. Nurse and P. Telford, »Measurement of $\sigma \times B r$ for $\left(Z / \gamma^{*}\right) \rightarrow \mu^{+} \mu^{-}$and $Z \rightarrow \mu^{+} \mu^{-}$in $p \bar{p}$ Collisions at $\sqrt{s}=1.96 \mathrm{TeV} \ll, \mathrm{D} \varnothing$ Note 4689 (2005); F. Déliot, G. Hesketh, P. Telford, B. Tuchming, »Measurement of $\sigma(p \bar{p} \rightarrow W X) \times B r(W \rightarrow \mu \nu)$ at $\sqrt{s}=1.96 \mathrm{TeV}$ ", DØ Note 4749 (2005); The DØ Collaboration, »Measurement of the Cross-section for Inclusive $W$ Production in the Muon Channel at $\sqrt{s}=1.96 \mathrm{TeV}$ Using the DØ Detector «, DØ Note 4750 (2005).

[175] G.Cowan, »Statistical Data Analysis«, Oxford University Press, Oxford, New York (1998); H. Tijms, »Understanding Probability, Chance Rules in Everyday Life «, Vrije Universiteit, Amsterdam, ISBN-10: 0521540364, The Netherlands (2004).

[176] R.J. Barlow, »Statistics, a guide to the use of statistical methods in the physical sciences «, John Wiley \& Sons, New York, U.S.A. (1997); R.J. Barlow, »Lecture 8: Signals, Backgrounds and Probabilities «, Lecture held at the SLAC Users Organisation: Lectures on Statistics and Numerical Methods in HEP, SLAC, Stanford, California, U.S.A. (2000);

[177] J. Neyman, Phil. Trans. Royal Soc. London, Series A 236 (1937), 333; Reprinted in A Selection of Early Statistical Papers on J. Neyman, University of California Press, Berkeley, U.S.A. 1967.

[178] T. Junk, »Confidence Level Computation for Combining Searches with Small Statistics «, Nucl. Instrum. Methods A 434 (1999), 435; [arXiv:hep-ex/9902006] (2000); A. L. Read, »Modified Frequentist Analysis of Search Result (the $C L_{S}$ method) «, Proceedings of the Workshop on Confidence Limits, CERN Yellow Report 2000-005, Eds. F James, L. Lyons and Y. Perrin; A. L. Read, »Presentation of Search Results: the $C L_{S}$-technique «; stacks.iop.org/JPhysG/28/2693 
[179] C ++ class TLimit from ROOT for limit computation, code adapted from the mclimit code from T. Junk; further details in [122].

[180] The DØ Collaboration (V.M. Abazov et al.), »Search for R-parity violating supersymmetry via the $L L \bar{E}$ couplings $\lambda_{121}, \lambda_{122}$ or $\lambda_{133}$ in pp collisions at $\sqrt{(s)}=1.96 \mathrm{TeV}$ ", To be published in Phys. Lett. B (2006); FERMILAB-PUB-06-089-E; [arXiv:hep-ex/0605005].

[181] The LEP2 SUSY Working Group (M. Berggren et al.), »Combined LEP Selectron, Smuon, and Stau Results $183-208 \mathrm{GeV}$ « http://lepsusy.web.cern.ch/lepsusy/www/sleptons_summer04/slep_final.html;

The ALEPH Collaboration (D. Buskulic et al.), »Search for Scalar Leptons in $e^{+} e^{-}$Collisions at Centre-of-mass Energies up to $209 \mathrm{GeV}$ ", Phys. Lett. B 526 (2002), 206; »Absolute Mass Lower Limit for the Lightest Neutralino of the MSSM from $e^{+} e^{-}$Data at $\sqrt{s}$ up to 209 GeV«, Phys. Lett. B 583 (2004), 247;

The DELPHI Collaboration (P. Abreu et al.), »Searches for Supersymmetric Particles in $e^{+} e^{-}$Collisions up to $208 \mathrm{GeV}$ and Interpretation of the Results within the MSSM «, Eur. Phys. J.C 31 (2003), 421; The L3 Collaboration (P. Achard et al.), »Search for Scalar Quarks and Scalar Leptons at LEP «, Phys. Lett. B 580 (2004), 37; The OPAL Collaboration (G. Abbiendi et al.), »Search for Anomalous Production of Dilepton Events with Missing Transverse Momentum in $e^{+} e^{-}$Collisions at $\sqrt{s}=183 \mathrm{GeV}$ to $209 \mathrm{GeV}$, Eur. Phys. J.C 32 (2004), 453. 


\section{Acknowledgements}

On these last pages I would like to thank the numerous people, who have helped and guided me during these last four years of my Ph.D. studies. This work would not have been possible without your advice and continuous support.

First of all, I am very grateful to my supervisor, Prof. Dr. Thomas Hebbeker for providing me with the opportunity to spent one year at the actual location of the DØ experiment, at Fermilab near Chicago. Besides being a great experience on its own, I have profited a lot from many people there, especially the members of the New Phenomena Group. Thank you very much for giving me freedom and at the same time being there and encouraging me whenever it was necessary and... for always asking the right questions! Many thanks also to Prof. Dr. Martin Erdmann for immediately agreeing to be my second referee.

At the beginning of my studies, Dr. Serge Sushkov, who had just returned from Fermilab, helped me in taking the first steps and getting a little familiar with the $\mathrm{D} \varnothing$ data formats and parts of the $\mathrm{D} \varnothing$ reconstruction software. Later on, when I stayed at Fermilab, Dr. Martin Wegner, was always there to answer my countless questions. Many thanks for your advice and for simply being there, when I was feeling down or overwhelmed by stuff I didn't really understand.

During this time, I also enjoyed the good company and support of many people from our German Ghetto. First and foremost, I'd like to thank Dr. Ulla Blumenschein, my climbing and »12-days-through-the-Wild-West « holiday companion. A big thank you for this nice adventure and for just listening to me during a difficult time! Many thanks also to Dr. Tobias Golling (for organising some non-physics events), Dr. Meta Binder, Dr. Marc Hohlfeld, Dr. Johannes Elmsheuser, Dr. Markus Klute, Dr. Carsten Nöding, and Ingo Torchiani (especially for the spontaneous trip to the Florida's east coast beach in April 2005).

For useful physics advice, their continuous encouragement, and many fruitul discussions, I am grateful to the conveners of the New Phenomena Group: Dr. Volker Büscher, Dr. Jean-François Grivaz, and Dr. Arnd Meyer.

To Arnd, I'm also indepted for his continuous support and help with numerous other things. Thank you for producing quite a number of signal Monte Carlo samples, for explaining various hard- and software parts of the experiment, for saving my data from navala, for proof-reading nearly all my talks, notes and especially this Ph.D. thesis, for constructive critics, brilliant practical ideas, and perspicacious answers to almost any question I had. The biggest thank you, however, is for simply listening to me, whenever I needed someone to talk to, and for your endless patience. - Thank you very much!

Special thanks to all players of the winter and summer volleyball leagues at Fermilab: especially to Elizabeth, Kurt, Robin, Miro, Rick, Andrea, Mark, Licia, Adam, and Peter. I very much enjoyed playing with you. The short Chicago intermezzo was also nice, although the 1.5 hour drive on Sunday mornings was (and is) not exactly my favourite activity for this time of day. 
Many thanks also to my landlady, Evelyn Aponte, for the wonderful one-year-stay in Aurora, in a big town-home, with all comfort I could have wished for. I guess the billard table was of particular interest to most guests on whatever occasion, be it thanksgiving with a huuuge turkey, various movie nights or simply birthday parties. Good luck to you and, especially to Elise in the coming years! Thanks also for the good company to my roommates, Christina Galea and, later on, Jim Krauss.

Back in Aachen, the world got a little smaller again, but still the working atmosphere was great, thanks to the entire DØ Aachen group: Christian, Thomas, Oliver, Lotte, Philipp, Carsten, Jan, Volker, Daniel, Jens, Matthias, and Dr. Steffen Kappler. Special thanks to Christian for some enlightening discussions about various physics questions, in particular concerning $\not \mathbb{R}_{p}$-SUSY.

Our system administrator, Dr. Thomas Kress, is also not forgotten, since his quick responses to any problem were indispensable and ensured optimal working conditions. Even my private notebook got some polishing from the MaTas Mira and Fabian.

A big thank you goes to Carsten Hof for being a venturesome climbing partner in- and outdoors, for the $9 \mathrm{pm}$ coffee breaks, and for sharing Pizza or other unhealthy food with me during some long office night... Thanks to Michael for the fridge and to both of you for the usually open office and many intersting conversations about life in- and outside physics.

Muchísimas gracias a la vieja guardia del Club Latino: a Vera y Daniel, Alicia y Hans, Eduardo, Félix, y a todos los demás, por su amistad, por las fiestas, por una cultura differente y por las conexiones con un montón de sitios exóticos. Mil gracias también a Anke, a Claudia y a Katia por no olvidarse nunca de mí, sin importarles donde estuviéramos o la distancia que nos separase. También muchas gracias a Bea, por mandarme los mejores chistes y caricaturas - me hiciste reir un buen rato.

During the last one-and-a-half years a close and fruitful collaboration developed with two french colleagues, Dr. Anne-Marie Magnan and Dr. Anne-Catherine Le-Bihan. Many thanks to both of you, ladies, for the numerous long email discussions, where I usually learned a lot. (My email folders say that we exchanged over 1200(!) emails, not counting those from the EB and PLB reviews...) Even though we all had our hard times, including being blockheads sometimes, I very much enjoyed working with you - it has been a great experience!

I also like to extend very special thanks to Prof. Dr. Gérard Sajot, who shared the subtleties of $\not R_{p}$-SUSY with the three of us and offered help in many ways, from Monte Carlo production, over the calculation of most of the GaUginos $K$-factors for all our signal MCs, to the point of writing the first publication draft. Although nearly every sentence got changed about three times before the end, it was very helpful to have this early draft.

I am grateful to the Deutsche Forschungsgemeinschaft (DFG) for the financial support during the first three years of my studies. During this time a had been granted a scholarship within the graduate college »Elementary Particle Physics at the TeV Scale« that started at Aachen Technical University in October 2001.

Many thanks, muchas gracias und Dankeschön to all those, whom I did not mention explicitly here. I have not forgotten you - thanks for your friendship and understanding, even though I have hardly been there and never had much time...

For proof-reading, I thank again Dr. Arnd Meyer (as always), and Herbert Domhöver for carefully reviewing the English grammar, syntax and orthography. Special thanks go to Prof. Dr. Herbi Dreiner for explaining $\not R_{p^{-}}$SUSY in a way, that I could get the main concepts and for proof-reading my theory chapter. 
Zuletzt möchte ich mich ganz herzlich bei meiner Familie bedanken: bei meiner Schwester und René für diverse Besuche und Gegenbesuche, Ausflüge zum Weihnachtsmarkt, selbstgebastelte Adventskalender und die dichterische Unterstützung. Insbesondere aber danke ich meinen Eltern, die mich stets unterstützt haben und mich meinen Weg gehen liessen, selbst ohne zu wissen, womit ich gerade beschäftigt war. Danke für so manch' seeehr spontane Fahrt nach Aachen, für die stets offene Tür, (bzw. den entsprechenden Schlüssel), vor allem aber danke für Eure Liebe und Euer Vertrauen. - DANKE für alles!

According to » The Hitchhiker's Guide to the Galaxy«, the ultimate answer to life, the universe, and everything! 



\section{DANIELA KÄFER}

Curriculum Vitae

\section{Personal DATA}

Name:

Daniela Käfer

Address:

Püngelerstr. 2d, D-52074 Aachen

Email:

daniela.kaefer@physik.rwth-aachen.de

kaefer@fnal.gov

Date of Birth:

Place of Birth:

March 23, 1976

Nationality:

Aachen

Marital status:

German

single, no children

\section{$\underline{\text { SCHOOL EDUCATION }}$}

Aug. 1982 - Aug. 1986

Catholic Primary School in Kalterherberg

Aug. 1986 - May 1995

St. Michael-Gymnasium in Monschau

May 1995

Abitur

\section{UNIVERSITY EDUCATION}

Oct. 1995 - Mar. 2002

Study of physics at the Rheinisch-Westfälische Technische Hochschule (RWTH) Aachen, Germany

Mar. 1998

Diplom-Vorprüfung

Oct. 1998 - Jun. 1999

Study of physics at the Universidad Autónoma de Madrid (UAM), Madrid, Spain (Erasmus Fellowship)

May 2001 - Aug. 2001

Summer student at the European Center for Nuclear

Research (CERN), Switzerland

Mar. 2001 - Feb. 2002

Diploma Thesis at the RWTH Aachen

"Nachweis einzelner W-Bosonen mit dem L3-Detektor bei LEP im Energiebereich von $192 \mathrm{GeV}$ bis $208 \mathrm{GeV}$ " 1

Thesis Advisor: Prof. Dr. A. Böhm, RWTH

Second Referee: Prof. Dr. J. Mnich, DESY, (RWTH)

Mar. 2002

Undergraduate Degree: Diplom-Physikerin

Apr. 2002 - Apr. 2005

Scholarship of the DFG within the Graduate Seminar

"Elementarteilchenphysik an der TeV-Skala" at Aachen

Technical University (RWTH)

\footnotetext{
${ }^{1}$ http://www .physik.rwth-aachen.de/group/IIIphys/L3/diplom.html
} 
Sep. 2002

Oct. 2002 - Oct. 2003

Sep. 2004

Apr. 2002 - Sep. 2006

Oct. 2006
Annual graduate Seminar in Bad Honnef and "Joint Belgian-Dutch-German Summer School"

Research stay as a guest scientist at the Fermi National Accelerator Laboratory (FNAL), Chicago, U.S.A.

Work areas: monitoring software for the $\mathrm{D} \varnothing$ muon system; shifts: Muon, Data Aquisition (DAQ), and Calorimeter-Muon; Measurements of noise and leakage currents at silicon modules for the Tevatron Run IIb; Data analysis

Annual graduate Seminar in Bad Honnef and HEP school Herbstschule für Hochenergiephysik in Maria Laach

Dissertation

"Search for R-parity Violating Supersymmetry in Multilepton Final States with the DØ Detector"

Thesis Advisor: Prof. Dr. T. Hebbeker, RWTH

Second Referee: Prof. Dr. M. Erdmann, RWTH

Graduate Degree: Dr. rer. nat., RWTH Aachen

\section{LANGUAGES}

English

Spanish

French

German fluent in spoken and written

fluent in spoken; good in written

basic knowledge

native language

\section{TEACHING EXPERIENCE}

Oct. 1999 - Mar. 2001

Oct. 2003 - Sep. 2006 student assistant for lectures in Experimental Physics I-III responsible for the laboratory course experiment:

Mößbauer spectroscopy 


\section{Selbständigkeits-Erklärung}

Hiermit erkläre ich, dass ich die vorliegende Dissertation selbständig erarbeitet und ausschließlich die aufgeführten Referenzen verwendet habe. Alle Quellen und Zitate sind als solche kenntlich gemacht und die Literaturangaben sind nach meinem besten Wissen und Gewissen korrekt und vollständig wiedergegeben. 
